\title{
Visible-light-induced Dearomatization via [2+2] \\ Cycloaddition or 1,5-Hydrogen Atom Transfer: Divergent Reaction Pathways of Transient Diradicals
}

\author{
Min Zhu, ${ }^{\dagger},+$ Xiao Zhang, ${ }^{*}, \dagger, \S$ Chao Zheng, ${ }^{*, \dagger}$ and Shu-Li You*, ${ }^{* \dagger, \dagger}$ \\ ${ }^{\dagger}$ State Key Laboratory of Organometallic Chemistry, Center for Excellence in \\ Molecular Synthesis, Shanghai Institute of Organic Chemistry, University of Chinese \\ Academy of Sciences, Chinese Academy of Sciences, 345 Lingling Lu, Shanghai \\ 200032, China \\ $\star$ School of Physical Science and Technology, ShanghaiTech University, 100 Haike \\ Road, Shanghai 201210, China \\ ${ }^{\S}$ Fujian Key Laboratory of Polymer Science, Fujian Provincial Key Laboratory of \\ Advanced Materials Oriented Chemical Engineering, College of Chemistry and \\ Materials Science, Fujian Normal University, 32 Shangsan Lu, Fuzhou 350007, China \\ *Corresponding authors: zhangxiao@sioc.ac.cn, zhengchao@sioc.ac.cn, \\ slyou@sioc.ac.cn
}

\section{Table of Contents}

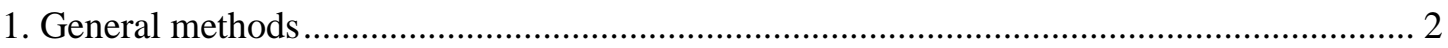

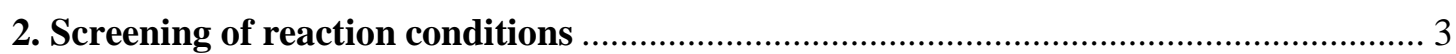

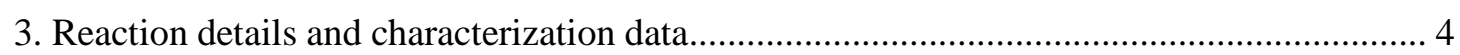

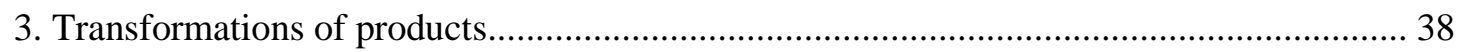

4. Experimental details for mechanistic studies ................................................................. 41

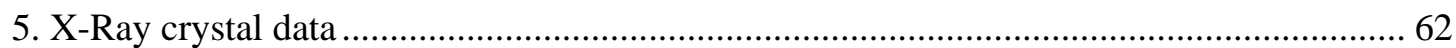

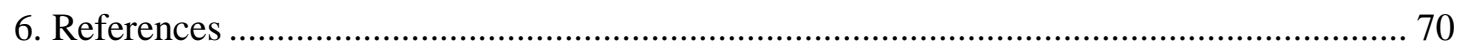

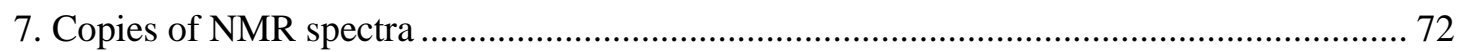




\section{General methods.}

Unless stated otherwise, all reactions were carried out in flame-dried glassware under a dry argon atmosphere. All solvents were purified and dried according to standard methods prior to use.

${ }^{1} \mathrm{H}$ and ${ }^{13} \mathrm{C}$ NMR spectra were recorded on an Agilent instrument $(400 \mathrm{MHz}$ and 100 $\mathrm{MHz}$, respectively) or an Agilent instrument (600 MHz and $150 \mathrm{MHz}$, respectively) and internally referenced to tetramethylsilane signal or residual protio solvent signals. ${ }^{19} \mathrm{~F}$ NMR spectra were recorded on an Agilent instrument $(376 \mathrm{MHz})$ and referenced relative to $\mathrm{CFCl}_{3}$. Data for ${ }^{1} \mathrm{H}$ NMR are recorded as follows: chemical shift $(\delta, \mathrm{ppm})$, multiplicity $(\mathrm{s}=$ singlet, $\mathrm{d}=$ doublet, $\mathrm{t}=$ triplet, $\mathrm{m}=$ multiplet or unresolved, $\mathrm{br}=$ broad singlet, coupling constant(s) in $\mathrm{Hz}$, integration). Data for ${ }^{13} \mathrm{C} N M R$ are reported in terms of chemical shift $(\delta, \mathrm{ppm})$.

Substrates 1, 3 were synthesized according to the literature procedures. ${ }^{1}$ Solvents are commercially available from Alfa Aesar, and were used without further purification. 


\section{Screening of reaction conditions}<smiles>CCOC(=O)c1cc2ccccc2n1C(=O)CC/C=N/OC</smiles>

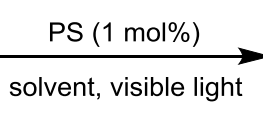

solvent, visible light

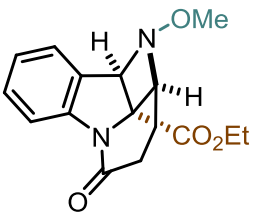

$2 a$

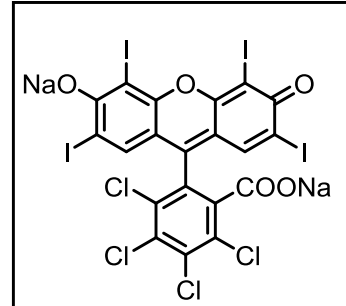

Rose Bengal (I) $\mathrm{E}_{1 / 2}\left(\mathrm{RB}^{*} / \mathrm{RB}^{-}\right)=+0.99 \mathrm{~V}$ $\mathrm{E}_{1 / 2}\left(\mathrm{RB}^{+} / \mathrm{RB}^{*}\right)=-0.68 \mathrm{~V}$ $\Delta G\left(\mathrm{~T}_{1}-\mathrm{S}_{0}\right)=40.9 \mathrm{kcal} / \mathrm{mol}$

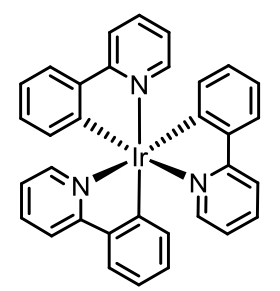

$$
\operatorname{Ir}(\text { ppy })_{3}(\mathbf{I V})
$$

$\mathrm{E}_{1 / 2}\left(\mathrm{Ir}^{\mathrm{III}} / \mathrm{Ir}^{\mathrm{II}}\right)=+0.31 \mathrm{~V}$ $\mathrm{E}_{1 / 2}\left(\mathrm{Ir}^{\mathrm{IV}} / \mathrm{Ir}^{\mathrm{III}}\right)=-1.73 \mathrm{~V}$ $\Delta G\left(\mathrm{~T}_{1}-\mathrm{S}_{0}\right)=57.8 \mathrm{kcal} / \mathrm{mol}$

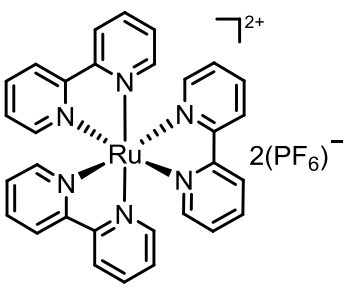

$\mathrm{Ru}(\mathrm{bpy})_{3}\left(\mathrm{PF}_{6}\right)_{2}$ (II) $\mathrm{E}_{1 / 2}\left(\mathrm{Ru}^{\mathrm{II}} / \mathrm{Ru}^{\mathrm{I}}\right)=+0.77 \mathrm{~V}$ $\mathrm{E}_{1 / 2}\left(\mathrm{Ru}^{\mathrm{III}} / \mathrm{Ru}^{\mathrm{II}}\right)=-0.81 \mathrm{~V}$ $\Delta G\left(\mathrm{~T}_{1}-\mathrm{S}_{0}\right)=46.5 \mathrm{kcal} / \mathrm{mol}$

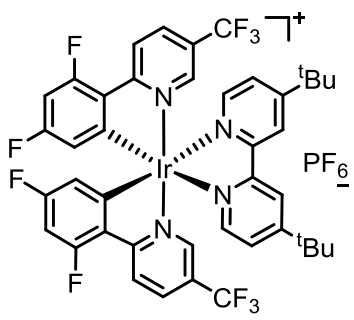

$\operatorname{Ir}\left(\mathrm{dFCF}_{3} \text { ppy }\right)_{2}(\mathrm{dtbbpy}) \mathrm{PF}_{6}(\mathbf{V})$ $\mathrm{E}_{1 / 2}\left(\mathrm{Ir}^{\mathrm{III}} / \mathrm{Ir}^{\mathrm{II}}\right)=+1.21 \mathrm{~V}$ $\mathrm{E}_{1 / 2}\left(\mathrm{Ir}^{\mathrm{IV}} / \mathrm{Ir}^{\mathrm{III}}\right)=-0.89 \mathrm{~V}$ $\Delta G\left(\mathrm{~T}_{1}-\mathrm{S}_{0}\right)=60.8 \mathrm{kcal} / \mathrm{mol}$

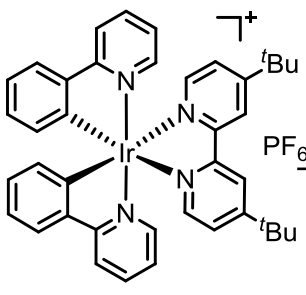

$\operatorname{Ir}(\mathrm{ppy})_{2}(\mathrm{dtbbpy}) \mathrm{PF}_{6}$ (III) $\mathrm{E}_{1 / 2}\left(\mathrm{Ir}^{\mathrm{III}} / \mathrm{Ir}^{\mathrm{II}}\right)=+0.66 \mathrm{~V}$ $\mathrm{E}_{1 / 2}\left(\mathrm{Ir}^{\mathrm{IV}} / \mathrm{Ir}^{\mathrm{III}}\right)=-0.96 \mathrm{~V}$ $\Delta G\left(\mathrm{~T}_{1}-\mathrm{S}_{0}\right)=49.2 \mathrm{kcal} / \mathrm{mol}$

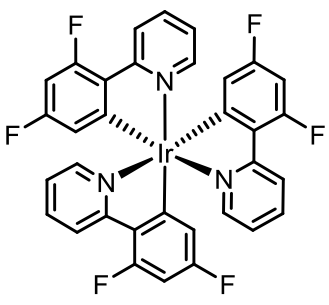

$\operatorname{Ir}(\mathrm{dFppy})_{3}(\mathbf{V I})$ $\mathrm{E}_{1 / 2}\left(\mathrm{Ir}^{\mathrm{III}} / \mathrm{Ir}^{\mathrm{II}}\right)=+0.43 \mathrm{~V}$ $\mathrm{E}_{1 / 2}\left(\mathrm{Ir}^{\mathrm{IV}} / \mathrm{Ir}^{\mathrm{III}}\right)=-1.39 \mathrm{~V}$ $\Delta G\left(\mathrm{~T}_{1}-\mathrm{S}_{0}\right)=63.5 \mathrm{kcal} / \mathrm{mol}$

\begin{tabular}{ccccc}
\hline entry & PS & solvent & time (h) & yield (\%) $^{\boldsymbol{b}}$ \\
\hline 1 & VI & MeOH & 3 & 9 \\
2 & VI & DCM & 3 & 95 \\
3 & VI & acetone & 3 & 90 \\
5 & VI & DMF & 3 & 85 \\
6 & VI & $\mathrm{CH}_{3} \mathrm{CN}$ & 3 & 12 \\
7 & VI & DMSO & 3 & 92 \\
8 & I & DCM & 5 & 0 \\
9 & II & DCM & 5 & 0 \\
10 & III & DCM & 5 & trace \\
11 & IV & DCM & 5 & 12 \\
12 & V & DCM & 3 & 94 \\
$\mathbf{1 3}^{c}$ & VI & DCM & $\mathbf{3}$ & quant. (98) \\
$14^{d}$ & - & DCM & 24 & 0 \\
$15^{e}$ & VI & DCM & 24 & 0 \\
\hline
\end{tabular}

${ }^{a}$ Reaction conditions: a solution of $1 \mathrm{a}(0.1 \mathrm{mmol})$ and PS $(1 \mathrm{~mol} \%)$ in solvent $(c=0.1 \mathrm{M})$ was irradiated by $24 \mathrm{~W}$ blue LEDs $(450 \mathrm{~nm})$ at room temperature under argon. PS = photosensitizer. ${ }^{b} \mathrm{NMR}$ yield with $\mathrm{CH}_{2} \mathrm{Br}_{2}$ as the internal standard. ${ }^{c} c=0.01 \mathrm{M}$. ${ }^{d}$ Absence of a photosensitizer. ${ }^{e}$ In dark. 


\section{Reaction details and characterization data}

\section{General procedure for the synthesis of the precursors for substrates ${ }^{1}$}

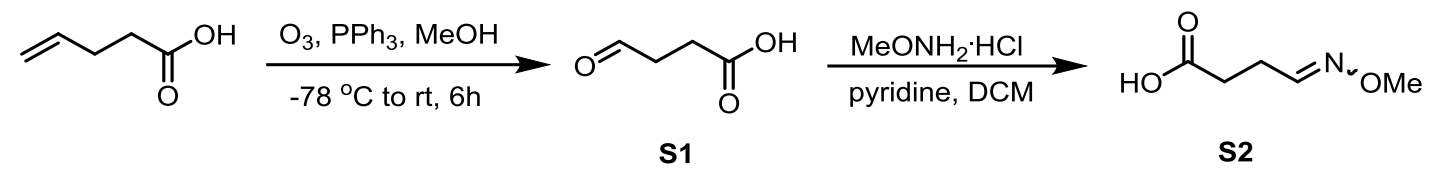

According to the previous report, ${ }^{1}$ corresponding carboxylic acids were prepared. A solution of allylacetic acid $(3.0 \mathrm{~g}, 30 \mathrm{mmol})$ in $\mathrm{MeOH}(30 \mathrm{~mL})$ was cooled to $-78{ }^{\circ} \mathrm{C}$. Ozone was bubbled through the solution until blue color persisted. When TLC indicated the disappearance of the starting material, the ozone inlet tube was replaced with nitrogen, which was bubbled through the solution for $30 \mathrm{~min}$ to remove any excess ozone. Triphenylphosphine $(16.0 \mathrm{~g}, 60 \mathrm{mmol})$ was added in one portion, the cooling bath was removed, and the mixture was stirred for $6 \mathrm{~h}$ at room temperature. Then the reaction mixture was concentrated under reduced pressure to afford $\mathbf{S 1}$ as a colorless oil, which was used in the subsequent step without further purification.

To a solution of $\mathbf{S 1}$ (above crude mixture) in DCM $(50 \mathrm{~mL})$ was added with methoxylamine hydrochloride $(5.0 \mathrm{~g}, 60 \mathrm{mmol})$. Then pyridine $(5.3 \mathrm{~mL}, 66 \mathrm{mmol})$ was added slowly with stirring at room temperature for 8 hours. After the reaction was complete, $\mathrm{NaOH}$ solution was then added to adjust $\mathrm{pH}>11$. Then the solution was extracted with DCM $(30 \mathrm{~mL} \times 3)$. The aqueous phase was acidified with $\mathrm{HCl}(2 \mathrm{~N})$ to adjust $\mathrm{pH}<2$. The aqueous solution was then extracted with EtOAc $(30 \mathrm{~mL} \times 3)$. The combined organic layers were washed with brine, dried over $\mathrm{Na}_{2} \mathrm{SO}_{4}$, filtered and concentrated by rotary evaporation to afford $\mathbf{S 2}$ as a colorless oil, which was used in the subsequent step without further purification.

\section{General procedure for the synthesis of substrates ${ }^{2}$}




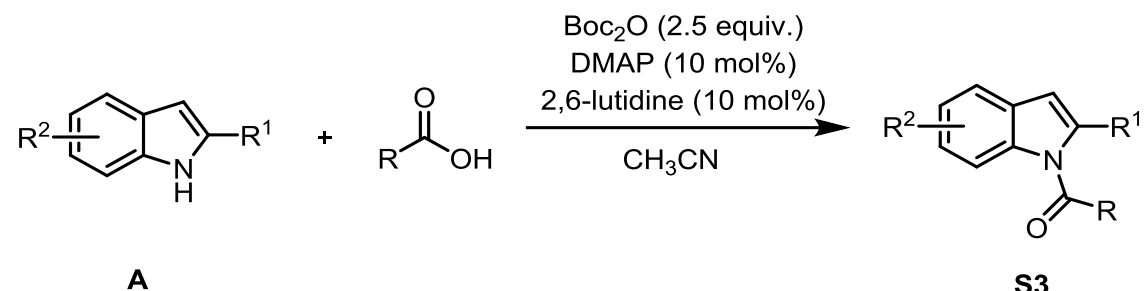

In a round bottom flask, $\mathbf{A}(20 \mathrm{mmol})$ was dissolved into reagent grade $\mathrm{CH}_{3} \mathrm{CN}$ (30.0 $\mathrm{mL}$ ). After the addition of substituted carboxylic acid (10 mmol), DMAP (122 mg, 1.0 mmol), 2,6-lutidine (0.12 mL, $1.0 \mathrm{mmol})$ and $\mathrm{Boc}_{2} \mathrm{O}(5.5 \mathrm{~mL}, 25.0 \mathrm{mmol})$ were added in sequence. After the reaction was complete (monitored by TLC), it was quenched with water. The aqueous layer was extracted with EtOAc $(30 \mathrm{~mL} \times 3)$. The combined organic layers were washed with brine, dried over $\mathrm{Na}_{2} \mathrm{SO}_{4}$, filtered and concentrated by rotary evaporation. Then the residue was purified by silica gel column chromatography $(\mathrm{PE} / \mathrm{EA}=40 / 1)$ to afford the desired product S3. The analytical data of the products are summarized below, and the data of minor isomers are indicated by an asterisk (*).

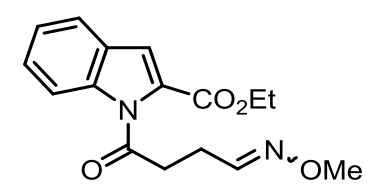

1a, yellow oil, $0.68 \mathrm{~g}, 57 \%$ yield (4 mmol scale), $E / Z=1.7 / 1 .{ }^{1} \mathrm{H}$ NMR (400 MHz, $\left.\mathrm{CDCl}_{3}\right) \delta 8.08-8.04(\mathrm{~m}, 1 \mathrm{H}), 7.61(\mathrm{~d}, J=8.0 \mathrm{~Hz}, 1 \mathrm{H}), 7.45-7.39(\mathrm{~m}, 2 \mathrm{H}), 7.35(\mathrm{~s}, 1 \mathrm{H})$, 7.30-7.25 (m, 1H), $6.75\left(\mathrm{t}, J=5.6 \mathrm{~Hz}, 1 \mathrm{H}^{*}\right), 4.40(\mathrm{q}, J=7.2 \mathrm{~Hz}, 2 \mathrm{H}), 3.84\left(\mathrm{~s}, 3 \mathrm{H}^{*}\right)$, $3.48(\mathrm{~s}, 3 \mathrm{H}), 3.09(\mathrm{t}, J=7.2 \mathrm{~Hz}, 2 \mathrm{H}), 2.79-2.67(\mathrm{~m}, 2 \mathrm{H}), 1.41(\mathrm{t}, J=7.2 \mathrm{~Hz}, 3 \mathrm{H}) .{ }^{13} \mathrm{C}$ NMR $\left(100 \mathrm{MHz}, \mathrm{CDCl}_{3}\right) \delta 173.5,173.2,161.7,149.1,147.9,138.6,138.3,129.3,127.9$, 127.8, 127.1, 127.0, 123.7, 123.6, 122.4, 122.3, 118.2, 118.1, 115.0, 114.8, 61.7, 61.63, $61.59,61.0,35.9,35.7,25.8,21.6,14.2$. IR (thin film): $v_{\max }\left(\mathrm{cm}^{-1}\right)=2980,2937,1709$, 1607, 1534, 1473, 1442, 1375, 1329, 1279, 1256, 1196, 1166, 1036, 1015, 840, 745, 656, 608, 570, 547, 440. HRMS (ESI) calcd for $\mathrm{C}_{16} \mathrm{H}_{18} \mathrm{~N}_{2} \mathrm{NaO}_{4}[\mathrm{M}+\mathrm{Na}]^{+}: 325.1159$. Found: 325.1154.

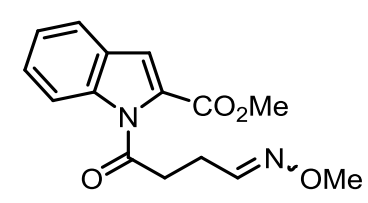


1b, yellow oil, $1.00 \mathrm{~g}, 70 \%$ yield (5 mmol scale), $E / Z=1.6 / 1 .{ }^{1} \mathrm{H}$ NMR $(400 \mathrm{MHz}$, $\left.\mathrm{CDCl}_{3}\right) \delta 8.07-8.03(\mathrm{~m}, 1 \mathrm{H}), 7.61(\mathrm{~d}, J=8.0 \mathrm{~Hz}, 1 \mathrm{H}), 7.45-7.39(\mathrm{~m}, 2 \mathrm{H}), 7.34(\mathrm{~s}, 1 \mathrm{H})$, 7.30-7.25 (m, 1H), $6.76\left(\mathrm{t}, J=5.6 \mathrm{~Hz}, 1 \mathrm{H}^{*}\right), 3.93$ (s, 3H), 3.84 (s, 3H*), 3.48 (s, 3H), $3.07(\mathrm{t}, J=6.8 \mathrm{~Hz}, 2 \mathrm{H}), 2.79-2.67(\mathrm{~m}, 2 \mathrm{H}) .{ }^{13} \mathrm{C} \mathrm{NMR}\left(100 \mathrm{MHz}, \mathrm{CDCl}_{3}\right) \delta 173.5,173.1$, 162.0, 149.1, 147.9, 138.6, 138.3, 128.8, 127.93, 127.86, 127.0, 126.9, 123.7, 123.6, $122.5,122.3,118.4,118.3,114.9,114.8,61.6,61.0,52.5,52.4,35.9,35.7,25.8,21.6$. IR (thin film): $v_{\max }\left(\mathrm{cm}^{-1}\right)=2951,1711,1606,1533,1474,1435,1374,1334,1256$, 1203, 1166, 1146, 1111, 1044, 946, 837, 745, 665, 608, 570, 440. HRMS (ESI) calcd for $\mathrm{C}_{15} \mathrm{H}_{16} \mathrm{~N}_{2} \mathrm{NaO}_{4}[\mathrm{M}+\mathrm{Na}]^{+}:$311.1002. Found: 311.1001 .

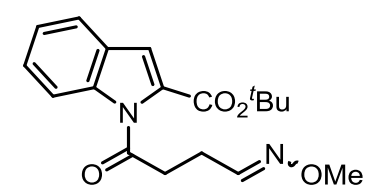

1c, colorless oil, $0.30 \mathrm{~g}, 42 \%$ yield ( $2 \mathrm{mmol}$ scale), $E / Z=1.6 / 1 .{ }^{1} \mathrm{H}$ NMR $(400 \mathrm{MHz}$, $\left.\mathrm{CDCl}_{3}\right) \delta 8.06(\mathrm{t}, J=8.8 \mathrm{~Hz}, 1 \mathrm{H}), 7.59(\mathrm{~d}, J=6.8 \mathrm{~Hz}, 1 \mathrm{H}), 7.40(\mathrm{~s}, 2 \mathrm{H}), 7.25(\mathrm{~s}, 2 \mathrm{H})$, $6.75\left(\mathrm{t}, J=4.8 \mathrm{~Hz}, 1 \mathrm{H}^{*}\right), 3.84\left(\mathrm{~s}, 3 \mathrm{H}^{*}\right), 3.49$ (s, 3H), 3.09 (d, $\left.J=6.0 \mathrm{~Hz}, 2 \mathrm{H}\right), 2.77-$ $2.69(\mathrm{~m}, 2 \mathrm{H}), 1.61(\mathrm{~s}, 9 \mathrm{H}) .{ }^{13} \mathrm{C} \mathrm{NMR}\left(100 \mathrm{MHz}, \mathrm{CDCl}_{3}\right) \delta 173.5,173.1,161.0,149.1$, $147.9,138.4,138.1,130.8,127.51,127.46,127.1,127.0,123.6,123.4,122.2$, 122.1, $117.41,117.36,114.9,114.8,82.7,82.6,61.6,61.0,35.9,35.7,28.03,28.00,25.8,21.6$. IR (thin film): $v_{\max }\left(\mathrm{cm}^{-1}\right)=2975,2940,1698,1533,1474,1441,1368,1320,1254$, 1217, 1158, 1142, 1092, 1045, 884, 867, 840, 780, 756, 743, 563. HRMS (ESI) calcd for $\mathrm{C}_{18} \mathrm{H}_{22} \mathrm{~N}_{2} \mathrm{NaO}_{4}[\mathrm{M}+\mathrm{Na}]^{+}:$353.1472. Found: 353.1469 .

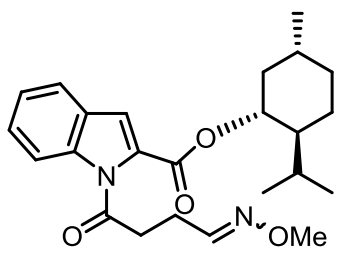

1d, brown oil, $0.86 \mathrm{~g}$, 56\% yield (4 mmol scale), $E / Z=1.6 / 1 .{ }^{1} \mathrm{H}$ NMR (400 MHz, $\left.\mathrm{CDCl}_{3}\right) \delta 8.09(\mathrm{~d}, J=8.4 \mathrm{~Hz}, 1 \mathrm{H}), 7.61(\mathrm{~d}, J=7.6 \mathrm{~Hz}, 1 \mathrm{H}), 7.44-7.40(\mathrm{~m}, 2 \mathrm{H}), 7.32-$ $7.24(\mathrm{~m}, 2 \mathrm{H}), 6.74\left(\mathrm{t}, J=5.2 \mathrm{~Hz}, 1 \mathrm{H}^{*}\right), 4.95(\mathrm{t}, J=10.0 \mathrm{~Hz}, 1 \mathrm{H}), 3.84\left(\mathrm{~s}, 3 \mathrm{H}^{*}\right), 3.51$ (s, 3H), 3.15-3.02 (m, 2H), 2.79-2.68 (m, 2H), 2.12 (d, $J=8.8 \mathrm{~Hz}, 1 \mathrm{H}), 1.98$ (d, $J=6.4$ $\mathrm{Hz}, 1 \mathrm{H}), 1.74(\mathrm{~d}, J=11.2 \mathrm{~Hz}, 2 \mathrm{H}), 1.56$ (t, $J=10.4 \mathrm{~Hz}, 2 \mathrm{H}), 1.21-1.08$ (m, 2H), 0.96$0.93(\mathrm{~m}, 7 \mathrm{H}), 0.82(\mathrm{~d}, J=6.8 \mathrm{~Hz}, 3 \mathrm{H}) .{ }^{13} \mathrm{C} \mathrm{NMR}\left(100 \mathrm{MHz}, \mathrm{CDCl}_{3}\right) \delta$ 173.5, 173.1, 
161.33, 161.29, 149.1, 147.9, 138.6, 138.4, 129.61, 129.57, 127.8, 127.7, 127.0, 126.9, $123.7,123.5,122.3,122.2,117.8,117.6,115.0,75.63$, 75.57, 61.6, 61.0, 47.1, 40.7, 36.0, 35.7, 34.1, 31.4, 26.4, 25.8, 23.3, 22.0, 21.6, 20.7, 16.2. IR (thin film): $v_{\max }\left(\mathrm{cm}^{-1}\right)$ =2955, 2869, 1707, 1536, 1443, 1379, 1280, 1257, 1201, 1166, 1145, 1096, 1047, 1007, 980, 954, 912, 882, 862, 838, 737, 703. HRMS (ESI) calcd for $\mathrm{C}_{24} \mathrm{H}_{32} \mathrm{~N} 2 \mathrm{NaO} 4$ $[\mathrm{M}+\mathrm{Na}]^{+}:$435.2254. Found: 435.2249.

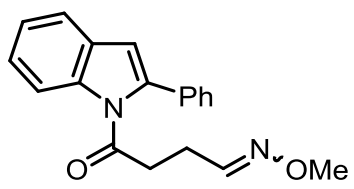

1e, colorless oil, $0.21 \mathrm{~g}, 17 \%$ yield (4 mmol scale), $E / Z=1.4 / 1 .{ }^{1} \mathrm{H}$ NMR (400 MHz, $\left.\mathrm{CDCl}_{3}\right) \delta 8.32-8.29(\mathrm{~m}, 1 \mathrm{H}), 7.55(\mathrm{~d}, J=7.2 \mathrm{~Hz}, 1 \mathrm{H}), 7.45-7.43(\mathrm{~m}, 5 \mathrm{H}), 7.36-7.23(\mathrm{~m}$, $3 \mathrm{H}), 6.62(\mathrm{~s}, 1 \mathrm{H}), 6.50\left(\mathrm{t}, J=5.2 \mathrm{~Hz}, 1 \mathrm{H}^{*}\right), 3.75\left(\mathrm{~s}, 3 \mathrm{H}^{*}\right), 3.56(\mathrm{~s}, 3 \mathrm{H}), 2.51-2.44(\mathrm{~m}$, 4H). ${ }^{13} \mathrm{C} \mathrm{NMR}\left(100 \mathrm{MHz}, \mathrm{CDCl}_{3}\right) \delta 173.4,173.3,149.2,148.1,139.4,139.2,137.8$, 134.1, 134.0, 129.00, 128.98, 128.80, 128.78, 128.72, 128.69, 128.62, 128.55, 125.1, 125.0, 123.7, 123.5, 120.41, 120.36, 115.74, 115.68, 111.7, 111.5, 61.6, 61.1, 36.3, 36.0, 25.3, 21.4. IR (thin film): $v_{\max }\left(\mathrm{cm}^{-1}\right)=2935,2816,1700,1602,1558,1491,1450,1368$, 1325, 1275, 1164, 1097, 1042, 879, 858, 816, 747, 698, 665, 628, 546, 490. HRMS (ESI) calcd for $\mathrm{C}_{19} \mathrm{H}_{18} \mathrm{~N}_{2} \mathrm{NaO}_{2}[\mathrm{M}+\mathrm{Na}]^{+}$: 329.1261. Found: 329.1260 .

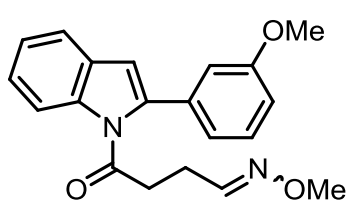

1f, brown oil, $0.30 \mathrm{~g}, 19 \%$ yield (5 mmol scale), $E / Z=1.3 / 1 .{ }^{1} \mathrm{H}$ NMR (400 MHz, $\left.\mathrm{CDCl}_{3}\right) \delta 8.32-8.28(\mathrm{~m}, 1 \mathrm{H}), 7.52(\mathrm{~d}, J=7.6 \mathrm{~Hz}, 1 \mathrm{H}), 7.33-7.21(\mathrm{~m}, 4 \mathrm{H}), 7.02-6.92(\mathrm{~m}$, $3 \mathrm{H}), 6.61(\mathrm{~s}, 1 \mathrm{H}), 6.51\left(\mathrm{~s}, 1 \mathrm{H}^{*}\right), 3.81(\mathrm{~s}, 3 \mathrm{H}), 3.75\left(\mathrm{~s}, 3 \mathrm{H}^{*}\right), 3.53(\mathrm{~s}, 3 \mathrm{H}), 2.53-2.43(\mathrm{~m}$, 4H). ${ }^{13} \mathrm{C} \mathrm{NMR}\left(100 \mathrm{MHz}, \mathrm{CDCl}_{3}\right) \delta 173.4,173.2,159.6,149.1,148.0,139.1,138.9$, $137.7,137.5,135.2,135.1,129.8,129.7,128.81,128.79$, 125.0, 124.9, 123.5, 123.4, 121.1, 120.33, 120.27, 115.6, 115.5, 114.09, 114.07, 114.0, 113.9, 111.6, 111.3, 61.4, 60.9, 55.2, 36.0, 35.7, 25.3, 21.3. IR (thin film): $v_{\max }\left(\mathrm{cm}^{-1}\right)=2936,1701,1580,1558$, 1485, 1447, 1368, 1324, 1277, 1224, 1162, 1085, 1040, 981, 858, 816, 782, 749, 699, 677, 591, 561, 459. HRMS (ESI) calcd for $\mathrm{C}_{20} \mathrm{H}_{20} \mathrm{~N}_{2} \mathrm{NaO}_{3}[\mathrm{M}+\mathrm{Na}]^{+}: 359.13661$. Found: 359.13621. 


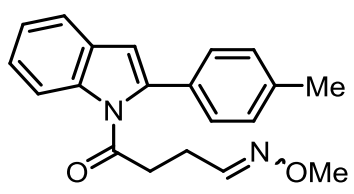

1g, colorless oil, $0.10 \mathrm{~g}$, 7\% yield (5 mmol scale), $E / Z=1.3 / 1 .{ }^{1} \mathrm{H}$ NMR (400 MHz, $\left.\mathrm{CDCl}_{3}\right) \delta 8.32-8.28(\mathrm{~m}, 1 \mathrm{H}), 7.53(\mathrm{~d}, J=7.2 \mathrm{~Hz}, 1 \mathrm{H}), 7.35-7.24(\mathrm{~m}, 7 \mathrm{H}), 6.58(\mathrm{~s}, 1 \mathrm{H})$, 6.52-6.50 (m, 1H*), $3.76\left(\mathrm{~s}, 3 \mathrm{H}^{*}\right), 3.56(\mathrm{~s}, 3 \mathrm{H}), 2.51-2.44(\mathrm{~m}, 4 \mathrm{H}), 2.41(\mathrm{~s}, 3 \mathrm{H}) .{ }^{13} \mathrm{C}$ NMR $\left(100 \mathrm{MHz}, \mathrm{CDCl}_{3}\right) \delta 173.5,173.4,149.3,148.2,139.5,139.3,138.6,138.5,137.7$, $137.6,131.1,131.0,129.48,129.46,129.04,129.01,128.58,128.55,124.9,124.8$, 123.6, 123.5, 120.3, 120.2, 115.7, 115.6, 111.4, 111.1, 61.5, 61.1, 36.2, 36.0, 25.3, 21.4, 21.3. IR (thin film): $v_{\max }\left(\mathrm{cm}^{-1}\right)=2934,2816,1700,1587,1503,1448,1368,1324$, 1278, 1213, 1165, 1108, 1043, 1021, 953, 937, 880, 858, 809, 747, 676, 640, 627, 597. HRMS (ESI) calcd for $\mathrm{C}_{20} \mathrm{H}_{20} \mathrm{~N}_{2} \mathrm{NaO}_{2}[\mathrm{M}+\mathrm{Na}]^{+}:$343.1417. Found: 343.1420 .

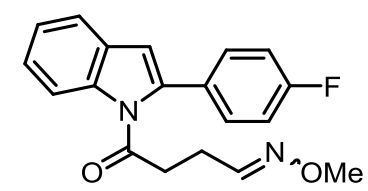

1h, colorless oil, $0.30 \mathrm{~g}, 25 \%$ yield (4 mmol scale), $E / Z=1.5 / 1 .{ }^{1} \mathrm{H}$ NMR $(400 \mathrm{MHz}$, $\left.\mathrm{CDCl}_{3}\right) \delta 8.28-8.27(\mathrm{~m}, 1 \mathrm{H}), 7.52-7.11(\mathrm{~m}, 8 \mathrm{H}), 6.72-6.35(\mathrm{~m}, 1 \mathrm{H}), 3.75\left(\mathrm{~s}, 3 \mathrm{H}^{*}\right), 3.54$ (s, 3H), 2.75-2.12 (m, 4H). ${ }^{13} \mathrm{C}$ NMR $\left(100 \mathrm{MHz}, \mathrm{CDCl}_{3}\right) \delta 172.9,172.8,162.6$ (d, $J=$ $247.0 \mathrm{~Hz}), 148.9,147.8,138.1,138.0,137.5,137.4,130.3$ (d, J = 8.0 Hz), 130.1, 128.8, 125.1, 125.0, 123.6, 123.5, $120.3(\mathrm{~d}, J=6.0 \mathrm{~Hz}), 115.7(\mathrm{~d}, J=22.0 \mathrm{~Hz}), 111.7(\mathrm{~d}, J=$ $25.0 \mathrm{~Hz}), 61.4,61.0,36.03,35.91,25.1,21.2 .{ }^{19} \mathrm{~F} \mathrm{NMR}\left(376 \mathrm{MHz}, \mathrm{CDCl}_{3}\right) \delta-112.0 \sim$ -112.3 (m, 1F). IR (thin film): $v_{\max }\left(\mathrm{cm}^{-1}\right)=2937,1702,1592,1562,1501,1448,1368$, 1324, 1279, 1222, 1158, 1094, 1043, 1017, 953, 938, 883, 840, 814, 796, 736, 677, 620, 596. HRMS (ESI) calcd for $\mathrm{C}_{19} \mathrm{H}_{17} \mathrm{~N}_{2} \mathrm{NaO}_{2} \mathrm{~F}[\mathrm{M}+\mathrm{Na}]^{+}$: 347.1166. Found: 347.1160.

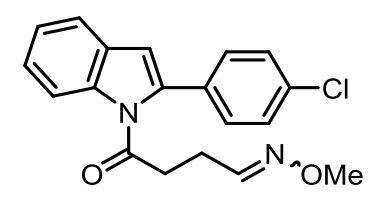

1i, colorless oil, $0.50 \mathrm{~g}, 33 \%$ yield (4 mmol scale), $E / Z=1.6 / 1 .{ }^{1} \mathrm{H}$ NMR (400 MHz, $\left.\mathrm{CDCl}_{3}\right) \delta 8.25(\mathrm{~d}, J=8.4 \mathrm{~Hz}, 1 \mathrm{H}), 7.52(\mathrm{~d}, J=7.6 \mathrm{~Hz}, 1 \mathrm{H}), 7.41-7.31(\mathrm{~m}, 5 \mathrm{H}), 7.28-$ $7.23(\mathrm{~m}, 2 \mathrm{H}), 6.59(\mathrm{~s}, 1 \mathrm{H}), 6.55\left(\mathrm{t}, J=5.2 \mathrm{~Hz}, 1 \mathrm{H}^{*}\right), 3.76\left(\mathrm{~s}, 3 \mathrm{H}^{*}\right), 3.54(\mathrm{~s}, 3 \mathrm{H}), 2.52-$ 
$2.45(\mathrm{~m}, 4 \mathrm{H}) .{ }^{13} \mathrm{C} \mathrm{NMR}\left(100 \mathrm{MHz}, \mathrm{CDCl}_{3}\right) \delta 172.9,172.8,148.8,147.7,138.1,137.9$, 137.7, 137.6, 134.5, 134.4, 132.5, 132.4, 129.8, 128.94, 128.90, 128.80, 128.77, 125.3, 125.1, 123.7, 123.5, 120.5, 120.4, 115.6, 112.1, 111.9, 61.5, 61.0, 36.1, 36.0, 25.2, 21.3. IR (thin film): $v_{\max }\left(\mathrm{cm}^{-1}\right)=2935,2816,1701,1566,1486,1447,1367,1323,1277$, 1164, 1089, 1042, 1014, 858, 835, 811, 748, 718, 673, 634, 575. HRMS (ESI) calcd for $\mathrm{C}_{19} \mathrm{H}_{17} \mathrm{~N}_{2} \mathrm{NaO}_{2} \mathrm{Cl}[\mathrm{M}+\mathrm{Na}]^{+}: 363.0871$ Found: 363.0865 .

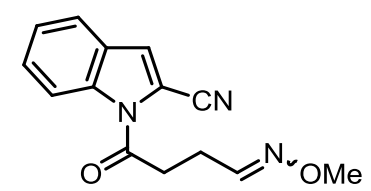

1j, white solid, $0.31 \mathrm{~g}, 30 \%$ yield (4 mmol scale), $E / Z=1.3 / 1$, m.p. $=101.0-104.3{ }^{\circ} \mathrm{C}$. ${ }^{1} \mathrm{H}$ NMR $\left(400 \mathrm{MHz}, \mathrm{CDCl}_{3}\right) \delta 8.26(\mathrm{t}, J=9.6 \mathrm{~Hz}, 1 \mathrm{H}), 7.62(\mathrm{~d}, J=7.6 \mathrm{~Hz}, 1 \mathrm{H}), 7.53-$ $7.49(\mathrm{~m}, 2 \mathrm{H}), 7.39(\mathrm{~s}, 1 \mathrm{H}), 7.36(\mathrm{t}, J=7.6 \mathrm{~Hz}, 1 \mathrm{H}), 6.85\left(\mathrm{t}, J=5.6 \mathrm{~Hz}, 1 \mathrm{H}^{*}\right), 3.89(\mathrm{~s}$, $\left.3 \mathrm{H}^{*}\right), 3.75$ (s, 3H), 3.40-3.35 (m, 2H), 2.83-2.72 (m, 2H). $\left.{ }^{13} \mathrm{C} \mathrm{NMR} \mathrm{(100} \mathrm{MHz,} \mathrm{CDCl}_{3}\right)$ $\delta 170.0,169.9,148.7,147.7,136.5,128.9,128.8,127.22,127.18,124.7,124.6,123.8$, 123.5, 122.1, 116.6, 116.5, 113.73, 113.71, 107.5, 107.4, 61.7, 61.3, 34.0, 33.9, 24.4, 20.7. IR (thin film): $v_{\max }\left(\mathrm{cm}^{-1}\right)=2960,222,1719,1532,1439,1414,1377,1331$, 1281, 1199, 1166, 1142, 1103, 1031, 1004, 896, 874, 842, 760, 650, 622. HRMS (ESI) calcd for $\mathrm{C}_{14} \mathrm{H}_{13} \mathrm{~N}_{3} \mathrm{NaO}_{2}[\mathrm{M}+\mathrm{Na}]^{+}$: 278.0900. Found: 278.0899 .

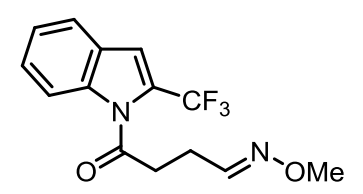

1k, brown oil, $0.10 \mathrm{~g}, 20 \%$ yield (1.8 mmol scale), E/Z > 20/1. ${ }^{1} \mathrm{H}$ NMR (400 MHz, $\left.\mathrm{CDCl}_{3}\right) \delta 7.88(\mathrm{~d}, J=8.4 \mathrm{~Hz}, 1 \mathrm{H}), 7.66(\mathrm{~d}, J=7.6 \mathrm{~Hz}, 1 \mathrm{H}), 7.53(\mathrm{t}, J=4.4 \mathrm{~Hz}, 1 \mathrm{H})$, $7.47(\mathrm{t}, J=8.0 \mathrm{~Hz}, 1 \mathrm{H}), 7.33(\mathrm{t}, J=7.6 \mathrm{~Hz}, 1 \mathrm{H}), 7.23(\mathrm{~s}, 1 \mathrm{H}), 3.74(\mathrm{~s}, 3 \mathrm{H}), 3.31(\mathrm{t}, J=$ $6.8 \mathrm{~Hz}, 2 \mathrm{H}), 2.80-2.75(\mathrm{~m}, 2 \mathrm{H}) .{ }^{13} \mathrm{C} \mathrm{NMR}\left(100 \mathrm{MHz}, \mathrm{CDCl}_{3}\right) \delta 170.3,147.9,136.5$, 127.22, 127.15, 123.8, 122.8, 120.9 (q, $J=266.0 \mathrm{~Hz}), 115.1,114.82,114.77,61.4,34.8$, 24.9. ${ }^{19} \mathrm{~F}$ NMR (376 MHz, $\left.\mathrm{CDCl}_{3}\right) \delta-57.8$ (s, 3F). IR (thin film): $v_{\max }\left(\mathrm{cm}^{-1}\right)=3103$, 2941, 1720, 1589, 1564, 1457, 1425, 1383, 1348, 1328, 1285, 1195, 1176, 1133, 1072, 1037, 866, 752, 741, 723, 705, 676, 627. HRMS (ESI) calcd for $\mathrm{C}_{14} \mathrm{H}_{13} \mathrm{~F}_{3} \mathrm{~N}_{2} \mathrm{NaO}_{2}$ $[\mathrm{M}+\mathrm{Na}]^{+}:$321.0821. Found: 321.0827. 


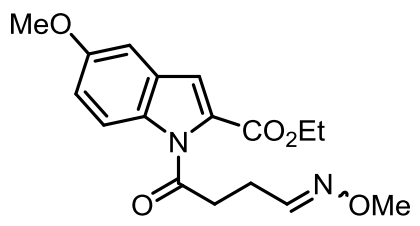

11, yellow oil, $0.61 \mathrm{~g}, 46 \%$ yield (4 mmol scale), $E / Z=1.4 / 1 .{ }^{1} \mathrm{H}$ NMR (400 MHz, $\left.\mathrm{CDCl}_{3}\right) \delta$ 7.99-7.97 (m, 1H), 7.43-7.36 (m, 1H), 7.24 (s, 1H), 7.05-7.00 (m, 2H), 6.75 $\left(\mathrm{t}, J=5.2 \mathrm{~Hz}, 1 \mathrm{H}^{*}\right), 4.38(\mathrm{q}, J=6.8 \mathrm{~Hz}, 2 \mathrm{H}), 3.84-3.52(\mathrm{~m}, 6 \mathrm{H}), 3.52\left(\mathrm{~m}, 3 \mathrm{H}^{*}\right), 3.07$ $(\mathrm{t}, J=6.8 \mathrm{~Hz}, 2 \mathrm{H}), 2.77-2.67(\mathrm{~m}, 2 \mathrm{H}), 1.40(\mathrm{t}, J=6.8 \mathrm{~Hz}, 3 \mathrm{H}) .{ }^{13} \mathrm{C} \mathrm{NMR}(100 \mathrm{MHz}$, $\left.\mathrm{CDCl}_{3}\right) \delta 173.0,172.7,161.5,156.3,156.2,149.1,147.9,133.4,133.2,129.6,127.7$, 127.6, 117.9, 117.8, 117.4, 117.3, 115.6, 115.9, 103.5, 103.4, 61.5, 61.5, 60.9, 55.4, 35.6, 35.4, 25.6, 21.5, 14.1. IR (thin film): $v_{\max }\left(\mathrm{cm}^{-1}\right)=2935,1709,1614,1536,1465$, $1447,1375,1284,1260,1215,1191,1162,1032,853,813,759,737,665,436$. HRMS (ESI) calcd for $\mathrm{C}_{17} \mathrm{H}_{20} \mathrm{~N}_{2} \mathrm{NaO}_{5}[\mathrm{M}+\mathrm{Na}]^{+}:$355.1264. Found: 355.1264 .

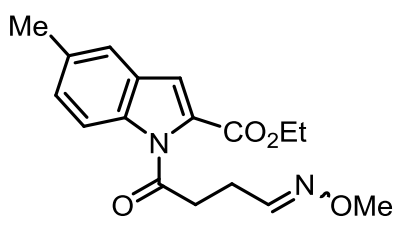

1m, yellow oil, $0.86 \mathrm{~g}$, $69 \%$ yield (4 mmol scale), $E / Z=1.5 / 1 .{ }^{1} \mathrm{H}$ NMR (400 MHz, $\left.\mathrm{CDCl}_{3}\right) \delta 7.91(\mathrm{t}, J=8.8 \mathrm{~Hz}, 1 \mathrm{H}), 7.38(\mathrm{t}, J=4.8 \mathrm{~Hz}, 1 \mathrm{H}), 7.33(\mathrm{~s}, 1 \mathrm{H}), 7.22-7.19(\mathrm{~m}$, $2 \mathrm{H}), 6.73\left(\mathrm{t}, J=5.2 \mathrm{~Hz}, 1 \mathrm{H}^{*}\right), 4.37(\mathrm{q}, J=7.2 \mathrm{~Hz}, 2 \mathrm{H}), 3.83\left(\mathrm{~s}, 3 \mathrm{H}^{*}\right), 3.51(\mathrm{~s}, 3 \mathrm{H})$, 3.07-3.04 (m, 2H), 2.76-2.63 (m, 2H), $2.39(\mathrm{~s}, 3 \mathrm{H}), 1.38(\mathrm{t}, J=7.2 \mathrm{~Hz}, 3 \mathrm{H}) .{ }^{13} \mathrm{C} \mathrm{NMR}$ $\left(100 \mathrm{MHz}, \mathrm{CDCl}_{3}\right) \delta 173.0,172.8,161.5,149.0,147.8,136.7,136.5,133.1,132.9$, 129.2, 129.1, 127.13, 127.08, 121.8, 121.7, 117.8, 117.7, 114.5, 114.4, 61.4, 61.3, 60.8, 35.6, 35.4, 25.5, 21.4, 20.9, 13.98, 13.96. IR (thin film): $v_{\max }\left(\mathrm{cm}^{-1}\right)=2980,2937,2818$, 1710, 1539, 1462, 1375, 1280, 1201, 1159, 1096, 1036, 998, 877, 841, 808, 754, 665, 634, 586, 432. HRMS (ESI) calcd for $\mathrm{C}_{17} \mathrm{H}_{20} \mathrm{~N}_{2} \mathrm{NaO}_{4}[\mathrm{M}+\mathrm{Na}]^{+}$: 339.1315. Found: 339.1310 .

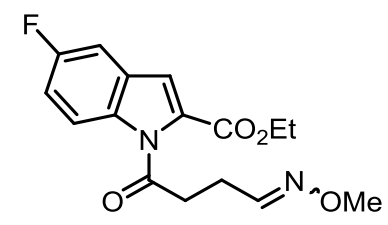

1n, yellow oil, $0.80 \mathrm{~g}, 60 \%$ yield (4 mmol scale), $E / Z=1.6 / 1 .{ }^{1} \mathrm{H}$ NMR $(400 \mathrm{MHz}$, $\left.\mathrm{CDCl}_{3}\right) \delta 8.06-8.02(\mathrm{~m}, 1 \mathrm{H}), 7.40-7.39(\mathrm{~m}, 1 \mathrm{H})$, 7.28-7.23 (m, 2H), 7.17-7.12 (m, 1H), 
6.75-6.73 (m, 1H*), 4.43-4.37 (m, 2H), $3.83\left(\mathrm{~s}, 3 \mathrm{H}^{*}\right), 3.46(\mathrm{~s}, 3 \mathrm{H}), 3.09-3.06(\mathrm{~m}, 2 \mathrm{H})$, 2.76-2.68 (m, 2H), $1.41(\mathrm{t}, J=6.8 \mathrm{~Hz}, 3 \mathrm{H}) .{ }^{13} \mathrm{C} \mathrm{NMR}\left(100 \mathrm{MHz}, \mathrm{CDCl}_{3}\right) \delta 173.3,172.9$, $161.3,159.3(\mathrm{~d}, J=239.0 \mathrm{~Hz}), 148.9,147.7,134.9,134.7,130.6,127.7,127.6,127.5$, $117.4,117.2,116.3(\mathrm{~d}, J=9.0 \mathrm{~Hz}), 116.2,116.1,116.0,115.9,115.8,115.7,107.2(\mathrm{~d}$, $J=23.0 \mathrm{~Hz}), 107.1$ (d, $J=24.0 \mathrm{~Hz}), 106.9,61.8,61.7,61.5,60.9,35.7,35.5,25.8,21.6$, 14.1. ${ }^{19} \mathrm{~F}$ NMR $\left(376 \mathrm{MHz}, \mathrm{CDCl}_{3}\right) \delta-119.0 \sim-119.3(\mathrm{~m}, 1 \mathrm{~F})$. IR (thin film): $v_{\max }\left(\mathrm{cm}^{-}\right.$ $\left.{ }^{1}\right)=2939,1712,1617,1589,1537,1463,1443,1376,1328,1280,1243,1204,1169$, 1103, 1045, 997, 956, 862, 810, 754, 665, 599, 509. HRMS (ESI) calcd for $\mathrm{C}_{16} \mathrm{H}_{17} \mathrm{~N}_{2} \mathrm{NaFO}_{4}[\mathrm{M}+\mathrm{Na}]^{+}:$343.1065. Found: 343.1055.

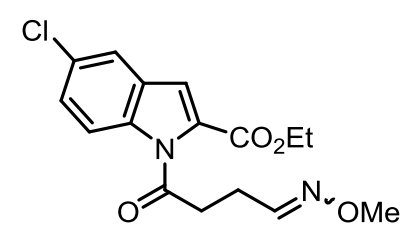

1o, colorless oil, $0.30 \mathrm{~g}, 30 \%$ yield (3 mmol scale), $E / Z=2.1 / 1 .{ }^{1} \mathrm{H}$ NMR $(400 \mathrm{MHz}$, $\left.\mathrm{CDCl}_{3}\right) \delta 7.96(\mathrm{t}, J=9.2 \mathrm{~Hz}, 1 \mathrm{H}), 7.53(\mathrm{~s}, 1 \mathrm{H}), 7.39-7.32(\mathrm{~m}, 2 \mathrm{H}), 7.22(\mathrm{~d}, J=5.2 \mathrm{~Hz}$, $1 \mathrm{H}), 6.74\left(\mathrm{t}, J=5.6 \mathrm{~Hz}, 1 \mathrm{H}^{*}\right), 4.39$ (q, $\left.J=7.2 \mathrm{~Hz}, 2 \mathrm{H}\right), 3.83\left(\mathrm{~s}, 3 \mathrm{H}^{*}\right), 3.45(\mathrm{~s}, 3 \mathrm{H}), 3.06$ $(\mathrm{t}, J=6.4 \mathrm{~Hz}, 2 \mathrm{H}), 2.77-2.66(\mathrm{~m}, 2 \mathrm{H}), 1.41(\mathrm{t}, J=7.2 \mathrm{~Hz}, 3 \mathrm{H}) .{ }^{13} \mathrm{C}$ NMR $(100 \mathrm{MHz}$, $\left.\mathrm{CDCl}_{3}\right) \delta 173.2,172.8,161.2,148.8,147.6,136.7,136.4,130.3,129.0,128.9,127.9$, $127.84,127.78,127.7,121.5,121.4,116.8,116.5,116.1,115.9,61.8,61.7,61.5,60.9$, 35.6, 35.5, 25.7, 21.5, 14.0. IR (thin film): $v_{\max }\left(\mathrm{cm}^{-1}\right)=2936,1705,1529,1437,1377$, 1329, 1274, 1191, 1045, 998, 915, 862, 811, 798, 761, 731, 660, 546, 422. HRMS (ESI) calcd for $\mathrm{C}_{16} \mathrm{H}_{17} \mathrm{~N}_{2} \mathrm{NaClO}_{4}[\mathrm{M}+\mathrm{Na}]^{+}:$359.0769. Found: 359.0766 .

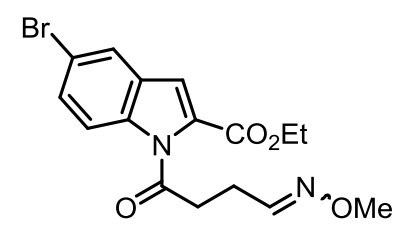

1p, brown oil, $0.72 \mathrm{~g}, 52 \%$ yield (4 mmol scale), $E / Z=1.8 / 1 .{ }^{1} \mathrm{H}$ NMR (400 MHz, $\left.\mathrm{CDCl}_{3}\right) \delta 7.96-7.94(\mathrm{~m}, 1 \mathrm{H}), 7.74(\mathrm{~s}, 1 \mathrm{H}), 7.50(\mathrm{~d}, J=8.8 \mathrm{~Hz}, 1 \mathrm{H}), 7.39(\mathrm{t}, J=4.4 \mathrm{~Hz}$, 1H), $7.25(\mathrm{~s}, 1 \mathrm{H}), 6.74\left(\mathrm{t}, J=5.6 \mathrm{~Hz}, 1 \mathrm{H}^{*}\right), 4.40(\mathrm{q}, J=7.2 \mathrm{~Hz}, 2 \mathrm{H}), 3.84\left(\mathrm{~s}, 3 \mathrm{H}^{*}\right), 3.46$ $(\mathrm{s}, 3 \mathrm{H}), 3.07(\mathrm{t}, J=6.8 \mathrm{~Hz}, 2 \mathrm{H}), 2.77-2.67(\mathrm{~m}, 2 \mathrm{H}), 1.42(\mathrm{t}, J=7.2 \mathrm{~Hz}, 3 \mathrm{H}) .{ }^{13} \mathrm{C} \mathrm{NMR}$ $\left(100 \mathrm{MHz}, \mathrm{CDCl}_{3}\right) \delta 173.4,173.0,161.3,148.9,147.7,137.2,136.9,130.6,130.5$, 130.2, 128.6, 128.5, 124.8, 124.7, 116.8, 116.61, 116.56, 116.4, 61.93, 61.86, 61.7, 61.0, 
35.8, 35.7, 25.8, 21.6, 14.2. IR (thin film): $v_{\max }\left(\mathrm{cm}^{-1}\right)=2981,2937,2818,1712,1531$, 1438, 1375, 1273, 1186, 1165, 1095, 1045, 997, 907, 874, 808, 752, 666, 578. HRMS (ESI) calcd for $\mathrm{C}_{16} \mathrm{H}_{17} \mathrm{~N}_{2} \mathrm{BrNaO}_{4}[\mathrm{M}+\mathrm{Na}]^{+}$: 403.0264. Found: 403.0262.

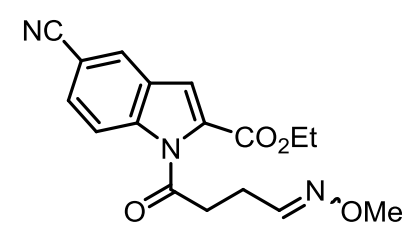

1q, white solid, $0.31 \mathrm{~g}, 23 \%$ yield ( 4 mmol scale), $E / Z=1.9 / 1$, m.p. $=79.7-81.6{ }^{\circ} \mathrm{C} .{ }^{1} \mathrm{H}$ NMR (400 MHz, $\left.\mathrm{CDCl}_{3}\right) \delta 8.09(\mathrm{t}, J=8.8 \mathrm{~Hz}, 1 \mathrm{H}), 7.97(\mathrm{~m}, 1 \mathrm{H}), 7.63(\mathrm{~d}, J=8.8 \mathrm{~Hz}$, $1 \mathrm{H}), 7.38-7.33(\mathrm{~m}, 1 \mathrm{H}), 6.76(\mathrm{t}, J=5.2 \mathrm{~Hz}, 1 \mathrm{H}), 4.44(\mathrm{q}, J=7.2 \mathrm{~Hz}, 2 \mathrm{H}), 3.83(\mathrm{~s}, 3 \mathrm{H})$, 3.35 (s, 3H*), 3.10 (q, $J=7.2 \mathrm{~Hz}, 2 \mathrm{H}), 2.79-2.71(\mathrm{~m}, 2 \mathrm{H}), 1.44(\mathrm{t}, J=7.2 \mathrm{~Hz}, 3 \mathrm{H}) .{ }^{13} \mathrm{C}$ $\mathrm{NMR}\left(100 \mathrm{MHz}, \mathrm{CDCl}_{3}\right) \delta 173.4,173.0,160.8,148.4,147.3,139.6,139.3,131.0,129.9$, 129.7, 127.3, 127.2, 126.6, 126.5, 118.8, 118.7, 116.4, 116.3, 115.7, 115.5, 106.9, 106.7, 62.1, 62.0, 61.5, 60.7, 35.81, 35.76, 25.7, 21.4, 14.0. IR (thin film): $v_{\max }\left(\mathrm{cm}^{-1}\right)=2938$, 2226, 1719, 1611, 1543, 1459, 1377, 1280, 1200, 1153, 1096, 1054, 1026, 904, 873, 827, 792, 766, 740, 696, 667, 607. HRMS (ESI) calcd for $\mathrm{C}_{17} \mathrm{H}_{17} \mathrm{~N}_{3} \mathrm{NaO}_{4}[\mathrm{M}+\mathrm{Na}]^{+}$: 350.1111. Found: 350.1112.

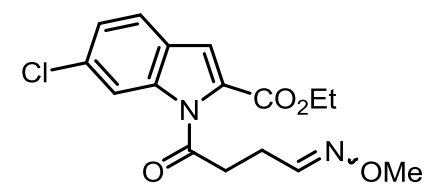

1r, yellow oil, $0.51 \mathrm{~g}, 60 \%$ yield (4 mmol scale), $E / Z=1.6 / 1 .{ }^{1} \mathrm{H}$ NMR $(400 \mathrm{MHz}$, $\left.\mathrm{CDCl}_{3}\right) \delta 8.13-8.12(\mathrm{~m}, 1 \mathrm{H}), 7.52(\mathrm{~d}, J=8.4 \mathrm{~Hz}, 1 \mathrm{H}), 7.39(\mathrm{t}, J=4.4 \mathrm{~Hz}, 1 \mathrm{H}), 7.31(\mathrm{~s}$, $1 \mathrm{H}), 7.26-7.23(\mathrm{~m}, 1 \mathrm{H}), 6.75\left(\mathrm{t}, J=5.6 \mathrm{~Hz}, 1 \mathrm{H}^{*}\right), 4.40(\mathrm{q}, J=7.2 \mathrm{~Hz}, 2 \mathrm{H}), 3.84(\mathrm{~s}$, $\left.3 \mathrm{H}^{*}\right), 3.46(\mathrm{~s}, 3 \mathrm{H}), 3.09-3.05(\mathrm{~m}, 2 \mathrm{H}), 2.78-2.67(\mathrm{~m}, 2 \mathrm{H}), 1.41(\mathrm{t}, J=7.2 \mathrm{~Hz}, 3 \mathrm{H}) .{ }^{13} \mathrm{C}$ NMR $\left(100 \mathrm{MHz}, \mathrm{CDCl}_{3}\right) \delta 173.5,161.3,148.9,147.7,138.9,134.0,133.8,129.7,125.4$, 125.3, 124.6, 124.4, 123.1, 123.0, 117.9, 117.6, 115.2, 115.1, 61.83, 61.75, 61.7, 61.0, 35.8, 35.7, 25.8, 21.6, 14.2, 14.1. IR (thin film): $v_{\max }\left(\mathrm{cm}^{-1}\right)=2938,1711,1604,1529$, 1458, 1417, 1375, 1331, 1299, 1245, 1190, 1161, 1119, 1044, 999, 936, 849, 802, 752, 666, 601, 457, 425. HRMS (ESI) calcd for $\mathrm{C}_{16} \mathrm{H}_{17} \mathrm{~N}_{2} \mathrm{NaClO}_{4}[\mathrm{M}+\mathrm{Na}]^{+}: 359.0769$. Found: 359.0764. 


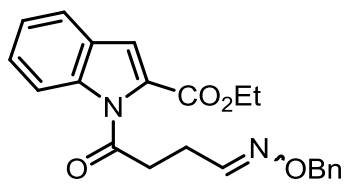

1s, yellow oil, $1.30 \mathrm{~g}, 68 \%$ yield (5 mmol scale), $E / Z=1.7 / 1 .{ }^{1} \mathrm{H}$ NMR $(400 \mathrm{MHz}$, $\left.\mathrm{CDCl}_{3}\right) \delta 8.07(\mathrm{~d}, J=8.4 \mathrm{~Hz}, 1 \mathrm{H}), 8.02\left(\mathrm{~d}, J=8.4 \mathrm{~Hz}, 1 \mathrm{H}^{*}\right), 7.59(\mathrm{~d}, J=7.6 \mathrm{~Hz}, 1 \mathrm{H})$, 7.47-7.38 (m, 2H), 7.33-7.23 (m, 5H), $7.16(\mathrm{~d}, J=6.4 \mathrm{~Hz}, 1 \mathrm{H}), 6.80(\mathrm{t}, J=5.2 \mathrm{~Hz}$, $\left.1 \mathrm{H}^{*}\right), 5.08\left(\mathrm{~s}, 2 \mathrm{H}^{*}\right), 4.63(\mathrm{~s}, 2 \mathrm{H}), 4.39-4.32(\mathrm{~m}, 2 \mathrm{H}), 3.09-3.05(\mathrm{~m}, 2 \mathrm{H}), 2.83-2.66(\mathrm{~m}$, $2 \mathrm{H}), 1.38(\mathrm{t}, J=7.2 \mathrm{~Hz}, 3 \mathrm{H}) .{ }^{13} \mathrm{C} \mathrm{NMR}\left(100 \mathrm{MHz}, \mathrm{CDCl}_{3}\right) \delta 173.4,173.0,161.6,149.7$, $148.4,138.5,138.2,137.7,137.2$, 129.2, 128.2, 128.1, 127.80, 127.78, 127.7, 127.6, 127.0, 126.9, 123.6, 123.5, 122.4, 122.3, 118.1, 118.0, 114.9, 114.8, 75.7, 75.3, 61.6, 61.5, 35.7, 35.6, 25.8, 21.8, 14.12, 14.09. IR (thin film): $v_{\max }\left(\mathrm{cm}^{-1}\right)=2981,1710,1606$, 1534, 1473, 1443, 1374, 1328, 1279, 1256, 1197, 1166, 1146, 1111, 1015, 902, 841, 734, 697, 615. HRMS (ESI) calcd for $\mathrm{C}_{22} \mathrm{H}_{22} \mathrm{~N}_{2} \mathrm{NaO}_{4}[\mathrm{M}+\mathrm{Na}]^{+}$: 401.1472. Found: 401.1464 .

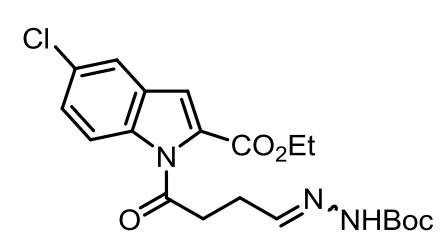

1t, colorless oil, $0.50 \mathrm{~g}, 96 \%$ yield (2 mmol scale), $E / Z=7 / 1 .{ }^{1} \mathrm{H}$ NMR (400 MHz, $\left.\mathrm{CDCl}_{3}\right) \delta 8.01\left(\mathrm{~d}, J=8.8 \mathrm{~Hz}, 1 \mathrm{H}^{*}\right), 7.96(\mathrm{~d}, J=9.2 \mathrm{~Hz}, 1 \mathrm{H}), 7.68-7.65(\mathrm{~m}, 1 \mathrm{H}), 7.59$ $(\mathrm{d}, J=2.0 \mathrm{~Hz}, 1 \mathrm{H}), 7.38-7.35(\mathrm{~m}, 1 \mathrm{H}), 7.32(\mathrm{br}, 1 \mathrm{H}), 7.26(\mathrm{~s}, 1 \mathrm{H}), 4.40(\mathrm{q}, J=7.2 \mathrm{~Hz}$, 2H), $3.16(\mathrm{t}, J=6.8 \mathrm{~Hz}, 2 \mathrm{H}), 3.07\left(\mathrm{t}, J=7.2 \mathrm{~Hz}, 2 \mathrm{H}^{*}\right), 2.78(\mathrm{q}, J=7.2 \mathrm{~Hz}, 2 \mathrm{H}), 2.68$ $\left(\mathrm{q}, J=7.2 \mathrm{~Hz}, 2 \mathrm{H}^{*}\right), 1.44(\mathrm{~s}, 9 \mathrm{H}), 1.41(\mathrm{t}, J=7.2 \mathrm{~Hz}, 3 \mathrm{H}) .{ }^{13} \mathrm{C} \mathrm{NMR}\left(100 \mathrm{MHz}, \mathrm{CDCl}_{3}\right)$ $\delta 173.3,172.7,161.8,161.2,136.3,135.3,130.2,128.8,128.5,127.9,127.6,125.8$, 125.2, 121.6, 121.4, 121.2, 116.4, 115.8, 113.2, 107.5, 81.0, 80.2, 61.7, 60.9, 35.8, 28.1, 28.02, 27.97, 27.9, 27.6, 25.1, 13.9. IR (thin film): $v_{\max }\left(\mathrm{cm}^{-1}\right)=3311,2977,1694,1522$, 1441, 1395, 1368, 1328, 1276, 1249, 1194, 1157, 1093, 1055, 915, 866, 799, 765, 732, 689, 662. HRMS (ESI) calcd for $\mathrm{C}_{20} \mathrm{H}_{24} \mathrm{~N}_{3} \mathrm{NaClO}_{5}[\mathrm{M}+\mathrm{Na}]^{+}:$444.1297. Found: 444.1287. 


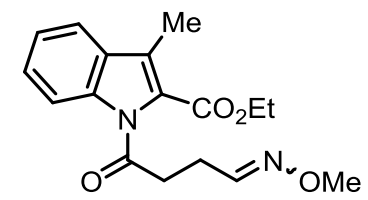

3a, colorless oil, $0.70 \mathrm{~g}, 49 \%$ yield (4 mmol scale), $E / Z=1.5 / 1 .{ }^{1} \mathrm{H}$ NMR (400 MHz, $\left.\mathrm{CDCl}_{3}\right) \delta 7.99(\mathrm{~d}, J=8.8 \mathrm{~Hz}, 1 \mathrm{H}), 7.95\left(\mathrm{~d}, J=8.4 \mathrm{~Hz}, 1 \mathrm{H}^{*}\right), 7.54(\mathrm{~d}, J=8.0 \mathrm{~Hz}, 1 \mathrm{H})$, 7.41-7.37 (m, 2H), 7.28-7.22 (m, 1H), $6.75\left(\mathrm{t}, J=5.6 \mathrm{~Hz}, 1 \mathrm{H}^{*}\right), 4.40(\mathrm{q}, J=7.2 \mathrm{~Hz}$, $2 \mathrm{H}), 3.84\left(\mathrm{~s}, 3 \mathrm{H}^{*}\right), 3.53(\mathrm{~s}, 3 \mathrm{H}), 3.03-2.99(\mathrm{~m}, 2 \mathrm{H}), 2.76-2.63(\mathrm{~m}, 2 \mathrm{H}), 2.46-2.43(\mathrm{~m}$, $3 \mathrm{H}), 1.40(\mathrm{t}, J=7.2 \mathrm{~Hz}, 3 \mathrm{H}) .{ }^{13} \mathrm{C} \mathrm{NMR}\left(100 \mathrm{MHz}, \mathrm{CDCl}_{3}\right) \delta 172.4,172.0,162.3,149.0$, $147.8,136.8,136.5,129.0,128.9,127.5,126.8,126.6,125.83,125.76,123.1,123.0$, 120.3, 120.2, 114.6, 114.4, 61.4, 61.29, 61.25, 60.8, 35.3, 35.1, 25.4, 21.3, 14.03, 14.00, 9.6, 9.5. IR (thin film): $v_{\max }\left(\mathrm{cm}^{-1}\right)=2981,2937,1703,1603,1558,1446,1400,1378$, 1319, 1285, 1257, 1201, 1168, 1147, 1127, 1093, 1070, 1042, 1017, 879, 744, 666. HRMS (ESI) calcd for $\mathrm{C}_{17} \mathrm{H}_{20} \mathrm{~N}_{2} \mathrm{NaO}_{4}[\mathrm{M}+\mathrm{Na}]^{+}: 339.1315$. Found: 339.1319.

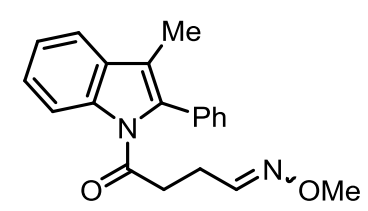

3b, colorless oil, $0.50 \mathrm{~g}, 61 \%$ yield (4 mmol scale), $E / Z=1.3 / 1 .{ }^{1} \mathrm{H} \mathrm{NMR}(400 \mathrm{MHz}$, $\left.\mathrm{CDCl}_{3}\right) \delta 8.40-8.37(\mathrm{~m}, 1 \mathrm{H}), 7.50-7.26(\mathrm{~m}, 8 \mathrm{H}), 7.21-7.18(\mathrm{~m}, 1 \mathrm{H}), 6.46(\mathrm{t}, J=5.2 \mathrm{~Hz}$, $\left.1 \mathrm{H}^{*}\right), 3.75\left(\mathrm{~s}, 3 \mathrm{H}^{*}\right), 3.59(\mathrm{~s}, 3 \mathrm{H}), 2.48-2.43(\mathrm{~m}, 1 \mathrm{H}), 2.38-2.28(\mathrm{~m}, 3 \mathrm{H}), 2.12(\mathrm{~s}, 3 \mathrm{H})$, $2.11\left(\mathrm{~s}, 3 \mathrm{H}^{*}\right) .{ }^{13} \mathrm{C} \mathrm{NMR}\left(100 \mathrm{MHz}, \mathrm{CDCl}_{3}\right) \delta 172.6,172.5,149.3,148.2,136.7,136.6$, $134.4,134.2,133.4,133.3,130.07,130.05,129.9,128.61,128.59,128.4,128.3,125.2$, 125.1, 123.3, 123.2, 118.48, 118.45, 118.2, 118.0, 116.0, 115.9, 61.4, 61.0, 35.8, 35.5, 25.0, 21.2, 9.1. IR (thin film): $v_{\max }\left(\mathrm{cm}^{-1}\right)=2935,1693,1602,1451,1368,1314,1285$, $1184,1155,1135,1064,1042,935,910,880,856,746,701,664,538,494$. HRMS (ESI) calcd for $\mathrm{C}_{20} \mathrm{H}_{20} \mathrm{~N}_{2} \mathrm{NaO}_{2}[\mathrm{M}+\mathrm{Na}]^{+}: 343.1417$. Found: 343.1412 .

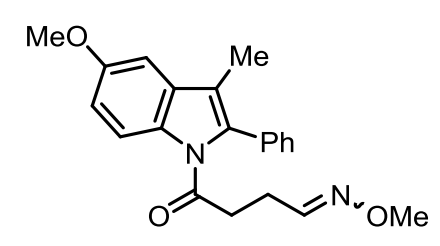

3c, colorless oil, $0.50 \mathrm{~g}, 36 \%$ yield (3 mmol scale), $E / Z=1.2 / 1 .{ }^{1} \mathrm{H}$ NMR (400 MHz, $\left.\mathrm{CDCl}_{3}\right) \delta 8.33-8.29(\mathrm{~m}, 1 \mathrm{H}), 7.48-7.36(\mathrm{~m}, 5 \mathrm{H}), 7.23-7.20(\mathrm{~m}, 1 \mathrm{H}), 6.95(\mathrm{~s}, 2 \mathrm{H}), 6.47$ 
(t, $\left.J=5.2 \mathrm{~Hz}, 1 \mathrm{H}^{*}\right), 3.86(\mathrm{~s}, 3 \mathrm{H}), 3.76$ (s, 3H*), 3.63 (s, 3H), 2.48-2.43 (m, 1H), 2.39$2.36(\mathrm{~m}, 1 \mathrm{H}), 2.33-2.27(\mathrm{~m}, 2 \mathrm{H}), 2.09$ (s, 3H), 2.08 (s, 3H*). ${ }^{13} \mathrm{C}$ NMR (100 MHz, $\left.\mathrm{CDCl}_{3}\right) \delta 172.14,172.11,156.33,156.27,149.5,148.5,135.1,135.0,133.5,133.4$, 131.43, 131.37, 131.1, 130.0, 128.6, 128.5, 128.4, 118.2, 118.0, 117.1, 117.0, 113.2, $113.1,101.5,101.4,61.4,61.0,55.5,35.6,35.3,25.0,21.2,9.2$. IR (thin film): $v_{\max }\left(\mathrm{cm}^{-}\right.$ $\left.{ }^{1}\right)=2932,1680,1609,1473,1451,1369,1285,1223,1175,1139,1047,1031,974$, 935, 887, 833, 809, 775, 726, 701, 664, 614. HRMS (ESI) calcd for $\mathrm{C}_{21} \mathrm{H}_{22} \mathrm{~N}_{2} \mathrm{NaO}_{3}$ $[\mathrm{M}+\mathrm{Na}]^{+}:$373.1523. Found: 373.1516.

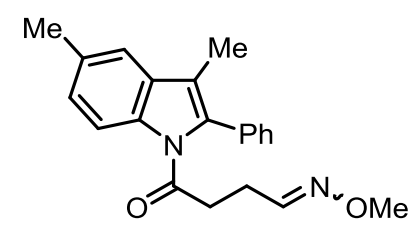

3d, colorless oil, $0.38 \mathrm{~g}, 31 \%$ yield (3 mmol scale), $E / Z=1.2 / 1 .{ }^{1} \mathrm{H}$ NMR (400 MHz, $\left.\mathrm{CDCl}_{3}\right) \delta 8.28-8.25(\mathrm{~m}, 1 \mathrm{H}), 7.47-7.35(\mathrm{~m}, 5 \mathrm{H}), 7.29(\mathrm{~s}, 1 \mathrm{H}), 7.22-7.15(\mathrm{~m}, 2 \mathrm{H}), 6.46$ $\left(\mathrm{t}, J=5.2 \mathrm{~Hz}, 1 \mathrm{H}^{*}\right), 3.76\left(\mathrm{~s}, 3 \mathrm{H}^{*}\right), 3.63(\mathrm{~s}, 3 \mathrm{H}), 2.54-2.41(\mathrm{~m}, 4 \mathrm{H}), 2.39-2.27(\mathrm{~m}, 3 \mathrm{H})$, 2.093 (s, 3H), 2.087 (s, 3H*). ${ }^{13} \mathrm{C} \mathrm{NMR}\left(100 \mathrm{MHz}, \mathrm{CDCl}_{3}\right) \delta 172.3,149.5,148.4,135.0$, $134.9,134.5,134.4,133.6,133.5,132.9,132.8,130.34,130.32,130.0,128.6,128.4$, $128.3,126.5,126.4,118.50,118.47,118.2$, 118.0, 115.8, 115.7, 61.4, 61.0, 35.7, 35.4, 25.0, 21.3, 9.1. IR (thin film): $v_{\max }\left(\mathrm{cm}^{-1}\right)=2934,2815,1687,1611,1459,1420,1371$, 1339, 1319, 1289, 1183, 1142, 1060, 1033, 943, 930, 877, 860, 813, 776, 726, 701, 595. HRMS (ESI) calcd for $\mathrm{C}_{21} \mathrm{H}_{22} \mathrm{~N}_{2} \mathrm{NaO}_{2}[\mathrm{M}+\mathrm{Na}]^{+}: 357.1574$. Found: 357.1566 .

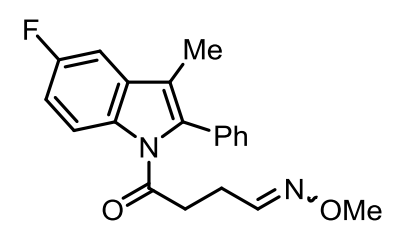

3e, yellow oil, $0.78 \mathrm{~g}$, $61 \%$ yield (4 mmol scale), $E / Z=1.2 / 1 .{ }^{1} \mathrm{H}$ NMR (400 MHz, $\left.\mathrm{CDCl}_{3}\right) \delta 8.37-8.32(\mathrm{~m}, 1 \mathrm{H}), 7.48-7.41(\mathrm{~m}, 3 \mathrm{H}), 7.38-7.36(\mathrm{~m}, 2 \mathrm{H}), 7.21(\mathrm{t}, J=4.8 \mathrm{~Hz}$, $1 \mathrm{H}), 7.11(\mathrm{~d}, J=8.4 \mathrm{~Hz}, 1 \mathrm{H}), 7.02(\mathrm{td}, J=9.2,2.4 \mathrm{~Hz}, 1 \mathrm{H}), 6.46\left(\mathrm{t}, J=5.2 \mathrm{~Hz}, 1 \mathrm{H}^{*}\right)$, $3.75\left(\mathrm{~s}, 3 \mathrm{H}^{*}\right), 3.60(\mathrm{~s}, 3 \mathrm{H}), 2.47-2.43(\mathrm{~m}, 1 \mathrm{H}), 2.38-2.36(\mathrm{~m}, 1 \mathrm{H}), 2.33-2.27(\mathrm{~m}, 2 \mathrm{H})$, $2.07(\mathrm{~s}, 3 \mathrm{H}), 2.06\left(\mathrm{~s}, 3 \mathrm{H}^{*}\right) .{ }^{13} \mathrm{C} \mathrm{NMR}\left(100 \mathrm{MHz}, \mathrm{CDCl}_{3}\right) \delta 172.4,172.3,159.4(\mathrm{~d}, J=$ $239.0 \mathrm{~Hz}), 149.2$, 148.1, 135.9, 135.8, 133.04, 133.01, 132.97, 132.9, 131.1 (d, $J=9.0$ $\mathrm{Hz}), 129.9,128.7,128.6,117.8,117.6,117.3,117.2,117.1,112.5$ (d, $J=25.0 \mathrm{~Hz})$, 
$112.4(\mathrm{~d}, J=25.0 \mathrm{~Hz}), 104.1(\mathrm{~d}, J=5.0 \mathrm{~Hz}), 103.9(\mathrm{~d}, J=5.0 \mathrm{~Hz}), 61.4,60.9,35.6$, 35.4, 25.0, 21.2, 9.0. $\left.{ }^{19} \mathrm{~F} \mathrm{NMR} \mathrm{(376} \mathrm{MHz,} \mathrm{CDCl}_{3}\right) \delta-119.1 \sim-119.3(\mathrm{~m}, 1 \mathrm{~F})$. IR (thin film): $v_{\max }\left(\mathrm{cm}^{-1}\right)=2937,2817,1695,1613,1596,1467,1451,1369,1284,1201,1166$, 1139, 1116, 1042, 943, 881, 849, 808, 775, 727, 700, 665, 603, 569. HRMS (ESI) calcd for $\mathrm{C}_{20} \mathrm{H}_{19} \mathrm{~N}_{2} \mathrm{NaFO}_{2}[\mathrm{M}+\mathrm{Na}]^{+}:$361.1323. Found: 361.1320 .

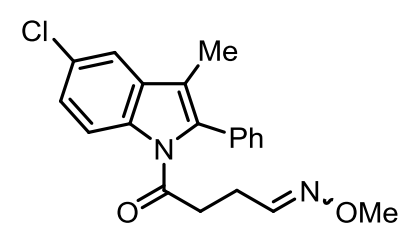

3f, colorless oil, $0.50 \mathrm{~g}, 56 \%$ yield (3 mmol scale), $E / Z=1.3 / 1 .{ }^{1} \mathrm{H}$ NMR (400 MHz, $\left.\mathrm{CDCl}_{3}\right) \delta 8.31-8.27(\mathrm{~m}, 1 \mathrm{H}), 7.48-7.44(\mathrm{~m}, 4 \mathrm{H})$, 7.38-7.37 (m, 2H), 7.27-7.24 (m, 1H), $7.21(\mathrm{t}, J=4.8 \mathrm{~Hz}, 1 \mathrm{H}), 6.46\left(\mathrm{t}, J=5.2 \mathrm{~Hz}, 1 \mathrm{H}^{*}\right), 3.75\left(\mathrm{~s}, 3 \mathrm{H}^{*}\right), 3.59(\mathrm{~s}, 3 \mathrm{H}), 2.47-2.43$ (m, 1H), 2.39-2.36 (m, 1H), 2.33-2.27 (m, 2H), $2.08(\mathrm{~s}, 3 \mathrm{H}), 2.07\left(\mathrm{~s}, 3 \mathrm{H}^{*}\right) .{ }^{13} \mathrm{C} \mathrm{NMR}$ $\left(100 \mathrm{MHz} \mathrm{CDCl}_{3}\right) \delta 172.6,172.4,149.2,148.1,135.7,135.6,135.01,134.95,132.90$, $132.87,131.4,129.9,128.8,128.7,125.1,125.0,118.2,118.1,117.5,117.3,117.2$, 117.1, 61.4, 61.0, 35.7, 35.5, 25.0, 21.2, 9.0. IR (thin film): $v_{\max }\left(\mathrm{cm}^{-1}\right)=2936,1684$, 1590, 1446, 1373, 1342, 1277, 1248, 1182, 1135, 1033, 925, 888, 846, 822, 768, 740, 707, 665, 594, 559, 491. HRMS (ESI) calcd for $\mathrm{C}_{20} \mathrm{H}_{19} \mathrm{~N}_{2} \mathrm{NaClO}_{2}[\mathrm{M}+\mathrm{Na}]^{+}: 377.1027$. Found: 377.1024.

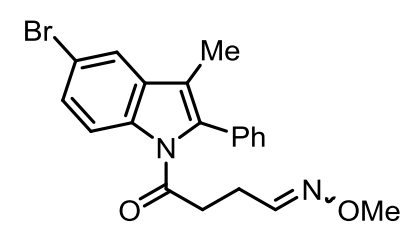

3g, colorless oil, $0.52 \mathrm{~g}, 35 \%$ yield (3 mmol scale), $E / Z=1.5 / 1 .{ }^{1} \mathrm{H}$ NMR (400 MHz, $\left.\mathrm{CDCl}_{3}\right) \delta 8.26-8.22(\mathrm{~m}, 1 \mathrm{H}), 7.61(\mathrm{~s}, 1 \mathrm{H}), 7.48-7.36(\mathrm{~m}, 6 \mathrm{H}), 7.20(\mathrm{t}, J=4.8 \mathrm{~Hz}, 1 \mathrm{H})$, $6.46\left(\mathrm{t}, J=5.2 \mathrm{~Hz}, 1 \mathrm{H}^{*}\right), 3.75\left(\mathrm{~s}, 3 \mathrm{H}^{*}\right), 3.59$ (s, 3H), 2.47-2.42 (m, 1H), 2.39-2.36 (m, 1H), 2.32-2.26 (m, 2H), $2.08(\mathrm{~s}, 3 \mathrm{H}), 2.07$ (s, 3H*). $\left.{ }^{13} \mathrm{C} \mathrm{NMR} \mathrm{(100} \mathrm{MHz,} \mathrm{CDCl}_{3}\right) \delta$ 172.6, 172.5, 149.2, 148.1, 135.5, 135.40, 135.37, 135.3, 132.83, 132.81, 131.9, 129.92, 129.90, 128.8, 128.7, 127.8, 127.7, 121.3, 121.2, 117.6, 117.5, 117.4, 117.2, 116.5, 116.4, 61.5, 61.0, 35.7, 35.5, 25.0, 21.2, 9.1. IR (thin film): $v_{\max }\left(\mathrm{cm}^{-1}\right)=2933,2813$, 1688, 1588, 1444, 1371, 1335, 1290, 1182, 1140, 1034, 924, 863, 840, 815, 762, 737, 
704, 660, 614, 588, 554. HRMS (ESI) calcd for $\mathrm{C}_{20} \mathrm{H}_{19} \mathrm{~N}_{2} \mathrm{NaBrO}_{2}[\mathrm{M}+\mathrm{Na}]^{+}$: 421.0522 . Found: 421.0520.

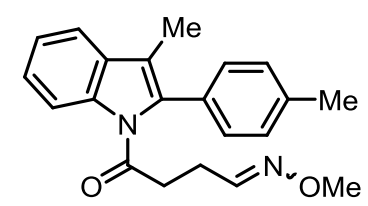

3h, brown oil, $0.42 \mathrm{~g}, 32 \%$ yield (4 mmol scale), $E / Z=1.2 / 1 .{ }^{1} \mathrm{H}$ NMR (400 MHz, $\left.\mathrm{CDCl}_{3}\right) \delta 8.40-8.37(\mathrm{~m}, 1 \mathrm{H}), 7.47(\mathrm{~d}, J=7.6 \mathrm{~Hz}, 1 \mathrm{H}), 7.34-7.22(\mathrm{~m}, 7 \mathrm{H}), 6.47(\mathrm{t}, J=$ $\left.5.2 \mathrm{~Hz}, 1 \mathrm{H}^{*}\right), 3.75$ (s, 3H*), 3.61 (s, 3H), 2.48-2.30 (m, 4H), $2.41(\mathrm{~s}, 3 \mathrm{H}), 2.11(\mathrm{~s}, 3 \mathrm{H})$, $2.10\left(\mathrm{~s}, 3 \mathrm{H}^{*}\right) .{ }^{13} \mathrm{C} \mathrm{NMR}\left(100 \mathrm{MHz}, \mathrm{CDCl}_{3}\right) \delta 172.7,172.6,149.5,148.4,138.3,138.2$, 136.7, 136.6, 134.5, 134.4, 130.34, 130.31, 130.14, 130.12, 129.8, 129.3, 125.1, 125.0, 123.3, 123.2, 118.39, 118.36, 118.0, 117.8, 116.0, 115.9, 61.4, 60.9, 35.8, 35.5, 25.0, 21.2, 9.1. IR (thin film): $v_{\max }\left(\mathrm{cm}^{-1}\right)=2919,1693,1509,1451,1368,1313,1285,1183$, 1155, 1134, 1109, 1043, 935, 911, 881, 841, 824, 792, 747, 702, 683, 628, 506. HRMS (ESI) calcd for $\mathrm{C}_{21} \mathrm{H}_{22} \mathrm{~N}_{2} \mathrm{NaO}_{2}[\mathrm{M}+\mathrm{Na}]^{+}: 357.1574$. Found: 357.1576 .

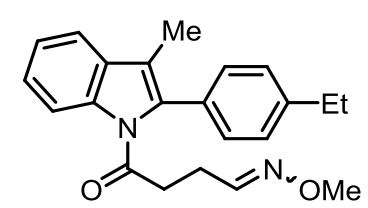

3i, brown oil, $0.32 \mathrm{~g}$, 24\% yield (4 mmol scale), $E / Z=1.2 / 1 .{ }^{1} \mathrm{H}$ NMR (400 MHz, $\left.\mathrm{CDCl}_{3}\right) \delta 8.40-8.37(\mathrm{~m}, 1 \mathrm{H}), 7.47(\mathrm{~d}, J=7.2 \mathrm{~Hz}, 1 \mathrm{H}), 7.33-7.27(\mathrm{~m}, 6 \mathrm{H}), 7.20-7.17(\mathrm{~m}$, $1 \mathrm{H}), 6.45\left(\mathrm{t}, J=5.2 \mathrm{~Hz}, 1 \mathrm{H}^{*}\right), 3.74\left(\mathrm{~s}, 3 \mathrm{H}^{*}\right), 3.59$ (s, 3H), $2.70(\mathrm{q}, J=7.6 \mathrm{~Hz}, 2 \mathrm{H})$, 2.48-2.43 (m, 1H), 2.38-2.28 (m, 3H), $2.11(\mathrm{~s}, 3 \mathrm{H}), 2.10\left(\mathrm{~s}, 3 \mathrm{H}^{*}\right), 1.28(\mathrm{t}, J=7.6 \mathrm{~Hz}$, $3 \mathrm{H}) .{ }^{13} \mathrm{C} \mathrm{NMR}\left(100 \mathrm{MHz}, \mathrm{CDCl}_{3}\right) \delta 172.7,172.6,149.4,148.3,144.6,144.4,136.7$, $136.6,134.5$, 134.4, 130.50, 130.49, 130.12, 130.10, 129.82, 129.80, 128.1, 125.0, 124.9, 123.2, 123.1, 118.4, 118.3, 117.9, 117.7, 115.94, 115.85, 61.3, 60.9, 35.8, 35.4, 28.5, 25.0, 21.2, 15.2, 15.2, 9.1. IR (thin film): $v_{\max }\left(\mathrm{cm}^{-1}\right)=2964,2933,1694,1451$, 1410, 1368, 1314, 1284, 1183, 1155, 1135, 1044, 935, 911, 881, 833, 747, 682, 629, 539, 515, 455. HRMS (ESI) calcd for $\mathrm{C}_{22} \mathrm{H}_{24} \mathrm{~N} 2 \mathrm{NaO}_{2}[\mathrm{M}+\mathrm{Na}]^{+}:$371.1730. Found: 371.1723. 


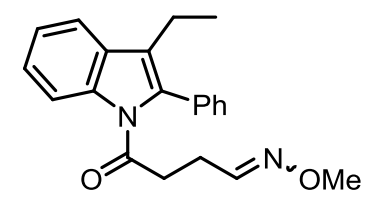

3j, yellow oil, $0.27 \mathrm{~g}$, 25\% yield (3 mmol scale), $E / Z=1.2 / 1 .{ }^{1} \mathrm{H}$ NMR (400 MHz, $\left.\mathrm{CDCl}_{3}\right) \delta 8.43-8.40(\mathrm{~m}, 1 \mathrm{H}), 7.55(\mathrm{~d}, J=7.2 \mathrm{~Hz}, 1 \mathrm{H}), 7.45-7.26(\mathrm{~m}, 7 \mathrm{H}), 7.22-7.19(\mathrm{~m}$, $1 \mathrm{H}), 6.47\left(\mathrm{t}, J=5.2 \mathrm{~Hz}, 1 \mathrm{H}^{*}\right), 3.75\left(\mathrm{~s}, 3 \mathrm{H}^{*}\right), 3.61(\mathrm{~s}, 3 \mathrm{H}), 2.58-2.53(\mathrm{~m}, 2 \mathrm{H}), 2.48-2.43$ $(\mathrm{m}, 1 \mathrm{H}), 2.39-2.36(\mathrm{~m}, 1 \mathrm{H}), 2.33-2.26(\mathrm{~m}, 2 \mathrm{H}), 1.18-1.15(\mathrm{~m}, 3 \mathrm{H}) .{ }^{13} \mathrm{C}$ NMR $(100 \mathrm{MHz}$, $\left.\mathrm{CDCl}_{3}\right) \delta 172.6,172.5,149.4,148.3,136.9,136.8,133.9,133.8,133.37,133.35,129.90$, 129.88, 129.11, 129.09, 128.6, 128.5, 128.4, 125.1, 125.0, 124.4, 124.2, 123.3, 123.2, 118.62, 118.59, 116.24, 116.16, 61.4, 61.0, 35.8, 35.5, 25.0, 21.2, 17.4, 14.84, 14.82. IR (thin film): $v_{\max }\left(\mathrm{cm}^{-1}\right)=2964,2933,2873,1694,1603,1452,1369,1319,1285$, 1183, 1156, 1135, 1081, 1040, 908, 880, 859, 746, 701, 670, 497, 431. HRMS (ESI) calcd for $\mathrm{C}_{21} \mathrm{H}_{22} \mathrm{~N}_{2} \mathrm{NaO}_{2}[\mathrm{M}+\mathrm{Na}]^{+}:$357.1574. Found: 357.1570 .

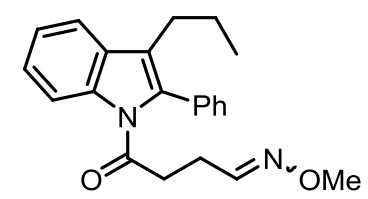

3k, yellow oil, $0.40 \mathrm{~g}, 33 \%$ yield (4 mmol scale), $E / Z=1.2 / 1 .{ }^{1} \mathrm{H}$ NMR (400 MHz, $\left.\mathrm{CDCl}_{3}\right) \delta 8.44-8.40(\mathrm{~m}, 1 \mathrm{H}), 7.54(\mathrm{~d}, J=7.2 \mathrm{~Hz}, 1 \mathrm{H}), 7.44-7.24(\mathrm{~m}, 7 \mathrm{H}), 7.21-7.18(\mathrm{~m}$, $1 \mathrm{H}), 6.45\left(\mathrm{t}, J=5.2 \mathrm{~Hz}, 1 \mathrm{H}^{*}\right), 3.74\left(\mathrm{~s}, 3 \mathrm{H}^{*}\right), 3.59$ (s, 3H), 2.53-2.42 (m, 3H), 2.37-2.34 $(\mathrm{m}, 1 \mathrm{H}), 2.31-2.24(\mathrm{~m}, 2 \mathrm{H}), 1.62-1.57(\mathrm{~m}, 2 \mathrm{H}), 0.85(\mathrm{~d}, J=7.2 \mathrm{~Hz}, 3 \mathrm{H}) .{ }^{13} \mathrm{C}$ NMR $(100$ $\left.\mathrm{MHz}, \mathrm{CDCl}_{3}\right) \delta 172.53,172.46,149.3,148.2,136.8,136.7,134.3,134.2,133.39$, $133.35,129.98,129.96,129.39,129.37,128.5,128.43,128.35,125.0,124.9,123.2$, 123.1, 122.8, 122.6, 118.74, 118.71, 116.13, 116.05, 61.3, 60.9, 35.7, 35.5, 26.1, 24.9, 23.22, 23.19, 21.1, 14.0. IR (thin film): $v_{\max }\left(\mathrm{cm}^{-1}\right)=2957,2933,2869,1694,1452$, 1370, 1317, 1285, 1184, 1155, 1136, 1089, 1043, 937, 916, 880, 855, 746, 701, 640. HRMS (ESI) calcd for $\mathrm{C}_{22} \mathrm{H}_{24} \mathrm{~N}_{2} \mathrm{NaO}_{2}[\mathrm{M}+\mathrm{Na}]^{+}$: 371.1730. Found: 371.1727.

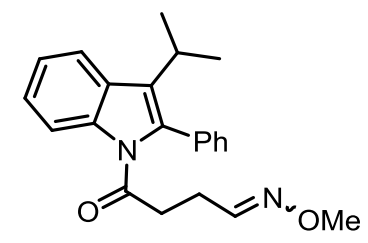


31, coloeless oil, $0.22 \mathrm{~g}, 17 \%$ yield (4 mmol scale), $E / Z=2.1 / 1 .{ }^{1} \mathrm{H}$ NMR (400 MHz, $\left.\mathrm{CDCl}_{3}\right) \delta 8.46-8.42(\mathrm{~m}, 1 \mathrm{H}), 7.74(\mathrm{~d}, J=7.6 \mathrm{~Hz}, 1 \mathrm{H}), 7.48-7.41(\mathrm{~m}, 3 \mathrm{H}), 7.38-7.24(\mathrm{~m}$, 4H), 7.22-7.20 (m, 1H), $6.46\left(\mathrm{t}, J=5.2 \mathrm{~Hz}, 1 \mathrm{H}^{*}\right), 3.76\left(\mathrm{~s}, 3 \mathrm{H}^{*}\right), 3.63(\mathrm{~s}, 3 \mathrm{H}), 2.96-2.87$ (m, 1H), 2.47-2.35 (m, 2H), 2.28-2.21 (m, 2H), $1.34(\mathrm{~d}, J=6.8 \mathrm{~Hz}, 6 \mathrm{H}) .{ }^{13} \mathrm{C}$ NMR $(100$ $\left.\mathrm{MHz}, \mathrm{CDCl}_{3}\right) \delta 172.70,172.65,149.5,148.4,137.23,137.18,133.7,133.1,133.0$, $130.3,128.7,128.6,127.8,127.7,127.6,124.8,124.7,123.0,122.9,120.3,116.4,116.3$ $61.4,61.0,35.9,35.6,25.9,25.0,22.2,21.2$. IR (thin film): $v_{\max }\left(\mathrm{cm}^{-1}\right)=2961,2932$, 2871, 2816, 1694, 1601, 1447, 1371, 1280, 1197, 1157, 1130, 1088, 1044, 915, 880, 856, 799, 746, 701, 602, 497, 434. HRMS (ESI) calcd for $\mathrm{C}_{22} \mathrm{H}_{24} \mathrm{~N}_{2} \mathrm{NaO}_{2}[\mathrm{M}+\mathrm{Na}]^{+}$: 371.1730. Found: 371.1728 .

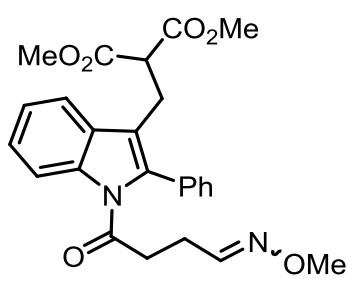

3m, yellow oil, $0.25 \mathrm{~g}, 28 \%$ yield ( $2 \mathrm{mmol}$ scale), $E / Z=1.6 / 1 .{ }^{1} \mathrm{H}$ NMR $(400 \mathrm{MHz}$, $\left.\mathrm{CDCl}_{3}\right) \delta 8.37(\mathrm{t}, J=7.6 \mathrm{~Hz}, 1 \mathrm{H}), 7.56-7.46(\mathrm{~m}, 4 \mathrm{H}), 7.43-7.41(\mathrm{~m}, 2 \mathrm{H}), 7.37-7.29(\mathrm{~m}$, 2H), $7.21(\mathrm{t}, J=5.2 \mathrm{~Hz}, 1 \mathrm{H}), 6.46\left(\mathrm{t}, J=5.2 \mathrm{~Hz}, 1 \mathrm{H}^{*}\right), 3.75(\mathrm{~s}, 1 \mathrm{H}), 3.62-3.51(\mathrm{~m}, 1 \mathrm{H})$, $3.59(\mathrm{~s}, 2 \mathrm{H}), 3.54(\mathrm{~s}, 6 \mathrm{H}), 3.27-3.24(\mathrm{~m}, 2 \mathrm{H}), 2.47-2.36(\mathrm{~m}, 2 \mathrm{H}), 2.31-2.25(\mathrm{~m}, 2 \mathrm{H})$. ${ }^{13} \mathrm{C}$ NMR $\left(100 \mathrm{MHz} \mathrm{CDCl}_{3}\right) \delta 172.7,172.6,168.89,168.86,149.2,148.1,136.62$, $136.57,135.9,135.8,132.58,132.55,130.00,129.97,129.0,128.9,128.8,128.5,125.4$, $125.3,123.5,123.3,118.6,118.5,117.9,117.7,116.1,116.0,61.4,61.0,52.4,51.18$, 51.15, 35.7, 35.5, 24.9, 23.5, 21.1. IR (thin film): $v_{\max }\left(\mathrm{cm}^{-1}\right)=2951,2849,1733,1699$, 1445, 1369, 1278, 1183, 1151, 1040, 910, 881, 854, 802, 750, 701, 636, 560, 487, 447. HRMS (ESI) calcd for $\mathrm{C}_{25} \mathrm{H}_{26} \mathrm{~N}_{2} \mathrm{NaO}_{6}[\mathrm{M}+\mathrm{Na}]^{+}:$473.1683. Found: 473.1671.

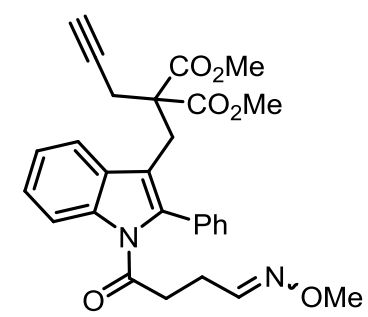

3n, yellow oil ${ }^{3}, 0.20 \mathrm{~g}, 21 \%$ yield ( $\left.2 \mathrm{mmol} \mathrm{scale}\right), E / Z=1.6 / 1 .{ }^{1} \mathrm{H}$ NMR $(400 \mathrm{MHz}$, $\left.\mathrm{CDCl}_{3}\right) \delta 8.24(\mathrm{t}, J=8.8 \mathrm{~Hz}, 1 \mathrm{H}), 7.68(\mathrm{~d}, J=7.6 \mathrm{~Hz}, 1 \mathrm{H}), 7.51-7.44(\mathrm{~m}, 5 \mathrm{H}), 7.35-$ 
$7.28(\mathrm{~m}, 1 \mathrm{H}), 7.27-7.23(\mathrm{~m}, 1 \mathrm{H}), 7.17(\mathrm{t}, J=5.2 \mathrm{~Hz}, 1 \mathrm{H}), 6.42\left(\mathrm{t}, J=5.2 \mathrm{~Hz}, 1 \mathrm{H}^{*}\right)$, $3.75\left(\mathrm{~s}, 3 \mathrm{H}^{*}\right), 3.63(\mathrm{~s}, 2 \mathrm{H}), 3.61\left(\mathrm{~s}, 2 \mathrm{H}^{*}\right), 3.56(\mathrm{~s}, 3 \mathrm{H}), 3.47(\mathrm{~s}, 6 \mathrm{H}), 2.52-2.49(\mathrm{~m}, 2 \mathrm{H})$, 2.44-2.34 (m, 2H), 2.20-2.14 (m, 2H), 1.74 (s, 1H), 1.73 (s, $\left.1 \mathrm{H}^{*}\right) .{ }^{13} \mathrm{C}$ NMR (100 MHz, $\left.\mathrm{CDCl}_{3}\right) \delta 173.4,173.2,170.2,149.1,148.0,137.4,137.3,136.3,136.2,132.5,132.4$, 130.82 , 130.80, 129.4, 129.2, 128.94, 128.86, 125.3, 125.2, 123.2, 123.0, 119.20, 119.16, 115.4, 115.3, 115.2, 115.1, 78.74, 78.69, 71.0, 61.5, 61.0, 58.0, 57.9, 52.6, 35.9, 35.7, 26.0, 25.2, 23.1, 21.3. IR (thin film): $v_{\max }\left(\mathrm{cm}^{-1}\right)=3285,2951,2869,1733,1700$, 1604, 1444, 1367, 1288, 1197, 1139, 1040, 972, 877, 809, 751, 701, 641, 580, 516. HRMS (ESI) calcd for $\mathrm{C}_{28} \mathrm{H}_{28} \mathrm{~N}_{2} \mathrm{NaO}_{6}[\mathrm{M}+\mathrm{Na}]^{+}:$511.1840. Found: 511.1848.

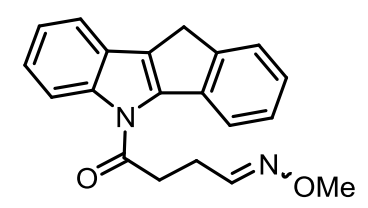

3o, brown solid, $0.65 \mathrm{~g}, 54 \%$ yield (4 mmol scale), $E / Z=1.2 / 1$, m.p. $=109.7-111.7^{\circ} \mathrm{C}$. ${ }^{1} \mathrm{H}$ NMR (400 MHz, $\left.\mathrm{CDCl}_{3}\right) \delta 8.06(\mathrm{~d}, J=5.2 \mathrm{~Hz}, 1 \mathrm{H}), 8.04$ (d, $\left.J=5.2 \mathrm{~Hz}, 1 \mathrm{H}^{*}\right), 7.42$ $(\mathrm{t}, J=5.2 \mathrm{~Hz}, 1 \mathrm{H}), 7.22-7.12(\mathrm{~m}, 3 \mathrm{H}), 7.08-6.96(\mathrm{~m}, 4 \mathrm{H}), 6.71\left(\mathrm{t}, J=5.2 \mathrm{~Hz}, 1 \mathrm{H}^{*}\right)$, $3.88\left(\mathrm{~s}, 3 \mathrm{H}^{*}\right), 3.77(\mathrm{~s}, 3 \mathrm{H}), 2.85(\mathrm{~s}, 2 \mathrm{H}), 2.82\left(\mathrm{~s}, 2 \mathrm{H}^{*}\right), 2.65-2.58(\mathrm{~m}, 3 \mathrm{H}), 2.49-2.44$ (m, 1H). ${ }^{13} \mathrm{C} \mathrm{NMR}\left(100 \mathrm{MHz}, \mathrm{CDCl}_{3}\right) \delta 169.48,169.46,149.5,148.6,146.9,146.8$, $143.7,143.6,138.1,137.9,135.20,135.18,128.38,128.36,126.30,126.29,126.26$, $126.2,124.84,124.81,124.39,124.37,123.2$, 123.1, 123.0, 122.6, 118.78, 118.76, 114.8, 114.6, 61.5, 61.1, 34.5, 34.2, 28.84, 28.81, 24.4, 20.8. IR (thin film): $v_{\max }\left(\mathrm{cm}^{-1}\right)$ =2929, 2812, 1696, 1604, 1449, 1416, 1387, 1342, 1311, 1283, 1209, 1173, 1108, 1080, 1043, 919, 885, 855, 813, 792, 761, 732, 670, 611. HRMS (ESI) calcd for $\mathrm{C}_{20} \mathrm{H}_{18} \mathrm{~N}_{2} \mathrm{NaO}_{2}[\mathrm{M}+\mathrm{Na}]^{+}:$341.1261. Found: 341.1264.

General procedure for visible-light-induced dearomatization reaction (using 1a as an example). 


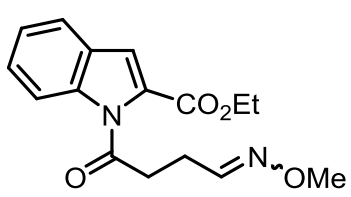

$1 a$

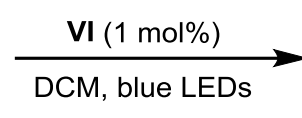

DCM, blue LEDs

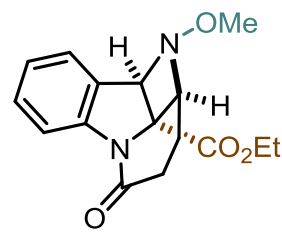

$2 \mathbf{a}$

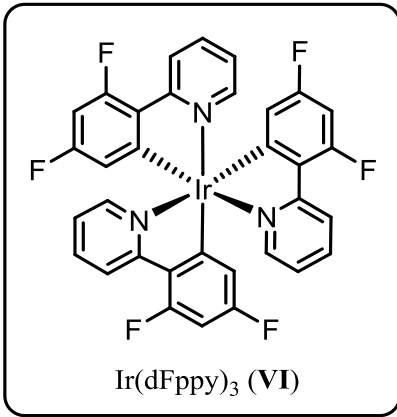

To a flame-dried sealed tube were added indole derivative $1 \mathrm{a}(60.0 \mathrm{mg}, 0.2 \mathrm{mmol})$ and photosensitizer VI $(1.5 \mathrm{mg}, 0.002 \mathrm{mmol})$ and anhydrous DCM $(20 \mathrm{~mL})$. The reaction mixture was degassed via freeze-pump-thaw for 3 cycles. After the reaction mixture was thoroughly degassed, the vial was sealed and positioned approximately $10 \mathrm{~cm}$ from $24 \mathrm{~W}$ blue LEDs $(450 \mathrm{~nm})$. Then the reaction mixture was stirred at room temperature for the indicated time (monitored by TLC) under argon atmosphere. Afterwards, the reaction mixture was concentrated by rotary evaporation. Then the residue was purified by silica gel column chromatography $(\mathrm{PE} / \mathrm{EtOAc}=8 / 1)$ to afford the desired product 2a. The analytical data of the products are summarized below, and the data of minor isomers are indicated by an asterisk $(*)$.

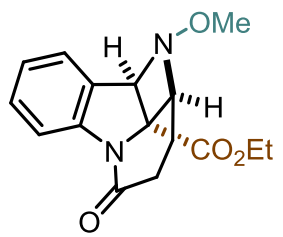

2a, colorless oil, $69.0 \mathrm{mg}$, 98\% yield. ${ }^{1} \mathrm{H}$ NMR $\left(400 \mathrm{MHz}, \mathrm{CDCl}_{3}\right) \delta 7.87(\mathrm{~d}, J=7.6$ $\mathrm{Hz}, 1 \mathrm{H}), 7.34-7.28(\mathrm{~m}, 2 \mathrm{H}), 7.11(\mathrm{t}, J=7.6 \mathrm{~Hz}, 1 \mathrm{H}), 4.80(\mathrm{~s}, 1 \mathrm{H}), 4.46-4.43(\mathrm{~m}, 1 \mathrm{H})$, 4.30-4.17 (m, 2H), 3.49 (s, 3H), 2.50-2.44 (m, 2H), 2.38-2.29 (m, 1H), 2.09-2.02 (m, $1 \mathrm{H}), 1.28(\mathrm{t}, J=7.2 \mathrm{~Hz}, 3 \mathrm{H}) .{ }^{13} \mathrm{C} \mathrm{NMR}\left(100 \mathrm{MHz}, \mathrm{CDCl}_{3}\right) \delta 170.2,168.0,146.5,129.8$, $129.5,124.6,123.7,118.4,74.0,67.1,66.1,62.4,61.4,32.8,26.5,14.0$. IR (thin film): $v_{\max }\left(\mathrm{cm}^{-1}\right)=2934,2851,1733,1686,1604,1462,1367,1293,1228,1140,1107,1081$, 1060, 1035, 979, 881, 862, 815, 752, 665, 600, 555. HRMS (ESI) calcd for $\mathrm{C}_{16} \mathrm{H}_{18} \mathrm{~N}_{2} \mathrm{NaO}_{4}[\mathrm{M}+\mathrm{Na}]^{+}:$325.1159. Found: 325.1160. 


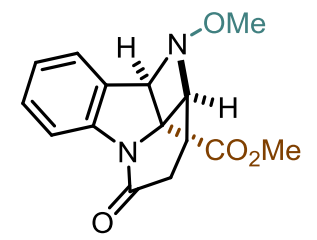

2b, colorless oil, $57.0 \mathrm{mg}, 98 \%$ yield. ${ }^{1} \mathrm{H}$ NMR (400 MHz, $\left.\mathrm{CDCl}_{3}\right) \delta 7.87(\mathrm{~d}, J=8.0$ $\mathrm{Hz}, 1 \mathrm{H}), 7.35-7.29$ (m, 2H), $7.11(\mathrm{t}, J=7.6 \mathrm{~Hz}, 1 \mathrm{H}), 4.80(\mathrm{~s}, 1 \mathrm{H}), 4.48-4.44(\mathrm{~m}, 1 \mathrm{H})$, $3.78(\mathrm{~s}, 3 \mathrm{H}), 3.49(\mathrm{~s}, 3 \mathrm{H}), 2.51-2.43(\mathrm{~m}, 2 \mathrm{H}), 2.36-2.28(\mathrm{~m}, 1 \mathrm{H}), 2.11-2.01(\mathrm{~m}, 1 \mathrm{H}) .{ }^{13} \mathrm{C}$ NMR (100 MHz, $\left.\mathrm{CDCl}_{3}\right) \delta 170.3,168.5,146.6,129.8,129.6,124.7,123.8,118.5,74.2$, 67.1, 66.1, 61.5, 53.2, 32.8, 26.5. IR (thin film): $v_{\max }\left(\mathrm{cm}^{-1}\right)=2955,1743,1685,1603$, 1460, 1435, 1361, 1289, 1224, 1134, 1107, 1075, 1034, 1003, 937, 906, 869, 809, 761, 733, 697, 639. HRMS (ESI) calcd for $\mathrm{C}_{15} \mathrm{H}_{16} \mathrm{~N}_{2} \mathrm{NaO}_{4}[\mathrm{M}+\mathrm{Na}]^{+}:$311.1002. Found: 311.1003 .

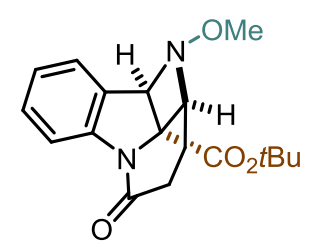

2c, brown oil, $41.0 \mathrm{mg}, 63 \%$ yield. ${ }^{1} \mathrm{H}$ NMR $\left(400 \mathrm{MHz}, \mathrm{CDCl}_{3}\right) \delta 7.87(\mathrm{~d}, J=8.0 \mathrm{~Hz}$, 1H), 7.34-7.27 (m, 2H), $7.10(\mathrm{t}, J=7.6 \mathrm{~Hz}, 1 \mathrm{H}), 4.75(\mathrm{~s}, 1 \mathrm{H}), 4.41-4.38(\mathrm{~m}, 1 \mathrm{H}), 3.48$ (s, 3H), 2.50-2.43 (m, 2H), 2.38-2.29 (m, 1H), 2.08-1.99 (m, 1H), $1.46(\mathrm{~s}, 9 \mathrm{H}) .{ }^{13} \mathrm{C}$ NMR (100 MHz, $\left.\mathrm{CDCl}_{3}\right) \delta 170.3,167.0,146.7,130.0,129.5,124.6,123.8,118.4,83.5$, 74.0, 67.2, 66.7, 61.4, 32.8, 27.8, 26.5. IR (thin film): $v_{\max }\left(\mathrm{cm}^{-1}\right)=2927,1726,1679$, 1601, 1461, 1364, 1310, 1291, 1244, 1150, 1106, 1080, 1061, 1034, 978, 884, 836, 788, 757, 730, 694, 644. HRMS (ESI) calcd for $\mathrm{C}_{18} \mathrm{H}_{22} \mathrm{~N}_{2} \mathrm{NaO}_{4}[\mathrm{M}+\mathrm{Na}]^{+}:$353.1472. Found: 353.1464 .

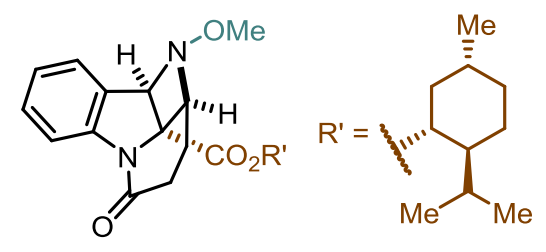

2d, colorless oil, $69.8 \mathrm{mg}, 87 \%$ yield, 1.1/1 dr. ${ }^{1} \mathrm{H}$ NMR $\left(400 \mathrm{MHz}, \mathrm{CDCl}_{3}\right) \delta 7.86(\mathrm{~d}$, $J=7.6 \mathrm{~Hz}, 1 \mathrm{H}), 7.34-7.28(\mathrm{~m}, 2 \mathrm{H}), 7.12-7.10(\mathrm{~m}, 1 \mathrm{H}), 4.76-4.71(\mathrm{~m}, 2 \mathrm{H}), 4.47-4.42$ (m, 1H), 3.49 (s, 3H), 2.50-2.47 (m, 2H), 2.37-2.22 (m, 1H), 2.10-1.94 (m, 2H), 1.69$1.42(\mathrm{~m}, 5 \mathrm{H}), 1.08-0.97(\mathrm{~m}, 2 \mathrm{H}), 0.93-0.78(\mathrm{~m}, 7 \mathrm{H}), 0.70(\mathrm{~d}, J=6.8 \mathrm{~Hz}, 3 \mathrm{H}), 0.65$ (d, 
$\left.J=6.8 \mathrm{~Hz}, 3 \mathrm{H}^{*}\right) .{ }^{13} \mathrm{C} \mathrm{NMR}\left(100 \mathrm{MHz}, \mathrm{CDCl}_{3}\right) \delta 170.2,170.0,167.6,167.5,146.6$, 129.9, 129.8, 129.52, 129.49, 124.60, 124.58, 123.8, 123.7, 118.43, 118.39, 76.9, 76.8, 74.3, 74.1, 67.01, 66.95, 66.4, 66.3, 61.5, 46.7, 46.6, 40.5, 40.4, 33.94, 33.91, 32.9, 32.8, $31.3,26.62,26.55,26.5,26.4,23.6,23.3,21.9,20.6,20.4,16.4,16.2$. IR (thin film): $v_{\max }\left(\mathrm{cm}^{-1}\right)=2954,2869,1728,1690,1605,1462,1367,1292,1230,1180,1140,1081$, 1060, 1034, 980, 949, 909, 751, 665, 598, 557, 465, 428. HRMS (ESI) calcd for $\mathrm{C}_{24} \mathrm{H}_{32} \mathrm{~N}_{2} \mathrm{NaO}_{4}[\mathrm{M}+\mathrm{Na}]^{+}:$435.2254. Found: 435.2246.

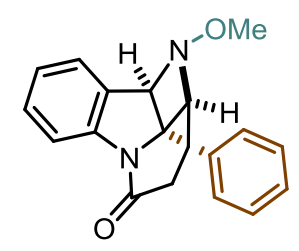

2e, colorless oil, $63.2 \mathrm{mg}, 97 \%$ yield. ${ }^{1} \mathrm{H}$ NMR $\left(400 \mathrm{MHz}, \mathrm{CDCl}_{3}\right) \delta 7.99(\mathrm{~d}, J=8.0$ $\mathrm{Hz}, 1 \mathrm{H}), 7.44$ (d, $J=7.6 \mathrm{~Hz}, 2 \mathrm{H}), 7.39-7.26(\mathrm{~m}, 5 \mathrm{H}), 7.09$ (t, $J=7.6 \mathrm{~Hz}, 1 \mathrm{H}), 4.57-$ 4.54 (m, 2H), 3.54 (s, 3H), 2.53-2.37 (m, 2H), 2.22-2.11 (m, 2H). ${ }^{13} \mathrm{C} \mathrm{NMR} \mathrm{(100} \mathrm{MHz,}$ $\left.\mathrm{CDCl}_{3}\right) \delta 170.7,146.7,138.2,130.4,129.3,128.9,128.1,125.3,124.7,124.2,118.4$, 78.9, 70.1, 68.6, 61.3, 32.6, 27.2. IR (thin film): $v_{\max }\left(\mathrm{cm}^{-1}\right)=2929,1676,1604,1473$, 1379, 1315, 1289, 1244, 1186, 1139, 1102, 1082, 1062, 992, 975, 871, 826, 766, 751, 701, 671, 607, 557. HRMS (ESI) calcd for $\mathrm{C}_{19} \mathrm{H}_{18} \mathrm{~N}_{2} \mathrm{NaO}_{2}[\mathrm{M}+\mathrm{Na}]^{+}: 329.1261$. Found: 329.1256.

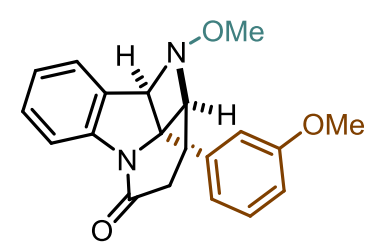

2f, yellow oil, $50.5 \mathrm{mg}, 97 \%$ yield. ${ }^{1} \mathrm{H}$ NMR (400 $\left.\mathrm{MHz} \mathrm{CDCl}_{3}\right) \delta 7.97(\mathrm{~d}, J=8.0 \mathrm{~Hz}$, 1H), 7.35-7.26 (m, 3H), 7.09 (t, $J=7.6 \mathrm{~Hz}, 1 \mathrm{H}), 7.03(\mathrm{~d}, J=7.6 \mathrm{~Hz}, 1 \mathrm{H}), 6.96(\mathrm{~s}, 1 \mathrm{H})$, $6.82(\mathrm{~d}, J=8.4 \mathrm{~Hz}, 1 \mathrm{H}), 4.55-4.53(\mathrm{~m}, 2 \mathrm{H}), 3.78$ (s, 3H), 3.54 (s, 3H), 2.51-2.36 (m, $2 \mathrm{H}), 2.23-2.13(\mathrm{~m}, 2 \mathrm{H}) .{ }^{13} \mathrm{C} \mathrm{NMR}\left(100 \mathrm{MHz}, \mathrm{CDCl}_{3}\right) \delta 170.7,160.0,146.7,140.0$, $130.4,130.1,129.4,124.7,124.2$, 118.5, 117.7, 112.8, 111.7, 78.9, 70.1, 68.6, 61.4, 55.3, 32.6, 27.2. IR (thin film): $v_{\max }\left(\mathrm{cm}^{-1}\right)=2971,2935,1677,1604,1581,1489,1462$, 1431, 1372, 1315, 1289, 1247, 1170, 1139, 1083, 1052, 1010, 974, 782, 756, 711, 692 , 661, 614. HRMS (ESI) calcd for $\mathrm{C}_{20} \mathrm{H}_{20} \mathrm{~N}_{2} \mathrm{NaO}_{3}[\mathrm{M}+\mathrm{Na}]^{+}$: 359.1366. Found: 359.1358. 


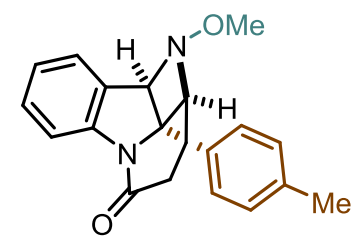

2g, brown oil, $47.7 \mathrm{mg}, 95 \%$ yield. ${ }^{1} \mathrm{H}$ NMR (400 $\left.\mathrm{MHz} \mathrm{CDCl}_{3}\right) \delta 7.98(\mathrm{~d}, J=8.0 \mathrm{~Hz}$, $1 \mathrm{H}), 7.35-7.31(\mathrm{~m}, 3 \mathrm{H}), 7.26(\mathrm{~d}, J=6.8 \mathrm{~Hz}, 1 \mathrm{H}), 7.18(\mathrm{~d}, J=7.6 \mathrm{~Hz}, 2 \mathrm{H}), 7.09$ (t, $J=$ $7.6 \mathrm{~Hz}, 1 \mathrm{H}), 4.54-4.51(\mathrm{~m}, 2 \mathrm{H}), 3.54(\mathrm{~s}, 3 \mathrm{H}), 2.52-2.45(\mathrm{~m}, 1 \mathrm{H}), 2.40(\mathrm{~d}, J=8.8 \mathrm{~Hz}$, $1 \mathrm{H}), 2.33(\mathrm{~s}, 3 \mathrm{H}), 2.22-2.12(\mathrm{~m}, 2 \mathrm{H}) .{ }^{13} \mathrm{C} \mathrm{NMR}\left(100 \mathrm{MHz}, \mathrm{CDCl}_{3}\right) \delta$ 170.7, 146.7, 138.0, 135.3, 130.5, 129.6, 129.3, 125.3, 124.7, 124.2, 118.4, 79.0, 70.2, 68.5, 61.4, 32.6, 27.2, 20.9. IR (thin film): $v_{\max }\left(\mathrm{cm}^{-1}\right)=2932,1678,1603,1513,1462,1372,1315$, 1291, 1247, 1216, 1189, 1140, 1082, 1062, 1035, 1005, 975, 815, 748, 665, 616, 573. HRMS (ESI) calcd for $\mathrm{C}_{20} \mathrm{H}_{20} \mathrm{~N}_{2} \mathrm{NaO}_{2}[\mathrm{M}+\mathrm{Na}]^{+}: 343.1417$. Found: 343.1411.

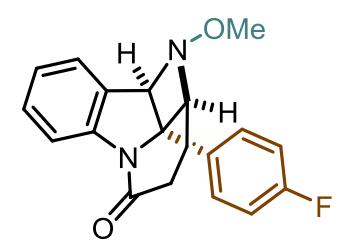

2h, colorless oil, $64.0 \mathrm{mg}$, 98\% yield. ${ }^{1} \mathrm{H}$ NMR $\left(400 \mathrm{MHz}, \mathrm{CDCl}_{3}\right) \delta 7.98(\mathrm{~d}, J=8.0$ $\mathrm{Hz}, 1 \mathrm{H}), 7.43-7.39$ (m, 2H), 7.35 (t, $J=8.0 \mathrm{~Hz}, 1 \mathrm{H}), 7.29-7.26(\mathrm{~m}, 1 \mathrm{H}), 7.13-7.04$ (m, $3 \mathrm{H}), 4.54-4.50(\mathrm{~m}, 2 \mathrm{H}), 3.54(\mathrm{~s}, 3 \mathrm{H}), 2.54-2.48(\mathrm{~m}, 1 \mathrm{H}), 2.44-2.38(\mathrm{~m}, 1 \mathrm{H}), 2.23-2.08$ $(\mathrm{m}, 2 \mathrm{H}) .{ }^{13} \mathrm{C}$ NMR $\left(100 \mathrm{MHz}, \mathrm{CDCl}_{3}\right) \delta 170.6,162.4(\mathrm{~d}, J=246.0 \mathrm{~Hz}), 146.6,134.1$ $(\mathrm{d}, J=3.0 \mathrm{~Hz}), 130.2,129.4,127.2(\mathrm{~d}, J=8.0 \mathrm{~Hz}), 124.8,124.3,118.5,115.9(\mathrm{~d}, J=$ 22.0 Hz), 79.0, 70.1, 68.2, 61.4, 32.6, 27.1. $\left.{ }^{19} \mathrm{~F} \mathrm{NMR} \mathrm{(376} \mathrm{MHz}, \mathrm{CDCl}_{3}\right) \delta-113.7$ 113.8 (m, 1F). IR (thin film): $v_{\max }\left(\mathrm{cm}^{-1}\right)=2936,1675,1605,1509,1473,1442,1378$, 1290, 1223, 1162, 1141, 1105, 1065, 1032, 995, 975, 839, 767, 616, 573, 557, 526. HRMS (ESI) calcd for $\mathrm{C}_{19} \mathrm{H}_{17} \mathrm{~N}_{2} \mathrm{NaFO}_{2}[\mathrm{M}+\mathrm{Na}]^{+}: 347.1166$. Found: 347.1160 .

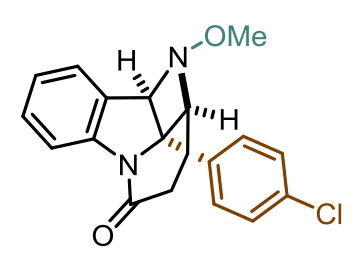

2i, yellow oil, $70.2 \mathrm{mg}, 97 \%$ yield. ${ }^{1} \mathrm{H}$ NMR (400 MHz, $\left.\mathrm{CDCl}_{3}\right) \delta 7.97(\mathrm{~d}, J=8.0 \mathrm{~Hz}$, $1 \mathrm{H}), 7.39-7.33(\mathrm{~m}, 5 \mathrm{H}), 7.28-7.26(\mathrm{~m}, 1 \mathrm{H}), 7.11(\mathrm{t}, J=7.6 \mathrm{~Hz}, 1 \mathrm{H}), 4.53-4.50(\mathrm{~m}, 2 \mathrm{H})$, $3.53(\mathrm{~s}, 3 \mathrm{H}), 2.54-2.40(\mathrm{~m}, 2 \mathrm{H}), 2.23-2.04(\mathrm{~m}, 2 \mathrm{H}) .{ }^{13} \mathrm{C} \mathrm{NMR}\left(100 \mathrm{MHz}, \mathrm{CDCl}_{3}\right) \delta$ 
$170.5,146.5,136.8,134.2,130.1,129.5,129.1,126.8,124.9,124.2,118.5,78.9,70.0$, 68.2, 61.4, 32.6, 27.1. IR (thin film): $v_{\max }\left(\mathrm{cm}^{-1}\right)=2933,1679,1602,1492,1462,1370$, 1315, 1291, 1247, 1216, 1176, 1140, 1092, 1062, 1034, 872, 825, 750, 664, 612, 558, 519. HRMS (ESI) calcd for $\mathrm{C}_{19} \mathrm{H}_{17} \mathrm{~N}_{2} \mathrm{NaClO}_{2}[\mathrm{M}+\mathrm{Na}]^{+}$: 363.0871 . Found: 363.0868 .

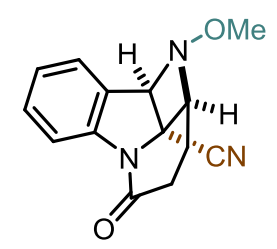

2j, colorless oil, $45.7 \mathrm{mg}, 90 \%$ yield. ${ }^{1} \mathrm{H} \mathrm{NMR}\left(400 \mathrm{MHz}, \mathrm{CDCl}_{3}\right) \delta 7.82(\mathrm{~d}, J=8.0 \mathrm{~Hz}$, 1H), 7.40-7.33 (m, 2H), $7.18(\mathrm{t}, J=7.6 \mathrm{~Hz}, 1 \mathrm{H}), 4.86(\mathrm{~s}, 1 \mathrm{H}), 4.42-4.39(\mathrm{~m}, 1 \mathrm{H}), 3.47$ (s, 3H), 2.66-2.46 (m, 3H), 2.14-2.05 (m, 1H). $\left.{ }^{13} \mathrm{C} \mathrm{NMR} \mathrm{(100} \mathrm{MHz,} \mathrm{CDCl}_{3}\right) \delta 169.2$, 145.6, 130.2, 128.5, 125.5, 123.9, 118.9, 115.2, 75.3, 68.1, 62.0, 54.8, 33.0, 26.2. IR (thin film): $v_{\max }\left(\mathrm{cm}^{-1}\right)=2920,1691,1601,1460,1442,1357,1310,1290,1266,1137$, 1082, 1058, 1039, 974, 883, 867, 846, 767, 712, 670, 645, 620. HRMS (ESI) calcd for $\mathrm{C}_{14} \mathrm{H}_{13} \mathrm{~N}_{3} \mathrm{NaO}_{2}[\mathrm{M}+\mathrm{Na}]^{+}: 278.0900$. Found: 278.0901 .

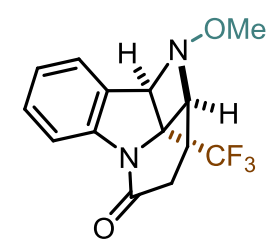

2k, yellow oil, $28.0 \mathrm{mg}$, 47\% yield. ${ }^{1} \mathrm{H} \mathrm{NMR}\left(400 \mathrm{MHz}, \mathrm{CDCl}_{3}\right) \delta 7.84(\mathrm{~d}, J=8.0 \mathrm{~Hz}$, $1 \mathrm{H}), 7.37-7.32(\mathrm{~m}, 2 \mathrm{H}), 7.15(\mathrm{t}, J=7.6 \mathrm{~Hz}, 1 \mathrm{H}), 4.69(\mathrm{~s}, 1 \mathrm{H}), 4.37-4.34(\mathrm{~m}, 1 \mathrm{H}), 3.50$ (s, 3H), 2.53-2.48 (m, 3H), 2.16-2.10 (m, 1H). $\left.{ }^{13} \mathrm{C} \mathrm{NMR} \mathrm{(100} \mathrm{MHz,} \mathrm{CDCl}_{3}\right) \delta 170.9$, 147.2, 129.8, 129.4, 125.3, 123.9, 122.8 (q, $J=277.0 \mathrm{~Hz}), 119.1,71.8,63.6,61.7,31.9$, 26.0. ${ }^{19} \mathrm{~F} \mathrm{NMR}\left(376 \mathrm{MHz}, \mathrm{CDCl}_{3}\right) \delta-77.0$ (s, 3F). IR (thin film): $v_{\max }\left(\mathrm{cm}^{-1}\right)=2921$, 2851, 1693, 1603, 1463, 1354, 1334, 1313, 1292, 1238, 1175, 1157, 1087, 1066, 869, 835, 758, 715, 669, 642, 619, 561. HRMS (ESI) calcd for $\mathrm{C}_{14} \mathrm{H}_{13} \mathrm{~N}_{2} \mathrm{NaF}_{3} \mathrm{O}_{2}[\mathrm{M}+\mathrm{Na}]^{+}$: 321.0821. Found: 321.0820 .

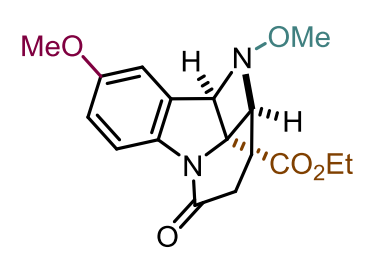


2l, colorless oil, $69.0 \mathrm{mg}$, 99\% yield. ${ }^{1} \mathrm{H}$ NMR (400 MHz, $\left.\mathrm{CDCl}_{3}\right) \delta 7.77(\mathrm{~d}, J=8.8 \mathrm{~Hz}$, $1 \mathrm{H}), 6.87-6.82(\mathrm{~m}, 2 \mathrm{H}), 4.75(\mathrm{~s}, 1 \mathrm{H}), 4.44-4.41(\mathrm{~m}, 1 \mathrm{H}), 4.30-4.17(\mathrm{~m}, 2 \mathrm{H}), 3.79$ (s, $3 \mathrm{H}), 3.49$ (s, 3H), 2.50-2.43 (m, 2H), 2.36-2.27 (m, 1H), 2.10-2.01 (m, 1H), 1.28 (t, $J$ $=7.2 \mathrm{~Hz}, 3 \mathrm{H}) .{ }^{13} \mathrm{C} \mathrm{NMR}\left(100 \mathrm{MHz}, \mathrm{CDCl}_{3}\right) \delta 170.1,168.1,157.0,140.1,131.3,119.0$, 113.6, 110.4, 74.0, 67.2, 66.6, 62.4, 61.5, 55.7, 32.6, 26.6, 14.0. IR (thin film): $v_{\max }\left(\mathrm{cm}^{-}\right.$ $\left.{ }^{1}\right)=2939,1728,1673,1593,1477,1369,1320,1298,1237,1168,1137,1115,1062$, 1034, 981, 897, 863, 812, 770, 691, 628, 599. HRMS (ESI) calcd for $\mathrm{C}_{17} \mathrm{H}_{20} \mathrm{~N}_{2} \mathrm{NaO}_{5}$ $[\mathrm{M}+\mathrm{Na}]^{+}:$355.1264. Found: 355.1261.

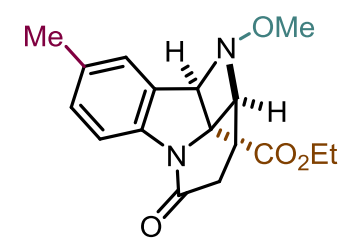

2m, colorless oil, $68.9 \mathrm{mg}$, 98\% yield. ${ }^{1} \mathrm{H}$ NMR (400 MHz, $\left.\mathrm{CDCl}_{3}\right) \delta 7.74$ (d, $J=8.0$ $\mathrm{Hz}, 1 \mathrm{H}), 7.13-7.11(\mathrm{~m}, 2 \mathrm{H}), 4.76(\mathrm{~s}, 1 \mathrm{H}), 4.45-4.41(\mathrm{~m}, 1 \mathrm{H}), 4.30-4.16(\mathrm{~m}, 2 \mathrm{H}), 3.50$ (s, 3H), 2.50-2.42 (m, 2H), 2.36-2.27 (m, 1H), $2.33(\mathrm{~s}, 3 \mathrm{H}), 2.09-2.00(\mathrm{~m}, 1 \mathrm{H}), 1.27$ (t, $J=7.2 \mathrm{~Hz}, 3 \mathrm{H}) .{ }^{13} \mathrm{C} \mathrm{NMR}\left(100 \mathrm{MHz}, \mathrm{CDCl}_{3}\right) \delta 170.2,168.1,144.3,134.4,129.92$, 129.88, 124.4, 118.1, 74.1, 67.1, 66.3, 62.4, 61.4, 32.7, 26.6, 21.0, 14.0. IR (thin film): $v_{\max }\left(\mathrm{cm}^{-1}\right)=2936,1733,1684,1483,1442,1363,1292,1219,1147,1131,1094,1079$, 1060, 1035, 979, 864, 818, 750, 664, 613, 581, 524. HRMS (ESI) calcd for $\mathrm{C}_{17} \mathrm{H}_{20} \mathrm{~N}_{2} \mathrm{NaO}_{4}[\mathrm{M}+\mathrm{Na}]^{+}$: 339.1315. Found: 339.1311.

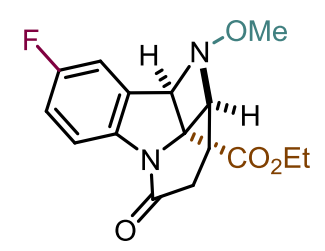

2n, colorless oil, $63.0 \mathrm{mg}$, 98\% yield. ${ }^{1} \mathrm{H} \mathrm{NMR}\left(400 \mathrm{MHz}, \mathrm{CDCl}_{3}\right) \delta 7.81(\mathrm{dd}, J=9.2$, $4.8 \mathrm{~Hz}, 1 \mathrm{H}), 7.03-7.00(\mathrm{~m}, 2 \mathrm{H}), 4.77(\mathrm{~s}, 1 \mathrm{H}), 4.47-4.43(\mathrm{~m}, 1 \mathrm{H}), 4.32-4.19(\mathrm{~m}, 2 \mathrm{H})$, 3.49 (s, 3H), 2.50-2.45 (m, 2H), 2.38-2.29 (m, 1H), 2.10-2.02 (m, 1H), $1.29(\mathrm{t}, J=7.2$ $\mathrm{Hz}, 3 \mathrm{H}) .{ }^{13} \mathrm{C} \mathrm{NMR}\left(100 \mathrm{MHz}, \mathrm{CDCl}_{3}\right) \delta 170.2,167.8,159.8(\mathrm{~d}, J=242.0 \mathrm{~Hz}), 142.6$ $(\mathrm{d}, J=2.0 \mathrm{~Hz}), 131.6(\mathrm{~d}, J=8.0 \mathrm{~Hz}), 119.3,115.6,111.3(\mathrm{~d}, J=24.0 \mathrm{~Hz}), 73.5,67.4$, 66.7, 62.6, 61.6, 32.6, 26.5, 14.0. ${ }^{19} \mathrm{~F}$ NMR $\left(376 \mathrm{MHz}, \mathrm{CDCl}_{3}\right) \delta-117.8 \sim-117.9(\mathrm{~m}$, 1F). IR (thin film): $v_{\max }\left(\mathrm{cm}^{-1}\right)=2931,1723,1679,1608,1471,1369,1301,1269,1230$, 
1126, 1101, 1062, 1034, 982, 958, 861, 821, 786, 766, 694, 612, 593. HRMS (ESI) calcd for $\mathrm{C}_{16} \mathrm{H}_{17} \mathrm{~N}_{2} \mathrm{NaFO}_{4}[\mathrm{M}+\mathrm{Na}]^{+}: 343.1065$. Found: 343.1064 .

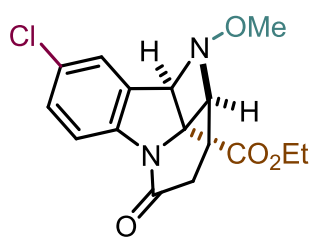

2o, white solid, $63.0 \mathrm{mg}$, 98\% yield, m.p. $=113.8-116.4{ }^{\circ} \mathrm{C} .{ }^{1} \mathrm{H} \mathrm{NMR}\left(400 \mathrm{MHz}, \mathrm{CDCl}_{3}\right)$ $\delta 7.80(\mathrm{~d}, J=8.0 \mathrm{~Hz}, 1 \mathrm{H}), 7.30-7.27(\mathrm{~m}, 2 \mathrm{H}), 4.77(\mathrm{~s}, 1 \mathrm{H}), 4.47-4.43(\mathrm{~m}, 1 \mathrm{H}), 4.31-$ $4.18(\mathrm{~m}, 2 \mathrm{H}), 3.49(\mathrm{~s}, 3 \mathrm{H}), 2.52-2.45(\mathrm{~m}, 2 \mathrm{H}), 2.37-2.29(\mathrm{~m}, 1 \mathrm{H}), 2.09-2.01(\mathrm{~m}, 1 \mathrm{H})$, $1.29(\mathrm{t}, J=7.2 \mathrm{~Hz}, 3 \mathrm{H}) .{ }^{13} \mathrm{C} \mathrm{NMR}\left(100 \mathrm{MHz}, \mathrm{CDCl}_{3}\right) \delta 170.2,167.7,145.2,131.7$, 129.7, 129.3, 124.1, 119.3, 73.5, 67.4, 66.4, 62.6, 61.7, 32.7, 26.5, 14.0. IR (thin film): $v_{\max }\left(\mathrm{cm}^{-1}\right)=2919,2849,1725,1681,1464,1365,1301,1270,1239,1171,1142,1061$, 1034, 981, 954, 919, 889, 864, 822, 767, 737, 707, 671, 608. HRMS (ESI) calcd for $\mathrm{C}_{16} \mathrm{H}_{17} \mathrm{~N}_{2} \mathrm{NaClO}_{4}[\mathrm{M}+\mathrm{Na}]^{+}:$359.0769. Found: 357.0765 .

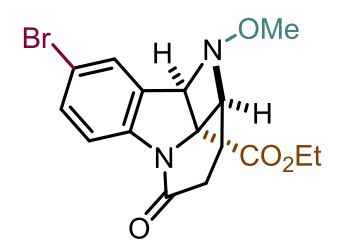

2p, colorless oil, $71.3 \mathrm{mg}$, 94\% yield. ${ }^{1} \mathrm{H}$ NMR (400 MHz, $\left.\mathrm{CDCl}_{3}\right) \delta 7.75(\mathrm{~d}, J=8.4$ $\mathrm{Hz}, 1 \mathrm{H}), 7.45-7.42$ (m, 2H), 4.78 (s, 1H), 4.47-4.43 (m, 1H), 4.31-4.18 (m, 2H), 3.49 (s, 3H), 2.51-2.45 (m, 2H), 2.37-2.28 (m, 1H), 2.09-2.01 (m, 1H), $1.29(\mathrm{t}, J=7.2 \mathrm{~Hz}$, $3 \mathrm{H}) .{ }^{13} \mathrm{C} \mathrm{NMR}\left(100 \mathrm{MHz}, \mathrm{CDCl}_{3}\right) \delta 170.2,167.7,145.6,132.3,132.0,126.9,119.8$, 117.2, 73.5, 67.4, 66.4, 62.6, 61.7, 32.7, 26.5, 14.0. IR (thin film): $v_{\max }\left(\mathrm{cm}^{-1}\right)=2920$, 2849, 1727, 1682, 1597, 1462, 1364, 1301, 1272, 1238, 1169, 1142, 1090, 1058, 1034, 982, 955, 916, 864, 821, 766, 727, 707. HRMS (ESI) calcd for $\mathrm{C}_{16} \mathrm{H}_{17} \mathrm{~N}_{2} \mathrm{NaBrO}_{4}$ $[\mathrm{M}+\mathrm{Na}]^{+}:$403.0264. Found: 403.0257.

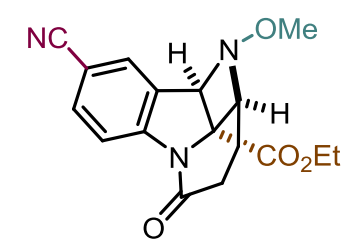


2q, colorless oil, $59.1 \mathrm{mg}$, 97\% yield. ${ }^{1} \mathrm{H}$ NMR $\left(400 \mathrm{MHz}, \mathrm{CDCl}_{3}\right) \delta 7.97(\mathrm{~d}, J=8.4$ $\mathrm{Hz}, 1 \mathrm{H}), 7.66(\mathrm{~d}, J=8.4 \mathrm{~Hz}, 1 \mathrm{H}), 7.58(\mathrm{~s}, 1 \mathrm{H}), 4.85$ (s, 1H), 4.51-4.48 (m, 1H), 4.33$4.21(\mathrm{~m}, 2 \mathrm{H}), 3.49$ (s, 3H), 2.55-2.48 (m, 2H), 2.41-2.32 (m, 1H), 2.11-2.01 (m, 1H), $1.30(\mathrm{t}, J=7.2 \mathrm{~Hz}, 3 \mathrm{H}) .{ }^{13} \mathrm{C} \mathrm{NMR}\left(100 \mathrm{MHz}, \mathrm{CDCl}_{3}\right) \delta 170.3,167.2,150.0,134.5$, $131.2,127.5,118.6,107.8,73.1,67.7,66.2,62.8,61.8,32.8,26.3,14.0$. IR (thin film): $v_{\max }\left(\mathrm{cm}^{-1}\right)=2937,2224,1736,1696,1611,1474,1439,1349,1298,1233,1143,1093$, 1060, 1034, 980, 865, 835, 753, 707, 665, 608, 492. HRMS (ESI) calcd for $\mathrm{C}_{17} \mathrm{H}_{17} \mathrm{~N}_{3} \mathrm{NaO}_{4}[\mathrm{M}+\mathrm{Na}]^{+}: 350.1111$. Found: 350.1105 .

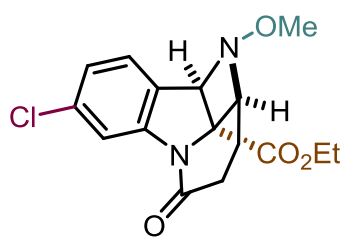

2r, colorless oil, $65.0 \mathrm{mg}, 96 \%$ yield. ${ }^{1} \mathrm{H}$ NMR (400 MHz, $\left.\mathrm{CDCl}_{3}\right) \delta 7.89$ (s, 1H), 7.21 (d, $J=7.6 \mathrm{~Hz}, 1 \mathrm{H}), 7.09$ (d, $J=8.0 \mathrm{~Hz}, 1 \mathrm{H}), 4.77(\mathrm{~s}, 1 \mathrm{H}), 4.46-4.43(\mathrm{~m}, 1 \mathrm{H}), 4.32-4.19$ (m, 2H), $3.48(\mathrm{~s}, 3 \mathrm{H}), 2.52-2.45(\mathrm{~m}, 2 \mathrm{H}), 2.37-2.28(\mathrm{~m}, 1 \mathrm{H}), 2.09-2.01(\mathrm{~m}, 1 \mathrm{H}), 1.29(\mathrm{t}$, $J=7.2 \mathrm{~Hz}, 3 \mathrm{H}) .{ }^{13} \mathrm{C} \mathrm{NMR}\left(100 \mathrm{MHz}, \mathrm{CDCl}_{3}\right) \delta 170.2,167.7,147.6,135.4,128.5$, $124.7,124.4,118.9,73.4,67.3,66.7,62.6,61.6,32.7,26.4,14.0$. IR (thin film): $v_{\max }$ $\left(\mathrm{cm}^{-1}\right)=2921,1728,1682,1599,1464,1417,1368,1295,1234,1183,1137,1090$, 1061, 1032, 986, 962, 874, 826, 772, 743, 685, 627, 594. HRMS (ESI) calcd for $\mathrm{C}_{16} \mathrm{H}_{17} \mathrm{~N}_{2} \mathrm{NaClO}_{4}[\mathrm{M}+\mathrm{Na}]^{+}: 359.0769$. Found: 359.0768 .

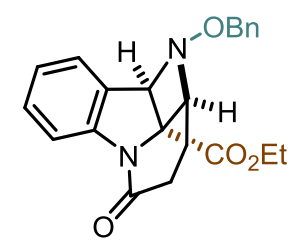

2s, colorless oil, $68.5 \mathrm{mg}, 90 \%$ yield. ${ }^{1} \mathrm{H}$ NMR (400 MHz, $\left.\mathrm{CDCl}_{3}\right) \delta 7.82(\mathrm{~d}, J=8.0$ $\mathrm{Hz}, 1 \mathrm{H}), 7.30-7.26(\mathrm{~m}, 6 \mathrm{H}), 6.99$ (t, $J=7.6 \mathrm{~Hz}, 1 \mathrm{H}), 6.80$ (d, J=7.2 Hz, 1H), 4.82 (s, $1 \mathrm{H}), 4.63(\mathrm{~s}, 2 \mathrm{H}), 4.49-4.46(\mathrm{~m}, 1 \mathrm{H}), 4.28-4.14(\mathrm{~m}, 2 \mathrm{H}), 2.41-2.36(\mathrm{~m}, 1 \mathrm{H}), 2.29-2.13$ $(\mathrm{m}, 2 \mathrm{H}), 1.83-1.70(\mathrm{~m}, 1 \mathrm{H}), 1.26(\mathrm{t}, J=7.2 \mathrm{~Hz}, 3 \mathrm{H}) .{ }^{13} \mathrm{C} \mathrm{NMR}\left(100 \mathrm{MHz}, \mathrm{CDCl}_{3}\right) \delta$ 170.4, 168.1, 146.4, 138.0, 129.6, 129.3, 128.6, 128.3, 127.9, 124.4, 123.8, 118.2, 76.7, 74.4, 67.8, 66.4, 62.4, 32.8, 26.0, 14.0. IR (thin film): $v_{\max }\left(\mathrm{cm}^{-1}\right)=2921,2850,1725$, 1682, 1602, 1461, 1369, 1298, 1237, 1185, 1141, 1107, 1073, 1029, 1019, 976, 945, 
882, 865, 758, 737, 695. HRMS (ESI) calcd for $\mathrm{C}_{22} \mathrm{H}_{22} \mathrm{~N}_{2} \mathrm{NaO}_{4}[\mathrm{M}+\mathrm{Na}]^{+}$: 401.1472. Found: 401.1469.

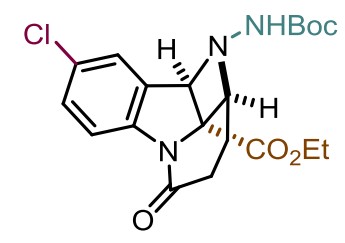

2t, colorless oil, $19.7 \mathrm{mg}, 33 \%$ yield. ${ }^{1} \mathrm{H}$ NMR $\left(400 \mathrm{MHz}, \mathrm{CDCl}_{3}\right) \delta 8.04(\mathrm{~d}, J=8.8 \mathrm{~Hz}$, 1H), $7.42(\mathrm{~s}, 1 \mathrm{H}), 7.33$ (d, $J=8.8 \mathrm{~Hz}, 1 \mathrm{H}), 5.68(\mathrm{~s}, 1 \mathrm{H}), 4.36-4.20(\mathrm{~m}, 3 \mathrm{H}), 3.89$ (d, $J$ $=12.0 \mathrm{~Hz}, 1 \mathrm{H}), 2.53-2.30(\mathrm{~m}, 3 \mathrm{H}), 1.57(\mathrm{~s}, 9 \mathrm{H}), 1.51-1.45(\mathrm{~m}, 1 \mathrm{H}), 1.28(\mathrm{t}, J=7.2 \mathrm{~Hz}$, $3 \mathrm{H}) .{ }^{13} \mathrm{C} \mathrm{NMR}\left(100 \mathrm{MHz}, \mathrm{CDCl}_{3}\right) \delta 169.4,169.2,141.3,130.3,129.8,129.5,127.1$, 126.1, 117.4, 82.1, 70.6, 63.1, 62.8, 31.2, 28.4, 21.9, 14.0. IR (thin film): $v_{\max }\left(\mathrm{cm}^{-1}\right)=$ 3209, 2920, 2850, 1737, 1682, 1660, 1473, 1383, 1310, 1235, 1196, 1163, 1138, 1033, $1003,914,890,861,822,759,730,697,653$. HRMS (ESI) calcd for $\mathrm{C}_{20} \mathrm{H}_{24} \mathrm{~N}_{3} \mathrm{NaClO}_{5}$ $[\mathrm{M}+\mathrm{Na}]^{+}:$444.1297. Found: 444.1291.

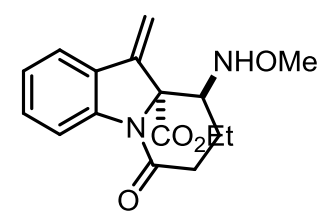

4a, yellow solid, $57.9 \mathrm{mg}$, 95\% yield, m.p. $=90.3-92.5^{\circ} \mathrm{C} .{ }^{1} \mathrm{H} \mathrm{NMR}\left(400 \mathrm{MHz}, \mathrm{CDCl}_{3}\right)$ $\delta 8.29(\mathrm{~d}, J=8.0 \mathrm{~Hz}, 1 \mathrm{H}), 7.46(\mathrm{~d}, J=7.2 \mathrm{~Hz}, 1 \mathrm{H}), 7.34(\mathrm{t}, J=8.0 \mathrm{~Hz}, 1 \mathrm{H}), 7.09$ (t, $J$ $=7.6 \mathrm{~Hz}, 1 \mathrm{H}), 5.97-5.52(\mathrm{br}, 1 \mathrm{H}), 5.75(\mathrm{~s}, 1 \mathrm{H}), 5.43(\mathrm{~s}, 1 \mathrm{H}), 4.26-4.13(\mathrm{~m}, 2 \mathrm{H}), 4.10-$ 4.09 (m, 1H), 3.41 (s, 3H), 2.74-2.66 (m, 1H), 2.56-2.50 (m, 1H), 2.31-2.26 (m, 1H), 1.97-1.89 (m, 1H), $1.22(\mathrm{t}, J=7.2 \mathrm{~Hz}, 3 \mathrm{H}) .{ }^{13} \mathrm{C} \mathrm{NMR}\left(100 \mathrm{MHz}, \mathrm{CDCl}_{3}\right) \delta$ 169.6, 168.1, 144.3, 141.7, 130.7, 126.0, 124.0, 120.4, 117.5, 105.1, 75.0, 63.0, 62.7, 57.7, 28.7, 20.8, 13.8. IR (thin film): $v_{\max }\left(\mathrm{cm}^{-1}\right)=3222,2987,2905,1741,1653,1596,1461,1414$, 1379, 1314, 1295, 1239, 1188, 1171, 1024, 985, 946, 909, 780, 758, 669, 529. HRMS (ESI) calcd for $\mathrm{C}_{17} \mathrm{H}_{20} \mathrm{~N}_{2} \mathrm{NaO}_{4}[\mathrm{M}+\mathrm{Na}]^{+}$: 339.1315. Found: 339.1312.

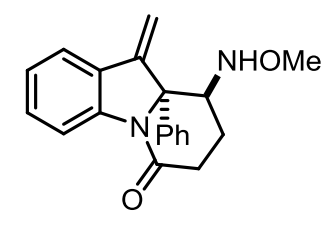


4b, brown solid, $62.5 \mathrm{mg}$, 96\% yield, m.p. $=127.8-130.1{ }^{\circ} \mathrm{C} .{ }^{1} \mathrm{H}$ NMR $(400 \mathrm{MHz}$, $\left.\mathrm{CDCl}_{3}\right) \delta 8.40(\mathrm{~d}, J=8.0 \mathrm{~Hz}, 1 \mathrm{H}), 7.40-7.25(\mathrm{~m}, 7 \mathrm{H}), 7.07(\mathrm{t}, J=7.6 \mathrm{~Hz}, 1 \mathrm{H}), 5.95(\mathrm{~s}$, $1 \mathrm{H}), 5.61(\mathrm{~s}, 1 \mathrm{H}), 5.36(\mathrm{~s}, 1 \mathrm{H}), 4.10(\mathrm{~s}, 1 \mathrm{H}), 3.50(\mathrm{~s}, 3 \mathrm{H}), 2.73-2.64(\mathrm{~m}, 1 \mathrm{H}), 2.41-2.34$ (m, 1H), 2.18-2.13 (m, 1H), 1.68-1.61 (m, 1H). ${ }^{13} \mathrm{C}$ NMR (100 MHz, $\left.\mathrm{CDCl}_{3}\right) \delta 169.2$, 147.3, 143.9, 142.1, 130.6, 129.0, 127.8, 126.4, 124.3, 124.2, 120.9, 117.8, 102.8, 73.9, 63.1, 59.7, 28.3, 19.4. IR (thin film): $v_{\max }\left(\mathrm{cm}^{-1}\right)=2919,1664,1597,1461,1381,1352$, 1299, 1259, 1188, 1011, 984, 953, 929, 886, 844, 797, 778, 755, 697, 543, 515, 472. HRMS (ESI) calcd for $\mathrm{C}_{20} \mathrm{H}_{20} \mathrm{~N}_{2} \mathrm{NaO}_{2}[\mathrm{M}+\mathrm{Na}]^{+}: 343.1417$. Found: 343.1409 .

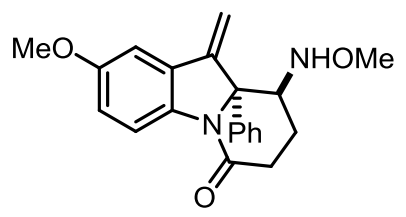

4c, yellow oil, $62.5 \mathrm{mg}, 90 \%$ yield. ${ }^{1} \mathrm{H} \mathrm{NMR}\left(400 \mathrm{MHz}, \mathrm{CDCl}_{3}\right) \delta 8.32(\mathrm{~d}, J=8.8 \mathrm{~Hz}$, 1H), 7.35-7.23 (m, 5H), 6.94-6.89 (m, 2H), $5.94(\mathrm{~s}, 1 \mathrm{H}), 5.59(\mathrm{~s}, 1 \mathrm{H}), 5.37(\mathrm{~s}, 1 \mathrm{H}), 4.08$ (s, 1H), 3.77 (s, 3H), 3.50 (s, 3H), 2.71-2.63 (m, 1H), 2.40-2.33 (m, 1H), 2.18-2.12 (m, $1 \mathrm{H}), 1.67-1.58(\mathrm{~m}, 1 \mathrm{H}) .{ }^{13} \mathrm{C} \mathrm{NMR}\left(100 \mathrm{MHz}, \mathrm{CDCl}_{3}\right) \delta 168.6,156.9,147.3,142.2$, 138.0, 129.0, 127.8, 127.6, 124.2, 118.7, 116.8, 105.3, 103.0, 74.2, 63.1, 59.7, 55.6, 28.1, 19.5. IR (thin film): $v_{\max }\left(\mathrm{cm}^{-1}\right)=3267,2938,1654,1588,1479,1380,1321,1279$, 1227, 1208, 1186, 1129, 1098, 1062, 1031, 1007, 984, 951, 927, 870, 840, 809, 758. HRMS (ESI) calcd for $\mathrm{C}_{21} \mathrm{H}_{22} \mathrm{~N}_{2} \mathrm{NaO}_{3}[\mathrm{M}+\mathrm{Na}]^{+}:$373.1523. Found: 373.1516.

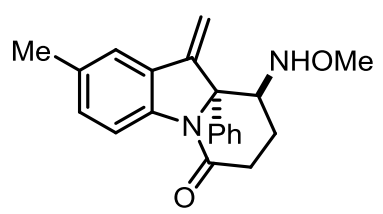

4d, yellow oil, $56.1 \mathrm{mg}, 85 \%$ yield. ${ }^{1} \mathrm{H} \mathrm{NMR}\left(400 \mathrm{MHz}, \mathrm{CDCl}_{3}\right) \delta 8.28(\mathrm{~d}, J=8.0 \mathrm{~Hz}$, 1H), 7.34-7.16 (m, 7H), $5.95(\mathrm{~s}, 1 \mathrm{H}), 5.58(\mathrm{~s}, 1 \mathrm{H}), 5.33(\mathrm{~s}, 1 \mathrm{H}), 4.08(\mathrm{~s}, 1 \mathrm{H}), 3.50$ (s, $3 \mathrm{H}), 2.72-2.63(\mathrm{~m}, 1 \mathrm{H}), 2.40-2.32(\mathrm{~m}, 1 \mathrm{H}), 2.30(\mathrm{~s}, 3 \mathrm{H}), 2.18-2.12(\mathrm{~m}, 1 \mathrm{H}), 1.68-1.59$ $(\mathrm{m}, 1 \mathrm{H}) .{ }^{13} \mathrm{C} \mathrm{NMR}\left(100 \mathrm{MHz}, \mathrm{CDCl}_{3}\right) \delta 168.9,147.3,142.2,141.8,134.0,131.4,129.0$, $127.8,126.5,124.2,121.2,117.5,102.5,74.1,63.1,59.7,28.2,21.0,19.4$. IR (thin film): $v_{\max }\left(\mathrm{cm}^{-1}\right)=2937,1651,1480,1444,1383,1348,1288,1213,1187,1153,1083$, 1063, 1035, 1011, 985, 950, 928, 880, 839, 820, 749, 698, 664. HRMS (ESI) calcd for $\mathrm{C}_{21} \mathrm{H}_{23} \mathrm{~N}_{2} \mathrm{O}_{2}[\mathrm{M}]^{+}:$335.1754. Found: 335.1747. 


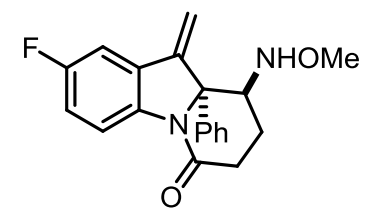

4e, white solid, $60.6 \mathrm{mg}$, 90\% yield, m.p. $=187.6-190.1{ }^{\circ} \mathrm{C} .{ }^{1} \mathrm{H} \mathrm{NMR}\left(400 \mathrm{MHz}, \mathrm{CDCl}_{3}\right)$ א 8.39-8.36 (m, 1H), 7.33-7.26 (m, 5H), 7.07-7.03 (m, 2H), $5.92(\mathrm{~s}, 1 \mathrm{H}), 5.60(\mathrm{~s}, 1 \mathrm{H})$, $5.42(\mathrm{~s}, 1 \mathrm{H}), 4.09(\mathrm{~s}, 1 \mathrm{H}), 3.50(\mathrm{~s}, 3 \mathrm{H}), 2.72-2.63(\mathrm{~m}, 1 \mathrm{H}), 2.40-2.33(\mathrm{~m}, 1 \mathrm{H}), 2.19-2.13$ (m, 1H), 1.68-1.60 (m, 1H). ${ }^{13} \mathrm{C}$ NMR (100 MHz, $\left.\mathrm{CDCl}_{3}\right) \delta 169.0,159.9$ (d, $J=242.0$ Hz), 146.6, 141.8, 140.1, 129.1, 128.2 (d, $J=9.0 \mathrm{~Hz}), 128.0,124.1,118.9,117.2$ (d, $J$ $=23.0 \mathrm{~Hz}), 107.2,104.2,74.4,63.1,59.7,28.1,19.5 .{ }^{19} \mathrm{~F} \mathrm{NMR}\left(376 \mathrm{MHz}, \mathrm{CDCl}_{3}\right) \delta-$ $117.9 \sim-118.0$ (m, 1F). IR (thin film): $v_{\max }\left(\mathrm{cm}^{-1}\right)=2921,1650,1600,1471,1375,1342$, 1274, 1192, 1089, 1037, 1016, 987, 946, 910, 894, 868, 840, 811, 747, 704, 664, 600. HRMS (ESI) calcd for $\mathrm{C}_{20} \mathrm{H}_{19} \mathrm{~N}_{2} \mathrm{NaFO}_{2}[\mathrm{M}+\mathrm{Na}]^{+}: 361.1323$. Found: 361.1324 .

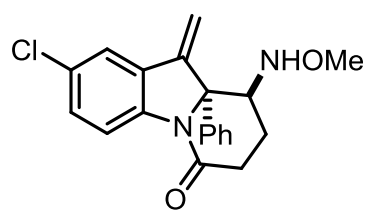

4f, colorless oil, $55.6 \mathrm{mg}, 84 \%$ yield. ${ }^{1} \mathrm{H}$ NMR $\left(400 \mathrm{MHz}, \mathrm{CDCl}_{3}\right) \delta 8.34(\mathrm{~d}, J=8.8 \mathrm{~Hz}$, 1H), 7.35-7.26 (m, 7H), $5.90(\mathrm{~s}, 1 \mathrm{H}), 5.62(\mathrm{~s}, 1 \mathrm{H}), 5.41(\mathrm{~s}, 1 \mathrm{H}), 4.09(\mathrm{~s}, 1 \mathrm{H}), 3.50$ (s, $3 \mathrm{H}), 2.72-2.63(\mathrm{~m}, 1 \mathrm{H}), 2.40-2.32(\mathrm{~m}, 1 \mathrm{H}), 2.19-2.13(\mathrm{~m}, 1 \mathrm{H}), 1.69-1.62(\mathrm{~m}, 1 \mathrm{H}) .{ }^{13} \mathrm{C}$ $\mathrm{NMR}\left(100 \mathrm{MHz}, \mathrm{CDCl}_{3}\right) \delta 169.2,146.3,142.4,141.8,130.4,129.7,129.2,128.2,128.1$, 124.1, 121.0, 118.8, 104.3, 74.4, 63.1, 59.7, 28.2, 19.5. IR (thin film): $v_{\max }\left(\mathrm{cm}^{-1}\right)=$ 2920, 1665, 1464, 1370, 1261, 1222, 1188, 1132, 1070, 1009, 983, 950, 915, 898, 876, 841, 813, 760, 726, 700, 662. HRMS (ESI) calcd for $\mathrm{C}_{20} \mathrm{H}_{19} \mathrm{~N}_{2} \mathrm{O}_{2} \mathrm{NaCl}[\mathrm{M}+\mathrm{Na}]^{+}$: 377.1027. Found: 377.1018 .

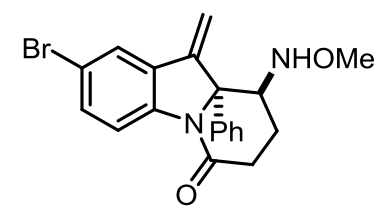

4g, yellow oil, $65.1 \mathrm{mg}, 81 \%$ yield. ${ }^{1} \mathrm{H}$ NMR $\left(400 \mathrm{MHz}, \mathrm{CDCl}_{3}\right) \delta 8.29(\mathrm{~d}, J=8.8 \mathrm{~Hz}$, 1H), $7.50(\mathrm{~s}, 1 \mathrm{H}), 7.45$ (d, $J=8.4 \mathrm{~Hz}, 1 \mathrm{H}), 7.34-7.26(\mathrm{~m}, 5 \mathrm{H}), 5.89(\mathrm{~s}, 1 \mathrm{H}), 5.61$ (s, $1 \mathrm{H}), 5.40(\mathrm{~s}, 1 \mathrm{H}), 4.09(\mathrm{~s}, 1 \mathrm{H}), 3.49(\mathrm{~s}, 3 \mathrm{H}), 2.71-2.62(\mathrm{~m}, 1 \mathrm{H}), 2.39-2.31(\mathrm{~m}, 1 \mathrm{H})$, 2.18-2.13 (m, 1H), 1.69-1.61 (m, 1H). $\left.{ }^{13} \mathrm{C} \mathrm{NMR} \mathrm{(100} \mathrm{MHz,} \mathrm{CDCl}_{3}\right) \delta 169.2,146.2$, 
$142.8,141.7,133.2,129.2,128.6,128.0,124.1,123.9,119.2,117.1,104.3,74.4,63.1$, 59.6, 28.3, 19.5. IR (thin film): $v_{\max }\left(\mathrm{cm}^{-1}\right)=2937,2808,1658,1590,1493,1462,1374$, 1347, 1264, 1191, 1159, 1083, 1060, 1034, 1010, 985, 949, 928, 880, 821, 755. HRMS (ESI) calcd for $\mathrm{C}_{20} \mathrm{H}_{19} \mathrm{~N}_{2} \mathrm{O}_{2} \mathrm{NaBr}[\mathrm{M}+\mathrm{Na}]^{+}$: 421.0522 . Found: 421.0524.

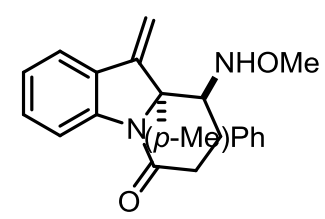

4h, colorless oil, $65.0 \mathrm{mg}, 96 \%$ yield. ${ }^{1} \mathrm{H}$ NMR (400 MHz, $\left.\mathrm{CDCl}_{3}\right) \delta 8.39$ (d, $J=8.0$ $\mathrm{Hz}, 1 \mathrm{H}), 7.40-7.33(\mathrm{~m}, 2 \mathrm{H}), 7.22(\mathrm{~d}, J=8.0 \mathrm{~Hz}, 2 \mathrm{H}), 7.11-7.04(\mathrm{~m}, 3 \mathrm{H}), 5.94(\mathrm{~s}, 1 \mathrm{H})$, $5.59(\mathrm{~s}, 1 \mathrm{H}), 5.34(\mathrm{~s}, 1 \mathrm{H}), 4.07(\mathrm{~s}, 1 \mathrm{H}), 3.49(\mathrm{~s}, 3 \mathrm{H}), 2.72-2.63(\mathrm{~m}, 1 \mathrm{H}), 2.41-2.34(\mathrm{~m}$, 1H), $2.28(\mathrm{~s}, 3 \mathrm{H}), 2.18-2.12(\mathrm{~m}, 1 \mathrm{H}), 1.71-1.62(\mathrm{~m}, 1 \mathrm{H}) .{ }^{13} \mathrm{C} \mathrm{NMR}\left(100 \mathrm{MHz}, \mathrm{CDCl}_{3}\right)$ $\delta 169.2,147.4,143.9,139.2,137.7,130.5,129.7,126.4,124.2,124.1,120.9,117.7$, 102.6, 73.8, 63.1, 59.7, 28.3, 20.8, 19.4. IR (thin film): $v_{\max }\left(\mathrm{cm}^{-1}\right)=2945,1654,1598$, 1509, 1461, 1417, 1384, 1348, 1300, 1215, 1187, 1152, 1115, 1093, 1066, 1009, 881, 843, 814, 793, 747. HRMS (ESI) calcd for $\mathrm{C}_{21} \mathrm{H}_{22} \mathrm{~N}_{2} \mathrm{O}_{2} \mathrm{Na}[\mathrm{M}+\mathrm{Na}]^{+}:$357.1574. Found: 357.1567 .

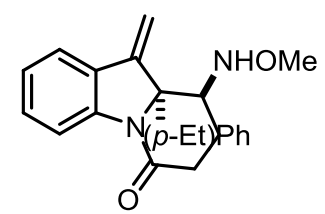

4i, colorless oil, $66.6 \mathrm{mg}, 95 \%$ yield. ${ }^{1} \mathrm{H}$ NMR $\left(400 \mathrm{MHz}, \mathrm{CDCl}_{3}\right) \delta 8.40(\mathrm{~d}, J=8.0 \mathrm{~Hz}$, $1 \mathrm{H}), 7.40-7.33(\mathrm{~m}, 2 \mathrm{H}), 7.24(\mathrm{~d}, J=8.4 \mathrm{~Hz}, 1 \mathrm{H}), 7.12(\mathrm{~d}, J=8.4 \mathrm{~Hz}, 1 \mathrm{H}), 7.06(\mathrm{t}, J=$ $7.6 \mathrm{~Hz}, 1 \mathrm{H}), 5.95$ (s, 1H), 5.60 (s, 1H), 5.35 (s, 1H), 4.07 (s, 1H), 3.49 (s, 3H), 2.73$2.56(\mathrm{~m}, 3 \mathrm{H}), 2.41-2.34(\mathrm{~m}, 1 \mathrm{H}), 2.18-2.12(\mathrm{~m}, 1 \mathrm{H}), 1.70-1.63(\mathrm{~m}, 1 \mathrm{H}), 1.18(\mathrm{t}, J=7.6$ $\mathrm{Hz}, 3 \mathrm{H}) .{ }^{13} \mathrm{C} \mathrm{NMR}\left(100 \mathrm{MHz}, \mathrm{CDCl}_{3}\right) \delta 169.3,147.4,143.9,139.3,130.5,128.5,126.5$, $124.2,124.1,120.9,117.7,102.6,73.8,63.1,59.7,28.3,28.1,19.4,15.2$. IR (thin film): $v_{\max }\left(\mathrm{cm}^{-1}\right)=2919,2848,1671,1638,1596,1510,1459,1378,1333,1296,1185,1150$, 1110, 1090, 1063, 1020, 930, 889, 829, 775, 752. HRMS (ESI) calcd for $\mathrm{C}_{22} \mathrm{H}_{25} \mathrm{~N}_{2} \mathrm{O}_{2}$ $[\mathrm{M}]^{+}:$349.1911. Found: 349.1906. 


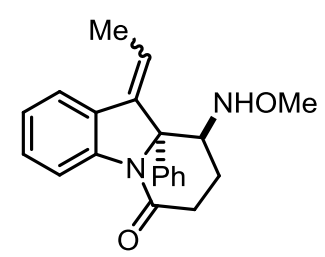

4j, colorless oil, $57.0 \mathrm{mg}$, 95\% yield, $E / Z=1.2 / 1 .{ }^{1} \mathrm{H} \mathrm{NMR}\left(400 \mathrm{MHz}, \mathrm{CDCl}_{3}\right) \delta 8.48$ $(\mathrm{d}, J=8.0 \mathrm{~Hz}, 1 \mathrm{H}), 8.37\left(\mathrm{~d}, J=8.0 \mathrm{~Hz}, 1 \mathrm{H}^{*}\right), 7.52-7.22(\mathrm{~m}, 7 \mathrm{H}), 7.09-7.02(\mathrm{~m}, 1 \mathrm{H})$, $6.10(\mathrm{q}, J=7.6 \mathrm{~Hz}, 1 \mathrm{H}), 5.99$ (q, $\left.J=7.2 \mathrm{~Hz}, 1 \mathrm{H}^{*}\right), 5.96-5.60$ (br, $\left.1 \mathrm{H}\right), 4.54$ (s, $\left.1 \mathrm{H}^{*}\right)$, $4.01(\mathrm{~s}, 1 \mathrm{H}), 3.513(\mathrm{~s}, 3 \mathrm{H}), 3.507$ (s, 3H*), 2.74-2.57 (m, 1H), 2.39-2.12 (m, 2H), 2.102.07 (m, 3H), 1.95-1.54 (m, 1H). $\left.{ }^{13} \mathrm{C} \mathrm{NMR} \mathrm{(100} \mathrm{MHz,} \mathrm{CDCl}_{3}\right) \delta$ 169.2, 169.0, 144.2, $142.8,142.7,141.5,138.9,138.7,129.3,129.1,128.9,128.8,128.2,127.7,127.6,126.9$, 125.0, 124.9, 124.1, 124.0, 119.5, 117.6, 117.5, 117.4, 115.9, 74.2, 74.0, 63.1, 63.0, 59.6, 57.3, 28.6, 28.3, 20.3, 19.3, 14.4, 13.7. IR (thin film): $v_{\max }\left(\mathrm{cm}^{-1}\right)=3002,2919$, 1646, 1594, 1459, 1413, 1388, 1348, 1292, 1224, 1183, 1083, 1010, 938, 926, 843, 807, 746, 699, 663, 629. HRMS (ESI) calcd for $\mathrm{C}_{21} \mathrm{H}_{22} \mathrm{~N}_{2} \mathrm{O}_{2} \mathrm{Na}[\mathrm{M}+\mathrm{Na}]^{+}: 357.1574$. Found: 357.1571 .

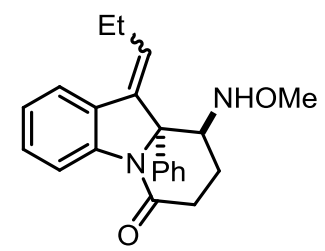

4k, colorless oil, $62.7 \mathrm{mg}$, 90\% yield, $E / Z=1.1 / 1 .{ }^{1} \mathrm{H}$ NMR $\left(400 \mathrm{MHz}, \mathrm{CDCl}_{3}\right) \delta 8.47$ $(\mathrm{d}, J=8.0 \mathrm{~Hz}, 1 \mathrm{H}), 8.37\left(\mathrm{~d}, J=8.0 \mathrm{~Hz}, 1 \mathrm{H}^{*}\right), 7.47-7.23(\mathrm{~m}, 7 \mathrm{H}), 7.08-7.03(\mathrm{~m}, 1 \mathrm{H})$, $5.96(\mathrm{t}, J=8.0 \mathrm{~Hz}, 1 \mathrm{H}), 5.85\left(\mathrm{t}, J=7.2 \mathrm{~Hz}, 1 \mathrm{H}^{*}\right), 5.89-5.63(\mathrm{br}, 1 \mathrm{H}), 4.51\left(\mathrm{~s}, 1 \mathrm{H}^{*}\right)$, $4.00(\mathrm{~s}, 1 \mathrm{H}), 3.52(\mathrm{~s}, 3 \mathrm{H}), 3.50\left(\mathrm{~s}, 3 \mathrm{H}^{*}\right), 2.75-2.33(\mathrm{~m}, 3 \mathrm{H}), 2.23-1.55(\mathrm{~m}, 3 \mathrm{H}), 1.18(\mathrm{t}$, $J=7.2 \mathrm{~Hz}, 3 \mathrm{H}), 1.05$ (t, $\left.J=7.2 \mathrm{~Hz}, 3 \mathrm{H}^{*}\right) .{ }^{13} \mathrm{C} \mathrm{NMR}\left(100 \mathrm{MHz}, \mathrm{CDCl}_{3}\right) \delta 169.3,169.1$, 144.1, 143.0, 142.7, 141.7, 137.4, 137.1, 129.3, 129.2, 128.9, 128.8, 128.0, 127.7, 127.6, 126.8, 125.4, 124.9, 124.8, 124.2, 124.1, 123.6, 119.6, 117.7, 117.3, 74.2, 73.9, 63.1, 63.0, 59.5, 57.8, 28.7, 28.3, 21.8, 21.4, 20.5, 19.3, 14.0. IR (thin film): $v_{\max }\left(\mathrm{cm}^{-1}\right)=$ 2932, 2873, 2808, 1657, 1593, 1474, 1458, 1417, 1386, 1348, 1312, 1289, 1226, 1185 , 1152, 1131, 1083, 1065, 1010, 832, 749, 698, 664. HRMS (ESI) calcd for $\mathrm{C}_{22} \mathrm{H}_{24} \mathrm{~N}_{2} \mathrm{O}_{2} \mathrm{Na}[\mathrm{M}+\mathrm{Na}]^{+}:$371.1730. Found: 371.1727. 


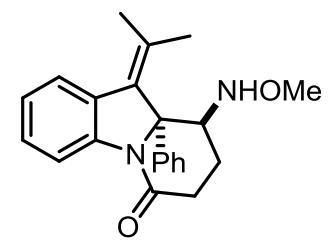

4l, white solid, $27.9 \mathrm{mg}, 40 \%$ yield, m.p. $=194.2-196.7^{\circ} \mathrm{C} .{ }^{1} \mathrm{H} \mathrm{NMR}\left(400 \mathrm{MHz}, \mathrm{CDCl}_{3}\right)$ $\delta 8.46(\mathrm{~d}, J=8.0 \mathrm{~Hz}, 1 \mathrm{H}), 7.52(\mathrm{~d}, J=7.6 \mathrm{~Hz}, 1 \mathrm{H}), 7.29-7.20(\mathrm{~m}, 6 \mathrm{H}), 7.06(\mathrm{t}, J=7.2$ $\mathrm{Hz}, 1 \mathrm{H}), 5.92(\mathrm{~s}, 1 \mathrm{H}), 4.59-4.57$ (m, 1H), $3.51(\mathrm{~s}, 3 \mathrm{H}), 2.61-2.53(\mathrm{~m}, 1 \mathrm{H}), 2.19-2.11(\mathrm{~m}$, 2H), 2.14 (s, 3H), 2.13 (s, 3H), 2.09-1.97 (m, 1H). $\left.{ }^{13} \mathrm{C} \mathrm{NMR} \mathrm{(100} \mathrm{MHz,} \mathrm{CDCl}_{3}\right) \delta 169.2$, $144.2,141.8,133.2,129.2,128.9,128.5,128.2,127.6,125.4,125.2,123.6,117.0,74.8$, 63.1, 57.6, 29.2, 24.6, 23.2, 21.0. IR (thin film): $v_{\max }\left(\mathrm{cm}^{-1}\right)=3226,2922,2853,1649$, 1587, 1460, 1398, 1297, 1254, 1179, 1128, 1086, 1027, 982, 867, 832, 752, 725, 701, $623,559,484,450$. HRMS (ESI) calcd for $\mathrm{C}_{22} \mathrm{H}_{24} \mathrm{~N}_{2} \mathrm{O}_{2} \mathrm{Na}[\mathrm{M}+\mathrm{Na}]^{+}: 371.1730$. Found: 371.1729 .

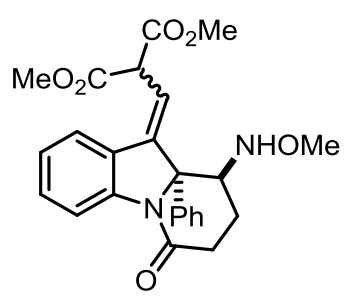

4m, yellow oil, $37.1 \mathrm{mg}, 62 \%$ yield, $E / Z=1.1 / 1$. For major isomer, ${ }^{1} \mathrm{H}$ NMR $(400 \mathrm{MHz}$, $\left.\mathrm{CDCl}_{3}\right) \delta 8.48(\mathrm{~d}, J=8.0 \mathrm{~Hz}, 1 \mathrm{H}), 7.54(\mathrm{~d}, J=8.0 \mathrm{~Hz}, 1 \mathrm{H}), 7.38(\mathrm{t}, J=7.6 \mathrm{~Hz}, 1 \mathrm{H})$, 7.33-7.29 (m, 3H), 7.27-7.22 (m, 2H), 7.09 (t, $J=7.2 \mathrm{~Hz}, 1 \mathrm{H}), 6.35-5.94$ (br, 1H), 6.15 $(\mathrm{d}, J=10.0 \mathrm{~Hz}, 1 \mathrm{H}), 4.83(\mathrm{~d}, J=9.6 \mathrm{~Hz}, 1 \mathrm{H}), 4.07(\mathrm{t}, J=2.8 \mathrm{~Hz}, 1 \mathrm{H}), 3.82(\mathrm{~s}, 3 \mathrm{H})$, $3.74(\mathrm{~s}, 3 \mathrm{H}), 3.54(\mathrm{~s}, 3 \mathrm{H}), 2.79-2.70(\mathrm{~m}, 1 \mathrm{H}), 2.46-2.39(\mathrm{~m}, 1 \mathrm{H}), 2.21-2.15(\mathrm{~m}, 1 \mathrm{H})$, 1.62-1.53 (m, 1H). ${ }^{13} \mathrm{C}$ NMR $\left(100 \mathrm{MHz}, \mathrm{CDCl}_{3}\right) \delta 169.3,168.0,167.7,144.9,142.5$, $141.5,130.9,129.0,127.9,124.9,124.43,124.42,124.39,118.2,113.4,74.7,62.9,59.4$, 53.3, 53.1, 50.6, 28.3, 19.2. IR (thin film): $v_{\max }\left(\mathrm{cm}^{-1}\right)=2920,2851,1733,1659,1595$, 1458, 1385, 1311, 1280, 1198, 1151, 1104, 1062, 1016, 929, 834, 752, 698, 636, 550, 514, 496. HRMS (ESI) calcd for $\mathrm{C}_{25} \mathrm{H}_{26} \mathrm{~N}_{2} \mathrm{O}_{6} \mathrm{Na}[\mathrm{M}+\mathrm{Na}]^{+}$: 473.1683 . Found: 473.1678. For minor isomer, ${ }^{1} \mathrm{H}$ NMR $\left(400 \mathrm{MHz}, \mathrm{CDCl}_{3}\right) \delta 8.38(\mathrm{~d}, J=8.4 \mathrm{~Hz}, 1 \mathrm{H}), 7.48(\mathrm{~d}, J=$ $7.6 \mathrm{~Hz}, 1 \mathrm{H}), 7.38(\mathrm{t}, J=8.0 \mathrm{~Hz}, 1 \mathrm{H}), 7.30-7.28(\mathrm{~m}, 4 \mathrm{H}), 7.27-7.22(\mathrm{~m}, 1 \mathrm{H}), 7.12$ (t, $J$ $=7.6 \mathrm{~Hz}, 1 \mathrm{H}), 6.18(\mathrm{~d}, J=11.6 \mathrm{~Hz}, 1 \mathrm{H}), 5.88(\mathrm{~s}, 1 \mathrm{H}), 4.71(\mathrm{~d}, J=11.2 \mathrm{~Hz}, 1 \mathrm{H}), 4.50-$ $4.48(\mathrm{~m}, 1 \mathrm{H}), 3.82(\mathrm{~s}, 3 \mathrm{H}), 3.56(\mathrm{~s}, 3 \mathrm{H}), 3.41(\mathrm{~s}, 3 \mathrm{H}), 2.52-2.45(\mathrm{~m}, 1 \mathrm{H}), 2.19-2.05(\mathrm{~m}$, $3 \mathrm{H}) .{ }^{13} \mathrm{C} \mathrm{NMR}\left(100 \mathrm{MHz}, \mathrm{CDCl}_{3}\right) \delta 169.0,168.0,167.2,144.1,143.4,140.7,130.7$, 
129.1, 128.2, 126.8, 125.1, 124.2, 120.6, 117.1, 111.4, 74.0, 62.8, 58.5, 53.4, 52.8, 50.6, 29.3, 21.3. IR (thin film): $v_{\max }\left(\mathrm{cm}^{-1}\right)=2920,2850,1734,1662,1595,1557,1463,1390$, 1250, 1194, 1149, 1086, 1019, 982, 850, 755, 734, 700, 564, 483, 438. HRMS (ESI) calcd for $\mathrm{C}_{25} \mathrm{H}_{26} \mathrm{~N}_{2} \mathrm{O}_{6} \mathrm{Na}[\mathrm{M}+\mathrm{Na}]^{+}:$473.1683. Found: 473.1672 .

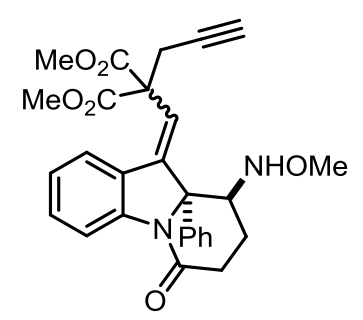

4n, colorless oil, $30.0 \mathrm{mg}, 50 \%$ yield, $E / Z=1.6 / 1 .{ }^{1} \mathrm{H} \mathrm{NMR}\left(400 \mathrm{MHz}, \mathrm{CDCl}_{3}\right) \delta 8.49$ (d, $J=8.4 \mathrm{~Hz}, 1 \mathrm{H}), 8.35\left(\mathrm{~d}, J=8.0 \mathrm{~Hz}, 1 \mathrm{H}^{*}\right), 7.64$ (d, $\left.J=7.6 \mathrm{~Hz}, 1 \mathrm{H}^{*}\right), 7.40-7.34(\mathrm{~m}$, 2H), 7.32-7.29 (m, 1H), 7.28-7.21 (m, 4H), $7.16\left(\mathrm{t}, J=7.2 \mathrm{~Hz}, 1 \mathrm{H}^{*}\right), 7.11\left(\mathrm{~s}, 1 \mathrm{H}^{*}\right)$, $7.05(\mathrm{t}, J=8.0 \mathrm{~Hz}, 1 \mathrm{H}), 6.68(\mathrm{~s}, 1 \mathrm{H}), 6.26(\mathrm{~s}, 1 \mathrm{H}), 5.84\left(\mathrm{~s}, 1 \mathrm{H}^{*}\right), 4.23(\mathrm{~d}, J=6.4 \mathrm{~Hz}$, $\left.1 \mathrm{H}^{*}\right), 4.09(\mathrm{t}, J=2.8 \mathrm{~Hz}, 1 \mathrm{H}), 3.84\left(\mathrm{~s}, 3 \mathrm{H}^{*}\right), 3.72(\mathrm{~s}, 3 \mathrm{H}), 3.67(\mathrm{~s}, 3 \mathrm{H}), 3.64\left(\mathrm{~s}, 3 \mathrm{H}^{*}\right)$, $3.52(\mathrm{~s}, 3 \mathrm{H}), 3.38\left(\mathrm{~s}, 3 \mathrm{H}^{*}\right), 3.36-3.10(\mathrm{~m}, 1 \mathrm{H}), 2.81-1.59(\mathrm{~m}, 1 \mathrm{H}), 2.48-2.14(\mathrm{~m}, 3 \mathrm{H})$, $2.03(\mathrm{t}, J=2.4 \mathrm{~Hz}, 1 \mathrm{H}), 1.97-1.88\left(\mathrm{~m}, 1 \mathrm{H}^{*}\right), 1.80\left(\mathrm{t}, J=2.4 \mathrm{~Hz}, 1 \mathrm{H}^{*}\right), 1.67-1.56(\mathrm{~m}$, 1H). ${ }^{13} \mathrm{C} \mathrm{NMR}\left(100 \mathrm{MHz}, \mathrm{CDCl}_{3}\right) \delta 170.2,169.5,169.4,169.1,145.4,144.2,142.0$, $141.5,141.4,139.4,130.7,130.5,128.82$, 128.77, 128.7, 128.4, 127.8, 126.4, 125.3, 124.6, 124.23, 124.17, 124.1, 120.1, 118.7, 118.2, 116.8, 116.7, 78.7, 78.1, 75.3, 73.7, 71.9, 71.4, 63.0, 62.9, 59.6, 57.2, 56.6, 56.1, 54.1, 53.7, 53.6, 53.5, 29.8, 29.7, 28.4, 24.8, 24.1, 21.9, 19.1. IR (thin film): $v_{\max }\left(\mathrm{cm}^{-1}\right)=3284,2921,2851,1734,1662,1594$, 1459, 1386, 1275, 1201, 1178, 1065, 1040, 972, 947, 886, 824, 755, 734, 697, 662, 573, 549. HRMS (ESI) calcd for $\mathrm{C}_{28} \mathrm{H}_{28} \mathrm{~N}_{2} \mathrm{O}_{6} \mathrm{Na}[\mathrm{M}+\mathrm{Na}]^{+}:$511.1840. Found: 511.1830.

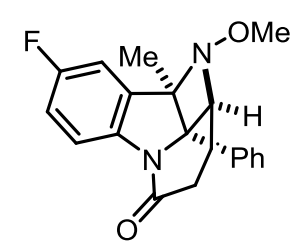

4e', white solid, $66.0 \mathrm{mg}$, 98\% yield, m.p. $=158.3-162.1{ }^{\circ} \mathrm{C}$, reaction time: $0.5 \mathrm{~h} .{ }^{1} \mathrm{H}$ NMR (400 MHz, $\left.\mathrm{CDCl}_{3}\right) \delta 7.95(\mathrm{dd}, J=8.8,4.8 \mathrm{~Hz}, 1 \mathrm{H}), 7.38-7.29(\mathrm{~m}, 3 \mathrm{H}), 7.22-7.20$ (m, 2H), $7.03(\mathrm{td}, J=9.2,2.8 \mathrm{~Hz}, 1 \mathrm{H}), 6.85(\mathrm{dd}, J=7.6,2.4 \mathrm{~Hz}, 1 \mathrm{H}), 4.60(\mathrm{dd}, J=8.8$, $3.2 \mathrm{~Hz}, 1 \mathrm{H}), 3.45$ (s, 3H), 2.54-2.46 (m, 1H), 2.41-2.36 (m, 1H), 2.20-2.12 (m, 1H), 2.07-1.99 (m, 1H), $1.27(\mathrm{~s}, 3 \mathrm{H}) .{ }^{13} \mathrm{C}$ NMR $\left(100 \mathrm{MHz}, \mathrm{CDCl}_{3}\right) \delta 170.3,160.1(\mathrm{~d}, J=$ 
$242.0 \mathrm{~Hz}$ ), 142.2, 137.5 (d, $J=7.0 \mathrm{~Hz}), 135.2,128.8,128.4,126.2,118.7(\mathrm{~d}, J=8.0$ Hz), 115.1 (d, $J=23.0 \mathrm{~Hz}), 109.4(\mathrm{~d}, J=24.0 \mathrm{~Hz}), 78.7,72.1,65.2,62.5,32.8,26.2$, 10.1. ${ }^{19} \mathrm{~F} \mathrm{NMR}\left(376 \mathrm{MHz}, \mathrm{CDCl}_{3}\right) \delta-117.9 \sim-118.0$ (m, 1F). IR (thin film): $v_{\max }\left(\mathrm{cm}^{-}\right.$ $\left.{ }^{1}\right)=2924,1675,1607,1476,1369,1313,1281,1258,1177,1140,1088,1062,1035$, 1003, 973, 915, 862, 829, 792, 773, 744, 695, 610, 589, 516. HRMS (ESI) calcd for $\mathrm{C}_{20} \mathrm{H}_{19} \mathrm{~N}_{2} \mathrm{O}_{2} \mathrm{NaF}[\mathrm{M}+\mathrm{Na}]^{+}:$361.1323. Found: 361.1326 .

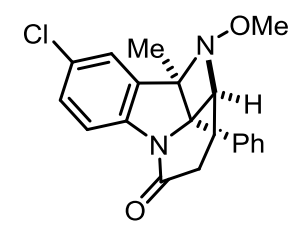

4f', viscous solid, $67.3 \mathrm{mg}$, 95\% yield, reaction time: $1.0 \mathrm{~h} .{ }^{1} \mathrm{H}$ NMR (400 MHz, $\left.\mathrm{CDCl}_{3}\right)$ $\delta 7.94(\mathrm{~d}, J=8.4 \mathrm{~Hz}, 1 \mathrm{H}), 7.38-7.31(\mathrm{~m}, 4 \mathrm{H}), 7.20-7.18(\mathrm{~m}, 2 \mathrm{H}), 7.11(\mathrm{~d}, J=2.0 \mathrm{~Hz}$, $1 \mathrm{H}), 4.60(\mathrm{dd}, J=9.2,3.2 \mathrm{~Hz}, 1 \mathrm{H}), 3.45(\mathrm{~s}, 3 \mathrm{H}), 2.54-2.47(\mathrm{~m}, 1 \mathrm{H}), 2.41-2.37(\mathrm{~m}, 1 \mathrm{H})$, 2.20-2.11 (m, 1H), 2.07-1.99 (m, 1H), $1.27(\mathrm{~s}, 3 \mathrm{H}) .{ }^{13} \mathrm{C} \mathrm{NMR}\left(100 \mathrm{MHz}, \mathrm{CDCl}_{3}\right) \delta$ 170.4, 144.8, 137.4, 135.1, 129.6, 128.91, 128.85, 128.5, 126.2, 122.3, 118.8, 78.8, 72.0, 65.2, 62.6, 32.9, 26.2, 10.1. IR (thin film): $v_{\max }\left(\mathrm{cm}^{-1}\right)=2933,1688,1598,1459,1381$, 1355, 1319, 1289, 1257, 1233, 1181, 1143, 1084, 1063, 1034, 1006, 974, 885, 827, 788, 760, 701, 644, 619, 600. HRMS (ESI) calcd for $\mathrm{C}_{20} \mathrm{H}_{19} \mathrm{~N}_{2} \mathrm{O}_{2} \mathrm{NaCl}[\mathrm{M}+\mathrm{Na}]^{+}:$377.1027. Found: 377.1039.

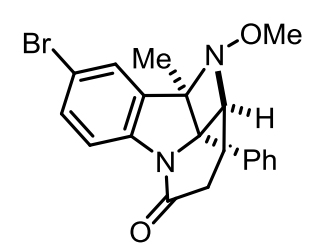

4g', yellow oil, $57.0 \mathrm{mg}, 71 \%$ yield, reaction time: 1.0 h. ${ }^{1} \mathrm{H}$ NMR (400 $\mathrm{MHz}, \mathrm{CDCl}_{3}$ ) $\delta 7.89(\mathrm{~d}, J=8.4 \mathrm{~Hz}, 1 \mathrm{H}), 7.47(\mathrm{dd}, J=8.4,2.0 \mathrm{~Hz}, 1 \mathrm{H}), 7.38-7.30(\mathrm{~m}, 3 \mathrm{H}), 7.25(\mathrm{~d}, J$ $=2.0 \mathrm{~Hz}, 1 \mathrm{H}), 7.20-7.18(\mathrm{~m}, 2 \mathrm{H}), 4.60(\mathrm{dd}, J=8.8,3.2 \mathrm{~Hz}, 1 \mathrm{H}), 3.45(\mathrm{~s}, 3 \mathrm{H}), 2.54-$ $2.46(\mathrm{~m}, 1 \mathrm{H}), 2.41-2.37(\mathrm{~m}, 1 \mathrm{H}), 2.20-2.11(\mathrm{~m}, 1 \mathrm{H}), 2.07-1.98(\mathrm{~m}, 1 \mathrm{H}), 1.27(\mathrm{~s}, 3 \mathrm{H})$. ${ }^{13} \mathrm{C}$ NMR (100 MHz, $\left.\mathrm{CDCl}_{3}\right) \delta 170.4,145.3,137.7,135.1,131.9,128.9,128.5,126.2$, 125.1, 119.3, 117.1, 78.8, 71.9, 65.2, 62.6, 32.9, 26.2, 10.1. IR (thin film): $v_{\max }\left(\mathrm{cm}^{-1}\right)$ =2931, 1678, 1595, 1465, 1362, 1323, 1260, 1229, 1178, 1143, 1090, 1062, 1045, 1006, 974, 879, 823, 787, 736, 696, 643, 612, 590, 570, 518. HRMS (ESI) calcd for $\mathrm{C}_{20} \mathrm{H}_{19} \mathrm{~N}_{2} \mathrm{O}_{2} \mathrm{NaBr}[\mathrm{M}+\mathrm{Na}]^{+}:$421.0522. Found: 421.0517. 


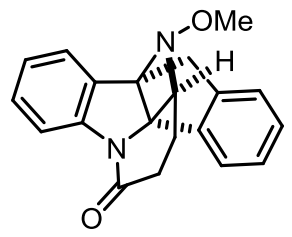

4o', colorless oil, $62.0 \mathrm{mg}$, 96\% yield, reaction time: $12 \mathrm{~h} .{ }^{1} \mathrm{H}$ NMR (400 MHz, $\mathrm{CDCl}_{3}$ ) $\delta 7.81(\mathrm{~d}, J=8.0 \mathrm{~Hz}, 1 \mathrm{H}), 7.33-7.28(\mathrm{~m}, 2 \mathrm{H}), 7.26-7.23(\mathrm{~m}, 4 \mathrm{H}), 7.11(\mathrm{t}, J=7.6 \mathrm{~Hz}$, $1 \mathrm{H}), 4.14(\mathrm{~d}, J=17.2 \mathrm{~Hz}, 1 \mathrm{H}), 4.06-3.99(\mathrm{~m}, 1 \mathrm{H}), 3.50$ (s, 3H), 3.34 (d, $J=17.2 \mathrm{~Hz}$, 1H), 2.64-2.47 (m, 3H), 2.19-2.07 (m, 1H). $\left.{ }^{13} \mathrm{C} \mathrm{NMR} \mathrm{(100} \mathrm{MHz,} \mathrm{CDCl}_{3}\right) \delta 171.4,147.0$, 145.1, 141.1, 134.8, 129.2, 128.7, 127.4, 126.2, 125.0, 123.2, 122.9, 119.0, 81.8, 73.7, 69.8, 60.9, 34.2, 32.4, 27.1. IR (thin film): $v_{\max }\left(\mathrm{cm}^{-1}\right)=2918,2857,1735,1675,1603$, 1461, 1361, 1287, 1222, 1183, 1145, 1097, 1077, 1055, 1032, 1004, 979, 873, 838, 820, 796, 750, 713, 671, 639, 603. HRMS (ESI) calcd for $\mathrm{C}_{20} \mathrm{H}_{18} \mathrm{~N}_{2} \mathrm{NaO}_{2}[\mathrm{M}+\mathrm{Na}]^{+}$: 341.1261. Found: 341.1263. 


\section{Transformations of products}

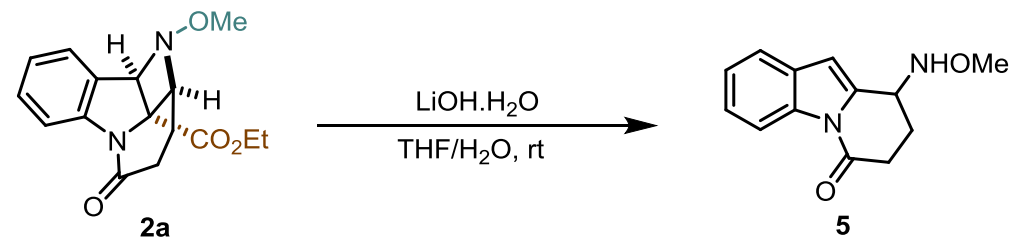

A solution of compound $2 \mathbf{a}(63.2 \mathrm{mg}, 0.2 \mathrm{mmol})$ in THF $(1 \mathrm{~mL})$ was slowly added to a flask containing solution of $\mathrm{LiOH} \cdot \mathrm{H}_{2} \mathrm{O}(28.0 \mathrm{mg}, 0.6 \mathrm{mmol})$ in water $(1 \mathrm{~mL})$. The resulting mixture was vigorously stirred at room temperature for $2 \mathrm{~h}$. THF was then evaporated under reduced pressure and the resulting aqueous solution was acidified with $\mathrm{HCl}(2 \mathrm{~N})$ to $\mathrm{pH}=2$. The mixture was extracted with EtOAc $(10 \mathrm{~mL} \times 3)$ and washed by saturated sodium carbonate solution $(10 \mathrm{~mL} \times 3)$. The combined organic layer was washed with brine, dried over $\mathrm{Na}_{2} \mathrm{SO}_{4}$, filtered and concentrated by rotary evaporation. Then the residue was purified by silica gel column chromatography $(\mathrm{PE} / \mathrm{EtOAc}=3 / 1)$ to afford the desired product $\mathbf{5}$.<smiles>CNC1CCC(=O)n2c1cc1ccccc12</smiles>

5, colorless oil, $33.1 \mathrm{mg}, 72 \%$ yield. ${ }^{1} \mathrm{H}$ NMR $\left(400 \mathrm{MHz}, \mathrm{CDCl}_{3}\right) \delta 8.46(\mathrm{~d}, J=8.4 \mathrm{~Hz}$, $1 \mathrm{H}), 7.51(\mathrm{~d}, J=7.6 \mathrm{~Hz}, 1 \mathrm{H}), 7.35-7.24(\mathrm{~m}, 2 \mathrm{H}), 6.57(\mathrm{~s}, 1 \mathrm{H}), 5.61(\mathrm{~s}, 1 \mathrm{H}), 4.43(\mathrm{t}, J=$ $4.4 \mathrm{~Hz}, 1 \mathrm{H}), 3.57(\mathrm{~s}, 3 \mathrm{H}), 3.17-3.09(\mathrm{~m}, 1 \mathrm{H}), 2.75-2.68(\mathrm{~m}, 1 \mathrm{H}), 2.25-2.20(\mathrm{~m}, 1 \mathrm{H}) .{ }^{13} \mathrm{C}$ NMR $\left(100 \mathrm{MHz}, \mathrm{CDCl}_{3}\right) \delta 169.0,136.4,135.0,129.1,125.0,124.0,120.4,116.5,107.2$, 62.8, 53.3, 30.3, 24.9. IR (thin film): $v_{\max }\left(\mathrm{cm}^{-1}\right)=2920,2806,1687,1595,1449,1374$, 1357, 1319, 1243, 1184, 1151, 1103, 1073, 1010, 996, 937, 896, 854, 822, 743, 698. HRMS (EI) calcd for $\mathrm{C}_{13} \mathrm{H}_{14} \mathrm{~N}_{2} \mathrm{O}_{2}[\mathrm{M}]^{+}:$230.1050. Found: 230.1050.

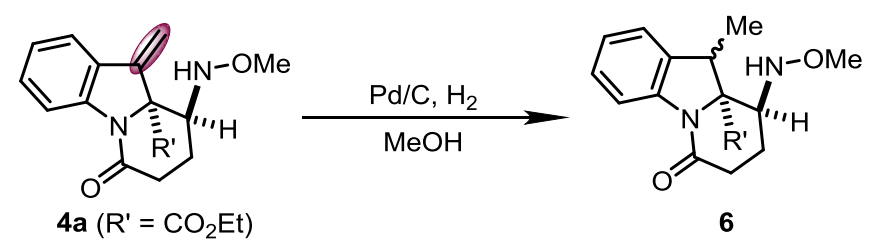


4a $(63.2 \mathrm{mg}, 0.2 \mathrm{mmol})$ and $\mathrm{Pd} / \mathrm{C}(7.0 \mathrm{mg}, 10 \% \mathrm{w})$ were added into a flask containing $\mathrm{MeOH}(10 \mathrm{~mL})$ under argon. The reaction mixture was stirred at room temperature under hydrogen atmosphere (1.0 atm). After the reaction was complete (monitored by TLC), the suspension was filtered and washed with dichloromethane. Then the filtrate was concentrated by rotary evaporation and the residue was purified by silica gel column chromatography $(\mathrm{PE} / \mathrm{EtOAc}=3 / 1)$ to afford the desired product 6 .

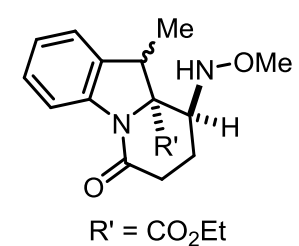

6, colorless oil, $50.6 \mathrm{mg}$, 85\% yield, $1 / 1 \mathrm{dr} .{ }^{1} \mathrm{H}$ NMR (400 MHz, $\left.\mathrm{CDCl}_{3}\right) \delta 8.22(\mathrm{~d}, J=$ $8.0 \mathrm{~Hz}, 1 \mathrm{H}), 8.18\left(\mathrm{~d}, J=8.0 \mathrm{~Hz}, 1 \mathrm{H}^{*}\right), 7.22(\mathrm{~d}, J=8.0 \mathrm{~Hz}, 1 \mathrm{H}), 7.12-7.04(\mathrm{~m}, 2 \mathrm{H})$, $5.58\left(\mathrm{~d}, J=6.0 \mathrm{~Hz}, 1 \mathrm{H}^{*}\right), 5.38(\mathrm{~d}, J=8.4 \mathrm{~Hz}, 1 \mathrm{H}), 4.28-3.62(\mathrm{~m}, 4 \mathrm{H}), 3.45(\mathrm{~s}, 3 \mathrm{H})$, 3.39 (s, 3H*), 2.65-2.40 (m, 2H), 2.31-2.20 (m, 1H), 1.96-1.73 (m, 1H), 1.68 (d, J= 7.6 $\mathrm{Hz}, 3 \mathrm{H}), 1.34$ (d, $\left.J=7.2 \mathrm{~Hz}, 3 \mathrm{H}^{*}\right), 1.26$ (t, $\left.J=7.2 \mathrm{~Hz}, 3 \mathrm{H}\right), 1.15$ (t, $\left.J=7.2 \mathrm{~Hz}, 3 \mathrm{H}^{*}\right)$. ${ }^{13} \mathrm{C}$ NMR $\left(100 \mathrm{MHz}, \mathrm{CDCl}_{3}\right) \delta 172.6,170.1,169.6,168.0,142.3,141.8,132.9,132.7$, $127.9,127.7,124.0,122.7,122.3,116.6,116.4,77.7,74.0,62.2,61.9,61.8,61.7,56.5$, $54.9,44.4,40.1,28.6,28.3,22.4,21.0,16.2,13.94,13.91,13.5$. IR (thin film): $v_{\max }\left(\mathrm{cm}^{-}\right.$ $\left.{ }^{1}\right)=2935,1727,1649,1598,1482,1458,1386,1314,1288,1246,1169,1132,1091$, 1054, 1020, 964, 920, 857, 762, 549, 453. HRMS (ESI) calcd for $\mathrm{C}_{17} \mathrm{H}_{22} \mathrm{~N}_{2} \mathrm{O}_{4} \mathrm{Na}$ $[\mathrm{M}+\mathrm{Na}]^{+}:$341.1472. Found: 341.1471.

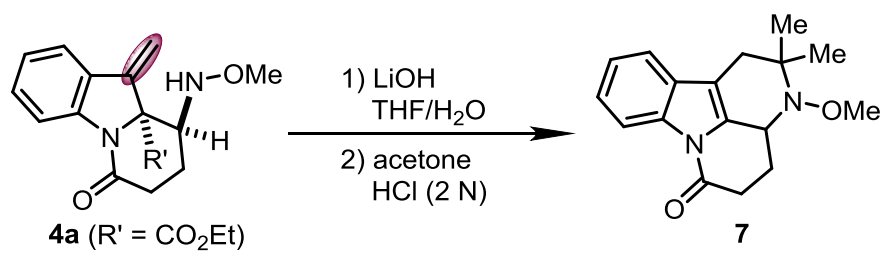

A solution of compound $\mathbf{2 a}(63.2 \mathrm{mg}, 0.2 \mathrm{mmol})$ in THF $(1 \mathrm{~mL})$ was slowly added to a flask containing solution of $\mathrm{LiOH} \cdot \mathrm{H}_{2} \mathrm{O}(28.0 \mathrm{mg}, 0.6 \mathrm{mmol})$ in water $(1 \mathrm{~mL})$. The resulting mixture was vigorously stirred at room temperature for $5 \mathrm{~h}$. THF was then evaporated under reduced pressure and the resulting aqueous solution was acidified with $\mathrm{HCl}(2 \mathrm{~N})$ to $\mathrm{pH}=2$. The mixture was diluted with acetone $(10 \mathrm{~mL})$ and washed 
by saturated sodium carbonate solution $(10 \mathrm{~mL} \times 3)$. The combined organic layer was washed with brine, dried over $\mathrm{Na}_{2} \mathrm{SO}_{4}$, filtered and concentrated by rotary evaporation. Then the residue was purified by silica gel column chromatography $(\mathrm{PE} / \mathrm{EtOAc}=3 / 1)$ to afford the desired product 7.

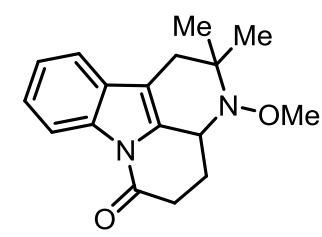

7, brown solid, $34.1 \mathrm{mg}, 61 \%$ yield, m.p. $=157.9-160.1{ }^{\circ} \mathrm{C}$, two rotamers were observed ${ }^{1} \mathrm{H}$ NMR (400 MHz, $\left.\mathrm{CDCl}_{3}\right) \delta 8.36(\mathrm{~d}, J=7.2 \mathrm{~Hz}, 1 \mathrm{H}), 7.42-7.36(\mathrm{~m}, 1 \mathrm{H}), 7.32-7.24$ (m, 2H), 4.25-3.91 (m, 1H), 3.66-3.55 (m, 3H), 2.91-2.77 (m, 3H), 2.63-1.98 (m, 3H), $1.45(\mathrm{~s}, 3 \mathrm{H}), 1.22-1.18(\mathrm{~m}, 3 \mathrm{H}) .{ }^{13} \mathrm{C} \mathrm{NMR}\left(100 \mathrm{MHz}, \mathrm{CDCl}_{3}\right) \delta 168.0,132.0,129.1$, $124.4,123.8,118.1,116.1,111.8,64.3,59.8,55.8,35.2,32.8,28.5,27.7,17.0$. IR (thin film): $v_{\max }\left(\mathrm{cm}^{-1}\right)=2962,2847,1701,1648,1453,1420,1360,1319,1252,1197,1165$, 1134, 1091, 1033, 839, 819, 761, 719, 690, 599, 555, 478, 465. HRMS (ESI) calcd for $\mathrm{C}_{17} \mathrm{H}_{21} \mathrm{~N}_{2} \mathrm{O}_{2}[\mathrm{M}+\mathrm{H}]^{+}:$285.1598. Found: 285.1592 . 


\section{Experimental details for mechanistic studies}

\subsection{Cyclic voltammetry}

A glassy carbon was used as the working electrode. A platinum wire was used as the auxillary electrode, and a SCE was used as the reference electrode. 0.1 M tetrabutylammonium in $\mathrm{CH}_{3} \mathrm{CN}$ was used as the supporting electrolyte. The scan rate was $100 \mathrm{mV} \mathrm{s}^{-1}$. Under these conditions, $\mathrm{E}_{1 / 2}=0.55 \mathrm{~V}$ for the $\left[\mathrm{Cp}_{2} \mathrm{Fe}\right]^{0,+}$ couple.

Cyclic voltammogram of substrate 1a.
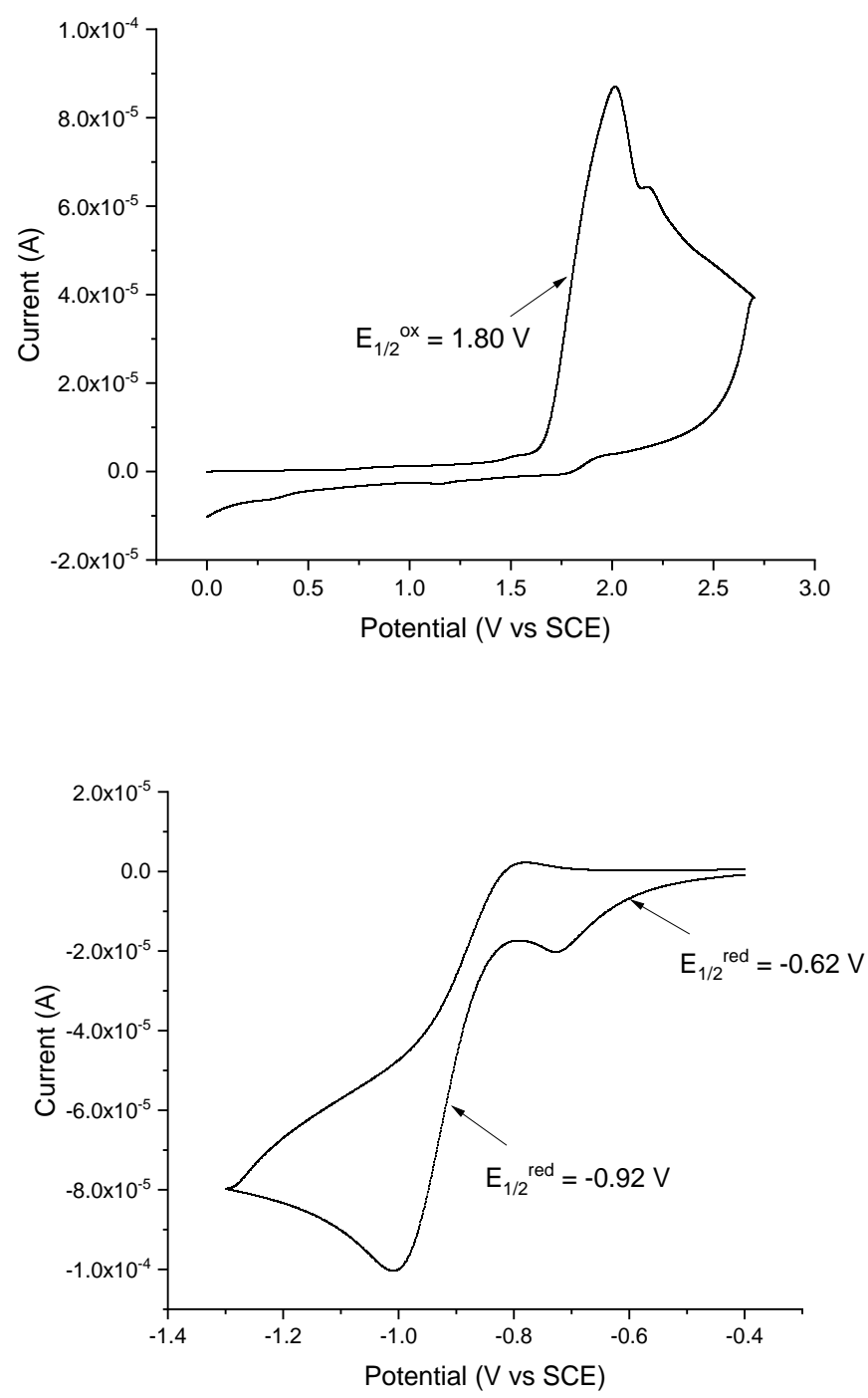


\subsection{Control experiment with triplet quencher}

2,5-Dimethylhexa-2,4-diene is known as a triplet quencher. ${ }^{4}$ The model reaction $\mathbf{1 a} \rightarrow$ 2a is significantly inhibited in the presence of 1.0 equivalent of 2,5-dimethylhexa-2,4diene. This reaction suggested that a triplet diradical intermediate might be involved in the reaction.

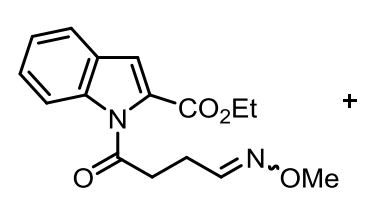

$1 \mathrm{a}$<smiles>CC(C)=CC=C(C)C</smiles>

(1.0 equiv.)

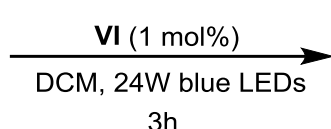

$3 \mathrm{~h}$

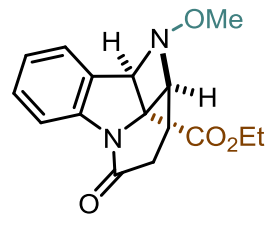

$2 a$

$54 \%$ yield

\subsection{Stern-Volmer luminescence quenching studies}

Stern-Volmer experiments were conducted on a Hitachi F4600 Fluorescence Spectrophotometer. Each component was prepared in DCM prior to each set of experiments. The solutions were irradiated at $335 \mathrm{~nm}$ and the luminescence measured at $491 \mathrm{~nm}$. The value for $I_{0} / I$ from each run was averaged to yield a value of $I_{0} / I$ for the experiment. Linear regression of $I_{0} / I$ against concentration was performed in Origin.

\begin{tabular}{|c|c|}
\hline species & concentration $(\mathrm{mM})$ \\
\hline $\operatorname{Ir}\left[(\mathrm{dFppy})_{3}\right]$ & 0.02 \\
\hline $\mathbf{1 a}$ & varied \\
\hline
\end{tabular}

\begin{tabular}{|c|c|c|c|c|}
\hline $\mathbf{1 a}\left(\times 10^{-5} \mathrm{M}\right)$ & 0 & 4 & 8 & 12 \\
\hline $\mathrm{I}_{1}$ & 2085 & 947.0 & 436.2 & 268.9 \\
\hline $\mathrm{I}_{2}$ & 2011 & 947.8 & 429 & 281.1 \\
\hline Average & 2048 & 947 & 432 & 275 \\
\hline $\mathrm{I}_{0} / \mathrm{I}$ & 1.00 & 2.16 & 4.74 & 7.44 \\
\hline
\end{tabular}




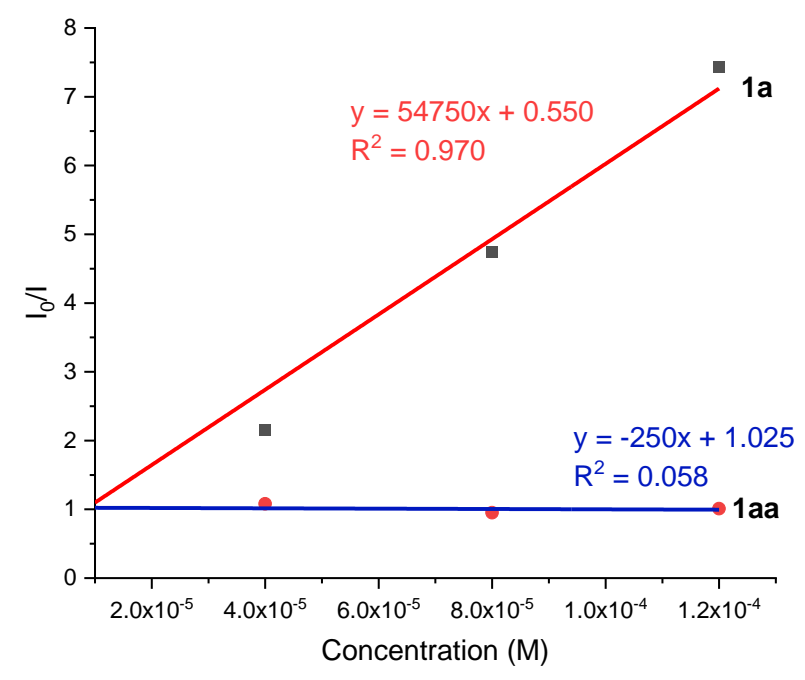

\begin{tabular}{|c|c|}
\hline species & concentration $(\mathrm{mM})$ \\
\hline $\operatorname{Ir}\left[(\mathrm{dFppy})_{3}\right]$ & 0.02 \\
\hline $\mathbf{1 a}^{\prime}$ & varied \\
\hline
\end{tabular}

\begin{tabular}{|c|c|c|c|c|}
\hline $1 \mathbf{a}^{\prime}\left(\times 10^{-5} \mathrm{M}\right)$ & 0 & 4 & 8 & 12 \\
\hline $\mathrm{I}_{1}$ & 1970 & 1814 & 2030 & 1905 \\
\hline $\mathrm{I}_{2}$ & 1906 & 1765 & 2032 & 1907 \\
\hline Average & 1938 & 1790 & 2031 & 1906 \\
\hline $\mathrm{I}_{0} / \mathrm{I}$ & 1.00 & 1.08 & 0.95 & 1.01 \\
\hline
\end{tabular}

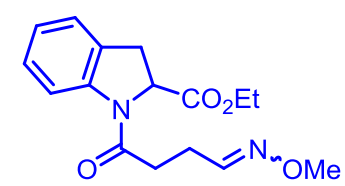

1a', colorless oil, $0.20 \mathrm{~g}, 17 \%$ yield (4 mmol scale), $E / Z=1.2 / 1$, two rotamers were observed. ${ }^{1} \mathrm{H}$ NMR $\left(400 \mathrm{MHz}, \mathrm{CDCl}_{3}\right) \delta 8.23(\mathrm{~d}, J=8.0 \mathrm{~Hz}, 1 \mathrm{H}), 7.99(\mathrm{~d}, J=8.4 \mathrm{~Hz}$, $\left.1 \mathrm{H}^{*}\right), 7.59-7.42(\mathrm{~m}, 1 \mathrm{H}), 7.23-7.14(\mathrm{~m}, 2 \mathrm{H}), 7.02(\mathrm{t}, J=7.6 \mathrm{~Hz}, 1 \mathrm{H}), 6.86-6.72(\mathrm{~m}$, $\left.1 \mathrm{H}^{*}\right)$, 5.16-4.89 (m, 1H), 4.26-4.20 (m, 2H), 3.87 (s, 3H), 3.79 (s, 3H*), 3.64-3.44 (m, 1H), 3.29-2.94 (m, 2H), 2.70-2.49 (m, 3H), $1.26(\mathrm{t}, J=7.2 \mathrm{~Hz}, 3 \mathrm{H}) .{ }^{13} \mathrm{C}$ NMR $(100$ $\left.\mathrm{MHz}, \mathrm{CDCl}_{3}\right) \delta 171.1,170.0,149.9,149.2,142.5,140.8,130.8,128.4,128.1,127.8$, 125.7, 124.4, 124.3, 124.1, 123.9, 123.3, 117.2, 115.6, 113.9, 62.1, 62.0, 61.5, 61.3, $61.2,60.51,60.45,60.3,33.5,33.4,33.0,32.8,31.63,31.56,31.3,24.7,20.9,14.0,13.9$. 
IR (thin film): $v_{\max }\left(\mathrm{cm}^{-1}\right)=2938,1785,1726,1658,1598,1480,1461,1406,1370$, 1312, 1270, 1222, 1200, 1015, 934, 871, 839, 753, 705, 656. HRMS (ESI) calcd for $\mathrm{C}_{16} \mathrm{H}_{20} \mathrm{~N}_{2} \mathrm{O}_{4} \mathrm{Na}[\mathrm{M}+\mathrm{Na}]^{+}:$327.1315. Found: 327.1309.

\subsection{Kinetic profile of the 1,5-HAT reaction}

Eight parallel reactions were set up accordingly. The content of $3 \mathbf{f}, \mathbf{4 f}$ and $4 \mathbf{f}^{\prime}$ (intramolecular [2+2] product) was monitored by ${ }^{1} \mathrm{H}$ NMR analysis of the crude reaction mixture over time.
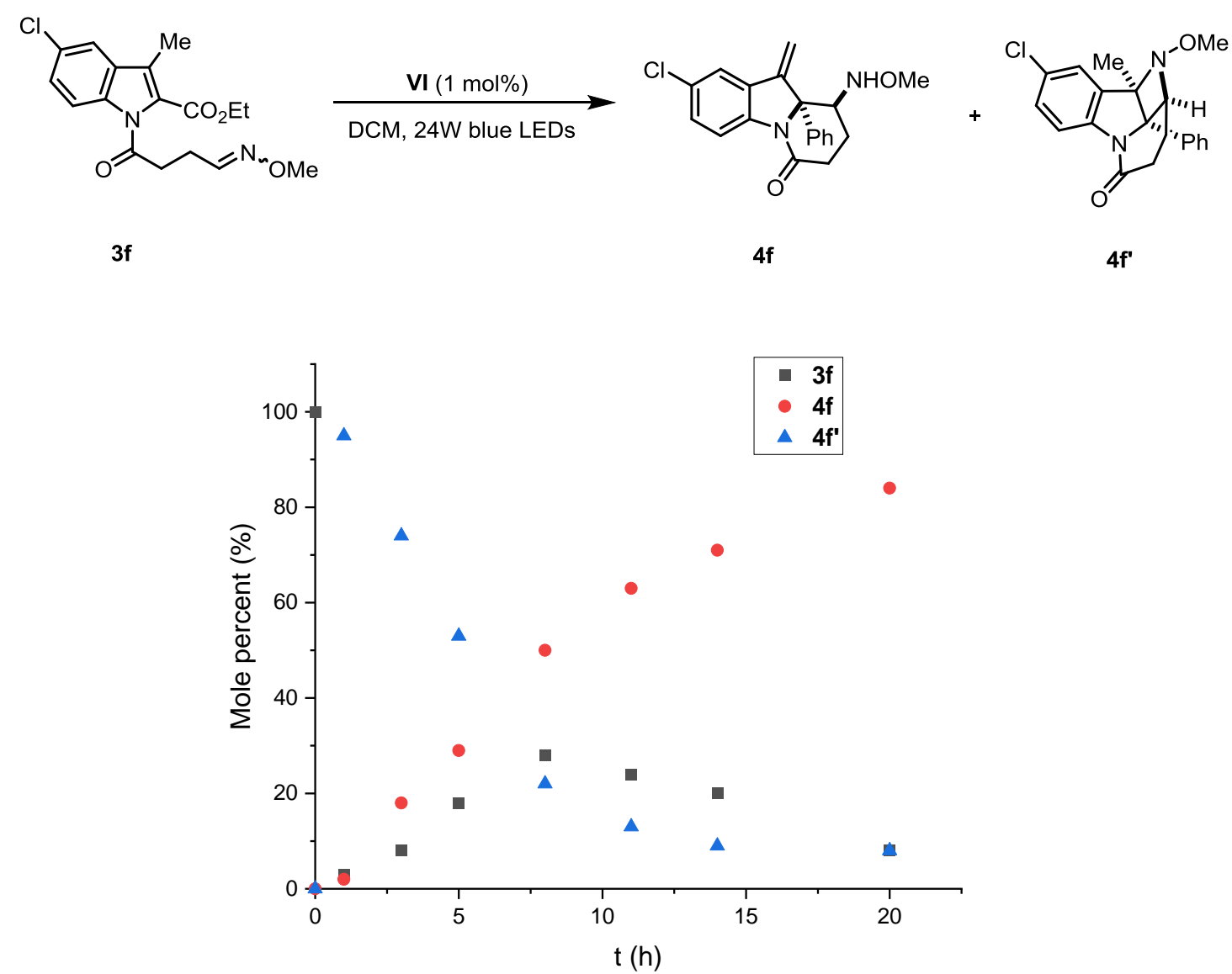

Figure S1. Kinetic profile of the reaction using $\mathbf{3 f}$ as starting material.

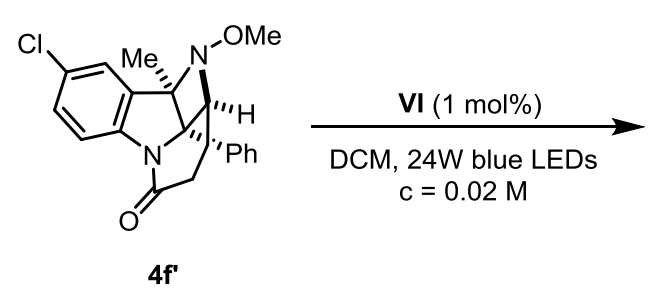

4f'

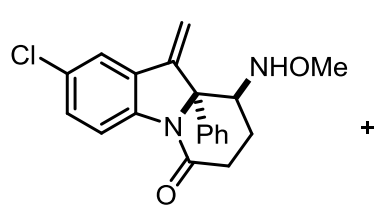

$4 f$

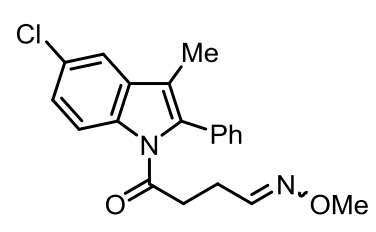

$3 \mathbf{f}$ 


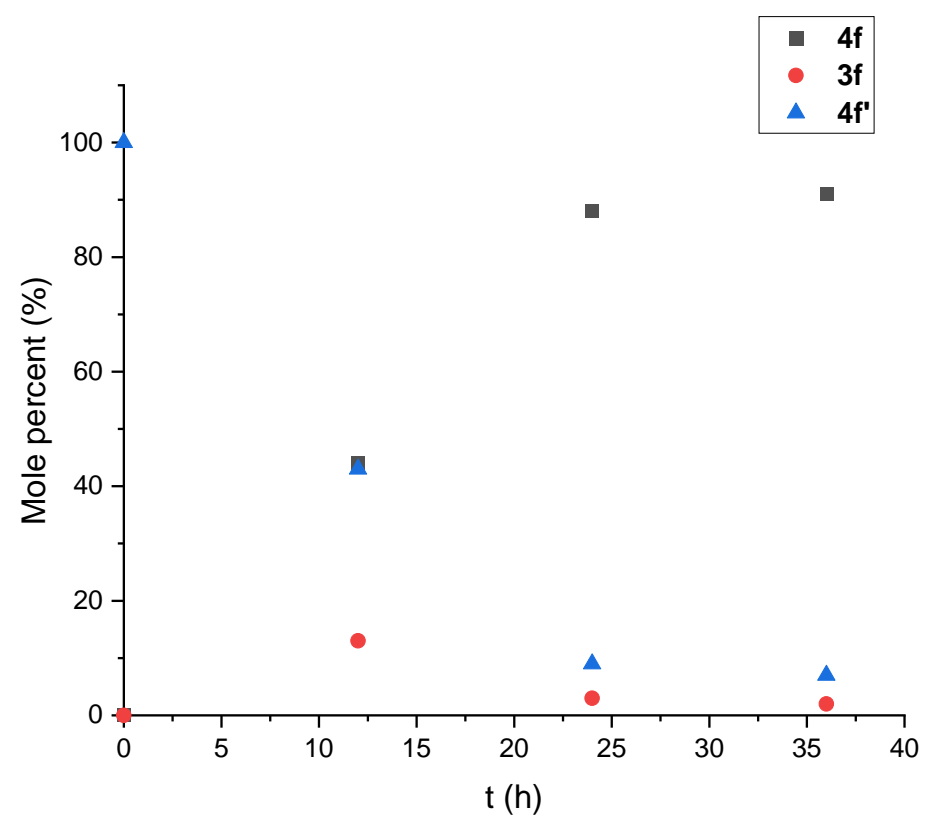

Figure S2. Kinetic profile of the reaction using $\mathbf{4 \mathbf { f } ^ { \prime }}$ as starting material

\subsection{Computational studies}

\section{Computational methods}

All the DFT calculations in this work were performed with Gaussian16 package. ${ }^{5}$ The density functional theory (DFT) method was employed using the (U)B3LYP functional. ${ }^{6}$ The standard 6-31G(d,p) basis sets were used for all atoms. Optimizations were conducted without any constraint using implicit solvation model (SMD) ${ }^{7}$ in dichloromethane $(\varepsilon=8.93)$. Frequency analyses (at $298.15 \mathrm{~K}$ and $1 \mathrm{~atm}$ ) were carried out to confirm each structure being a minimum (no imaginary frequency) or a transition state (only one imaginary frequency). The searching for minimal energy crossing points (MECP) were conducted using a modified version of Harvey's $\operatorname{code}^{8}\left(\right.$ sobMECP $^{9}$ ) interfaced with Gaussian16. The Gibbs free energies in dichloromethane $(\Delta G)$ were discussed throughout this paper unless otherwise specified. The 3D images of the calculated structures and the orbital diagrams were prepared using CYLView. ${ }^{10}$

Additional information on the reaction profile 
The excitation process of substrate 3a was first explored computationally. The calculated energy differences between $E-\mathbf{3 a}$ and $Z-3 \mathbf{a}$ are $0.4 \mathrm{kcal} / \mathrm{mol}$ (on ground state) and $0.1 \mathrm{kcal} / \mathrm{mol}$ (on triplet state), respectively. All the possible transition states for the first cyclization step are localized [including the facial selectivity for the two prochiral face, Si-Si or Si-Re, and the configuration of the oxime moiety, $E$ and Z]. The transition states TS1-(Si,Re,E) $(62.7 \mathrm{kcal} / \mathrm{mol})$ and TS1-(Si,Si,Z) $(64.5 \mathrm{kcal} / \mathrm{mol})$ are included in the main text as TS1 and TS1' leading to the products with different relative configuration.

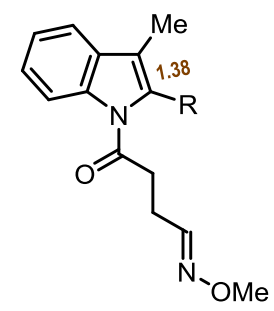

E-3a as $3 a$ in main text

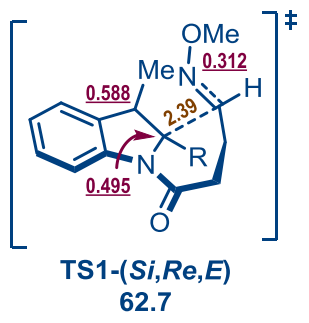
as TS1 in main text

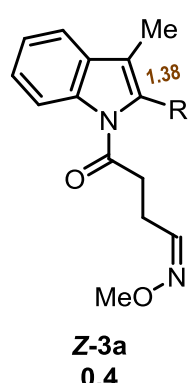

0.4

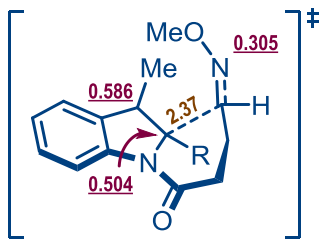

TS1-(Si,Re,Z) 63.2

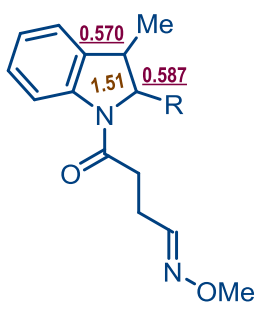

E-3a- $\mathrm{T}_{1}$

53.0 as $3 a-T_{1}$ in main text

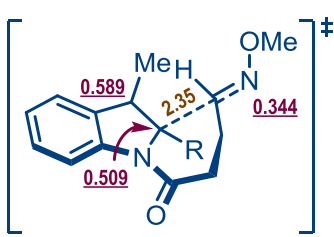

TS1-(Si,Si,E) 64.8

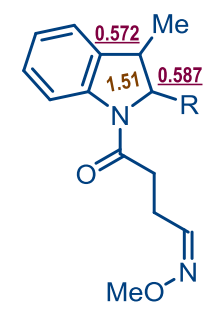

Z-3a-T,

53.1

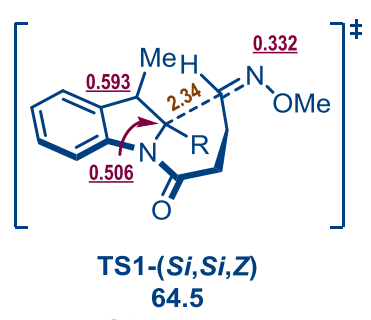

as TS1' in main text

Figure S3. The computational results for the excitation process of 3a. Calculated at (U)B3LYP/6-31G(d,p) level of theory. The Gibbs free energies $(\Delta G)$ or electronic energies $(\Delta E$, in parentheses) are in $\mathrm{kcal} / \mathrm{mol}$. The values in brown are bond distances in angstrom. The underlined values are Mulliken spin populations at certain atoms.

$\underline{\text { Cartesian coordinates and energies and of all optimized structures }}$

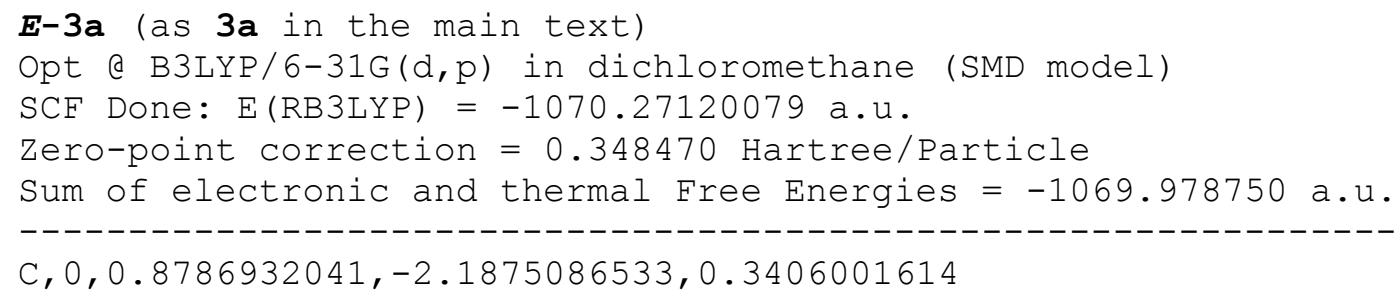


$C, 0,-0.3359878655,-2.2171304518,-0.3826924745$

C, $0,-1.0940173239,-1.0574782993,-0.5816722323$

$C, 0,-0.5949645652,0.1327511059,-0.0562650225$

$C, 0,0.6196238156,0.1804724652,0.6530385306$

C, $0,1.3628457022,-0.9754241463,0.857426353$

$\mathrm{C}, 0,1.4074059161,-3.5300471785,0.3891922612$

$\mathrm{C}, 0,0.5105024462,-4.3334417871,-0.2779451369$

$\mathrm{H}, 0,-2.019988677,-1.0819235302,-1.1378869775$

$\mathrm{H}, \mathrm{O},-1.1579302187,1.0488755541,-0.2095412404$

$\mathrm{H}, 0,0.9757294684,1.1297561957,1.0418186209$

$\mathrm{H}, 0,2.2973562415,-0.9477607362,1.4096787628$

$\mathrm{C}, 0,0.7427862638,-5.6972920833,-0.8002564531$

$0,0,0.4051215932,-6.0660246804,-1.913604905$

$0,0,1.3991287375,-6.4749941849,0.0771234052$

$\mathrm{C}, 0,1.7636920744,-7.8046680426,-0.393421373$

C, $0,2.4630235531,-8.5158553204,0.7447924695$

$\mathrm{H}, 0,2.4078994902,-7.6997387073,-1.2713233732$

$\mathrm{H}, 0,0.8515746889,-8.3237382563,-0.700699682$

$\mathrm{H}, 0,2.7351797456,-9.5266543739,0.4247730949$

$\mathrm{H}, 0,3.3779247817,-7.9926489479,1.0390069596$

$\mathrm{H}, 0,1.809731682,-8.5975105635,1.6188077182$

$\mathrm{N}, 0,-0.5794704793,-3.5550348345,-0.7509994316$

$\mathrm{C}, 0,-1.8397478857,-3.996059644,-1.2149553989$

$0,0,-2.5697654675,-3.2130906596,-1.80060264$

$\mathrm{C}, 0,-2.2828461768,-5.4150710557,-0.8970430107$

C, $0,-3.8142481743,-5.4907905085,-0.7518516063$

$\mathrm{H}, 0,-1.7970167434,-5.7764000015,0.0135469578$

$\mathrm{H}, 0,-1.9511954784,-6.0641032797,-1.7135502951$

C, $0,-4.2793552686,-6.9056502295,-0.5985216522$

$\mathrm{H}, 0,-4.1495814994,-4.9038121557,0.1084101669$

$\mathrm{H}, \mathrm{O},-4.2714166395,-5.0562761319,-1.6478722484$

$\mathrm{N}, 0,-4.9047948434,-7.262584095,0.4569993466$

$\mathrm{H}, 0,-4.075330685,-7.6229377361,-1.3987814444$

$0,0,-5.2608709547,-8.6226834243,0.4014036574$

$\mathrm{C}, 0,-5.9630101901,-8.9682434906,1.5941578388$

$\mathrm{H}, \mathrm{O},-6.8858995768,-8.3856275995,1.6985934918$

$\mathrm{H}, 0,-6.2102334678,-10.0277987011,1.4936994618$

$\mathrm{H}, 0,-5.3387975242,-8.8210813817,2.4834805304$

$\mathrm{C}, 0,2.7273754902,-3.8832901236,0.9984204151$

$\mathrm{H}, 0,2.6764078429,-3.8023019627,2.0914668063$

$\mathrm{H}, 0,3.0359504385,-4.8983421201,0.7528522144$

$\mathrm{H}, 0,3.502537889,-3.1859630027,0.6625077733$

\section{z-3a}

Opt a B3LYP/6-31G(d,p) in dichloromethane (SMD model)

$\mathrm{SCF}$ Done: $\mathrm{E}(\mathrm{RB} 3 \mathrm{LYP})=-1070.27007814 \mathrm{a} \cdot \mathrm{u}$.

Zero-point correction $=0.348562$ Hartree/Particle

Sum of electronic and thermal Free Energies = -1069.978055 a.u.

$C, 0,-3.245439144,-0.249044619,0.4434449884$

$C, 0,-2.3642885582,-1.2235739565,-0.0794760579$

C, $0,-2.7119686191,-2.5791017527,-0.1094644287$

$\mathrm{C}, 0,-3.9692045249,-2.9294547897,0.378455634$

C, $0,-4.8626239748,-1.9690607923,0.8887467488$

$C, 0,-4.5086115214,-0.6262194333,0.9262158538$

C, $0,-2.5985540719,1.0381511519,0.3536265194$ 
$C, 0,-1.3543396619,0.819030765,-0.1941985429$

$\mathrm{H}, \mathrm{O},-2.0387845838,-3.3213408489,-0.5136805856$

$\mathrm{H}, \mathrm{O},-4.267933995,-3.973408931,0.3527754875$

$\mathrm{H}, \mathrm{0},-5.8353121172,-2.2836738384,1.2548358722$

$\mathrm{H}, \mathrm{O},-5.1896547491,0.1196595258,1.3247717495$

$\mathrm{C}, 0,-0.4503800078,1.8186659154,-0.8018777158$

$0,0,0.1456849028,1.646857629,-1.8530638944$

$0,0,-0.3848040244,2.9524531201,-0.0833632185$

C, $0,0.4027690215,4.0325992521,-0.6622004422$

C, $0,0.3579040122,5.1981200401,0.3021417893$

$\mathrm{H}, 0,-0.0197681622,4.2892958016,-1.6380929712$

$\mathrm{H}, 0,1.4228296862,3.6704174981,-0.8180046262$

$\mathrm{H}, 0,0.9585412824,6.020678402,-0.0989093158$

$\mathrm{H}, \mathrm{O},-0.6654108701,5.5592808908,0.4425925935$

$\mathrm{H}, 0,0.7677184588,4.9184923692,1.2774608705$

$\mathrm{N}, 0,-1.1776818298,-0.5655592238,-0.457253525$

$\mathrm{C}, 0,0.0210801937,-1.2645574058,-0.7309595192$

$0,0,-0.0372671757,-2.4002699073,-1.1731207472$

C, $0,1.3482039592,-0.6093648935,-0.3863600426$

C, $0,2.3983418792,-1.6626995497,-0.0075035111$

$\mathrm{H}, 0,1.2191083853,0.1119455633,0.425694069$

$\mathrm{H}, 0,1.6822762079,-0.0434435811,-1.2617933216$

C, $0,3.7524204741,-1.0455214901,0.1899188617$

$\mathrm{H}, \mathrm{O}, 2.1070577956,-2.2002860371,0.8994929417$

$\mathrm{H}, \mathrm{0}, 2.4591996935,-2.4159879227,-0.8028092879$

$\mathrm{N}, 0,4.7169692398,-1.493276044,0.9031253981$

$\mathrm{H}, 0,3.9882207053,-0.11423968,-0.3252476481$

$0,0,4.3860273229,-2.7053904776,1.5336269468$

C, $0,5.4720564189,-3.1035752587,2.3695399879$

$\mathrm{H}, 0,5.6574529555,-2.3684854101,3.1614737614$

$\mathrm{H}, 0,5.1677518035,-4.0529753454,2.8165023398$

$\mathrm{H}, 0,6.3894308645,-3.2489564877,1.7875797738$

$\mathrm{C}, 0,-3.2565045554,2.3272912387,0.7322710406$

$\mathrm{H}, \mathrm{O},-3.3520121277,2.4006020707,1.8228926972$

$\mathrm{H}, \mathrm{0},-2.6940774211,3.1937727122,0.3879085193$

$\mathrm{H}, \mathrm{O},-4.2698347174,2.3751322305,0.3191286078$

\section{$E-3 a-T_{1}$}

Opt a UB3LYP/6-31G (d,p) in dichloromethane (SMD model)

$\mathrm{SCF}$ Done: $\mathrm{E}(\mathrm{UB} 3 \mathrm{LYP})=-1070.18215898 \mathrm{a} \cdot \mathrm{u}$.

Zero-point correction $=0.344983$ Hartree/Particle

Sum of electronic and thermal Free Energies = -1069.894322 a.u.

$C, 0,0.8364870685,-2.303617256,0.4337860498$

$\mathrm{C}, 0,-0.3066468306,-2.3190126098,-0.4327306373$

$\mathrm{C}, 0,-0.9340607287,-1.1694032139,-0.8406393775$

$\mathrm{C}, 0,-0.4334986697,0.0690886674,-0.3476670454$

$\mathrm{C}, 0,0.6646686982,0.1166293676,0.5155888201$

$\mathrm{C}, 0,1.3105985561,-1.0524032489,0.9145053386$

$C, 0,1.2795707497,-3.609938362,0.6486903302$

$\mathrm{C}, 0,0.4036369864,-4.4945570453,-0.2021327128$

$\mathrm{H}, 0,-1.7854488627,-1.1988999896,-1.50479057$

$\mathrm{H}, 0,-0.927200119,0.9872946852,-0.6492288347$

$\mathrm{H}, 0,1.0237576805,1.0764860372,0.8746298237$

$\mathrm{H}, 0,2.1699787736,-1.0148775538,1.5760389313$

C, $0,0.7350002709,-5.7731663539,-0.7975924172$ 
$0,0,0.2273061446,-6.2288252984,-1.8234108555$ $0,0,1.6890030496,-6.4271320025,-0.0865978322$ C, $0,2.1135989167,-7.7037489074,-0.6227649998$ $\mathrm{C}, 0,3.164367566,-8.2668562134,0.3112204872$ $\mathrm{H}, 0,2.5068253358,-7.5520754202,-1.6332398524$ $\mathrm{H}, 0,1.242541306,-8.3625090607,-0.698228734$ $\mathrm{H}, 0,3.5006920604,-9.2387941221,-0.0636535446$ $\mathrm{H}, 0,4.0335514665,-7.6047896822,0.3738245196$ $\mathrm{H}, 0,2.7606610982,-8.4090712194,1.3184659338$ $\mathrm{N}, 0,-0.619447114,-3.6989416832,-0.7142138888$ $\mathrm{C}, 0,-1.873960878,-4.1082633771,-1.2144261647$ $0,0,-2.5468296578,-3.317128288,-1.8646333052$ $\mathrm{C}, 0,-2.3848412923,-5.4847579297,-0.8328885466$ $\mathrm{C}, 0,-3.9216801211,-5.4881693999,-0.73221716$ $\mathrm{H}, \mathrm{0},-1.9357462516,-5.8057790192,0.1122351487$ $\mathrm{H}, 0,-2.0521424213,-6.2002837592,-1.5911714639$ $\mathrm{C}, 0,-4.4531195877,-6.8695189886,-0.5066737681$ $\mathrm{H}, 0,-4.2589398343,-4.8340793325,0.0775736277$ $\mathrm{H}, \mathrm{O},-4.3329500064,-5.0933335429,-1.6682335271$ $\mathrm{N}, 0,-5.1339484941,-7.1304136649,0.5427019669$ $\mathrm{H}, 0,-4.2496140425,-7.6460179934,-1.2497793149$ $0,0,-5.5469254448,-8.476042129,0.5591148233$ $\mathrm{C}, 0,-6.3064676963,-8.7140034924,1.7426746972$ $\mathrm{H}, 0,-7.2063161138,-8.0882882879,1.7738003609$ $\mathrm{H}, \mathrm{O},-6.5959569676,-9.7667554486,1.7003402494$ $\mathrm{H}, 0,-5.709535963,-8.5349315976,2.6448505209$ $\mathrm{C}, 0,2.3860235273,-4.0676740627,1.5276912471$ $\mathrm{H}, 0,2.0510727366,-4.8567631451,2.2118737244$ $\mathrm{H}, 0,3.2135013635,-4.4971453017,0.9489976351$ $\mathrm{H}, 0,2.7764551023,-3.2366575144,2.1206207169$

\section{$z-3 a-T_{1}$}

Opt a UB3LYP/6-31G $(\mathrm{d}, \mathrm{p})$ in dichloromethane (SMD model) SCF Done: $\mathrm{E}(\mathrm{UB} 3 \mathrm{LYP})=-1070.18103687 \mathrm{a} \cdot \mathrm{u}$. Zero-point correction $=0.345031$ Hartree/Particle Sum of electronic and thermal Free Energies = -1069.894056 a.u.

$C, 0,-3.1384659305,-0.1813005271,0.5370877839$

$\mathrm{C}, 0,-2.3070907549,-1.139948407,-0.13184158$

$\mathrm{C}, 0,-2.7287161898,-2.4179124352,-0.3981293752$

C, $0,-4.029940076,-2.7915126342,0.0427669333$

$\mathrm{C}, 0,-4.8558909837,-1.8875561469,0.7166224009$

$\mathrm{C}, 0,-4.4309288552,-0.5844772892,0.9706478058$

$C, 0,-2.4616330677,1.0355622935,0.6396156388$

C, $0,-1.1529562856,0.8489853102,-0.0857993759$

$\mathrm{H}, 0,-2.0941560412,-3.1229544795,-0.9148523272$

$\mathrm{H}, \mathrm{O},-4.373677735,-3.8031691872,-0.1474938466$

$\mathrm{H}, \mathrm{0},-5.8436648562,-2.2017321589,1.0402983773$

$\mathrm{H}, \mathrm{O},-5.0763962116,0.1208635204,1.4840117311$

$\mathrm{C}, 0,-0.3617172419,1.850320912,-0.7718471237$

$0,0,0.403591743,1.6262723887,-1.7108023204$

$0,0,-0.5629900426,3.0904243338,-0.2570208992$

$\mathrm{C}, 0,0.1553017516,4.1679315296,-0.9057032671$

C, $0,-0.2102585279,5.4551644411,-0.1966082483$

$\mathrm{H}, \mathrm{O},-0.1227674332,4.1935205986,-1.9642558854$

$\mathrm{H}, \mathrm{O}, 1.2290191105,3.9626443402,-0.8468593721$ 
$\mathrm{H}, 0,0.3247738387,6.2901258948,-0.6601545219$

$\mathrm{H}, \mathrm{O},-1.2839318,5.6546052972,-0.2682629736$

$\mathrm{H}, 0,0.067485555,5.4151093217,0.861218093$

$\mathrm{N}, 0,-1.0397373695,-0.5044454781,-0.3987356585$

C, $0,0.1301572169,-1.2346401108,-0.6989492638$

$0,0,0.0289069503,-2.3460591683,-1.2049880492$

$C, 0,1.4676467022,-0.6516786231,-0.2853895557$

C, $0,2.4631927657,-1.7634957974,0.0731901668$

$\mathrm{H}, 0,1.3331417437,0.0358690096,0.5557949355$

$\mathrm{H}, 0,1.8519891761,-0.0500155199,-1.1150765663$

$\mathrm{C}, 0,3.8367953018,-1.2147035614,0.328262308$

$\mathrm{H}, 0,2.1279330211,-2.3278492238,0.948288199$

$\mathrm{H}, 0,2.5131815268,-2.4837516975,-0.7531851376$

$\mathrm{N}, 0,4.7640820264,-1.7316257143,1.0441941947$

$\mathrm{H}, 0,4.1246822099,-0.2746699349,-0.1427817203$

$0,0,4.3669040042,-2.952965344,1.6174208095$

$\mathrm{C}, 0,5.4170122784,-3.4330069681,2.455843604$

$\mathrm{H}, 0,5.6211022654,-2.7383777404,3.2791365511$

$\mathrm{H}, \mathrm{O}, 5.0610027016,-4.3837287374,2.8599163306$

$\mathrm{H}, 0,6.3379335514,-3.598848713,1.8849164433$

C, $0,-2.910708595,2.2776146827,1.3193891068$

$\mathrm{H}, 0,-2.1463957799,2.6513312808,2.0113575108$

$\mathrm{H}, 0,-3.0966251906,3.0885171271,0.6037523267$

$\mathrm{H}, 0,-3.8305976228,2.0985418153,1.8818494667$

TS1- $(\boldsymbol{S} \boldsymbol{i}, \boldsymbol{R e}, \boldsymbol{E})$ (as $\mathbf{T S 1}$ in the main text)

Opt \& UB3LYP/6-31G $(\mathrm{d}, \mathrm{p})$ in dichloromethane (SMD model)

SCF Done: E (UB3LYP) $=-1070.17160047 \mathrm{a} \cdot \mathrm{u}$.

Zero-point correction $=0.345437$ Hartree/Particle

Sum of electronic and thermal Free Energies $=-1069.878800 \mathrm{a} \cdot \mathrm{u}$.

Imaginary Frequency $=-328.5725 \mathrm{~cm}^{-1}$

$0,0,-0.2557559091,2.2549607877,2.1234219174$

$0,0,0.5096637987,-3.6408113464,0.0128313477$

$0,0,-1.9526658584,-1.1427630683,-1.8704534773$

$0,0,-2.3798463689,0.8002038604,-0.7804435277$

$\mathrm{N}, 0,0.1259673573,0.8617624312,0.3568341273$

$\mathrm{N}, 0,0.5712279518,-2.6435093045,0.9768703293$

C, $0,1.4463105114,1.2906963912,0.0206471114$

C, $0,2.1915240942,2.3354452999,0.5243587279$

C, $0,3.506480051,2.5029353428,0.029815701$

C, $0,4.0292089972,1.6490492761,-0.9498119898$

C, $0,3.2672601435,0.6048383445,-1.468932434$

C, $0,1.9490620312,0.4068908614,-0.9864537513$

C, $0,0.9630574563,-0.5363196799,-1.3204703003$

C, $0,-0.2515976926,-0.1992964213,-0.5019497231$

$C, 0,-0.5725393,-1.9967458219,1.0479396223$

C, $0,-0.8033131782,-1.1812641234,2.292018609$

C, $0,-1.4925067723,0.1869630794,2.0998585696$

C, $0,-0.5359706693,1.2167983433,1.535146016$

C, $0,-1.5913395622,-0.2410324368,-1.12533802$

$C, 0,-3.7181835352,0.8051147076,-1.3482136236$

C, $0,-4.4102374592,2.0656273445,-0.8751687109$

C, $0,1.7691905419,-4.3200764118,-0.0517509176$

$\mathrm{H}, 0,1.7834703765,3.0002096045,1.2724496746$

$\mathrm{H}, 0,4.1149231235,3.3121921045,0.4214897818$ 
$\mathrm{H}, 0,3.6712940965,-0.050346604,-2.2341884098$

$\mathrm{H}, \mathrm{0},-1.4282519868,-2.3499360286,0.4715178068$

$\mathrm{H}, 0,0.1520783886,-1.0531436984,2.8112956$

$\mathrm{H}, 0,-1.4421383992,-1.7819250224,2.9529752412$

$\mathrm{H}, 0,-2.3862182642,0.082476635,1.4806500874$

$\mathrm{H}, 0,-1.8075903085,0.5754958105,3.070269853$

$\mathrm{H}, 0,-3.6333962214,0.7709450444,-2.4382582537$

$\mathrm{H}, \mathrm{O},-4.2413814171,-0.0987646236,-1.0214338329$

$\mathrm{H}, \mathrm{O},-4.4945666584,2.0876796872,0.2156673562$

$\mathrm{H}, 0,-5.419406199,2.1045765812,-1.2975908246$

$\mathrm{H}, 0,-3.8673234114,2.9580305904,-1.2009882113$

$\mathrm{H}, 0,2.0026219287,-4.7897099045,0.9095837273$

$\mathrm{H}, 0,2.573035618,-3.6306940207,-0.3283450697$

$\mathrm{H}, 0,1.6515784954,-5.0859045334,-0.8207311744$

$\mathrm{H}, 0,5.0398833393,1.8078664892,-1.3145256537$

C, $0,1.0101475147,-1.5634615397,-2.3921418223$

$\mathrm{H}, 0,0.3924418126,-1.2707861398,-3.2522192648$

$\mathrm{H}, 0,2.0345510843,-1.7073775608,-2.7468104373$

$\mathrm{H}, 0,0.6161728889,-2.5220240565,-2.0406144674$

\section{TS1- (Si, Re, Z)}

Opt a UB3LYP/6-31G(d,p) in dichloromethane (SMD model)

$\mathrm{SCF}$ Done: $\mathrm{E}(\mathrm{UB} 3 \mathrm{LYP})=-1070.17032769 \mathrm{a} \cdot \mathrm{u}$.

Zero-point correction $=0.345623$ Hartree/Particle

Sum of electronic and thermal Free Energies = -1069.878102 a.u.

Imaginary Frequency $=-322.5519 \mathrm{~cm}^{-1}$

$0,0,0.7601357845,-2.4041448369,2.1410709867$

$0,0,-1.7199122087,1.7891745429,2.2022670665$

$0,0,1.7529345513,1.5029483311,-1.63736251$

$0,0,2.5187790204,-0.4557084167,-0.7850914686$

$\mathrm{N}, 0,0.1187752377,-0.9805646137,0.4766992127$

$\mathrm{N}, 0,-0.8532578831,2.3343974682,1.2669312142$

C, $0,-1.1400367091,-1.5726901649,0.1526478924$

C, $0,-1.6951177308,-2.7587596694,0.5834700554$

$\mathrm{C}, 0,-2.9942052915,-3.0804805423,0.1258010186$

$\mathrm{C}, 0,-3.6889558525,-2.232390171,-0.7472911982$

$\mathrm{C}, 0,-3.1193000019,-1.0428497761,-1.1935608384$

$\mathrm{C}, 0,-1.81959188,-0.6899999168,-0.7481610248$

$\mathrm{C}, 0,-1.0058226337,0.4199169512,-1.0266953783$

C, $0,0.2910404254,0.1896185784,-0.3041654182$

$\mathrm{C}, 0,0.3668977033,1.8516927737,1.3871163992$

C, $0,0.8840683214,1.044914945,2.5520352089$

C, $0,1.7295124943,-0.1969564654,2.1835160222$

$\mathrm{C}, 0,0.8748713523,-1.3076087062,1.6041773898$

C, $0,1.5696506758,0.4856624228,-0.9800961908$

$\mathrm{C}, 0,3.8082871835,-0.202441363,-1.4067043732$

$\mathrm{C}, 0,4.7015517137,-1.3864468134,-1.1035178973$

C, $0,-3.0262942972,2.3460955022,2.0256440704$

$\mathrm{H}, \mathrm{0},-1.1553883761,-3.4143743065,1.2520567725$

$\mathrm{H}, \mathrm{O},-3.4552367759,-4.0040487303,0.4619962396$

$\mathrm{H}, 0,-3.6561050651,-0.3917747545,-1.8762385219$

$\mathrm{H}, 0,1.0833032722,2.3820632515,0.7648973137$

$\mathrm{H}, 0,0.053494452,0.7457721076,3.1927479025$

$\mathrm{H}, 0,1.5229530668,1.7143264853,3.1424018946$

$\mathrm{H}, 0,2.5421904292,0.0775876458,1.5077119388$ 
$\mathrm{H}, 0,2.1783250847,-0.6084175965,3.0897453447$

$\mathrm{H}, 0,3.6565078037,-0.071127578,-2.482019484$

$\mathrm{H}, 0,4.2139703689,0.7316704978,-1.0059191727$

$\mathrm{H}, 0,4.8499821152,-1.5066551988,-0.0260059691$

$\mathrm{H}, 0,5.6799341203,-1.2300929533,-1.5689689749$

$\mathrm{H}, 0,4.2758835429,-2.3122651605,-1.5022561791$

$\mathrm{H}, 0,-3.4256464748,2.10185034,1.0362016795$

$\mathrm{H}, \mathrm{O},-3.0068591768,3.4330837079,2.1570748598$

$\mathrm{H}, \mathrm{O},-3.6463811572,1.8891025844,2.7992844978$

$\mathrm{H}, 0,-4.6828777418,-2.5105746941,-1.085570123$

$\mathrm{C}, 0,-1.2747473511,1.5337599535,-1.975882052$

$\mathrm{H}, 0,-0.6997608947,1.4179003569,-2.9047799049$

$\mathrm{H}, 0,-2.3354076528,1.5661055077,-2.2410385227$

$\mathrm{H}, 0,-0.9938632545,2.5005268441,-1.5494879184$

\section{$\operatorname{TS} 1-(S i, S i, E)$}

Opt a UB3LYP/6-31G(d,p) in dichloromethane (SMD model)

$\mathrm{SCF}$ Done: $\mathrm{E}(\mathrm{UB} 3 \mathrm{LYP})=-1070.16788018 \mathrm{a} \cdot \mathrm{u}$.

Zero-point correction $=0.345308$ Hartree/Particle

Sum of electronic and thermal Free Energies = -1069.875481 a.u.

Imaginary Frequency $=-386.1822 \mathrm{~cm}^{-1}$

$0,0,0.7015072558,-2.3603374335,2.1199912558$

$0,0,0.2261338888,3.6606559897,-0.0515202883$

$0,0,1.5952151203,1.4041619893,-1.8709903628$

$0,0,2.4705591263,-0.3632903626,-0.7573132371$

$\mathrm{N}, 0,0.054866087,-0.9664597309,0.4342278083$

$\mathrm{N}, 0,0.9448902825,2.882847661,0.8375316516$

C, $0,-1.1564595143,-1.6320617148,0.0712279698$

$\mathrm{C}, 0,-1.6601186753,-2.8441516021,0.4923331915$

$\mathrm{C}, 0,-2.9273262038,-3.2378592086,-0.0001153341$

$C, 0,-3.6425175568,-2.4322233524,-0.8965682554$

C, $0,-3.1245157531,-1.2167564346,-1.3347097184$

$\mathrm{C}, 0,-1.8567073235,-0.7933269916,-0.8564381414$

$\mathrm{C}, 0,-1.0993017174,0.3560776418,-1.1184657155$

$\mathrm{C}, 0,0.1997089281,0.2019426032,-0.3652943661$

$\mathrm{C}, 0,0.2100476687,1.8841853308,1.2803179858$

$\mathrm{C}, 0,0.7506086498,1.1244573413,2.4736355226$

$\mathrm{C}, 0,1.6044546731,-0.1293309354,2.168948777$

$C, 0,0.794225496,-1.262862839,1.5794199232$

C, 0,1.4691030962,0.483167359,-1.0775994029

$C, 0,3.7449560237,-0.1221742101,-1.4136597444$

$\mathrm{C}, 0,4.7061436035,-1.1990055785,-0.9572176611$

C, $0,1.0888266604,4.6299606038,-0.658990435$

$\mathrm{H}, \mathrm{0},-1.107629815,-3.4662848699,1.1816526934$

$\mathrm{H}, \mathrm{O},-3.3470812631,-4.1831781271,0.3296274805$

$\mathrm{H}, 0,-3.674343457,-0.5988051091,-2.037414903$

$\mathrm{H}, \mathrm{O},-0.8634417102,1.8820687906,1.0873282201$

$\mathrm{H}, \mathrm{O},-0.0914197315,0.8417504273,3.1131625337$

$\mathrm{H}, 0,1.3775610718,1.809766102,3.0521911132$

$\mathrm{H}, 0,2.4344989916,0.1437981261,1.5151207967$

$\mathrm{H}, 0,2.0235489027,-0.5150280534,3.100624004$

$\mathrm{H}, 0,3.5887733969,-0.1477094842,-2.4960826214$

$\mathrm{H}, 0,4.092537751,0.880087614,-1.1453289666$

$\mathrm{H}, \mathrm{0}, 4.8633524513,-1.1600302598,0.1250166104$

$\mathrm{H}, 0,5.6732900152,-1.0522643443,-1.4486604743$ 
$\mathrm{H}, \mathrm{0}, 4.3352916917,-2.1943451756,-1.2201912145$

$\mathrm{H}, 0,1.8446360207,4.1365875859,-1.2759522411$

$\mathrm{H}, 0,1.5715657229,5.2480530924,0.10502897$

$\mathrm{H}, \mathrm{O}, 0.4412226041,5.2481724352,-1.2835539065$

$\mathrm{H}, \mathrm{O},-4.6105628087,-2.7646858219,-1.2600586709$

$\mathrm{C}, 0,-1.4322282493,1.4821795328,-2.0291011751$

$\mathrm{H}, 0,-0.8534301418,1.4294400098,-2.9610275084$

$\mathrm{H}, \mathrm{O},-2.4947640917,1.4695333903,-2.2888802361$

$\mathrm{H}, \mathrm{O},-1.1847115978,2.446008753,-1.5720428372$

TS1-(Si, $\boldsymbol{i}, \boldsymbol{E})$ (as TS1' in the main text)

Opt a UB3LYP/6-31G(d,p) in dichloromethane (SMD model)

$\mathrm{SCF}$ Done: $\mathrm{E}(\mathrm{UB} 3 \mathrm{LYP})=-1070.16820530 \mathrm{a} \cdot \mathrm{u}$.

Zero-point correction $=0.345619$ Hartree/Particle

Sum of electronic and thermal Free Energies = -1069.875906 a.u.

Imaginary Frequency $=-369.4041 \mathrm{~cm}^{-1}$

$0,0,1.4953147414,2.6007651199,1.5666438699$

$0,0,-3.2245657376,-0.4591185479,1.6310906217$

$0,0,-2.0168844421,-0.4621887796,-1.4577526318$

$0,0,-1.3585176303,1.6472830876,-0.9494128174$

$\mathrm{N}, 0,0.8922471757,0.7565735494,0.3664315778$

$\mathrm{N}, 0,-2.2121659144,-1.3892365783,1.4705394953$

C, $0,2.1968608246,0.2850073681,0.0237429942$

$\mathrm{C}, 0,3.4246945954,0.8878872185,0.1882491224$

C, $0,4.5744156279,0.1556780855,-0.1947036986$

$\mathrm{C}, 0,4.4699561634,-1.1340124224,-0.7339158769$

$C, 0,3.227896342,-1.7357211608,-0.9147007869$

C, $0,2.0553666227,-1.0266448255,-0.541064553$

$\mathrm{C}, 0,0.6989855517,-1.3690819307,-0.6042316584$

$C, 0,-0.0734220483,-0.1656906707,-0.1212156133$

$\mathrm{C}, 0,-1.0566549043,-0.9003521945,1.8700712114$

$\mathrm{C}, 0,-0.8776164149,0.3214532363,2.7471660632$

$\mathrm{C}, 0,-0.6832322112,1.6799904554,2.0317853769$

C, 0,0.6403577993,1.7627434576,1.2987599089

$\mathrm{C}, 0,-1.2369276738,0.3018621753,-0.90675953$

C, $0,-2.4904330948,2.1631805615,-1.6992432875$

$\mathrm{C}, 0,-2.3976717278,3.6743282422,-1.6863509749$

$\mathrm{C}, 0,-4.4551435218,-0.9964588934,1.1287794204$

$\mathrm{H}, 0,3.5041713261,1.8812405371,0.6065415854$

$\mathrm{H}, 0,5.5525003068,0.6073609929,-0.0611662736$

$\mathrm{H}, 0,3.1488491249,-2.7309104416,-1.3406703196$

$\mathrm{H}, 0,-0.2766506102,-1.6575141472,1.8816302641$

$\mathrm{H}, \mathrm{0},-0.0213861571,0.1409878151,3.4041819517$

$\mathrm{H}, \mathrm{O},-1.7659276742,0.4090941373,3.3803908727$

$\mathrm{H}, \mathrm{0},-1.5203090128,1.8699397535,1.3591326372$

$\mathrm{H}, 0,-0.6619100513,2.478242248,2.7766655869$

$\mathrm{H}, 0,-2.448410549,1.7610691546,-2.715697501$

$\mathrm{H}, 0,-3.4130034626,1.8052240349,-1.2318441884$

$\mathrm{H}, \mathrm{O},-2.4482816774,4.0675434539,-0.6663712012$

$\mathrm{H}, \mathrm{O},-3.2332887847,4.0931107253,-2.2562914068$

$\mathrm{H}, \mathrm{O},-1.4645262727,4.0147871119,-2.1453934799$

$\mathrm{H}, 0,-4.7334782124,-1.9028916779,1.6760857433$

$\mathrm{H}, 0,-4.3674126469,-1.2162744459,0.0613038631$

$\mathrm{H}, 0,-5.2035533468,-0.2187916155,1.2932468797$

$\mathrm{H}, 0,5.37105849,-1.6682503418,-1.0208929712$ 
$C, 0,0.0900535661,-2.6315301992,-1.1025359509$

$\mathrm{H}, 0,-0.3586800596,-2.4981047651,-2.0957702343$

$\mathrm{H}, 0,0.8415551822,-3.4230330874,-1.1746842442$

$\mathrm{H}, \mathrm{O},-0.7174745513,-2.9718392563,-0.4462171662$

\section{INT1}

Opt \& UB3LYP/6-31G(d,p) in dichloromethane (SMD model)

$\mathrm{SCF}$ Done: $\mathrm{E}(\mathrm{UB} 3 \mathrm{LYP})=-1070.21177920 \mathrm{a} \cdot \mathrm{u}$.

Zero-point correction $=0.348307$ Hartree/Particle

Sum of electronic and thermal Free Energies = -1069.917599 a.u.

$0,0,0.7560647616,-0.5038644312,2.9308220012$

$0,0,-3.4251525096,1.1112932086,-0.4778673704$

$0,0,0.8265553346,2.4521154838,-1.6160957165$

$0,0,2.1521095004,0.8595539381,-0.7103113041$

$\mathrm{N}, 0,0.1689188793,-0.2525059906,0.7339627944$

$\mathrm{N}, 0,-2.5041918054,0.8950894634,0.5096221779$

$\mathrm{C}, 0,0.040241904,-1.5967376639,0.3467537849$

$\mathrm{C}, 0,0.3221117811,-2.7547461152,1.0574328118$

$C, 0,0.0873545809,-3.986106516,0.4226436958$

$C, 0,-0.416134453,-4.0491810475,-0.8871649994$

C, $0,-0.6997920397,-2.8886808158,-1.5992365427$

C, $0,-0.4718291699,-1.6331052033,-0.9877435357$

$C, 0,-0.6684228455,-0.3205767837,-1.4580984325$

$\mathrm{C}, 0,-0.2200619437,0.6460368248,-0.3715967965$

C, $0,-1.3038192735,1.6477083239,0.1401843434$

C, $0,-0.7901857216,2.4024539494,1.3959258297$

C, $0,0.4266884725,1.7437306305,2.0886722545$

$C, 0,0.465607094,0.2301806504,1.9908117055$

C, $0,0.9765487279,1.4444224162,-0.9517519913$

$\mathrm{C}, 0,3.3239715162,1.4855113518,-1.313281785$

$\mathrm{C}, 0,4.5320936349,0.6707953874,-0.9067723759$

C, $0,-4.6273217114,0.3686672387,-0.2094304383$

$\mathrm{H}, \mathrm{O}, 0.7062258675,-2.7012797025,2.0663760162$

$\mathrm{H}, \mathrm{O}, 0.3022935122,-4.9049318795,0.9599233353$

$\mathrm{H}, \mathrm{O},-1.0908632757,-2.9390972526,-2.6107324271$

$\mathrm{H}, \mathrm{O},-1.5284984119,2.347258542,-0.6683904585$

$\mathrm{H}, 0,-1.6209313768,2.4620782867,2.104322451$

$\mathrm{H}, \mathrm{O},-0.5310142432,3.4273116773,1.1195634657$

$\mathrm{H}, 0,1.3587355076,2.1186810881,1.6467796979$

$\mathrm{H}, 0,0.4588395702,2.0000420668,3.1489906348$

$\mathrm{H}, 0,3.183596033,1.5029237365,-2.3978608225$

$\mathrm{H}, 0,3.3844252628,2.5183300592,-0.9586875348$

$\mathrm{H}, 0,4.6512173876,0.6578198729,0.1808146745$

$\mathrm{H}, 0,5.4310697071,1.1151330695,-1.3456618994$

$\mathrm{H}, 0,4.4496796968,-0.3606019566,-1.2623552906$

$\mathrm{H}, \mathrm{O},-5.0559567645,0.6765574721,0.748629313$

$\mathrm{H}, \mathrm{O},-4.4156595645,-0.7042472682,-0.1965385023$

$\mathrm{H}, 0,-5.3118764446,0.60959166,-1.0243388577$

$\mathrm{H}, \mathrm{O},-0.5854572722,-5.0175407558,-1.3491410973$

C, $0,-1.1916604093,0.1160418807,-2.7822339164$

$\mathrm{H}, 0,-1.2368232971,-0.7234137491,-3.4811529887$

$\mathrm{H}, \mathrm{0},-2.2027063039,0.5356338271,-2.692217102$

$\mathrm{H}, \mathrm{O},-0.5661126946,0.898997586,-3.2273799718$ 


\section{INT1'}

Opt \& UB3LYP/6-31G(d,p) in dichloromethane (SMD model)

SCF Done: $\mathrm{E}(\mathrm{UB} 3 \mathrm{LYP})=-1070.20871499 \mathrm{a} \cdot \mathrm{u}$.

Zero-point correction $=0.347941$ Hartree/Particle

Sum of electronic and thermal Free Energies = -1069.915341 a.u.

$0,0,-0.5718181557,0.5627089385,3.0845754175$

$0,0,2.3449984272,-3.3556701208,-0.4354580908$

$0,0,-1.4493558314,-1.9172312358,-1.6663108948$

$0,0,-2.3124101733,-0.1568878936,-0.5435953133$

$\mathrm{N}, 0,-0.110202891,0.3444165895,0.8563826344$

$\mathrm{N}, 0,1.322660792,-2.5953189652,-0.9423047938$

C, $0,0.3199333209,1.649903003,0.5622060661$

C, $0,0.3201982864,2.7849779061,1.3606302258$

C, $0,0.8292038146,3.9730280132,0.809211241$

C, $0,1.3227446113,4.0162898434,-0.5048871637$

C, $0,1.322883684,2.8782534584,-1.304662244$

C, $0,0.8152217258,1.6671491942,-0.7780468289$

$C, 0,0.6960052503,0.385185211,-1.3504990584$

$C, 0,0.048587773,-0.5353235654,-0.3244902571$

C, $0,0.9068424683,-1.7409155871,0.1600273434$

C, $0,0.1402028014,-2.5334455818,1.2501903753$

$C, 0,-0.7903111306,-1.6382235986,2.1077628536$

C, $0,-0.4767172189,-0.1509921494,2.0902009461$

C, $0,-1.3196856635,-0.9769974043,-0.9085732348$

C, $0,-3.6204696993,-0.4244896086,-1.1282958231$

C, $0,-4.5848270774,0.5973016235,-0.5665386426$

C, $0,2.8128696845,-4.2824946435,-1.4282956232$

$\mathrm{H}, 0,-0.0578616547,2.746552165,2.3724235073$

$\mathrm{H}, 0,0.8383328432,4.8733770375,1.4162021801$

$\mathrm{H}, \mathrm{O}, 1.7085254477,2.9123823322,-2.3189022802$

$\mathrm{H}, 0,1.8019374762,-1.2898214405,0.6092972813$

$\mathrm{H}, 0,0.872913889,-3.0359281444,1.8847791522$

$\mathrm{H}, \mathrm{O},-0.4518485749,-3.3117424211,0.7608941983$

$\mathrm{H}, \mathrm{O},-1.8268722481,-1.7387273549,1.7617838465$

$\mathrm{H}, \mathrm{O},-0.7830922175,-1.9468242358,3.1545325019$

$\mathrm{H}, \mathrm{O},-3.5320649891,-0.3536608208,-2.2163905859$

$\mathrm{H}, \mathrm{O},-3.9109702726,-1.4479992979,-0.8748980614$

$\mathrm{H}, \mathrm{O},-4.6553350187,0.5158176721,0.5223901891$

$\mathrm{H}, \mathrm{O},-5.5799187902,0.425689,-0.9891156845$

$\mathrm{H}, \mathrm{O},-4.2738635486,1.6148300379,-0.8215905617$

$\mathrm{H}, 0,3.1597475905,-3.7454302496,-2.3161906991$

$\mathrm{H}, 0,2.0175683897,-4.9815120513,-1.7035085548$

$\mathrm{H}, 0,3.6423679186,-4.8196447111,-0.9651590211$

$\mathrm{H}, 0,1.7092586191,4.9509955065,-0.9007550595$

C, $0,1.0778121464,-0.0297543806,-2.7299553919$

$\mathrm{H}, 0,1.1445693121,0.8393281994,-3.3916194347$

$\mathrm{H}, 0,2.0516087677,-0.5380339754,-2.7488820394$

$\mathrm{H}, 0,0.3537943755,-0.7333289639,-3.1528160573$

\section{MECP1}

Triplet state

$\mathrm{Sp}$ a UB3LYP/6-31G $(\mathrm{d}, \mathrm{p})$ in dichloromethane (SMD model)

SCF Done: E(UB3LYP) $=-1070.20391760 \mathrm{a} \cdot \mathrm{u}$.

Open-shelled singlet state

$\mathrm{Sp}$ e UB3LYP/6-31G $(\mathrm{d}, \mathrm{p})$ in dichloromethane (SMD model) 
SCF Done: E (UB3LYP) $=-1070.20392979 \mathrm{a} \cdot \mathrm{u}$.

\begin{tabular}{|c|c|c|}
\hline 0.66351 & -0.50803 & 2.89627 \\
\hline-3.10383 & 0.63739 & -0.51232 \\
\hline 0.7923 & 2.43012 & -1.72701 \\
\hline 2.14807 & 0.95583 & -0.67447 \\
\hline 0.15733 & -0.2349 & 0.68261 \\
\hline-2.47534 & 1.11594 & 0.61639 \\
\hline-0.131 & -1.56141 & 0.33108 \\
\hline 0.03697 & -2.73298 & 1.06178 \\
\hline-0.30064 & -3.94117 & 0.43794 \\
\hline-0.79527 & -3.97706 & -0.87801 \\
\hline-0.97379 & -2.8058 & -1.60297 \\
\hline-0.64331 & -1.57071 & -1.00088 \\
\hline-0.71461 & -0.24466 & -1.4835 \\
\hline-0.21646 & 0.66629 & -0.41234 \\
\hline-1.29928 & 1.75893 & 0.17118 \\
\hline-0.66206 & 2.50171 & 1.36511 \\
\hline 0.51352 & 1.75226 & 2.04612 \\
\hline 0.45081 & 0.24034 & 1.94844 \\
\hline 0.95865 & 1.47167 & -0.9976 \\
\hline 3.31527 & 1.59908 & -1.2671 \\
\hline 4.5314 & 0.8086 & -0.83788 \\
\hline-4.22218 & -0.18121 & -0.148 \\
\hline 0.42274 & -2.70162 & 2.07072 \\
\hline-0.16611 & -4.87025 & 0.98376 \\
\hline-1.35762 & -2.83334 & -2.61819 \\
\hline-1.47157 & 2.41984 & -0.68853 \\
\hline-1.45541 & 2.66881 & 2.09794 \\
\hline-0.31328 & 3.48489 & 1.04049 \\
\hline 1.46649 & 2.06111 & 1.59995 \\
\hline 0.57062 & 1.99856 & 3.10762 \\
\hline 3.18994 & 1.60522 & -2.35317 \\
\hline 3.35283 & 2.63544 & -0.91948 \\
\hline 4.63712 & 0.80599 & 0.25101 \\
\hline 5.42842 & 1.26318 & -1.27003 \\
\hline 4.46906 & -0.22707 & -1.18477 \\
\hline-4.93291 & 0.39436 & 0.45391 \\
\hline-3.8864 & -1.05717 & 0.41543 \\
\hline-4.68637 & -0.4915 & -1.08588 \\
\hline-1.04115 & -4.93288 & -1.3308 \\
\hline-1.1008 & 0.20748 & -2.84794 \\
\hline-1.52003 & -0.61983 & -3.42539 \\
\hline-1.85039 & 1.00526 & -2.79938 \\
\hline-0.24535 & 0.61064 & -3.40593 \\
\hline
\end{tabular}

\section{INT1-OSS}

Opt a UB3LYP/6-31G(d,p) in dichloromethane (SMD model)

$\mathrm{SCF}$ Done: $\mathrm{E}(\mathrm{UB} 3 \mathrm{LYP})=-1070.21416911 \mathrm{a} \cdot \mathrm{u}$.

Zero-point correction $=0.348266$ Hartree/Particle

Sum of electronic and thermal Free Energies $=-1069.917840 \mathrm{a} . u$.

$0,0,0.665114218,-0.5049118295,2.8997475695$

$0,0,-3.1418573963,0.6383806072,-0.4882923038$

$0,0,0.8133680467,2.4766650846,-1.6667069566$

$0,0,2.1486584879,0.9270036952,-0.7017137952$ 
$\mathrm{N}, 0,0.1472651915,-0.2272249626,0.6895960714$ $\mathrm{N}, 0,-2.4765759963,1.0790826293,0.6232179406$ C, $0,-0.1110960873,-1.5597705681,0.3244657525$ C, $0,0.0596840342,-2.7297375304,1.0536093771$ C, $0,-0.268751629,-3.9429988829,0.428516094$ $\mathrm{C}, 0,-0.7529440098,-3.9799281808,-0.889249705$ $\mathrm{C}, 0,-0.9263185345,-2.8074516388,-1.6176273616$ C, $0,-0.608032893,-1.5702610325,-1.0137456652$ C, $0,-0.6933654454,-0.2450021962,-1.4963390932$ C, $0,-0.2227479918,0.6820964671,-0.4076478523$ $\mathrm{C}, 0,-1.2900578601,1.738915927,0.1535953703$ C, $0,-0.6707859212,2.5045075608,1.344008197$ $C, 0,0.4865317946,1.7571478257,2.0576139469$ C, $0,0.4385425883,0.2437170525,1.9541679129$ C, $0,0.9678297924,1.4850909721,-0.980215172$ C, $0,3.3222716469,1.5704983852,-1.2826687595$ $\mathrm{C}, 0,4.5355078354,0.7825231826,-0.8400269314$ $\mathrm{C}, 0,-4.2804986121,-0.1524636119,-0.1109477346$ $\mathrm{H}, 0,0.4369112122,-2.6971129642,2.0659356166$ $\mathrm{H}, \mathrm{O},-0.1398175404,-4.8703085102,0.9787199366$ $\mathrm{H}, \mathrm{O},-1.3025117873,-2.8371675234,-2.6355910839$ $\mathrm{H}, 0,-1.5116378061,2.4076910736,-0.6858651368$ $\mathrm{H}, 0,-1.4740631881,2.6953621165,2.060115229$ $\mathrm{H}, 0,-0.3082475367,3.476272503,1.0015917313$ $\mathrm{H}, 0,1.4501139256,2.0722564575,1.6388494538$ $\mathrm{H}, 0,0.5137347316,2.0025778605,3.1204940516$ $\mathrm{H}, 0,3.2078582543,1.5756081231,-2.3703599622$ $\mathrm{H}, 0,3.354861288,2.6073300167,-0.9362665628$ $\mathrm{H}, 0,4.628656609,0.7807859951,0.2501877474$ $\mathrm{H}, 0,5.4361183233,1.2405551466,-1.2610895572$ $\mathrm{H}, 0,4.4815315745,-0.2532188667,-1.1884149012$ $\mathrm{H}, 0,-4.9545328029,0.4339730686,0.5203507308$ $\mathrm{H}, 0,-3.958128679,-1.0518959496,0.4214845892$ $\mathrm{H}, 0,-4.7777677335,-0.4234053788,-1.0436308933$ $\mathrm{H}, 0,-0.9937023026,-4.9361252575,-1.3446848817$ C, $0,-1.0919784855,0.2116228675,-2.8567622271$ $\mathrm{H}, 0,-1.507313677,-0.6165185569,-3.4368886428$ $\mathrm{H}, 0,-1.848955591,1.0023557522,-2.8046855313$ $\mathrm{H}, 0,-0.2429928474,0.6208256308,-3.4206577778$

\section{TS2}

Opt a UB3LYP/6-31G(d,p) in dichloromethane (SMD model)

SCF Done: $\mathrm{E}(\mathrm{UB} 3 \mathrm{LYP})=-1070.21004620 \mathrm{a} \cdot \mathrm{u}$.

Zero-point correction $=0.348528$ Hartree/Particle

Sum of electronic and thermal Free Energies = -1069.912385 a.u. Imaginary Frequency $=-231.9751 \mathrm{~cm}^{-1}$

$0,0,-0.7548336522,0.4946406029,3.0211094005$

$0,0,3.2804552298,-1.6525749049,-0.6860548145$

$0,0,-1.1432181643,-2.325804201,-1.608093634$

$0,0,-2.3029037084,-0.6481791191,-0.6319777203$

$\mathrm{N}, 0,-0.2193891391,0.2308889176,0.8119093055$

$\mathrm{N}, 0,2.3639089041,-0.8845562983,-0.0049752692$

$\mathrm{C}, 0,-0.0003495486,1.56621936,0.4229408753$

C, $0,-0.2562933691,2.7401354069,1.1189752934$

C, $0,0.0816581627,3.9533039107,0.4978276737$ 
$C, 0,0.6545792333,3.9848635268,-0.7808620455$ C, $0,0.9106075162,2.8024319865,-1.4746154587$ C, $0,0.5851955247,1.5733404047,-0.8737164924$ $\mathrm{C}, 0,0.7567149309,0.2296851495,-1.3263126337$ $\mathrm{C}, 0,0.0763626774,-0.6871983472,-0.3058432395$ C, $0,1.1393727071,-1.711216528,0.1041911451$ C, $0,0.940140544,-2.2735347861,1.5345834023$ C, $0,-0.3564137031,-1.7534736021,2.1921062984$ C, $0,-0.4811901297,-0.2430952388,2.0788282544$ C, $0,-1.1909104712,-1.333877495,-0.9061705028$ $\mathrm{C}, 0,-3.5350760392,-1.1411438256,-1.2367859243$ $\mathrm{C}, 0,-4.6547120307,-0.2233617383,-0.7975683677$ C, $0,4.5459284759,-0.9777335827,-0.7382114562$ $\mathrm{H}, 0,-0.6984977368,2.7105758354,2.1051511128$ $\mathrm{H}, \mathrm{O},-0.1106338593,4.8843128077,1.0229237416$ $\mathrm{H}, \mathrm{0}, 1.3543703024,2.8263204143,-2.4652041543$ $\mathrm{H}, 0,1.1545485768,-2.5092471531,-0.641986754$ $\mathrm{H}, 0,1.7973791228,-1.9652238577,2.1422369815$ $\mathrm{H}, 0,0.9284280847,-3.3664622619,1.5199798371$ $\mathrm{H}, \mathrm{0},-1.2317545906,-2.220195469,1.7213118256$ $\mathrm{H}, 0,-0.390568519,-2.0047994475,3.253897494$ $\mathrm{H}, 0,-3.4073202795,-1.1455645187,-2.3230862539$ $\mathrm{H}, 0,-3.6925681126,-2.1718787885,-0.9066089236$ $\mathrm{H}, \mathrm{O},-4.7631375728,-0.2265798476,0.291193588$ $\mathrm{H}, \mathrm{O},-5.5963415559,-0.5664415469,-1.2378314954$ $\mathrm{H}, \mathrm{0},-4.4747174428,0.8039386882,-1.1283146304$ $\mathrm{H}, 0,4.927571501,-0.802045401,0.2723320927$ $\mathrm{H}, 0,4.4496114271,-0.0244100303,-1.2674297238$ $\mathrm{H}, 0,5.2167855768,-1.6433219437,-1.2846609564$ $\mathrm{H}, \mathrm{O}, 0.8986661347,4.9397565124,-1.2368923294$ C, $0,1.1172481165,-0.2105339511,-2.7032431161$ $\mathrm{H}, 0,0.2247567304,-0.4137176934,-3.3122410161$ $\mathrm{H}, 0,1.6949645995,0.5648957228,-3.2141166293$ $\mathrm{H}, 0,1.7159928561,-1.1275444288,-2.6919763705$

\section{TS3}

Opt a UB3LYP/6-31G(d,p) in dichloromethane (SMD model) $\mathrm{SCF}$ Done: $\mathrm{E}(\mathrm{UB} 3 \mathrm{LYP})=-1070.18441698 \mathrm{a} \cdot \mathrm{u}$. Zero-point correction $=0.344832$ Hartree/Particle Sum of electronic and thermal Free Energies $=-1069.890310 \mathrm{a} \cdot \mathrm{u}$. Imaginary Frequency $=-1932.1191 \mathrm{~cm}^{-1}$

$0,0,-0.7564192543,0.1749150872,3.0885975863$ $0,0,3.959088318,-0.1242467208,-0.2690329915$ $\mathrm{N}, 0,-0.3564799753,0.1450481887,0.8438892244$ $\mathrm{N}, 0,2.6631674482,0.3786261742,-0.0855018304$ $\mathrm{C}, 0,-1.1577884615,1.2482143906,0.4748788054$ $\mathrm{C}, 0,-2.1080815394,1.9437235312,1.2162180921$ $C, 0,-2.6929017641,3.0750454666,0.6305052942$ $C, 0,-2.3349366208,3.5071288907,-0.6555420845$ $\mathrm{C}, 0,-1.3837482319,2.8099623859,-1.3956753984$ $\mathrm{C}, 0,-0.7937056888,1.663160101,-0.8361378475$ $\mathrm{C}, 0,0.1881884777,0.7672373571,-1.3514571803$ $\mathrm{C}, 0,0.3296198661,-0.3809900098,-0.3617167831$ C, $0,1.8041415637,-0.8073587849,-0.1139946365$ C, $0,1.853960338,-1.6519210401,1.1729113414$ 
$C, 0,1.2332020399,-0.9595960564,2.3844877217$

$\mathrm{C}, 0,-0.0644385014,-0.2058045359,2.1499117336$

C, $0,4.8986086218,0.9440142641,-0.17155786$

C, $0,1.2680085108,0.9840743915,-2.2493720551$

$\mathrm{H}, \mathrm{O}, 2.1762859838,0.9816289266,-1.3634856502$

$\mathrm{H}, \mathrm{0},-2.3724877765,1.6237079125,2.2136999493$

$\mathrm{H}, \mathrm{0},-3.4426661044,3.6264412585,1.1902931052$

$\mathrm{H}, \mathrm{0},-2.809586141,4.3873817188,-1.0785462658$

$\mathrm{H}, 0,-1.1072957859,3.1317152457,-2.3952259061$

$\mathrm{H}, 0,2.1089663769,-1.4542396506,-0.9456024821$

$\mathrm{H}, 0,1.3266711864,-2.5916623109,0.9686056797$

$\mathrm{H}, 0,2.8957403967,-1.9112083247,1.3795721465$

$\mathrm{H}, 0,1.0598905409,-1.6696708699,3.1976385319$

$\mathrm{H}, 0,1.9193890826,-0.1985626937,2.7775633422$

$\mathrm{H}, 0,4.746516792,1.6745591375,-0.9765863509$

$\mathrm{H}, 0,5.8855051895,0.4871562833,-0.2745768652$

$\mathrm{H}, 0,4.8190017917,1.4503109798,0.7967895273$

$\mathrm{H}, \mathrm{0}, 1.6066289258,0.1173411303,-2.8264597648$

$\mathrm{H}, 0,1.2481908569,1.9035445783,-2.8351709035$

C, $0,-0.4225195776,-1.6028019944,-0.9664665978$

$0,0,0.0259944319,-2.2531237902,-1.8906292953$

$0,0,-1.6127157197,-1.8067047255,-0.4025585619$

$C, 0,-2.4236288713,-2.8774635607,-0.972532369$

C, $0,-3.7101618917,-2.9414568437,-0.1791886741$

$\mathrm{H}, \mathrm{O},-1.8543844455,-3.8092592702,-0.9130354329$

$\mathrm{H}, \mathrm{O},-2.5995924428,-2.6501173244,-2.0278418432$

$\mathrm{H}, 0,-4.3437623277,-3.7374604878,-0.5831038728$

$\mathrm{H}, \mathrm{O},-3.5131239182,-3.1604801765,0.8745802555$

$\mathrm{H}, \mathrm{O},-4.2603246995,-1.9979732289,-0.2436738338$

\section{$4 a$}

Opt a B3LYP/6-31G(d,p) in dichloromethane (SMD model)

$\mathrm{SCF}$ Done: $\mathrm{E}(\mathrm{RB} 3 \mathrm{LYP})=-1070.25454001 \mathrm{a} \cdot \mathrm{u}$.

Zero-point correction $=0.351637$ Hartree/Particle

Sum of electronic and thermal Free Energies = -1069.955061 a.u.

$0,0,0.0241304915,-1.2950917619,2.98055728$

$0,0,-3.62529884,-1.0787404712,-1.0987561959$

$\mathrm{N}, 0,0.1902071753,-0.5236179499,0.8426478457$

$\mathrm{N}, 0,-2.2370333377,-1.1460103863,-0.6977813343$

C, $0,1.3545014366,-1.2203597561,0.4505391068$

$\mathrm{C}, 0,2.223225398,-1.9940643073,1.2230261112$

$\mathrm{C}, 0,3.2940501737,-2.6161929203,0.5732619456$

C, $0,3.4992819841,-2.4745033158,-0.804369874$

$\mathrm{C}, 0,2.625143724,-1.6980939417,-1.5668622009$

C, $0,1.5514322306,-1.069269479,-0.9354642544$

C, $0,0.4945909675,-0.2164097574,-1.4866739791$

$\mathrm{C}, 0,-0.3048253176,0.3078258134,-0.2781312451$

C, $0,-1.8423399486,0.2344262434,-0.3810663861$

$\mathrm{C}, 0,-2.4377554204,0.6788100983,0.9591706899$

C, $0,-1.8930194051,-0.1380712598,2.1402674253$

$\mathrm{C}, 0,-0.4720737743,-0.6776636709,2.0411906316$

$C, 0,-4.3118347119,-2.2088473228,-0.5749646569$

C, $0,0.2509031959,0.1071127066,-2.7629019809$

$\mathrm{H}, \mathrm{0},-1.7557705468,-1.4095592267,-1.5611314349$

$\mathrm{H}, 0,2.0631095451,-2.1040471801,2.2854406359$ 
$\mathrm{H}, 0,3.9820181408,-3.2200080196,1.1581379917$

$\mathrm{H}, 0,4.3424397951,-2.9659062435,-1.2800799358$

$\mathrm{H}, 0,2.7778181758,-1.5798013271,-2.6356194965$

$\mathrm{H}, 0,-2.1553970784,0.9280478149,-1.1706221675$

$\mathrm{H}, 0,-2.2202276954,1.7429316174,1.0963748139$

$\mathrm{H}, 0,-3.5258122629,0.5871074382,0.9130198507$

$\mathrm{H}, \mathrm{0},-1.9563102075,0.4301432875,3.0733662547$

$\mathrm{H}, \mathrm{0},-2.5010305903,-1.036358172,2.2941439723$

$\mathrm{H}, \mathrm{O},-3.9143929933,-3.1498715267,-0.9788559166$

$\mathrm{H}, \mathrm{O},-5.3526727245,-2.0962491625,-0.893675437$

$\mathrm{H}, \mathrm{O},-4.2664766358,-2.2452493995,0.520313564$

$\mathrm{H}, \mathrm{O},-0.5612410045,0.7711457725,-3.0391309598$

$\mathrm{H}, 0,0.8728332925,-0.2770015297,-3.5658934652$

$\mathrm{C}, 0,0.0992454638,1.80001768,-0.1136722306$

$0,0,-0.3838138061,2.6834057202,-0.7933726451$

$0,0,1.0467380642,1.9779810141,0.8059934053$

$\mathrm{C}, 0,1.5378843524,3.3414948981,0.9766676835$

C, $0,2.5969378768,3.3089400201,2.0564291453$

$\mathrm{H}, 0,0.6921066141,3.9800112599,1.2466776843$

$\mathrm{H}, 0,1.9352058081,3.6853532815,0.0175118118$

$\mathrm{H}, 0,2.9873535352,4.3201752516,2.2086630692$

$\mathrm{H}, 0,2.182325004,2.9540907156,3.004746738$

$\mathrm{H}, 0,3.429230936,2.6580189144,1.7725903$

\section{$4 a^{\prime}$}

Opt a B3LYP/6-31G (d,p) in dichloromethane (SMD model)

$\mathrm{SCF}$ Done: $\mathrm{E}(\mathrm{RB} 3 \mathrm{LYP})=-1070.24956005 \mathrm{a} \cdot \mathrm{u}$.

Zero-point correction $=0.351722$ Hartree/Particle

Sum of electronic and thermal Free Energies = -1069.947488 a.u.

$0,0,-0.5647459784,1.0632520642,3.0109029018$

$0,0,2.4445747976,-2.4356671247,-0.7399161385$

$0,0,-1.945028597,-2.0541609673,-1.3305335102$

$0,0,-2.4862303275,0.0529933998,-0.7154973977$

$\mathrm{N}, 0,-0.3128214292,0.3621803948,0.8459629283$

$\mathrm{N}, 0,1.6837802501,-1.4298162934,-0.0737866939$

C, $0,0.4910432623,1.4153403502,0.3317219044$

$\mathrm{C}, 0,0.6109931572,2.7201105927,0.801714011$

C, $0,1.4901227901,3.5757979856,0.1238656834$

$\mathrm{C}, 0,2.217122189,3.1405113771,-0.986159889$

C, $0,2.0871275574,1.8209595936,-1.4425824611$

$\mathrm{C}, 0,1.2273301138,0.9597394882,-0.7739504363$

$\mathrm{C}, 0,0.9132132672,-0.5009628913,-1.019110194$

$\mathrm{C}, 0,-0.3327165437,-0.7618992379,-0.1002776573$

$C, 0,0.4071297505,-1.9964766541,0.4508321527$

$C, 0,0.2915636903,-2.1963254712,1.9685474064$

$\mathrm{C}, 0,-0.8365931563,-1.3031455471,2.5376567446$

$\mathrm{C}, 0,-0.5879134647,0.1558600682,2.1866438279$

$\mathrm{C}, 0,-1.675450566,-1.0068783641,-0.7719568887$

$C, 0,-3.7764932132,-0.0826565515,-1.3791216764$

C, $0,-4.5193691243,1.2230581713,-1.1981352347$

$\mathrm{C}, 0,3.83177913,-2.242398407,-0.466485165$

$\mathrm{H}, 0,0.0445626242,3.0527215249,1.661009865$

$\mathrm{H}, 0,1.5974492091,4.6002775428,0.4686284146$

$\mathrm{H}, \mathrm{0}, 2.6554431584,1.4775686809,-2.3023226$

$\mathrm{H}, \mathrm{O}, \mathrm{O} .1110383448,-2.879773227,-0.1220605184$ 
$\mathrm{H}, 0,1.2434072164,-1.9226289181,2.4350197092$

$\mathrm{H}, 0,0.0910848015,-3.2423581168,2.2158425412$

$\mathrm{H}, \mathrm{0},-1.8041517807,-1.624023856,2.1298354754$

$\mathrm{H}, 0,-0.8970570937,-1.3723669976,3.6252777054$

$\mathrm{H}, 0,-3.6005001704,-0.3138996168,-2.4336152523$

$\mathrm{H}, \mathrm{O},-4.309289466,-0.9254021766,-0.9293600114$

$\mathrm{H}, \mathrm{0},-4.6813846204,1.4410739305,-0.1382134752$

$\mathrm{H}, 0,-5.4957803741,1.1540013564,-1.6881064366$

$\mathrm{H}, \mathrm{O},-3.9681860357,2.055283992,-1.6462423332$

$\mathrm{H}, 0,4.0370481289,-2.2800708614,0.609785413$

$\mathrm{H}, 0,4.1987876596,-1.2899081212,-0.8683849946$

$\mathrm{H}, \mathrm{O}, 4.3485880686,-3.0661684653,-0.9675922357$

$\mathrm{H}, 0,2.8845616009,3.8260511944,-1.4995592581$

C, $0,0.8590810236,-0.9499674154,-2.4654603667$

$\mathrm{H}, 0,0.1828317976,-0.3023336382,-3.0314124821$

$\mathrm{H}, 0,1.8542428675,-0.8809400461,-2.9130842675$

$\mathrm{H}, 0,0.516416535,-1.9817951115,-2.5576815697$ 


\section{X-Ray crystal data}

Figure S4. X-Ray crystal structure of 20 (The crystal was obtained by slow evaporation of the solution of DCM and PE) (CCDC 2011328).
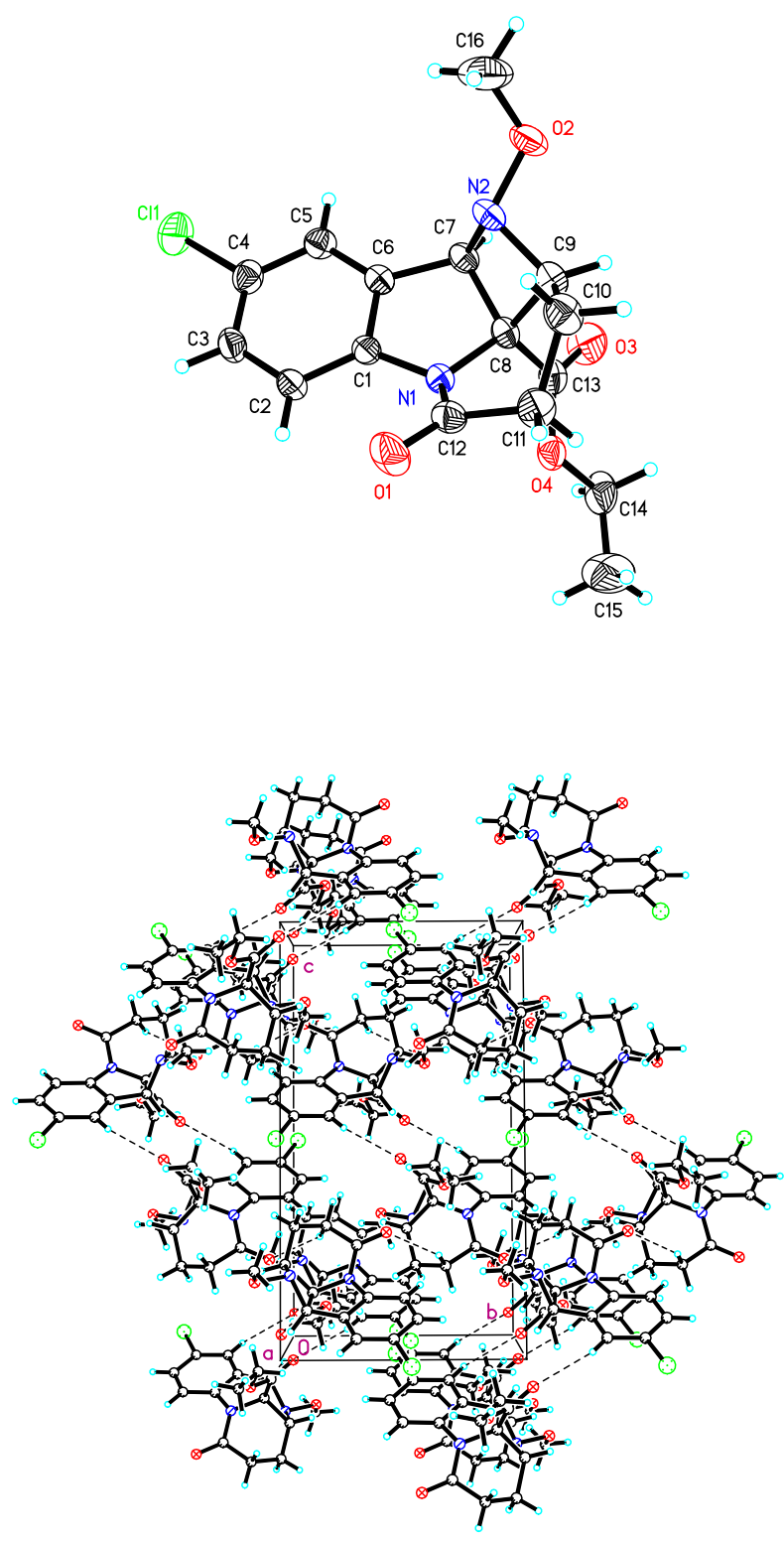

Table S1. Crystal data and structure refinement for mo_d8v191091_0m.

Identification code

Empirical formula

Formula weight mo_d8v191091_0m

C16 H17 Cl N2 O4

336.76 
Temperature

Wavelength

Crystal system

Space group

Unit cell dimensions

Volume

Z

Density (calculated)

Absorption coefficient

$\mathrm{F}(000)$

Crystal size

Theta range for data collection

Index ranges

Reflections collected

Independent reflections

Completeness to theta $=25.242^{\circ}$

Absorption correction

Max. and min. transmission

Refinement method

Data / restraints / parameters

Goodness-of-fit on $\mathrm{F}^{2}$

Final $\mathrm{R}$ indices [I $>2 \operatorname{sigma}(\mathrm{I})]$

$\mathrm{R}$ indices (all data)

Extinction coefficient

Largest diff. peak and hole
293(2) K

$0.71073 \AA$

Monoclinic

P 21/n

$\mathrm{a}=11.1555(3) \AA$

$\alpha=90^{\circ}$.

$\mathrm{b}=9.1674(3) \AA$

$\beta=94.8860(10)^{\circ}$.

$\mathrm{c}=16.4642(4) \AA$

$\gamma=90^{\circ}$.

1677.62(8) $\AA^{3}$

4

$1.333 \mathrm{Mg} / \mathrm{m}^{3}$

$0.248 \mathrm{~mm}^{-1}$

704

$0.200 \times 0.160 \times 0.130 \mathrm{~mm}^{3}$

2.124 to $25.999^{\circ}$.

$-13<=\mathrm{h}<=13,-11<=\mathrm{k}<=11,-19<=\mathrm{l}<=20$

23872

$3277[\mathrm{R}(\mathrm{int})=0.0287]$

$99.2 \%$

Semi-empirical from equivalents

0.7456 and 0.6396

Full-matrix least-squares on $\mathrm{F}^{2}$

3277 / 0 / 210

1.054

$\mathrm{R} 1=0.0432, \mathrm{wR} 2=0.1059$

$\mathrm{R} 1=0.0533, \mathrm{wR} 2=0.1142$

$\mathrm{n} / \mathrm{a}$

0.214 and -0.271 e. $\AA^{-3}$ 
Figure S5. X-Ray crystal structure of $\mathbf{4 b}$ (The crystal was obtained by slow evaporation of the solution of DCM and PE) (CCDC 2011329).
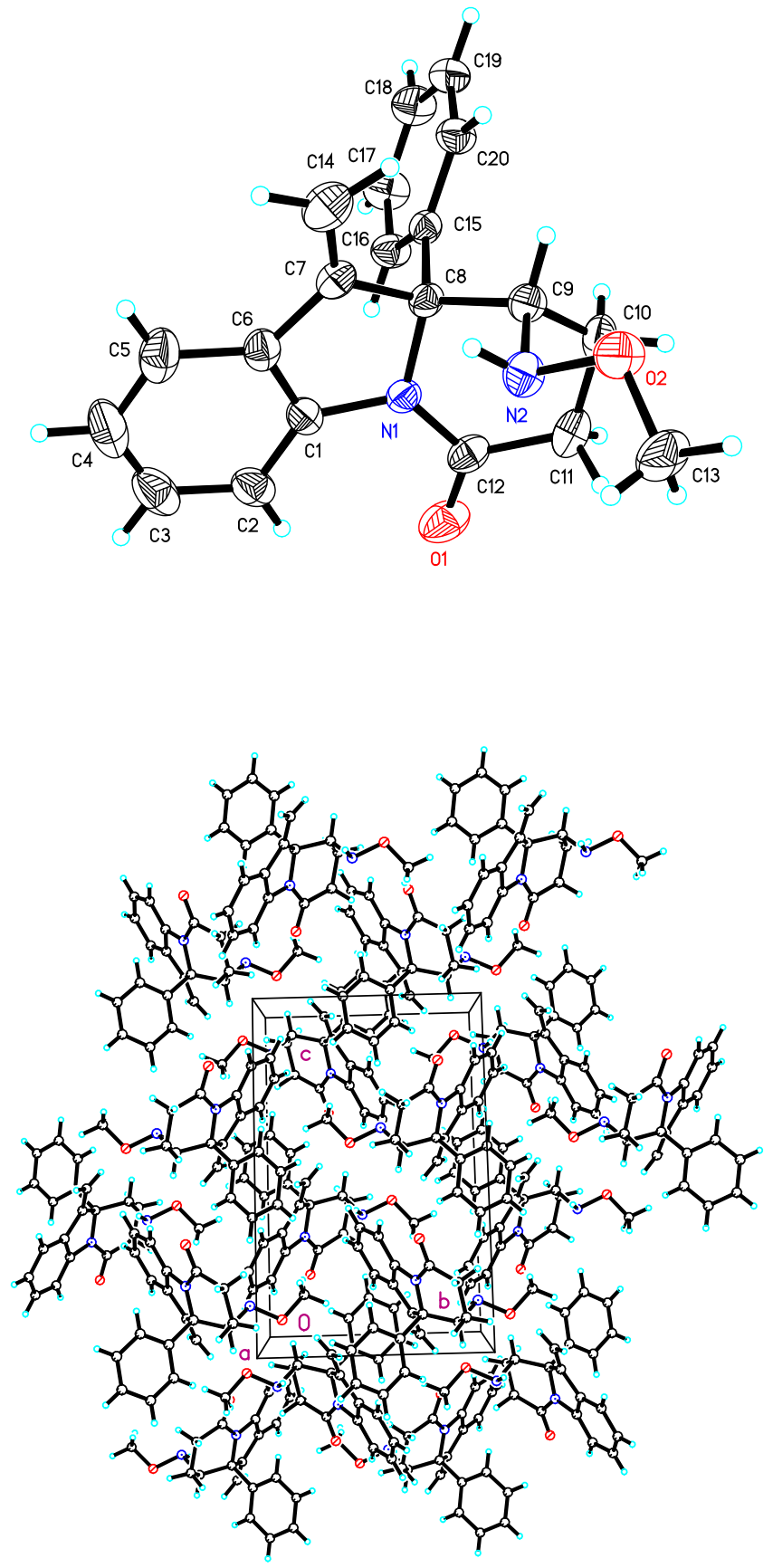

Table S2. Crystal data and structure refinement for mo_d8v20001_0m.

Identification code

Empirical formula mo_d8v20001_0m

$\mathrm{C} 20 \mathrm{H} 20 \mathrm{~N} 2 \mathrm{O} 2$ 
Formula weight

Temperature

Wavelength

Crystal system

Space group

Unit cell dimensions

Volume

Z

Density (calculated)

Absorption coefficient

$\mathrm{F}(000)$

Crystal size

Theta range for data collection

Index ranges

Reflections collected

Independent reflections

Completeness to theta $=25.242^{\circ}$

Absorption correction

Max. and min. transmission

Refinement method

Data / restraints / parameters

Goodness-of-fit on $\mathrm{F}^{2}$

Final $\mathrm{R}$ indices [I $>2 \operatorname{sigma}(\mathrm{I})]$

$\mathrm{R}$ indices (all data)

Extinction coefficient

Largest diff. peak and hole
320.38

293(2) K

$0.71073 \AA$

Monoclinic

P 21/c

$\begin{array}{ll}\mathrm{a}=12.058(3) \AA & \alpha=90^{\circ} . \\ \mathrm{b}=9.432(2) \AA & \beta=104.566(7)^{\circ} . \\ \mathrm{c}=14.999(4) \AA & \gamma=90^{\circ} .\end{array}$

$1651.0(7) \AA^{3}$

4

$1.289 \mathrm{Mg} / \mathrm{m}^{3}$

$0.084 \mathrm{~mm}^{-1}$

680

$0.170 \times 0.140 \times 0.100 \mathrm{~mm}^{3}$

2.575 to $24.999^{\circ}$.

$-14<=\mathrm{h}<=13,-11<=\mathrm{k}<=11,-15<=\mathrm{l}<=17$

19111

$2899[\mathrm{R}(\mathrm{int})=0.0894]$

$97.4 \%$

Semi-empirical from equivalents

0.7456 and 0.6356

Full-matrix least-squares on $\mathrm{F}^{2}$

2899 / 0 / 231

1.047

$\mathrm{R} 1=0.0644, \mathrm{wR} 2=0.1388$

$\mathrm{R} 1=0.1217, \mathrm{wR} 2=0.1707$

$0.011(2)$

0.158 and -0.188 e. $\AA^{-3}$ 
Figure S6. X-Ray crystal structure of $\mathbf{4} \mathbf{f}^{\prime}$ (The crystal was obtained by slow evaporation of the solution of DCM and PE) (CCDC 2011330).
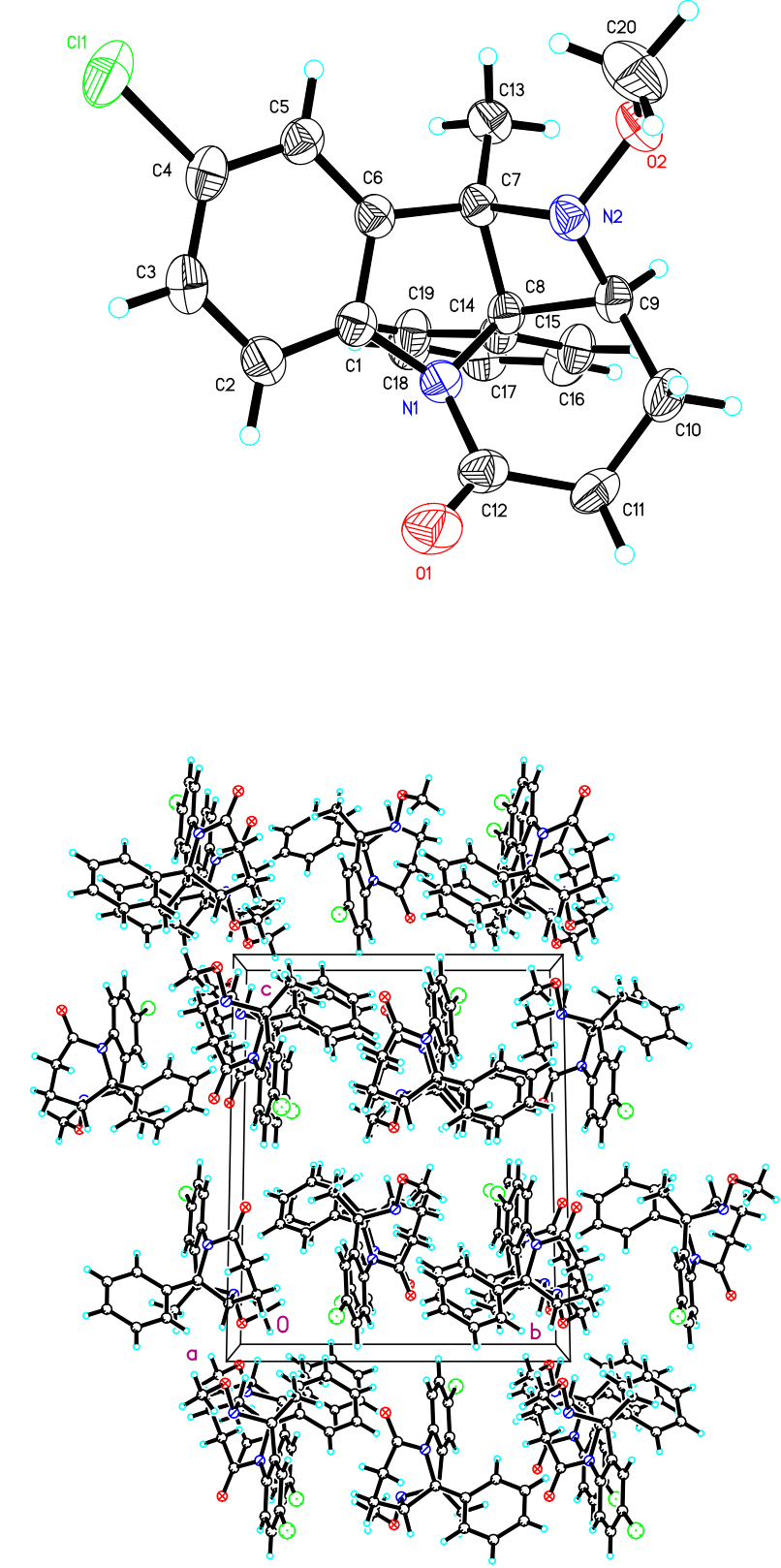

Table S3. Crystal data and structure refinement for mo_d8v20166_0m.

Identification code

Empirical formula

Formula weight

Temperature mo_d8v20166_0m

$\mathrm{C} 20 \mathrm{H} 19 \mathrm{ClN} 2 \mathrm{O} 2$

354.82

293(2) K 
Wavelength

Crystal system

Space group

Unit cell dimensions

Volume

Z

Density (calculated)

Absorption coefficient

$\mathrm{F}(000)$

Crystal size

Theta range for data collection

Index ranges

Reflections collected

Independent reflections

Completeness to theta $=25.242^{\circ}$

Absorption correction

Max. and min. transmission

Refinement method

Data / restraints / parameters

Goodness-of-fit on $\mathrm{F}^{2}$

Final R indices [I $>2 \operatorname{sigma(I)]~}$

$\mathrm{R}$ indices (all data)

Extinction coefficient

Largest diff. peak and hole
$0.71073 \AA$

Monoclinic

P 21/c

$\mathrm{a}=8.425(2) \AA$

$\alpha=90^{\circ}$.

$\mathrm{b}=13.085(3) \AA$

$\beta=104.597(7)^{\circ}$.

$c=16.325(4) \AA$

$\gamma=90^{\circ}$.

1741.6(8) $\AA^{3}$

4

$1.353 \mathrm{Mg} / \mathrm{m}^{3}$

$0.235 \mathrm{~mm}^{-1}$

744

$0.190 \times 0.150 \times 0.110 \mathrm{~mm}^{3}$

2.498 to $25.500^{\circ}$.

$-10<=\mathrm{h}<=8,-15<=\mathrm{k}<=15,-19<=\mathrm{l}<=19$

17015

$3236[\mathrm{R}($ int $)=0.0779]$

$99.8 \%$

Semi-empirical from equivalents

0.7456 and 0.5086

Full-matrix least-squares on $\mathrm{F}^{2}$

$3236 / 0 / 228$

1.036

$\mathrm{R} 1=0.0621, \mathrm{wR} 2=0.1531$

$\mathrm{R} 1=0.1090, \mathrm{wR} 2=0.1859$

$\mathrm{n} / \mathrm{a}$

0.267 and -0.218 e. $\AA^{-3}$ 
Figure S7. X-Ray crystal structure of 7 (The crystal was obtained by slow evaporation of the solution of DCM and PE) (CCDC 2011331).
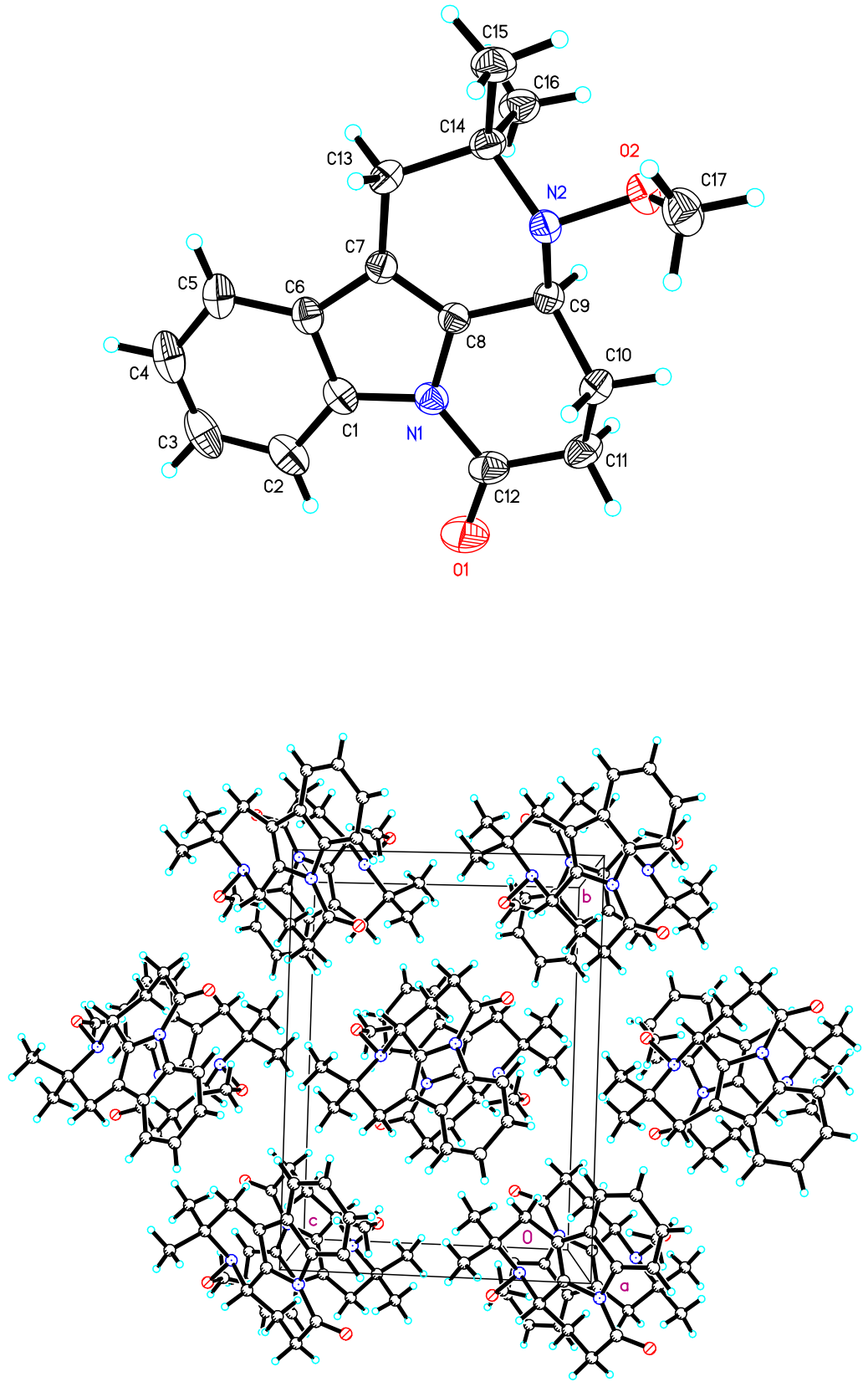

Table S4. Crystal data and structure refinement for d8v20127.

Identification code

Empirical formula

Formula weight d8v20127

C17 H20 N2 O2

284.35 
Temperature

Wavelength

Crystal system

Space group

Unit cell dimensions

Volume

Z

Density (calculated)

Absorption coefficient

$\mathrm{F}(000)$

Crystal size

Theta range for data collection

Index ranges

Reflections collected

Independent reflections

Completeness to theta $=25.242^{\circ}$

Absorption correction

Max. and min. transmission

Refinement method

Data / restraints / parameters

Goodness-of-fit on $\mathrm{F}^{2}$

Final $\mathrm{R}$ indices [I $>2 \operatorname{sigma}(\mathrm{I})]$

$\mathrm{R}$ indices (all data)

Extinction coefficient

Largest diff. peak and hole
293(2) K

$0.71073 \AA$

Monoclinic

P 21/c

$\mathrm{a}=11.2480(4) \AA$

$\alpha=90^{\circ}$.

$\mathrm{b}=13.4191(5) \AA$

$\beta=96.8590(10)^{\circ}$.

$c=9.9224(3) \AA$

$\gamma=90^{\circ}$.

1486.95(9) $\AA^{3}$

4

$1.270 \mathrm{Mg} / \mathrm{m}^{3}$

$0.084 \mathrm{~mm}^{-1}$

608

$0.190 \times 0.150 \times 0.120 \mathrm{~mm}^{3}$

3.001 to $26.000^{\circ}$.

$-13<=\mathrm{h}<=13,-13<=\mathrm{k}<=16,-12<=\mathrm{l}<=12$

14956

$2922[\mathrm{R}(\mathrm{int})=0.0393]$

$99.6 \%$

Semi-empirical from equivalents

0.7456 and 0.5790

Full-matrix least-squares on $\mathrm{F}^{2}$

2922 / 0 / 194

1.026

$\mathrm{R} 1=0.0414, \mathrm{wR} 2=0.1054$

$\mathrm{R} 1=0.0534, \mathrm{wR} 2=0.1159$

$0.033(7)$

0.178 and -0.138 e. $\AA^{-3}$ 


\section{References}

1. Lee, S.; Bae, M.; In, J.; Kim, J. H.; Kim, S. Asymmetric Total Synthesis of Lepadiformine C Using Memory of Chirality in an Intramolecular Ester Enolate Michael Addition. Org. Lett. 2017, 19, 254.

2. Umehara, A.; Ueda, H.; Tokuyama, H. Condensation of Carboxylic Acids with Non-Nucleophilic N-Heterocycles and Anilides Using Boc 2 O. J. Org. Chem. 2016, $81,11444$.

3. Zhu, M.; Zhou, K.; Zhang, X.; You, S.-L. Visible-Light-Promoted Cascade Alkene Trifluoromethylation and Dearomatization of Indole Derivatives via Intermolecular Charge Transfer. Org. Lett. 2018, 20, 4379.

4. Handbook of Photochemistry, $3^{\text {rd }}$ ed, Montalti, M.; Credi, A.; Prodi, L.; Gandolfi, M. T., Ed.; CRC, Taylor \& Francis Group, Boca Raton, FL, 2006.

5. Frisch, M. J.; Trucks, G. W.; Schlegel, H. B.; Scuseria, G. E.; Robb, M. A.; Cheeseman, J. R.; Scalmani, G.; Barone, V.; Pe-Tersson, G. A.; Nakatsuji, H.; Li, X.; Caricato, M.; Marenich, A. V.; Bloino, J.; Janesko, B. G.; Gomperts, R.; Mennucci, B.; Hratchian, H. P.; Ortiz, J. V.; Iz-Maylov, A. F.; Sonnenberg, J. L.; Williams-Young, D.; Ding, F.; Lipparini, F.; Egidi, F.; Goings, J.; Peng, B.; Petrone, A.; Henderson, T.; Ranasinghe, D.; Zakrzewski, V. G.; Gao, J.; Rega, N.; Zheng, G.; Liang, W.; Hada, M.; Ehara, M.; Toyota, K.; Fukuda, R.; Hasegawa, J.; Ishida, M.; Nakajima, T.; Honda, Y.; Kitao, O.; Nakai, H.; Vreven, T.; Throssell, K.; Montgomery, J. A., Jr.; Peralta, J. E.; Ogliaro, F.; Bearpark, M. J.; Heyd, J. J.; Brothers, E. N.; Kudin, K. N.; Staroverov, V. N.; Keith, T. A.; Kobayashi, R.; Normand, J.; Raghavachari, K.; Rendell, A. P.; Burant, J. C.; Iyengar, S. S.; Tomasi, J.; Cossi, M.; Millam, J. M.; Klene, M.; Adamo, C.; Cammi, R.; Ochterski, J. W.; Martin, R. L.; Morokuma, K.; Farkas, O.; Foresman, J. B.; Fox, D. J. Gaussian 16, revision A.03; Gaussian, Inc.: Wallingford, CT, 2016.

6. (a) Becke, A. D. Density-functional thermochemistry. III. The role of exact exchange. J. Chem. Phys. 1993, 98, 5648. (b) Lee, C.; Yang, W.; Parr, R. G. Development of the Colle-Salvetti correlation-energy formula into a functional of the electron density. Phys. Rev. B: Condens. Matter Mater. Phys. 1988, 37, 785. (c) Stephens, P. J.; Devlin, F. J.; Chabalowski, C. F.; Frisch, M. J. Ab Initio 
Calculation of Vibrational Absorption and Circular Dichroism Spectra Using Density Functional Force Fields. J. Phys. Chem. 1994, 98, 11623.

7. Marenich, A. V.; Cramer, C. J.; Truhlar, D. G. Universal Solvation Model Based on Solute Electron Density and on a Continuum Model of the Solvent Defined by the Bulk Dielectric Constant and Atomic Surface Tensions. J. Phys. Chem. B 2009, 113,6378 .

8. Harvey, J. N.; Aschi, M.; Schwarz, H.; Koch, W. The singlet and triplet states of phenyl cation. A hybrid approach for locating minimum energy crossing points between non-interacting potential energy surfaces. Theor. Chem. Acc. 1998, 99, 95.

9. Lu, T. sobMECP program; http://sobereva.com/286.

10. Legault, C. Y. CYLView, 1.0b; Université de Sherbrooke: Montreal, Québec, Canada, 2009; http://www.cylview.org. 


\section{Copies of NMR spectra}

${ }^{1} \mathrm{H}$ NMR Spectrum of $\mathbf{1 a}$

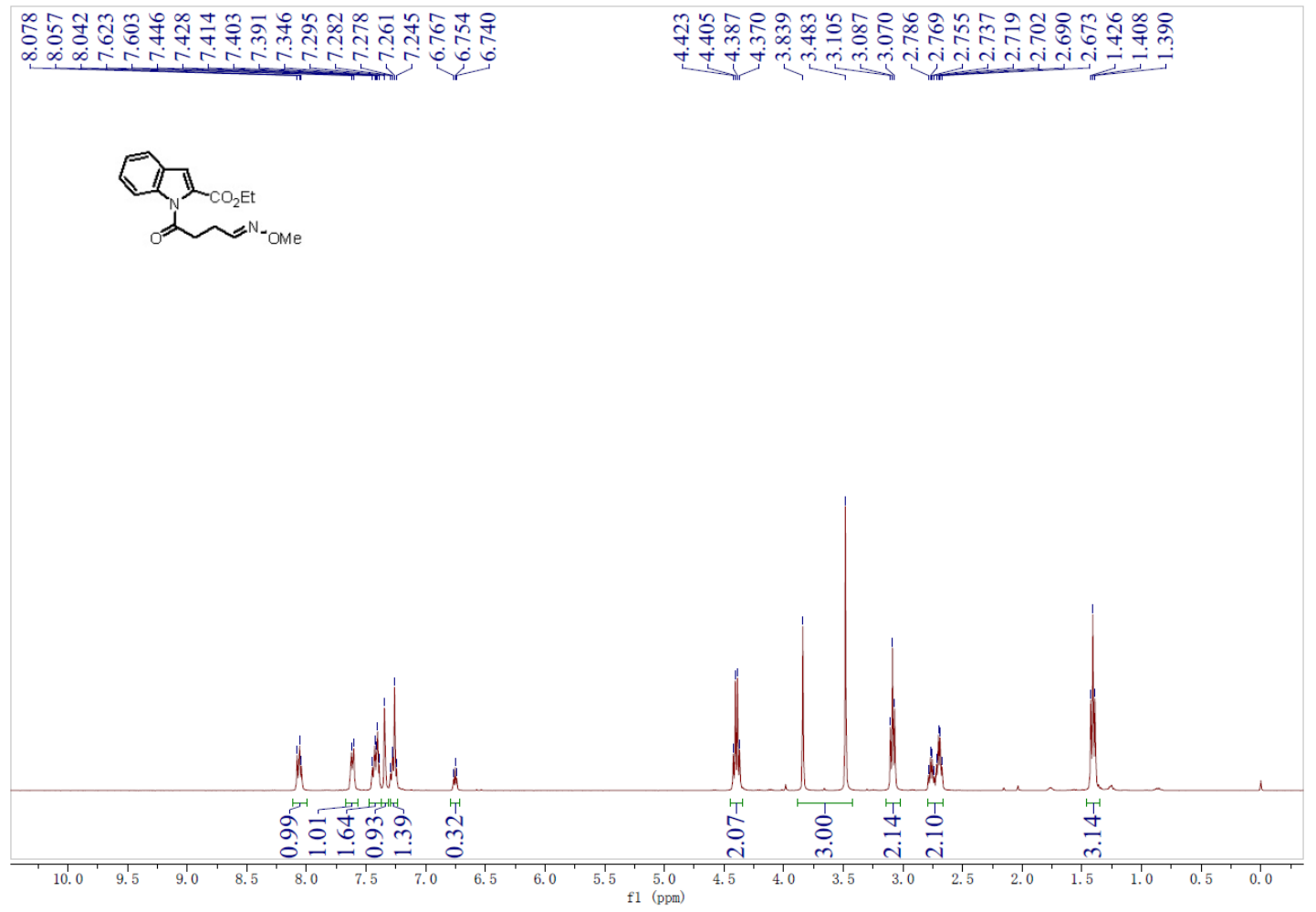

${ }^{13} \mathrm{C}$ NMR Spectrum of $\mathbf{1 a}$

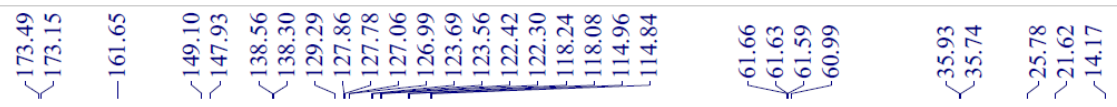<smiles>CCOc1cc2ccccc2n1C(=O)CCNCOC</smiles>

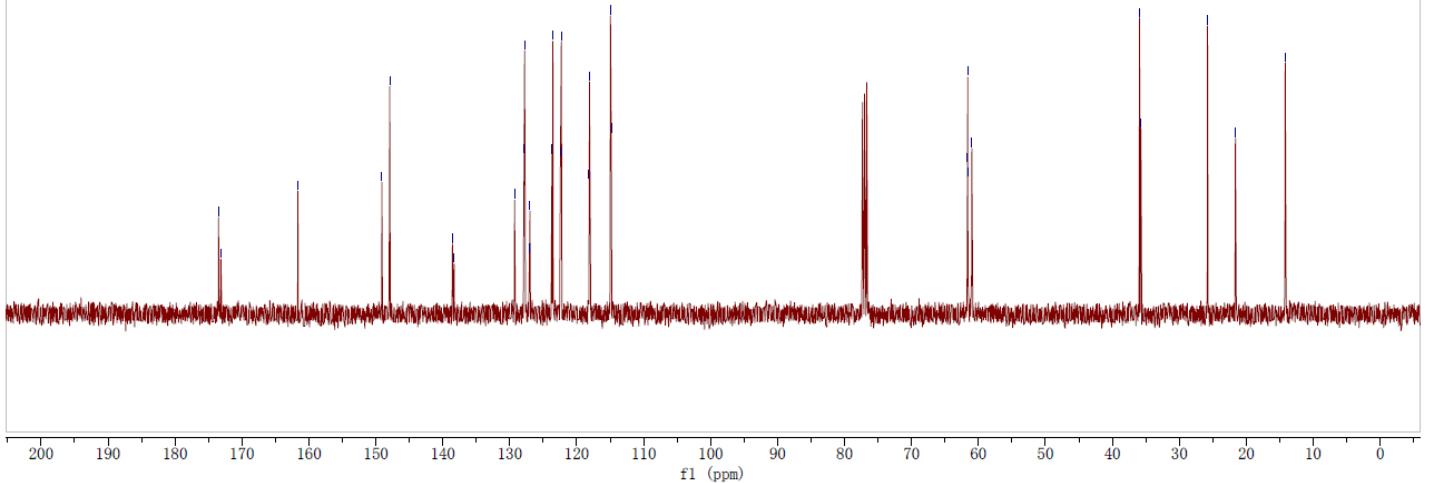


${ }^{1} \mathrm{H}$ NMR Spectrum of $\mathbf{1 b}$

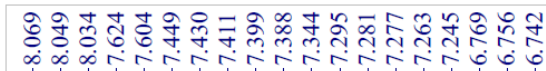

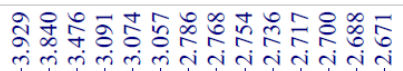

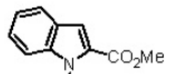

$\overbrace{}^{\mathrm{N}} \mathrm{N}_{\text {. }}^{\mathrm{OMme}}$

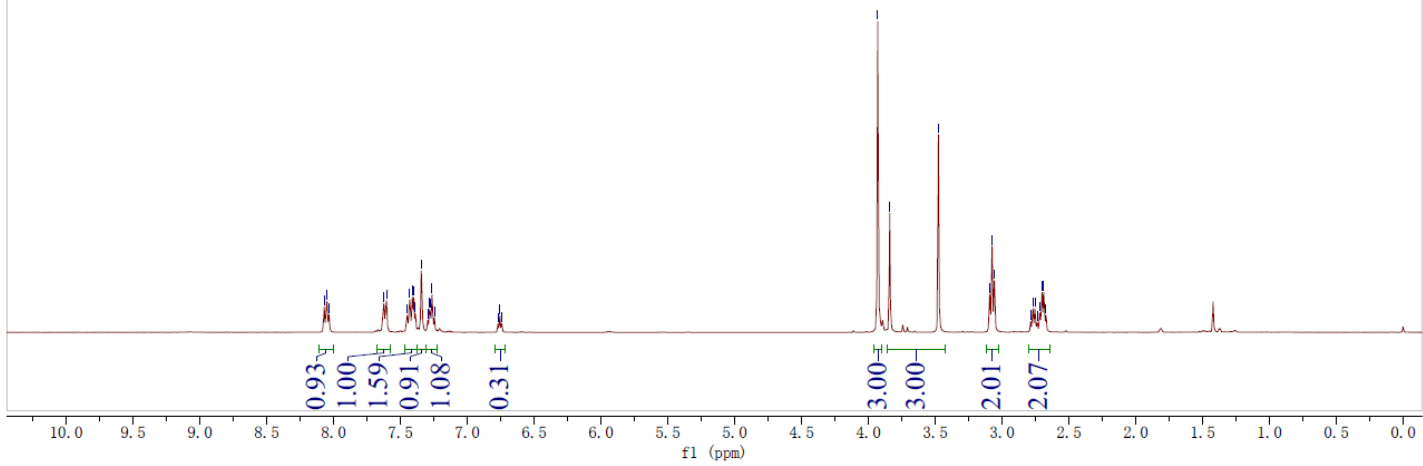

${ }^{13} \mathrm{C}$ NMR Spectrum of $\mathbf{1 b}$

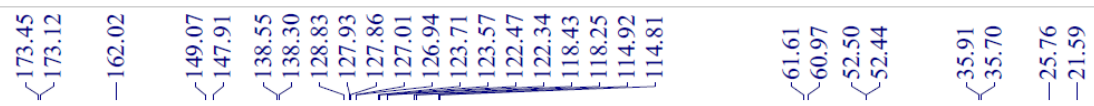
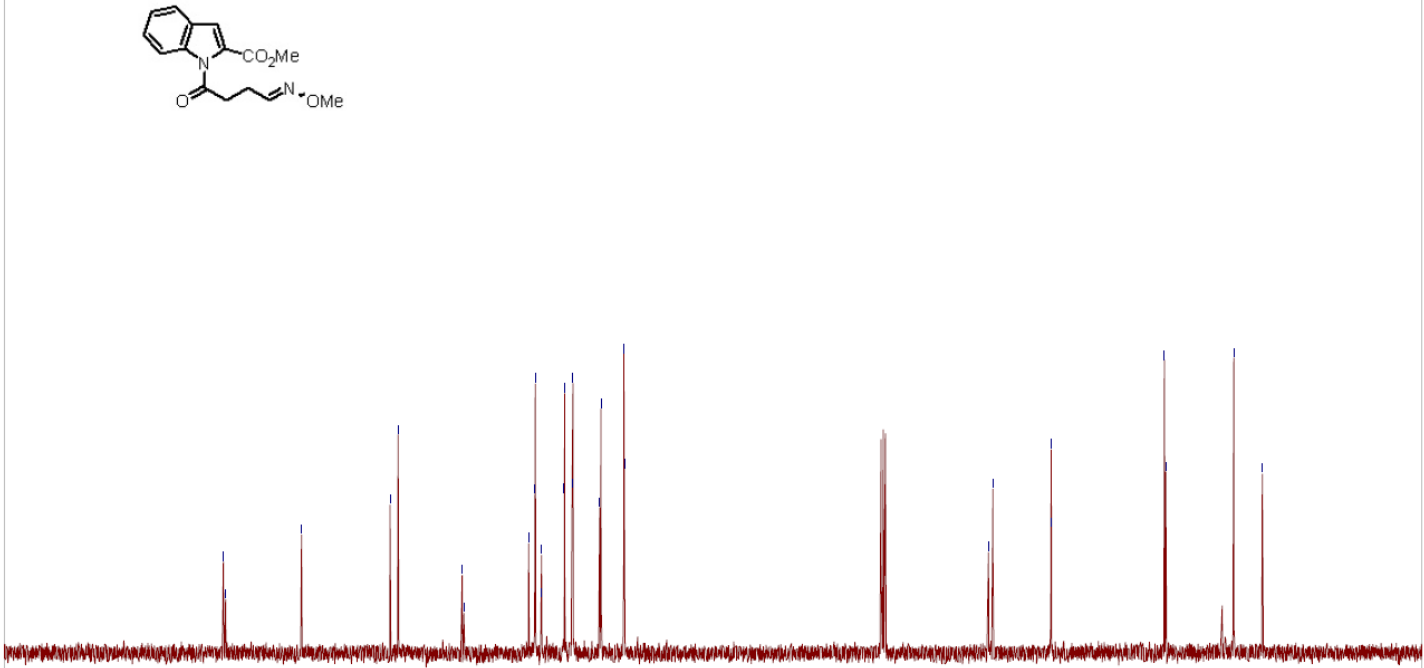

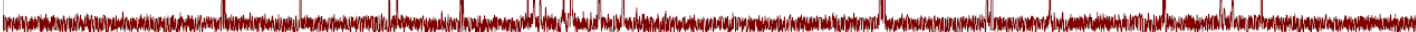

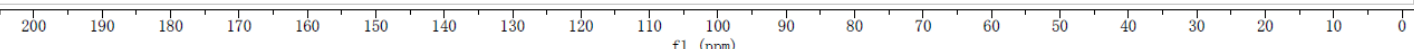


${ }^{1} \mathrm{H}$ NMR Spectrum of $\mathbf{1 c}$

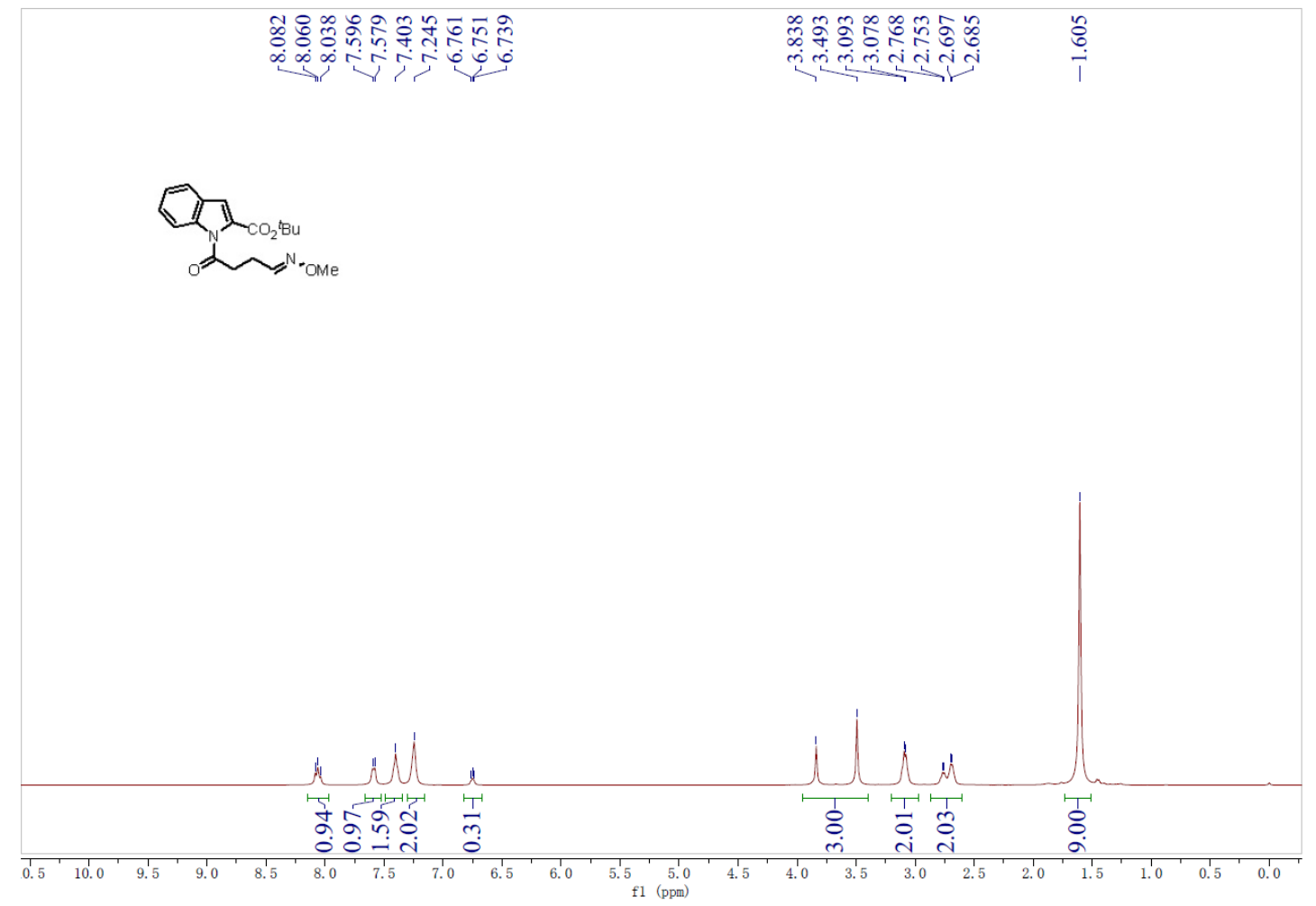

${ }^{13} \mathrm{C}$ NMR Spectrum of 1c

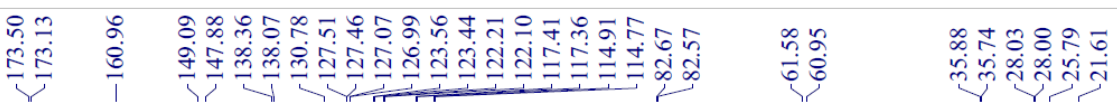
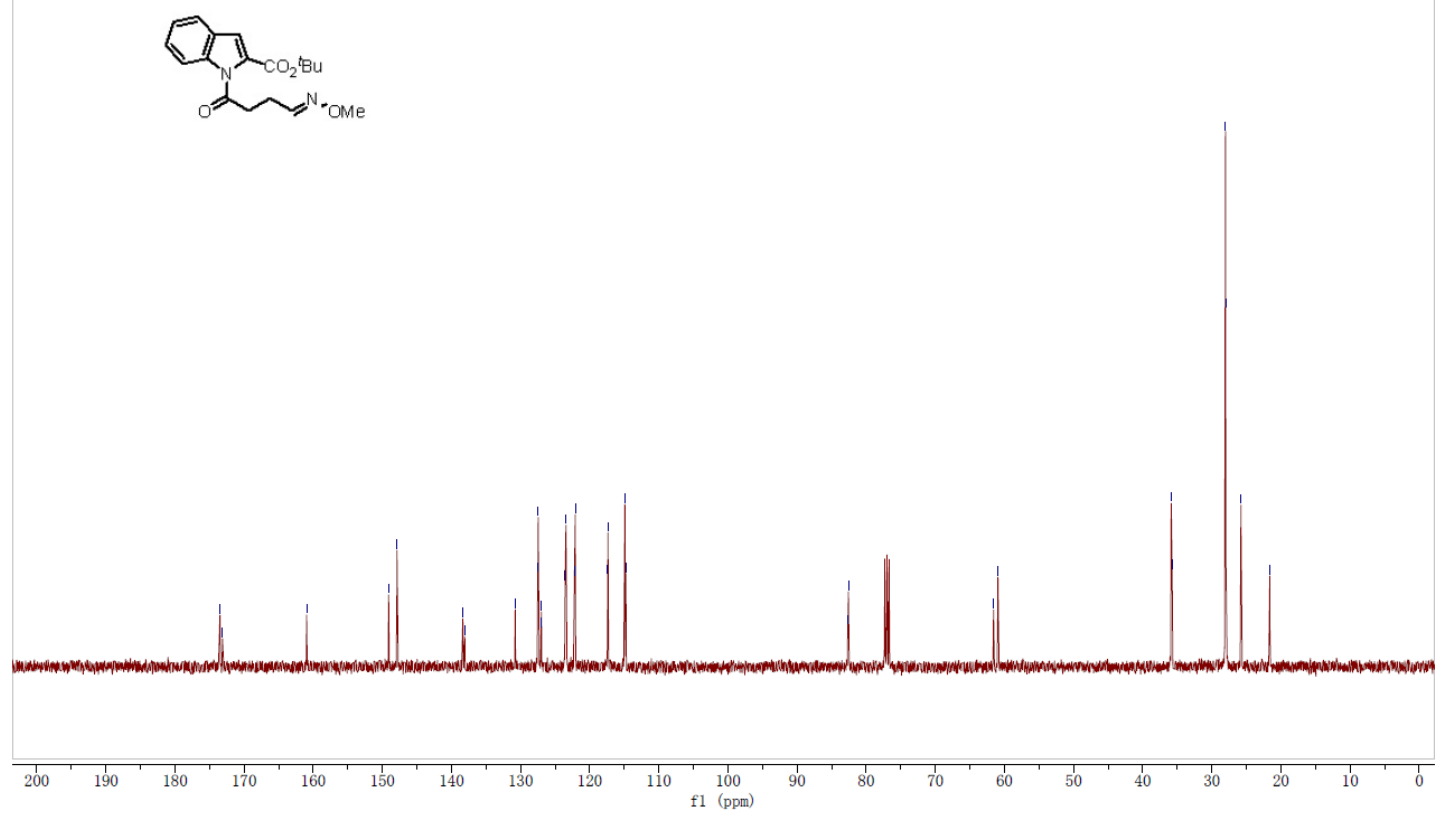
${ }^{1} \mathrm{H}$ NMR Spectrum of $\mathbf{1 d}$

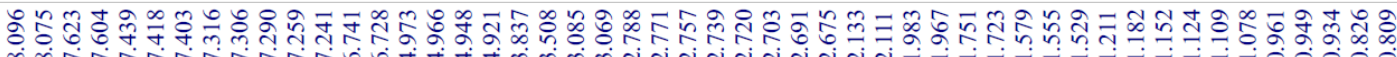

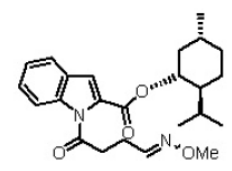

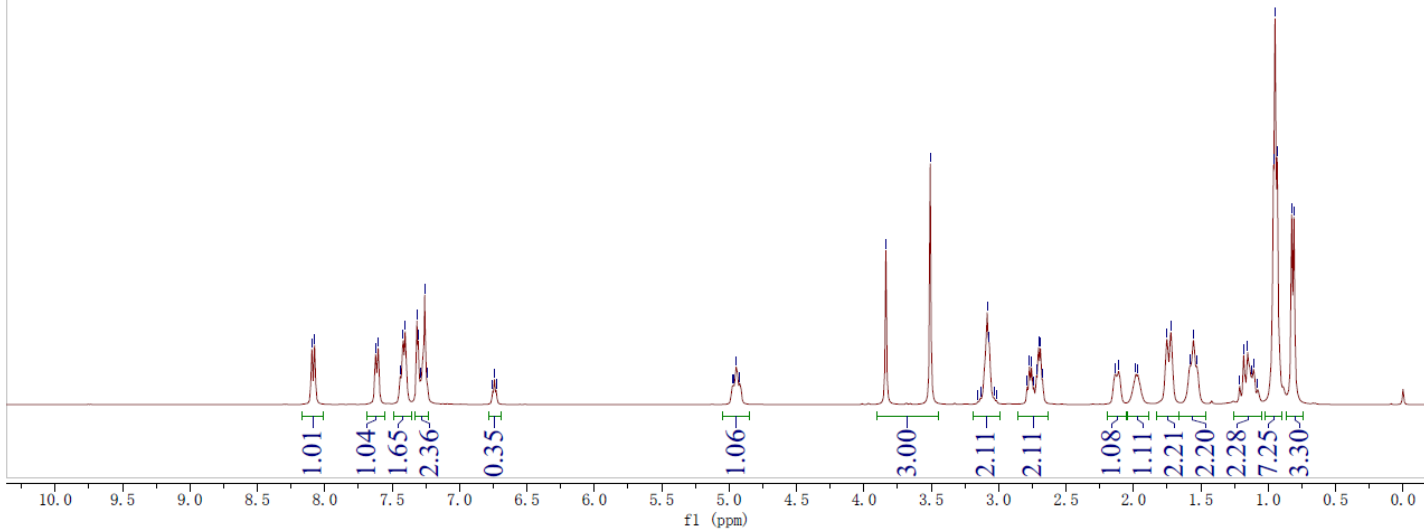

${ }^{13} \mathrm{C}$ NMR Spectrum of $\mathbf{1 d}$

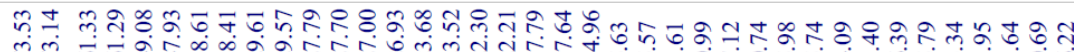

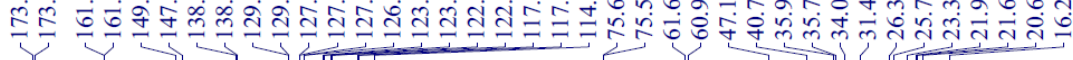

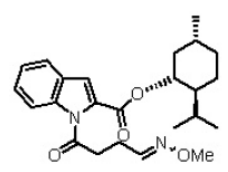

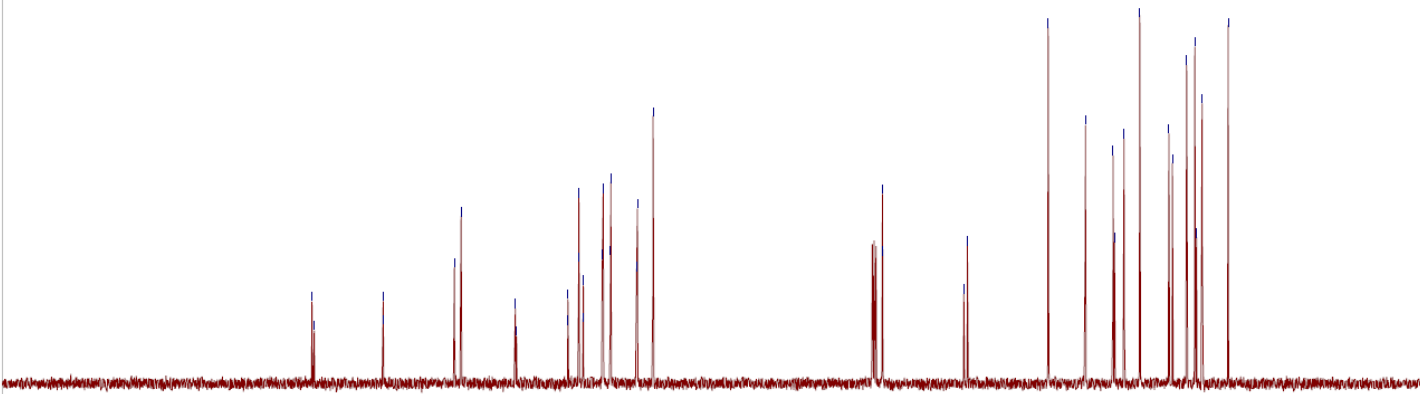

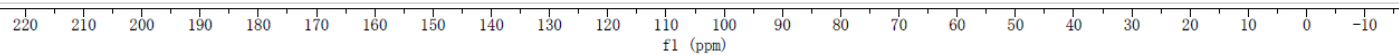


${ }^{1} \mathrm{H}$ NMR Spectrum of $\mathbf{1 e}$

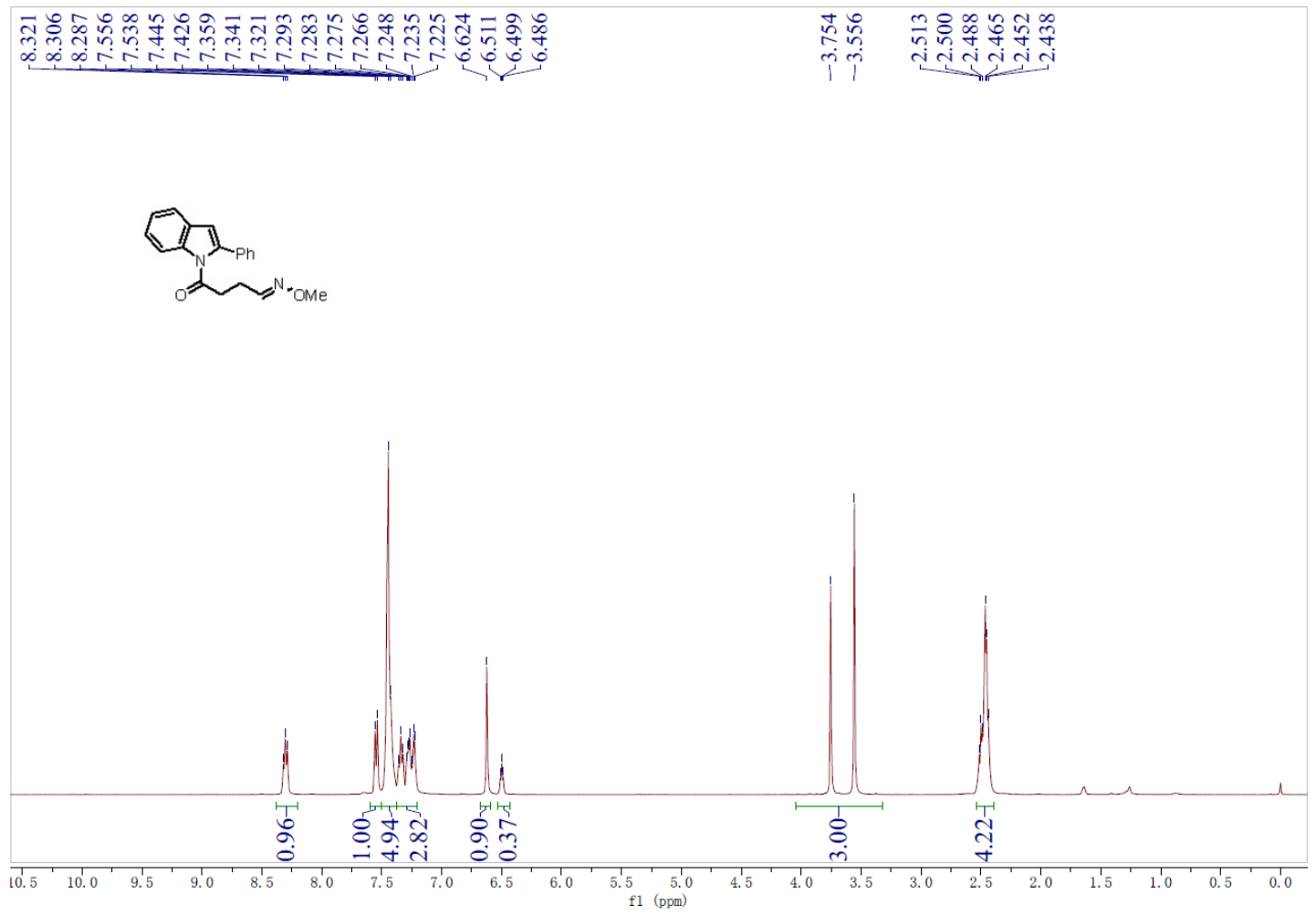

${ }^{13} \mathrm{C}$ NMR Spectrum of $\mathbf{1 e}$

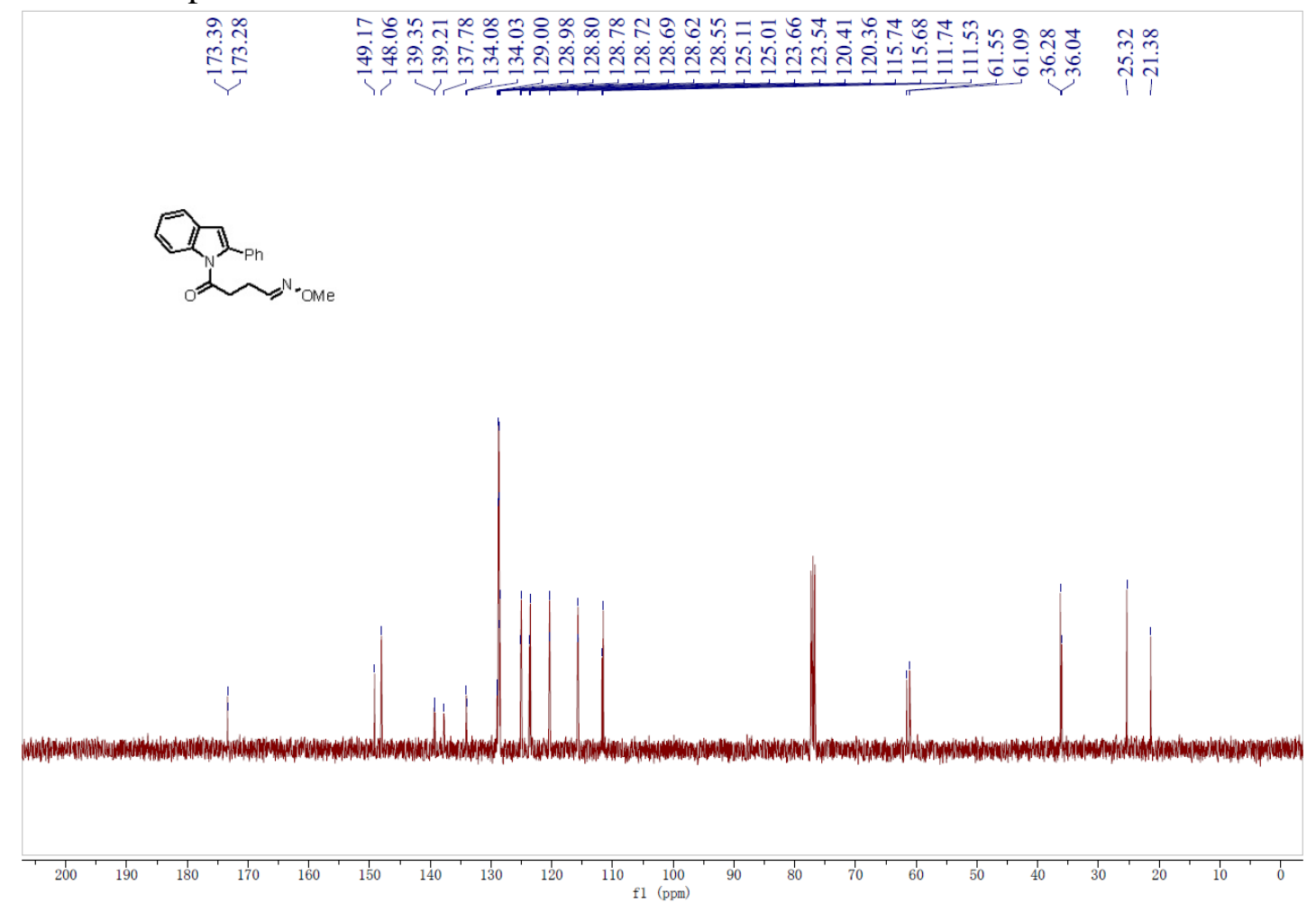


${ }^{1} \mathrm{H}$ NMR Spectrum of $\mathbf{1 f}$

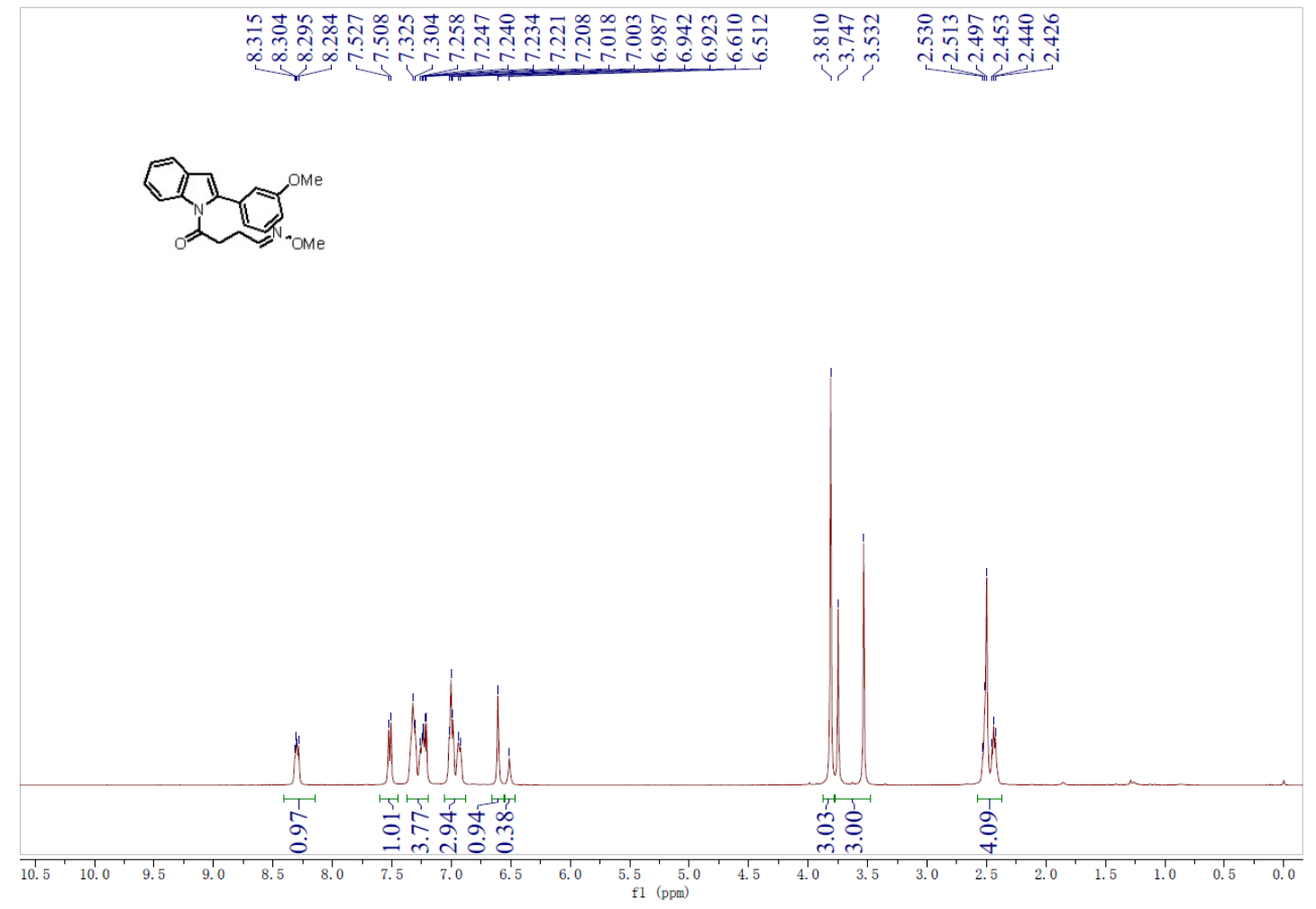

${ }^{13} \mathrm{C}$ NMR Spectrum of $\mathbf{1 f}$

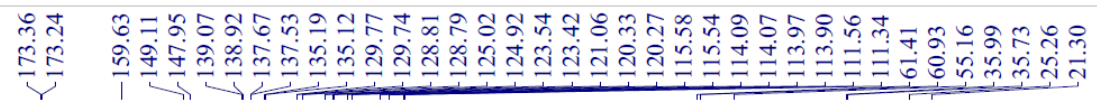

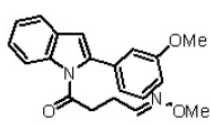
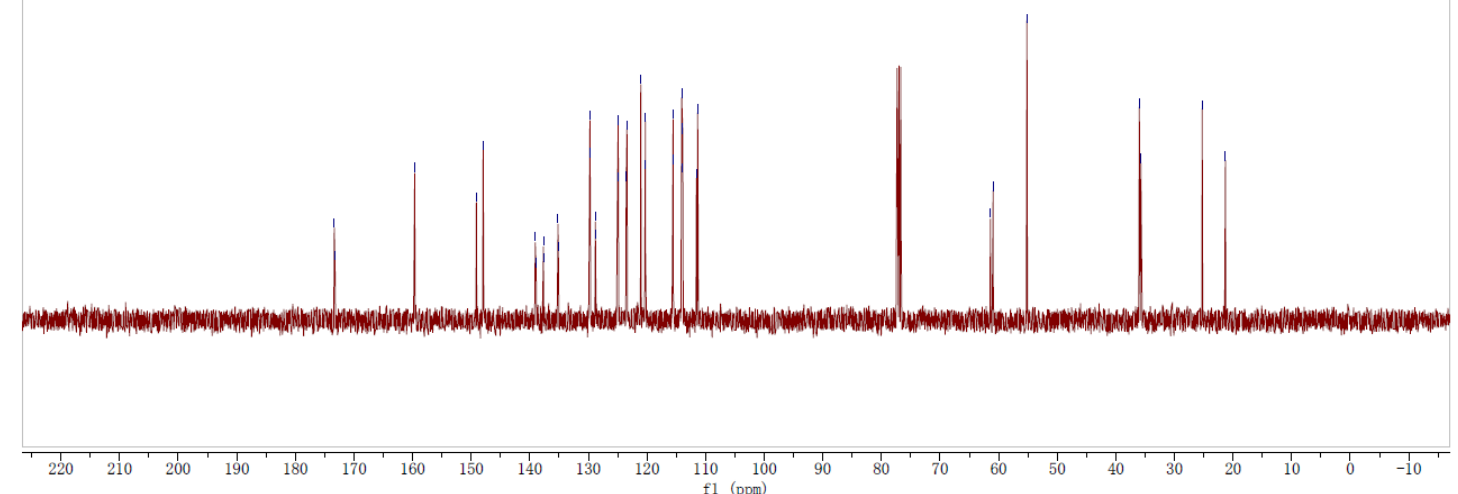
${ }^{1} \mathrm{H}$ NMR Spectrum of $\mathbf{1 g}$

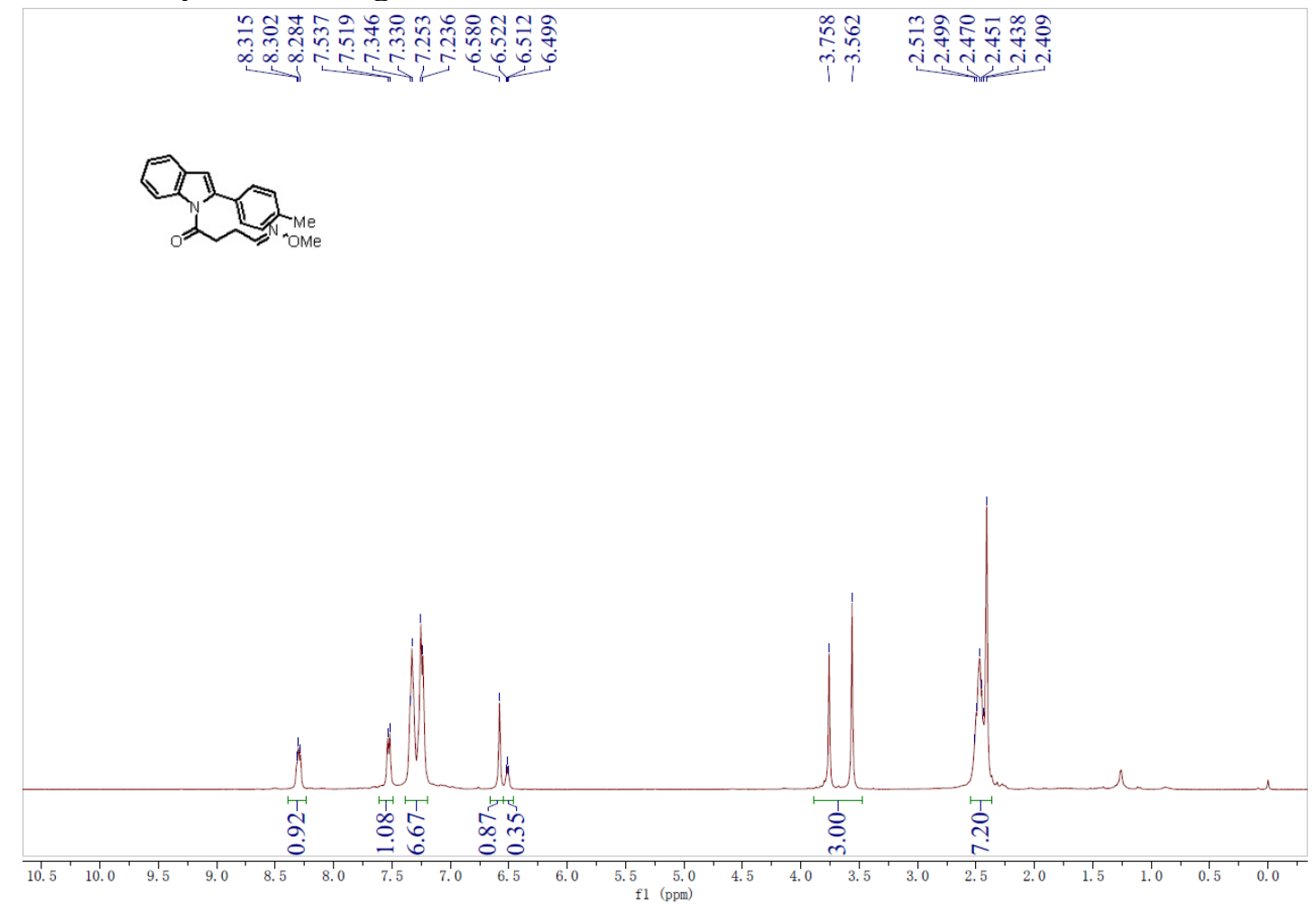

${ }^{13} \mathrm{C}$ NMR Spectrum of $\mathbf{1 g}$

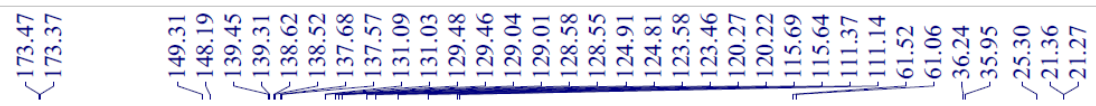
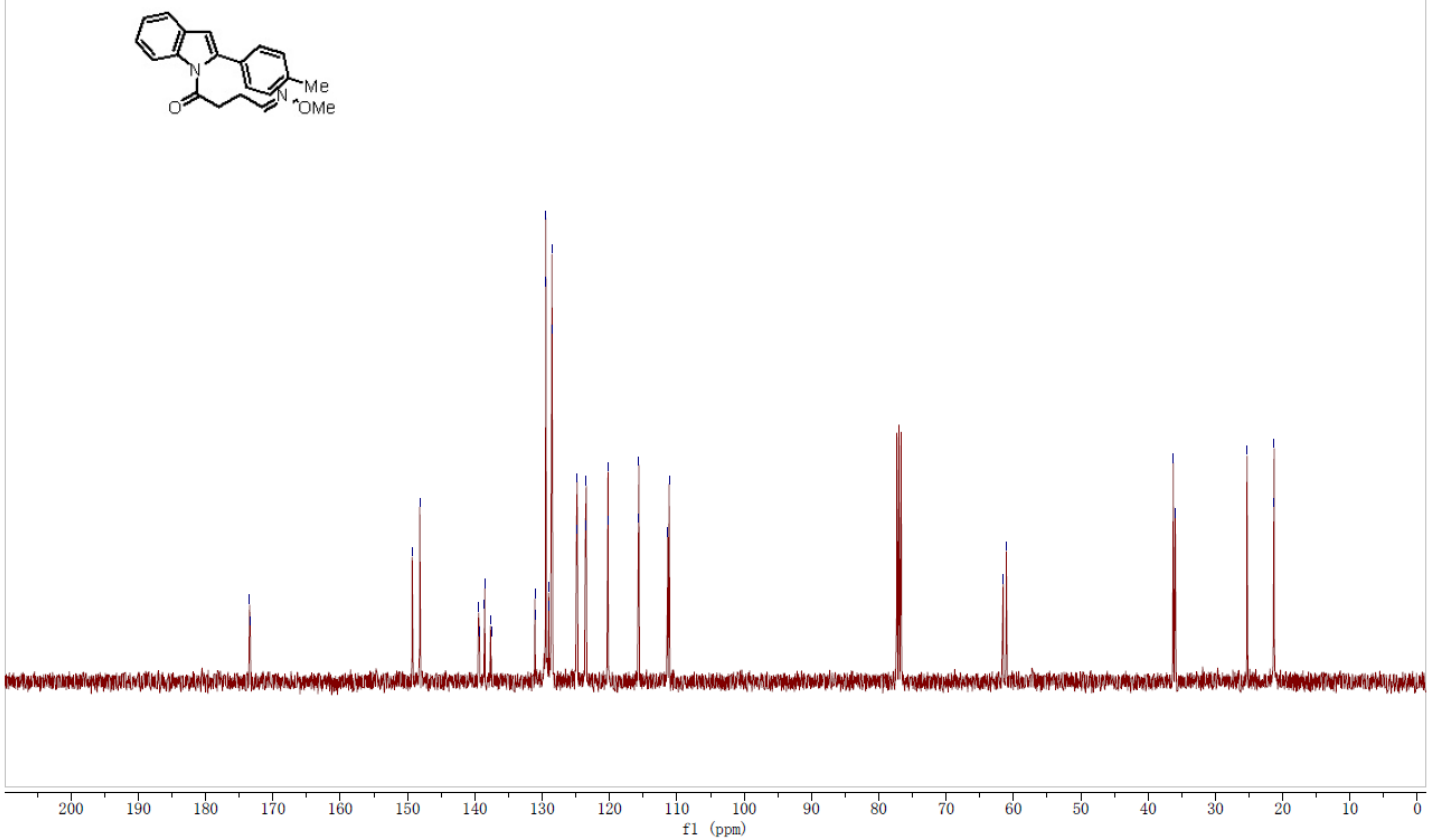

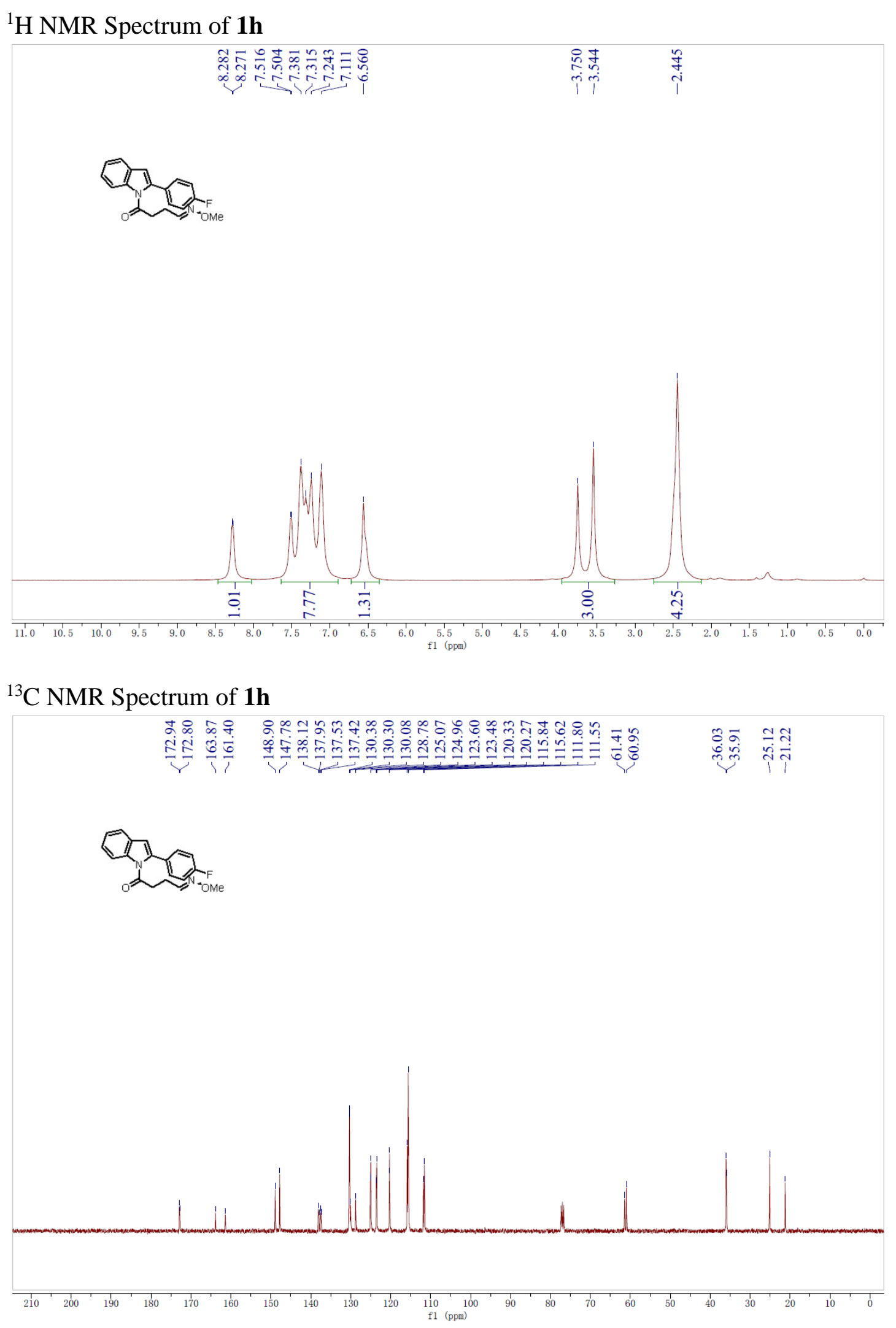

S79 
${ }^{19} \mathrm{~F}$ NMR Spectrum of $\mathbf{1 h}$

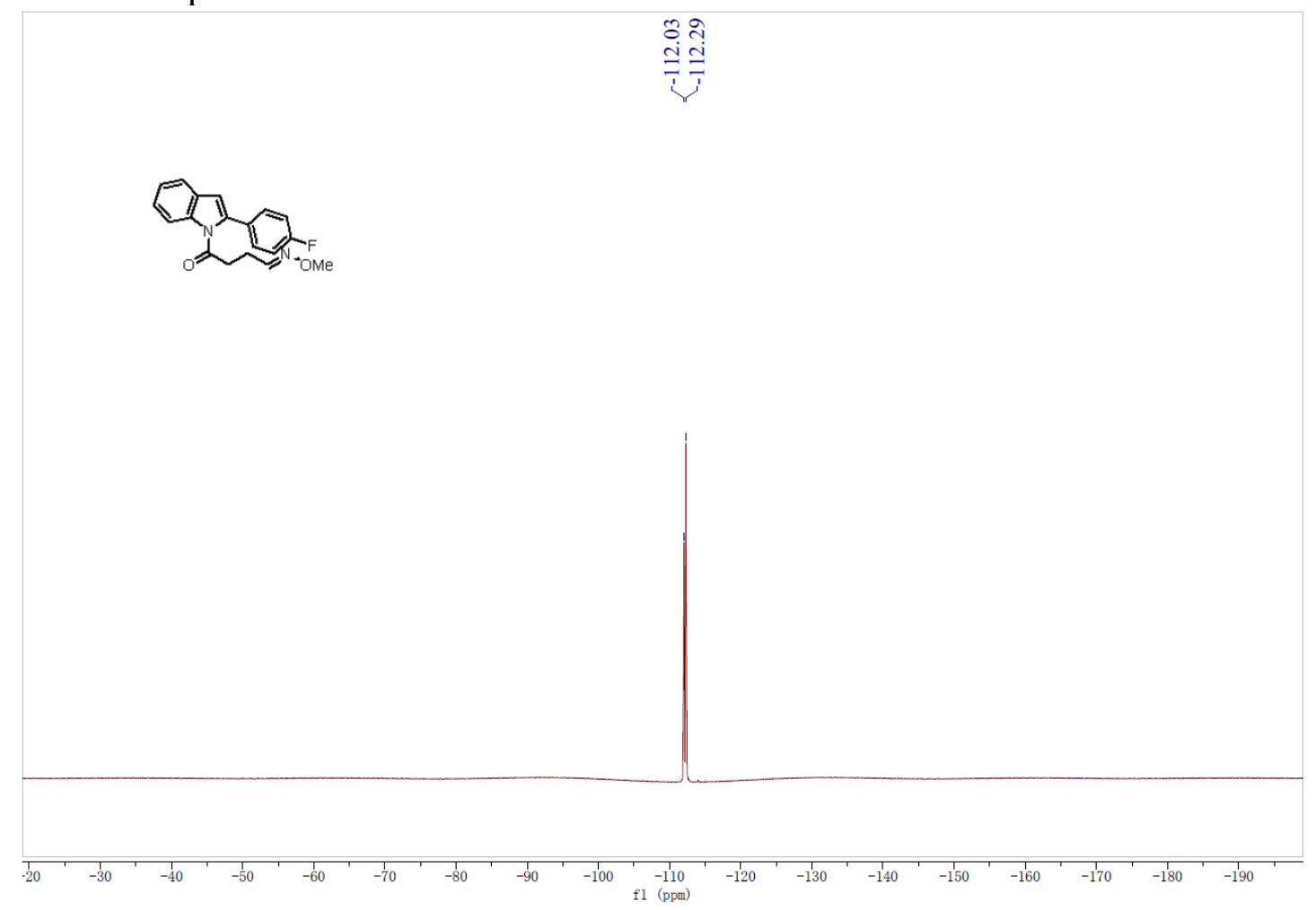

${ }^{1} \mathrm{H}$ NMR Spectrum of $\mathbf{1 i}$

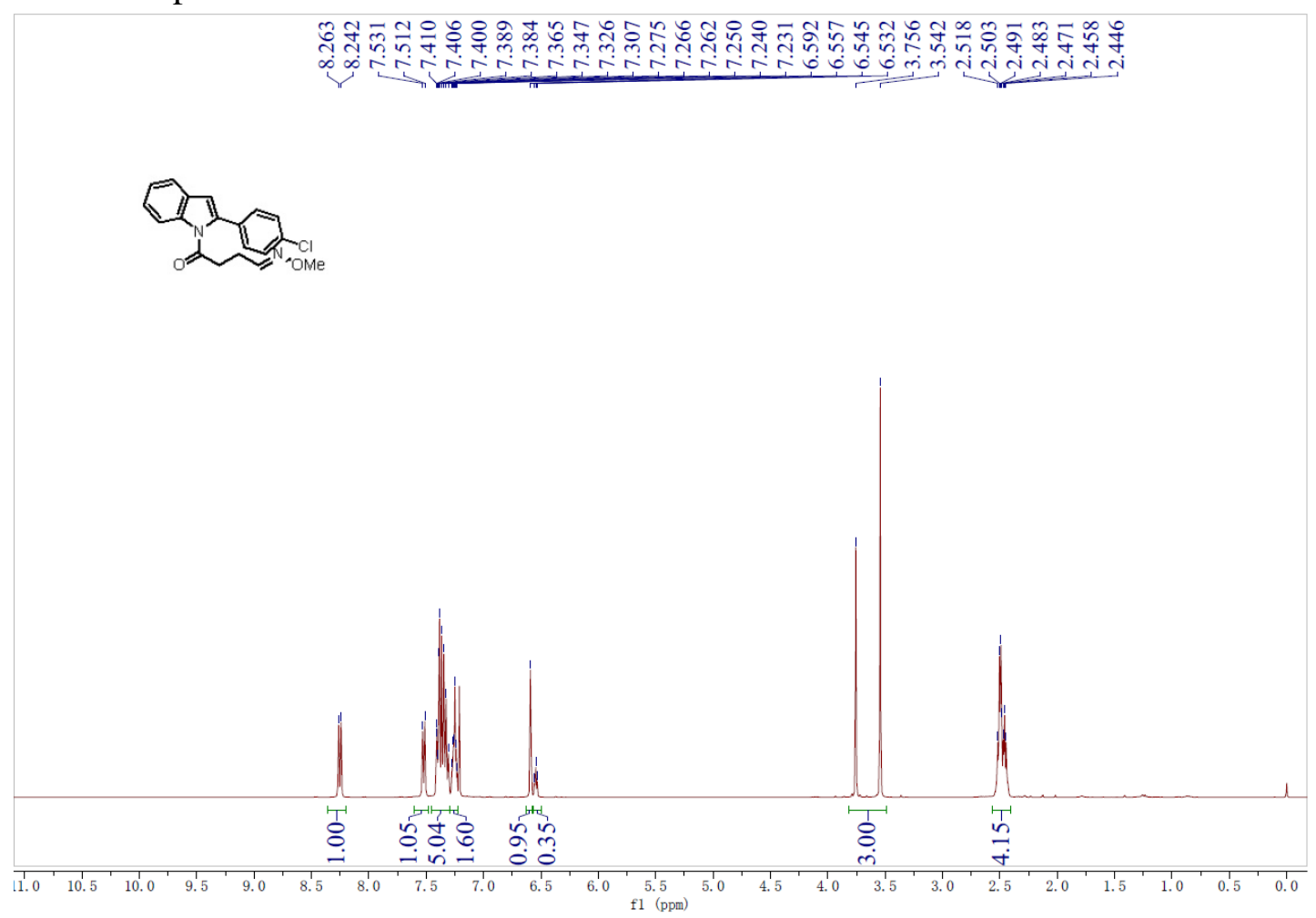

S80 
${ }^{13} \mathrm{C}$ NMR Spectrum of $\mathbf{1 i}$

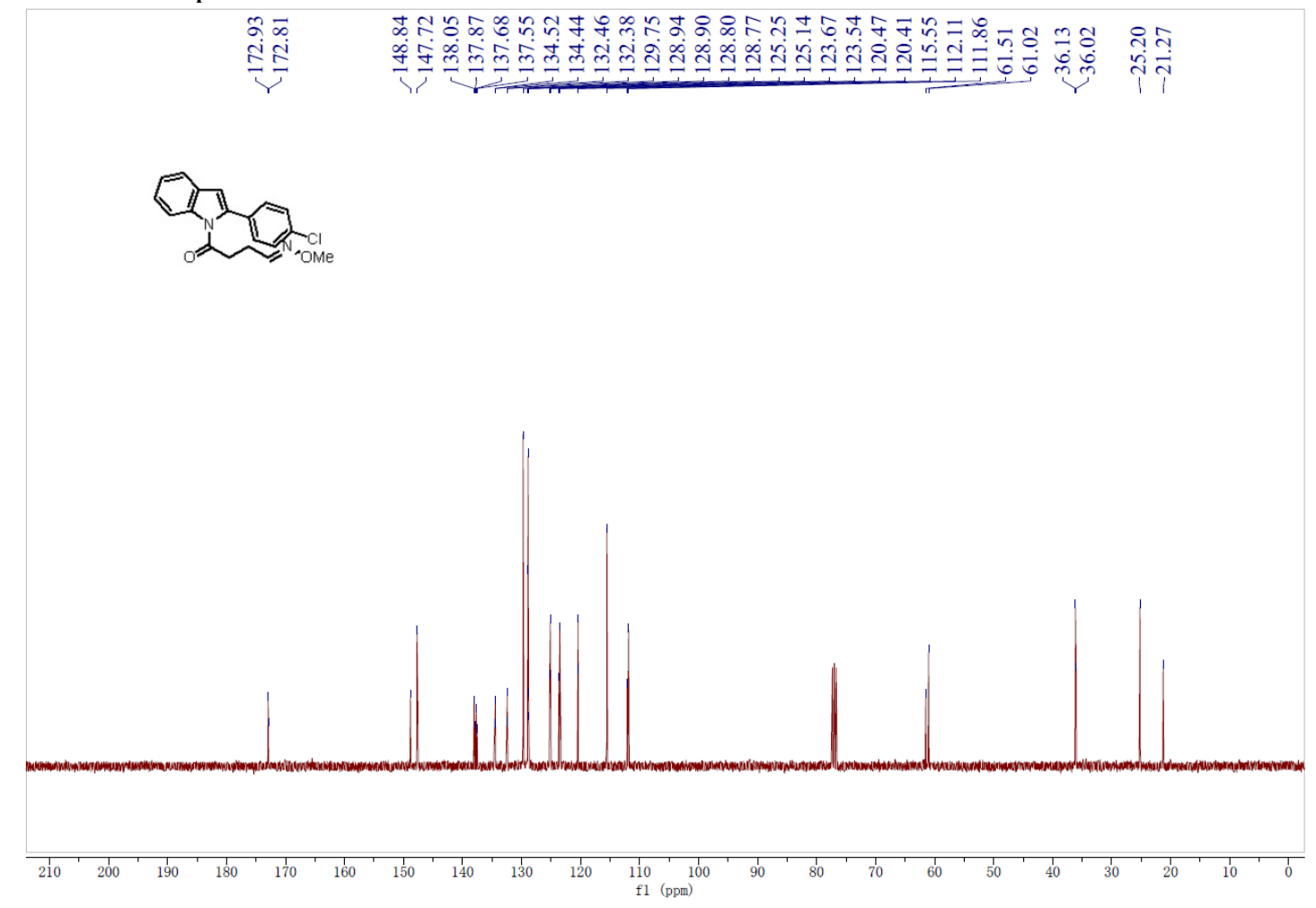

${ }^{1} \mathrm{H}$ NMR Spectrum of $\mathbf{1 j}$

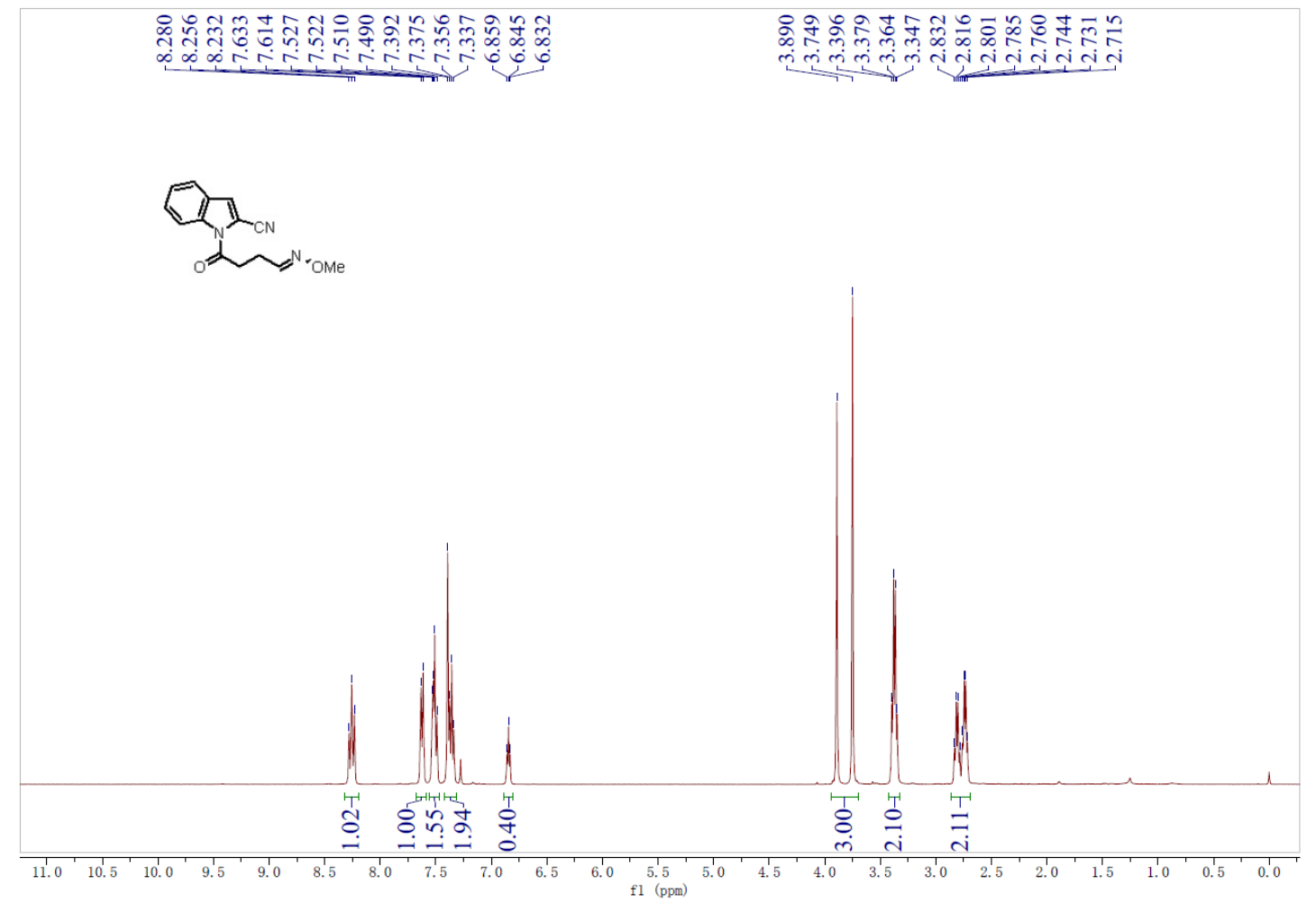


${ }^{13} \mathrm{C}$ NMR Spectrum of $\mathbf{1 j}$

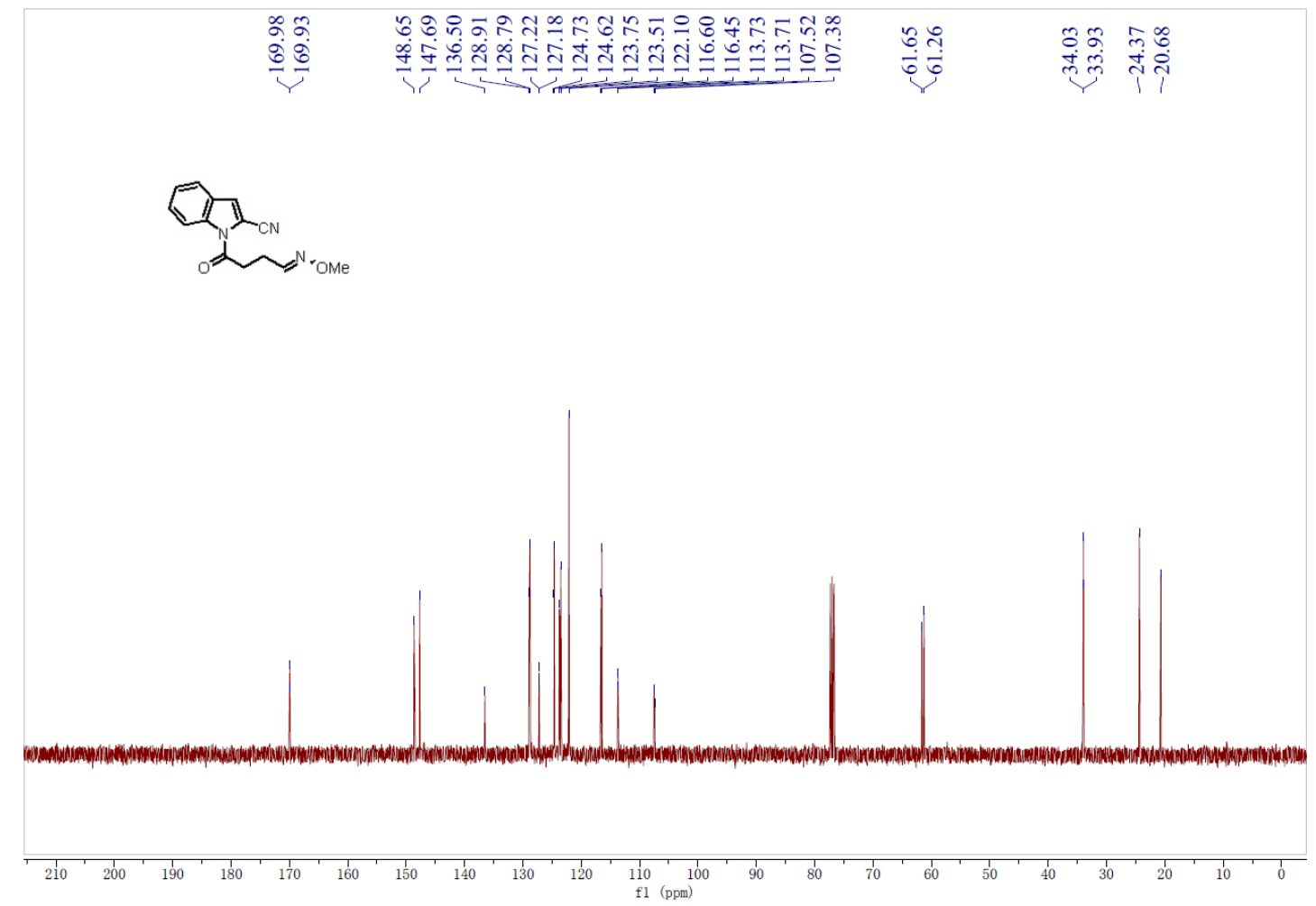

${ }^{1} \mathrm{H}$ NMR Spectrum of $\mathbf{1 k}$

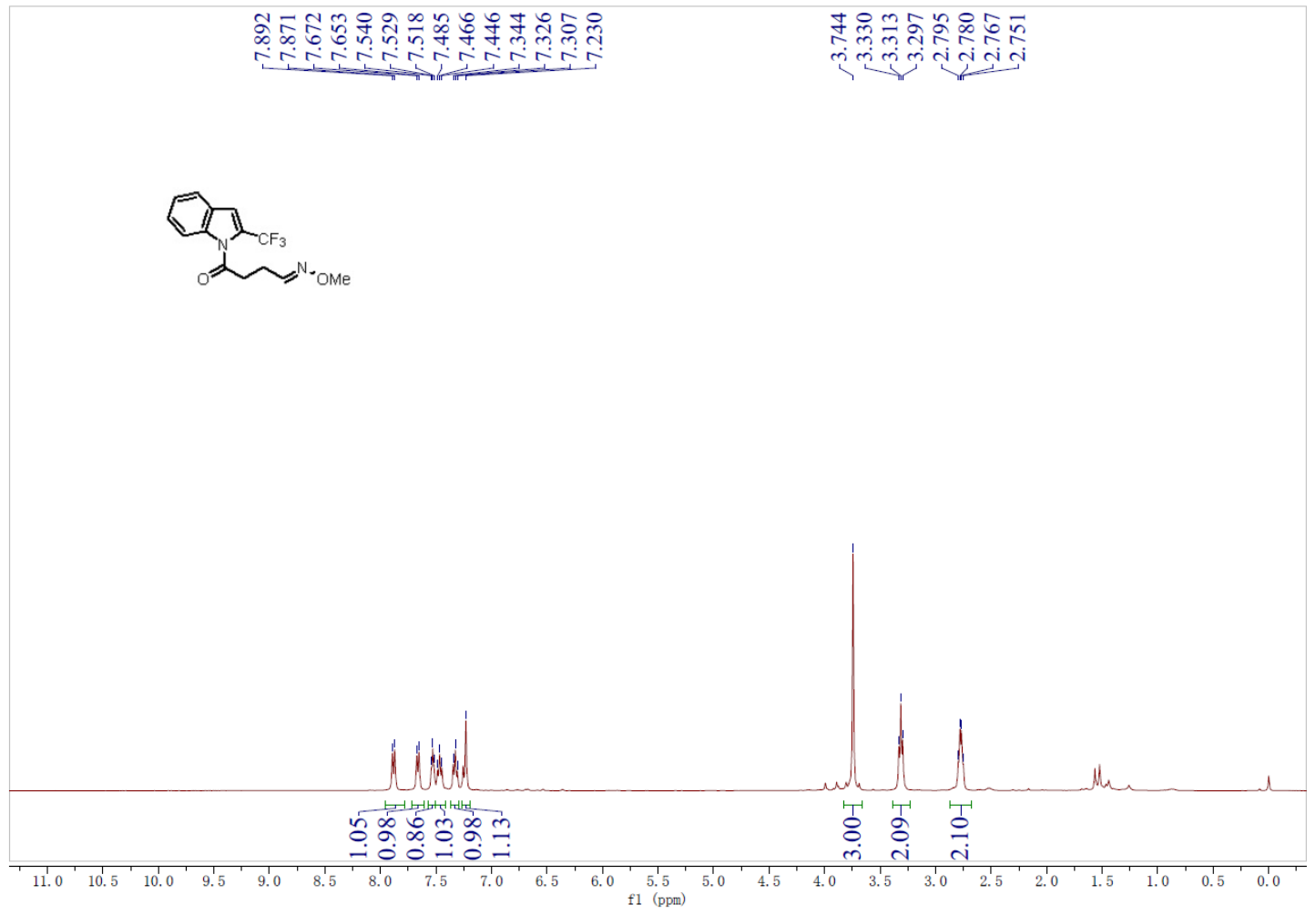


${ }^{13} \mathrm{C}$ NMR Spectrum of $\mathbf{1 k}$

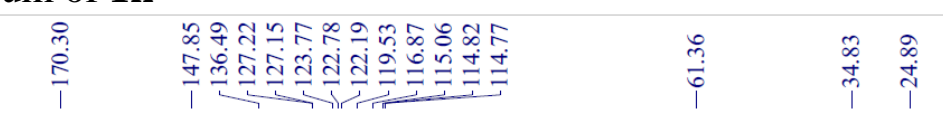
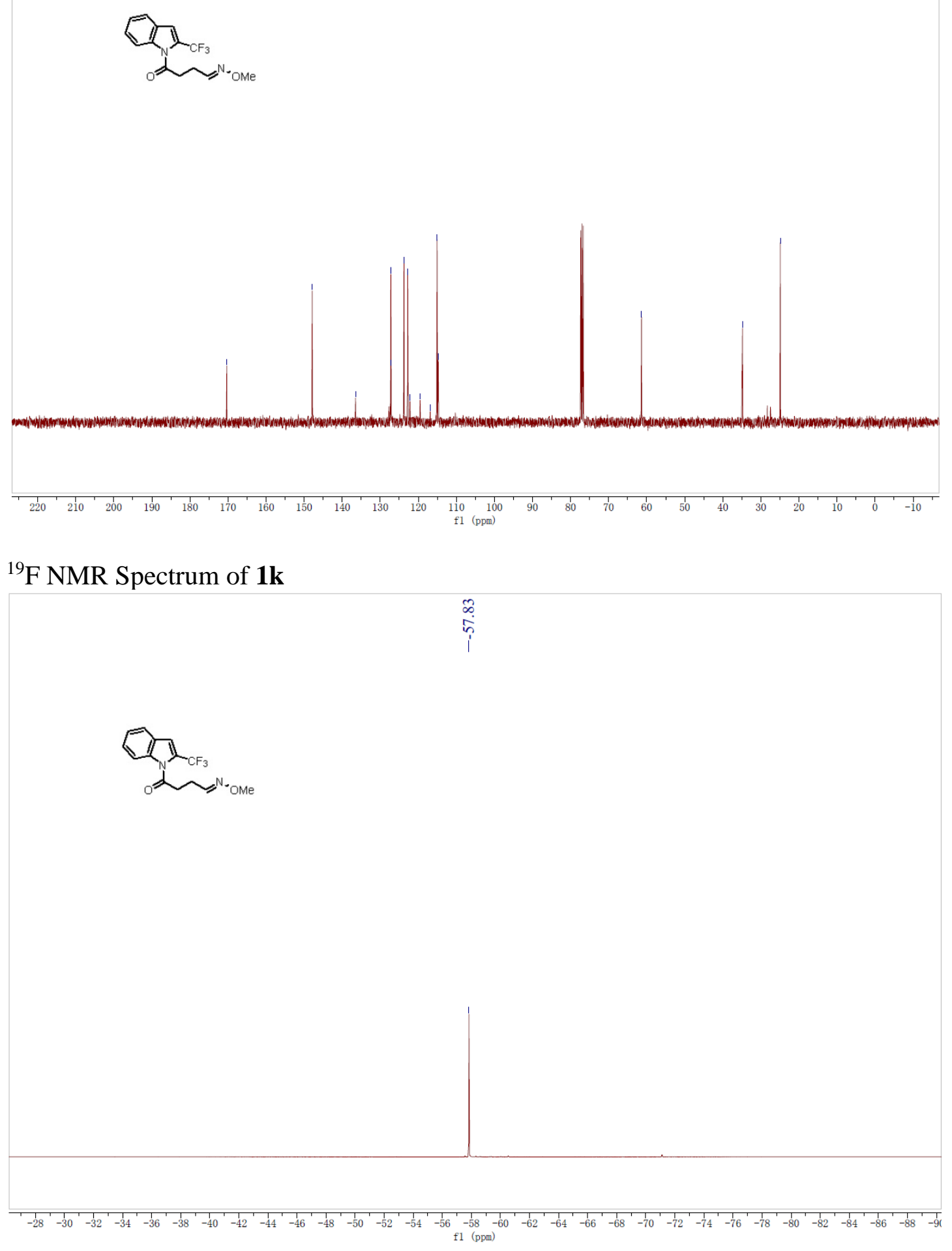

S83 
${ }^{1} \mathrm{H}$ NMR Spectrum of $\mathbf{1 l}$

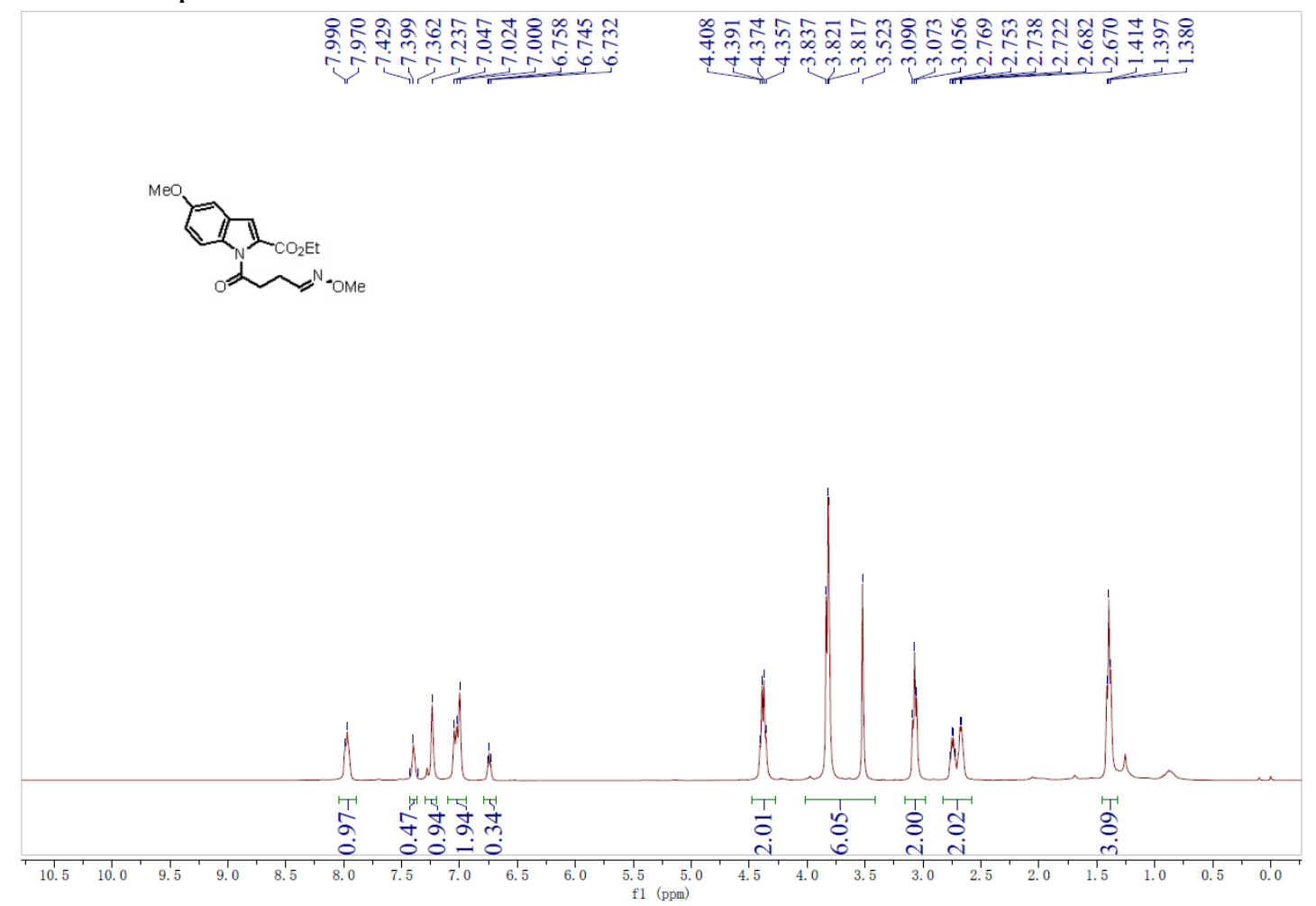

${ }^{13} \mathrm{C}$ NMR Spectrum of $\mathbf{1 l}$

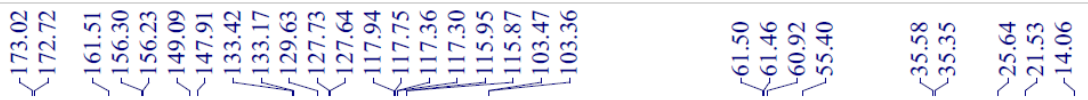

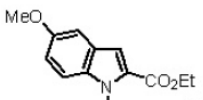

$\overbrace{}^{\mathrm{N}} \mathrm{COMMe}^{\mathrm{N}}$

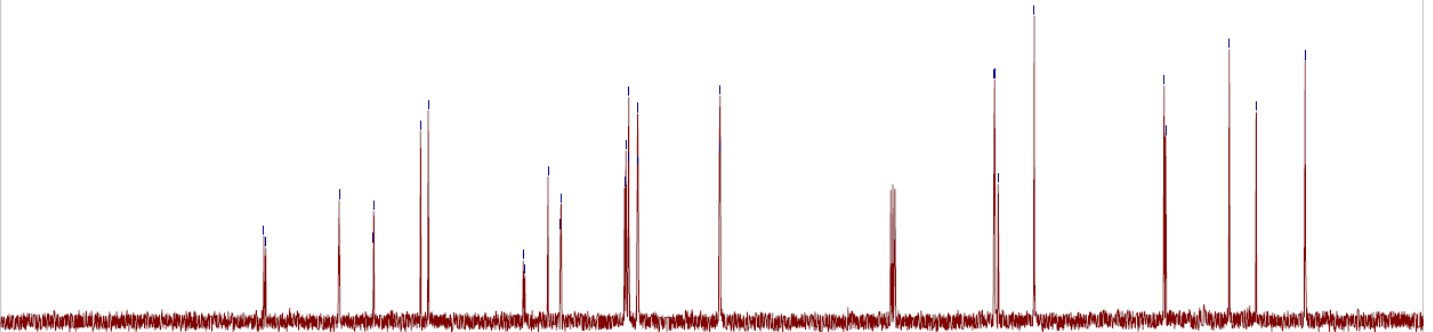

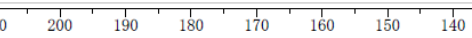

$\begin{array}{cc}110 & 100 \\ \mathrm{f} 1 & (\mathrm{ppm})\end{array}$ 
${ }^{1} \mathrm{H}$ NMR Spectrum of $\mathbf{1 m}$

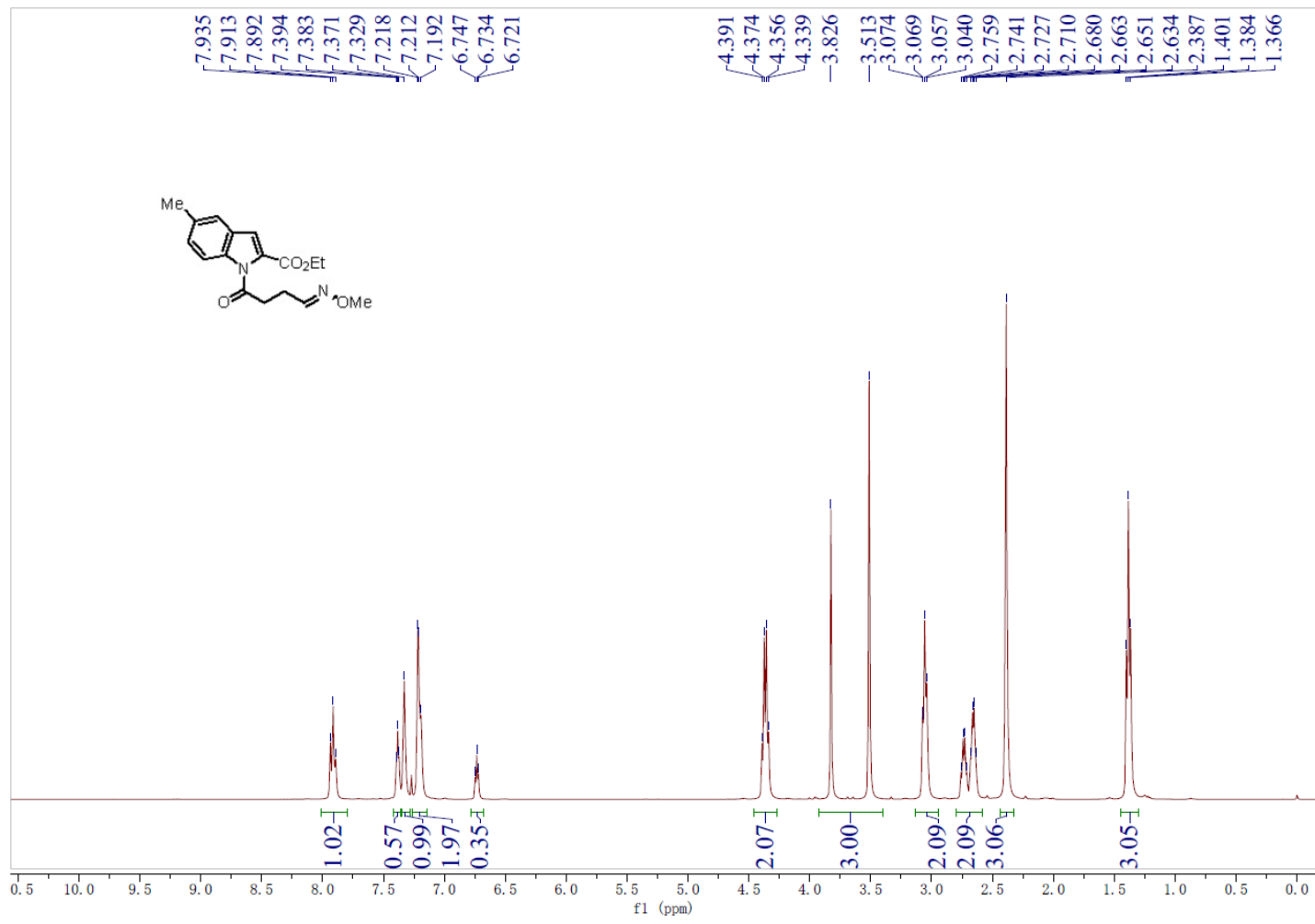

${ }^{13} \mathrm{C}$ NMR Spectrum of $\mathbf{1 m}$

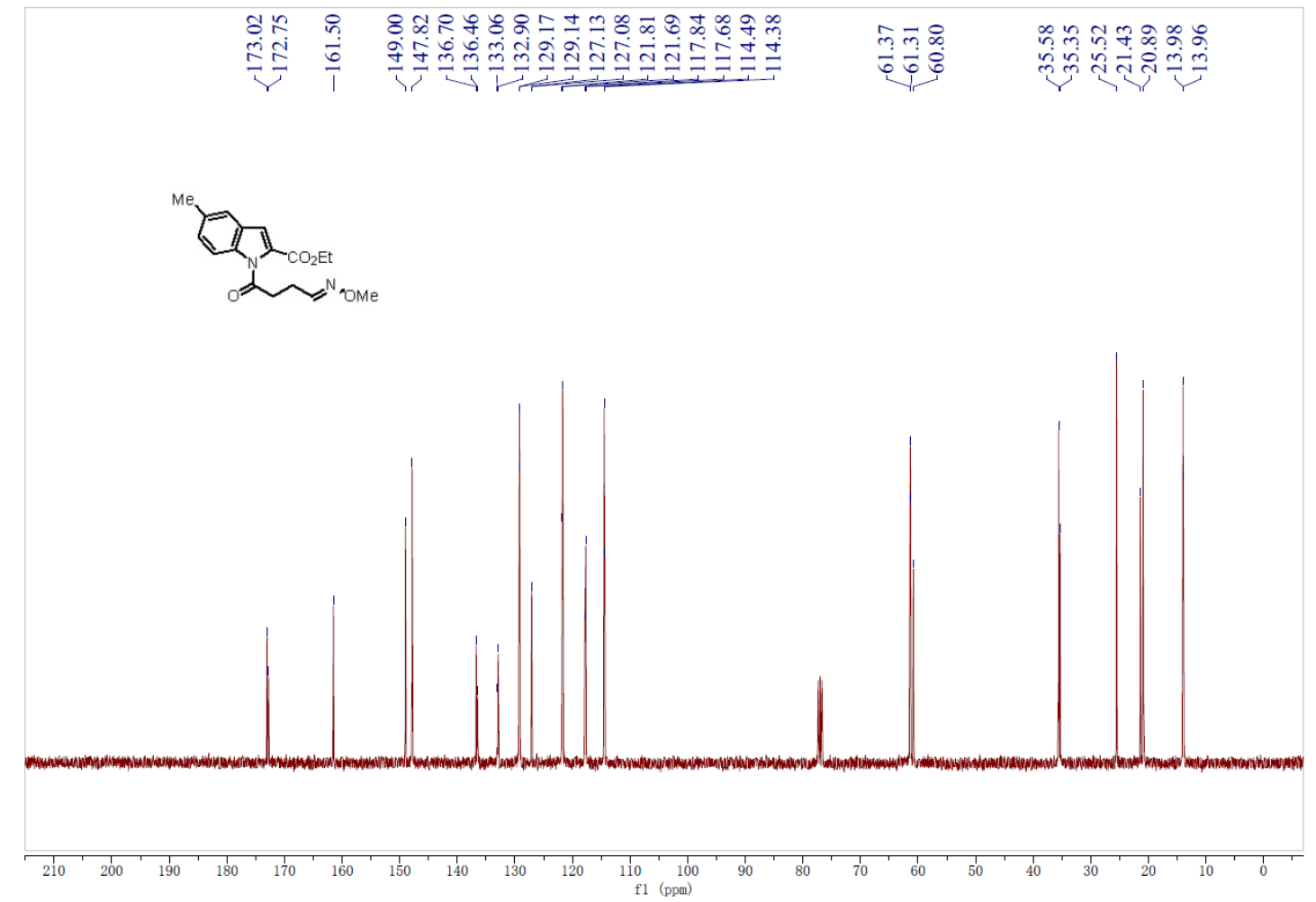


${ }^{1} \mathrm{H}$ NMR Spectrum of $\mathbf{1 n}$

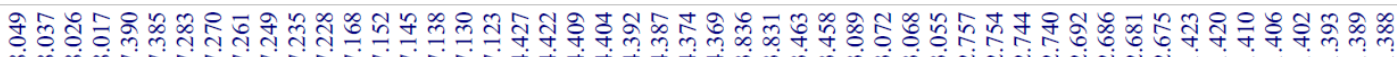

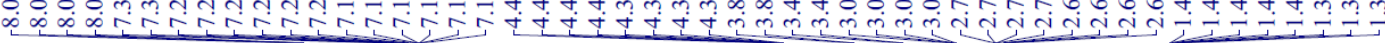
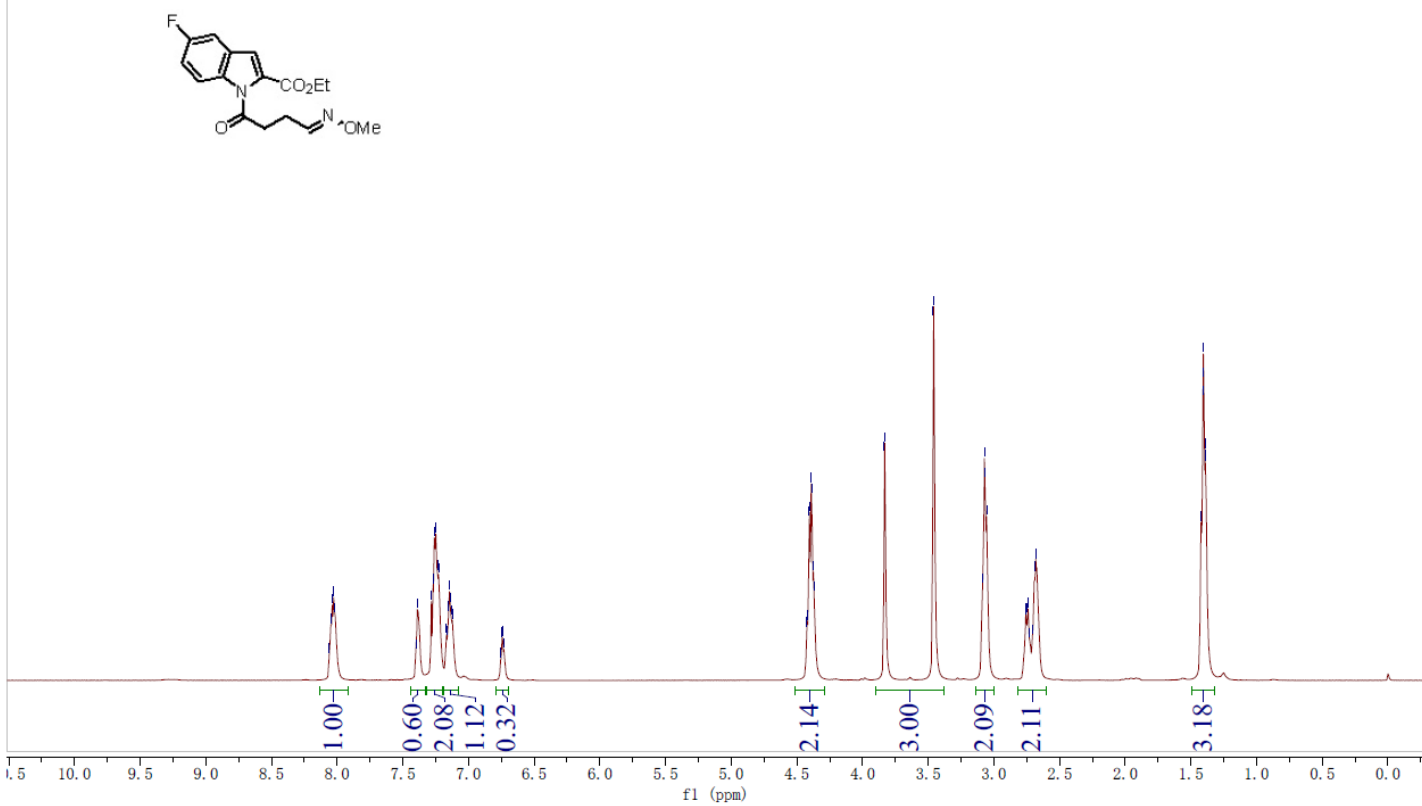

${ }^{13} \mathrm{C}$ NMR Spectrum of $\mathbf{1 n}$

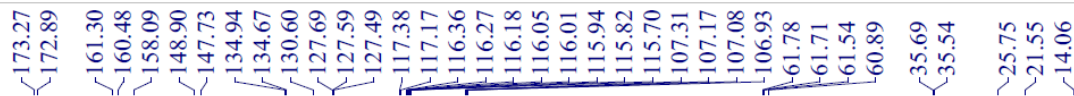

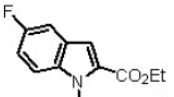

D

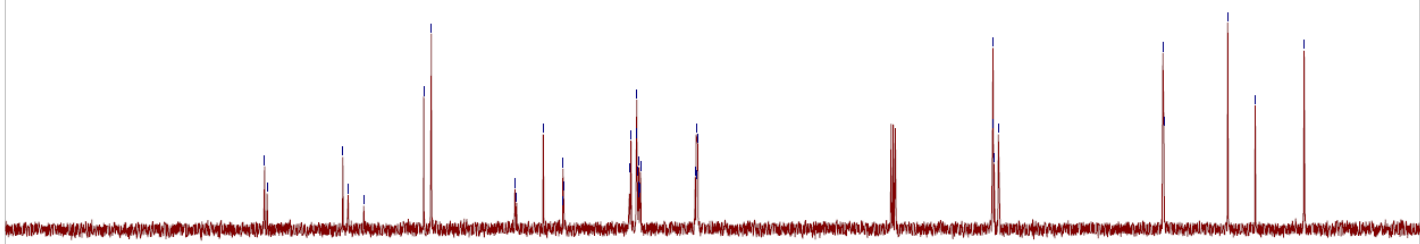

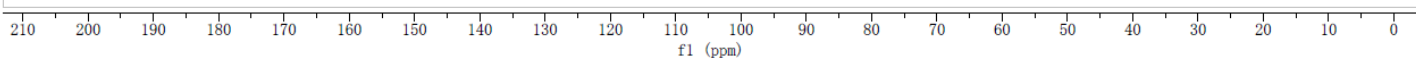


${ }^{19}$ F NMR Spectrum of $\mathbf{1 n}$

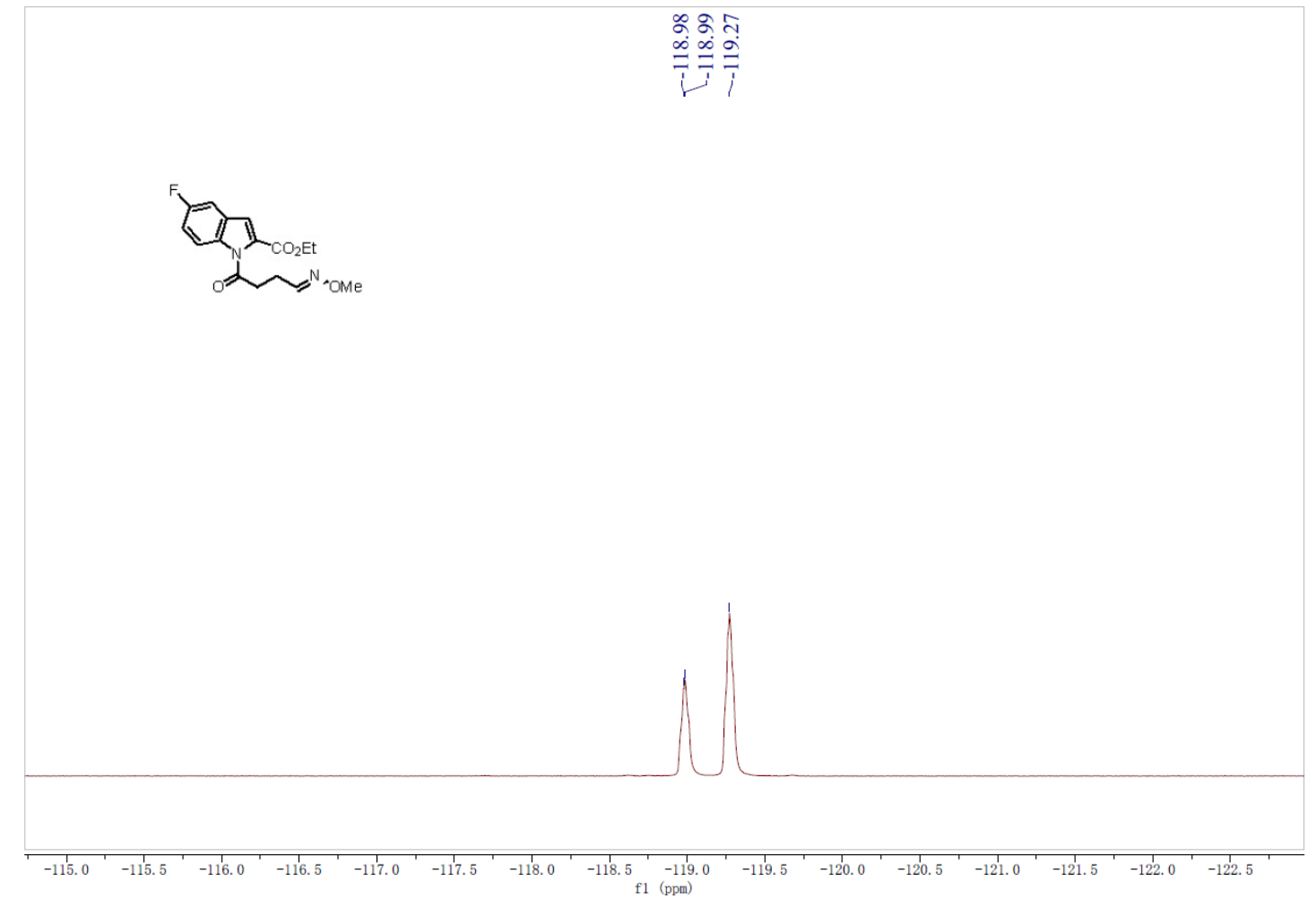


${ }^{1} \mathrm{H}$ NMR Spectrum of $\mathbf{1 0}$

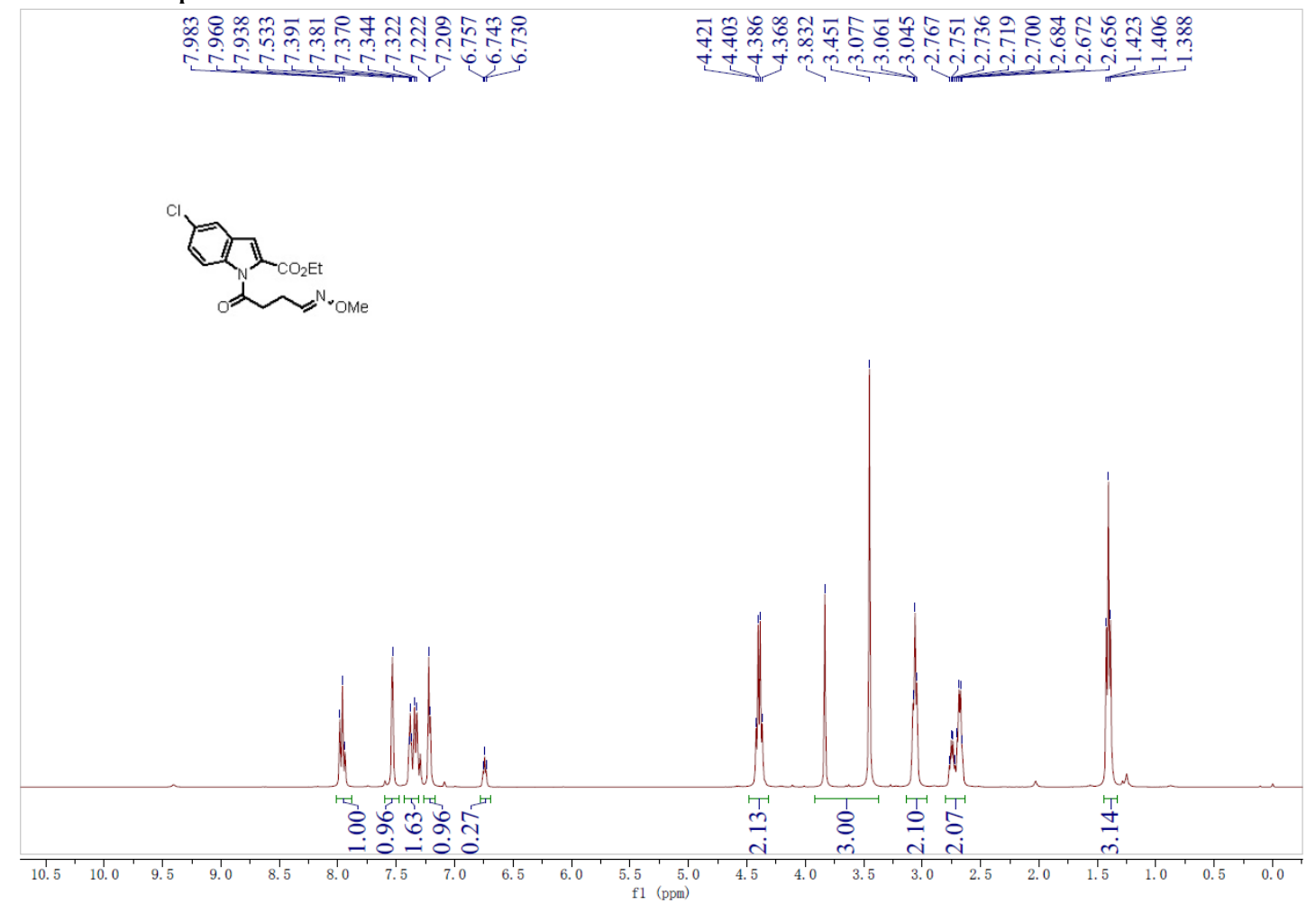

${ }^{13} \mathrm{C}$ NMR Spectrum of $\mathbf{1 o}$

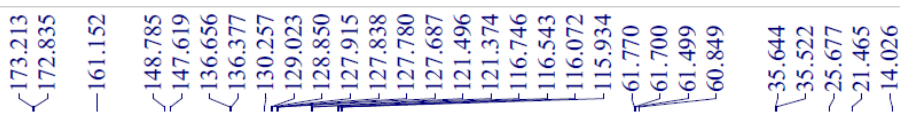
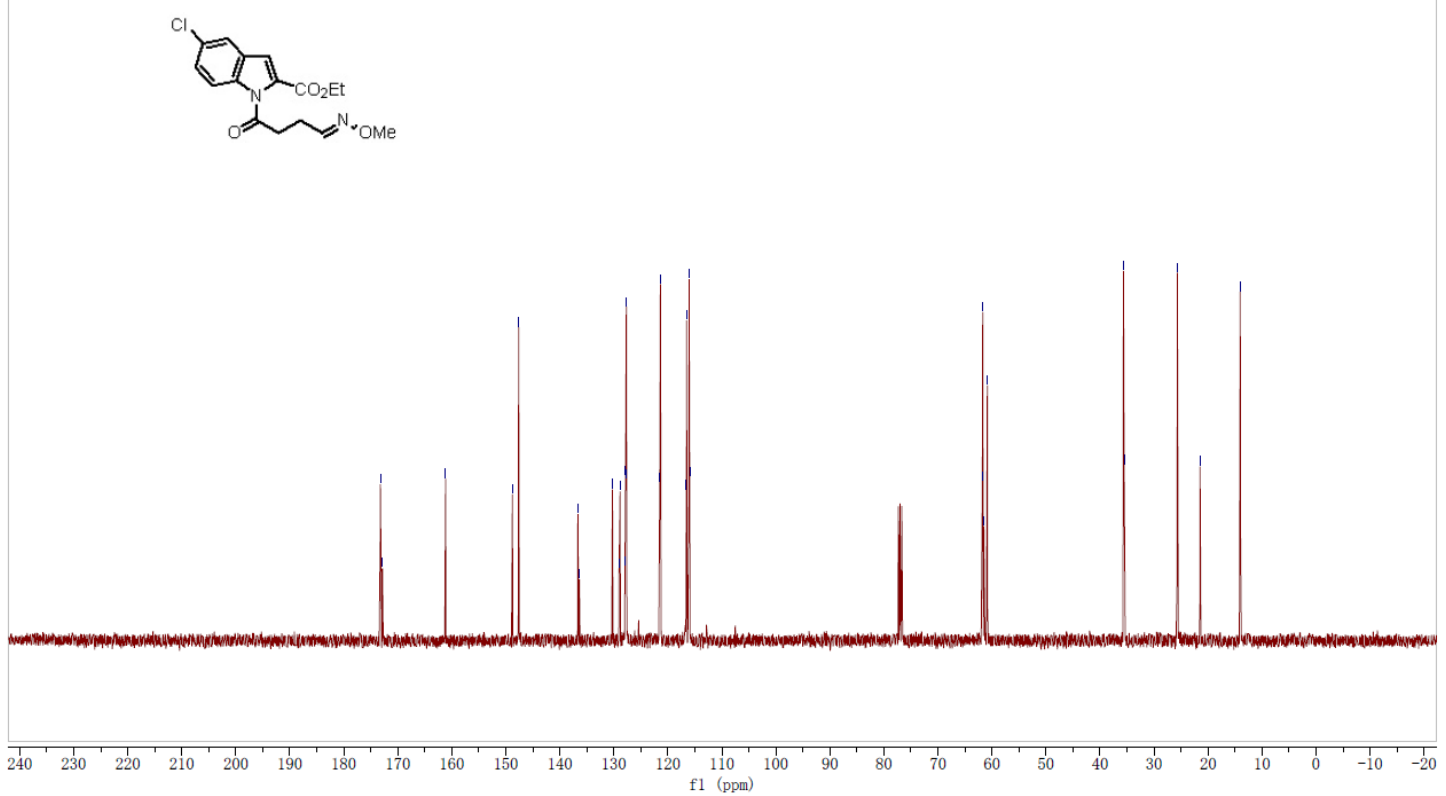
${ }^{1} \mathrm{H}$ NMR Spectrum of $\mathbf{1 p}$

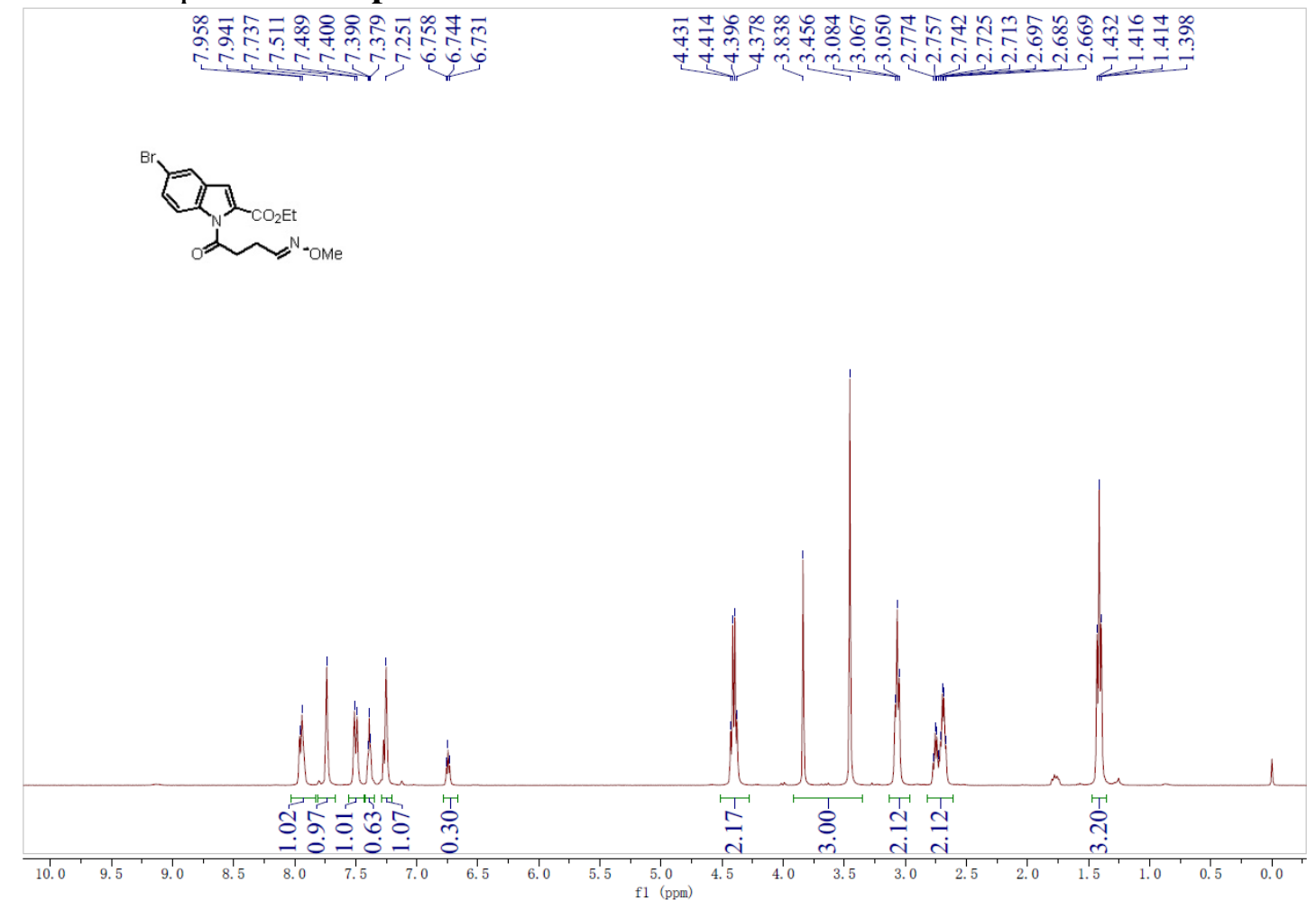

${ }^{13} \mathrm{C}$ NMR Spectrum of $\mathbf{1 p}$

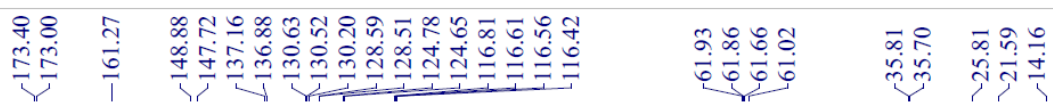
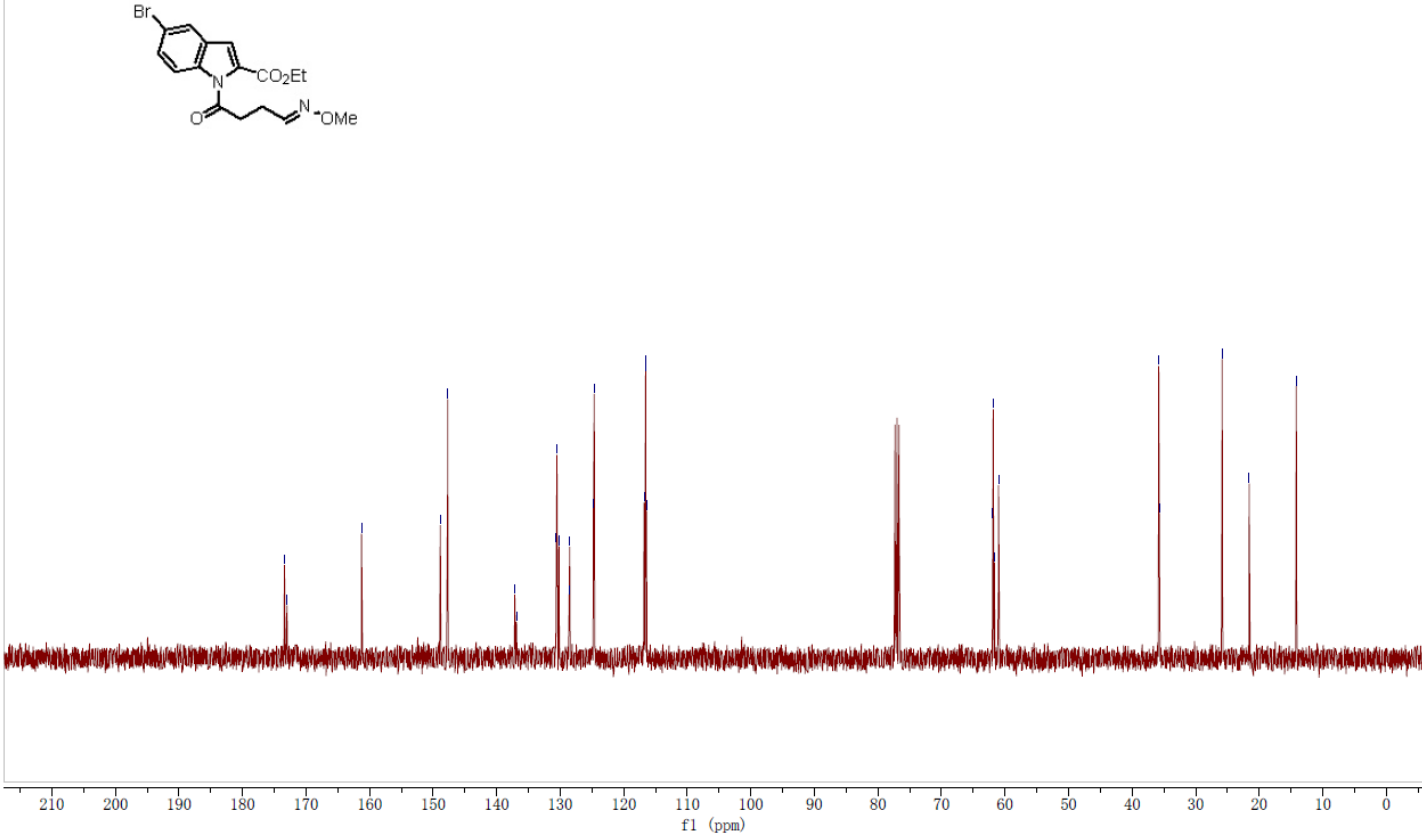
${ }^{1} \mathrm{H}$ NMR Spectrum of $\mathbf{1 q}$

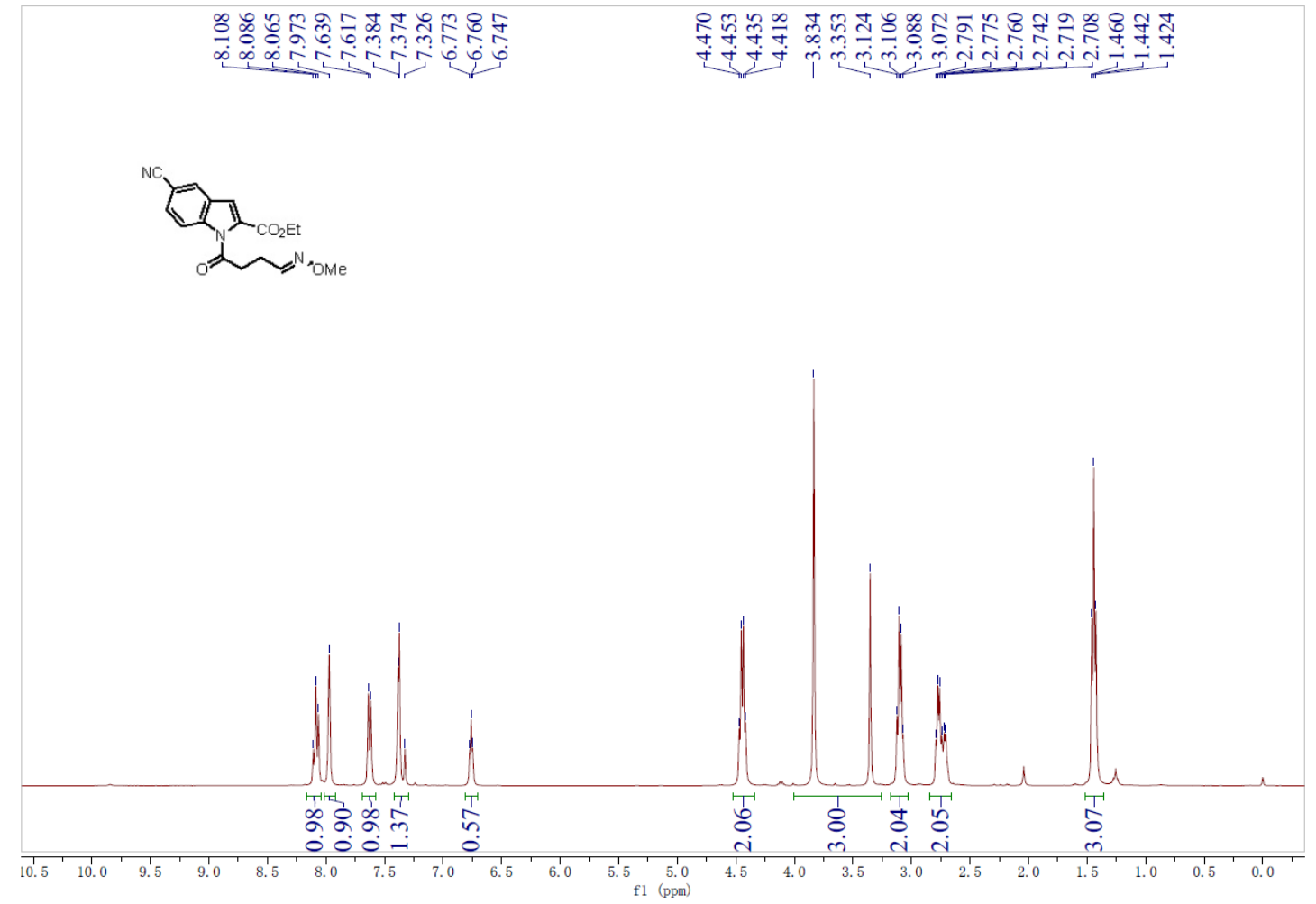

${ }^{13} \mathrm{C}$ NMR Spectrum of $\mathbf{1 q}$

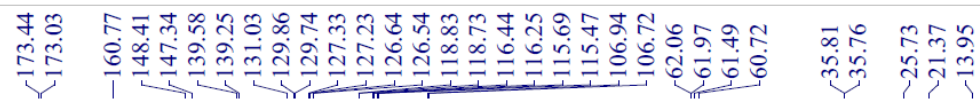
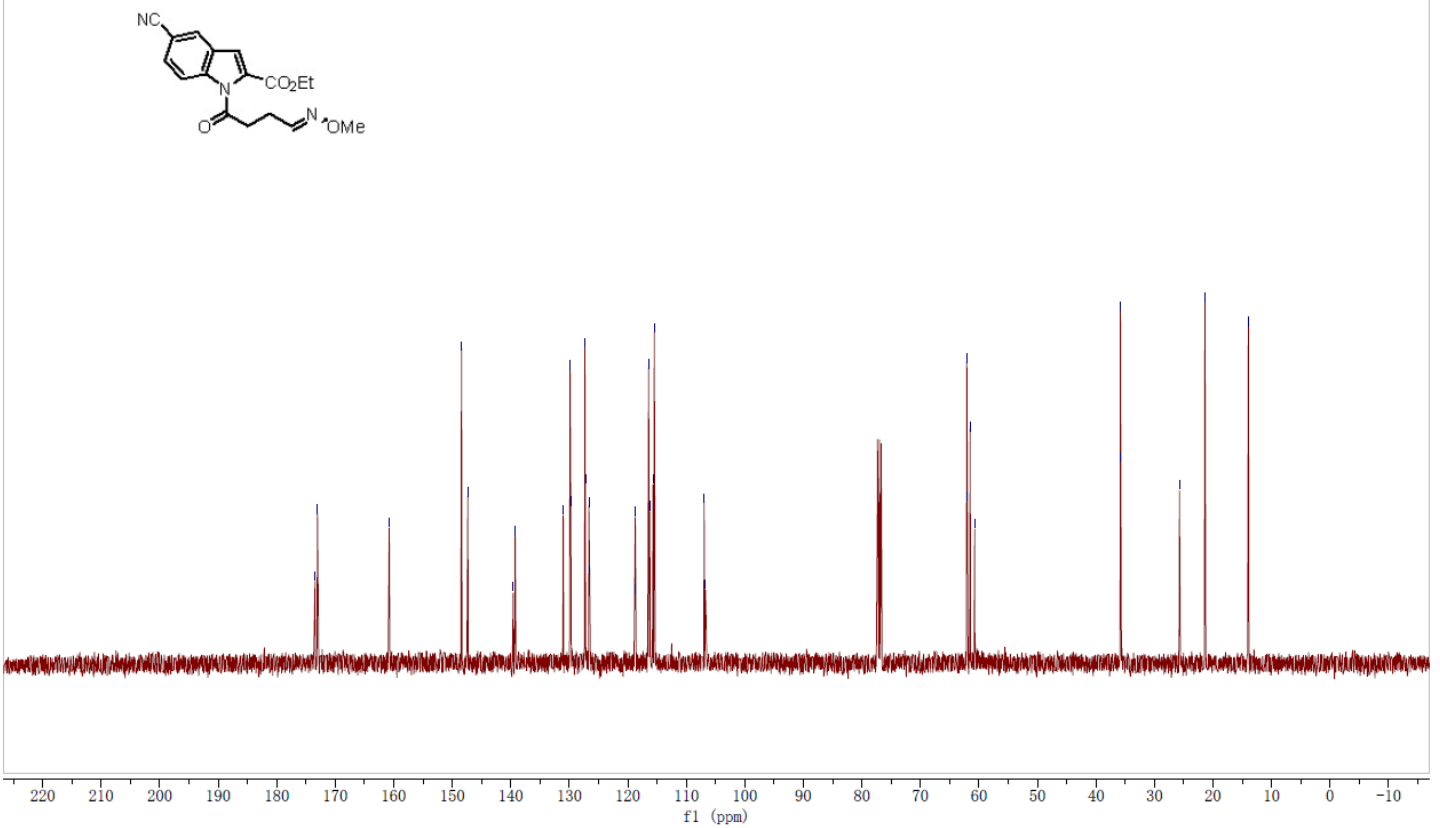
${ }^{1} \mathrm{H}$ NMR Spectrum of $\mathbf{1 r}$

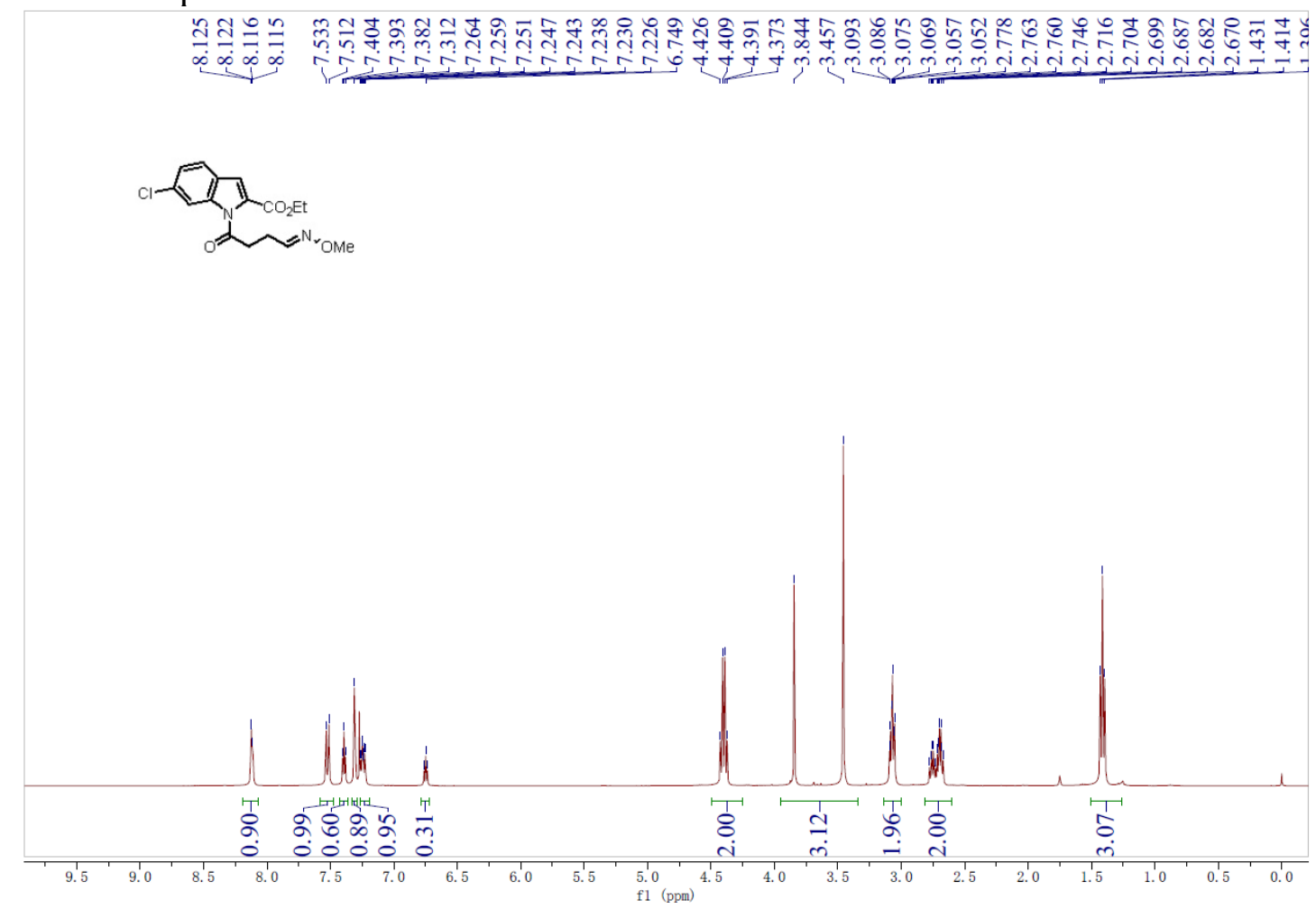

${ }^{13} \mathrm{C}$ NMR Spectrum of $\mathbf{1 r}$

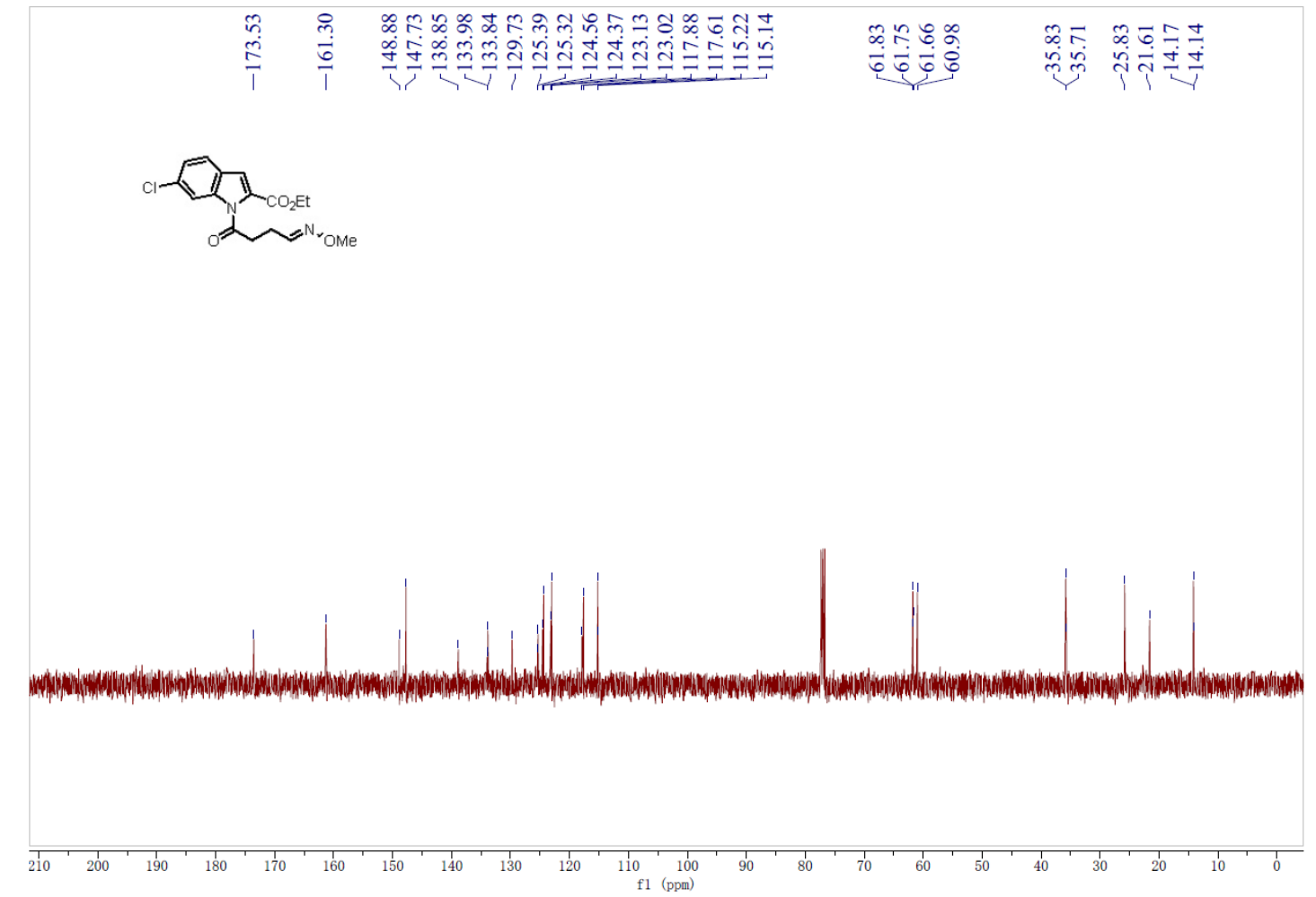




\section{${ }^{1} \mathrm{H}$ NMR Spectrum of $1 \mathrm{~s}$}

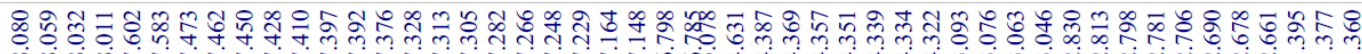 \\ o i}
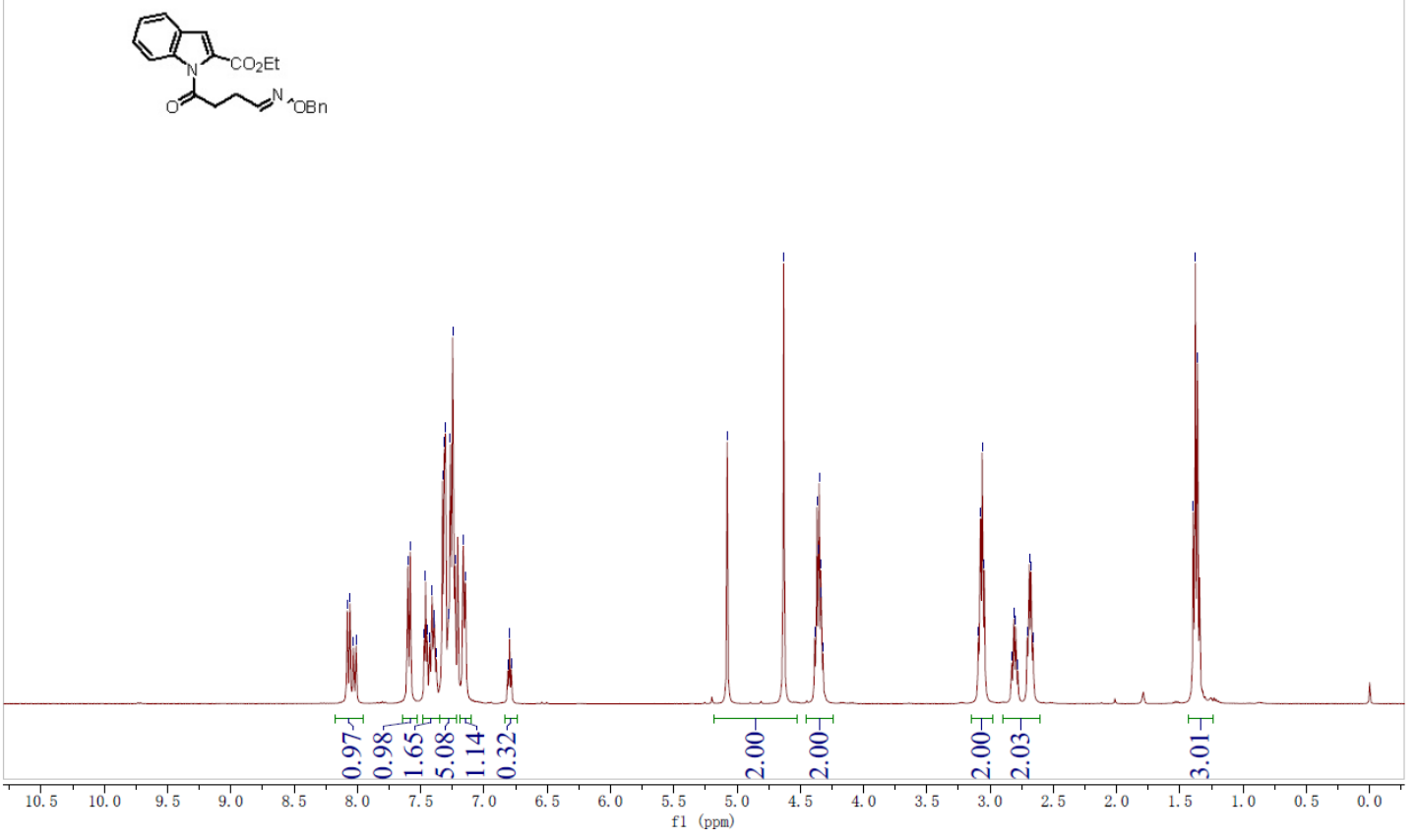

${ }^{13} \mathrm{C}$ NMR Spectrum of $1 \mathrm{~s}$

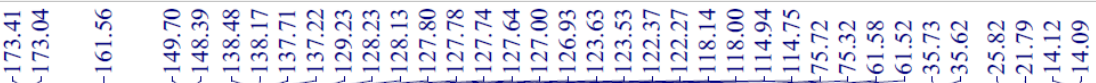
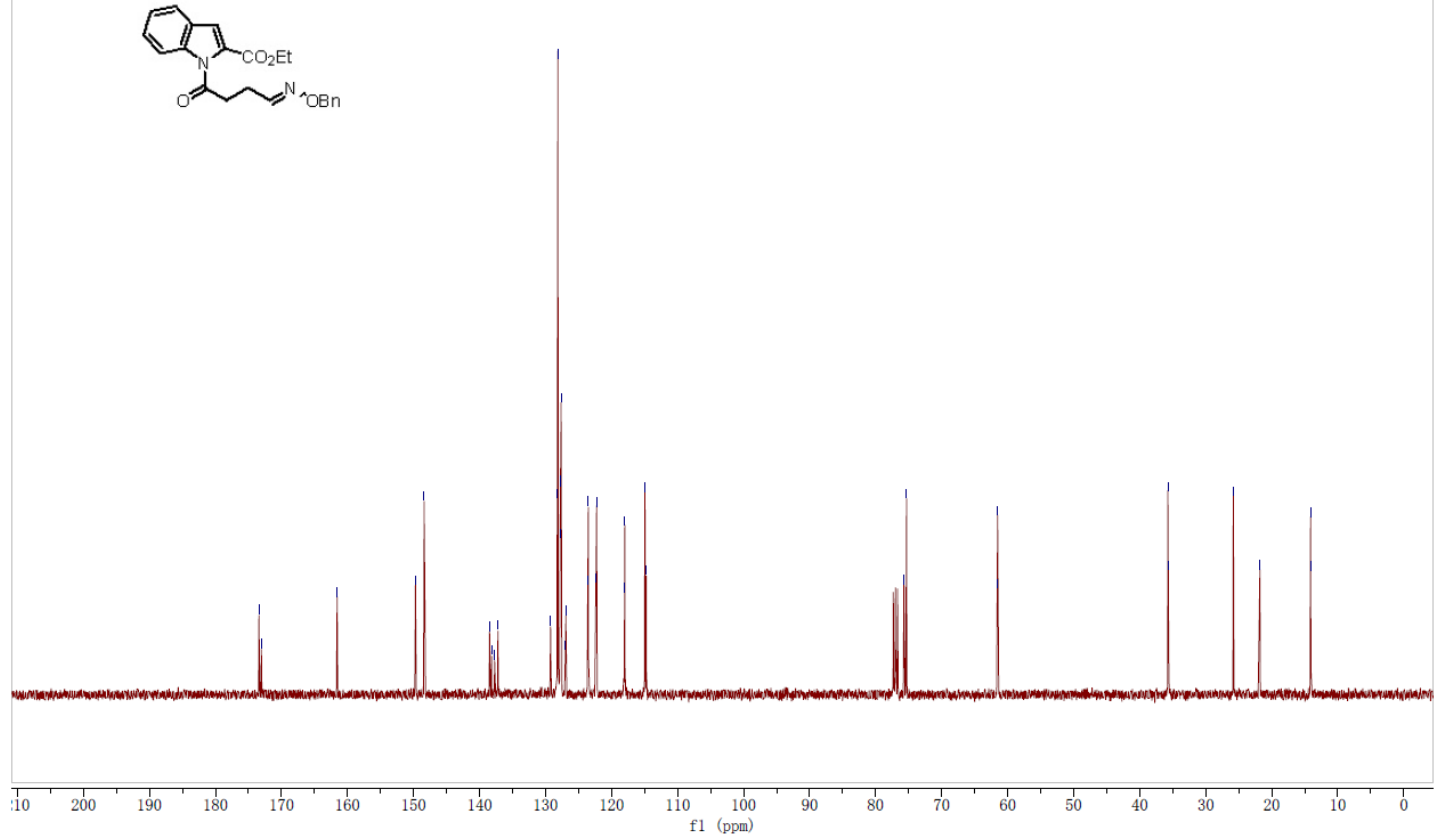
${ }^{1} \mathrm{H}$ NMR Spectrum of $\mathbf{1 t}$

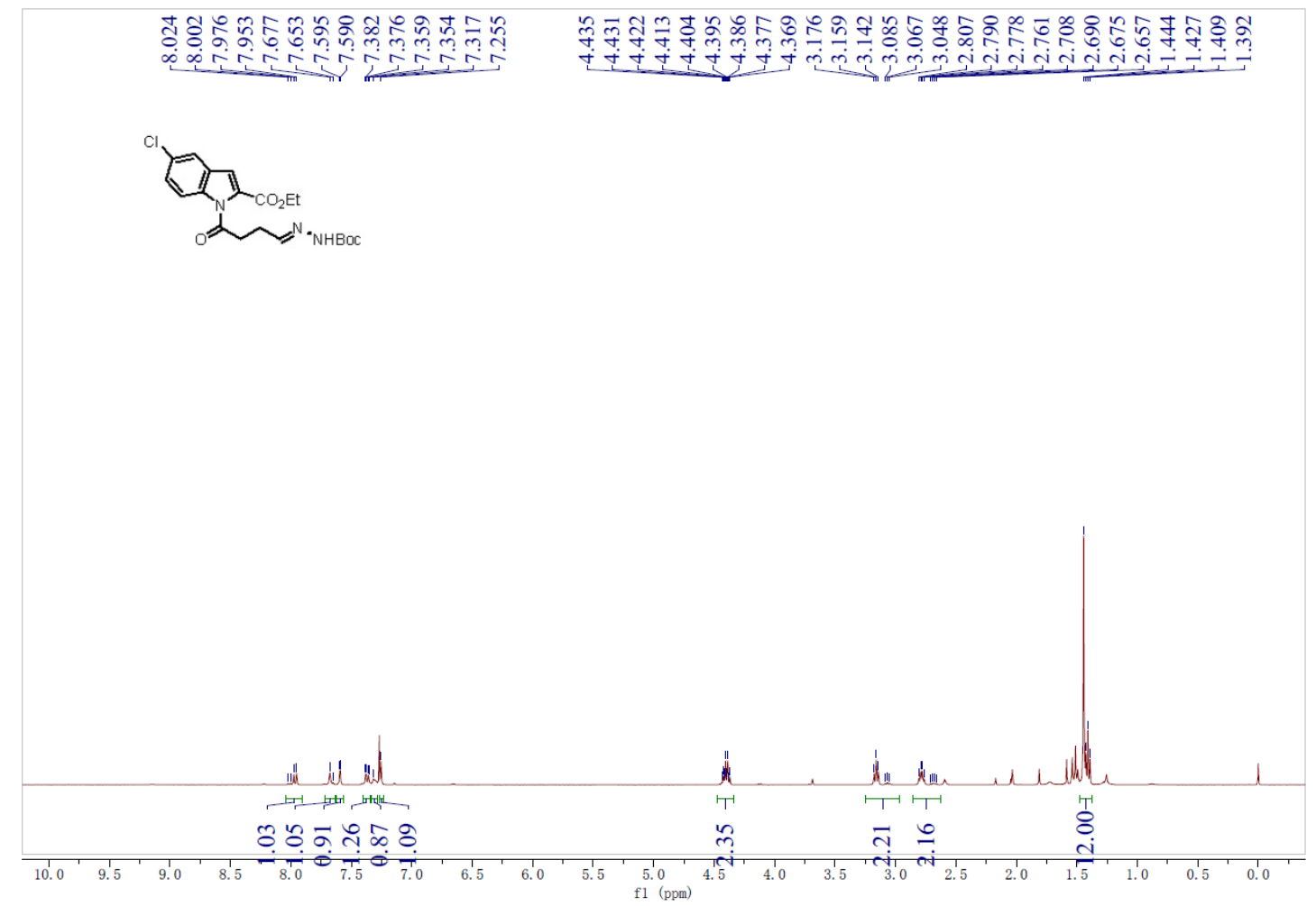

\section{${ }^{13} \mathrm{C}$ NMR Spectrum of $\mathbf{1 t}$}

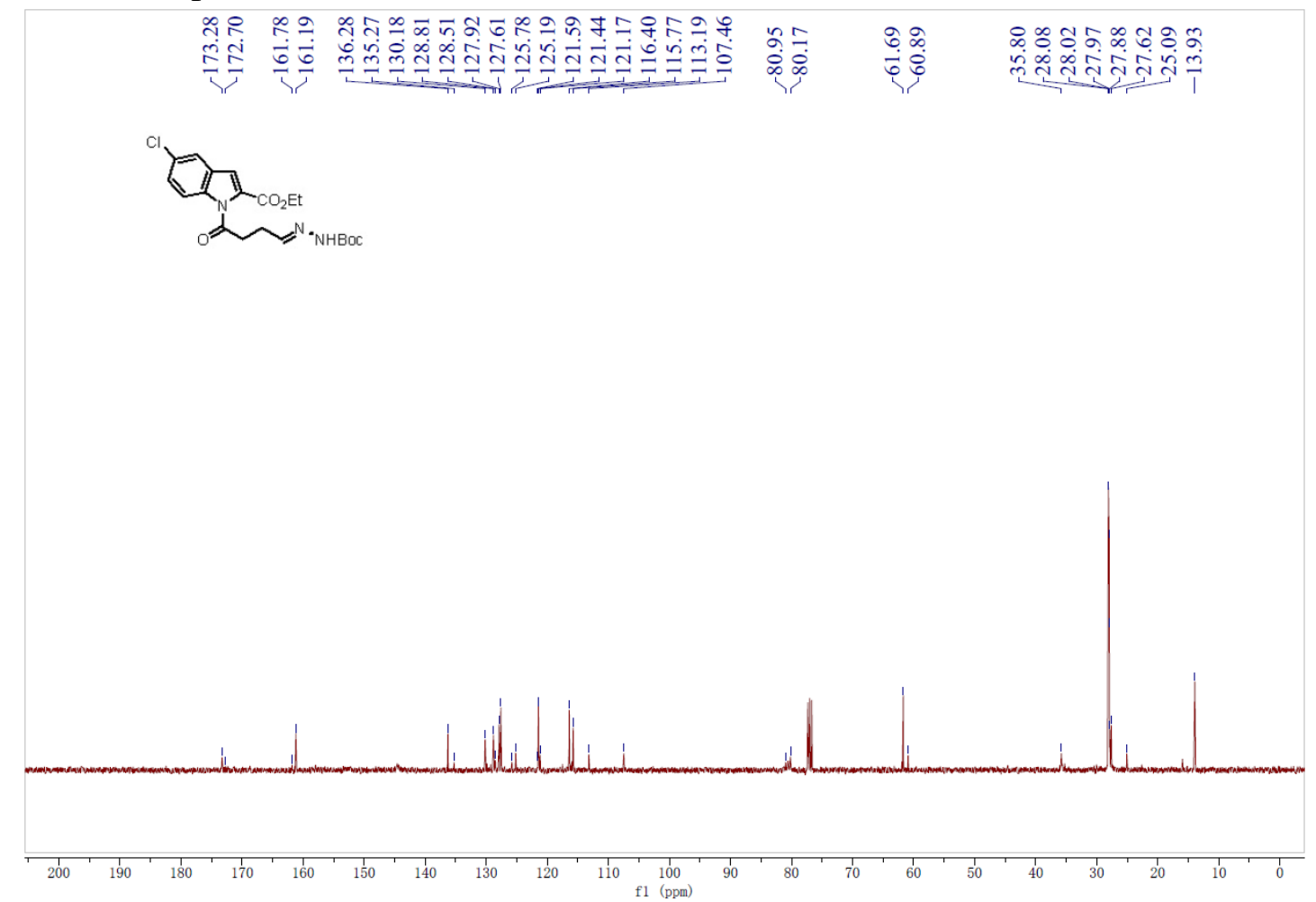


${ }^{1} \mathrm{H}$ NMR Spectrum of $\mathbf{3 a}$

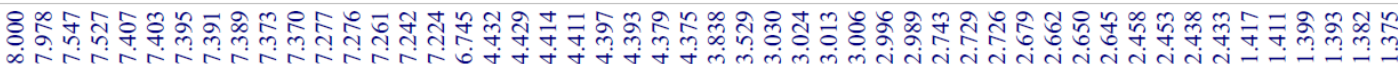
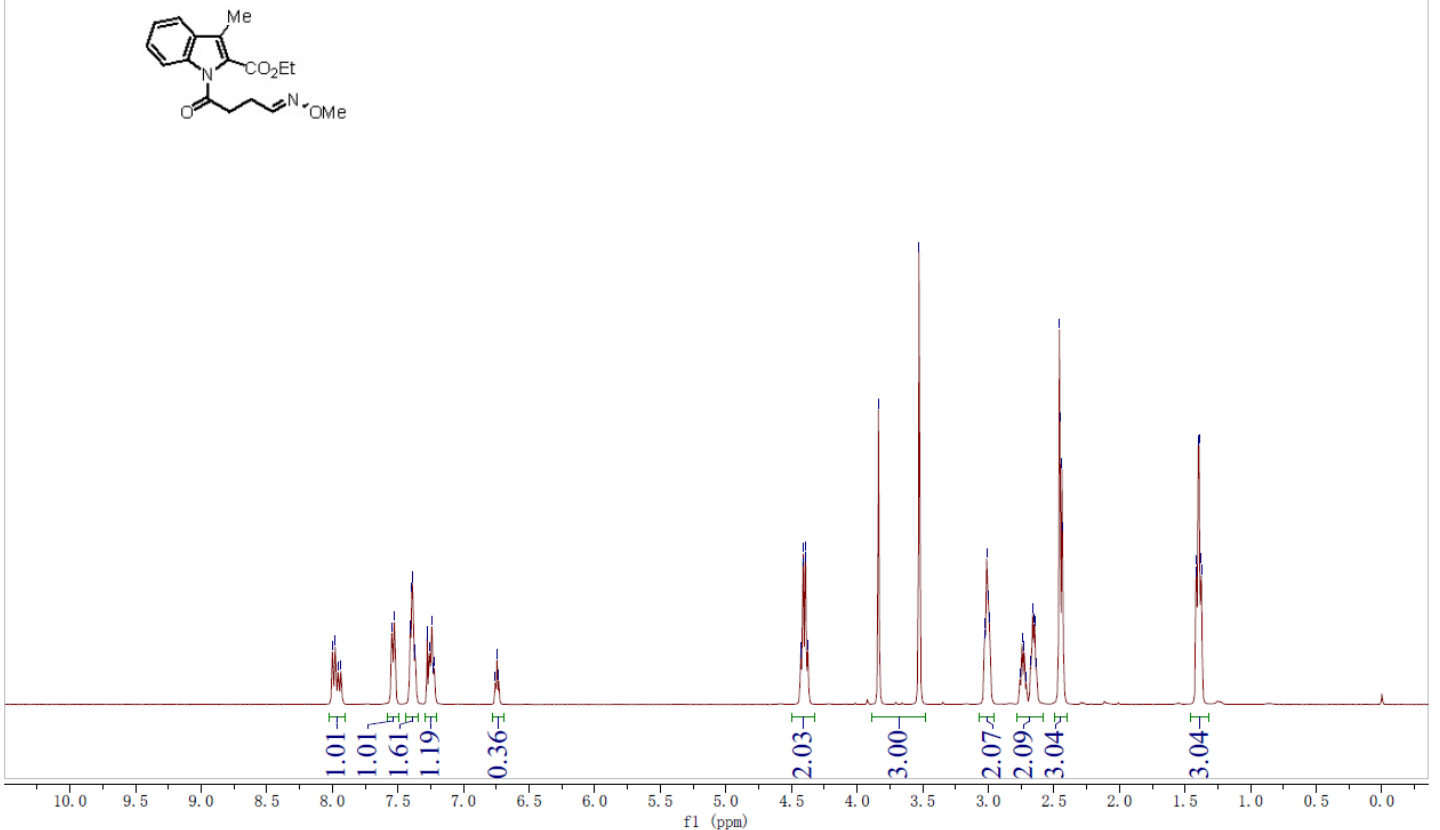

${ }^{13} \mathrm{C}$ NMR Spectrum of 3a

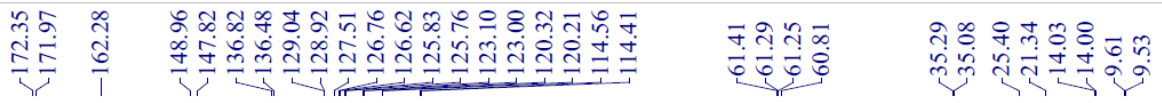

Dif

$\overbrace{}^{2} \mathrm{~N}_{\text {. OM }}$

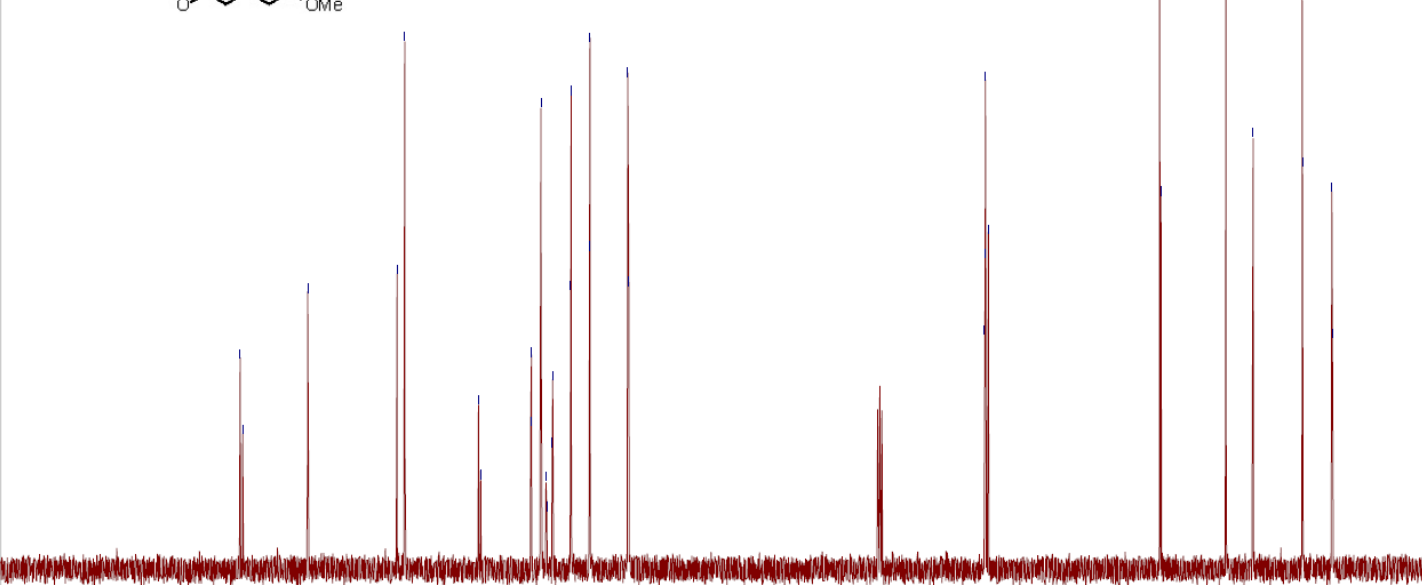

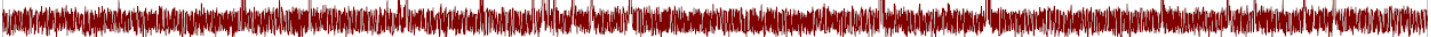

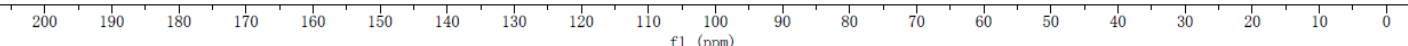


${ }^{1} \mathrm{H}$ NMR Spectrum of $\mathbf{3 b}$

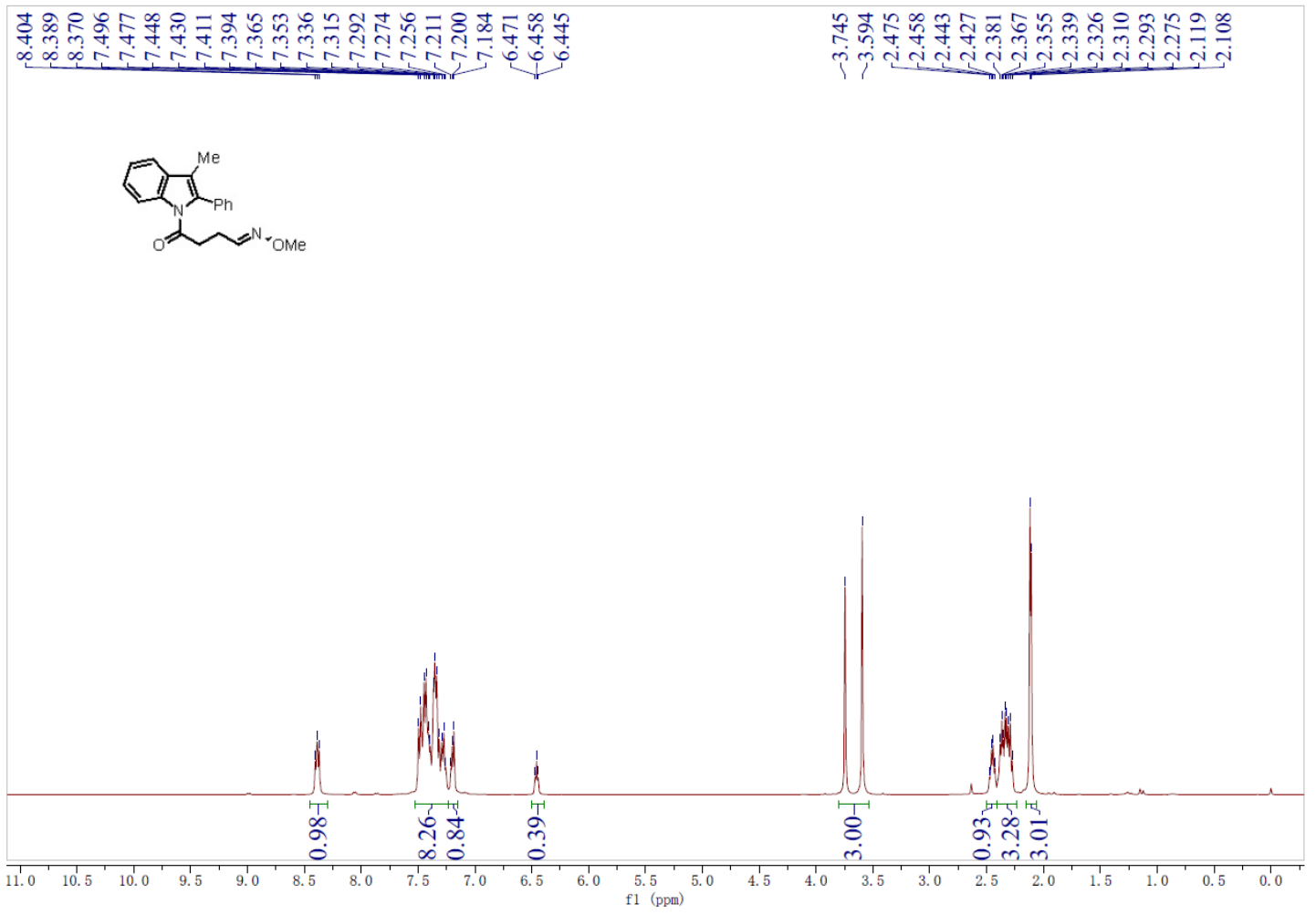

${ }^{13} \mathrm{C}$ NMR Spectrum of $\mathbf{3 b}$

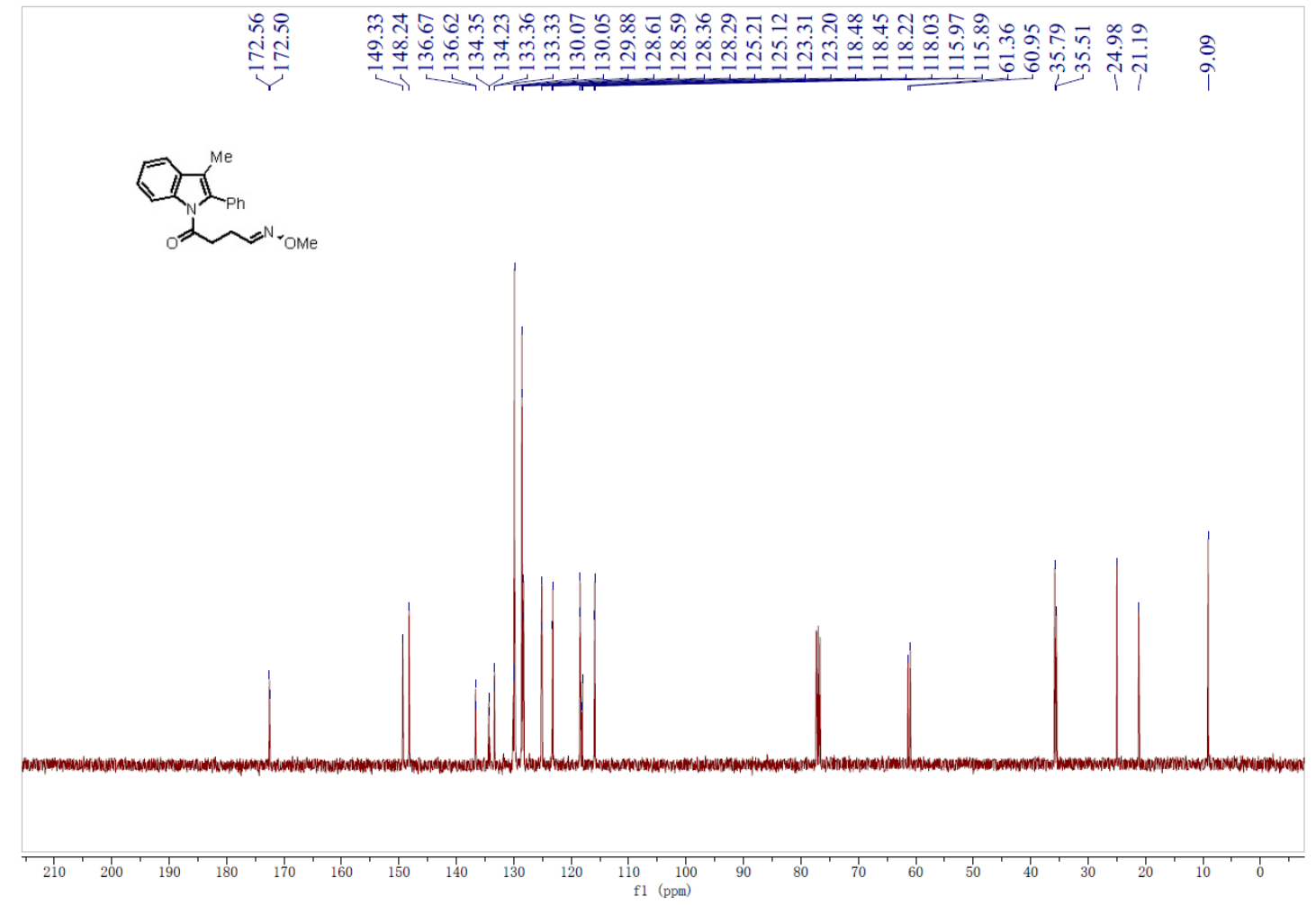


${ }^{1} \mathrm{H}$ NMR Spectrum of $\mathbf{3 c}$

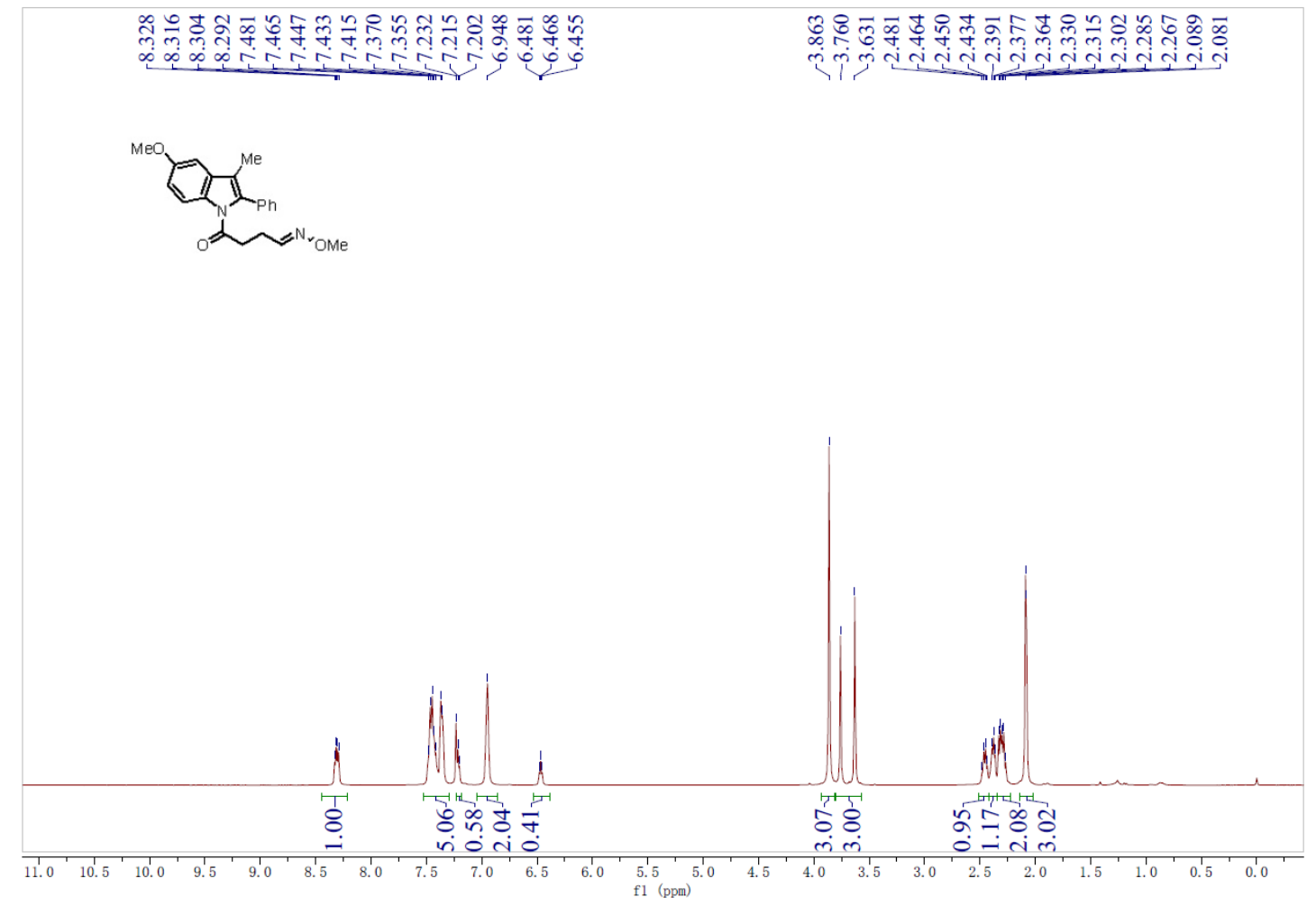

${ }^{13} \mathrm{C}$ NMR Spectrum of $\mathbf{3 c}$

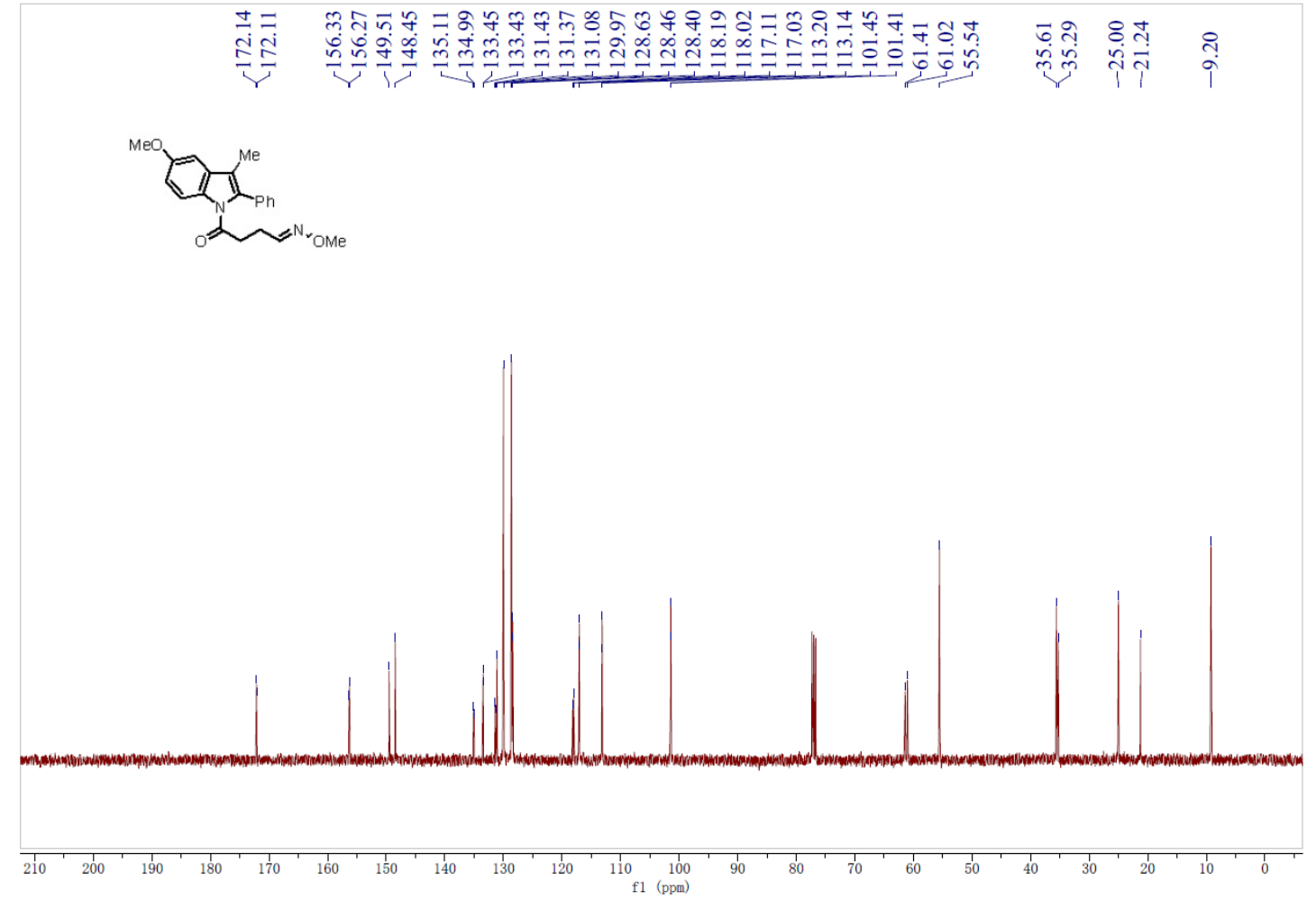


${ }^{1} \mathrm{H}$ NMR Spectrum of $\mathbf{3 d}$

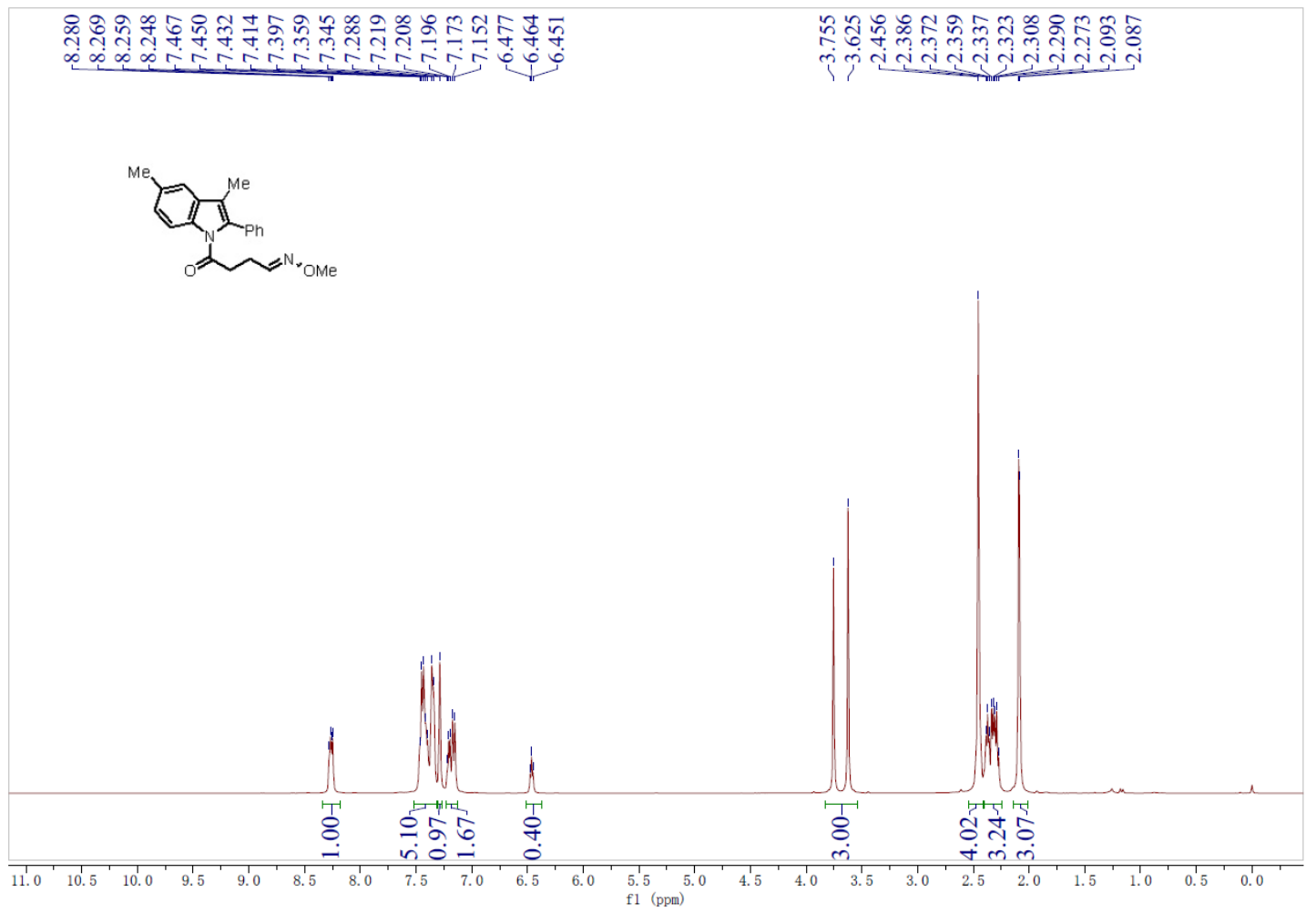

${ }^{13} \mathrm{C}$ NMR Spectrum of $\mathbf{3 d}$

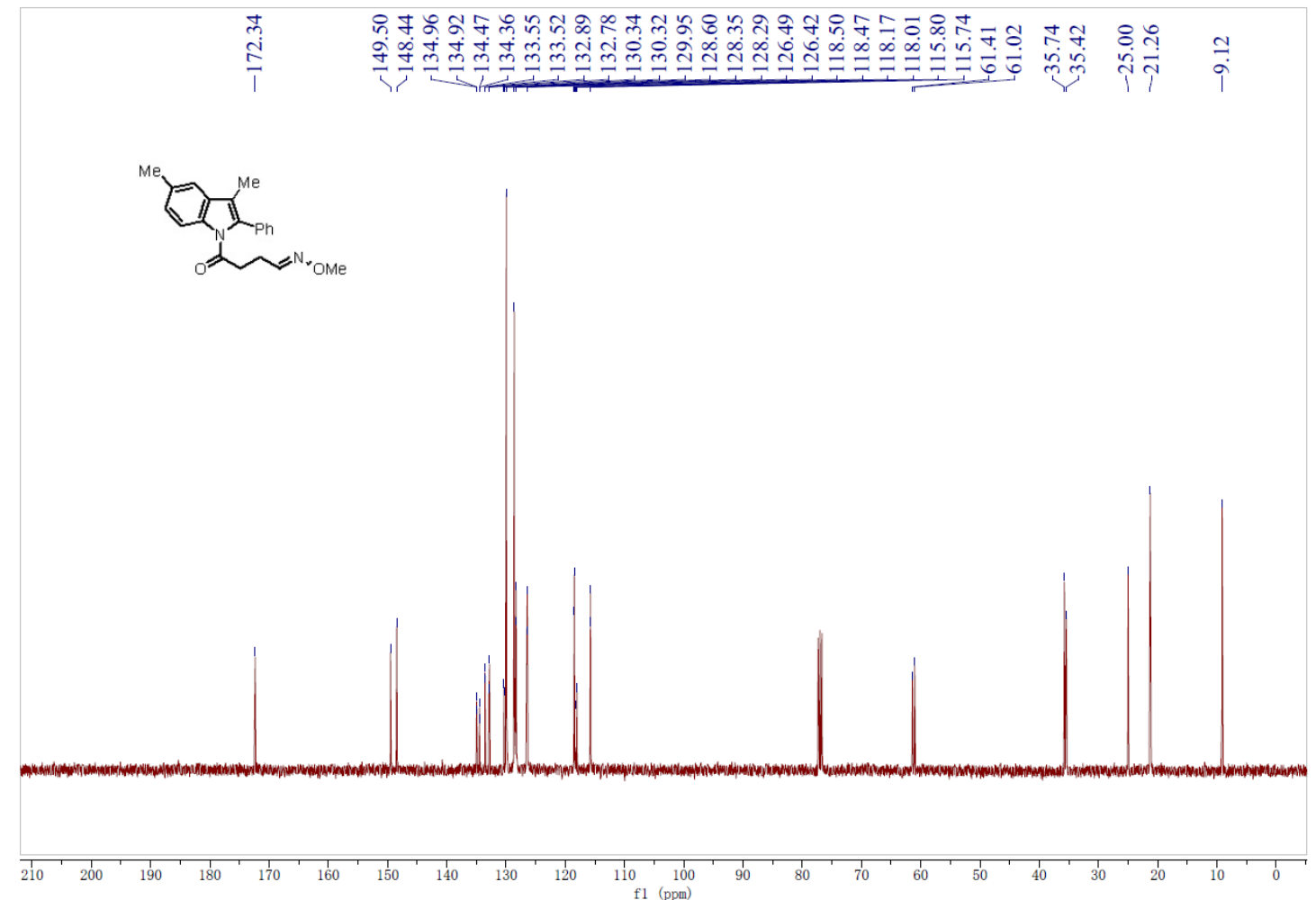




\section{${ }^{1} \mathrm{H}$ NMR Spectrum of $\mathbf{3 e}$}

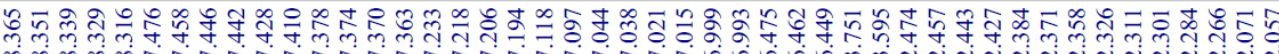
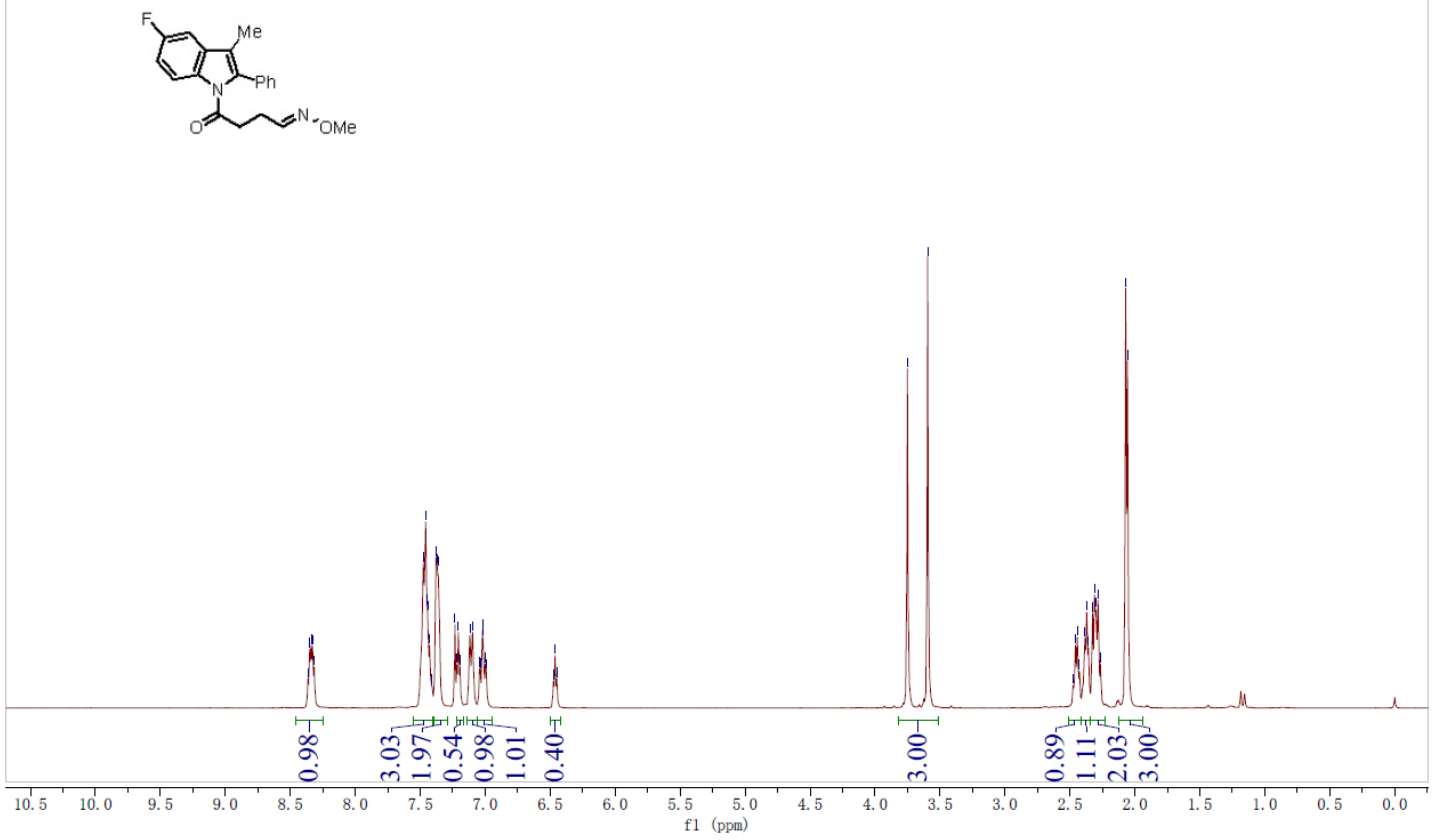

${ }^{13} \mathrm{C}$ NMR Spectrum of $\mathbf{3 e}$

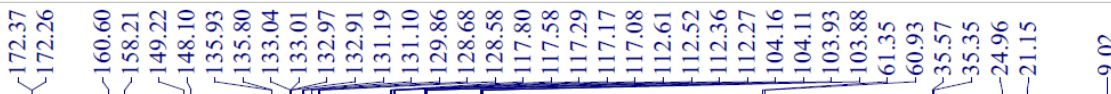
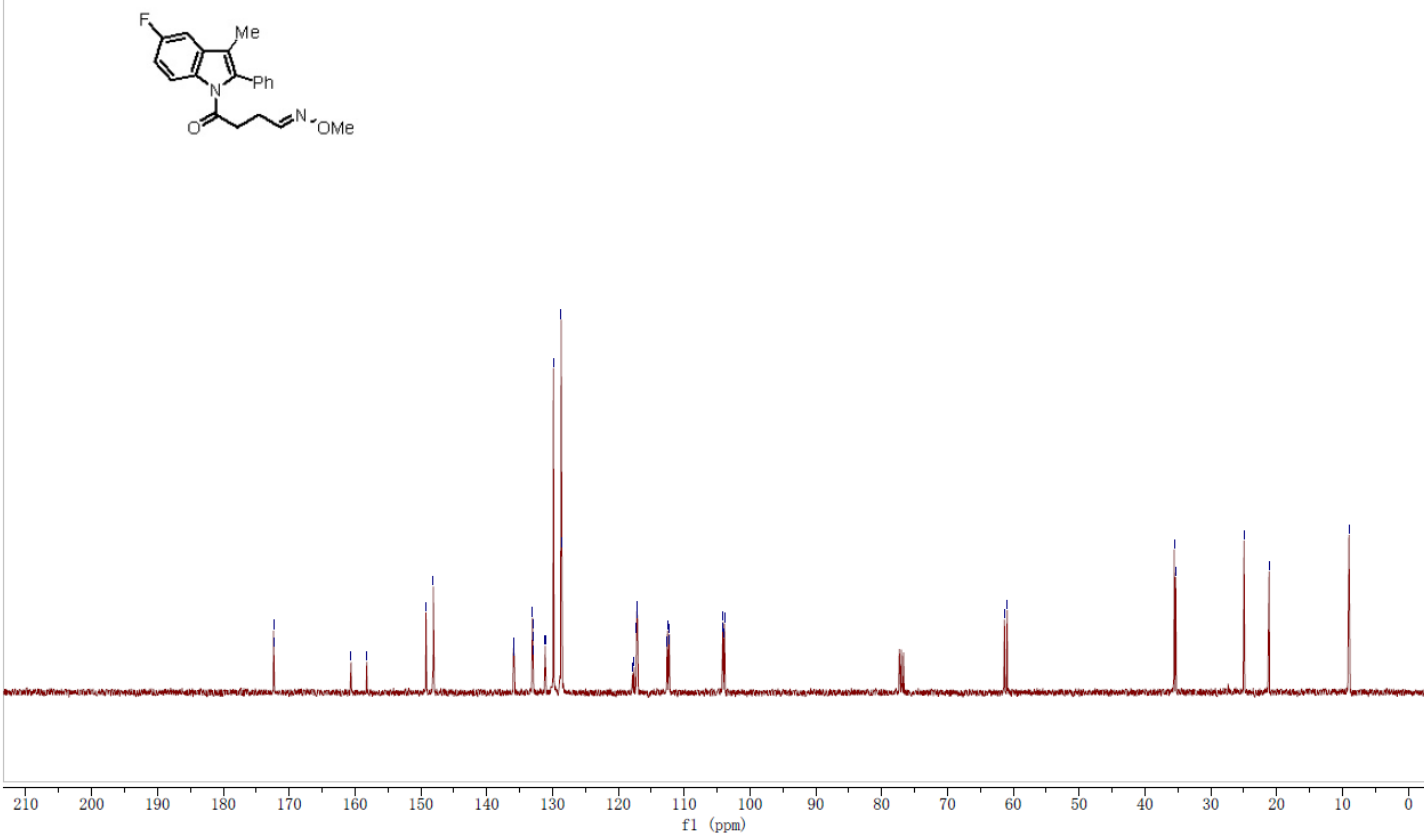
${ }^{19} \mathrm{~F}$ NMR Spectrum of $\mathbf{3 e}$

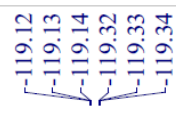

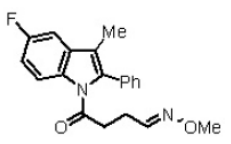

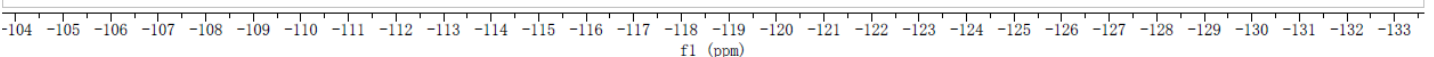

S99 
${ }^{1} \mathrm{H}$ NMR Spectrum of $\mathbf{3 f}$

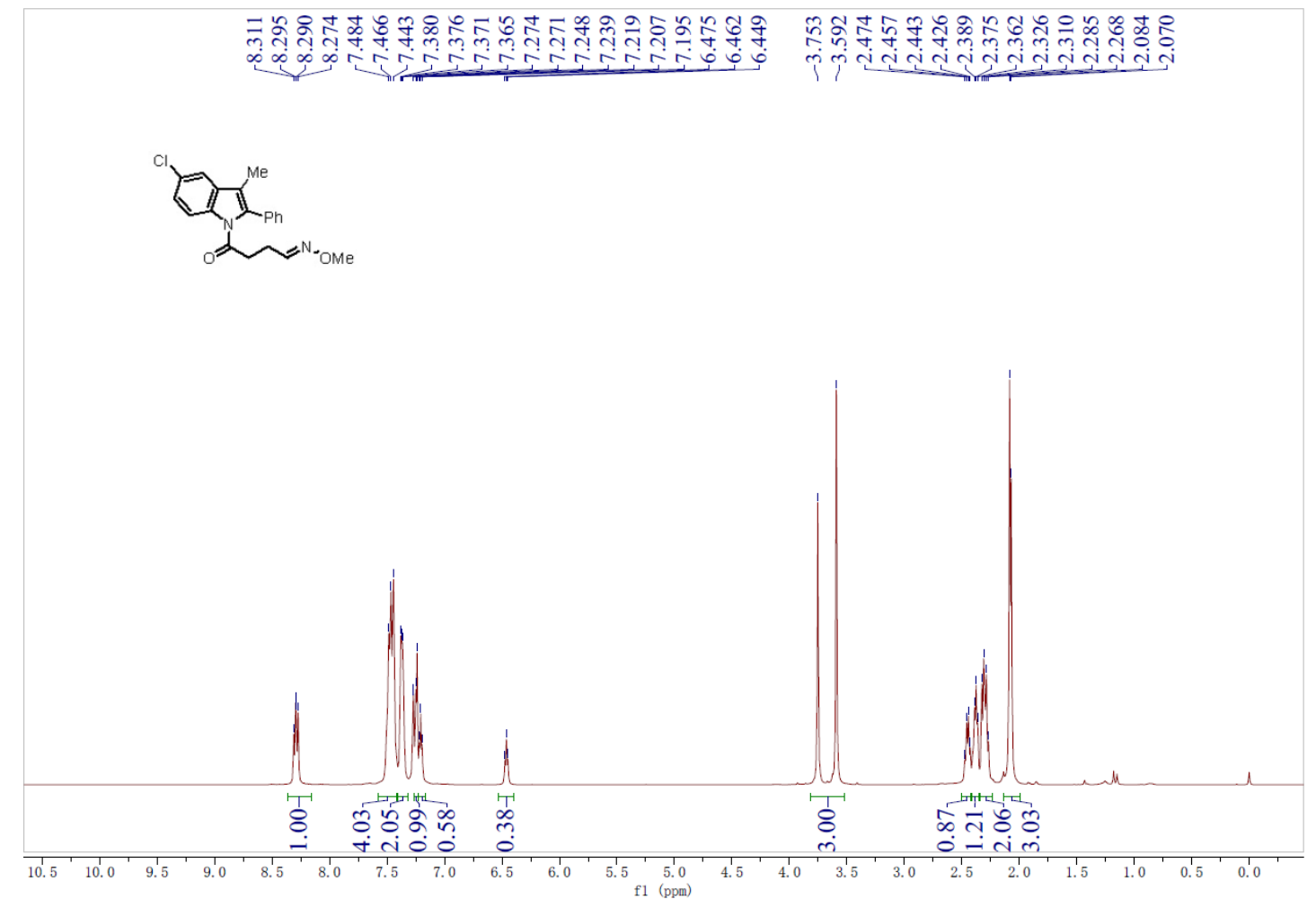

${ }^{13} \mathrm{C}$ NMR Spectrum of $\mathbf{3 f}$

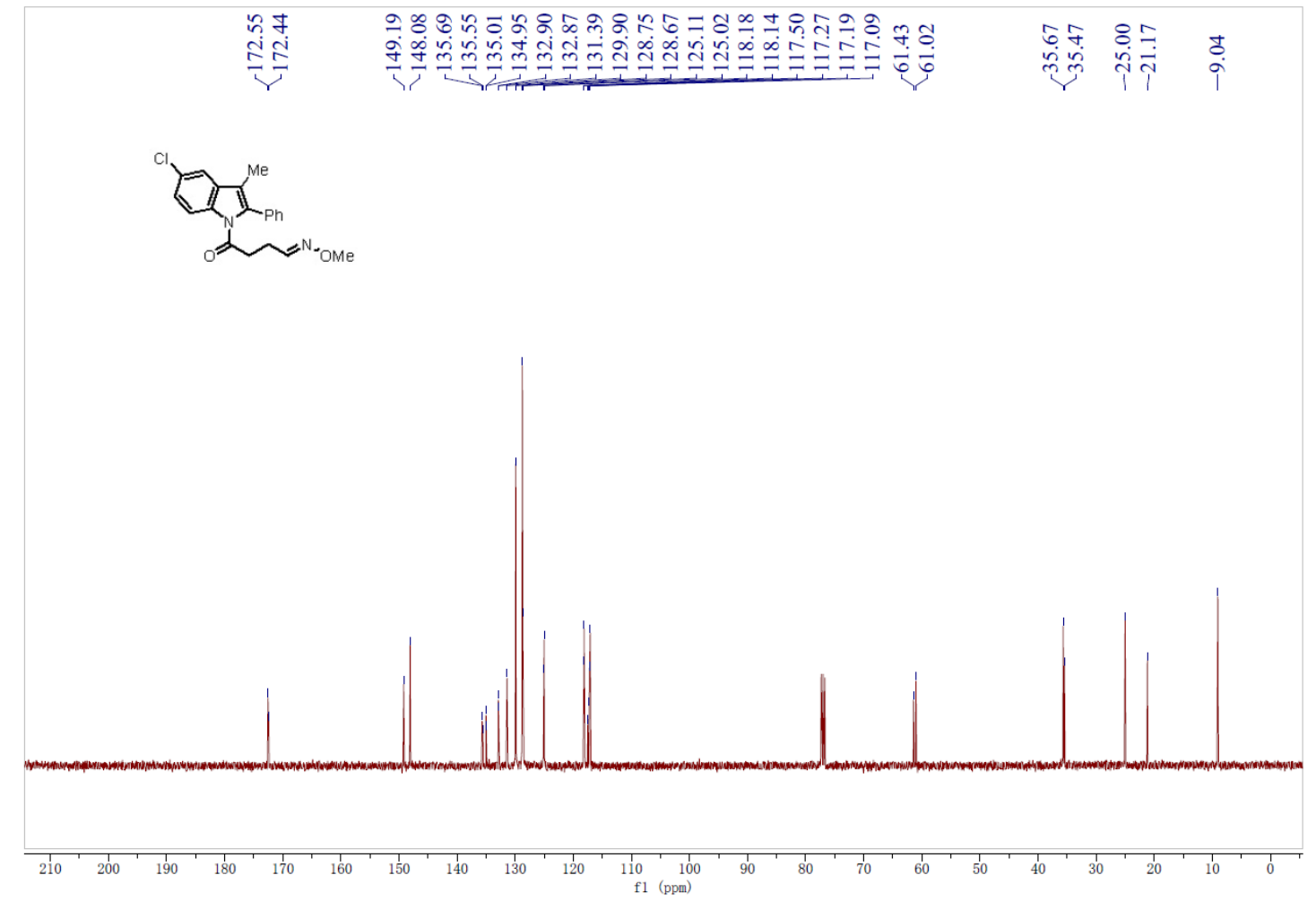


${ }^{1} \mathrm{H}$ NMR Spectrum of $\mathbf{3 g}$

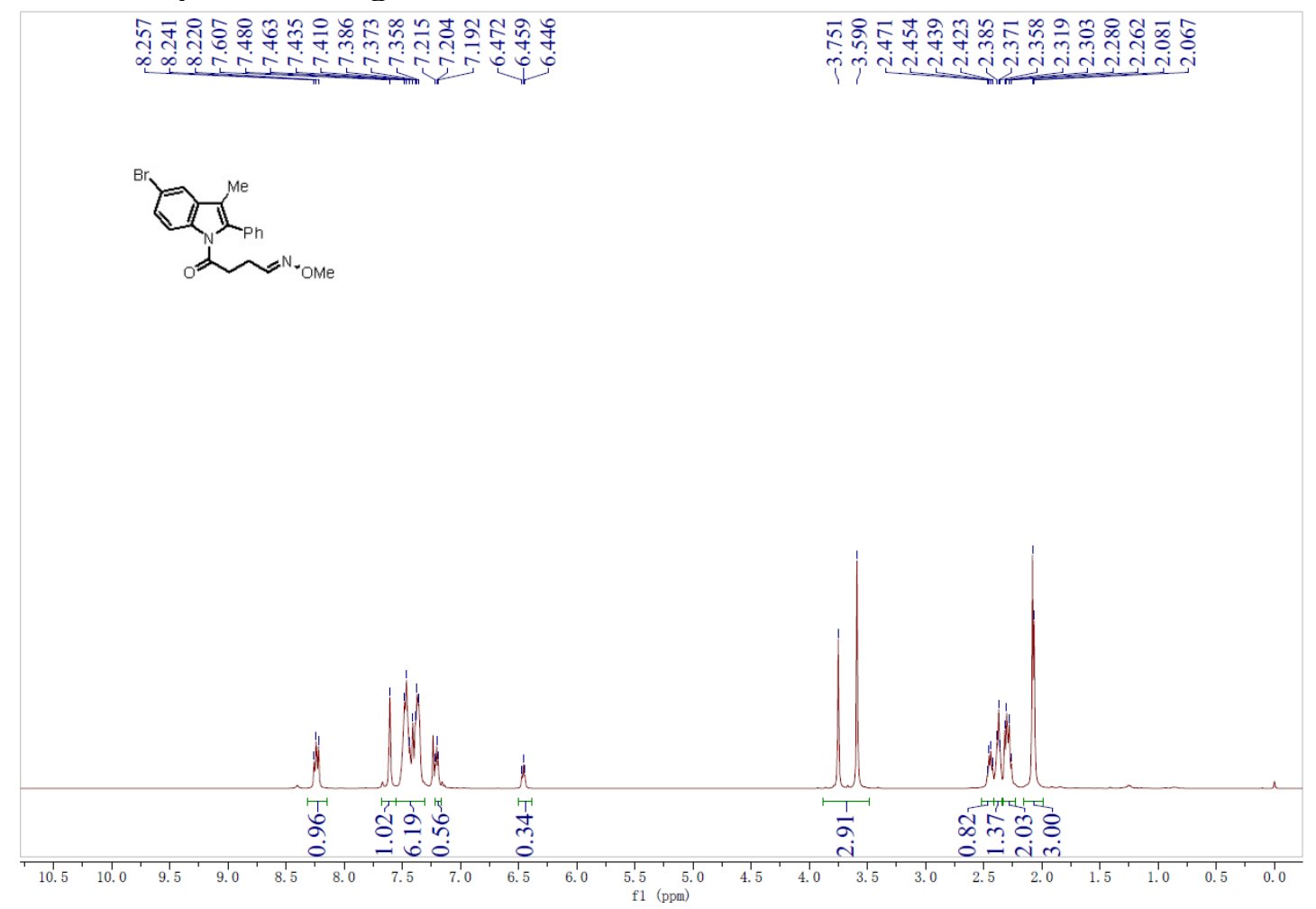

${ }^{13} \mathrm{C}$ NMR Spectrum of $\mathbf{3 g}$

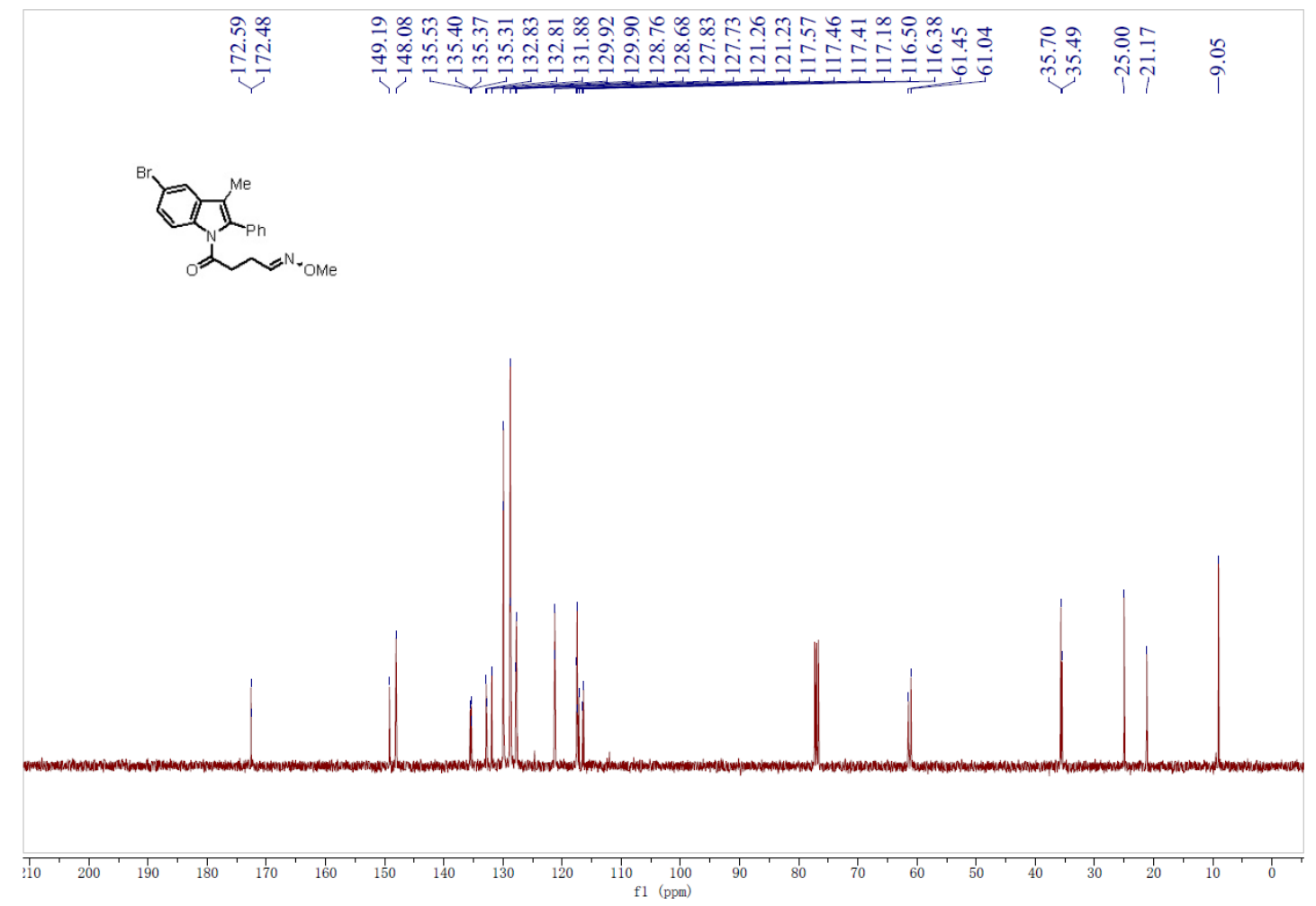


${ }^{1} \mathrm{H}$ NMR Spectrum of $\mathbf{3 h}$

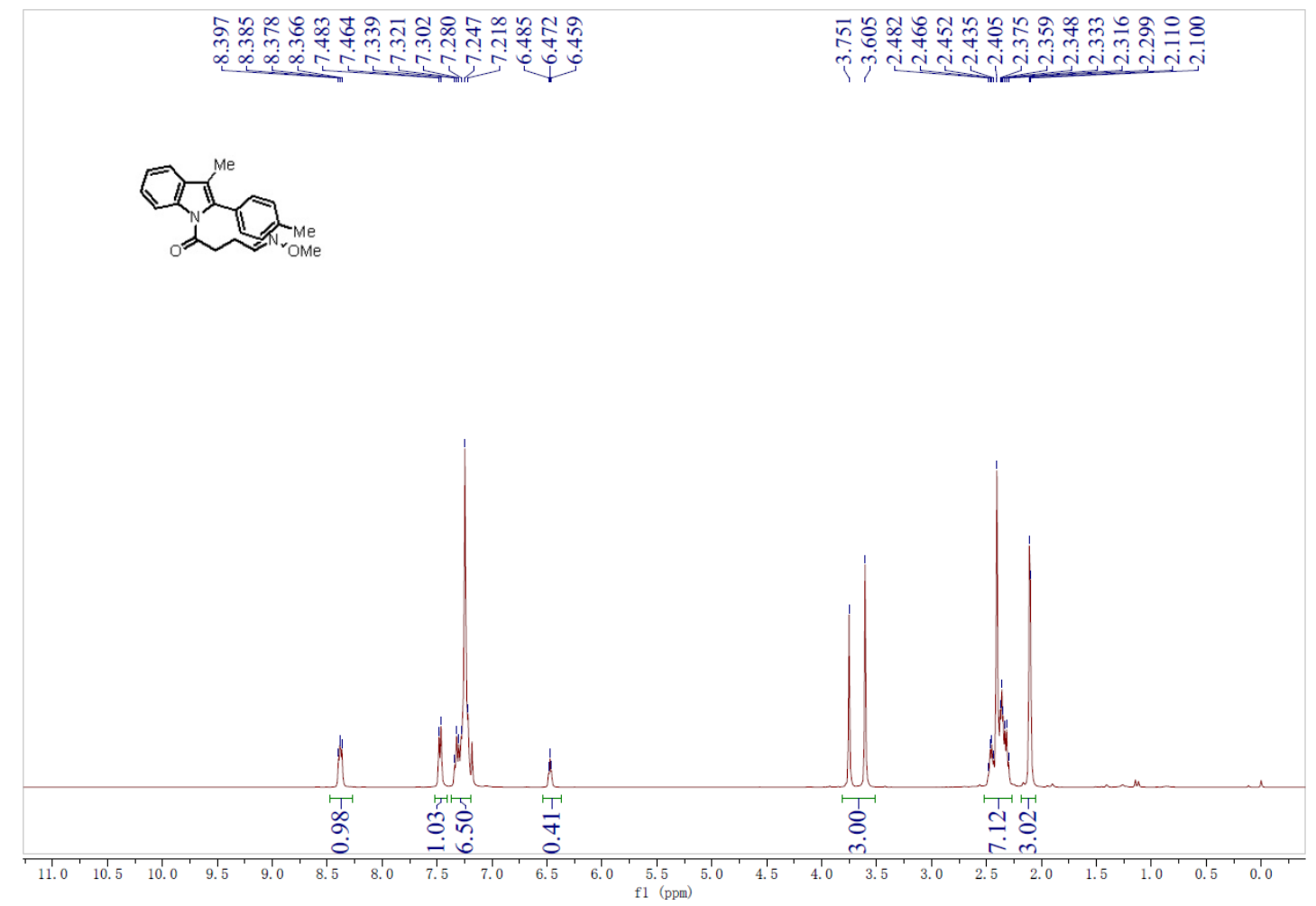

${ }^{13} \mathrm{C}$ NMR Spectrum of $\mathbf{3 h}$

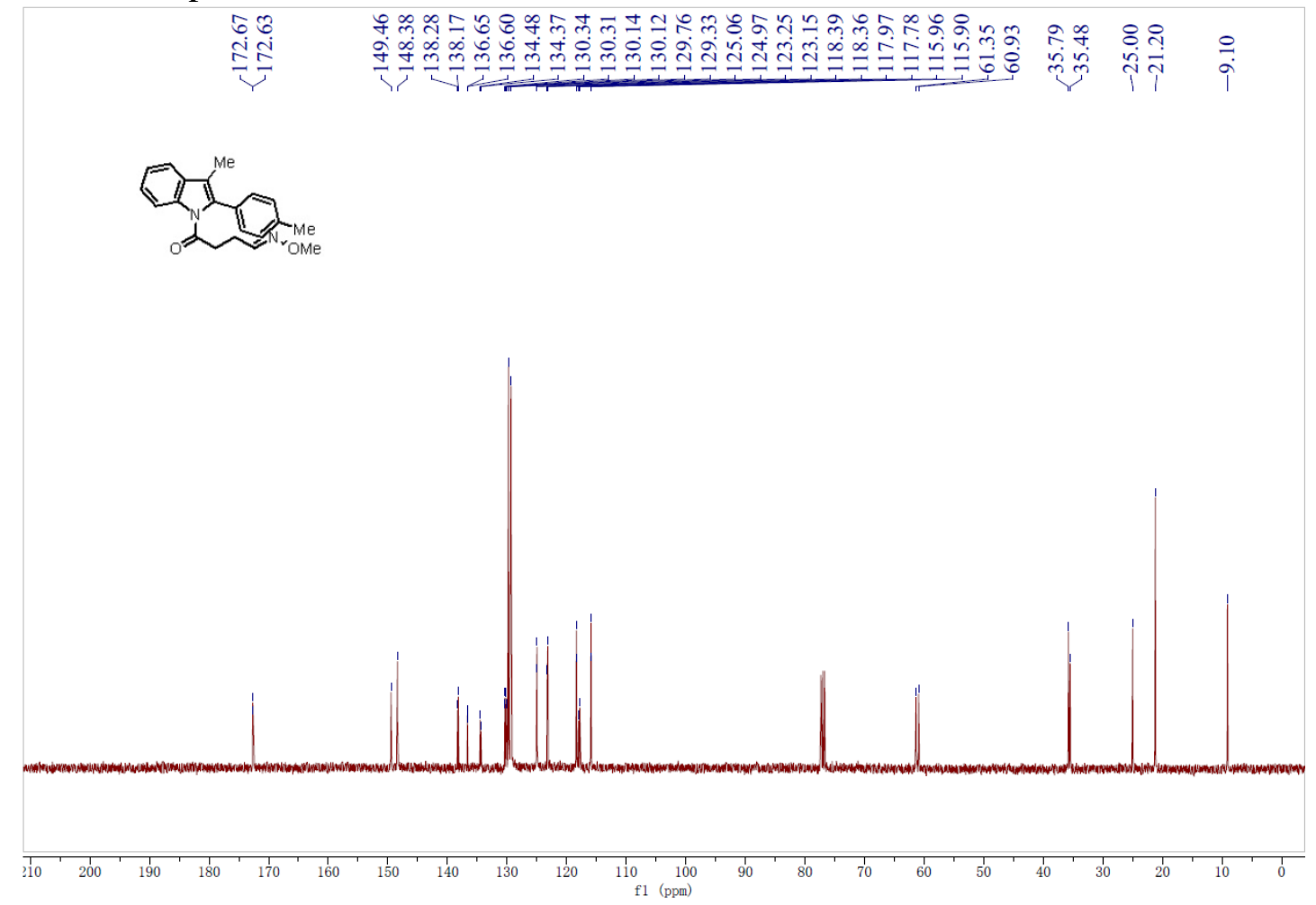


${ }^{1} \mathrm{H}$ NMR Spectrum of $\mathbf{3 i}$

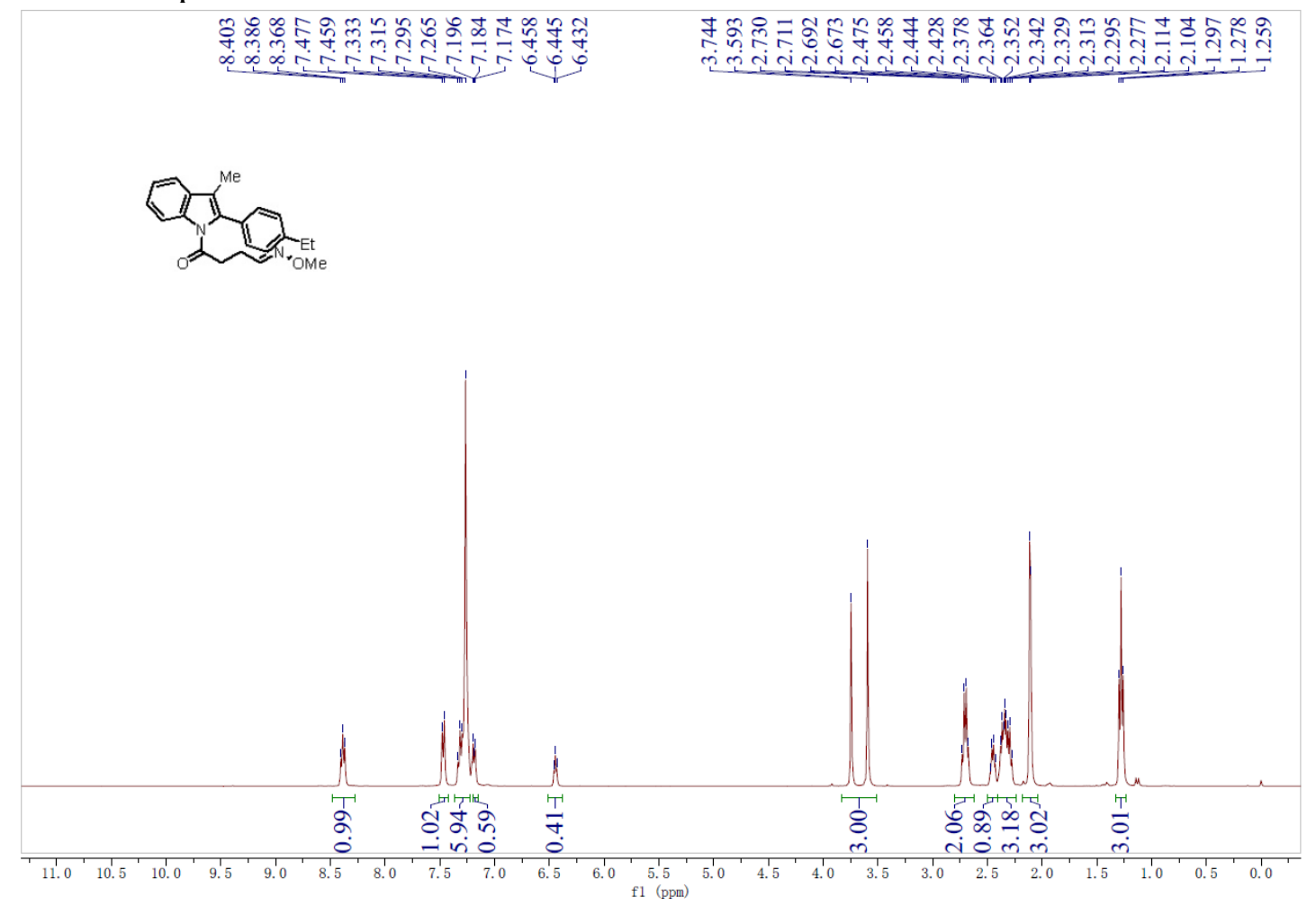

${ }^{13} \mathrm{C}$ NMR Spectrum of $\mathbf{3 i}$

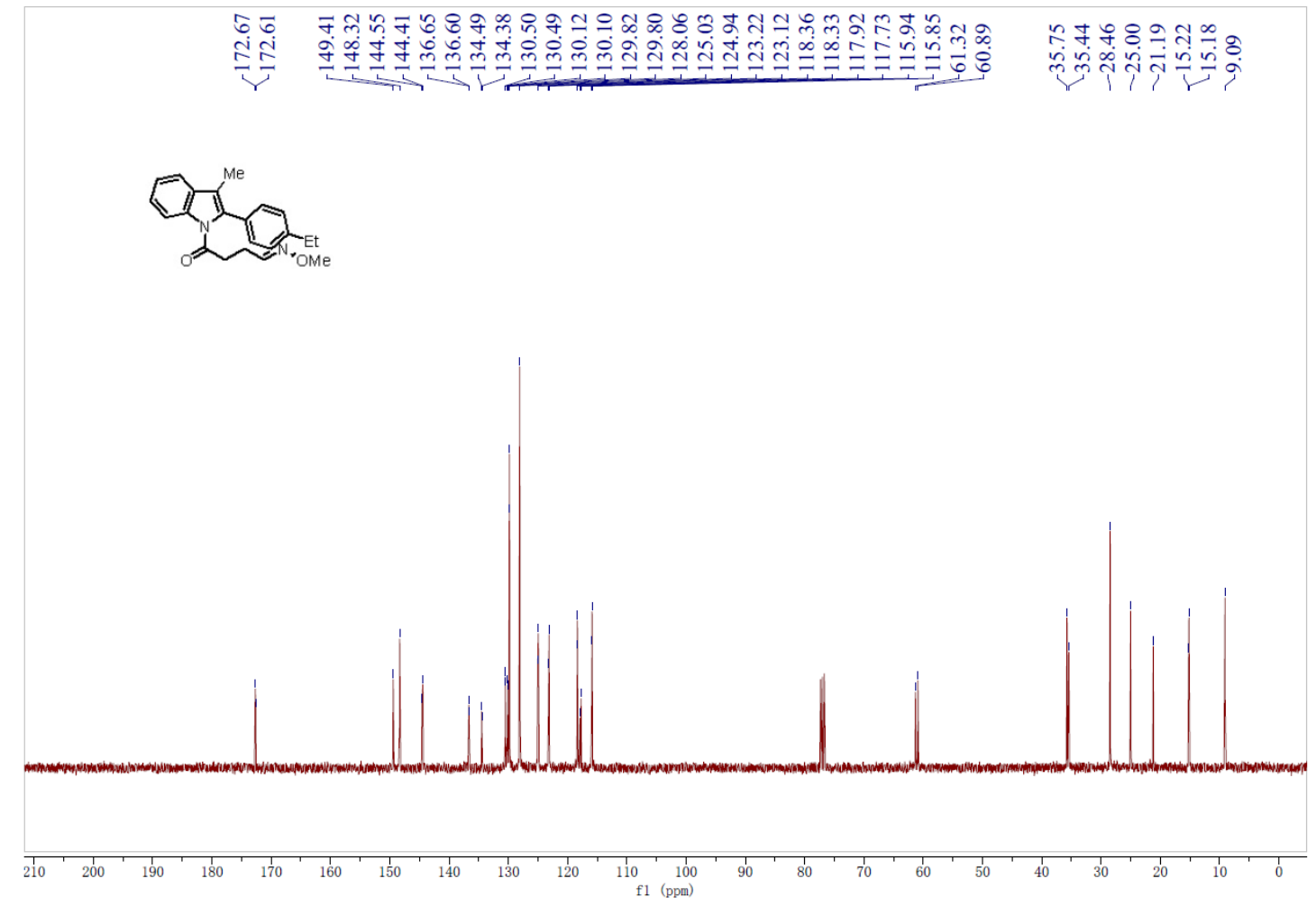


${ }^{1} \mathrm{H}$ NMR Spectrum of $\mathbf{3 j}$

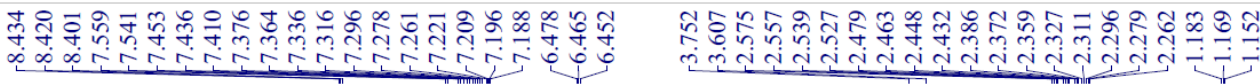
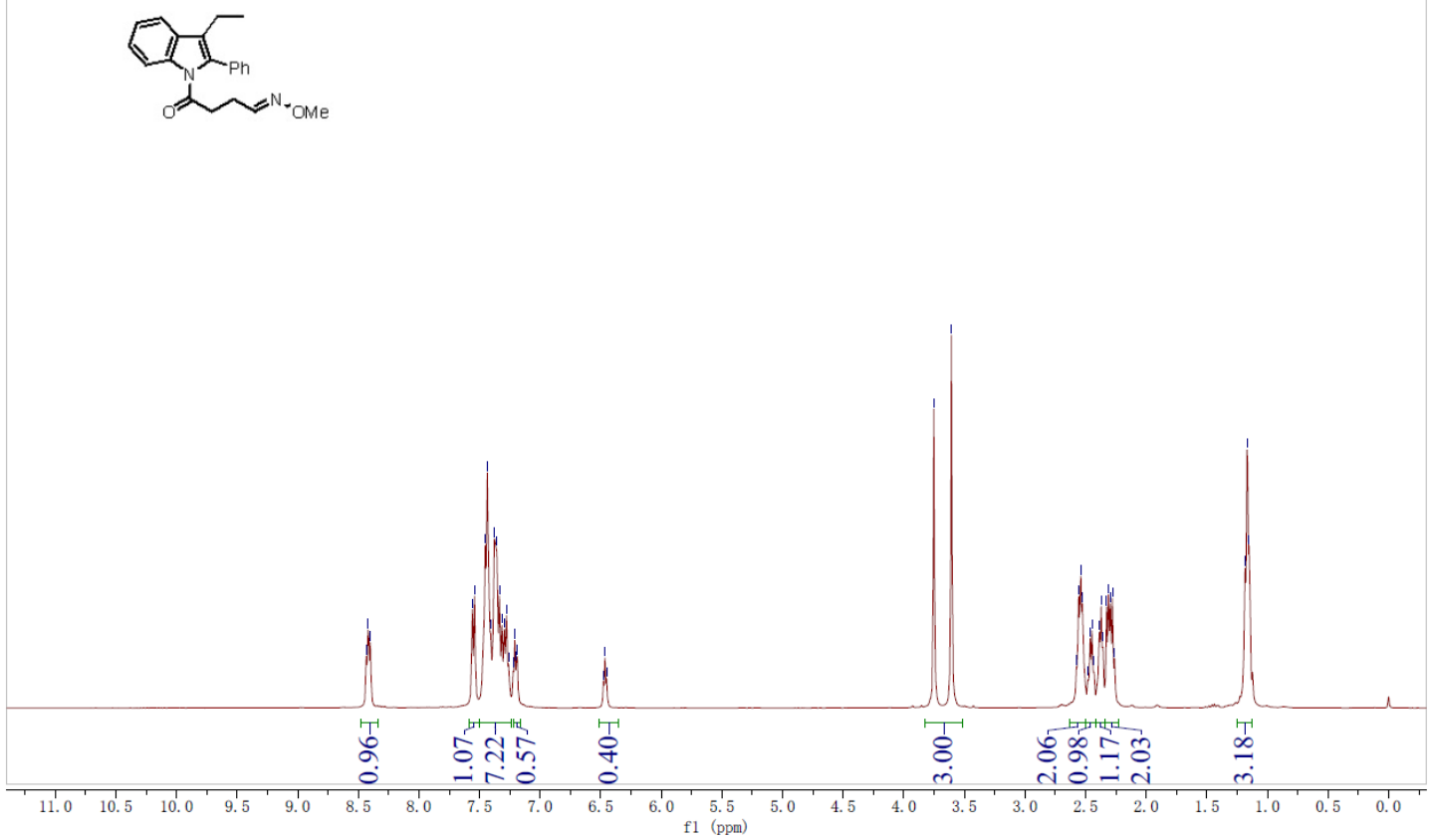

${ }^{13} \mathrm{C}$ NMR Spectrum of $\mathbf{3 j}$

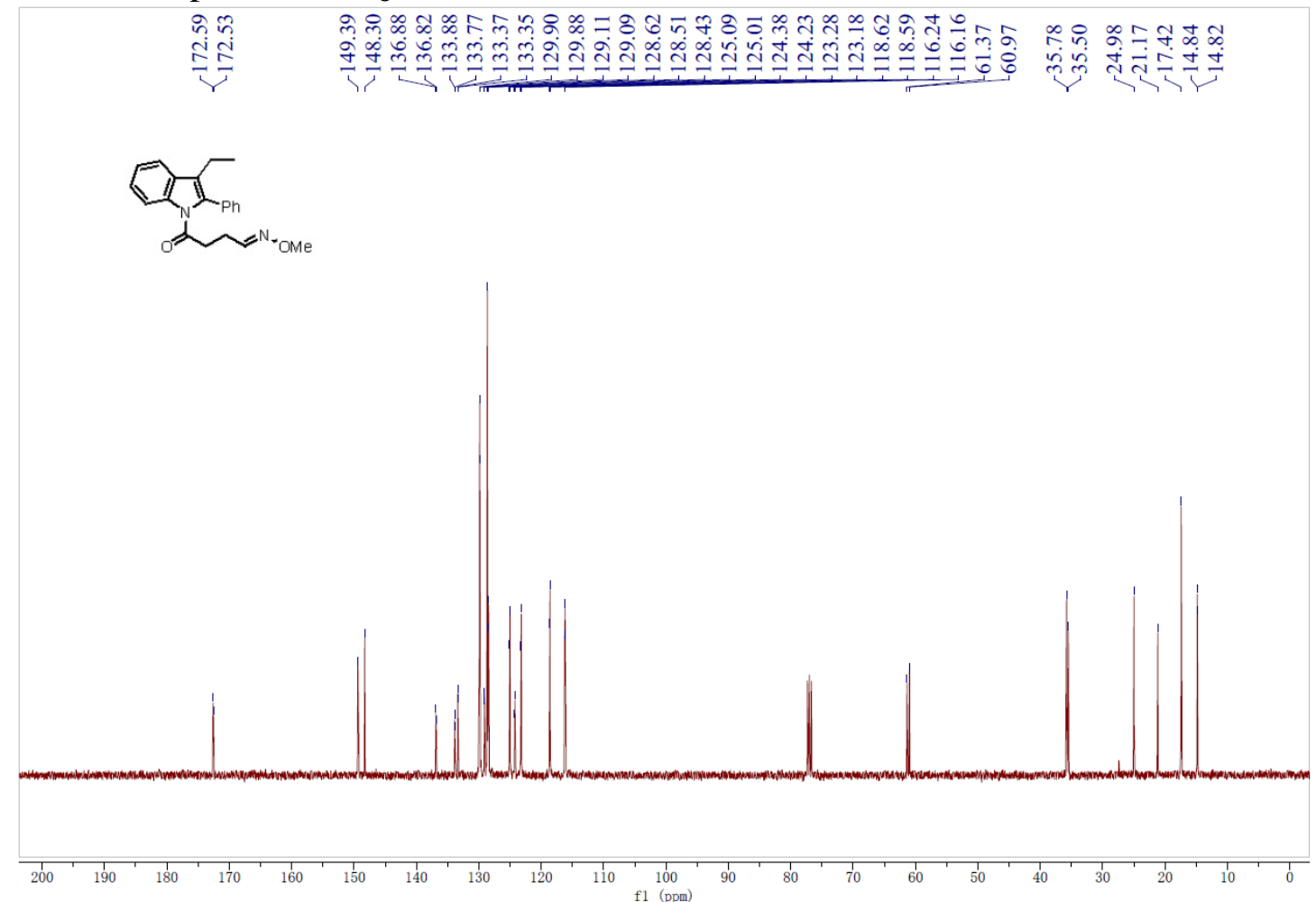


${ }^{1} \mathrm{H}$ NMR Spectrum of $\mathbf{3 k}$

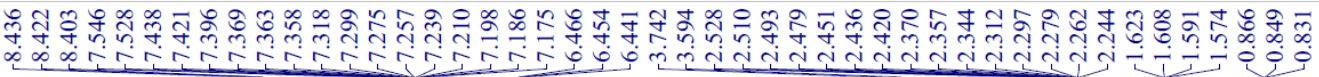
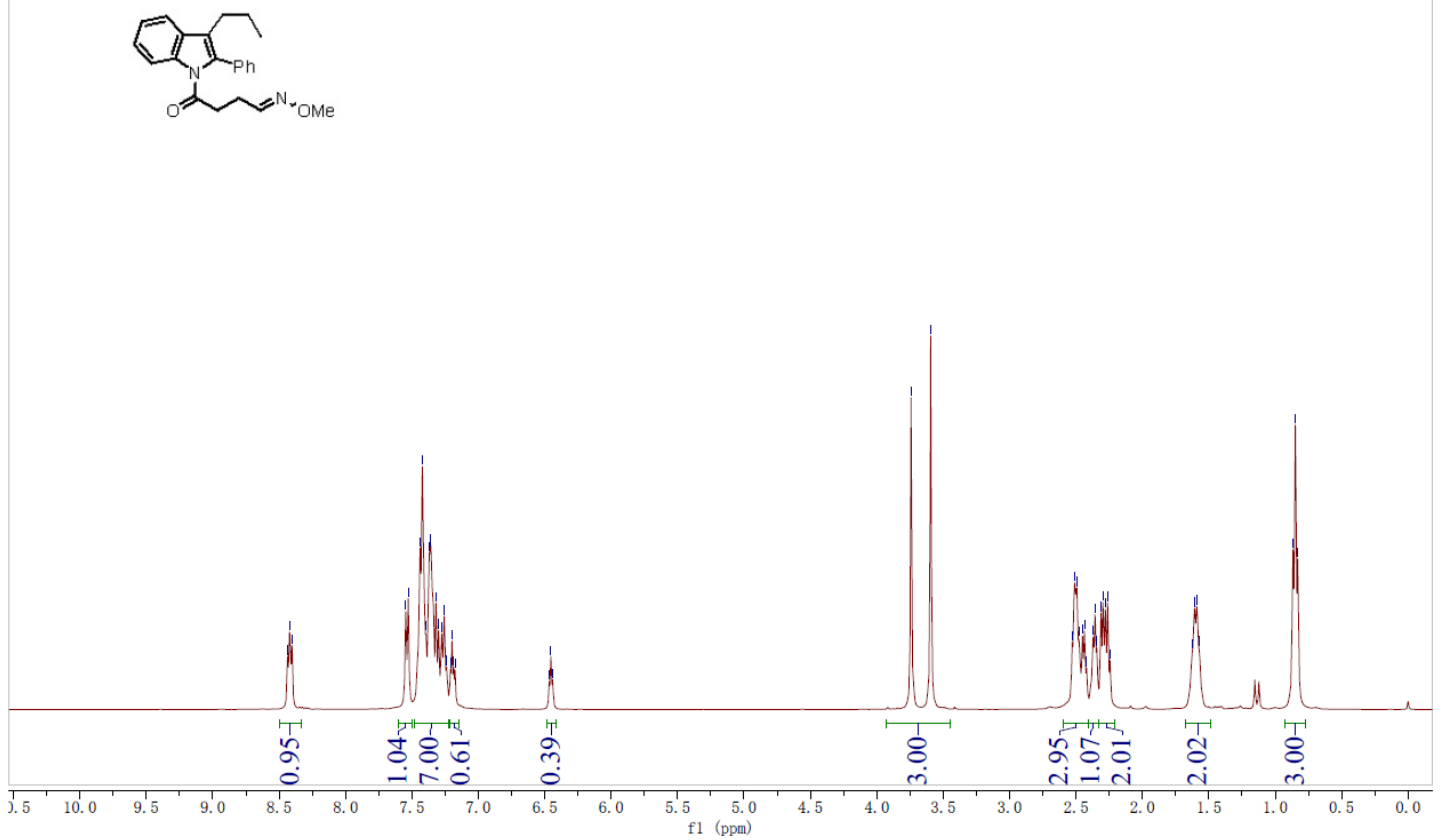

${ }^{13} \mathrm{C}$ NMR Spectrum of $\mathbf{3 k}$

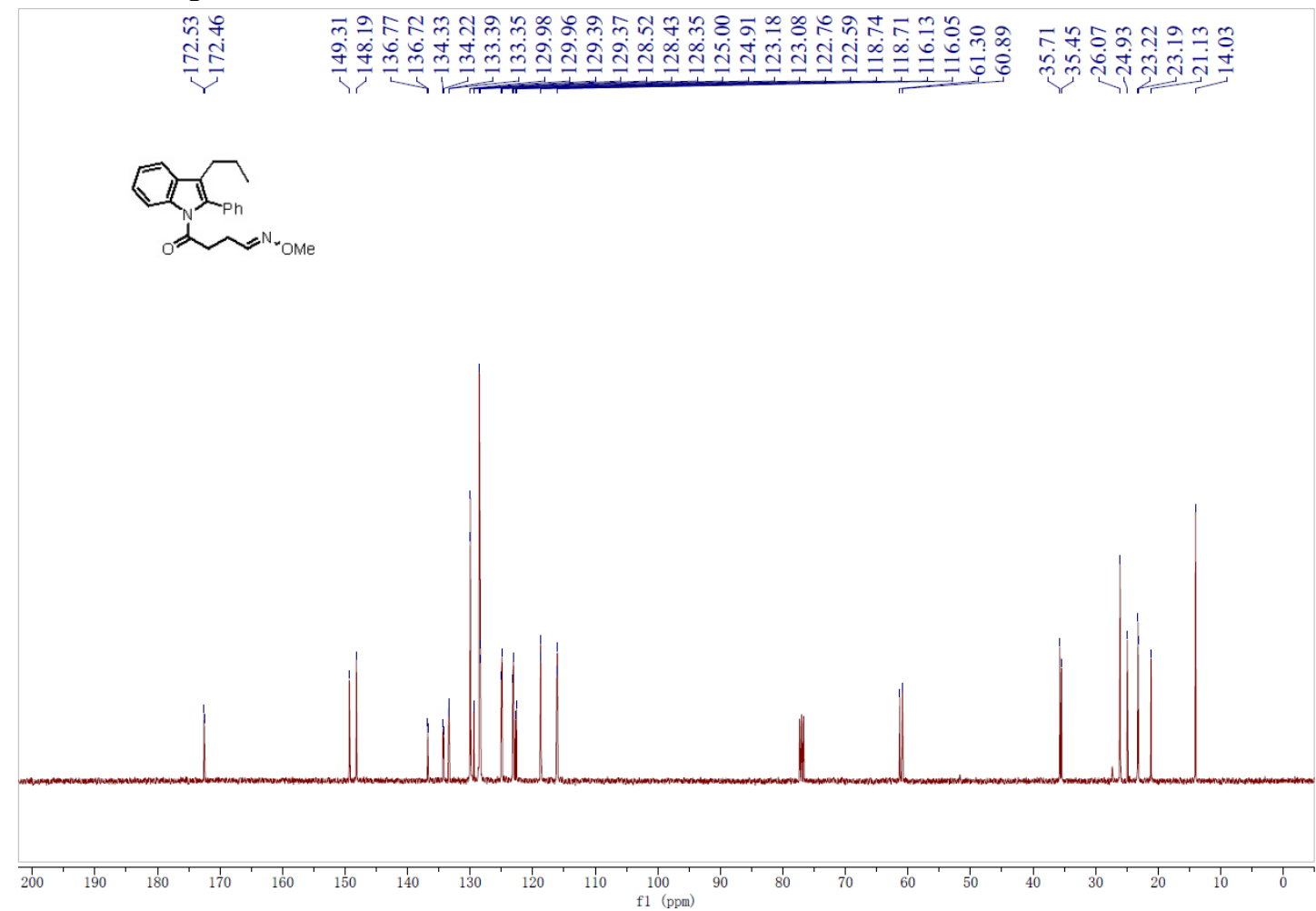


${ }^{1} \mathrm{H}$ NMR Spectrum of $\mathbf{3 l}$

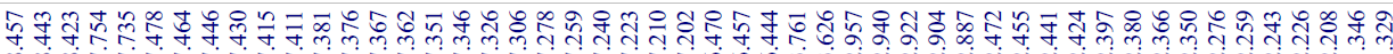

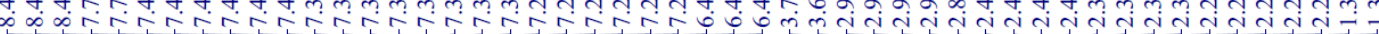
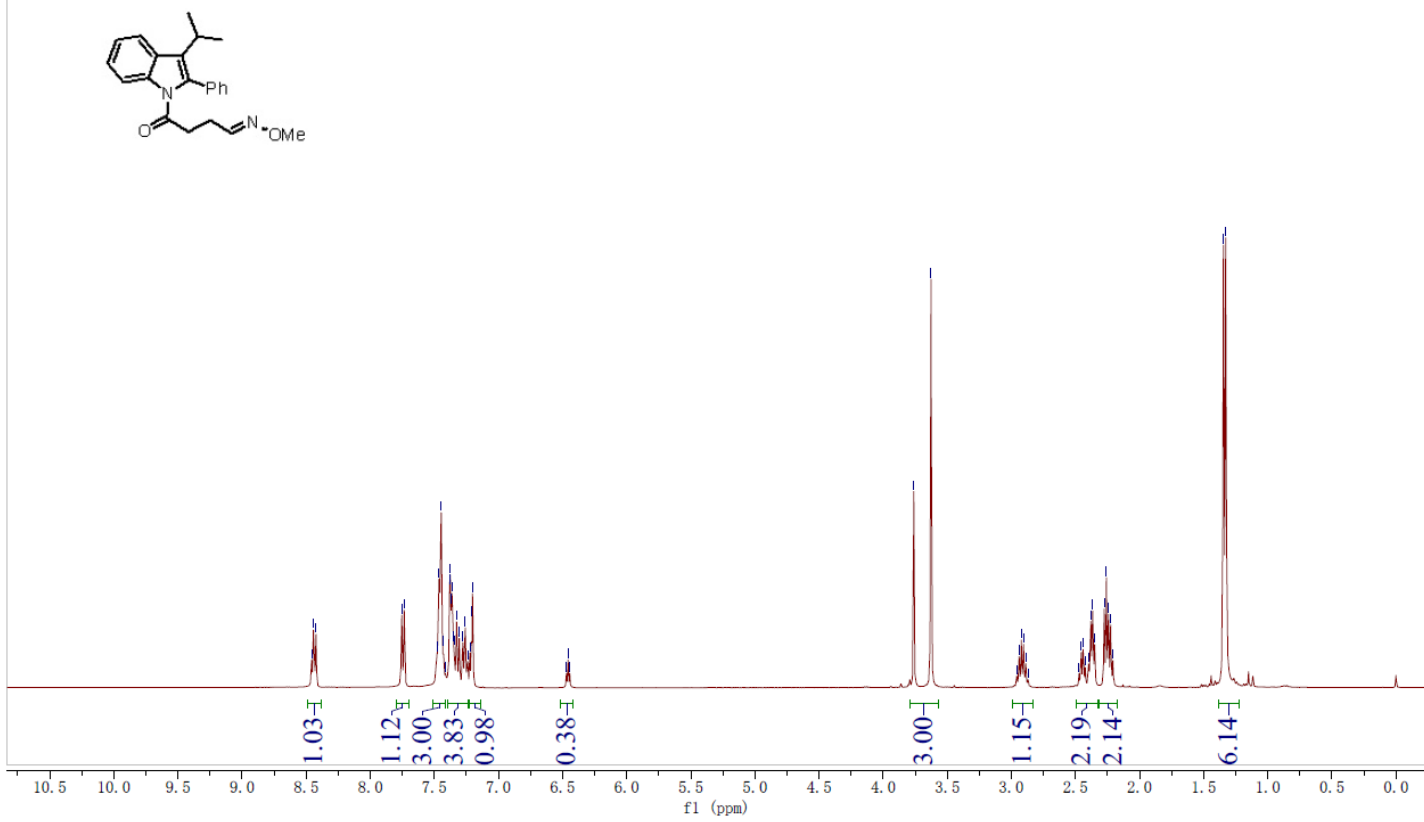

${ }^{13} \mathrm{C}$ NMR Spectrum of $\mathbf{3 1}$
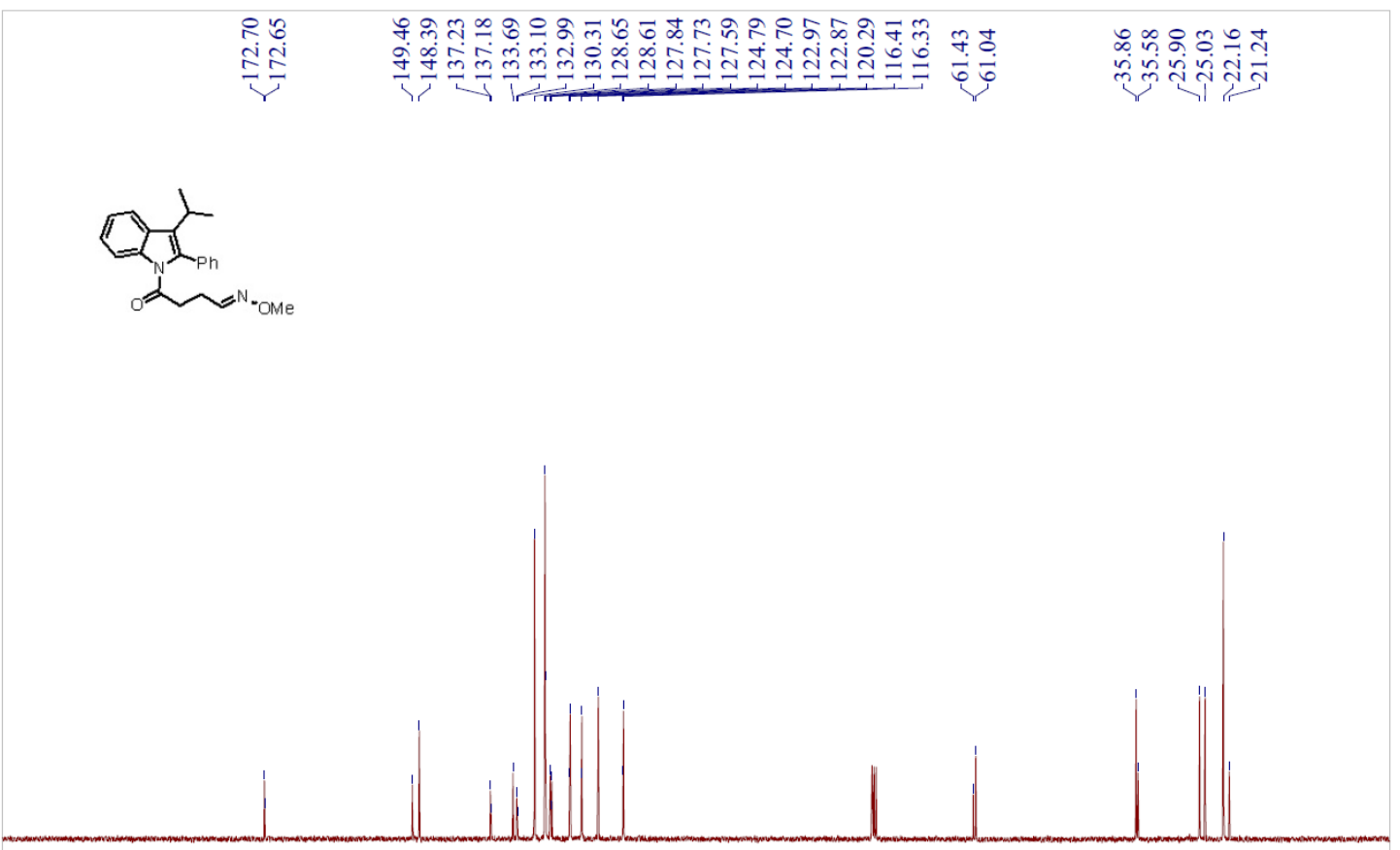

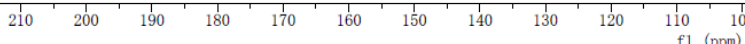


${ }^{1} \mathrm{H}$ NMR Spectrum of $\mathbf{3 m}$

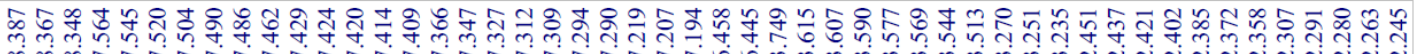

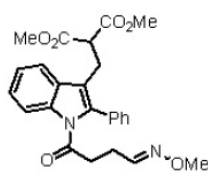

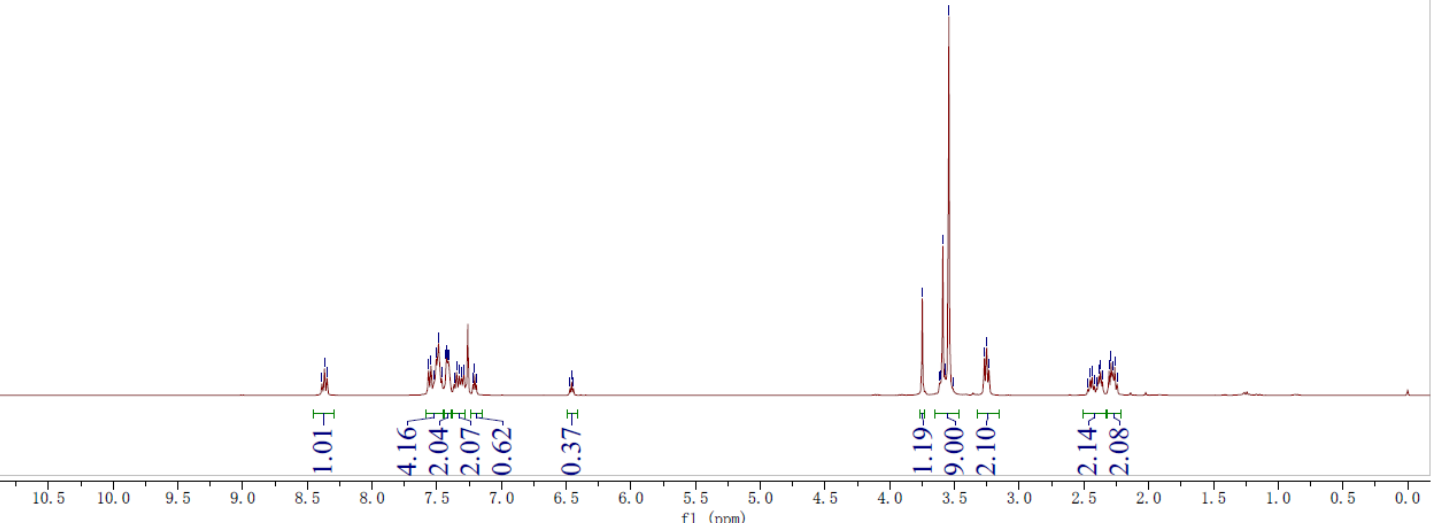

${ }^{13} \mathrm{C}$ NMR Spectrum of $\mathbf{3 m}$

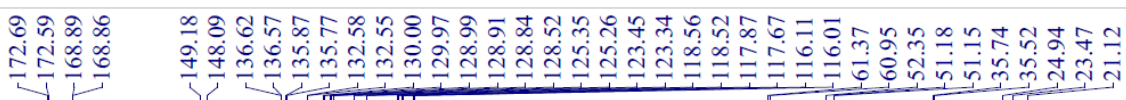
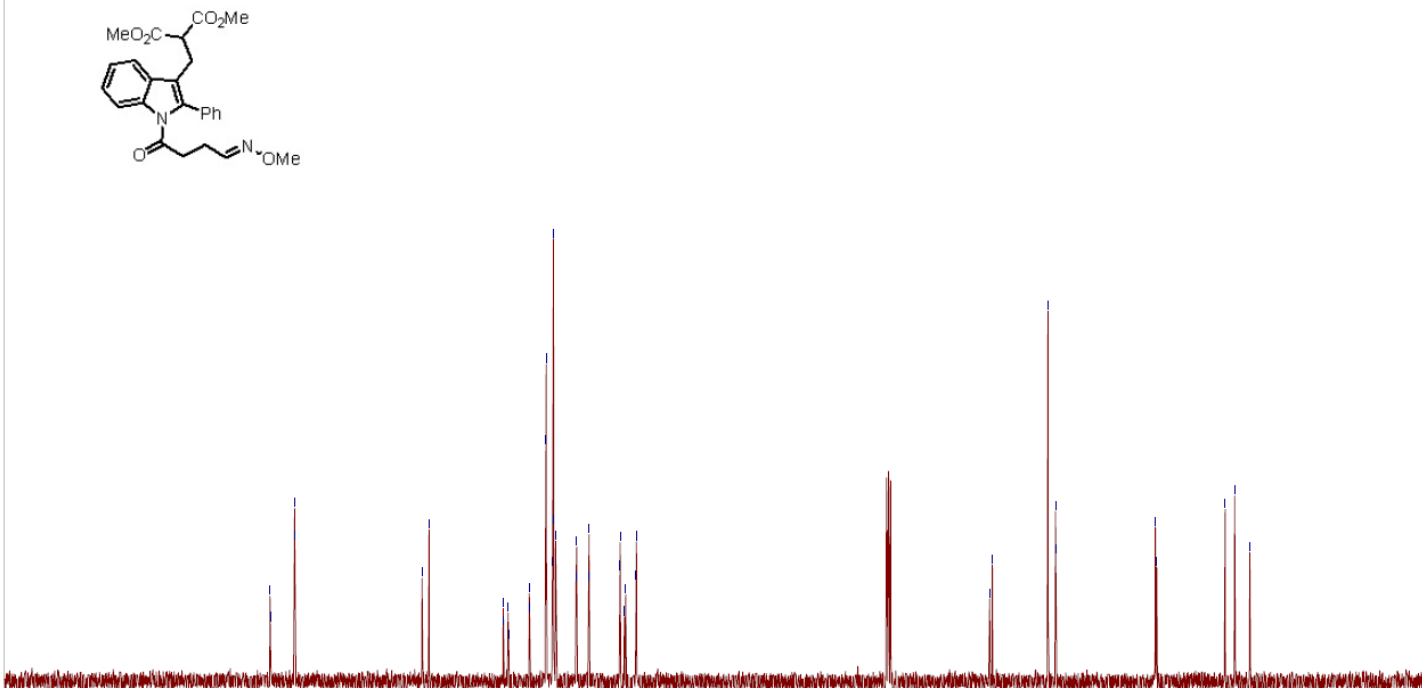

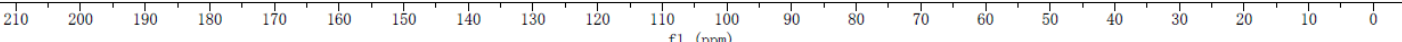


${ }^{1} \mathrm{H}$ NMR Spectrum of $\mathbf{3 n}$

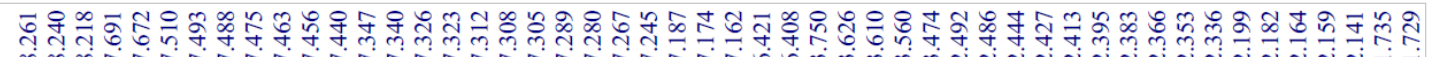

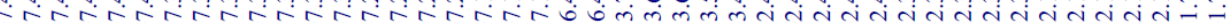

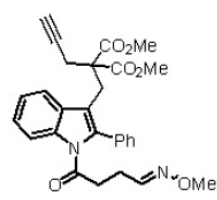

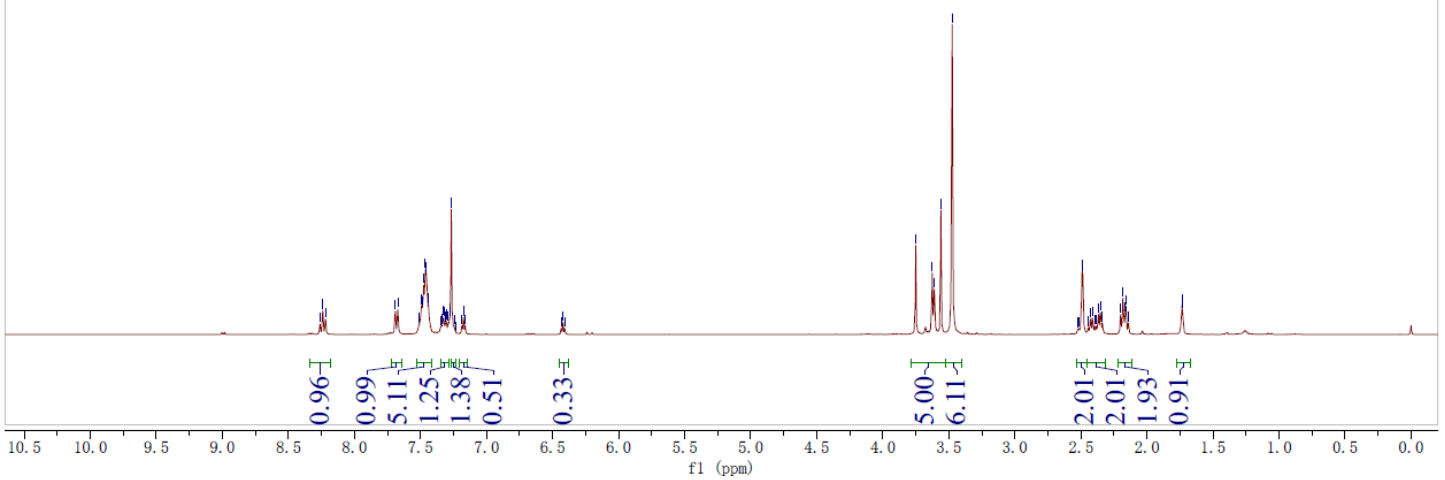

${ }^{13}$ C NMR Spectrum of $3 \mathbf{n}$

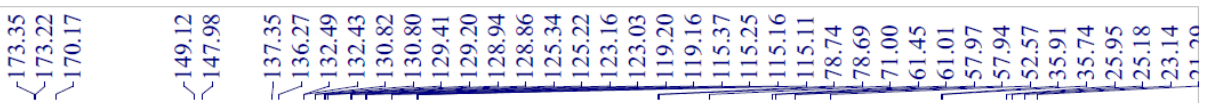<smiles>C=CCC(C)(COC)Cc1cccc2ccccc12</smiles>

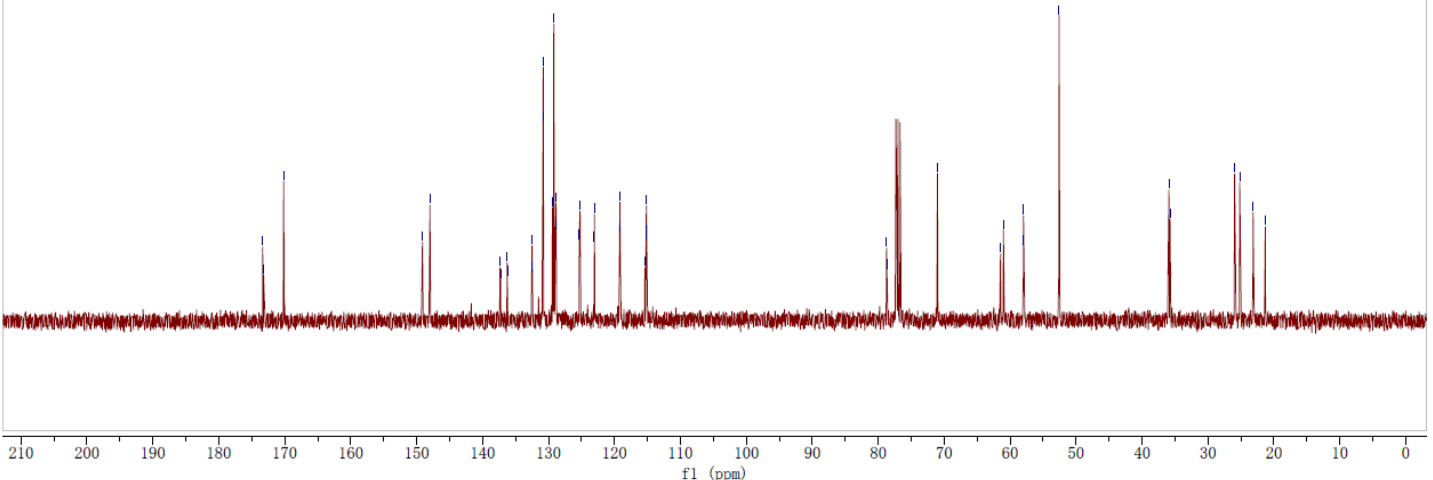


${ }^{1} \mathrm{H}$ NMR Spectrum of 30

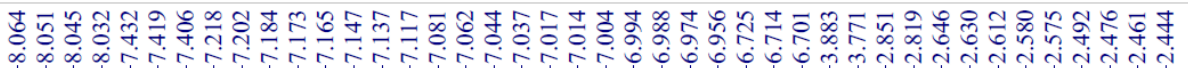
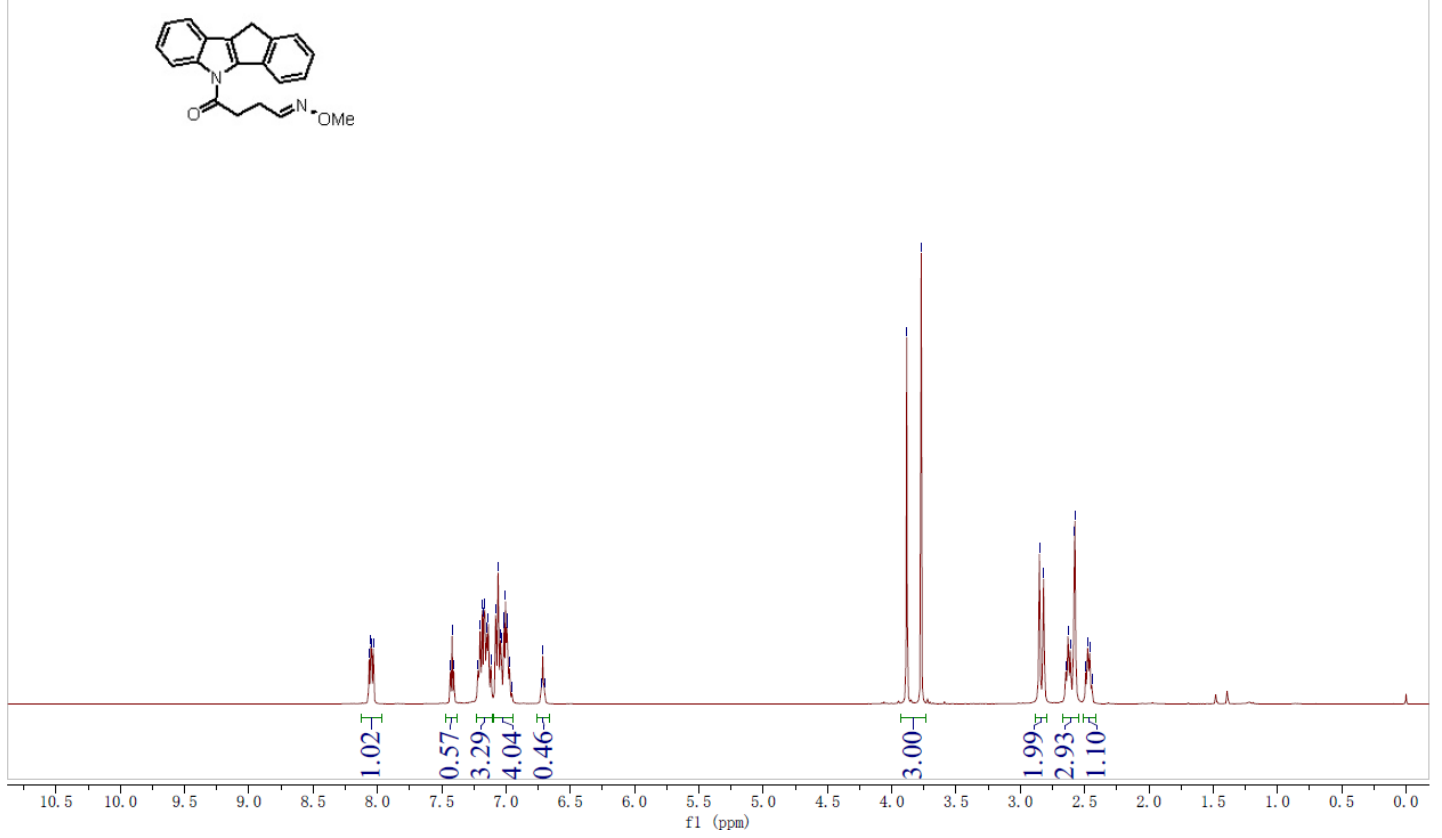

${ }^{13} \mathrm{C}$ NMR Spectrum of $\mathbf{3 o}$

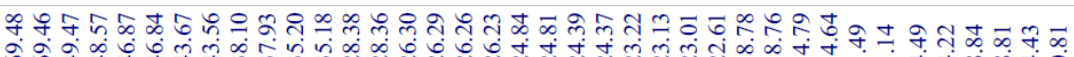

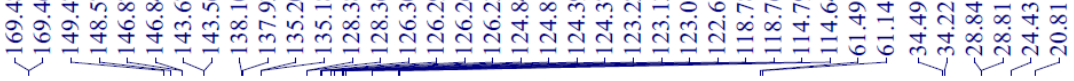
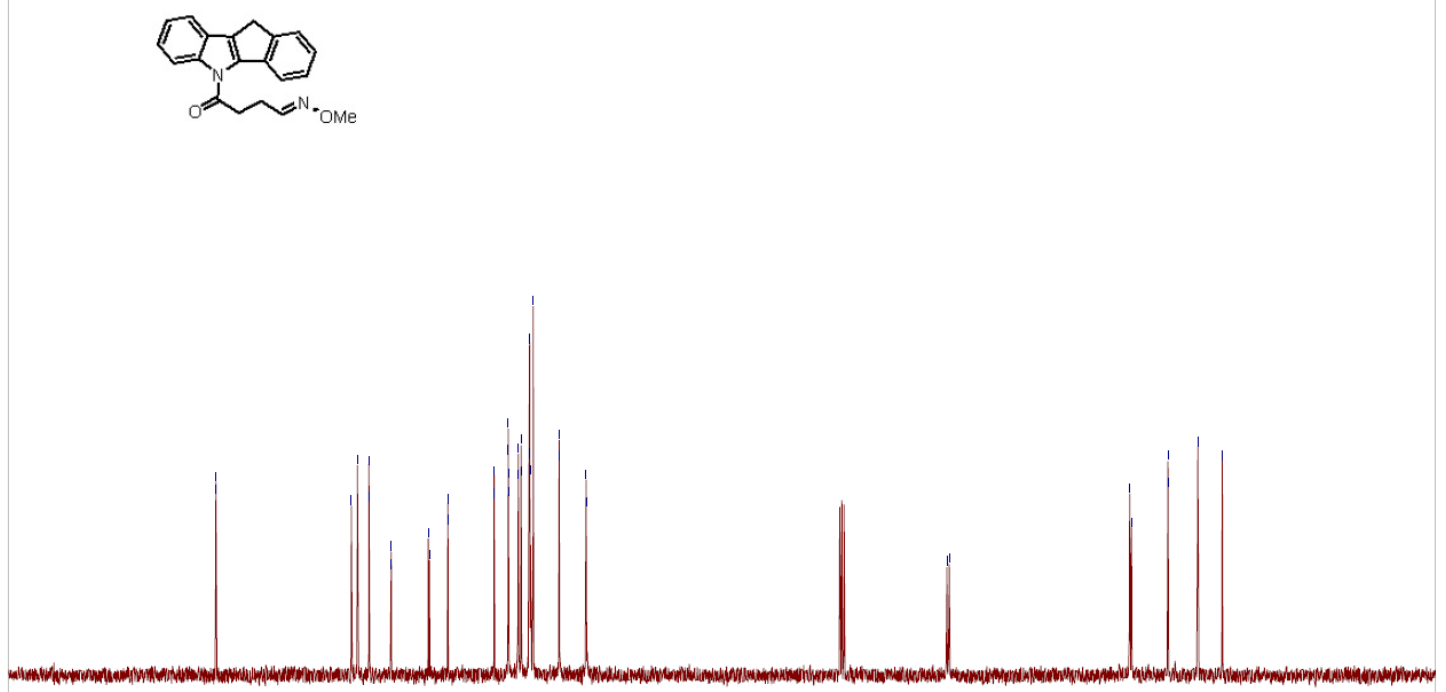

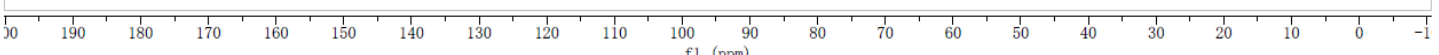


${ }^{1} \mathrm{H}$ NMR Spectrum of $\mathbf{2 a}$

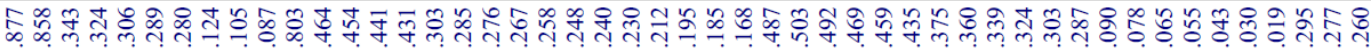

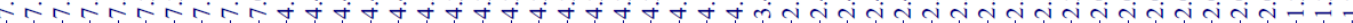

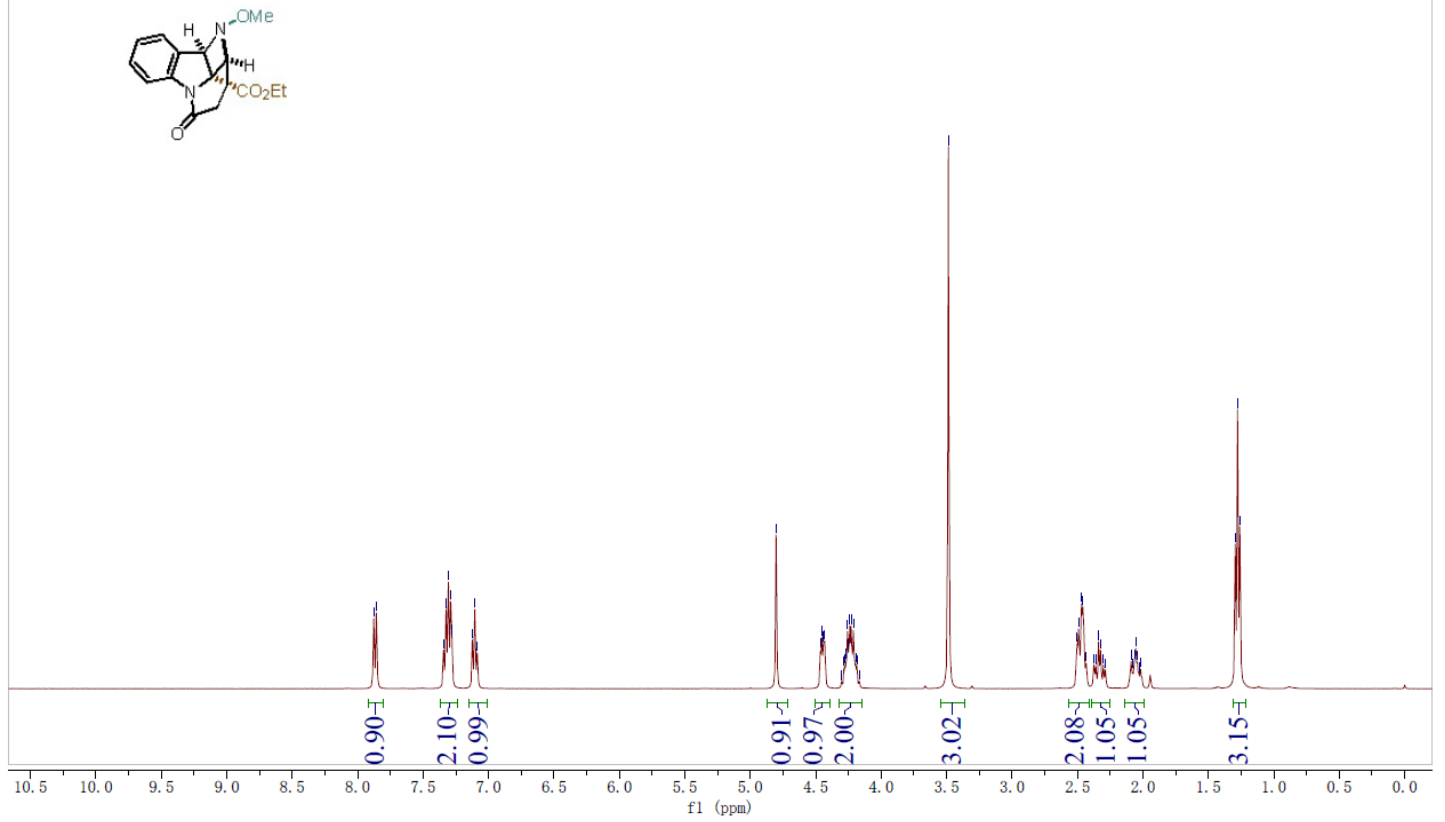

${ }^{13} \mathrm{C}$ NMR Spectrum of $\mathbf{2 a}$

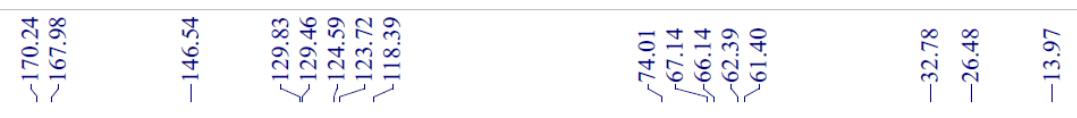

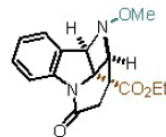

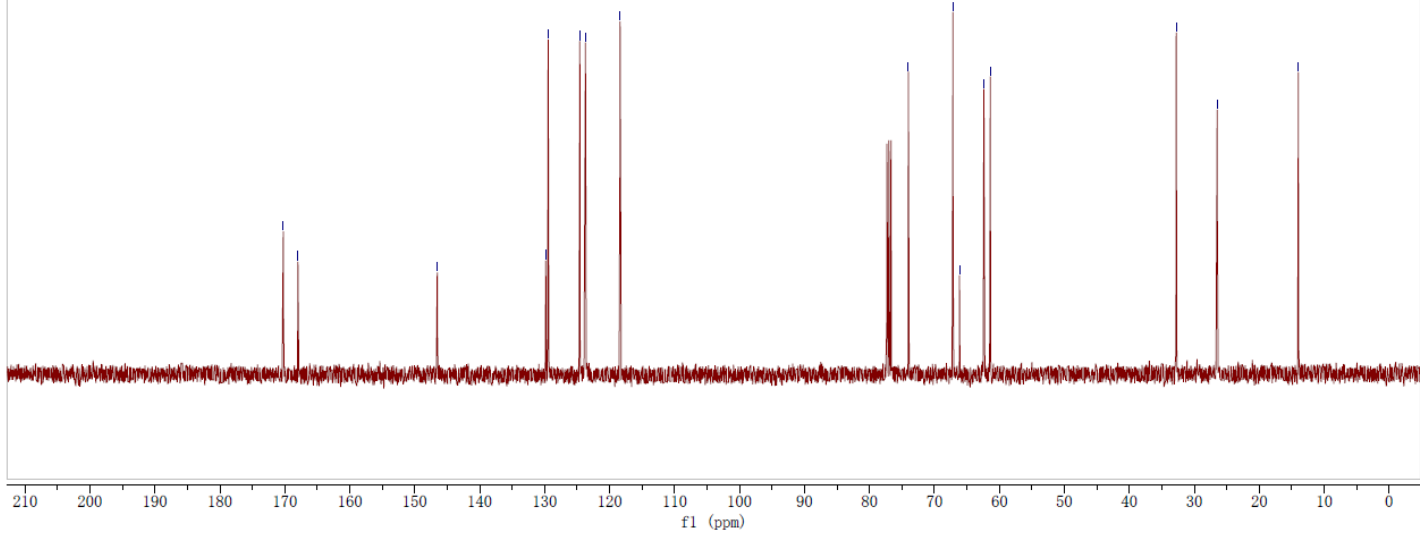


${ }^{1} \mathrm{H}$ NMR Spectrum of $\mathbf{2 b}$

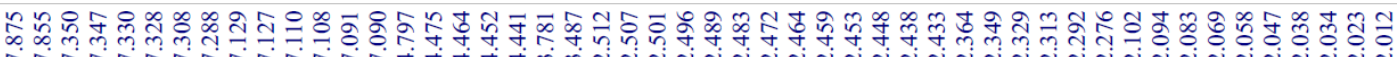

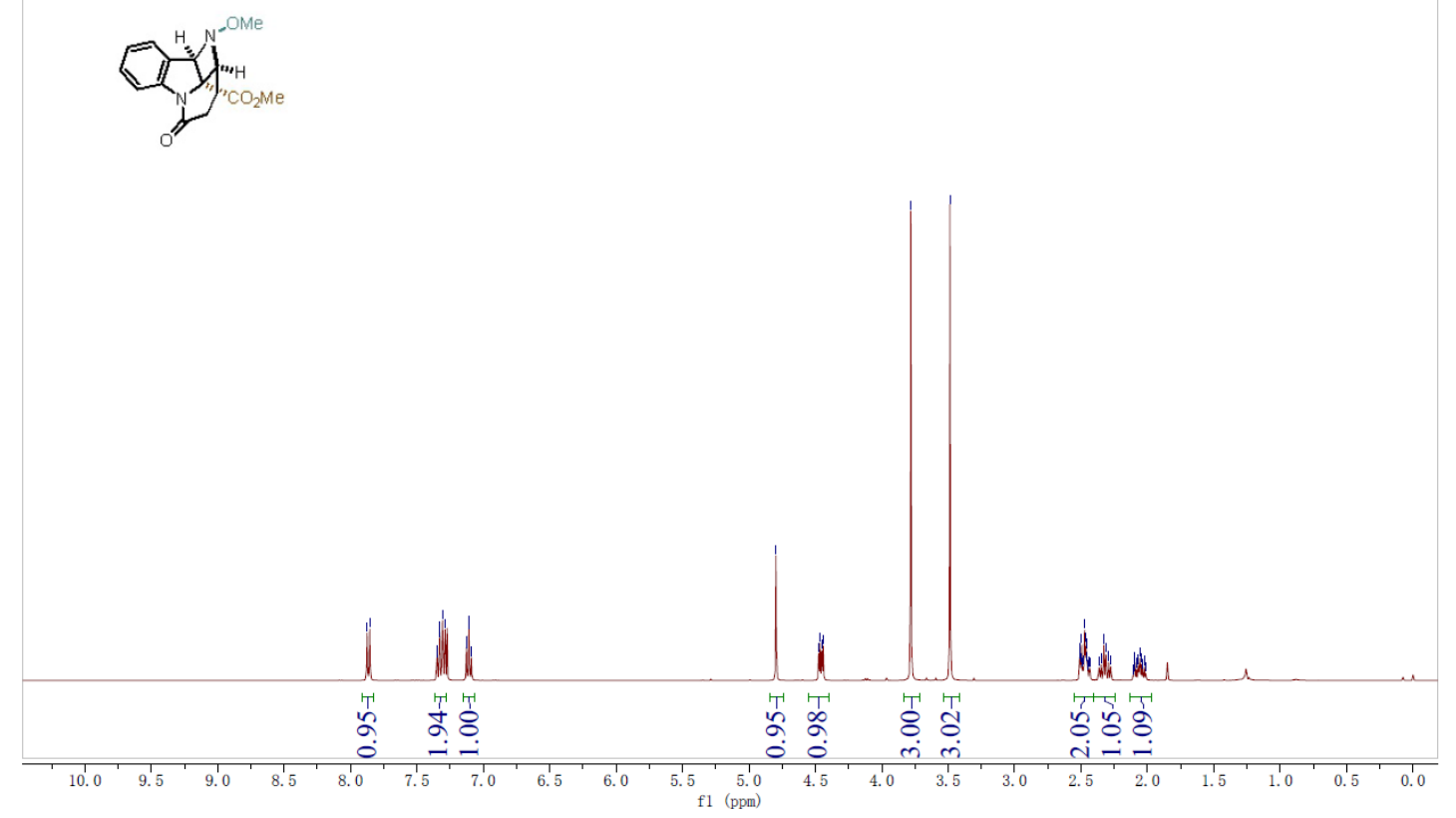

${ }^{13} \mathrm{C}$ NMR Spectrum of $\mathbf{2 b}$

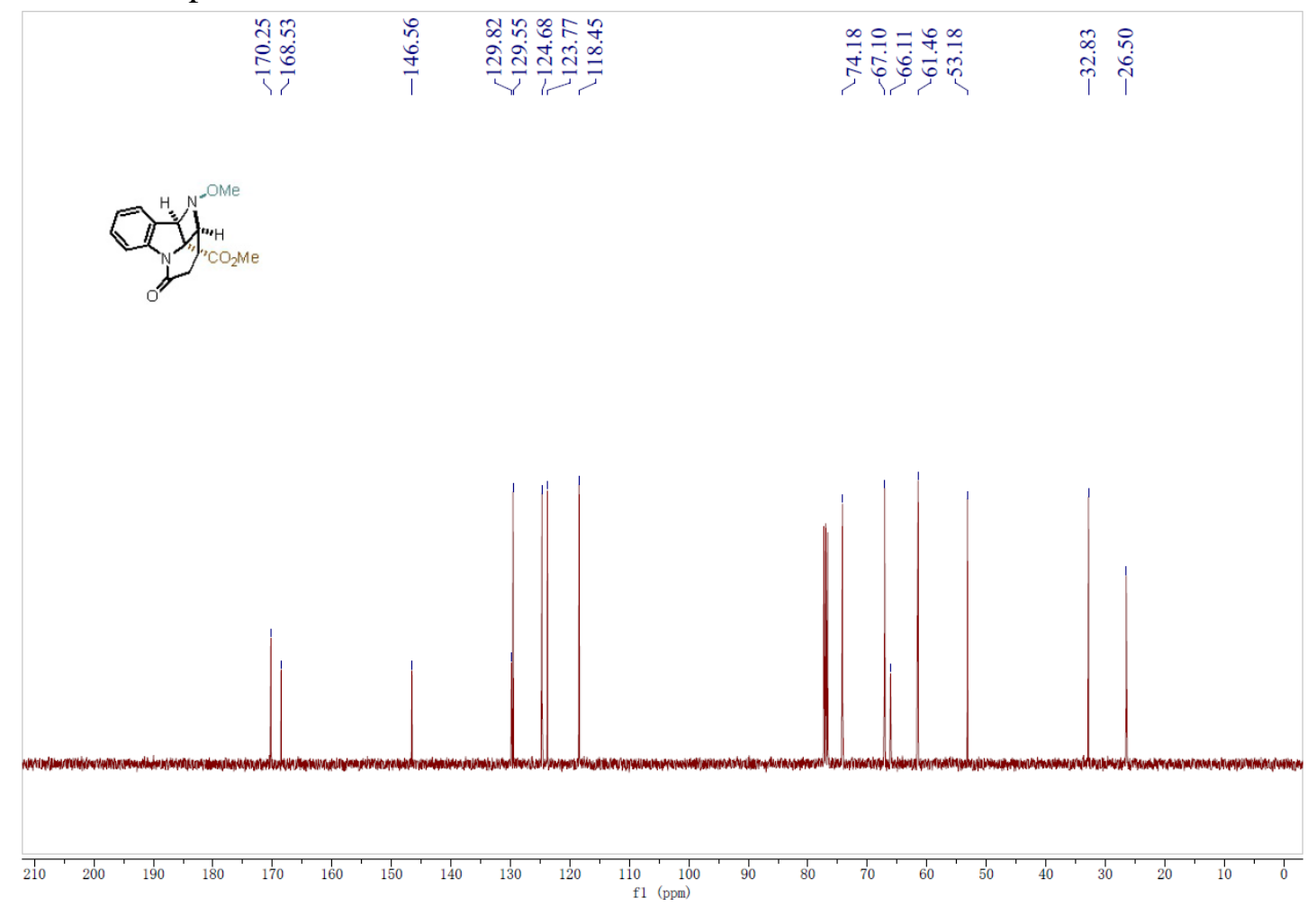


${ }^{1} \mathrm{H}$ NMR Spectrum of $\mathbf{2 c}$

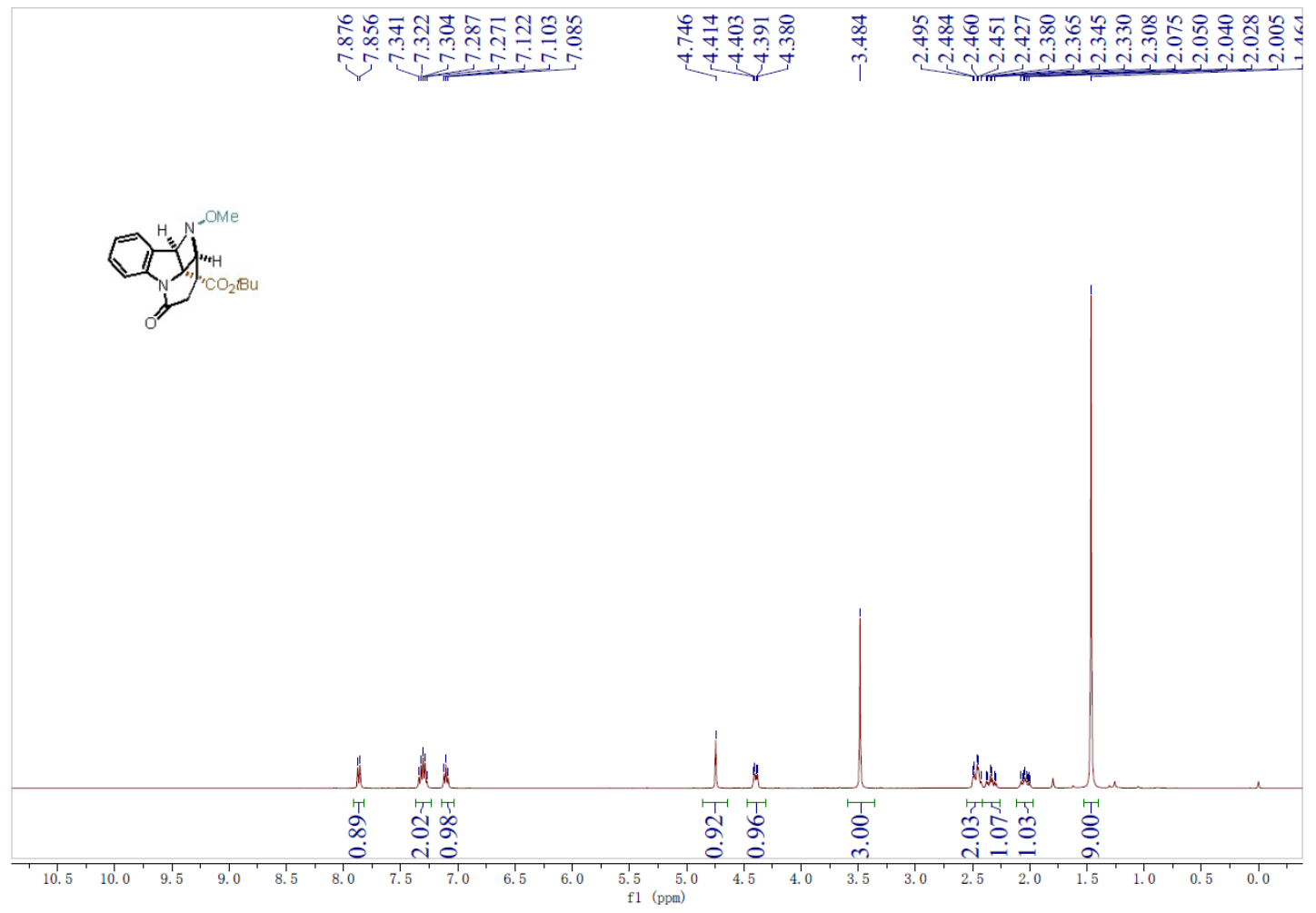

${ }^{13} \mathrm{C}$ NMR Spectrum of $\mathbf{2 c}$

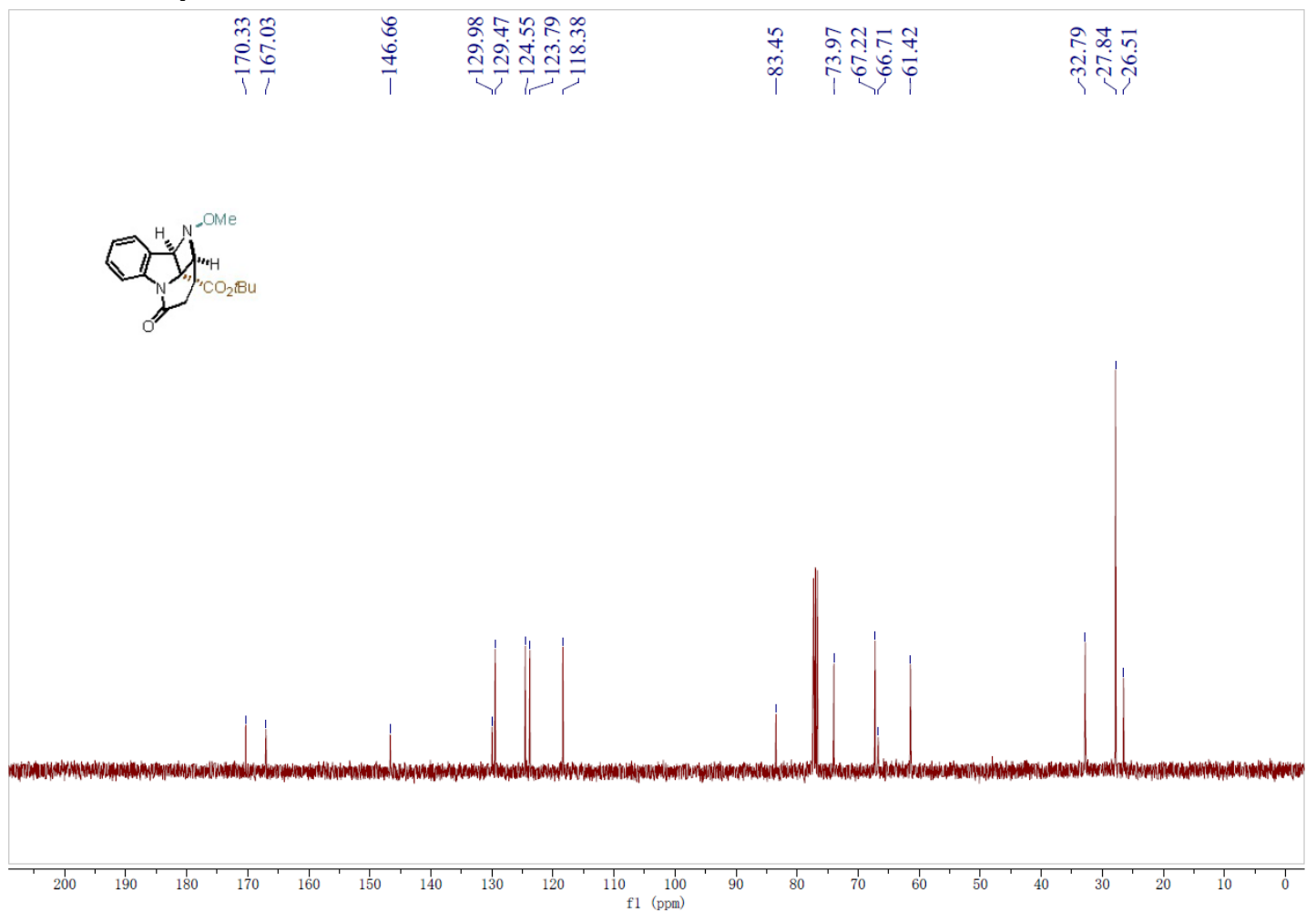


${ }^{1} \mathrm{H}$ NMR Spectrum of $\mathbf{2 d}$

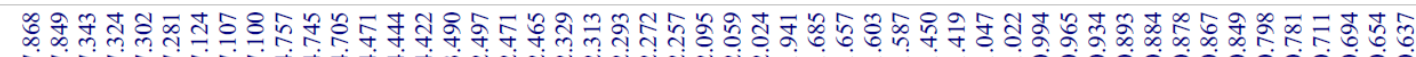

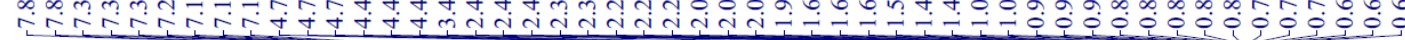

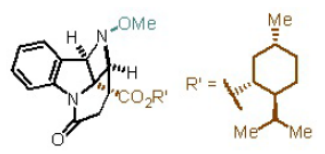

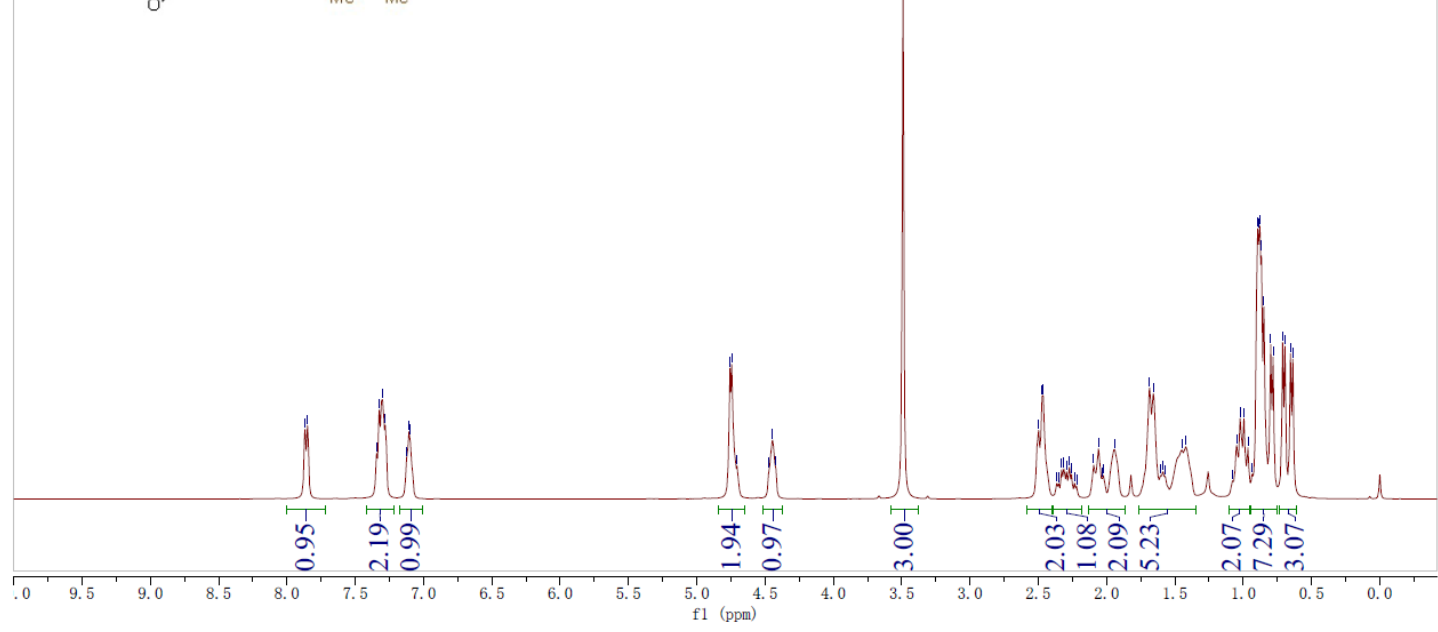

${ }^{13} \mathrm{C}$ NMR Spectrum of $\mathbf{2 d}$

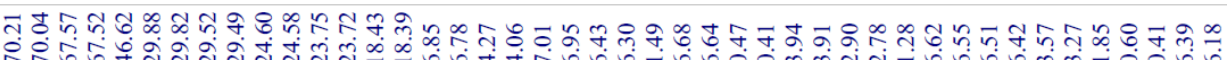

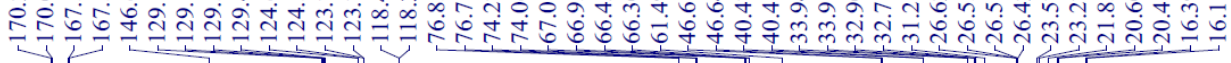

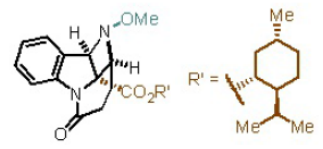
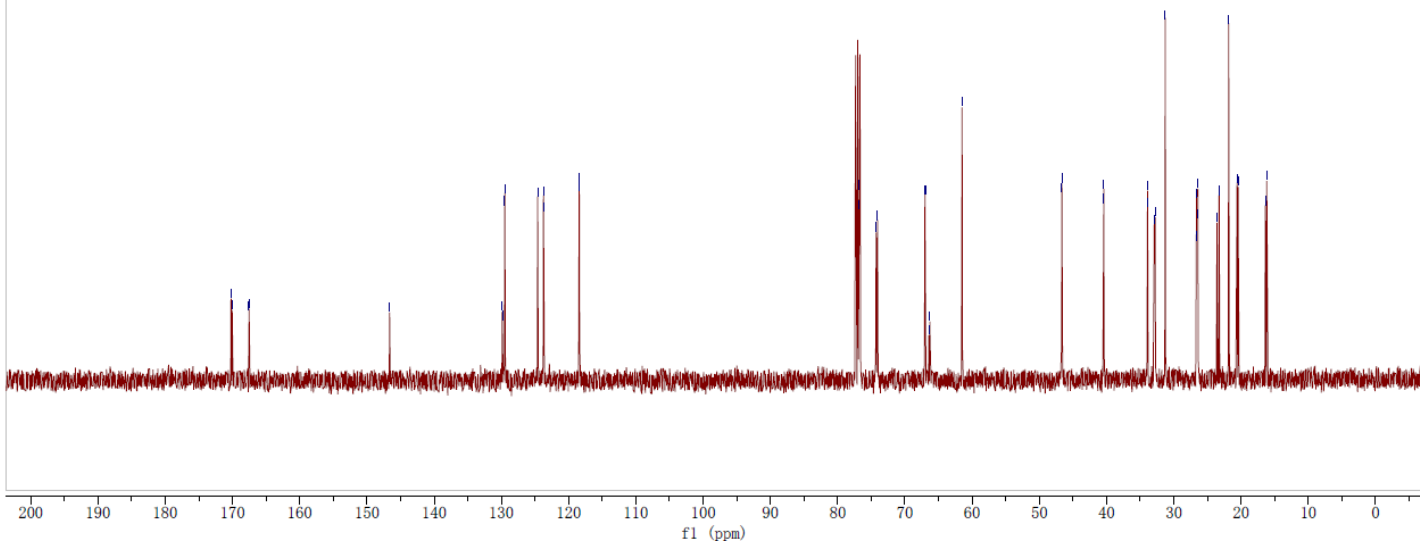
${ }^{1} \mathrm{H}$ NMR Spectrum of $\mathbf{2 e}$

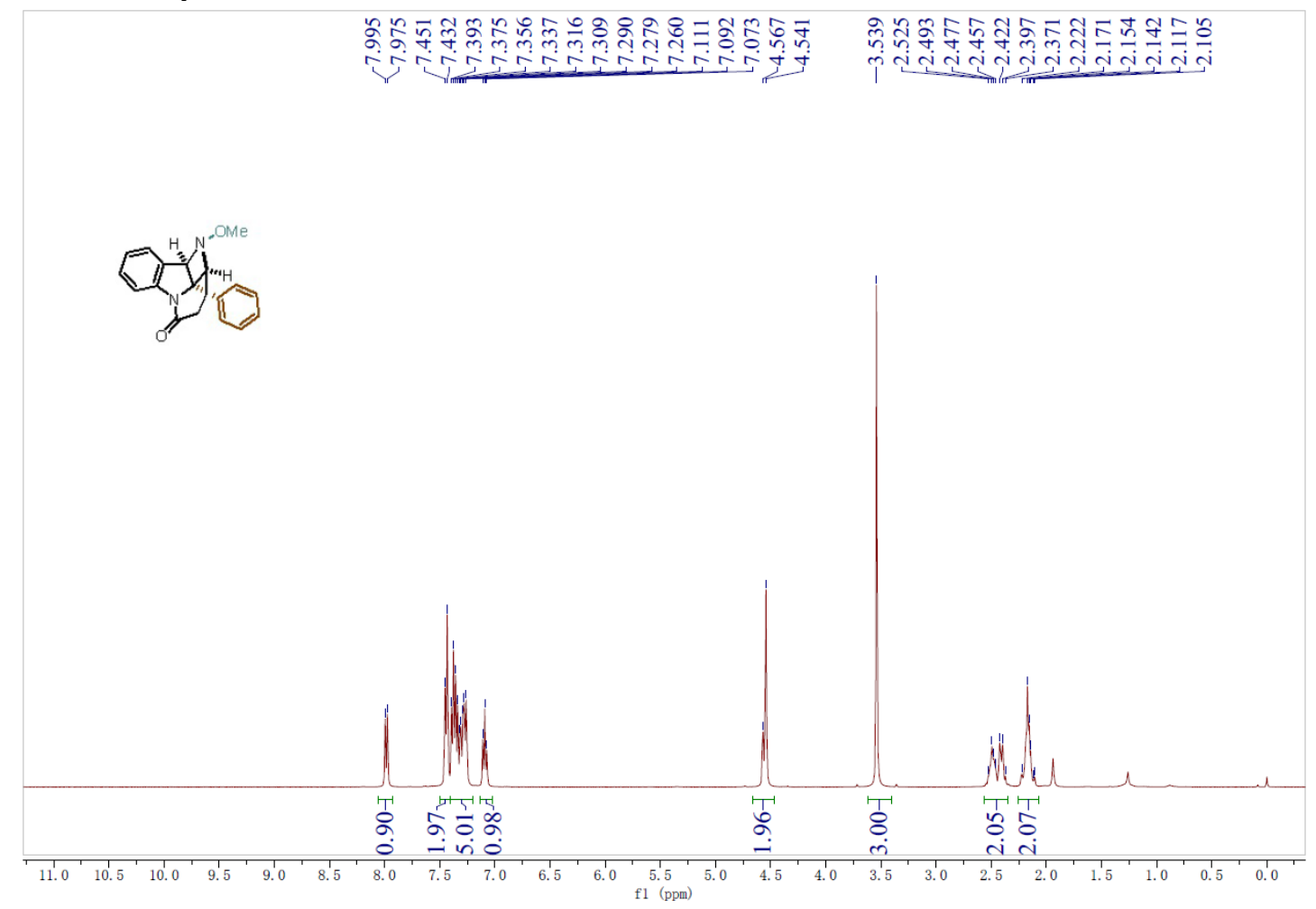

${ }^{13} \mathrm{C}$ NMR Spectrum of $\mathbf{2 e}$

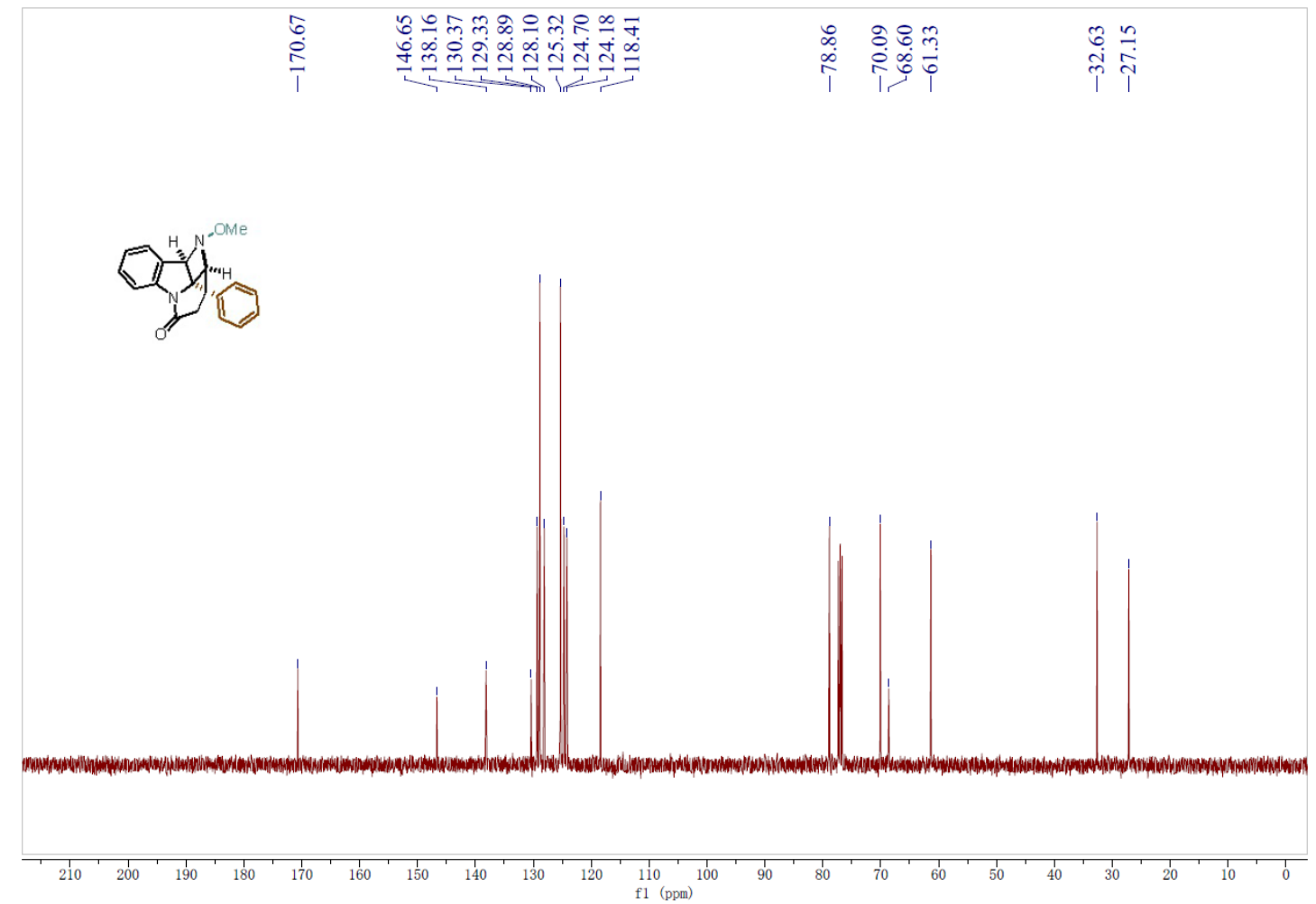


${ }^{1} \mathrm{H}$ NMR Spectrum of $\mathbf{2 f}$

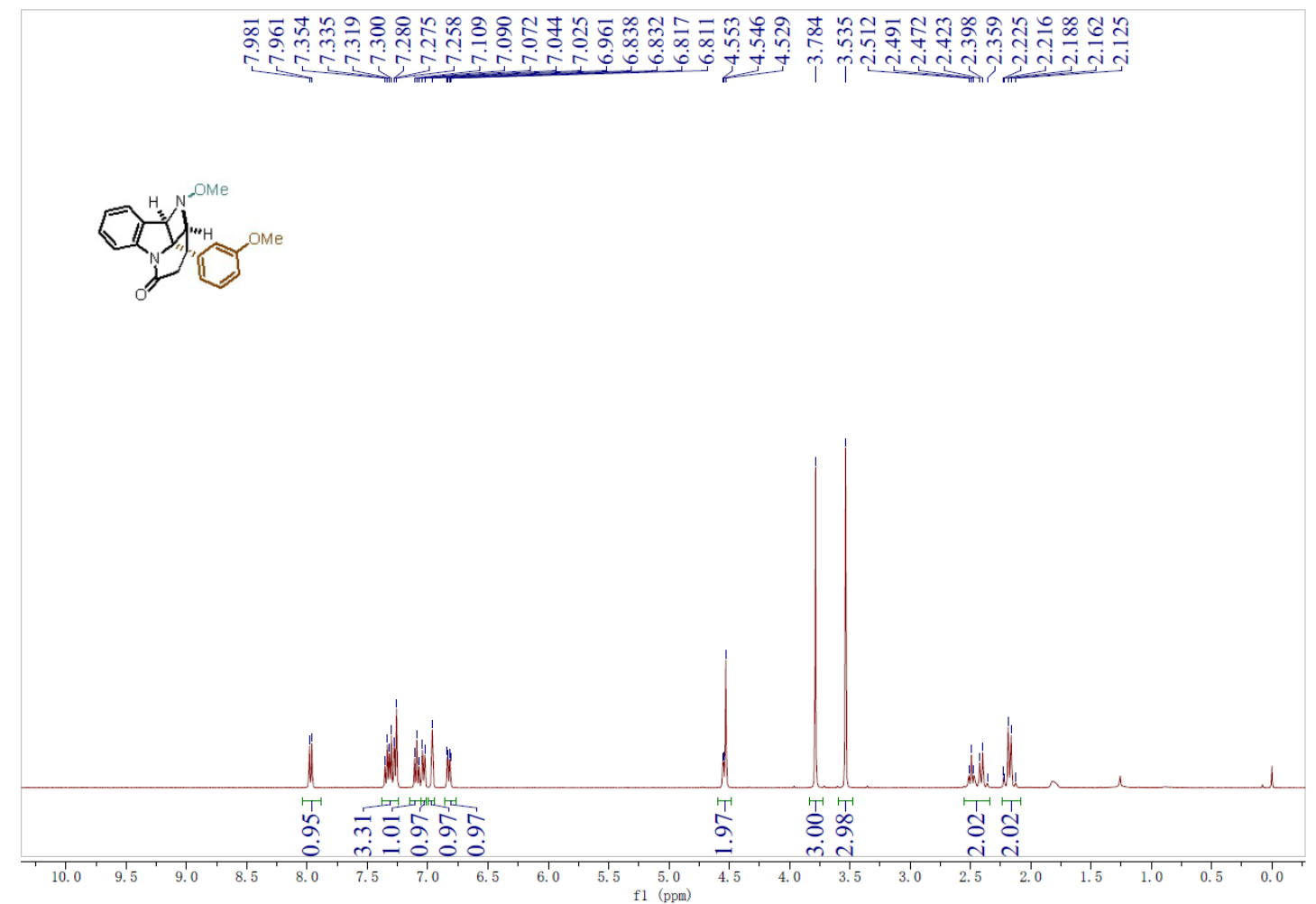

${ }^{13} \mathrm{C}$ NMR Spectrum of $\mathbf{2 f}$

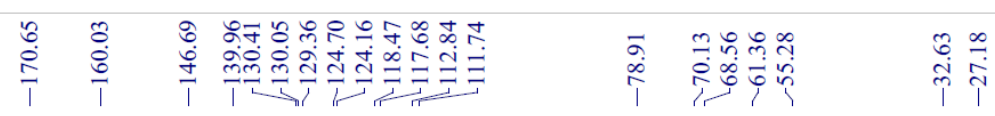

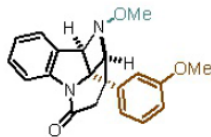
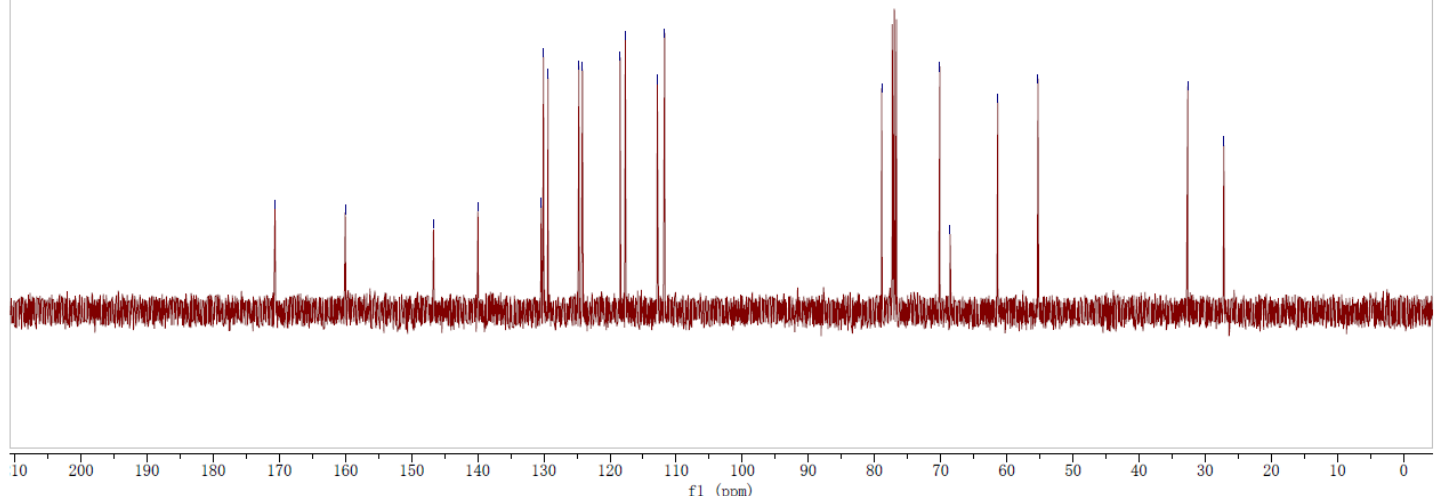
${ }^{1} \mathrm{H}$ NMR Spectrum of $\mathbf{2 g}$

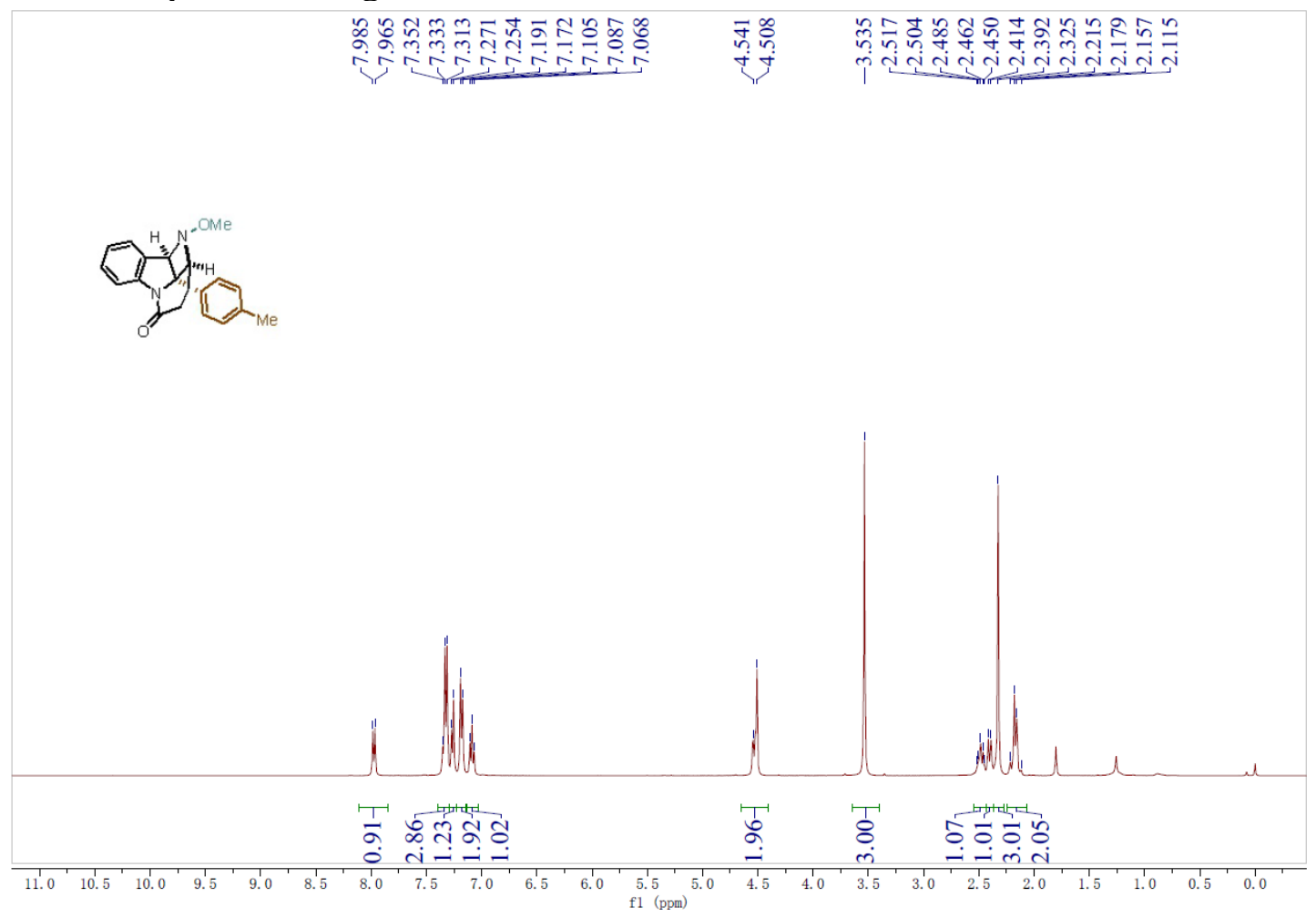

${ }^{13} \mathrm{C}$ NMR Spectrum of $\mathbf{2 g}$

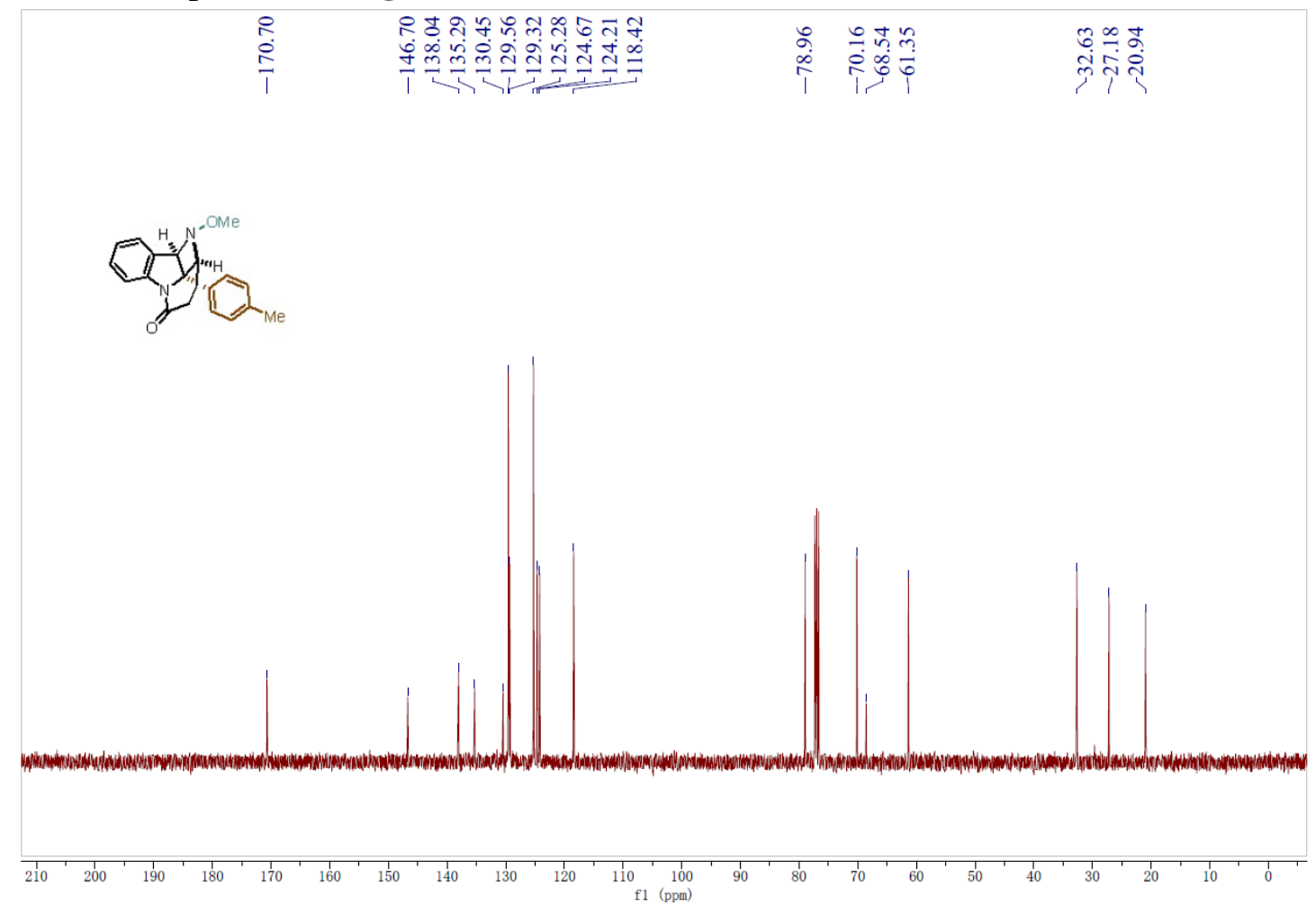


${ }^{1} \mathrm{H}$ NMR Spectrum of $\mathbf{2 h}$

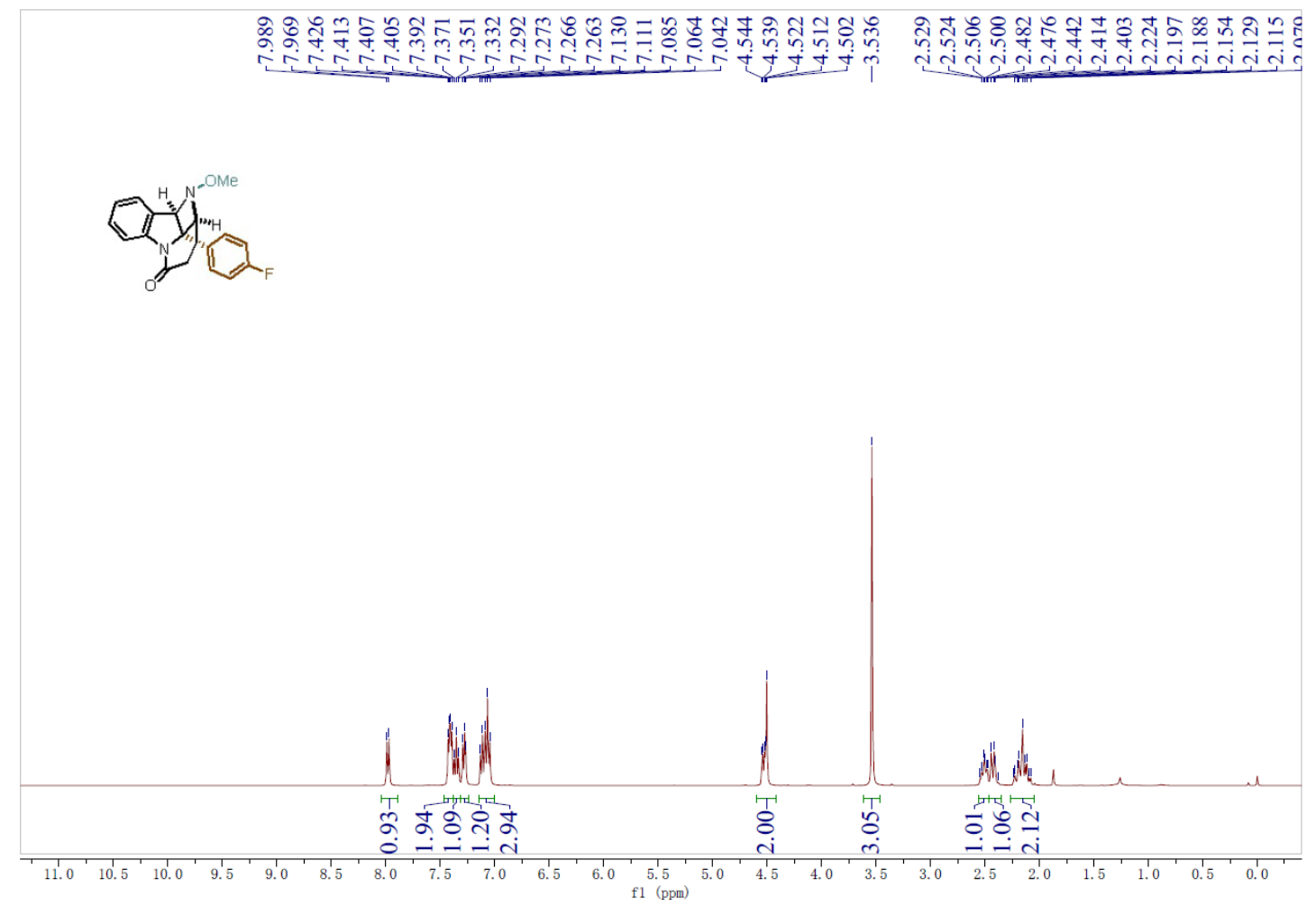

${ }^{13} \mathrm{C}$ NMR Spectrum of $\mathbf{2 h}$

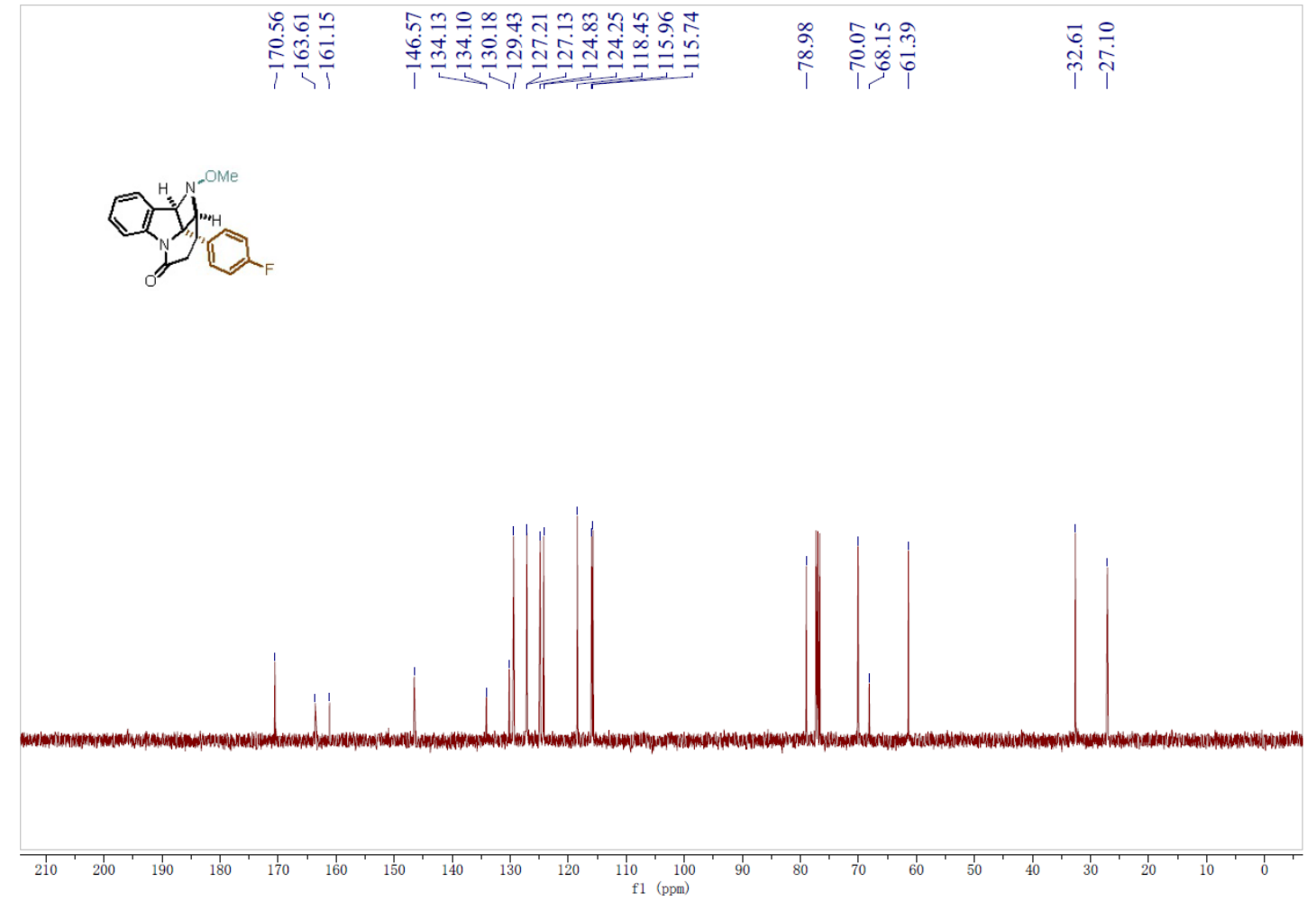


${ }^{19}$ F NMR Spectrum of $\mathbf{2 h}$

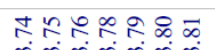

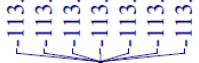

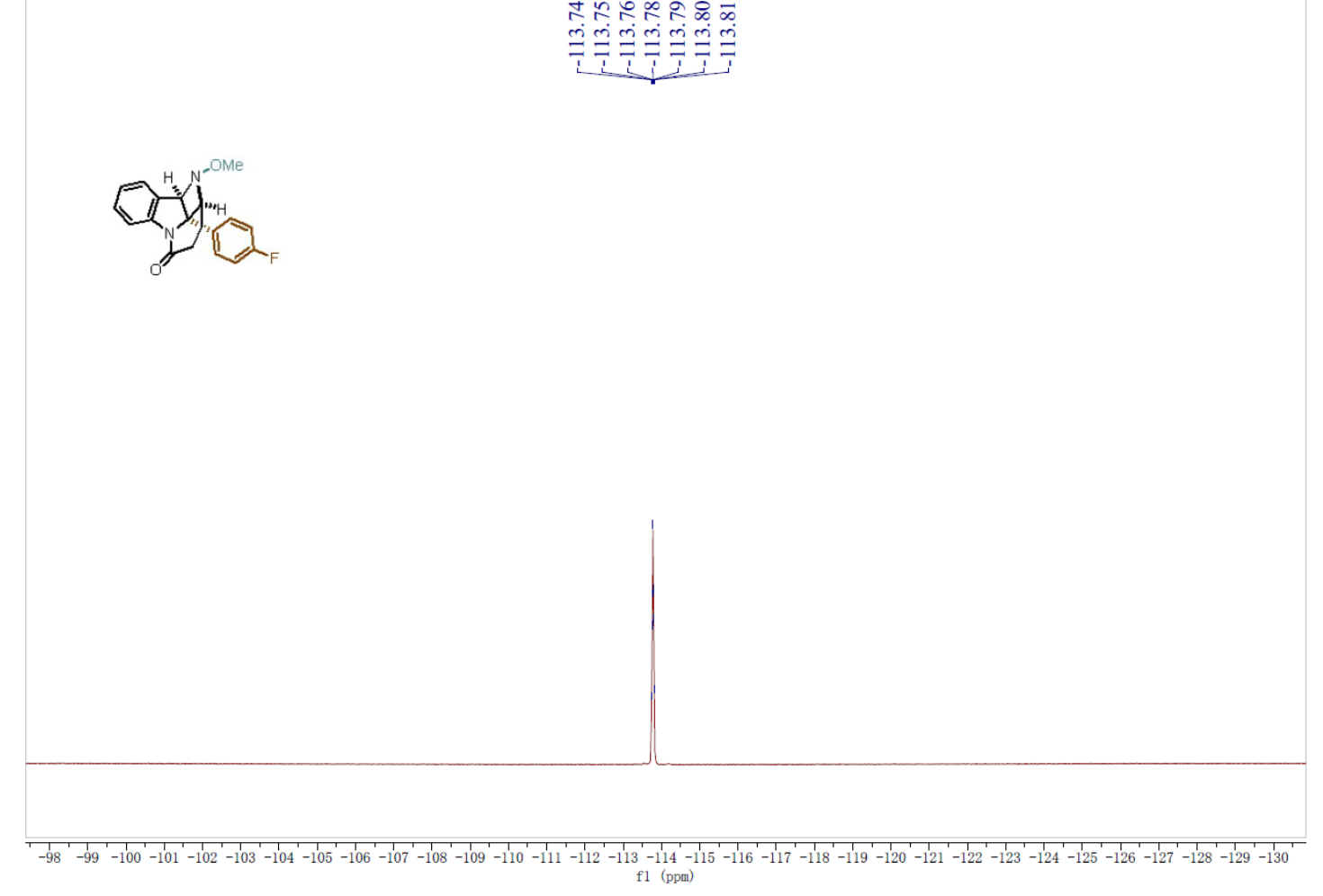


${ }^{1} \mathrm{H}$ NMR Spectrum of $\mathbf{2 i}$

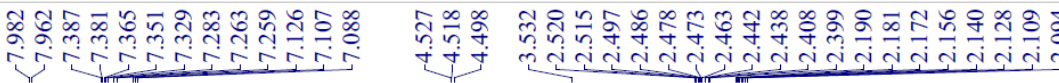
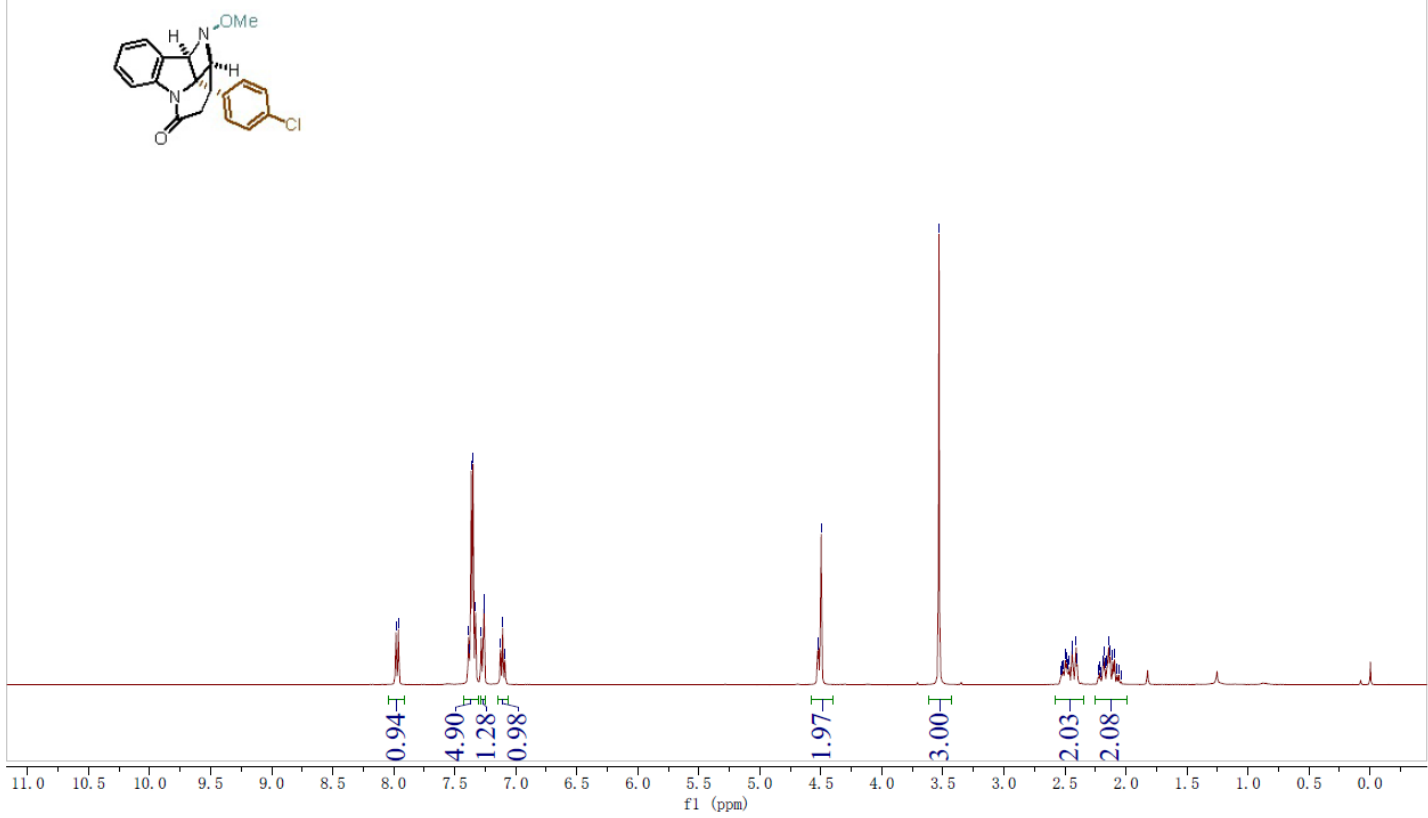

${ }^{13} \mathrm{C}$ NMR Spectrum of $\mathbf{2 i}$

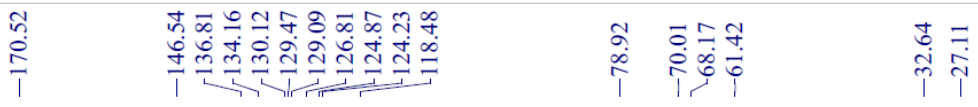

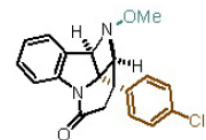

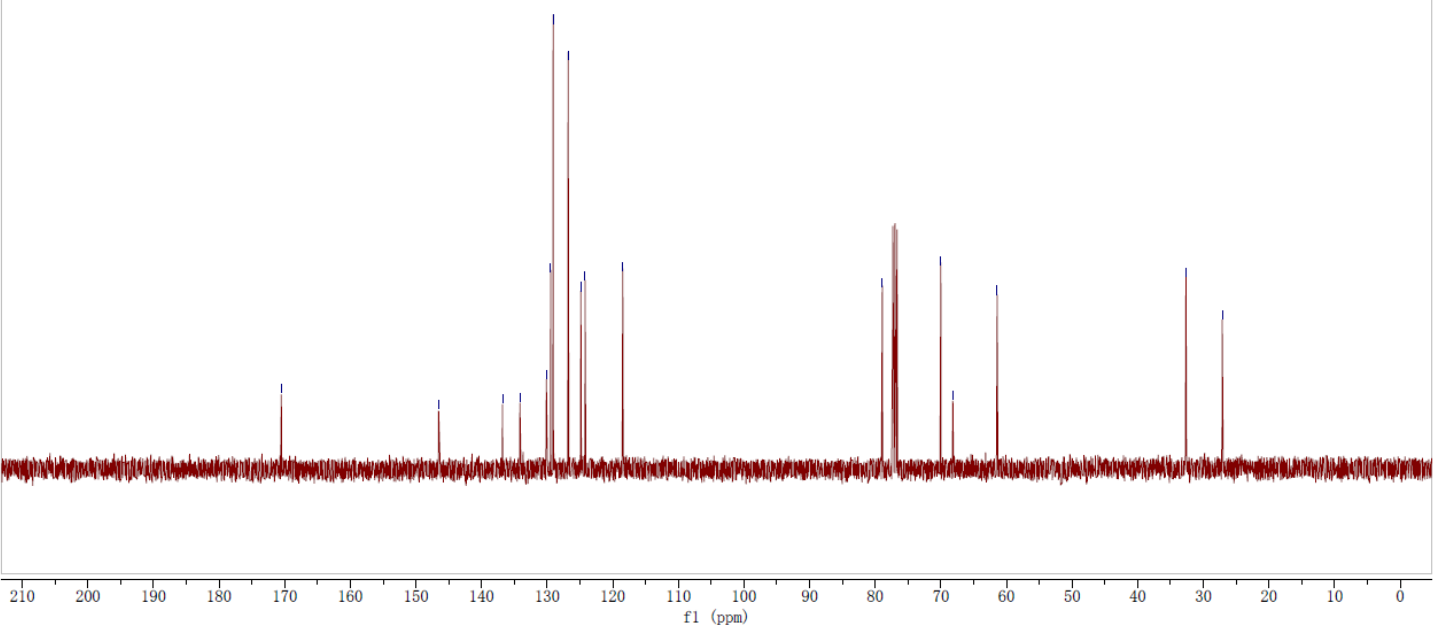


${ }^{1} \mathrm{H}$ NMR Spectrum of $\mathbf{2 j}$

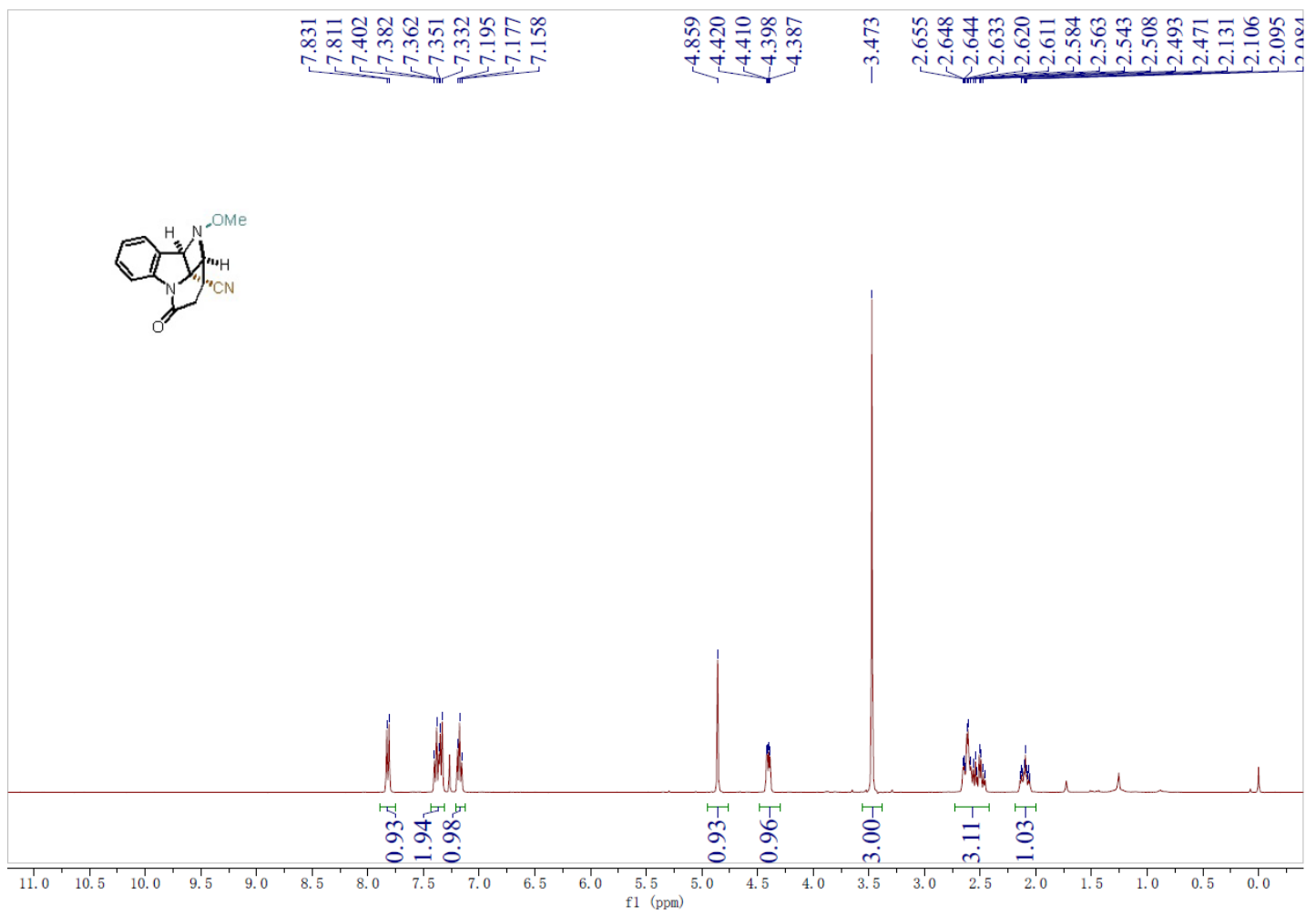

${ }^{13} \mathrm{C}$ NMR Spectrum of $\mathbf{2 j}$

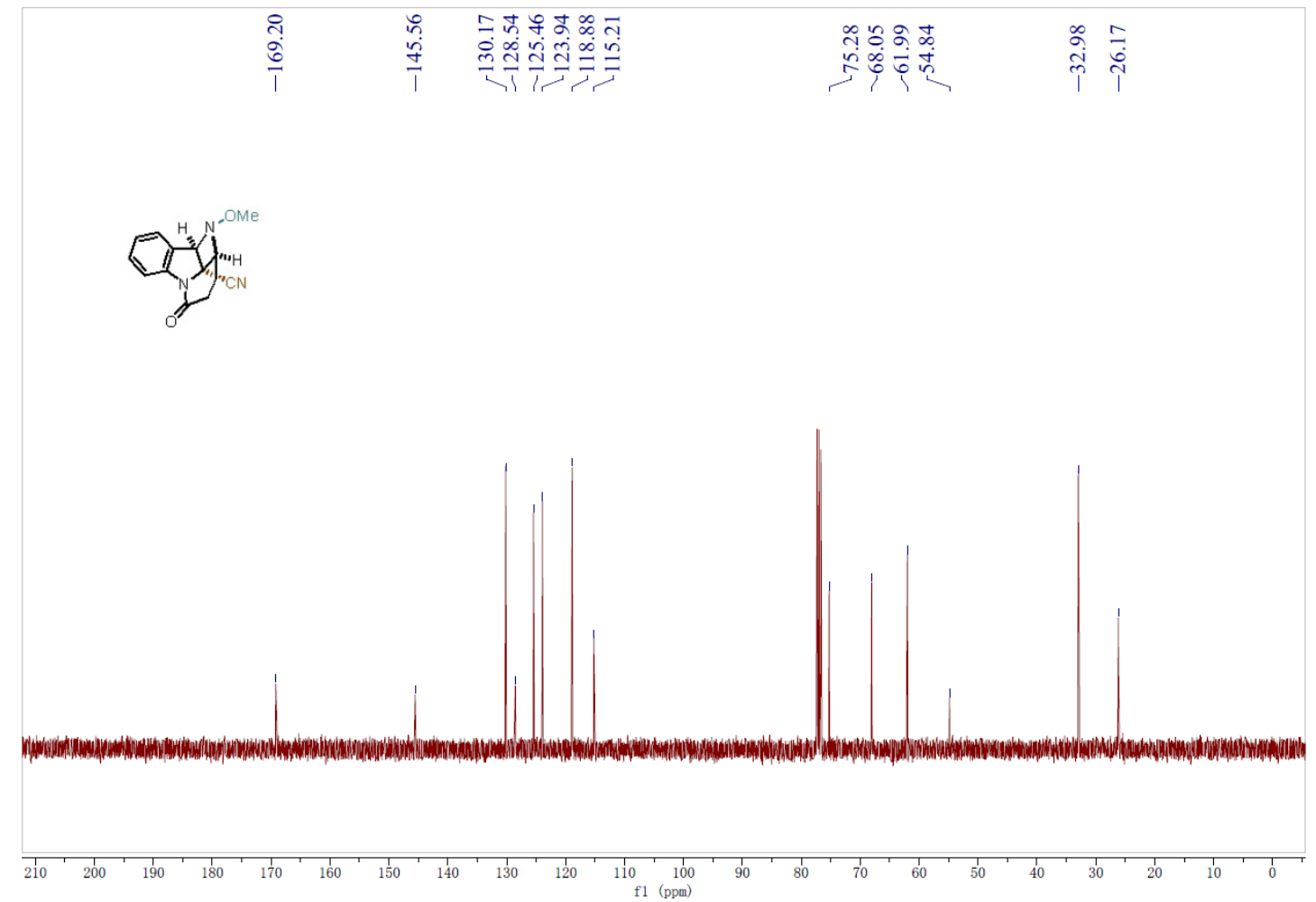


${ }^{1} \mathrm{H}$ NMR Spectrum of $\mathbf{2 k}$

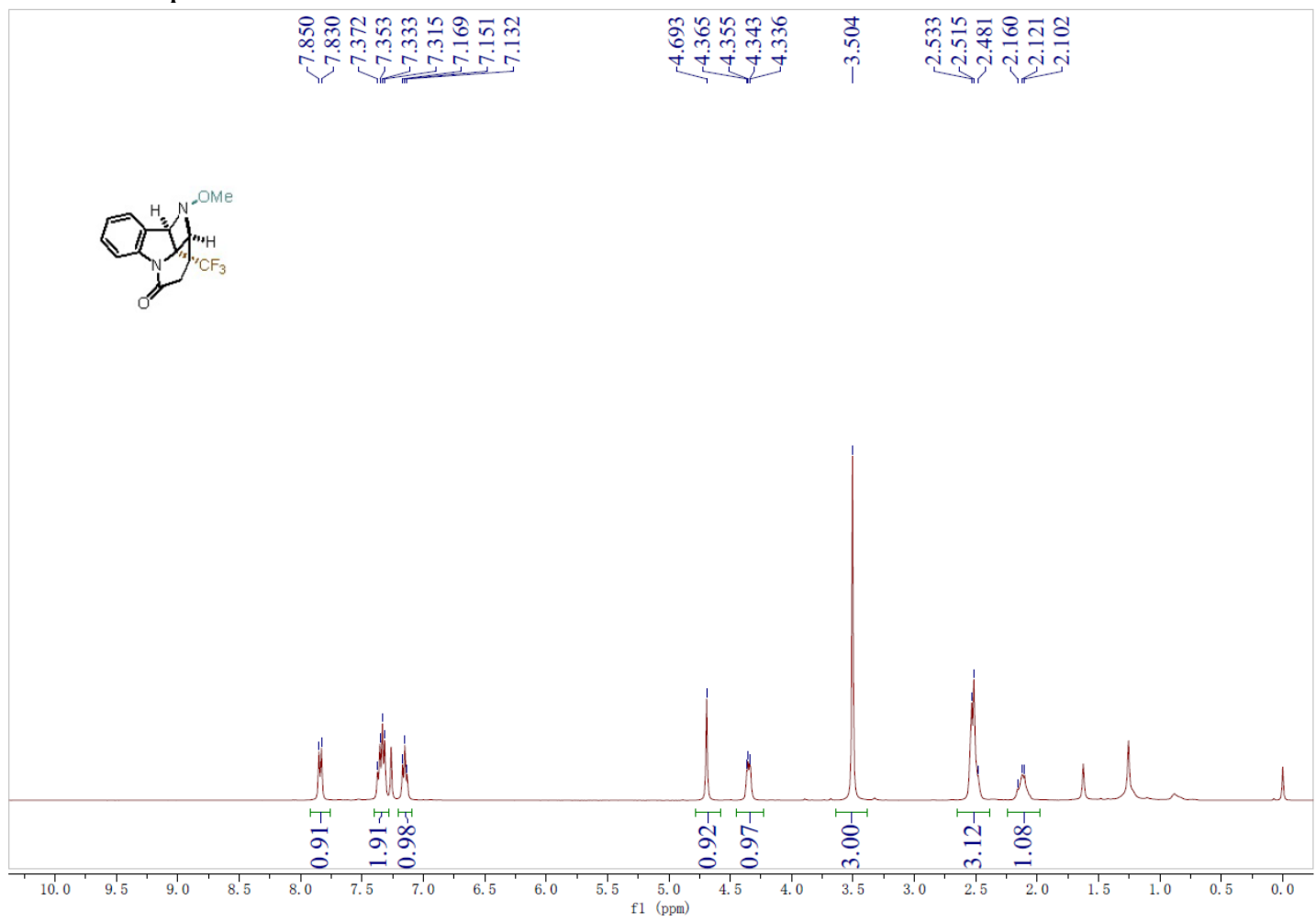

${ }^{13} \mathrm{C}$ NMR Spectrum of $\mathbf{2 k}$

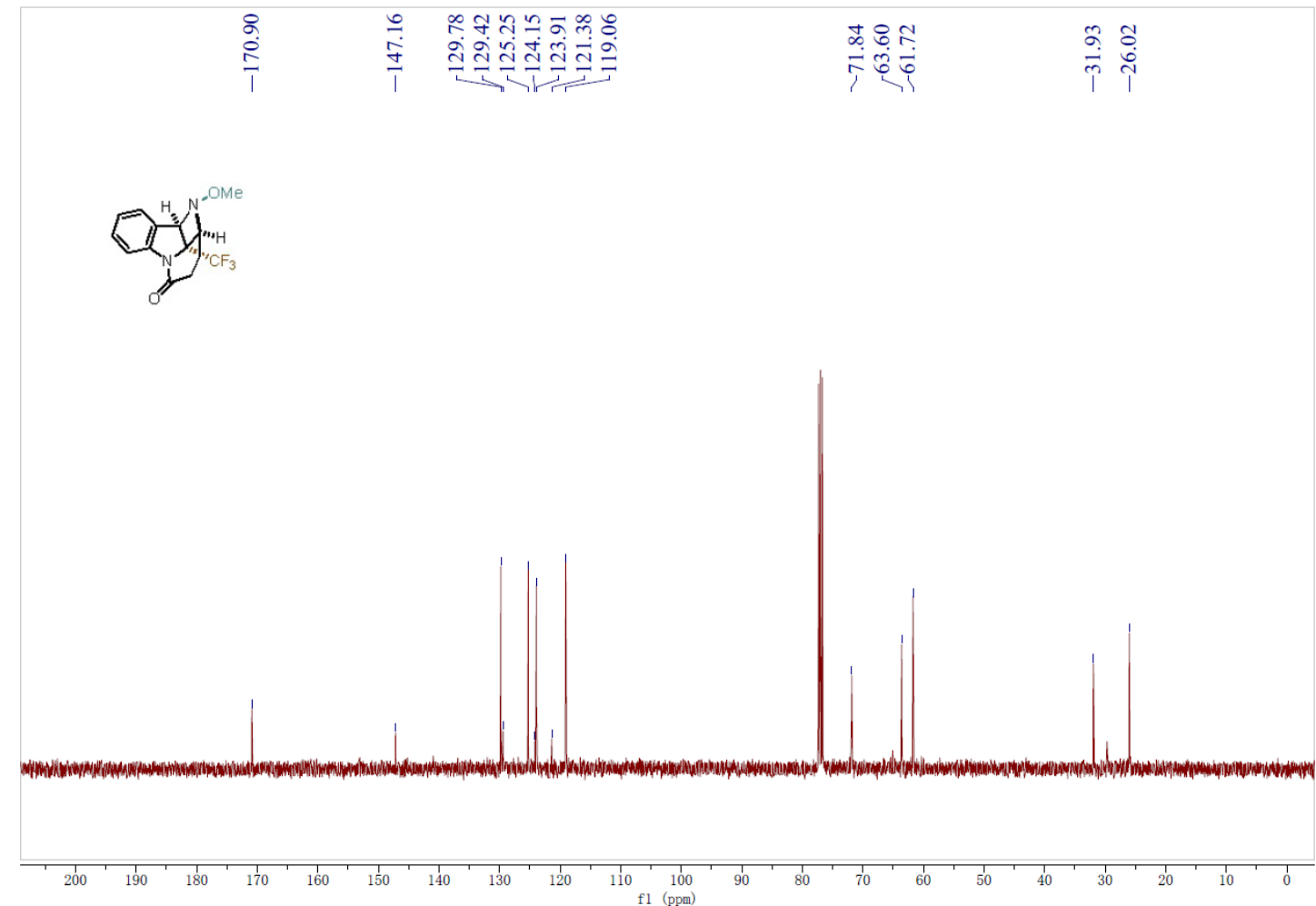


${ }^{19}$ F NMR Spectrum of $\mathbf{2 k}$

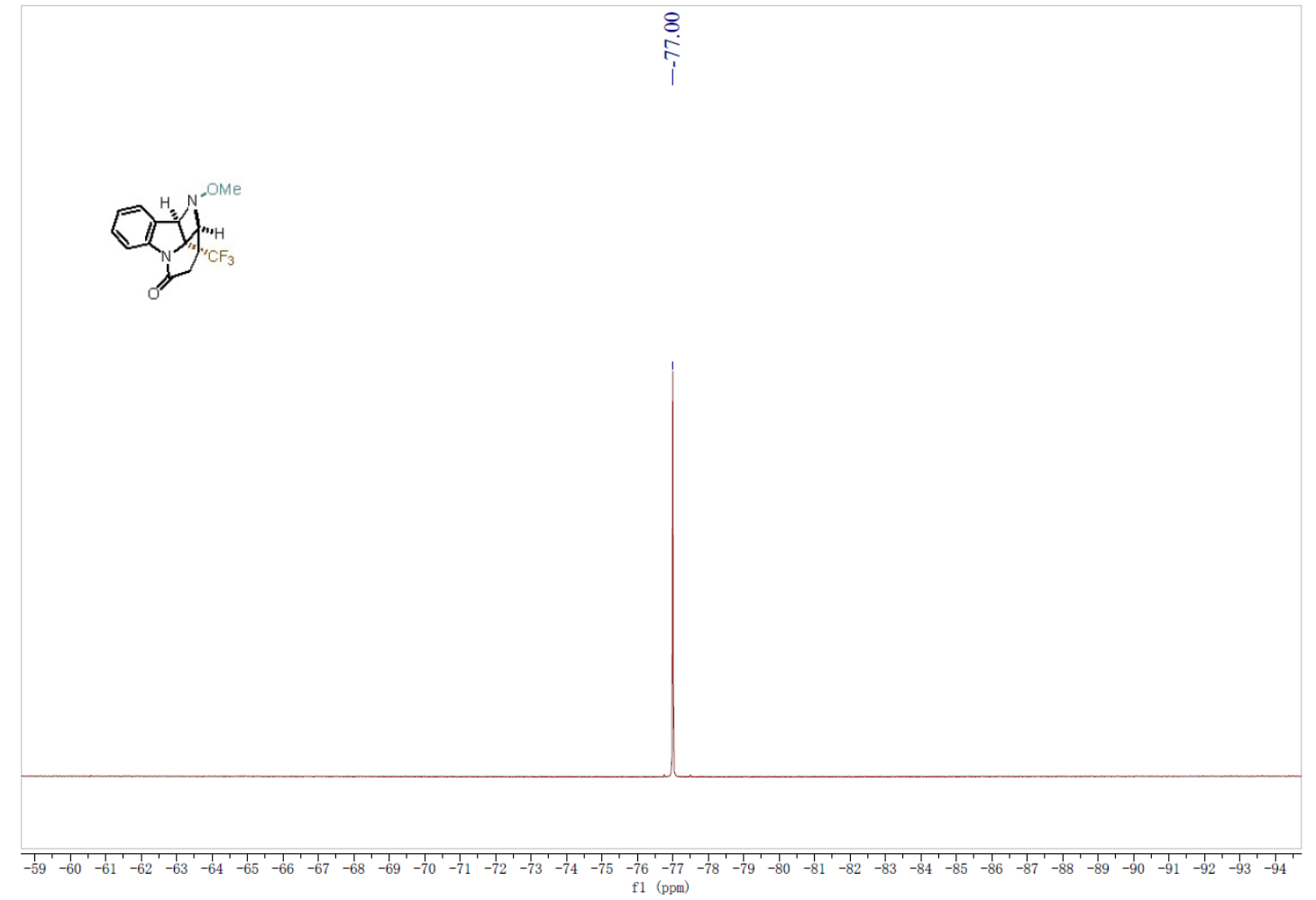


${ }^{1} \mathrm{H}$ NMR Spectrum of $\mathbf{2 l}$

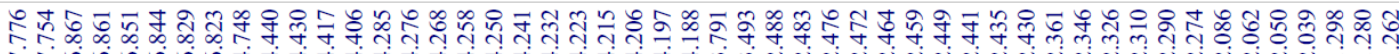

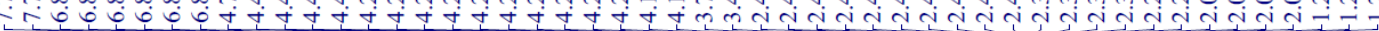

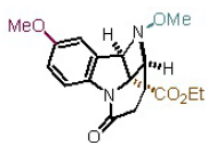

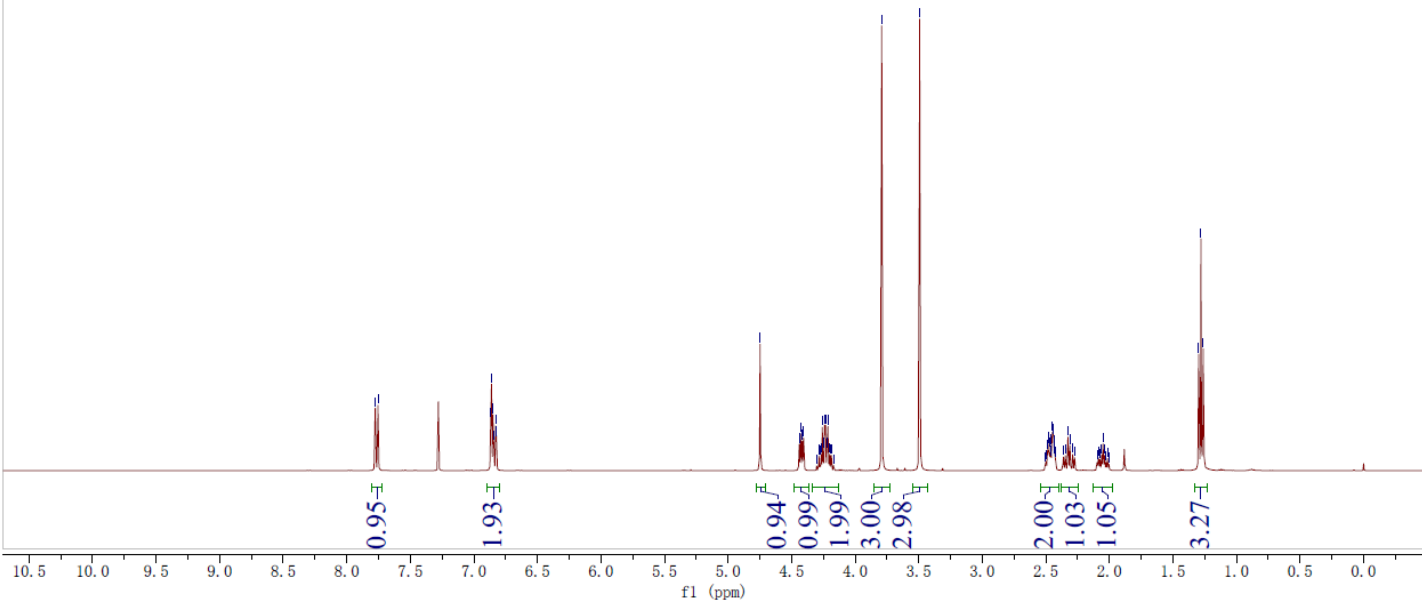

${ }^{13} \mathrm{C}$ NMR Spectrum of $\mathbf{2 l}$

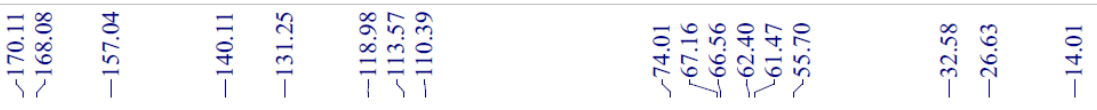

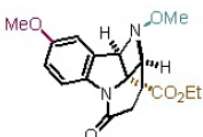
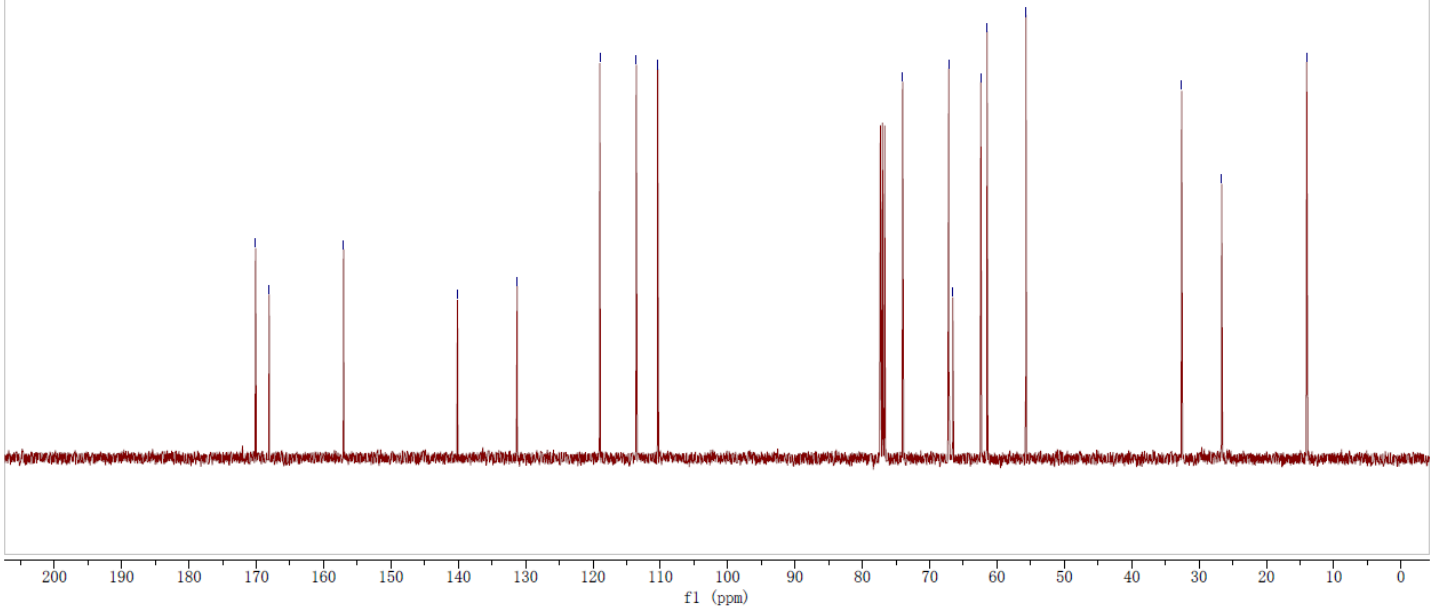
${ }^{1} \mathrm{H}$ NMR Spectrum of $\mathbf{2 m}$

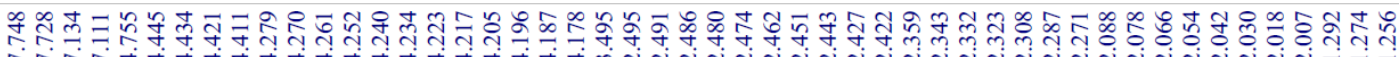

cind

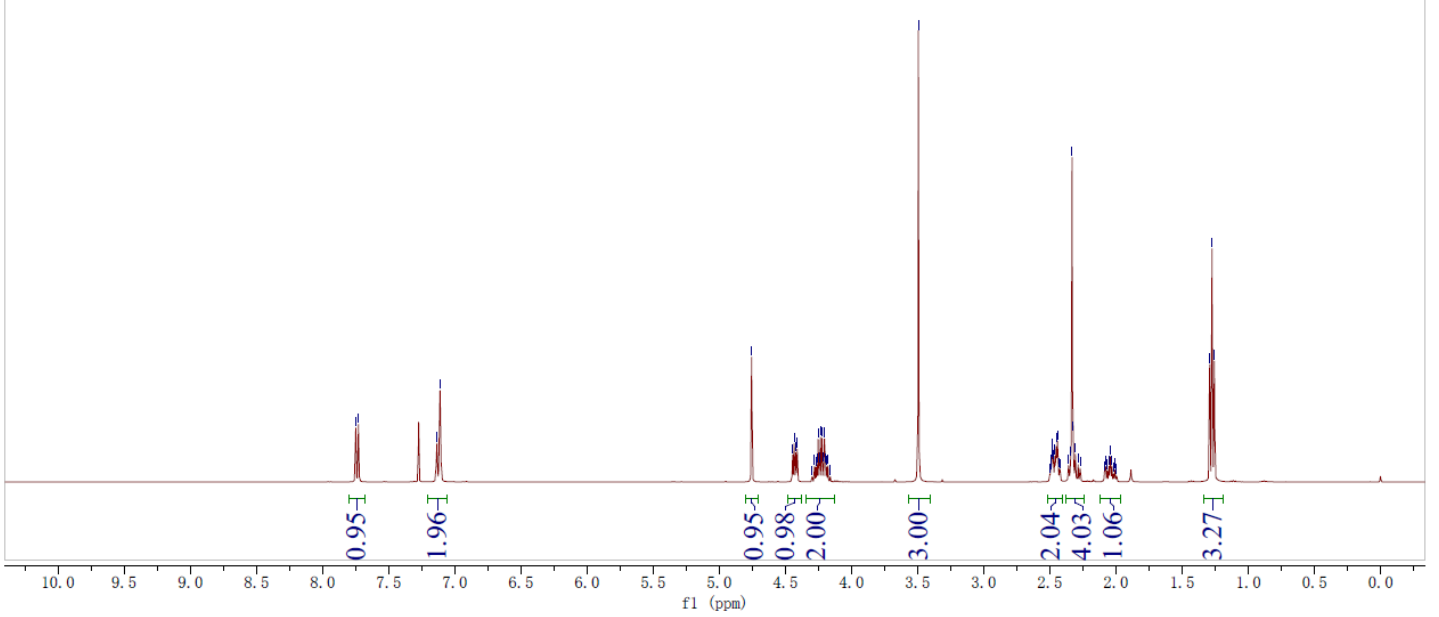

${ }^{13} \mathrm{C}$ NMR Spectrum of $\mathbf{2 m}$

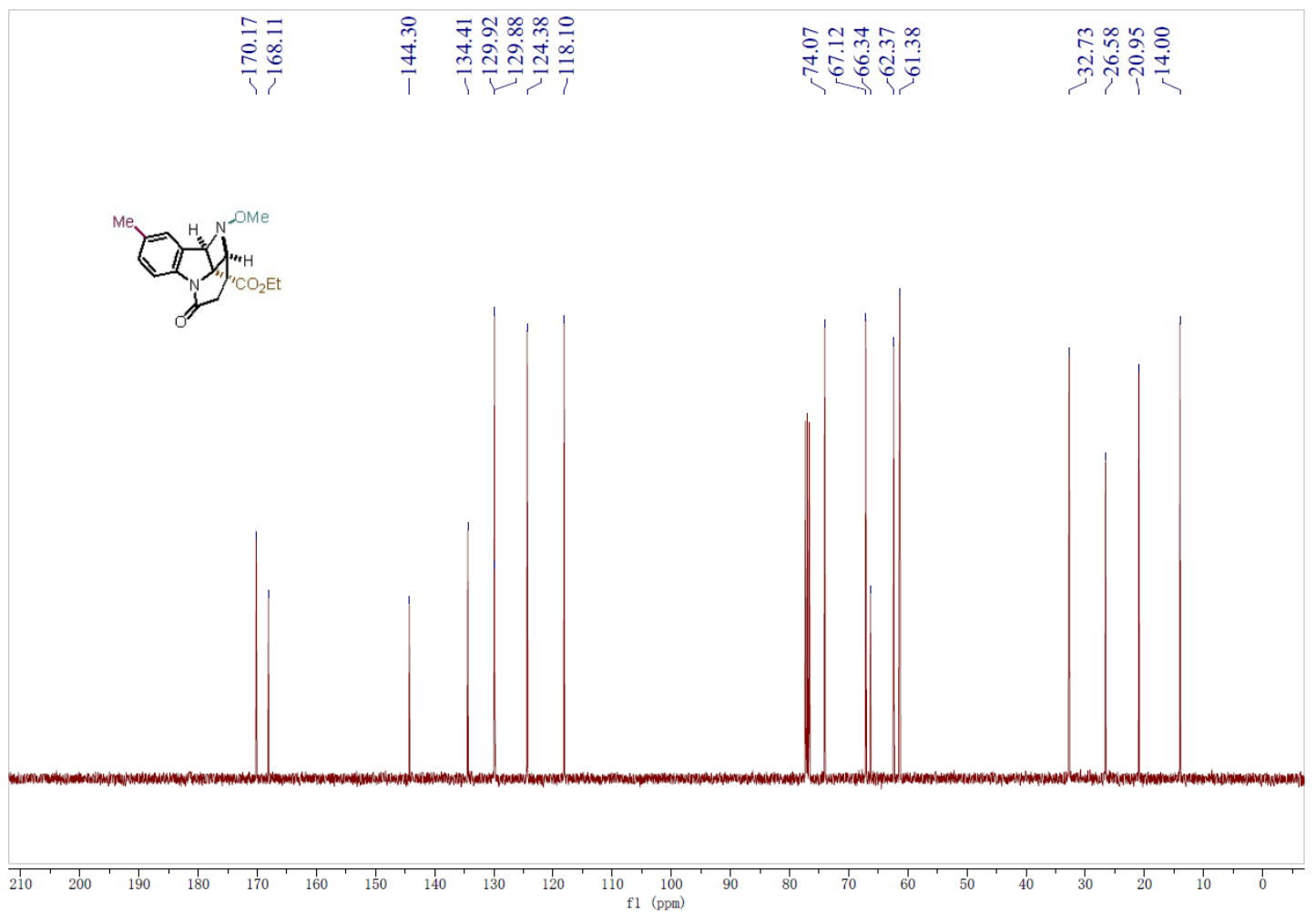


${ }^{1} \mathrm{H}$ NMR Spectrum of $\mathbf{2 n}$

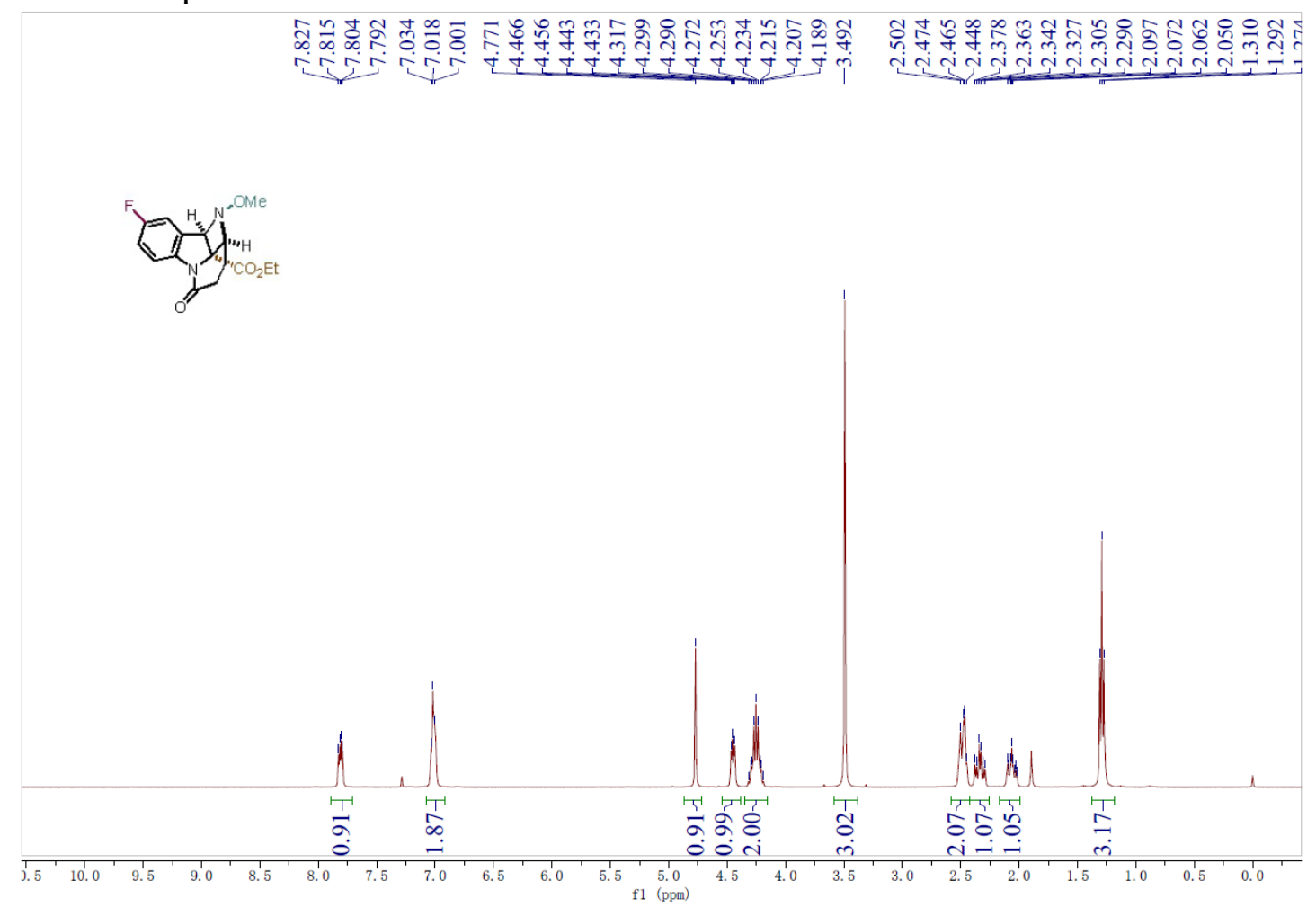

${ }^{13} \mathrm{C}$ NMR Spectrum of $\mathbf{2 n}$

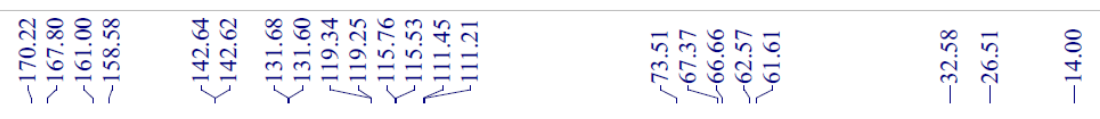

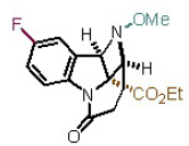
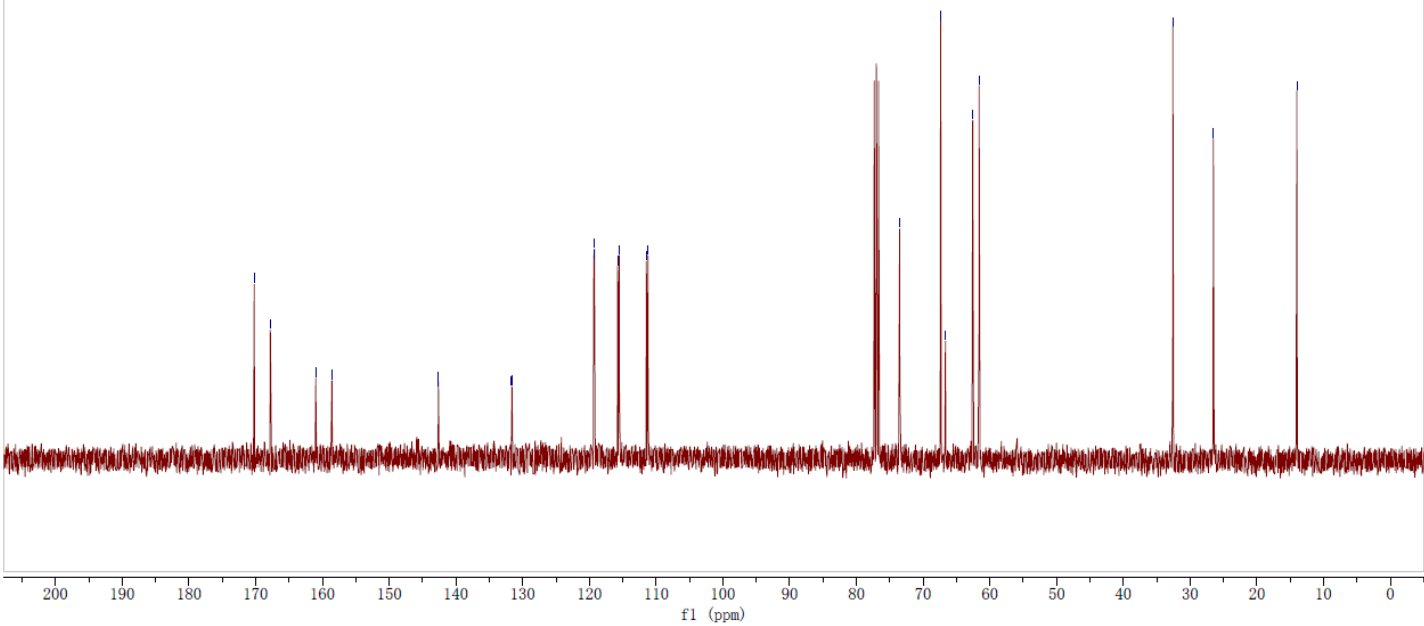
${ }^{19}$ F NMR Spectrum of $\mathbf{2 n}$

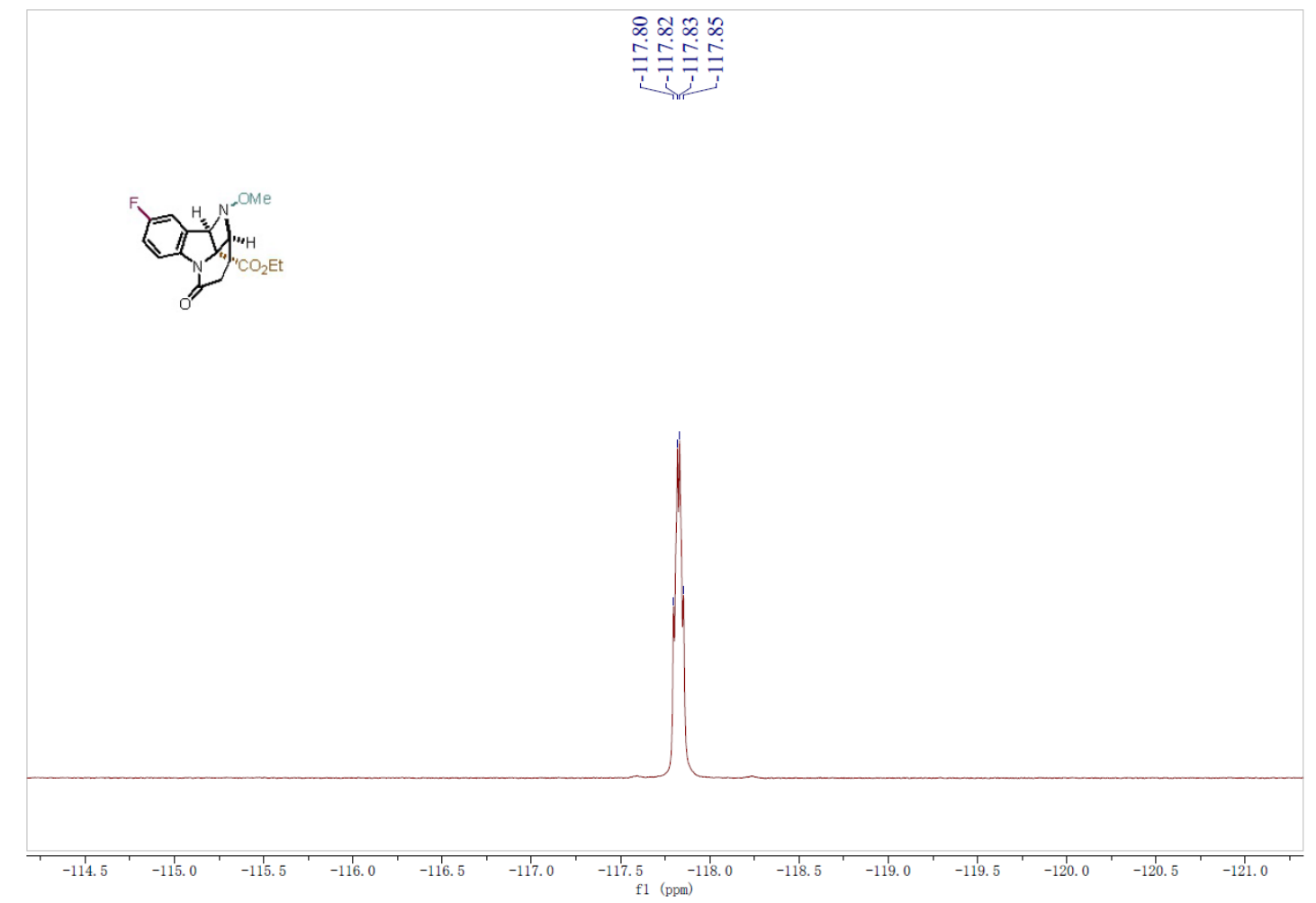


${ }^{1} \mathrm{H}$ NMR Spectrum of $\mathbf{2 0}$

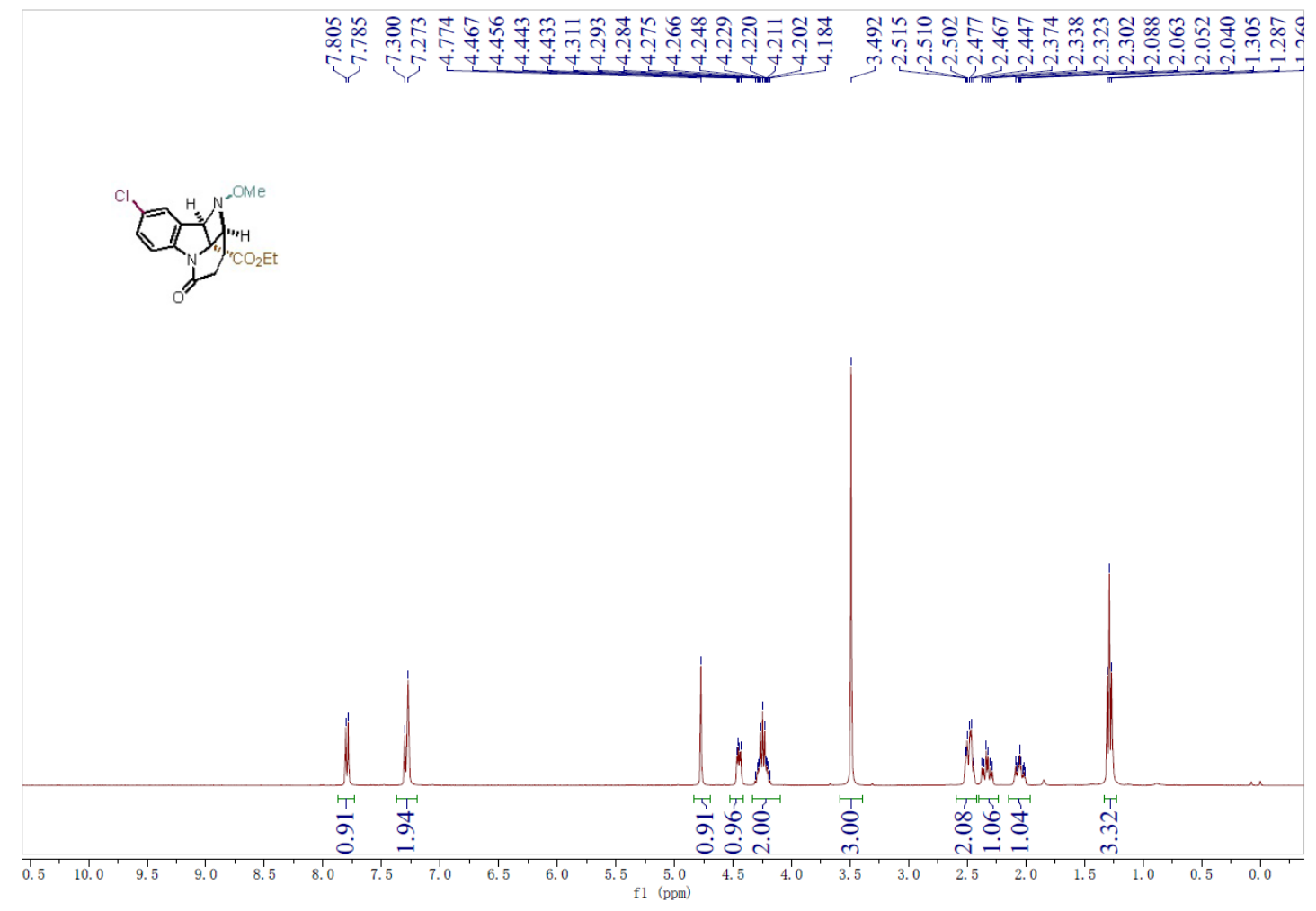

${ }^{13} \mathrm{C}$ NMR Spectrum of $\mathbf{2 0}$

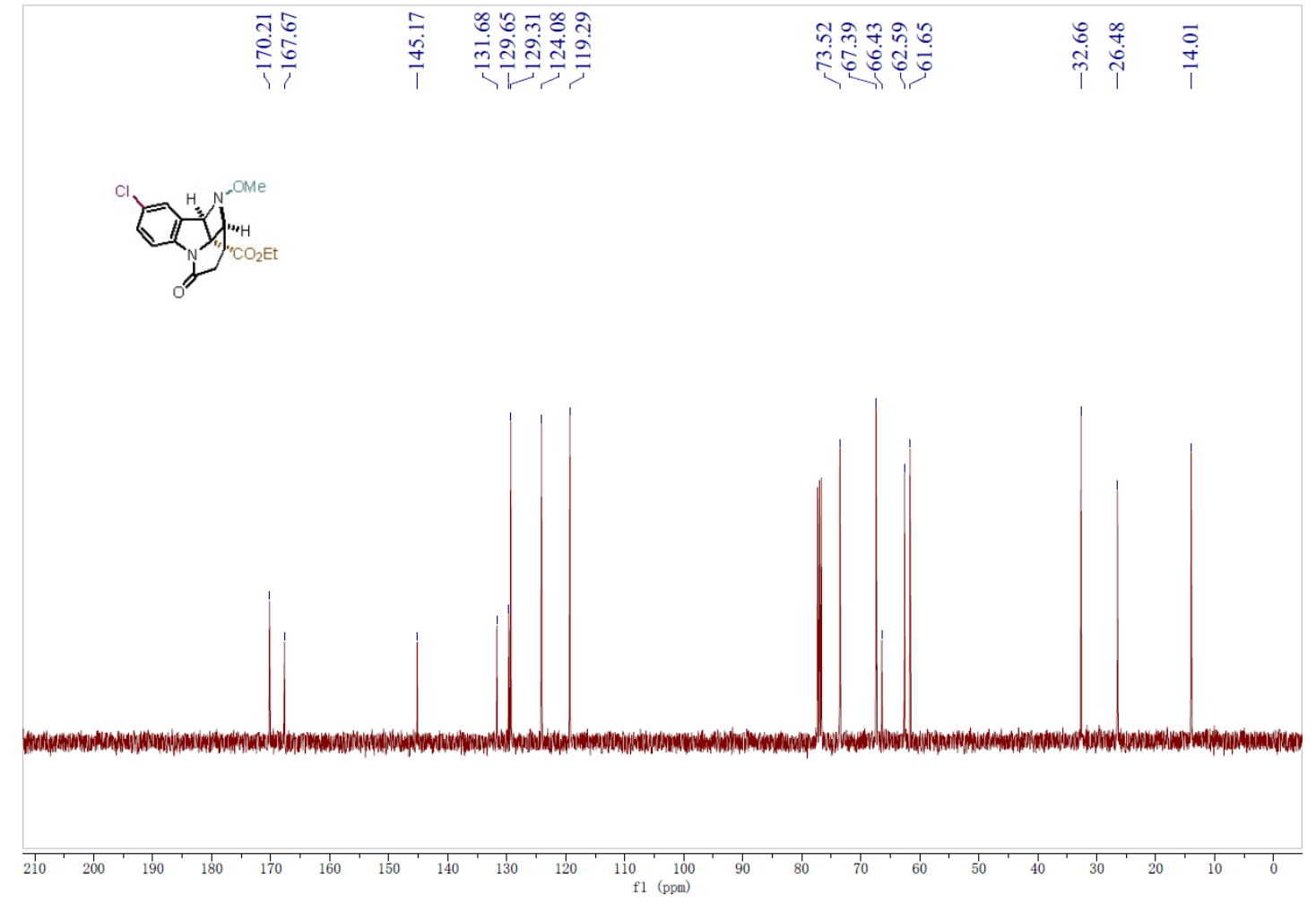


${ }^{1} \mathrm{H}$ NMR Spectrum of $\mathbf{2 p}$

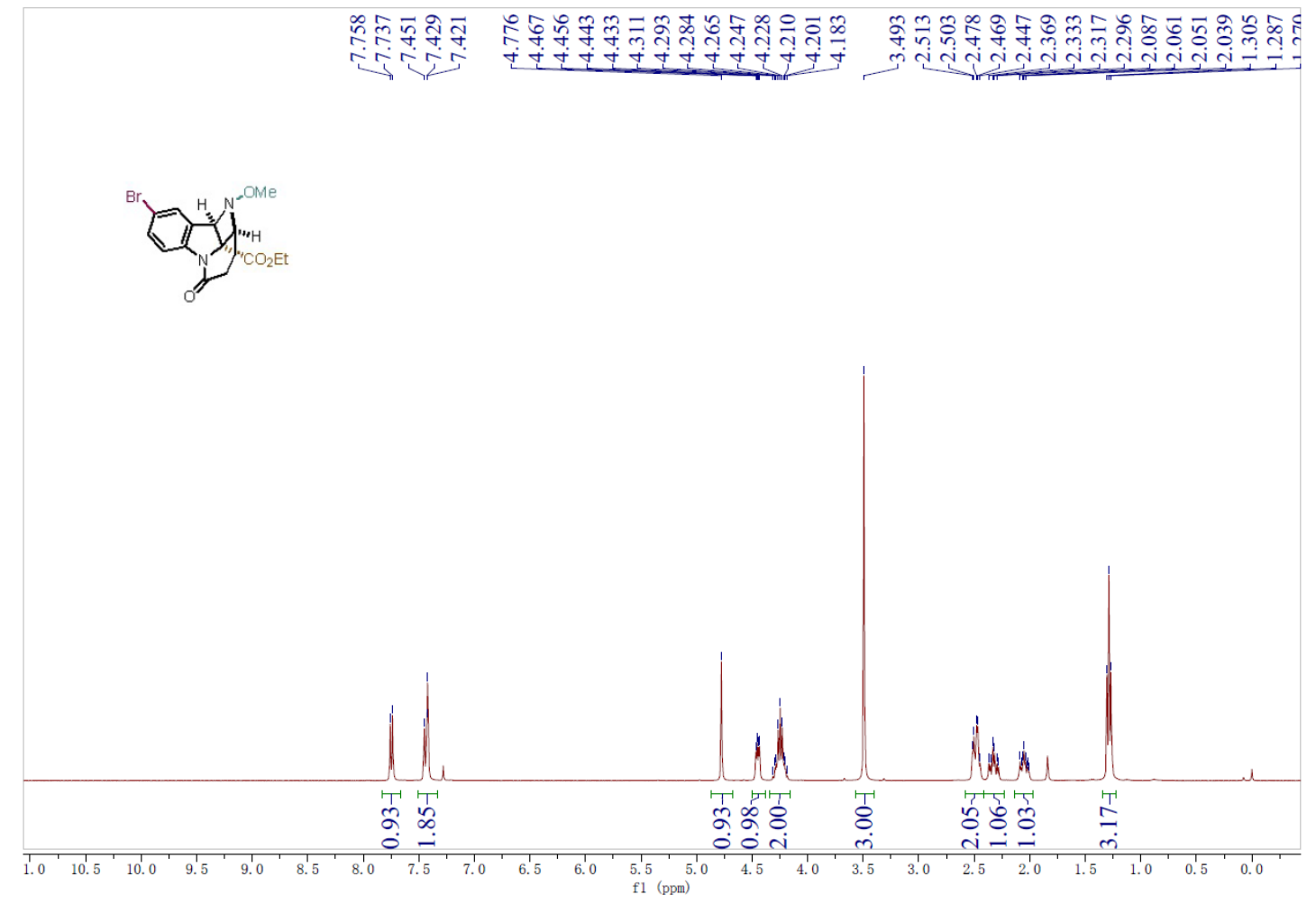

${ }^{13} \mathrm{C}$ NMR Spectrum of $\mathbf{2 p}$

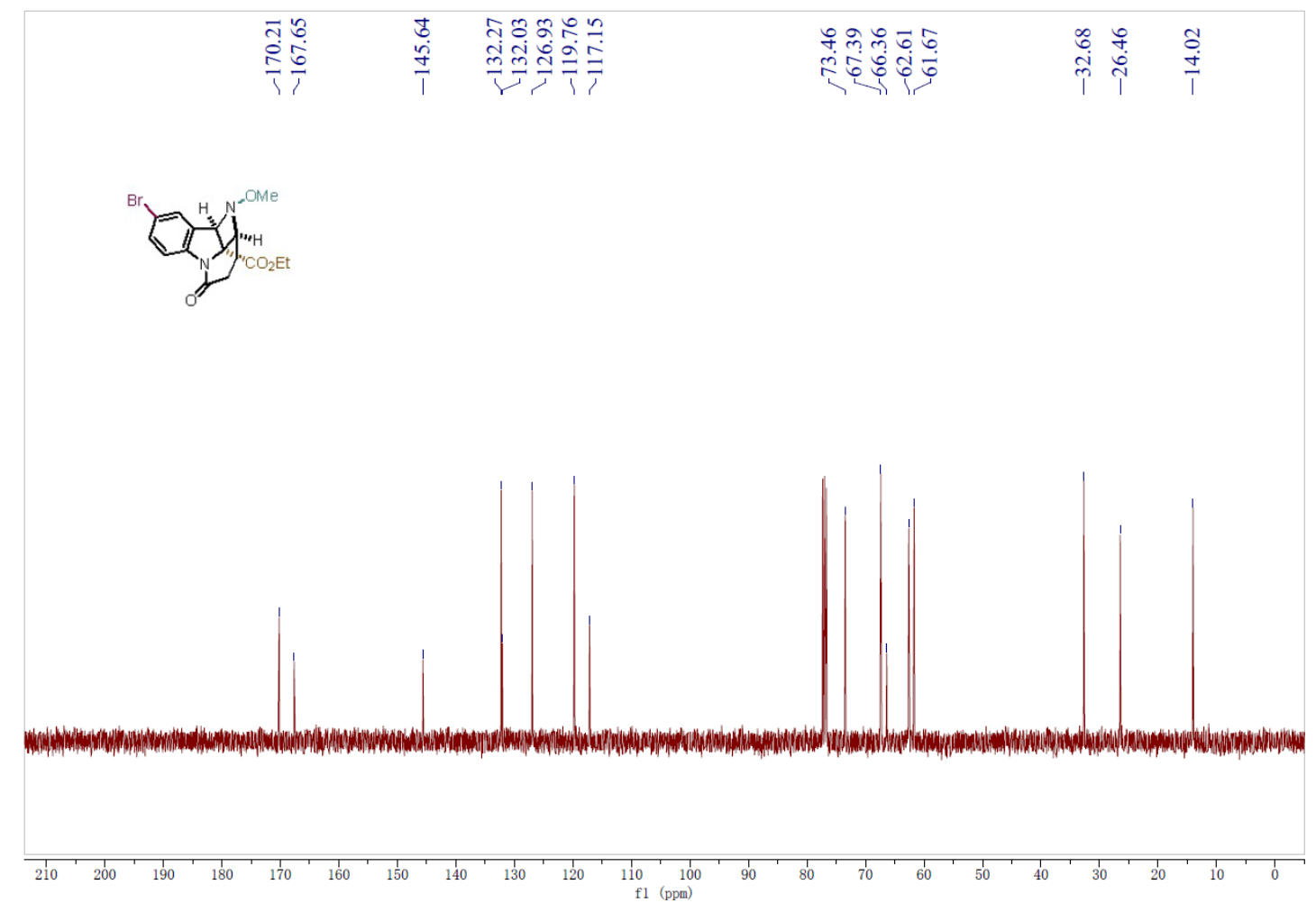


${ }^{1} \mathrm{H}$ NMR Spectrum of $\mathbf{2 q}$

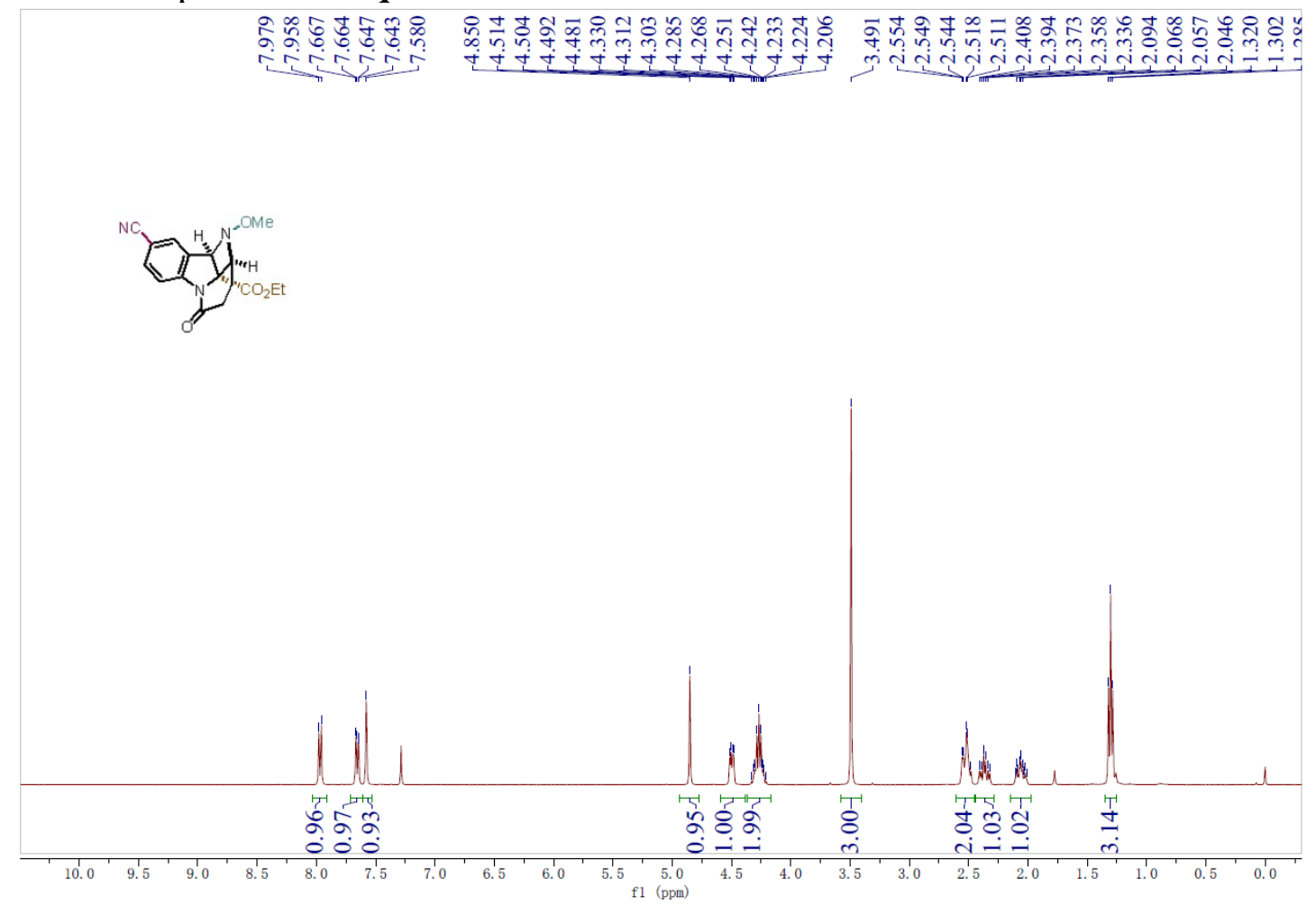

${ }^{13} \mathrm{C}$ NMR Spectrum of $\mathbf{2 q}$

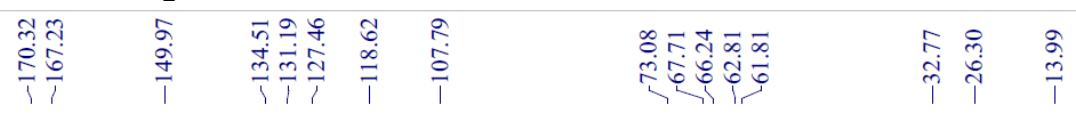

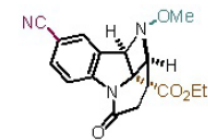
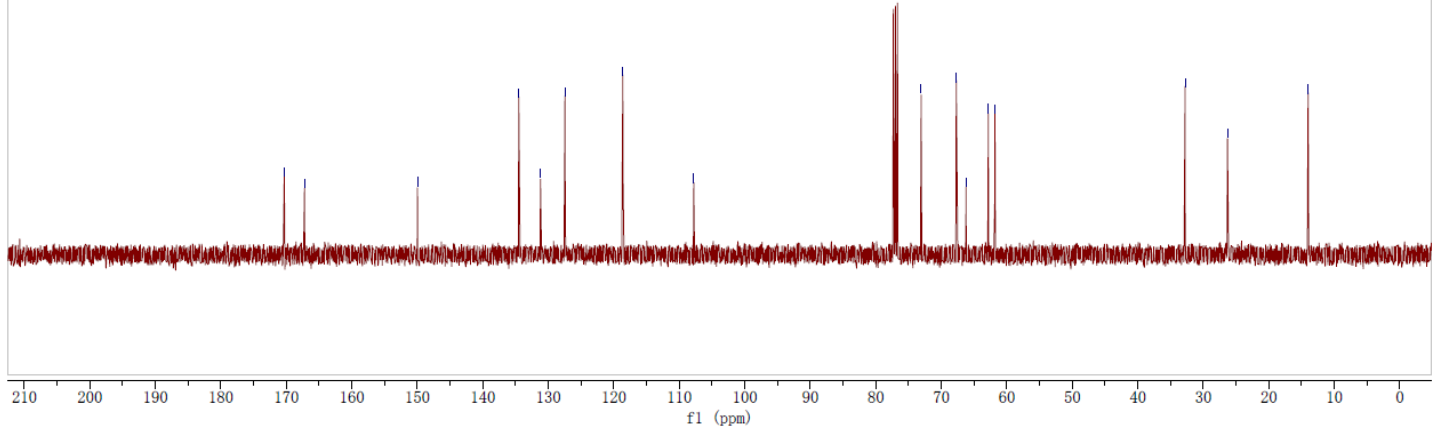
${ }^{1} \mathrm{H}$ NMR Spectrum of $\mathbf{2 r}$

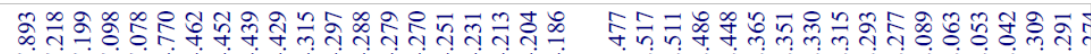

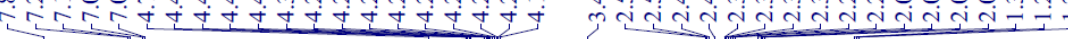
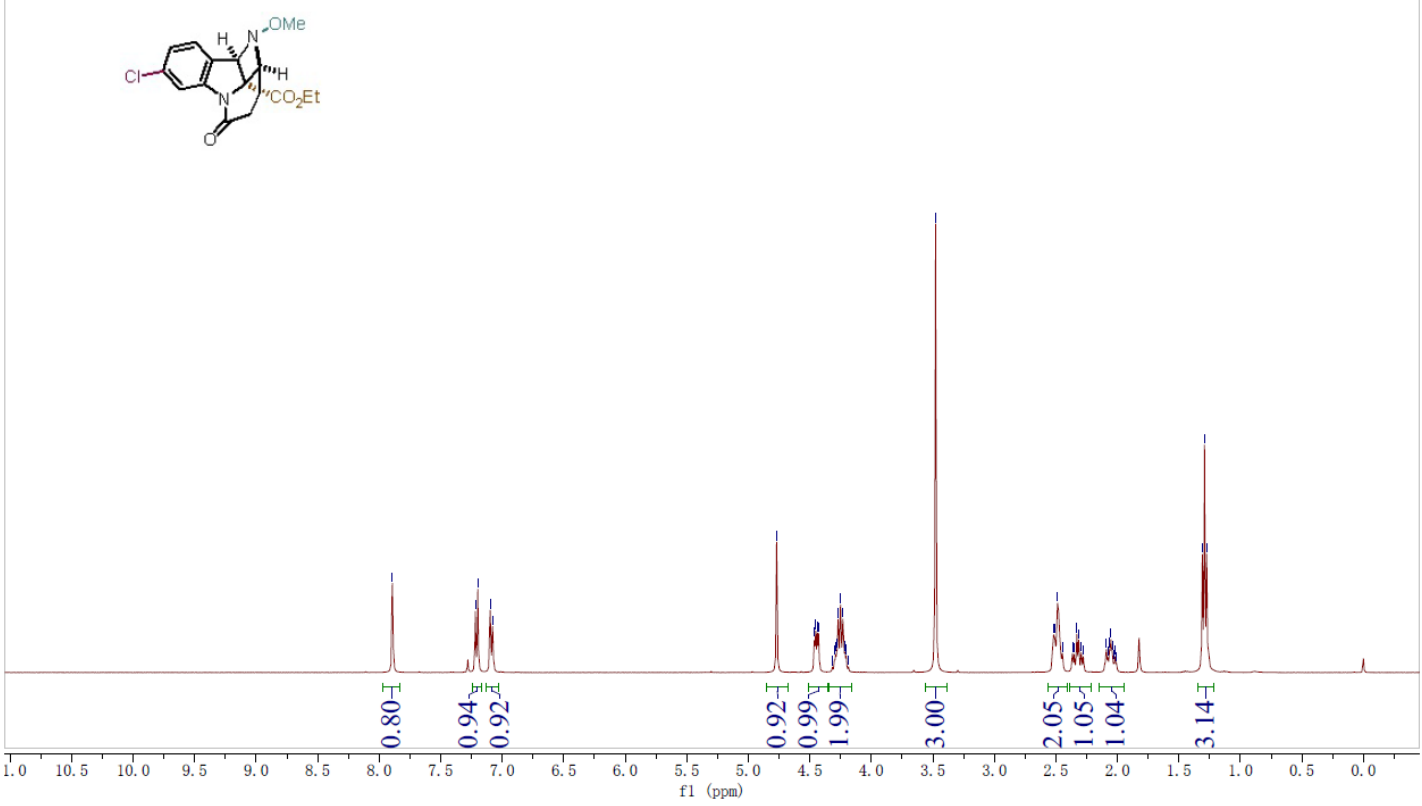

${ }^{13} \mathrm{C}$ NMR Spectrum of $\mathbf{2 r}$

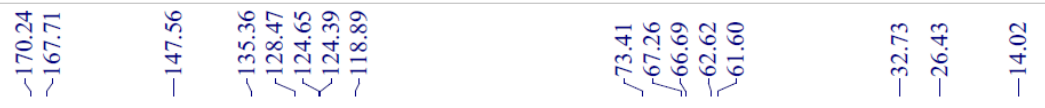
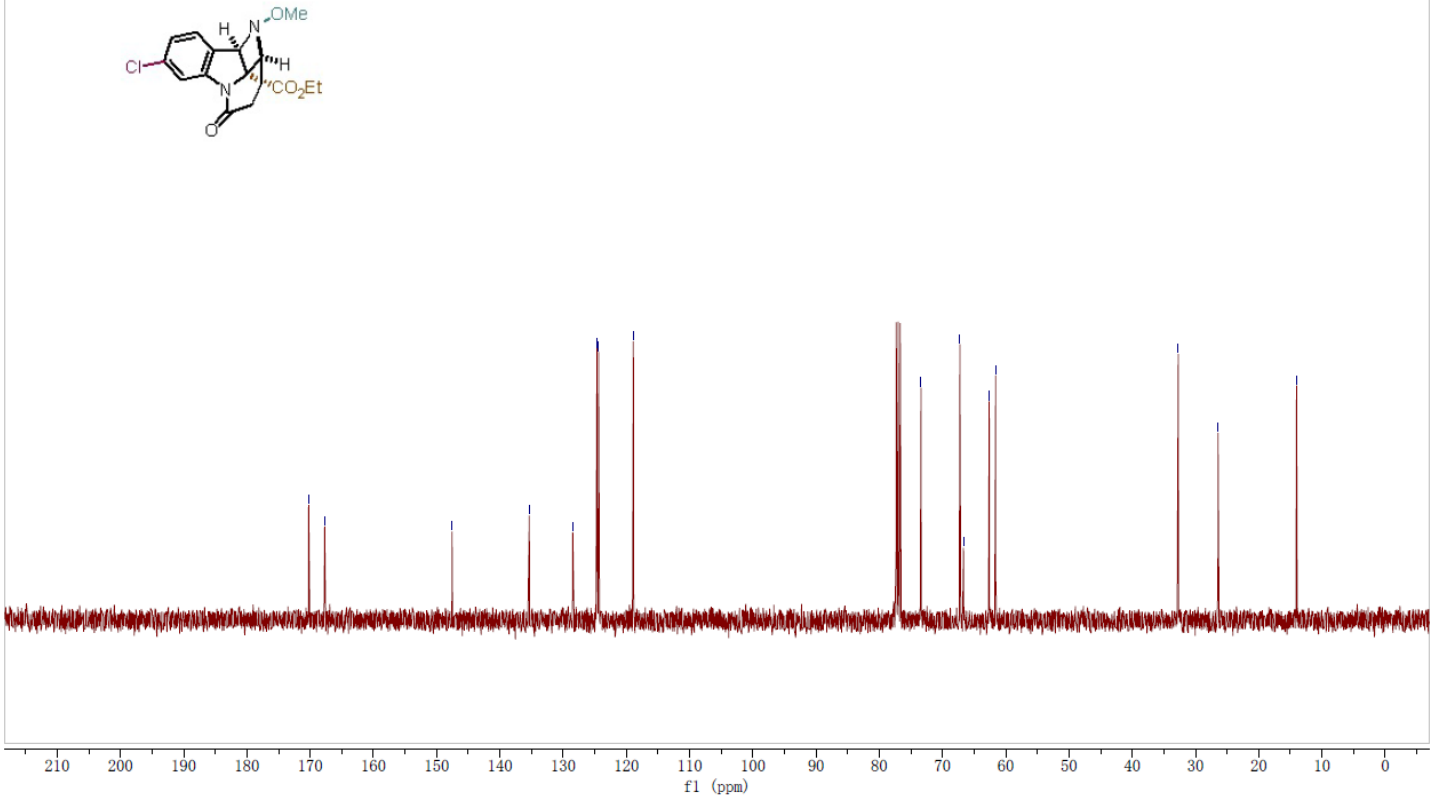
${ }^{1} \mathrm{H}$ NMR Spectrum of $\mathbf{2 s}$

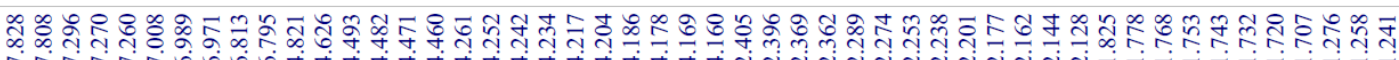

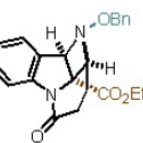

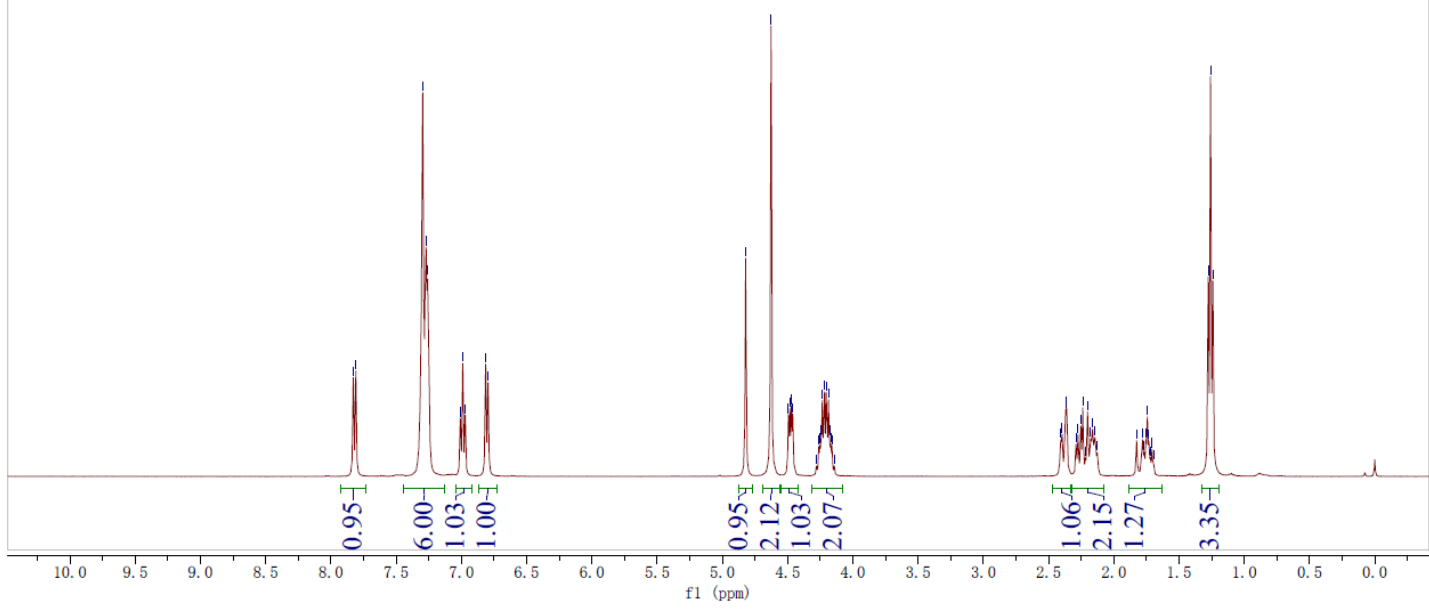

${ }^{13} \mathrm{C}$ NMR Spectrum of $2 \mathrm{~s}$

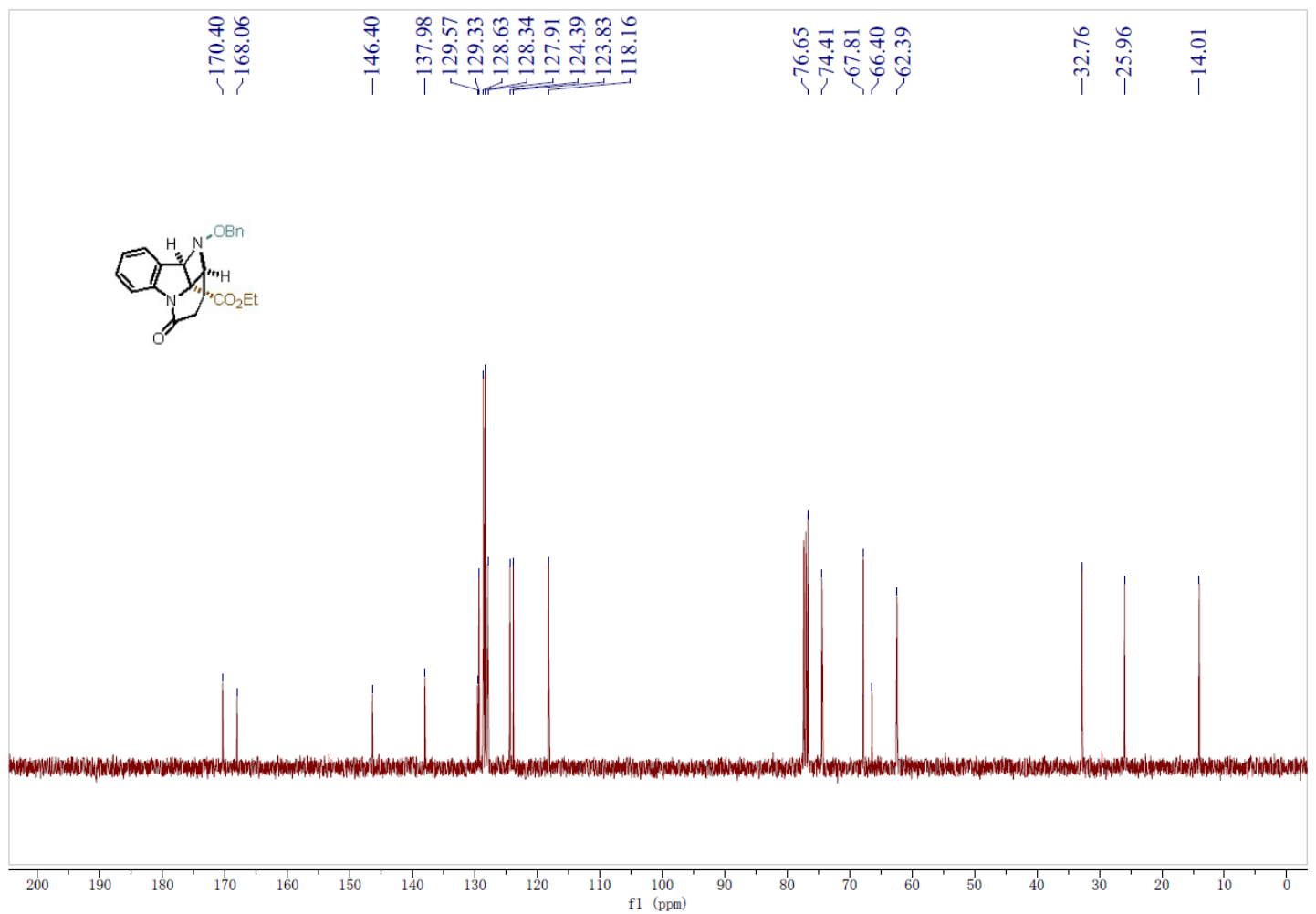


${ }^{1} \mathrm{H}$ NMR Spectrum of $\mathbf{2 t}$

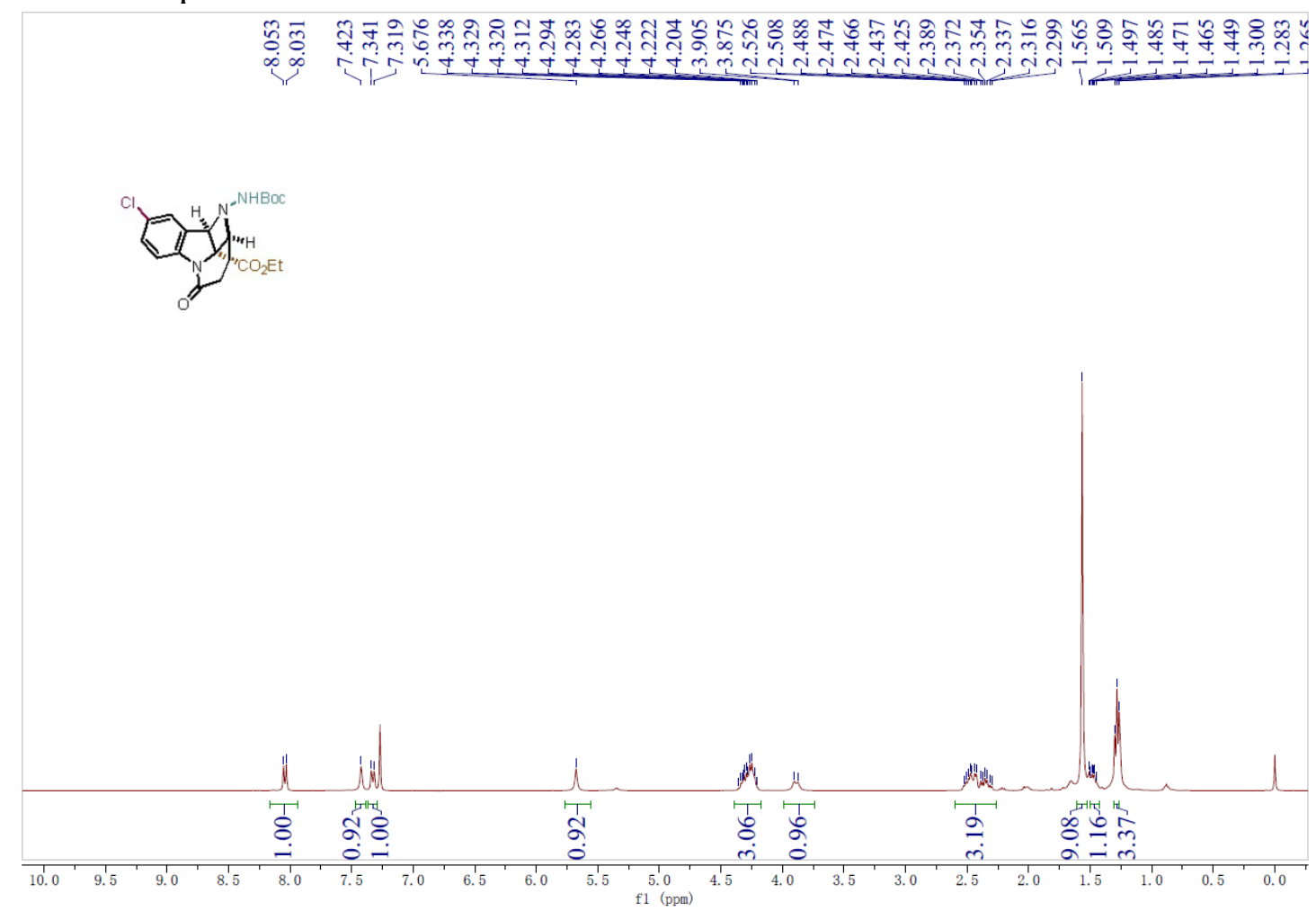

${ }^{13} \mathrm{C}$ NMR Spectrum of $\mathbf{2 t}$

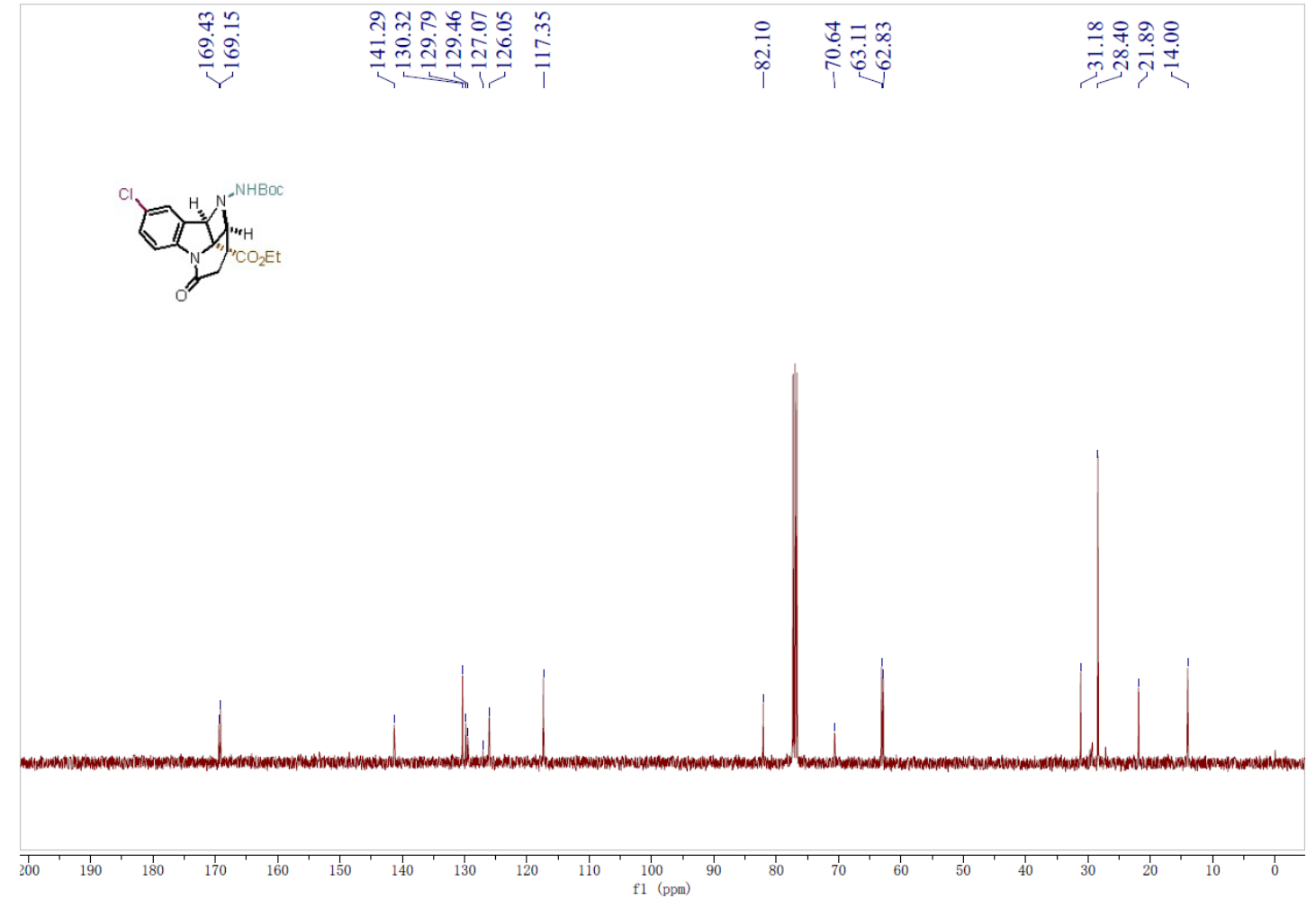


${ }^{1} \mathrm{H}$ NMR Spectrum of $\mathbf{4 a}$

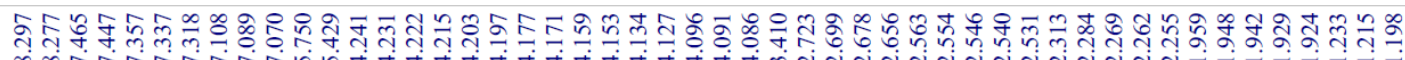

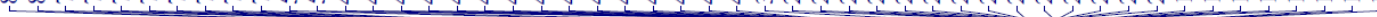<smiles>C=C1c2ccccc2N2C(=O)C=CC12NC</smiles>

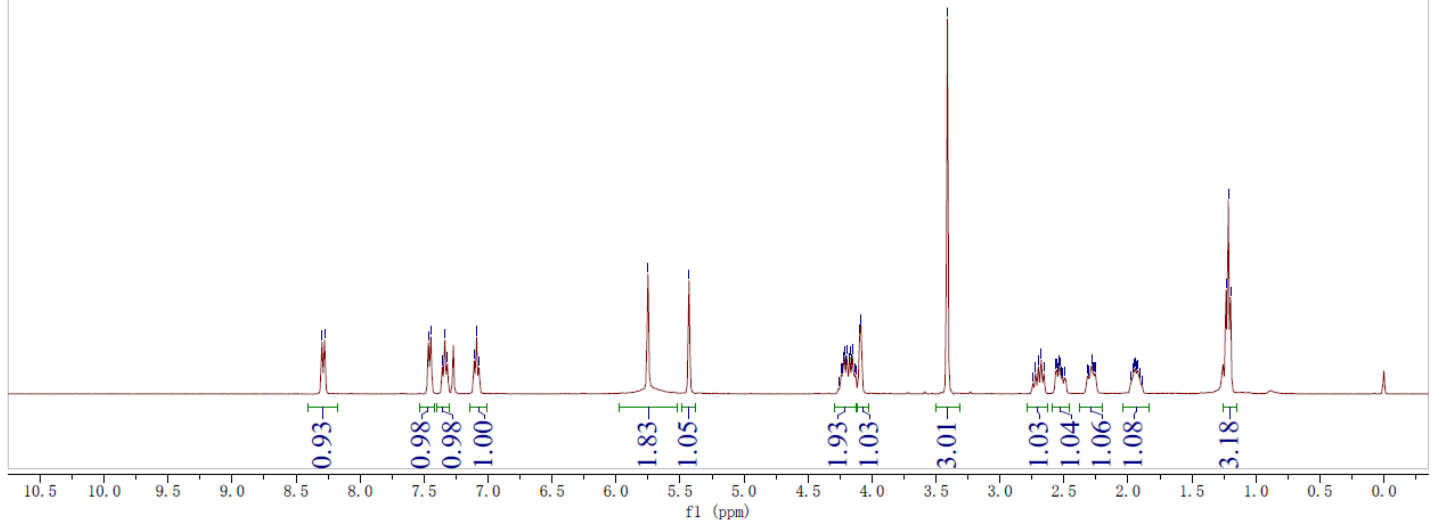

${ }^{13} \mathrm{C}$ NMR Spectrum of $\mathbf{4 a}$

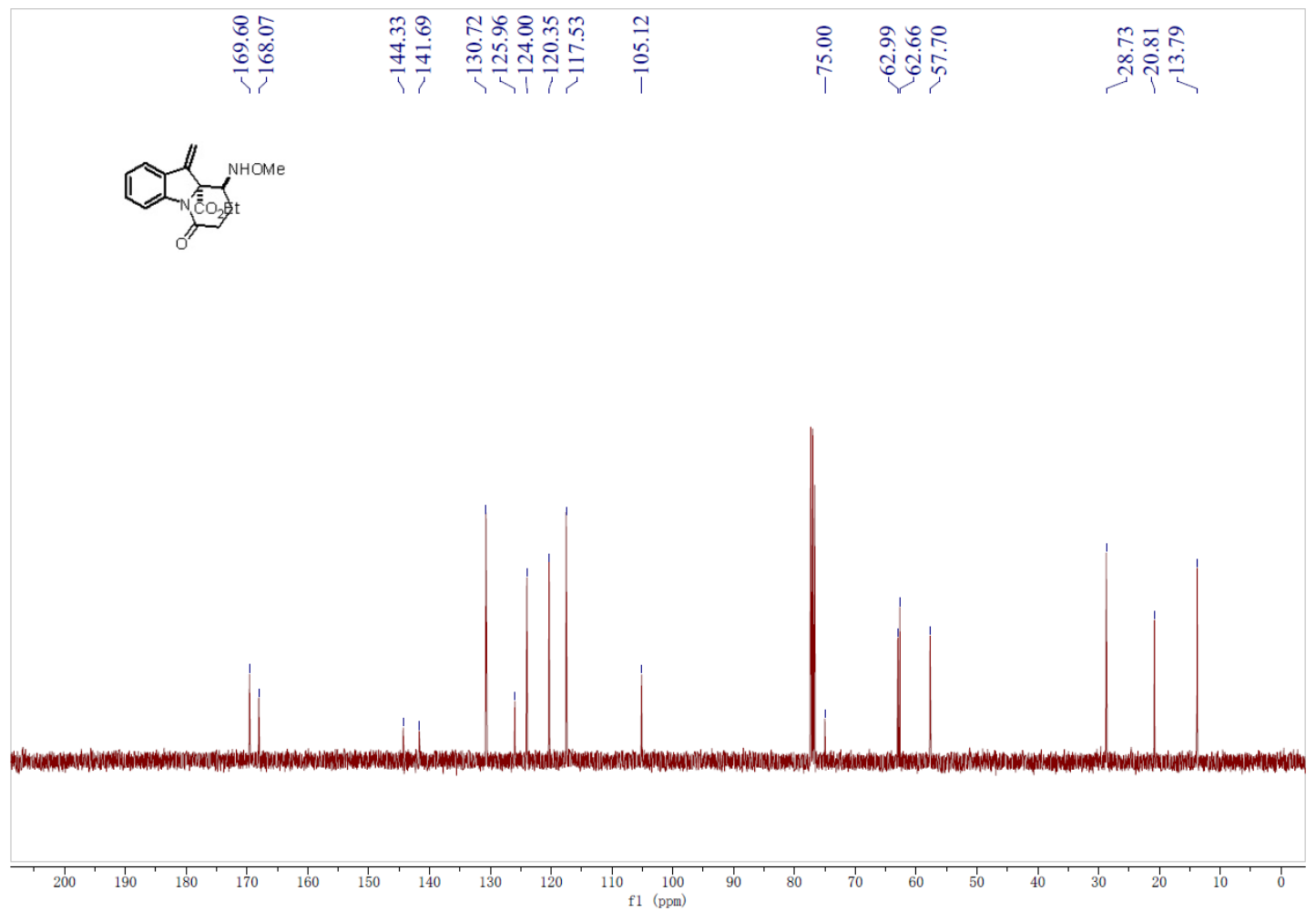


${ }^{1} \mathrm{H}$ NMR Spectrum of $\mathbf{4 b}$

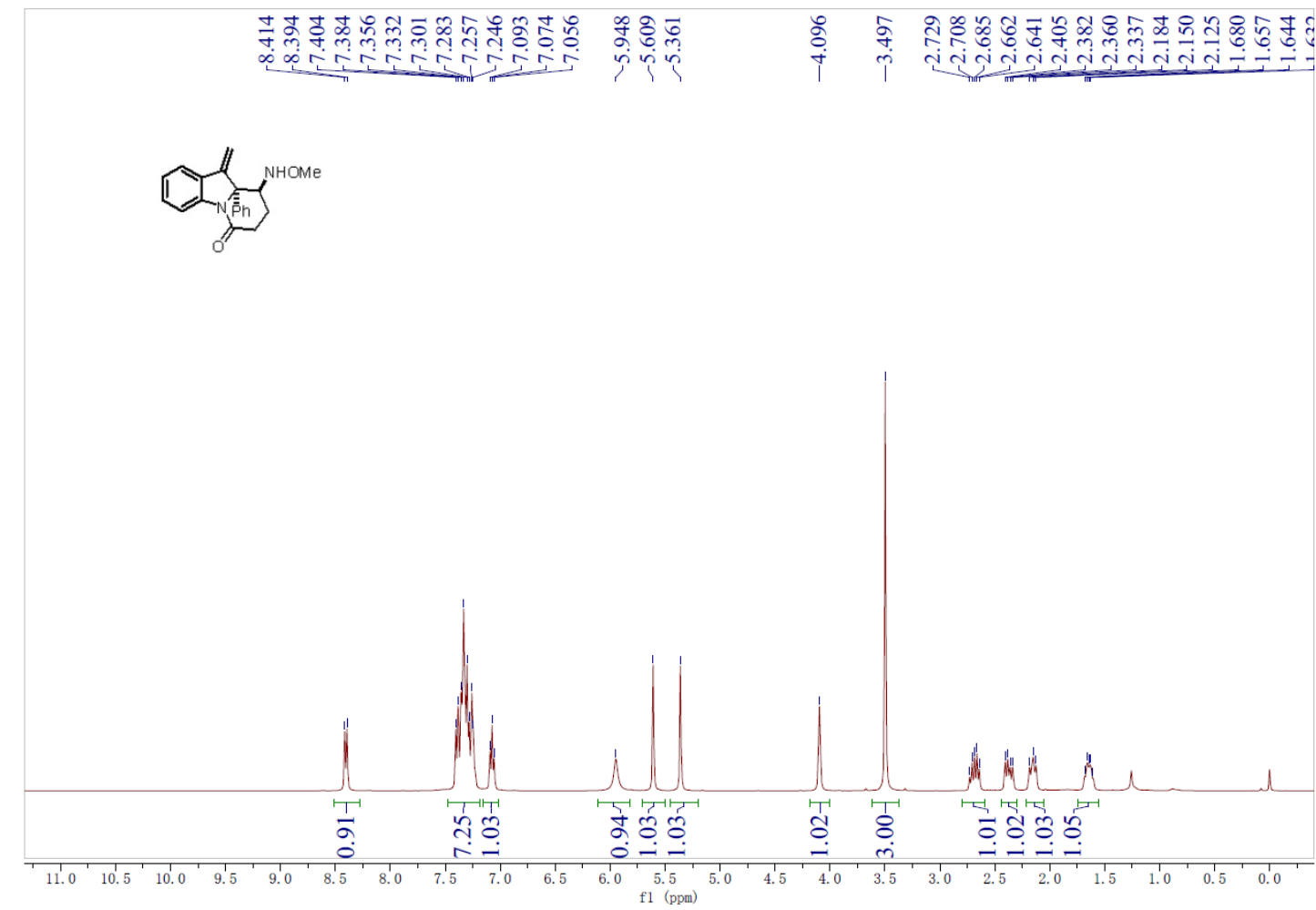

${ }^{13} \mathrm{C}$ NMR Spectrum of $\mathbf{4 b}$

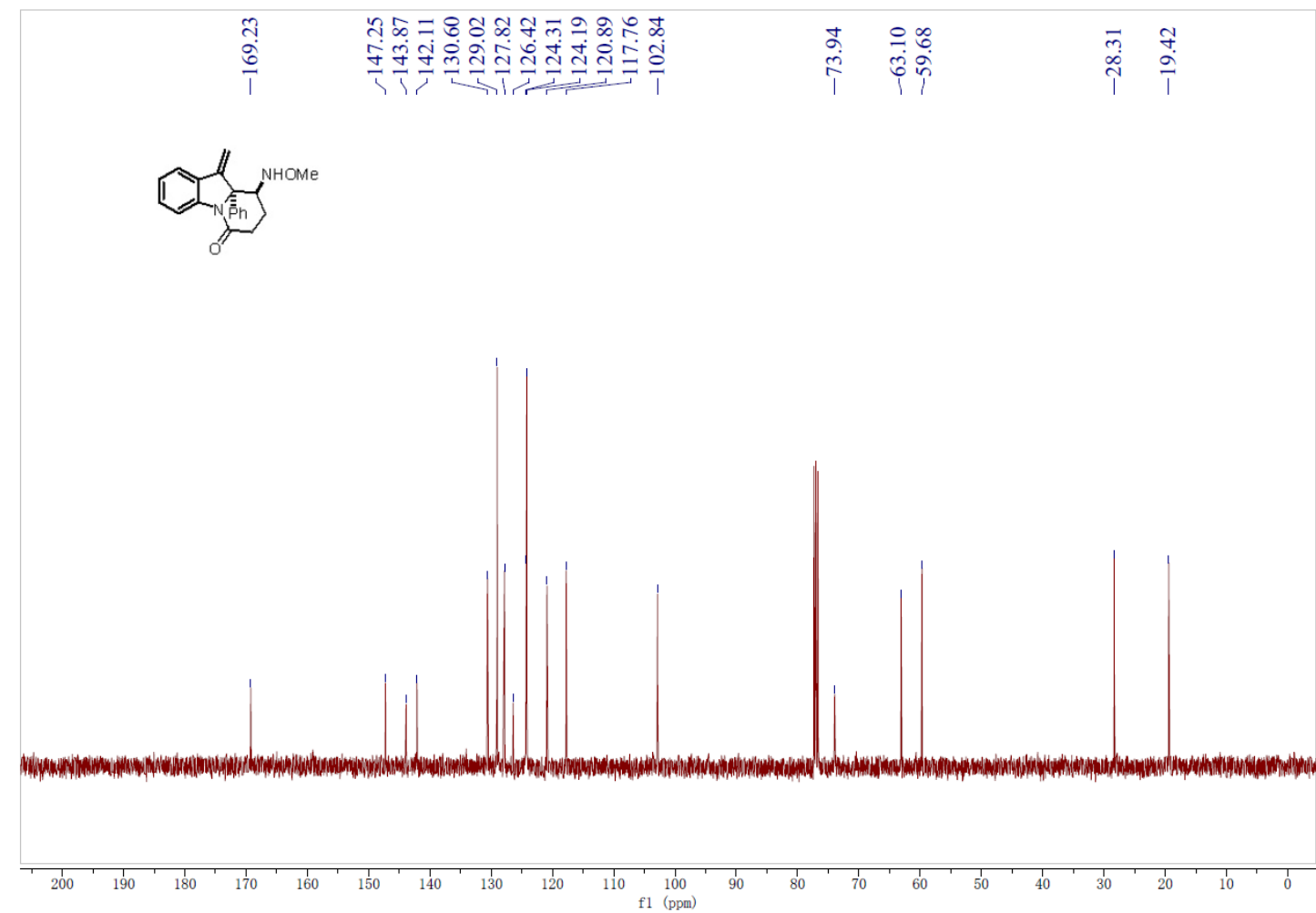


${ }^{1} \mathrm{H}$ NMR Spectrum of $\mathbf{4 c}$

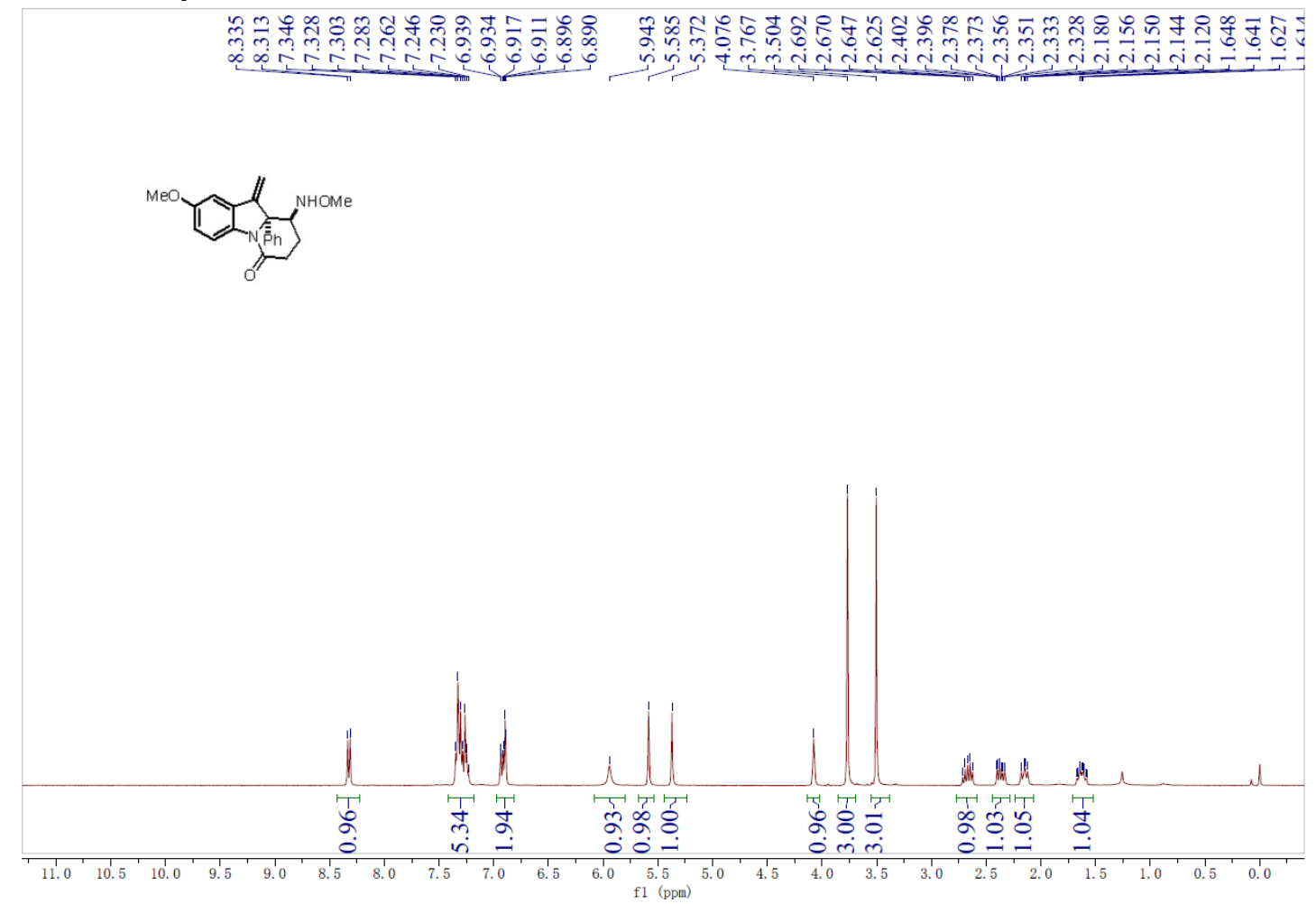

${ }^{13} \mathrm{C}$ NMR Spectrum of $\mathbf{4 c}$

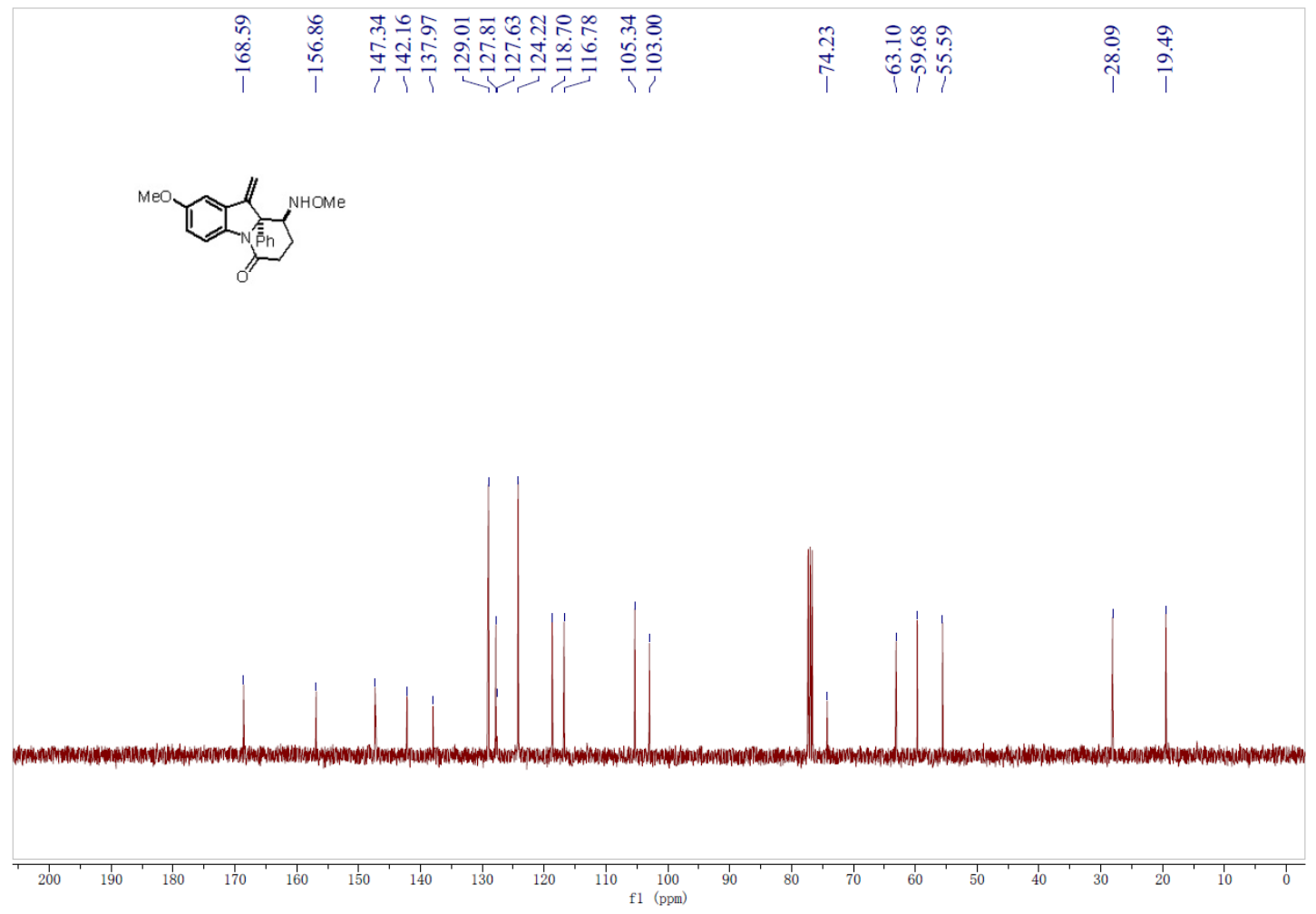


${ }^{1} \mathrm{H}$ NMR Spectrum of $\mathbf{4 d}$

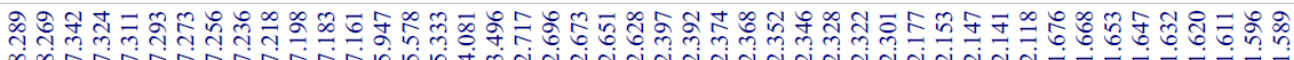

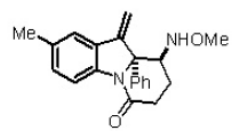

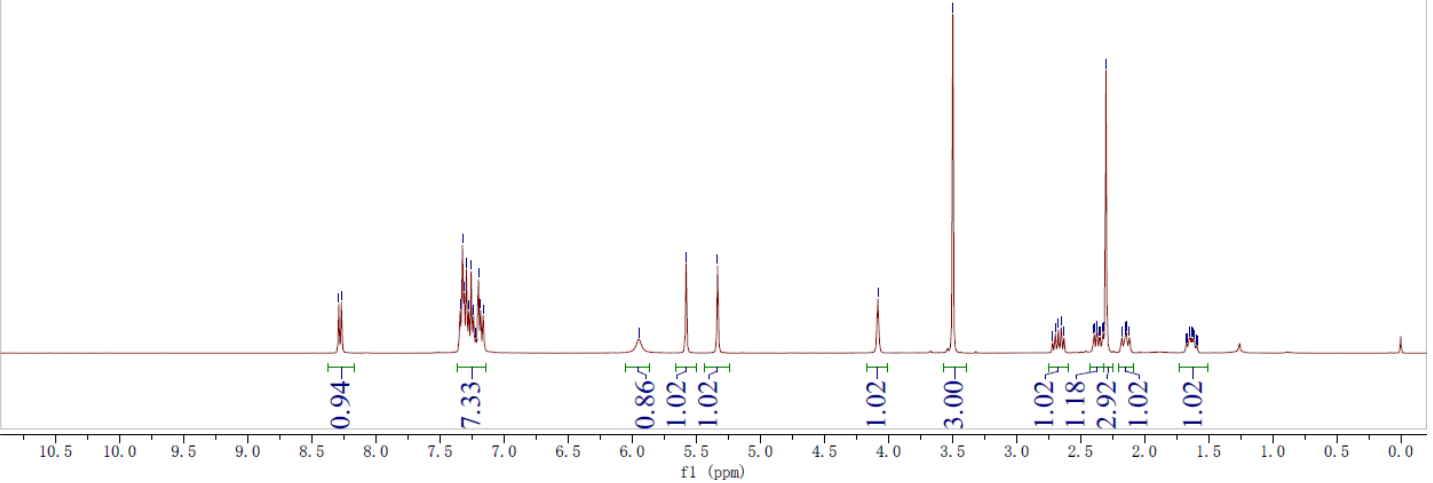

${ }^{13} \mathrm{C}$ NMR Spectrum of $\mathbf{4 d}$

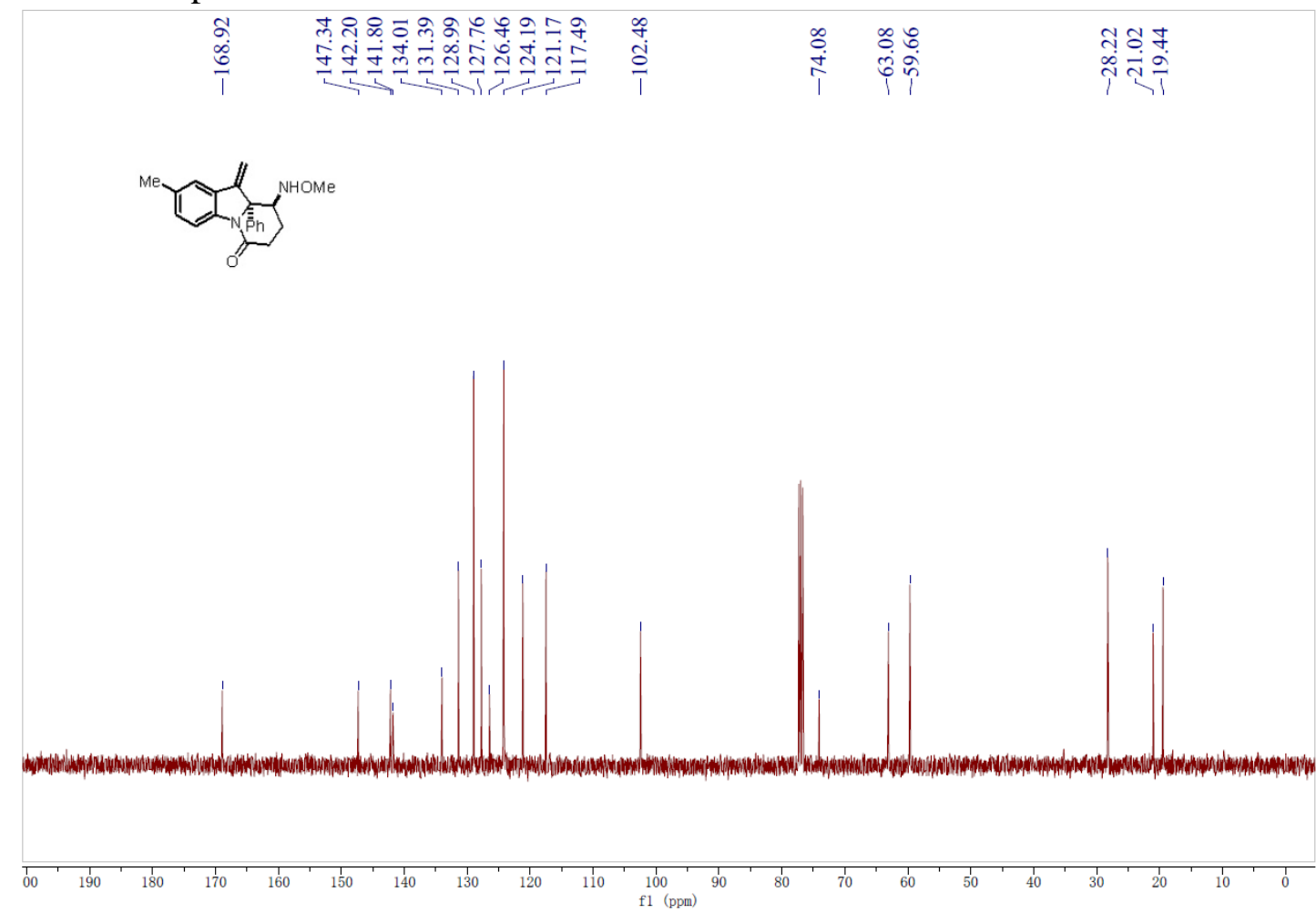


${ }^{1} \mathrm{H}$ NMR Spectrum of $\mathbf{4 e}$

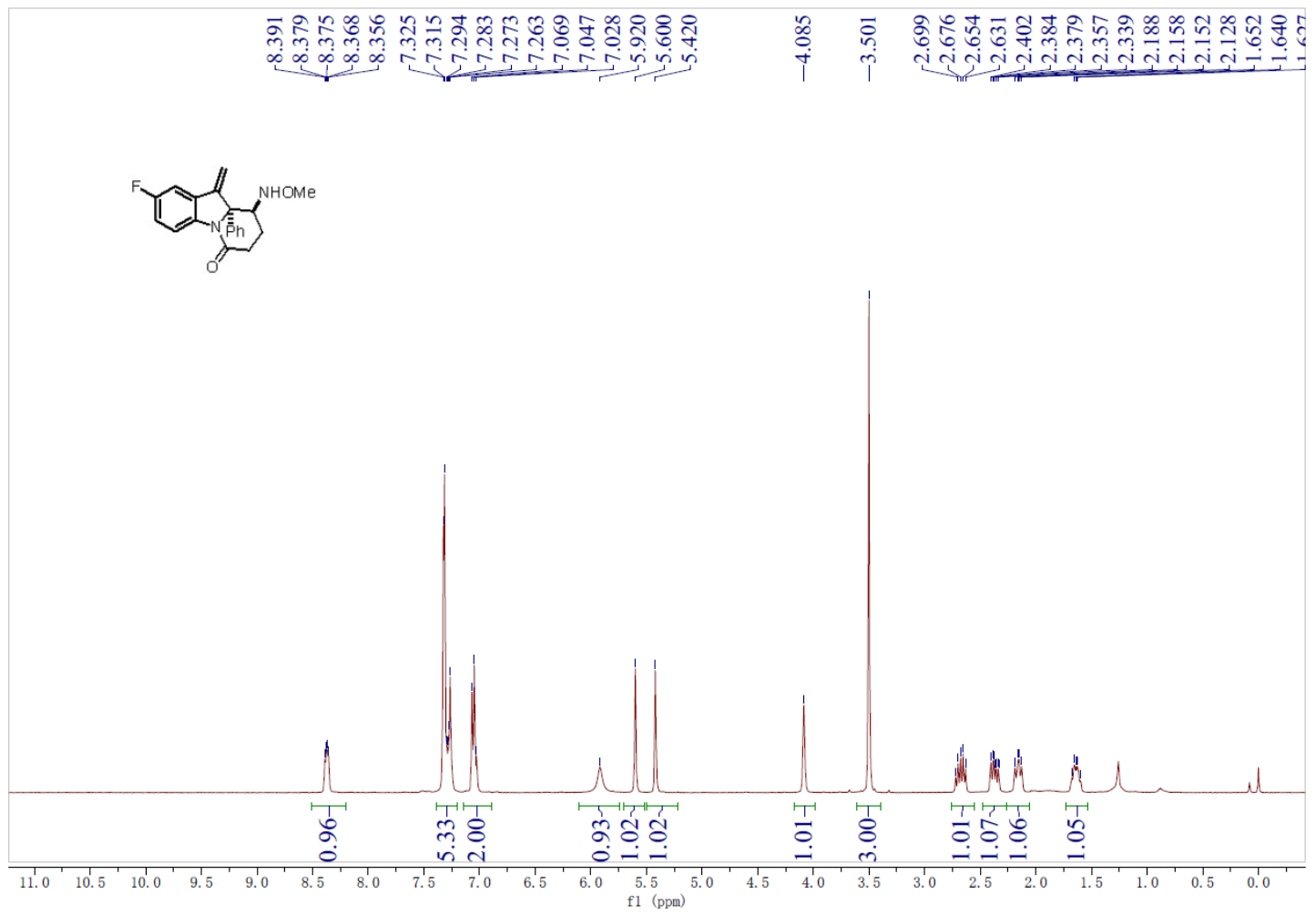

${ }^{13} \mathrm{C}$ NMR Spectrum of $\mathbf{4 e}$

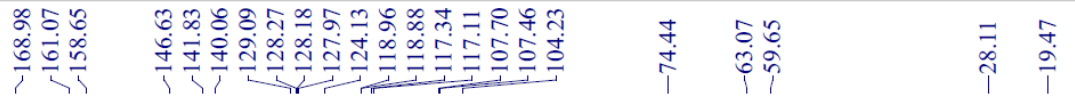

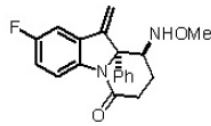
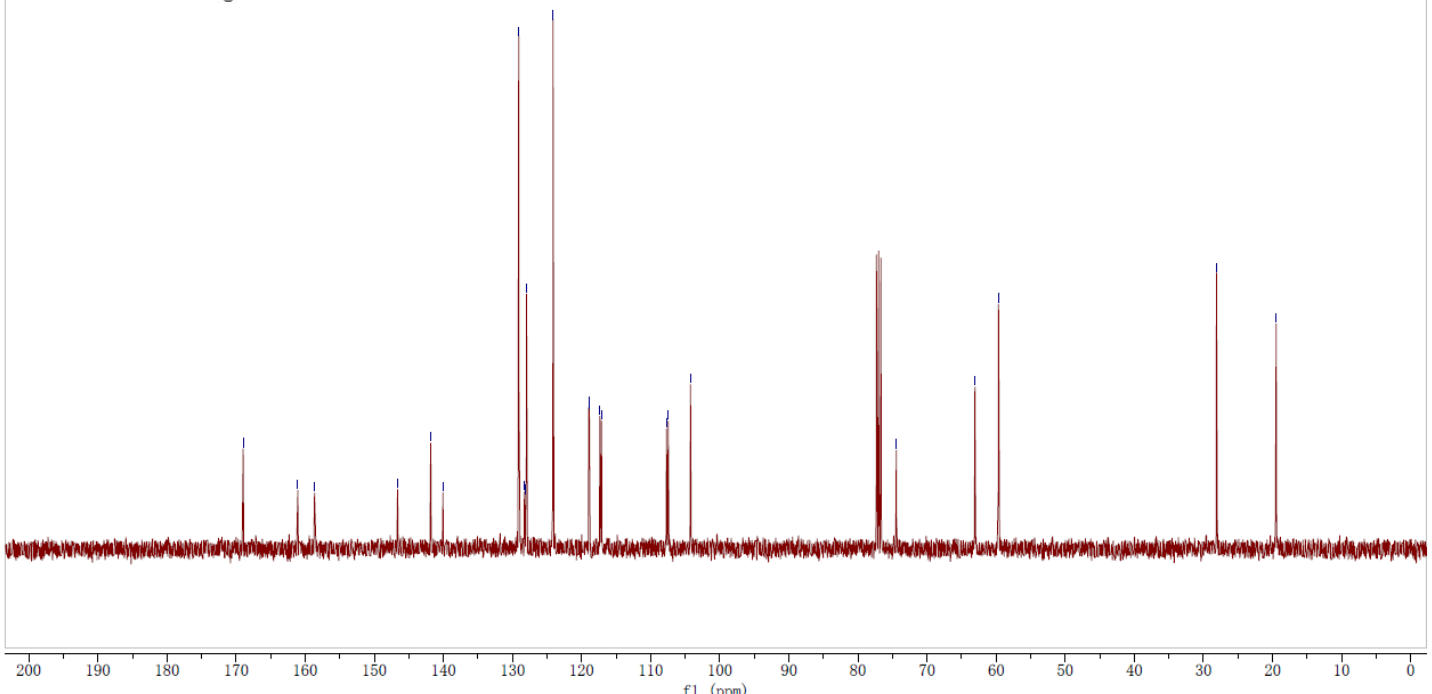
${ }^{19}$ F NMR Spectrum of $\mathbf{4 e}$

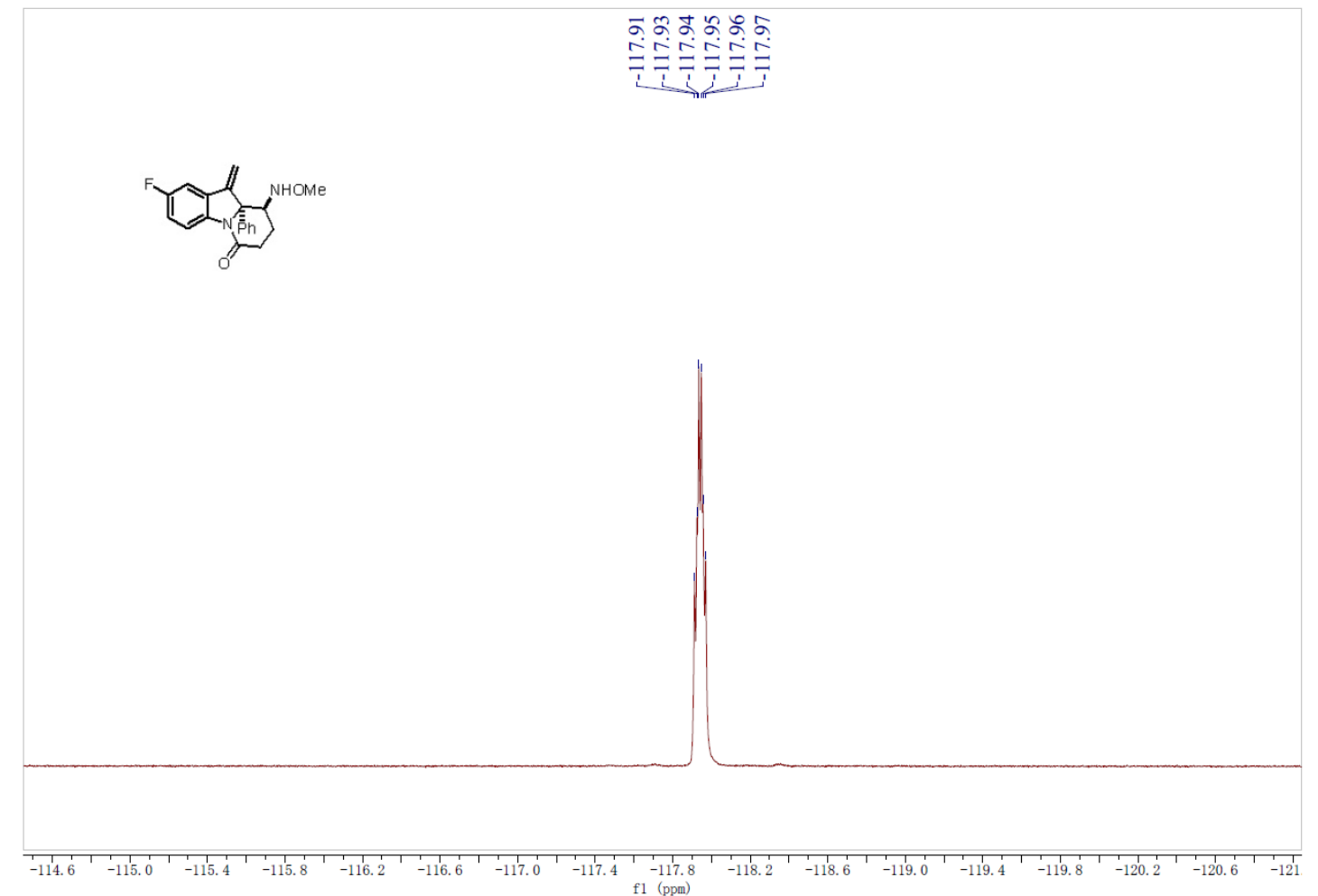


${ }^{1} \mathrm{H}$ NMR Spectrum of $\mathbf{4 f}$

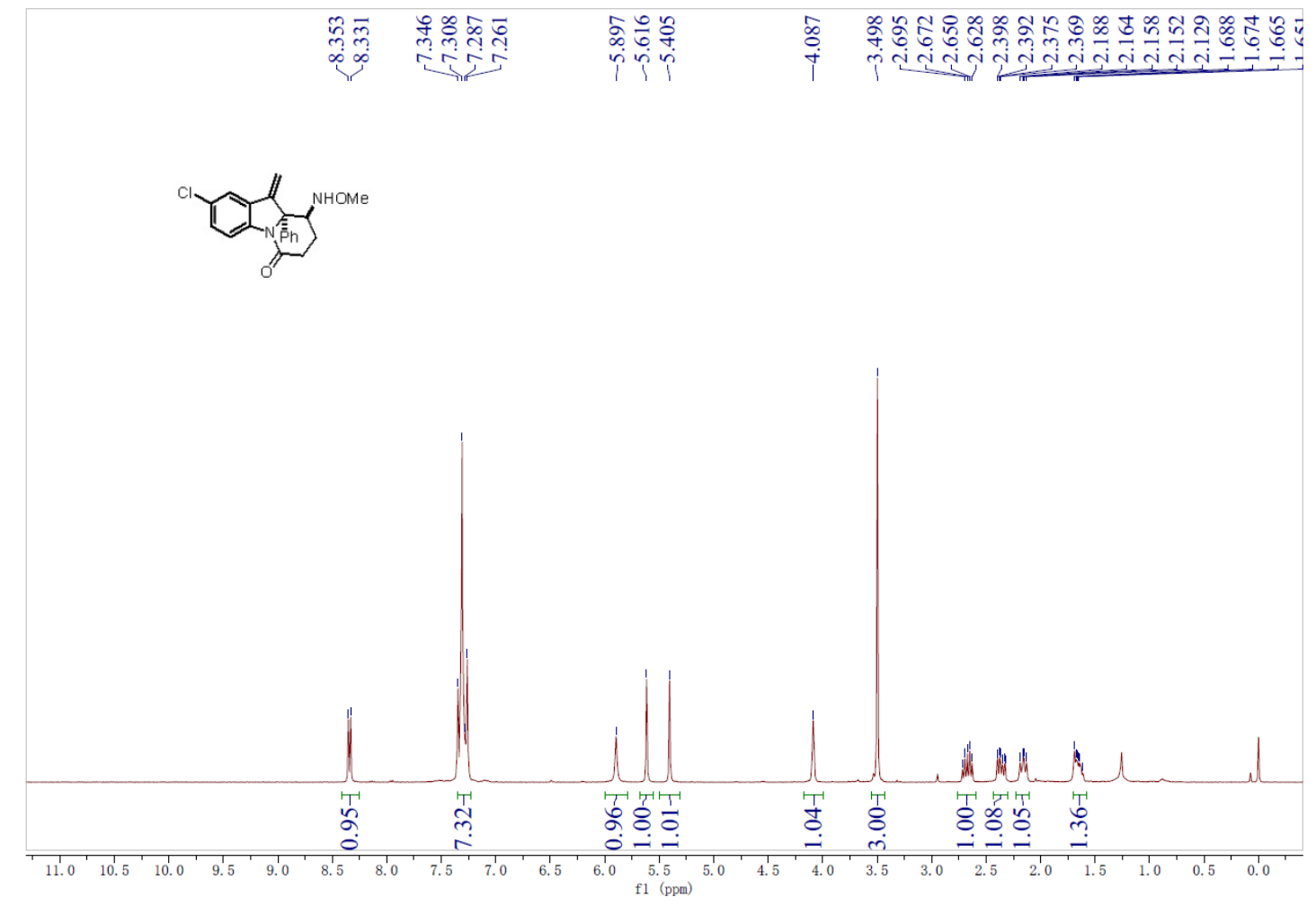

${ }^{13} \mathrm{C}$ NMR Spectrum of $\mathbf{4 f}$

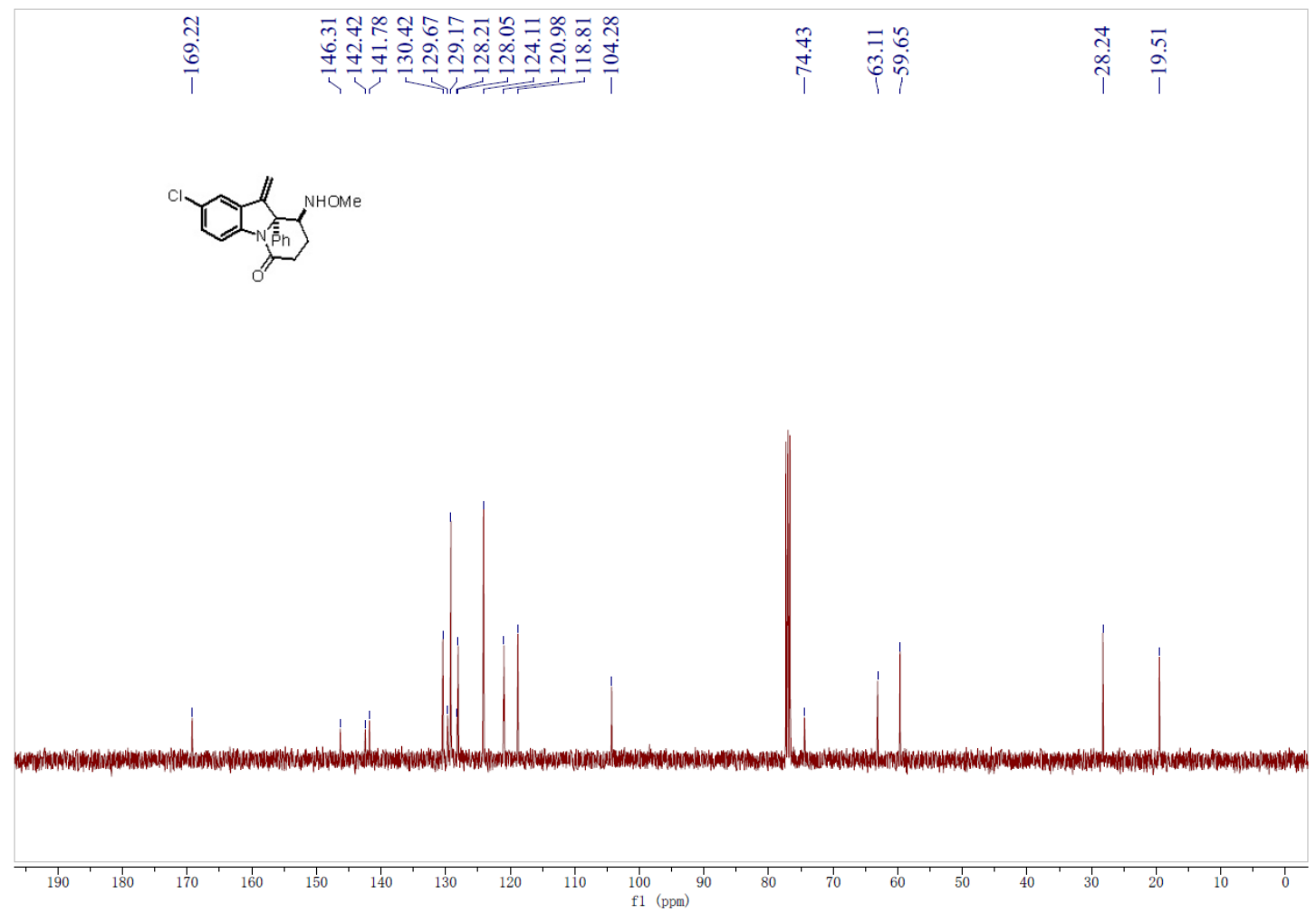


${ }^{1} \mathrm{H}$ NMR Spectrum of $\mathbf{4 g}$

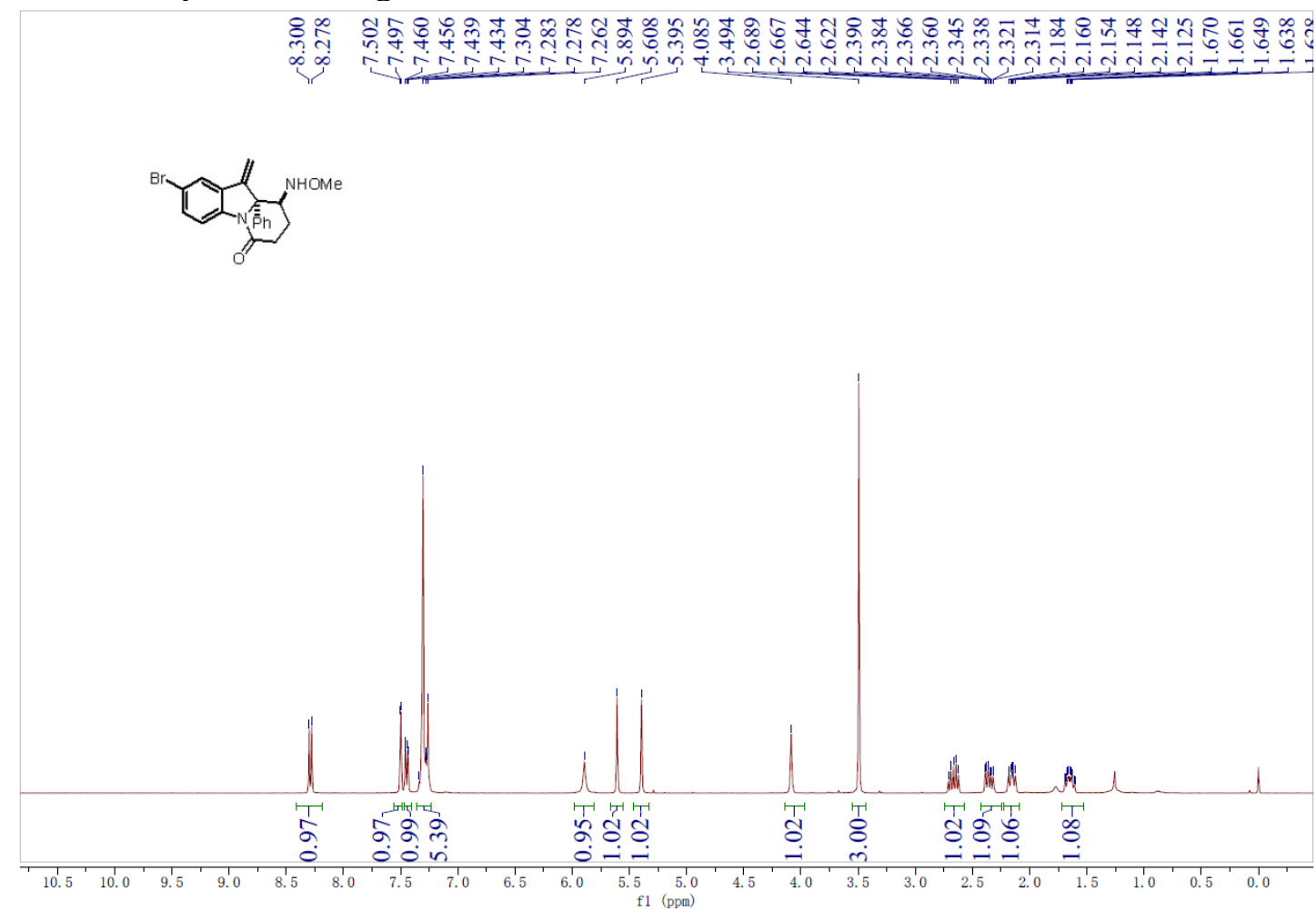

${ }^{13} \mathrm{C}$ NMR Spectrum of $\mathbf{4 g}$

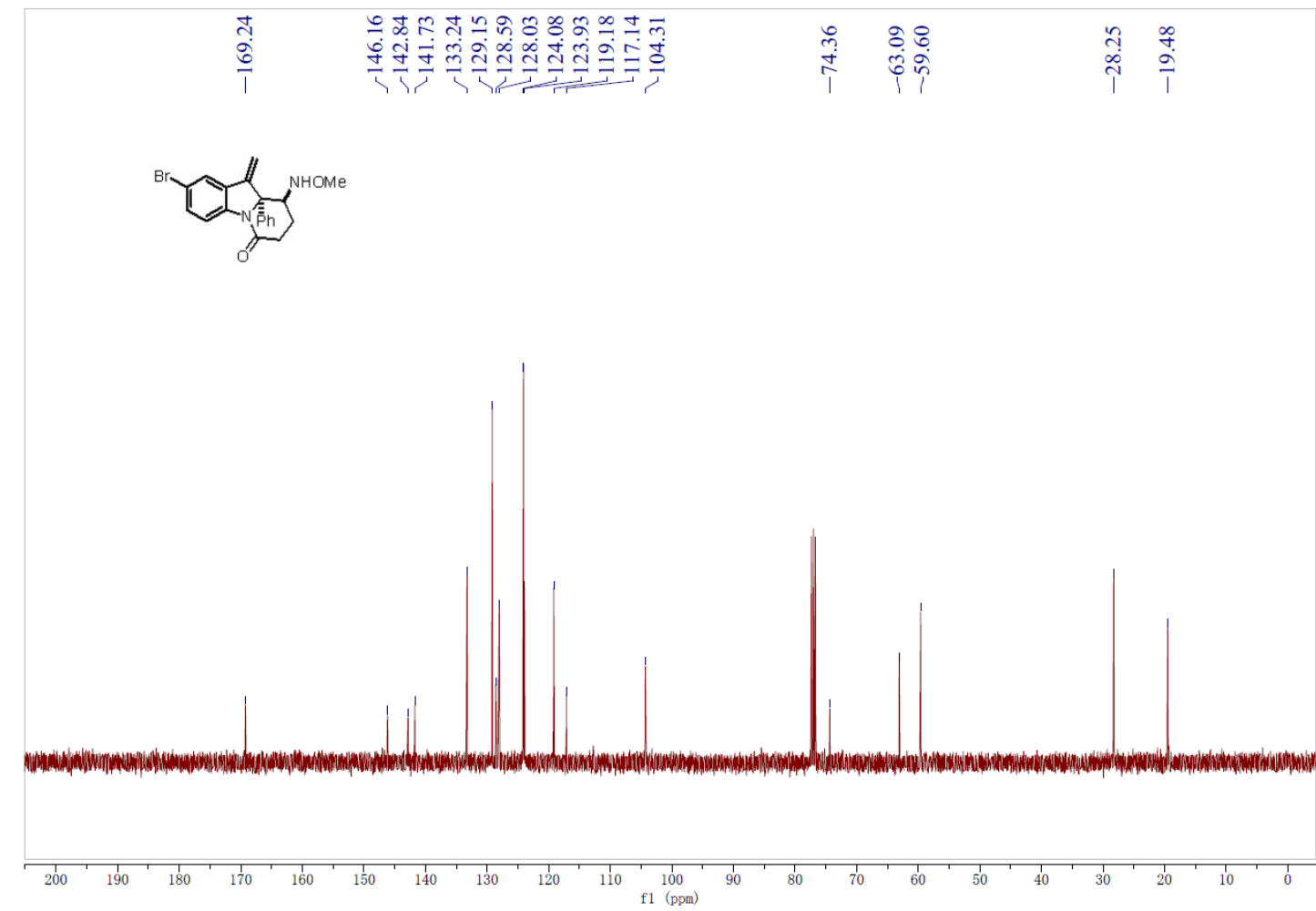


${ }^{1} \mathrm{H}$ NMR Spectrum of $\mathbf{4 h}$

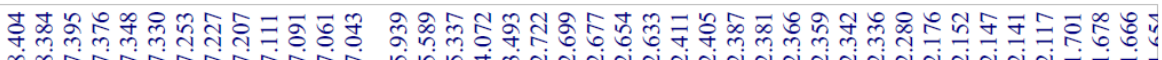
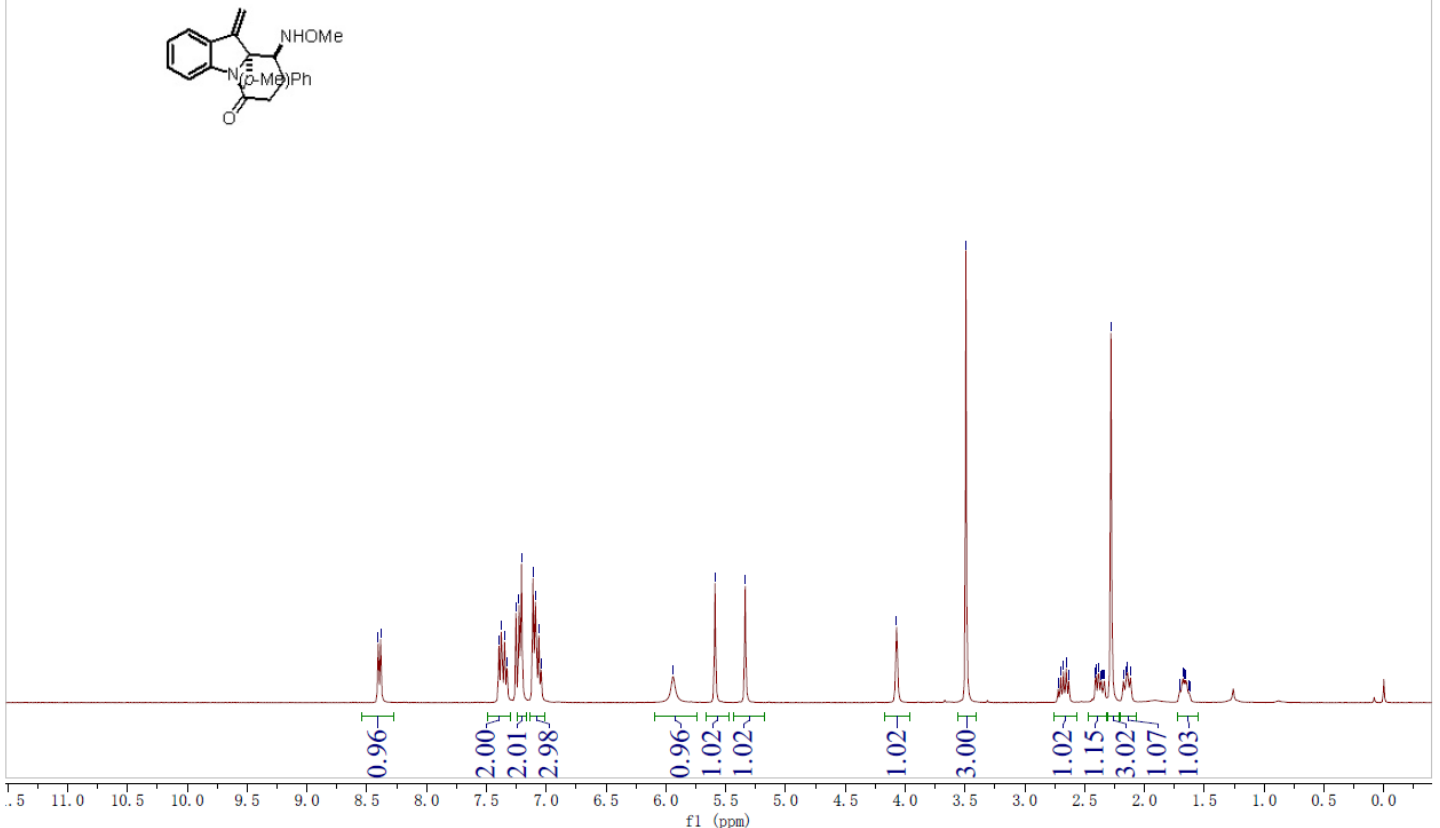

${ }^{13} \mathrm{C}$ NMR Spectrum of $\mathbf{4 h}$

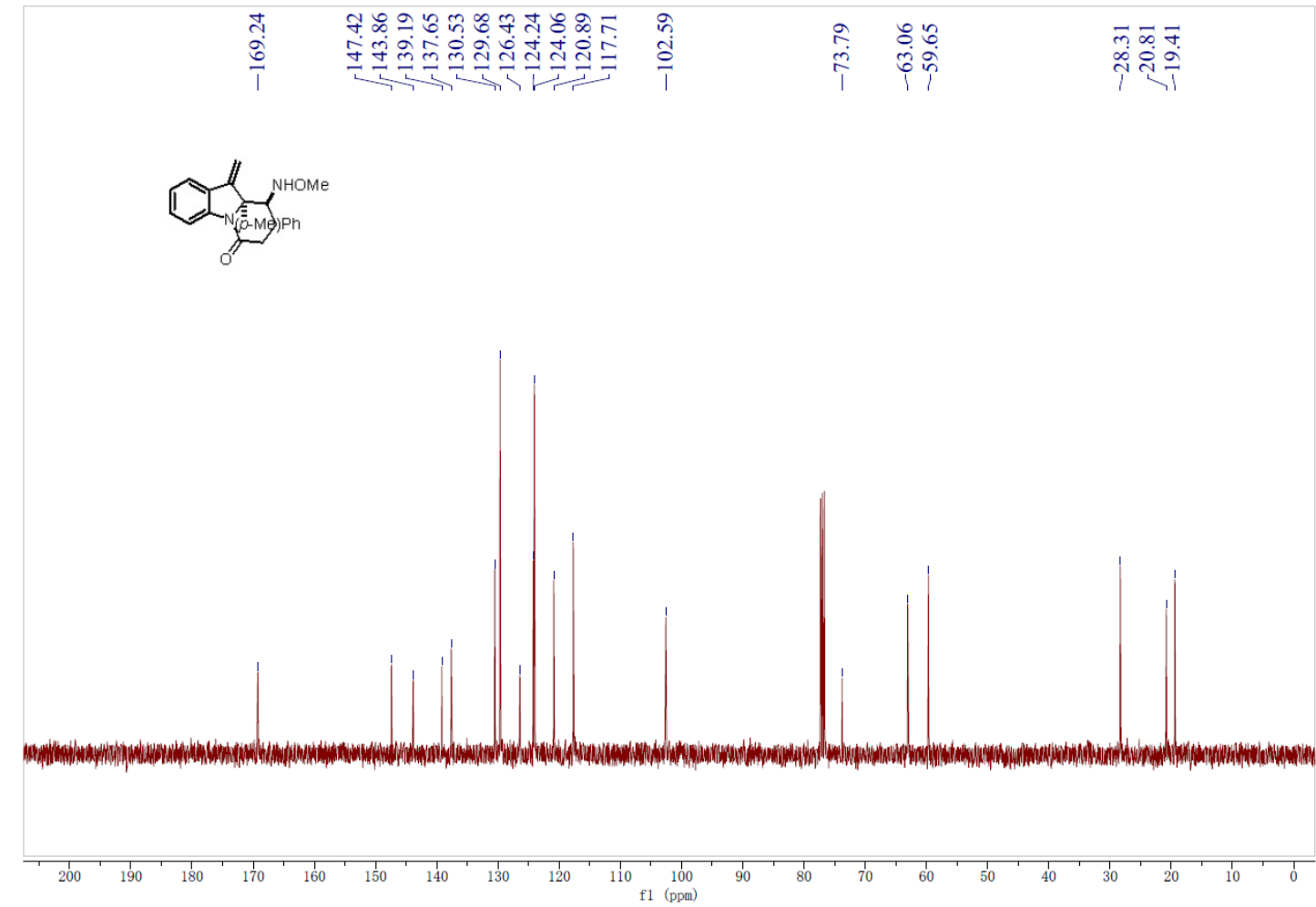


${ }^{1} \mathrm{H}$ NMR Spectrum of $\mathbf{4 i}$

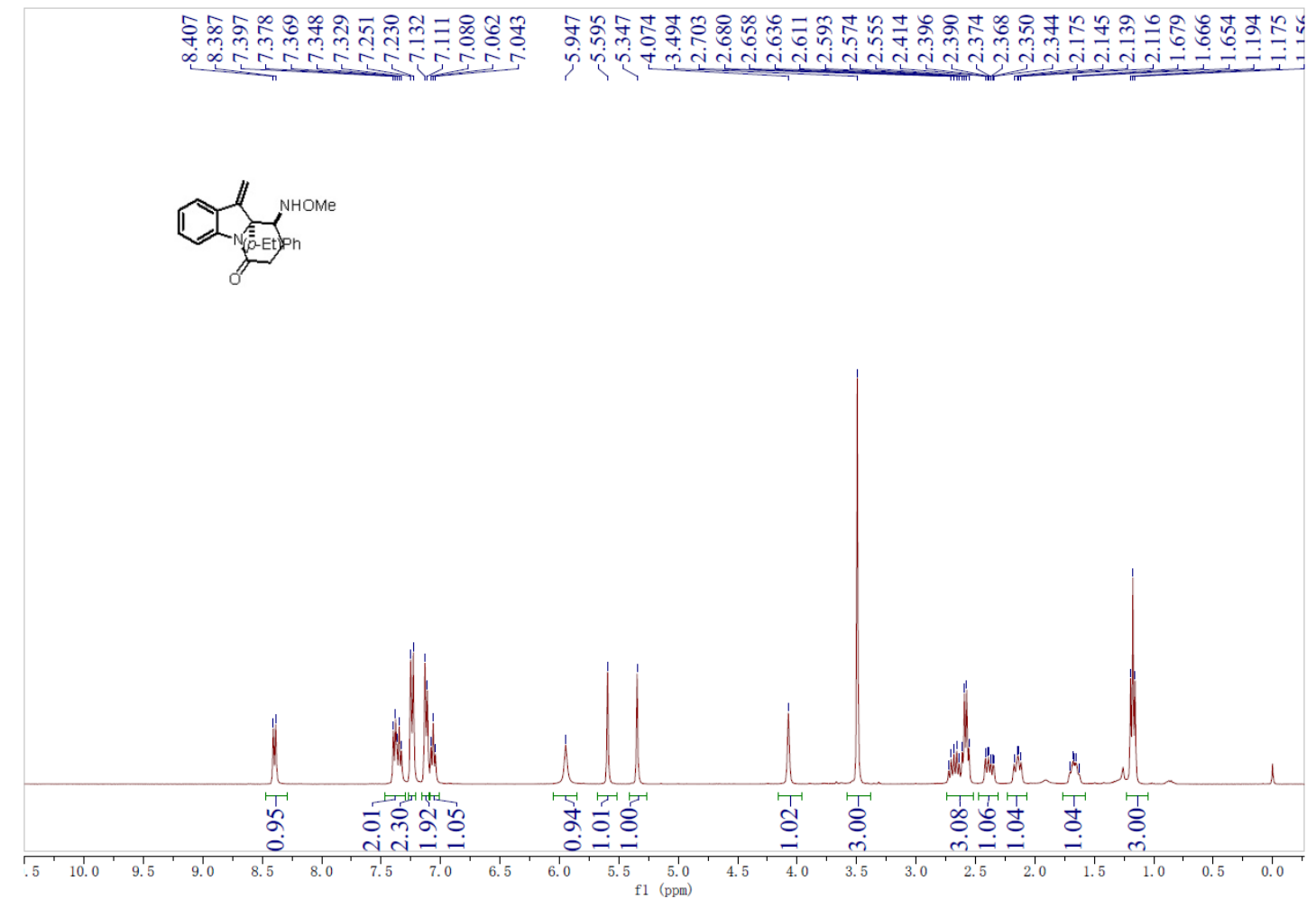

${ }^{13} \mathrm{C}$ NMR Spectrum of $\mathbf{4 i}$

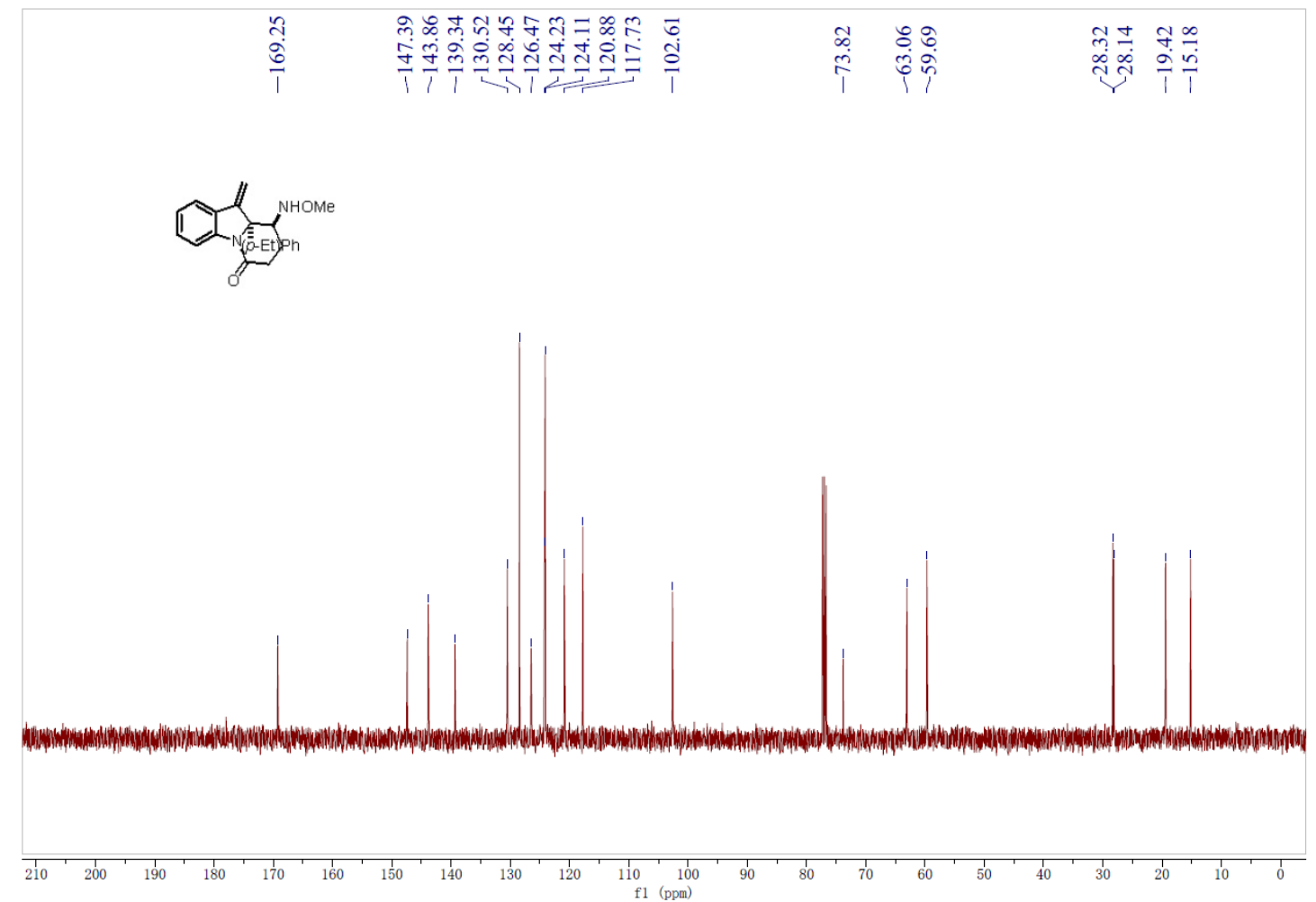


${ }^{1} \mathrm{H}$ NMR Spectrum of $\mathbf{4 j}$

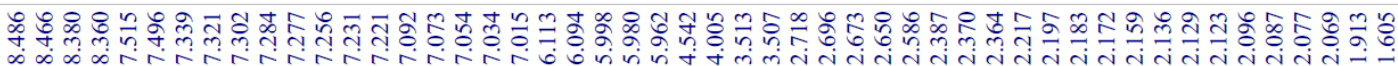

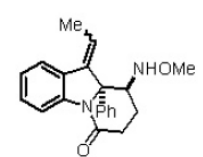

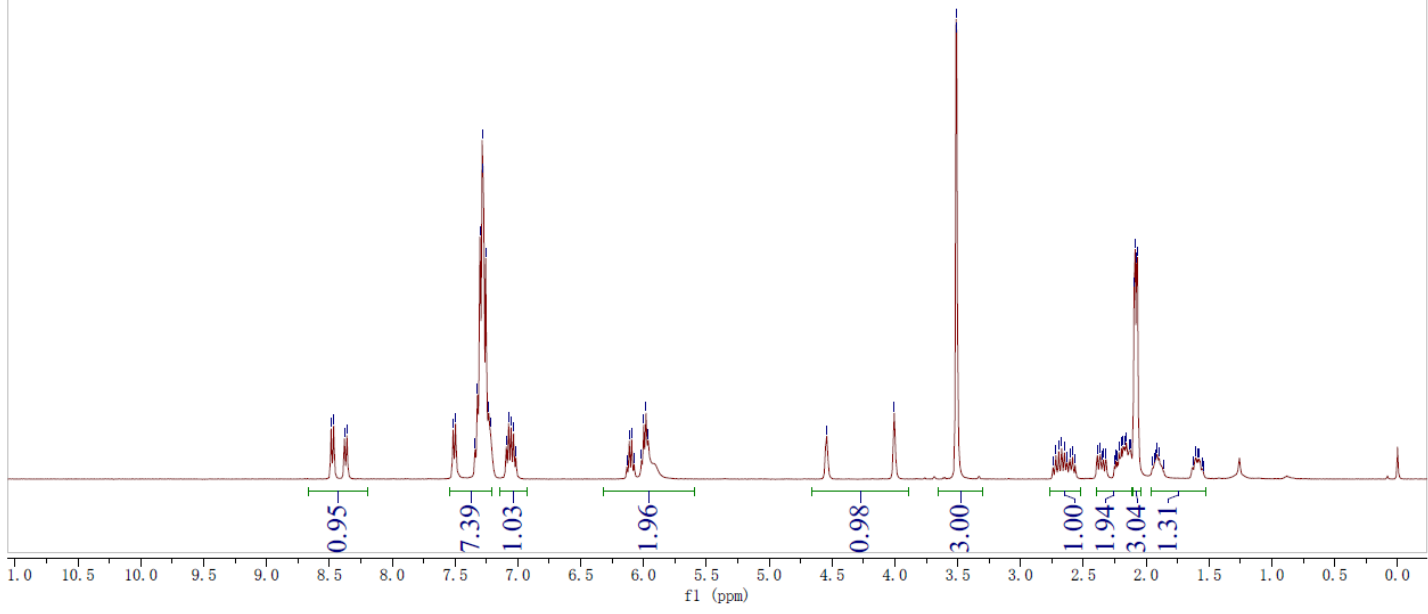

${ }^{13} \mathrm{C}$ NMR Spectrum of $\mathbf{4 j}$

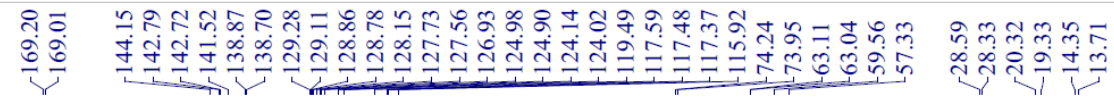<smiles>CC=C1c2ccccc2N2C(=O)CC[C@@H]12</smiles>

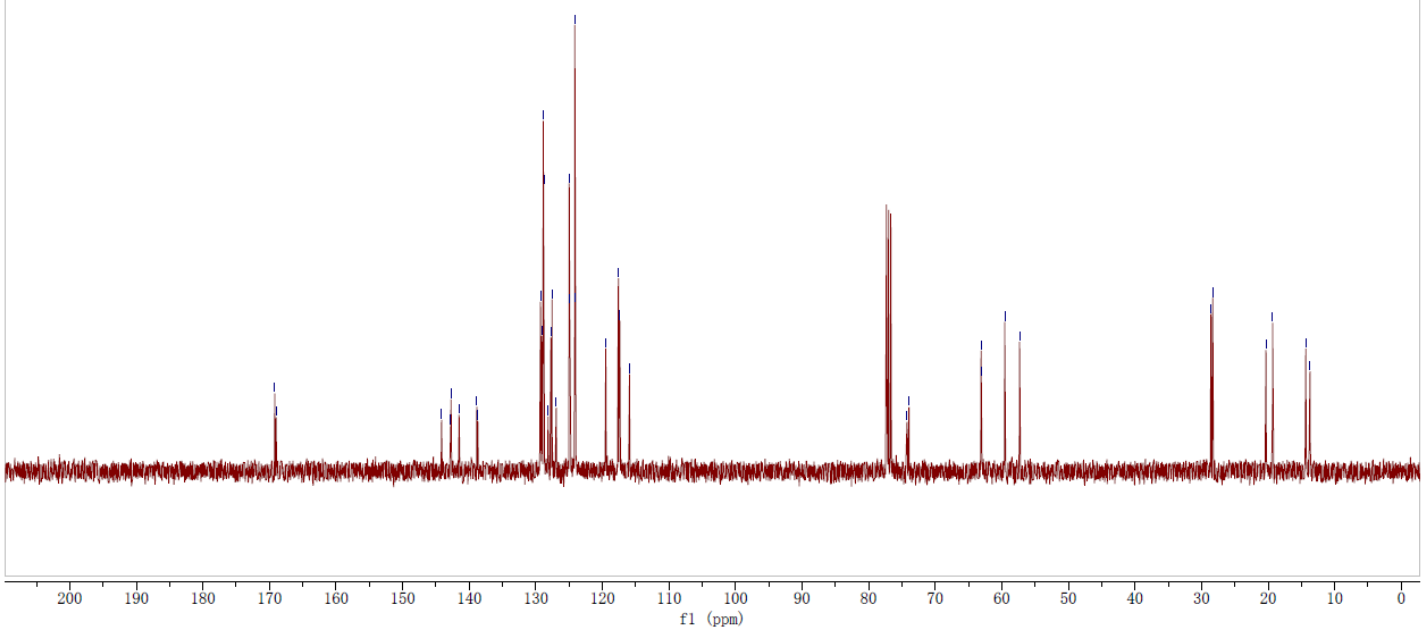


${ }^{1} \mathrm{H}$ NMR Spectrum of $\mathbf{4 k}$

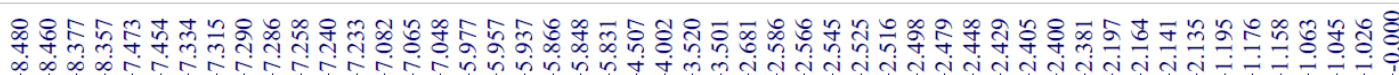

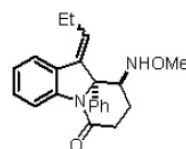

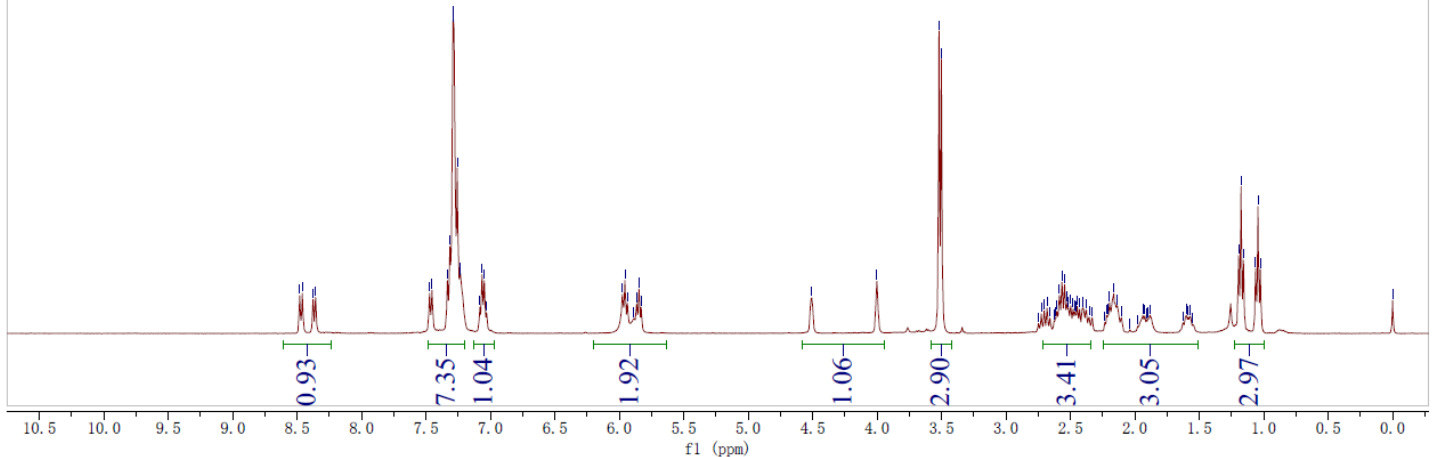

${ }^{13} \mathrm{C}$ NMR Spectrum of $\mathbf{4 k}$

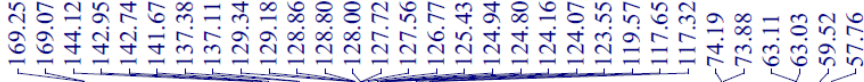

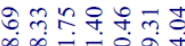

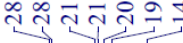

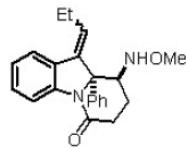

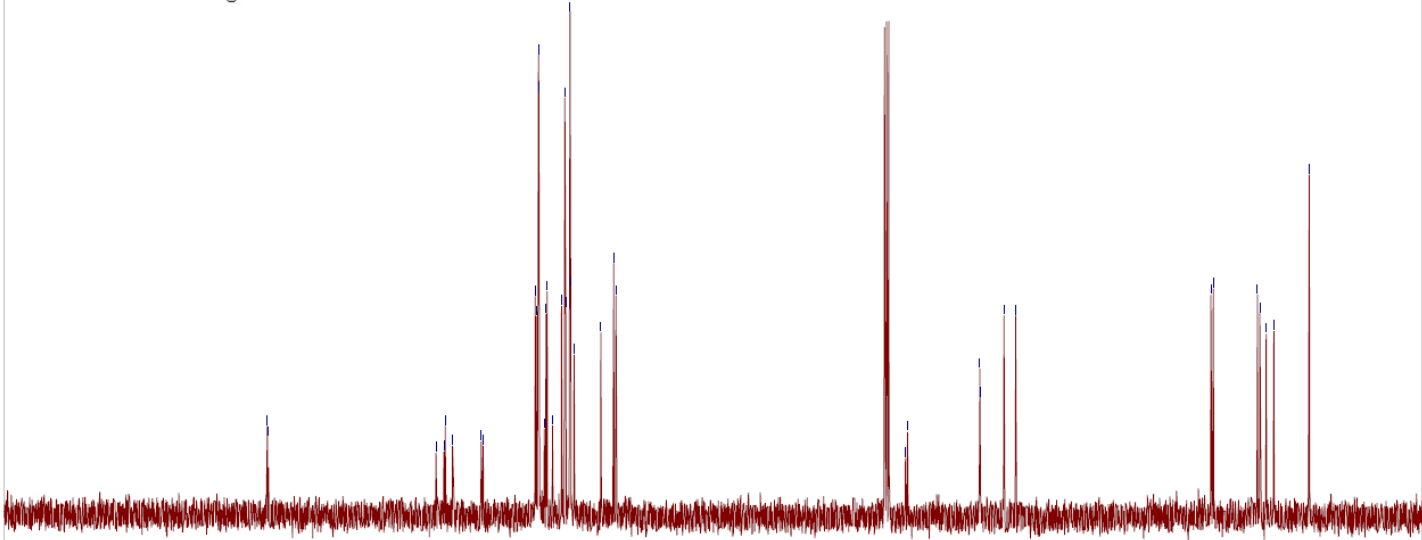

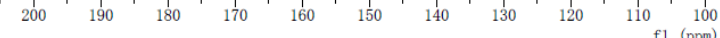


${ }^{1} \mathrm{H}$ NMR Spectrum of $4 \mathbf{l}$

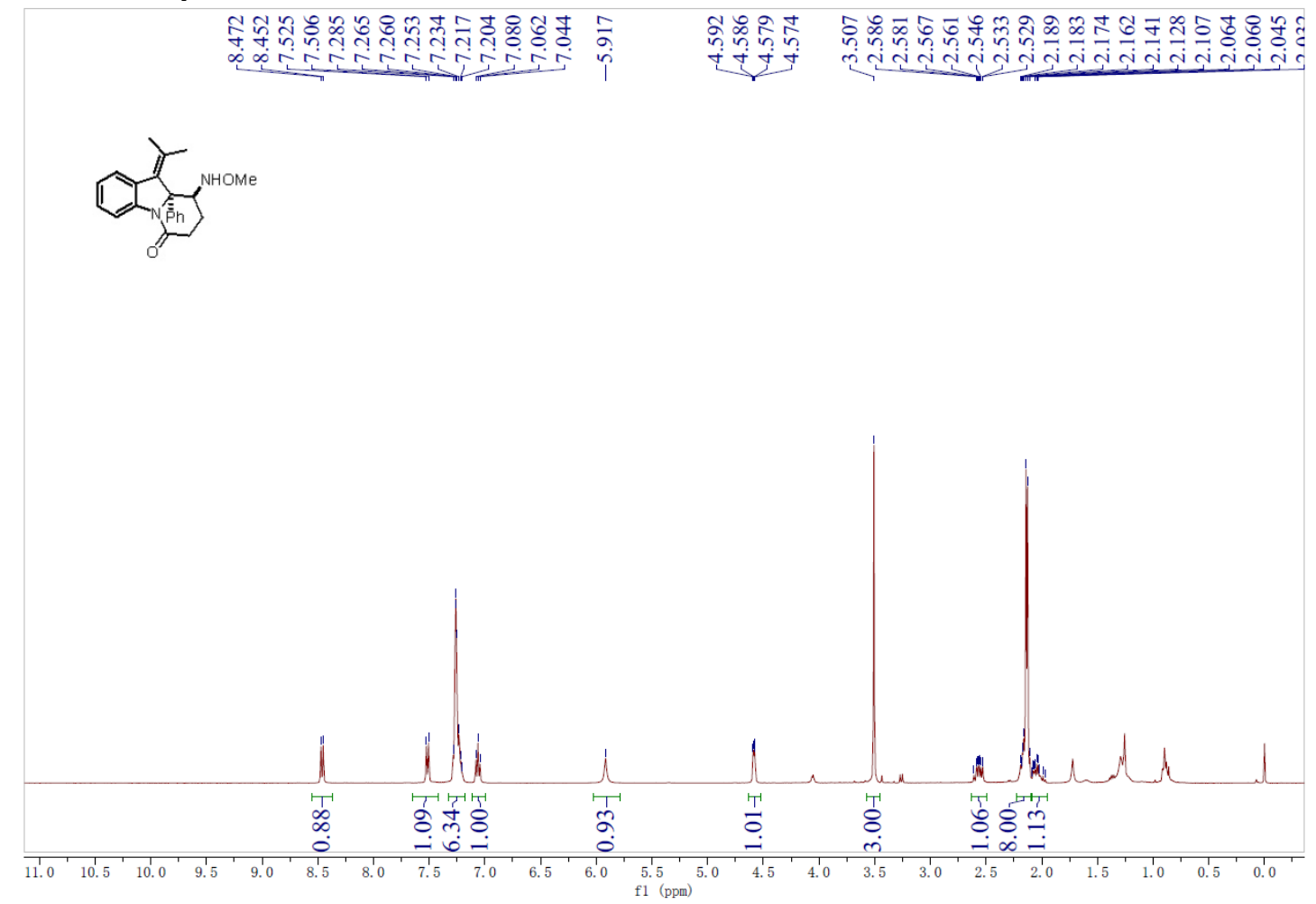

${ }^{13} \mathrm{C}$ NMR Spectrum of $4 \mathbf{I}$

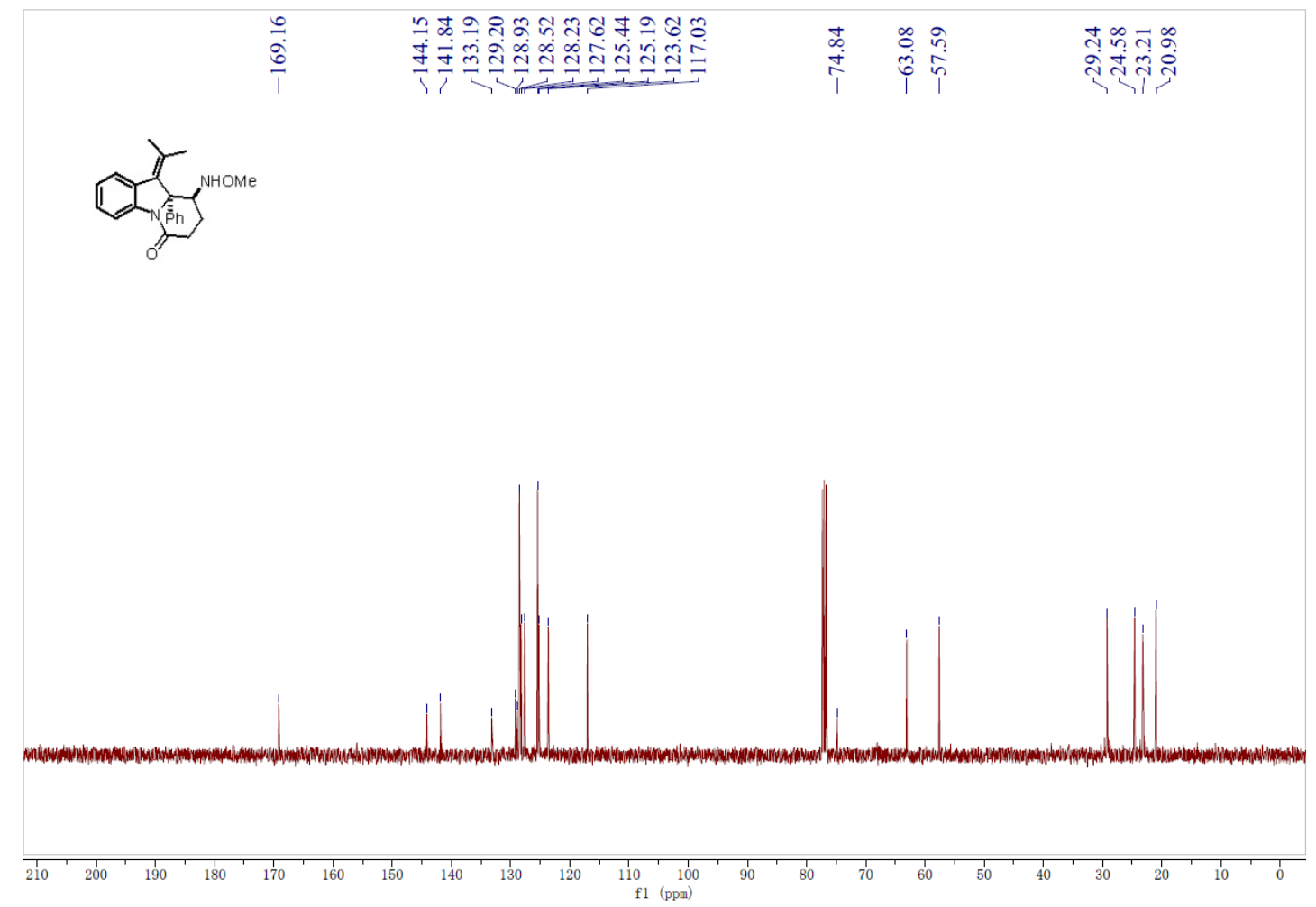


${ }^{1} \mathrm{H}$ NMR Spectrum of $\mathbf{4 m - m a j o r}$

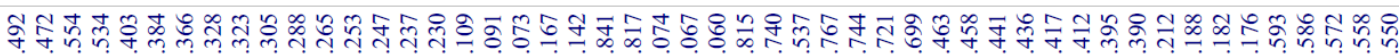

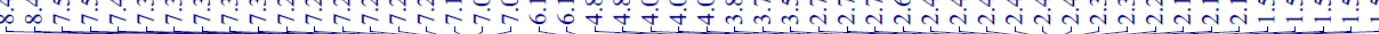<smiles>CNC1CCC(=O)N(c2ccccc2)C1=CC(C)=O</smiles>

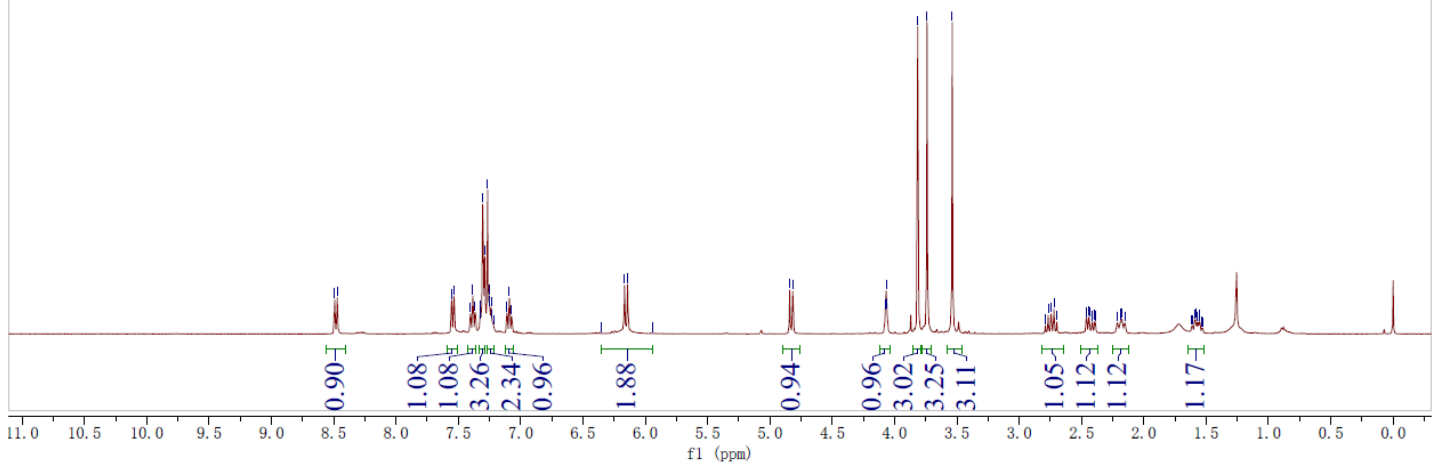

${ }^{13} \mathrm{C}$ NMR Spectrum of $\mathbf{4 m - m a j o r}$

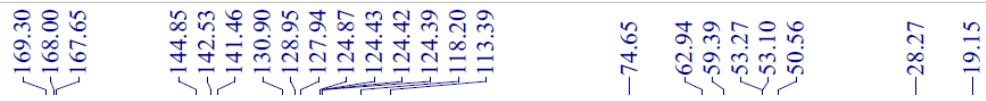

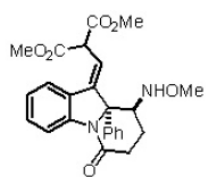

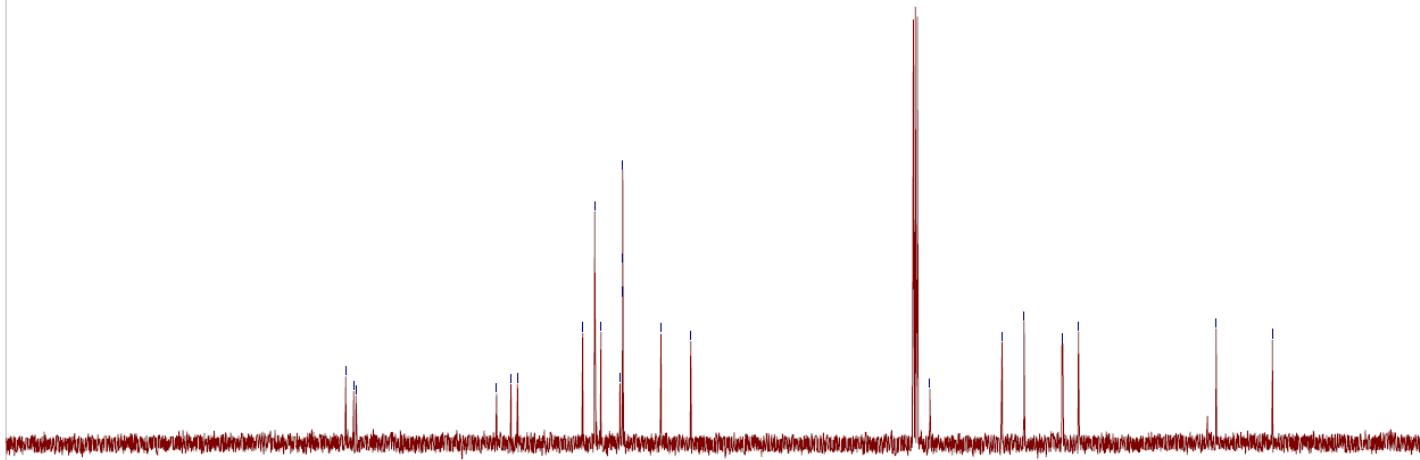

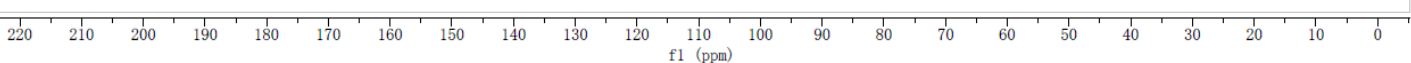


${ }^{1} \mathrm{H}$ NMR Spectrum of $\mathbf{4 m - m i n o r}$

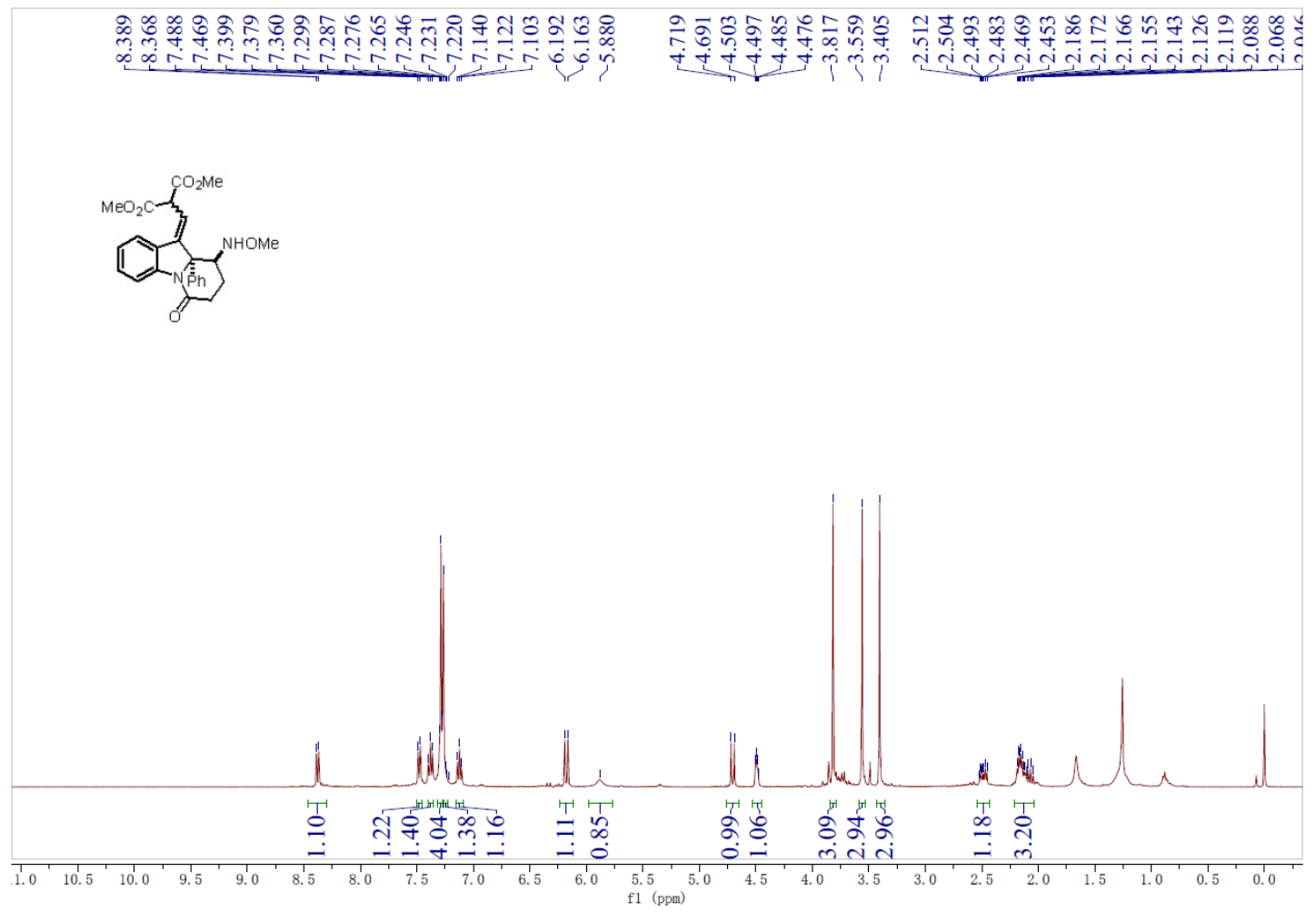

${ }^{13} \mathrm{C}$ NMR Spectrum of $\mathbf{4 m - m i n o r}$

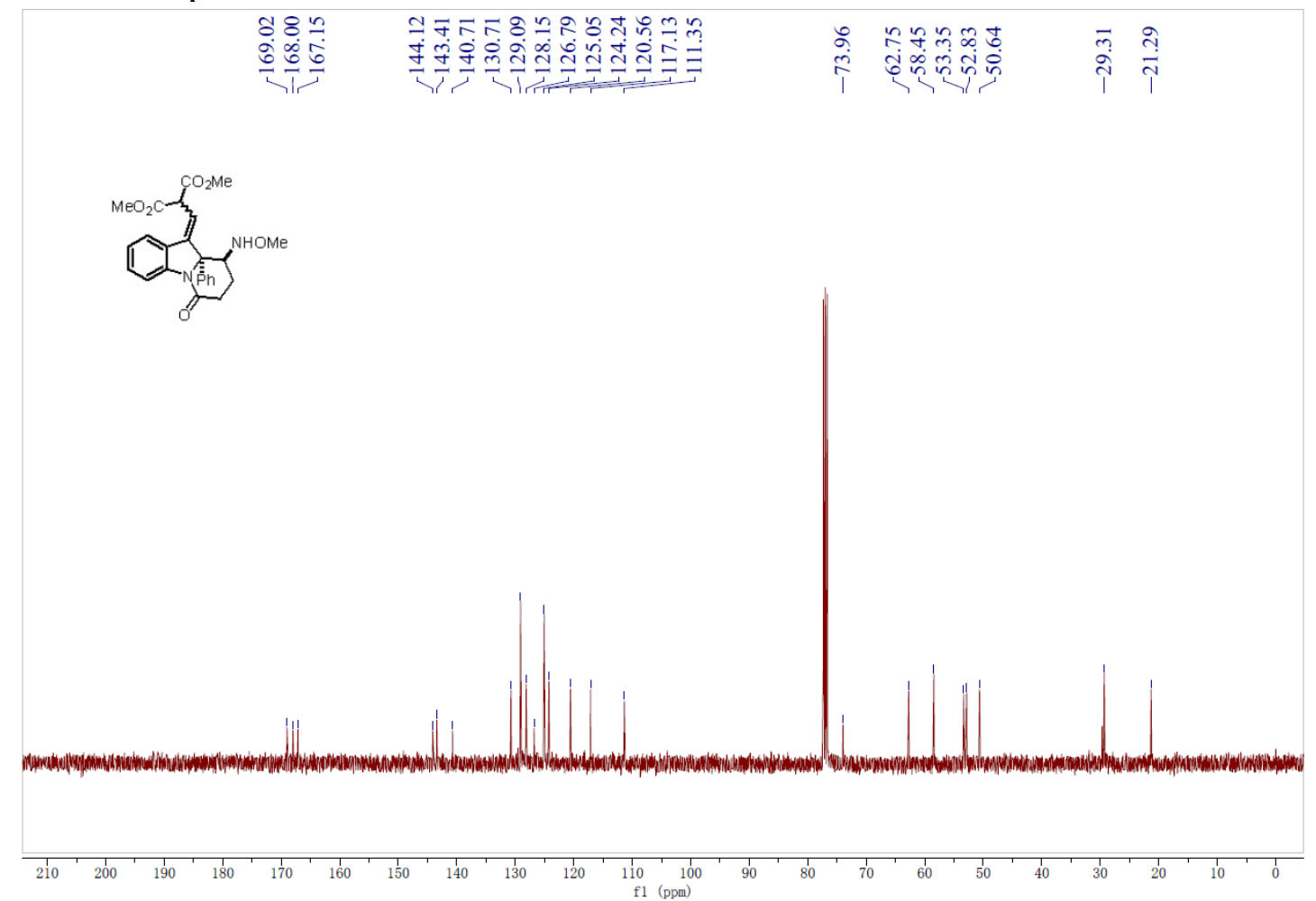


${ }^{1} \mathrm{H}$ NMR Spectrum of $\mathbf{4 n}$

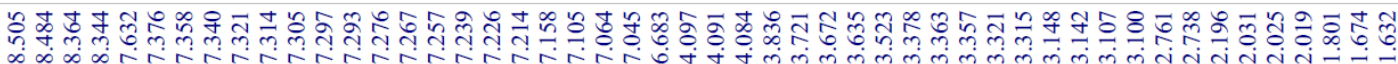

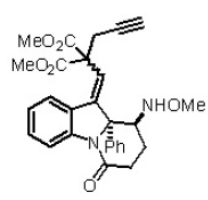

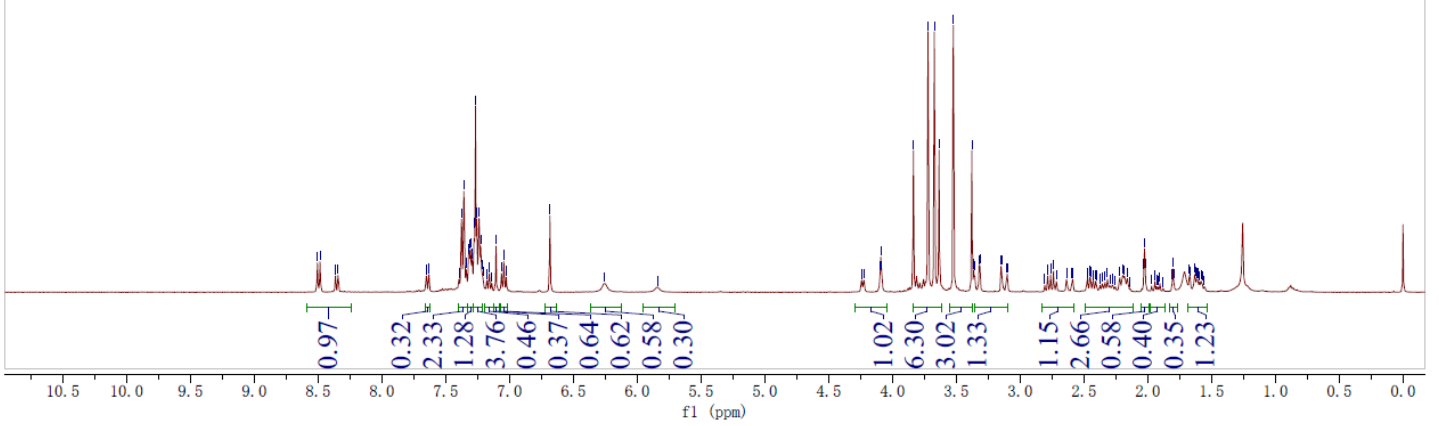

${ }^{13} \mathrm{C}$ NMR Spectrum of $\mathbf{4 n}$

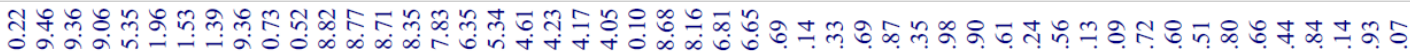

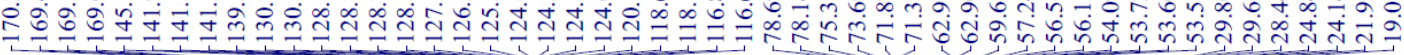

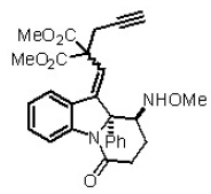

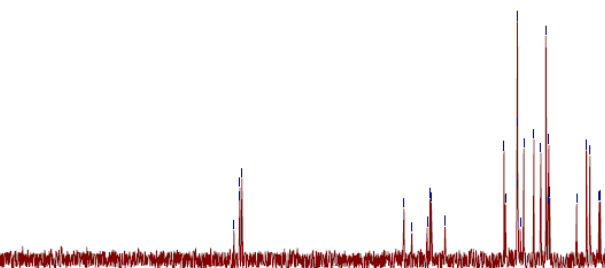

|.
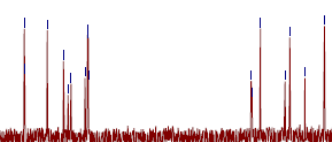
${ }^{1} \mathrm{H}$ NMR Spectrum of $\mathbf{4} \mathbf{e}^{\prime}$

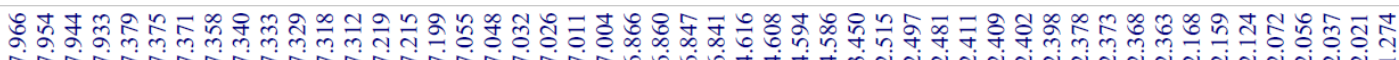

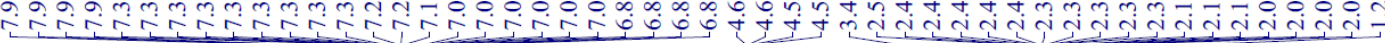

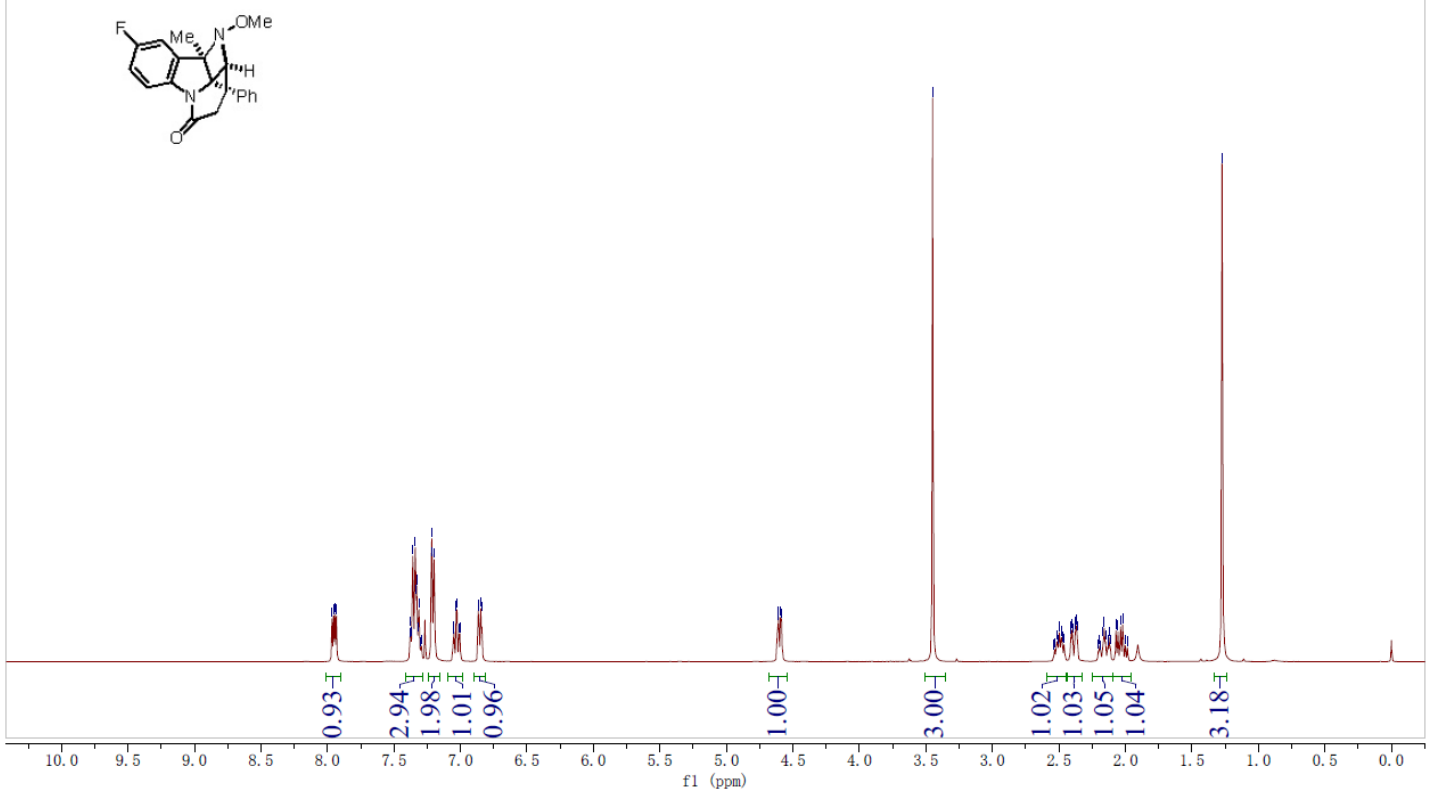

\section{${ }^{13} \mathrm{C}$ NMR Spectrum of $\mathbf{4} \mathbf{e}^{\prime}$}

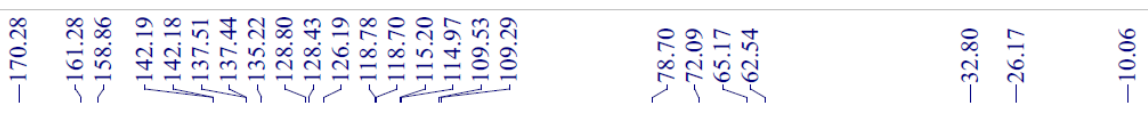

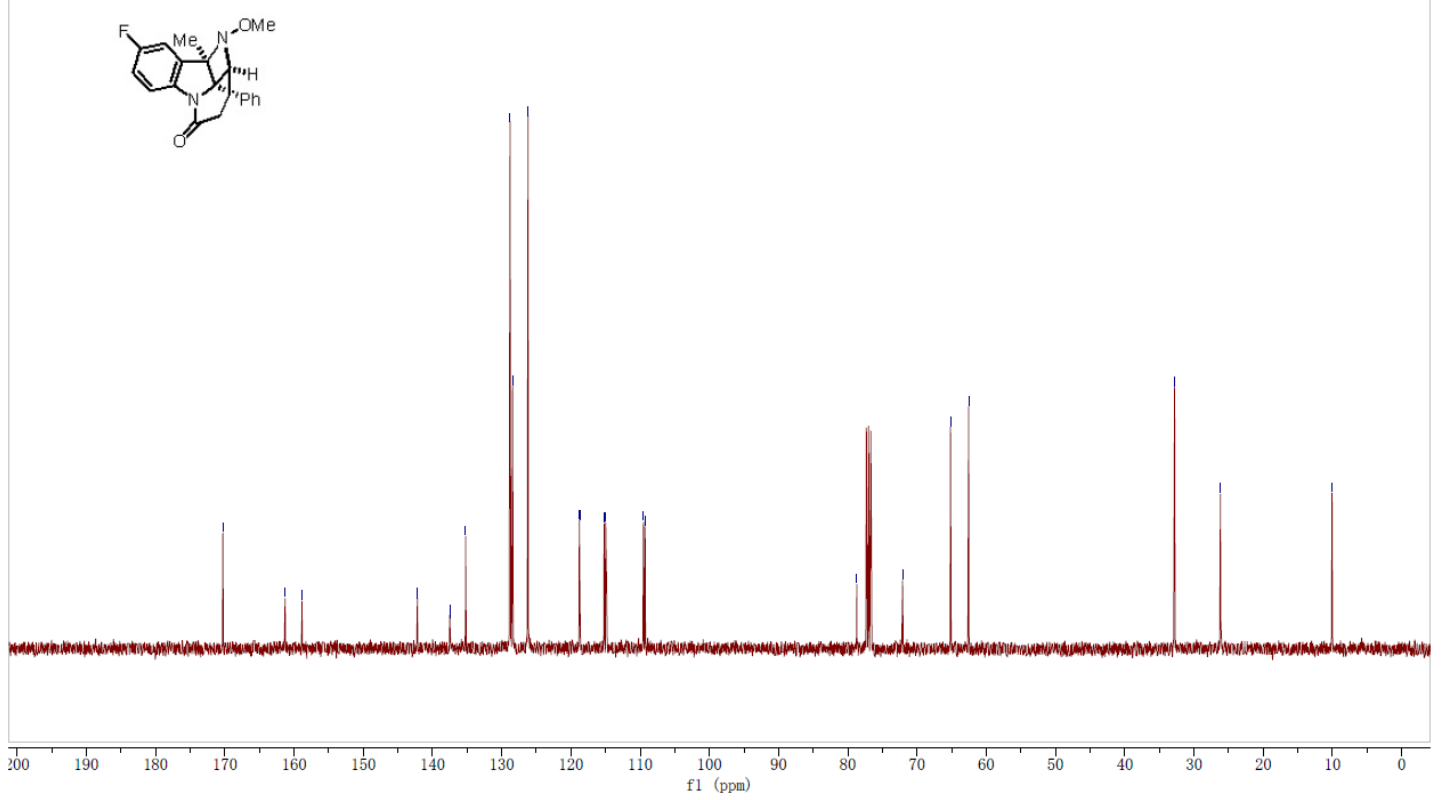


${ }^{19} \mathrm{~F}$ NMR Spectrum of $\mathbf{4} \mathbf{e}^{\prime}$

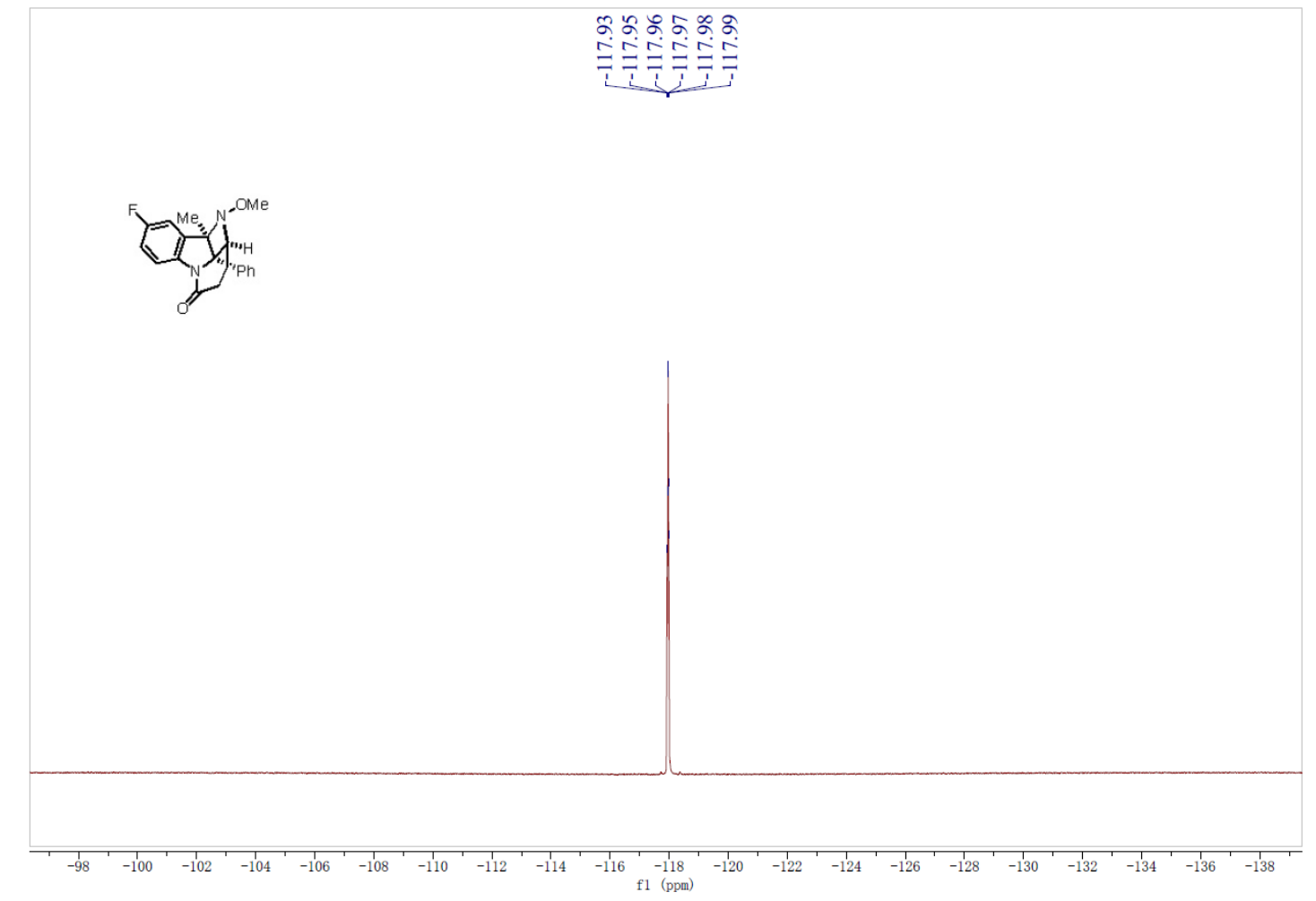


${ }^{1} \mathrm{H}$ NMR Spectrum of $\mathbf{4} \mathbf{f}^{\prime}$

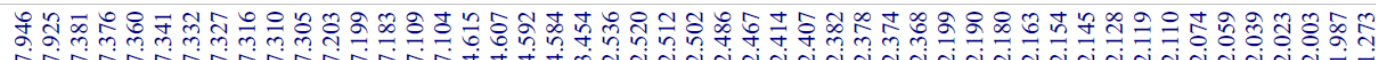
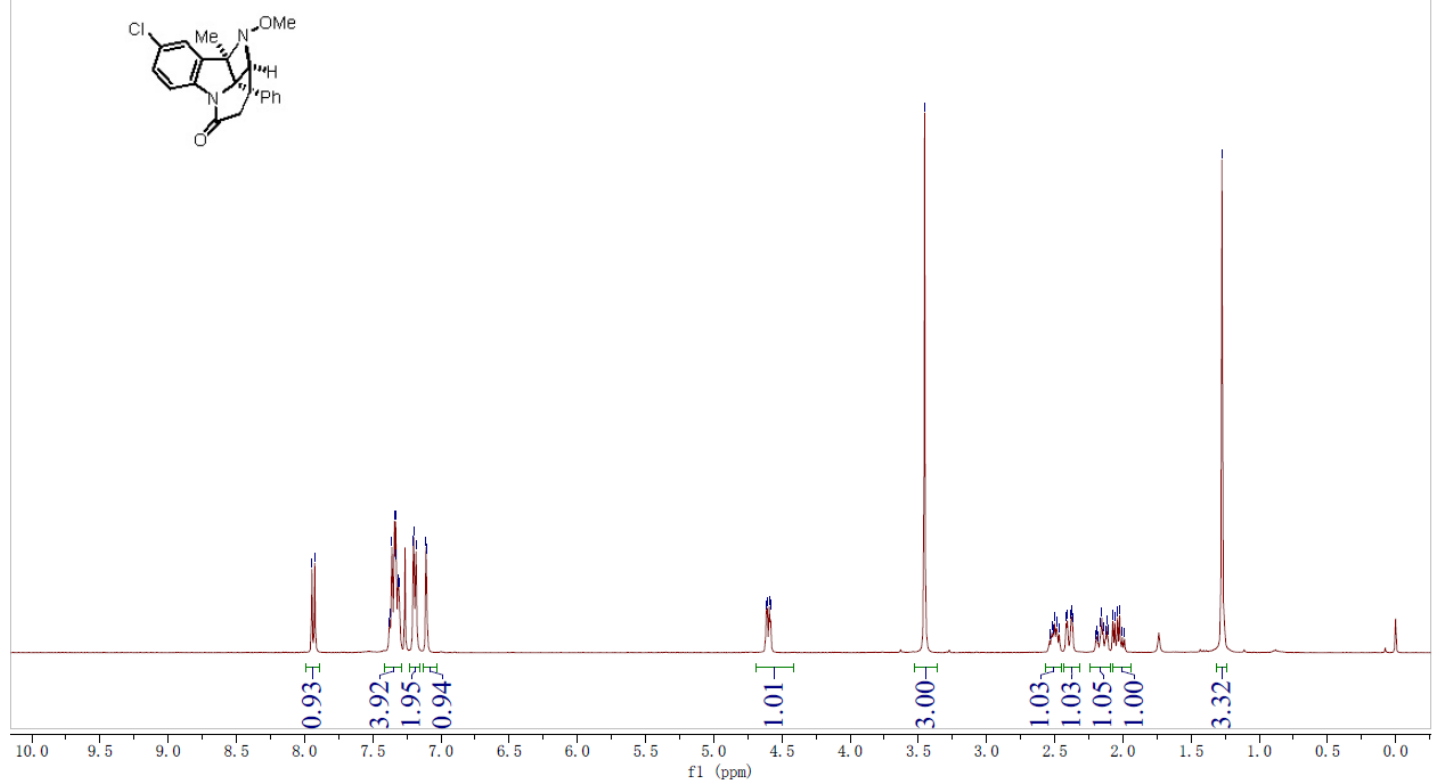

${ }^{13} \mathrm{C}$ NMR Spectrum of $\mathbf{4 f}^{4}$

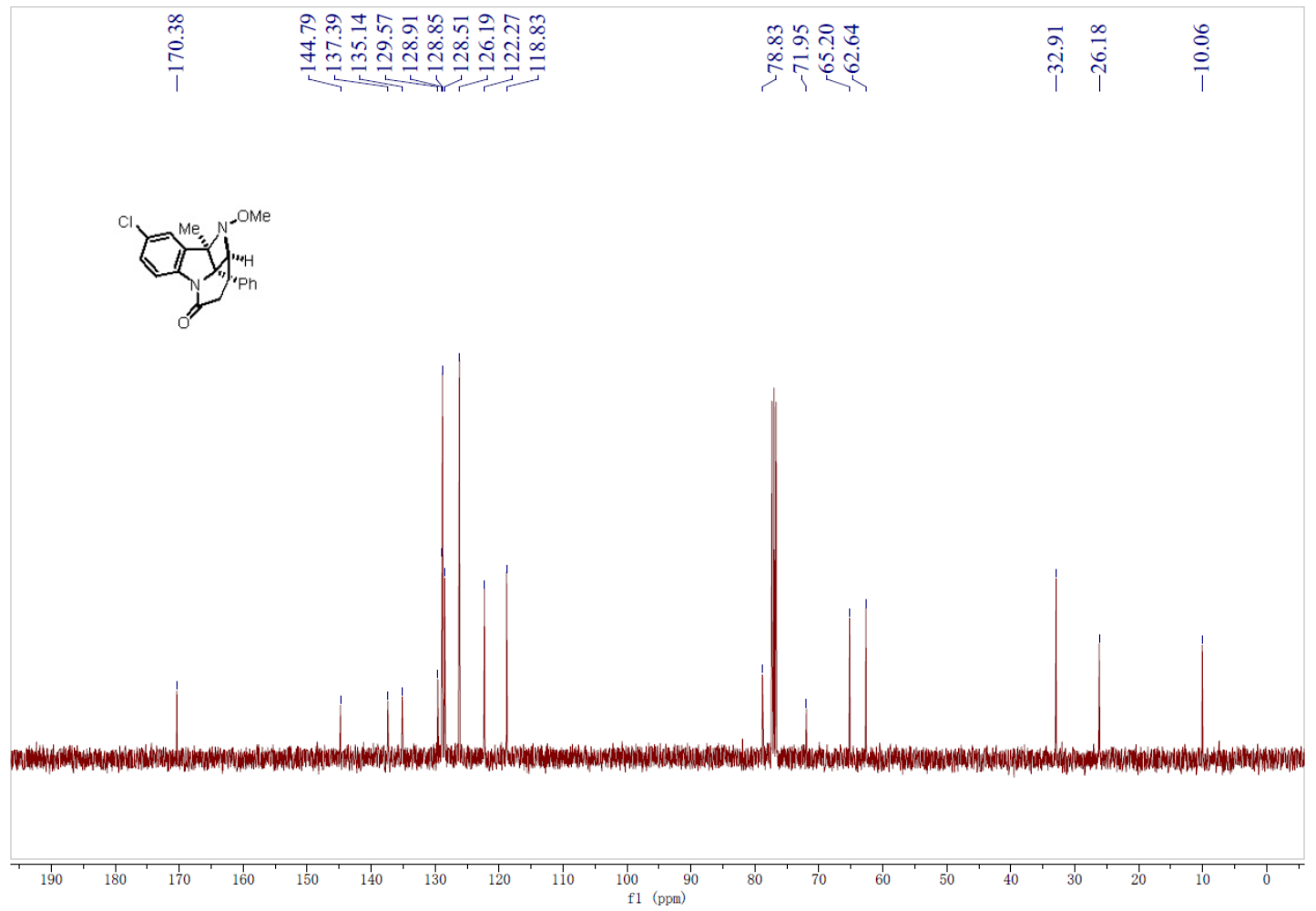


${ }^{1} \mathrm{H}$ NMR Spectrum of $\mathbf{4} \mathbf{g}^{\prime}$

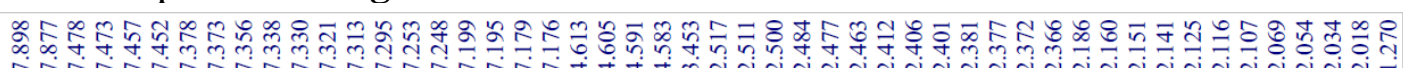
I

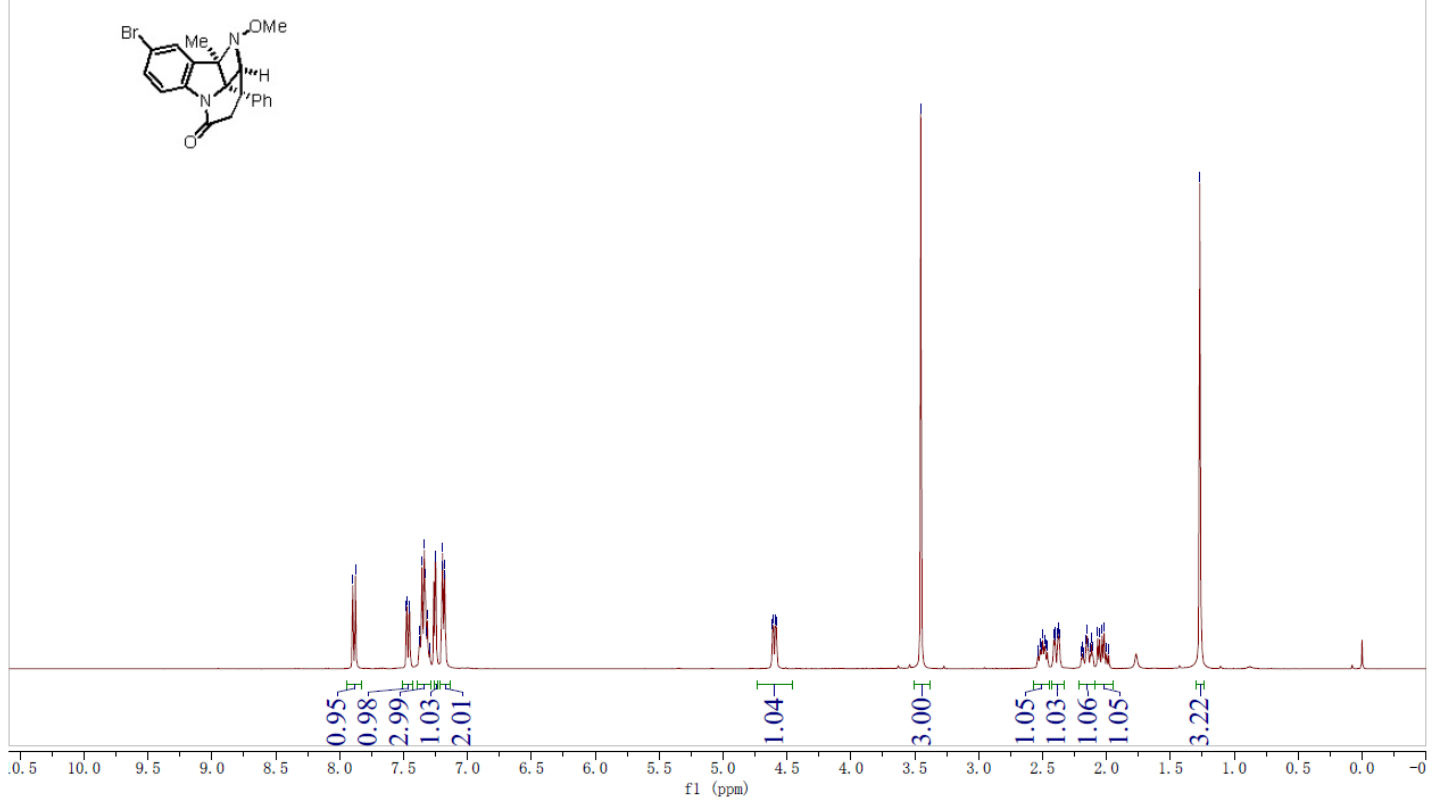

${ }^{13} \mathrm{C}$ NMR Spectrum of $\mathbf{4 9}$

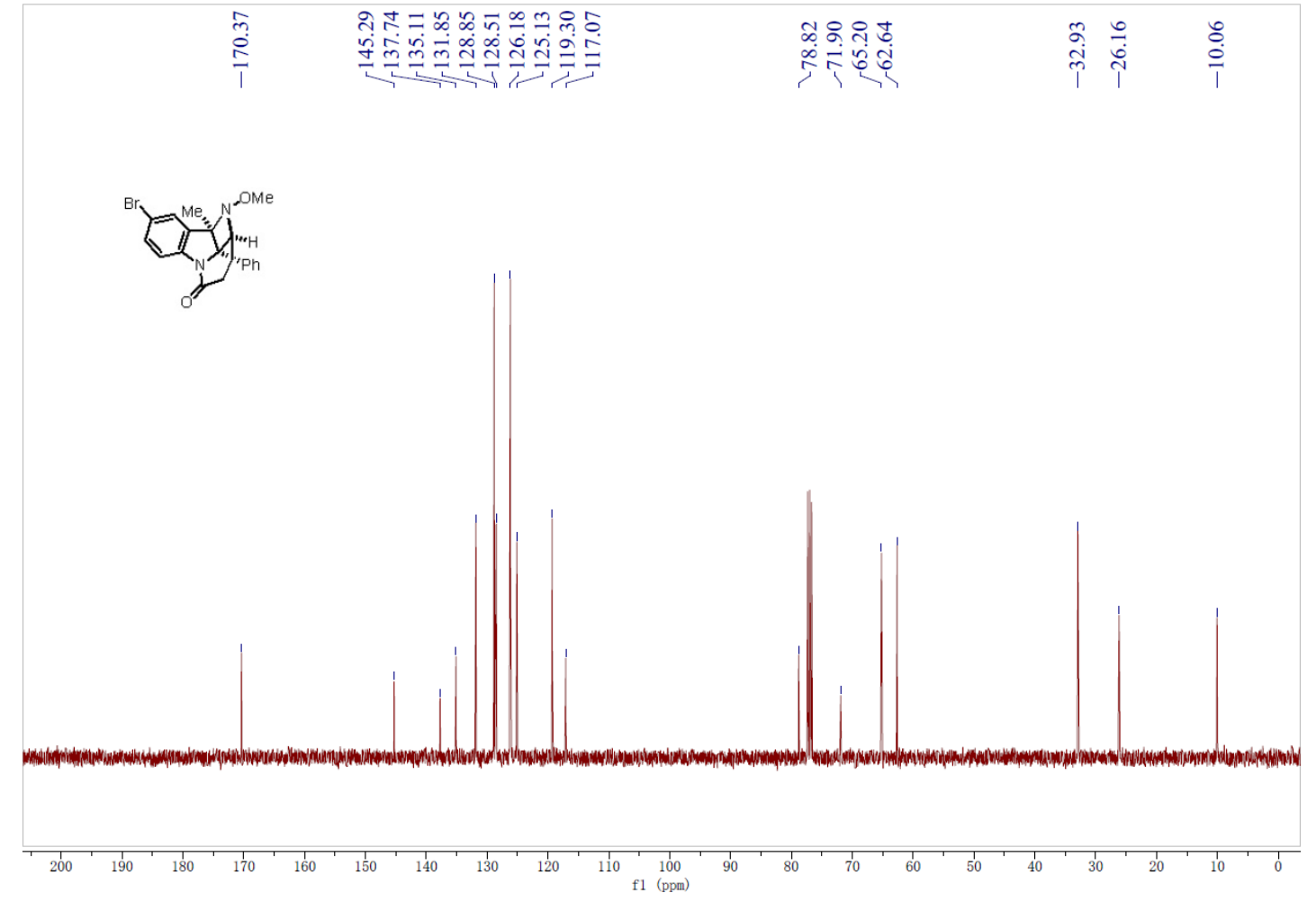


${ }^{1} \mathrm{H}$ NMR Spectrum of 4o'

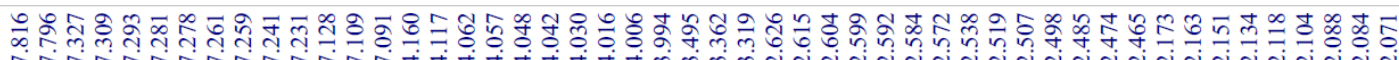

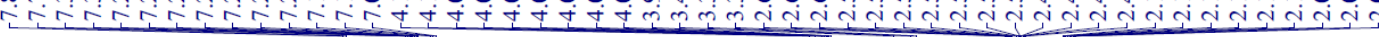
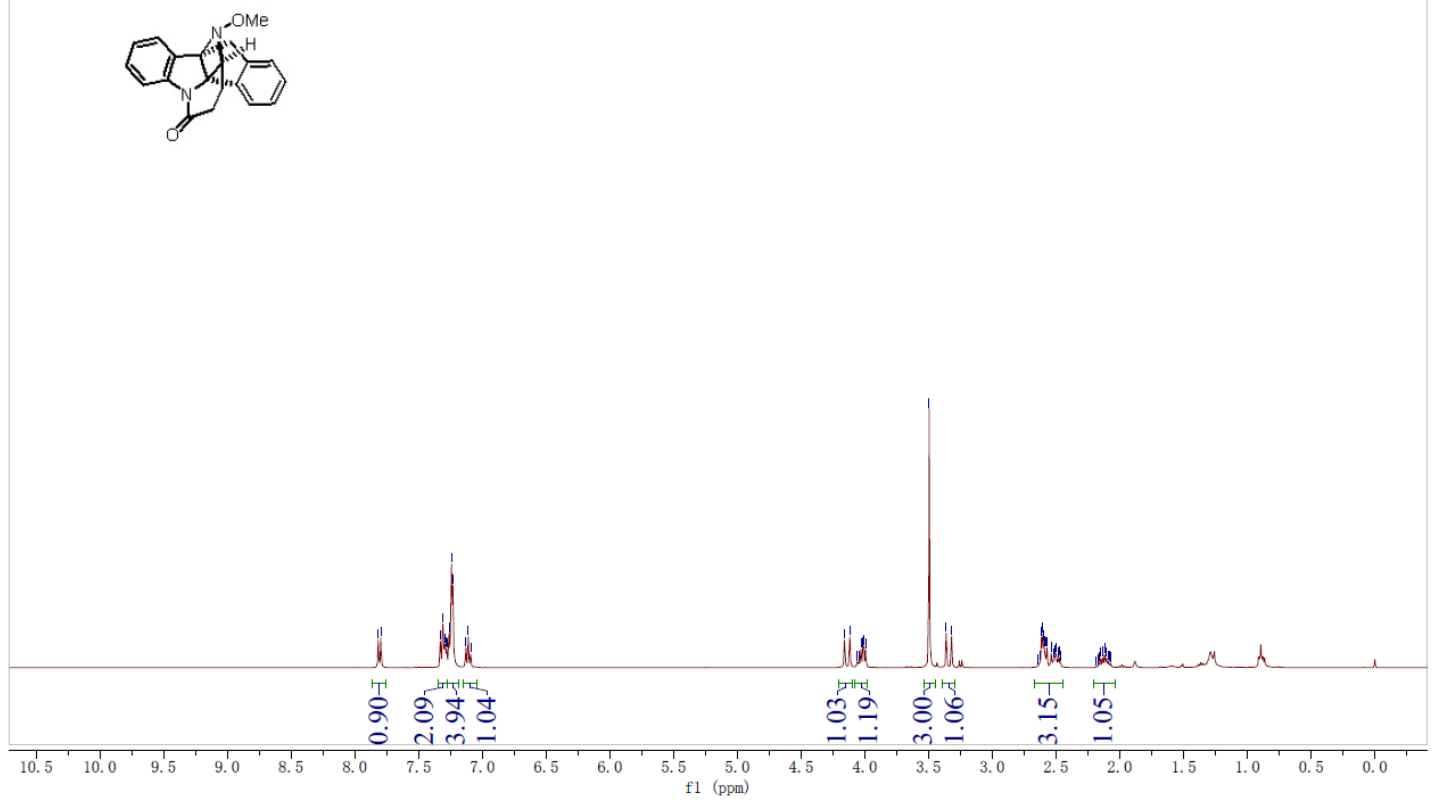

${ }^{13} \mathrm{C}$ NMR Spectrum of $\mathbf{4 0}{ }^{\prime}$

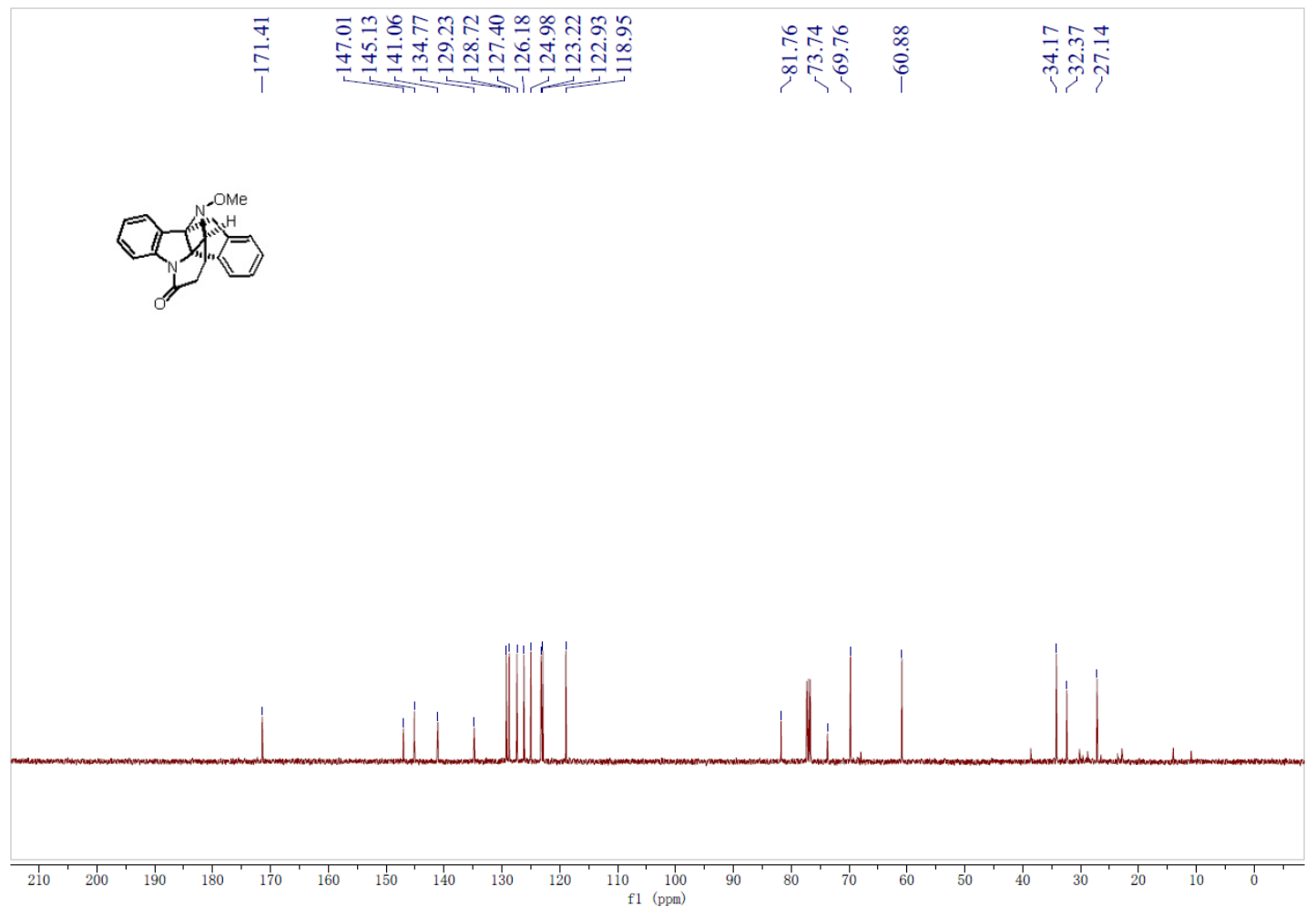


${ }^{1} \mathrm{H}$ NMR Spectrum of $\mathbf{5}$

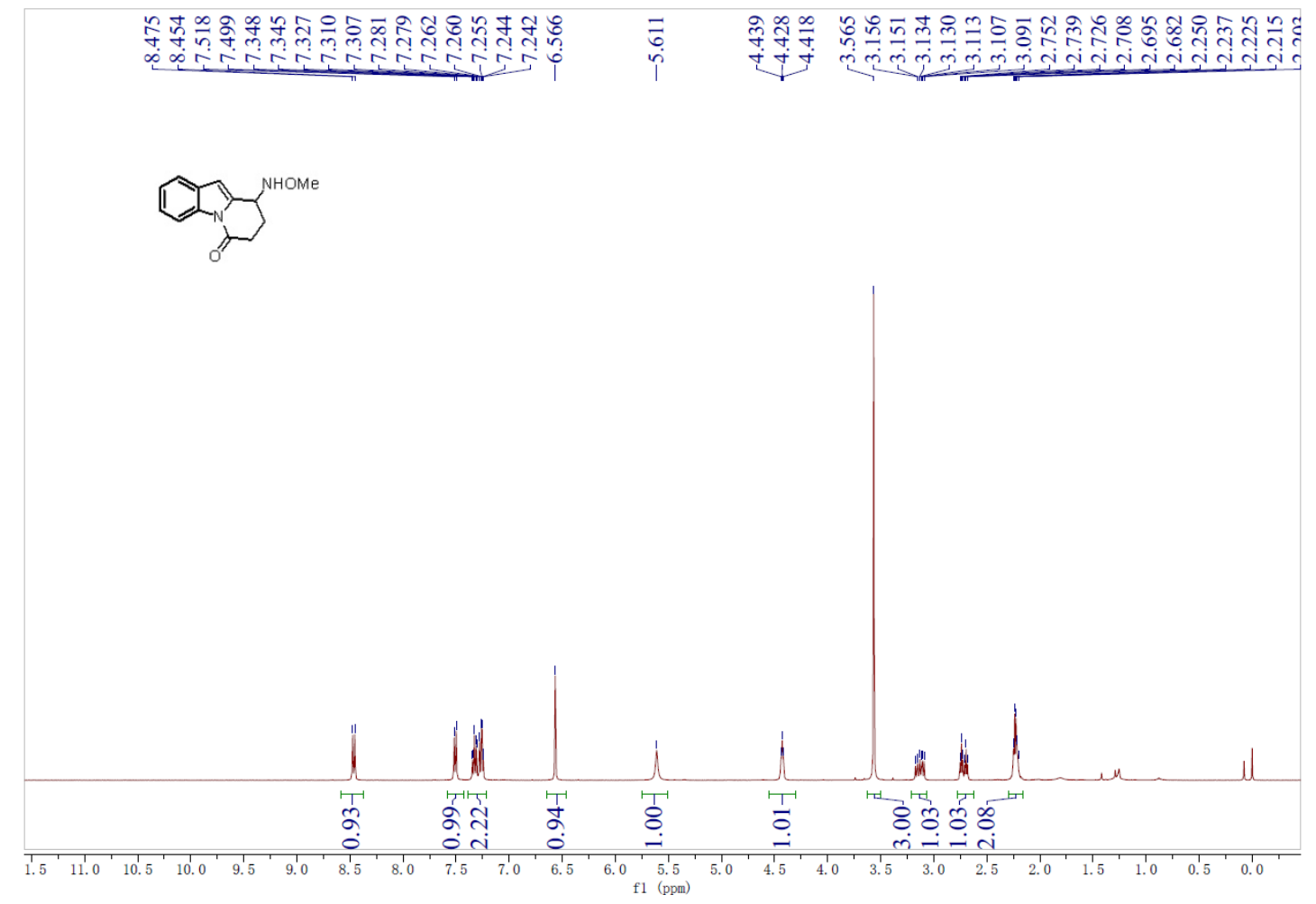

${ }^{13} \mathrm{C}$ NMR Spectrum of $\mathbf{5}$

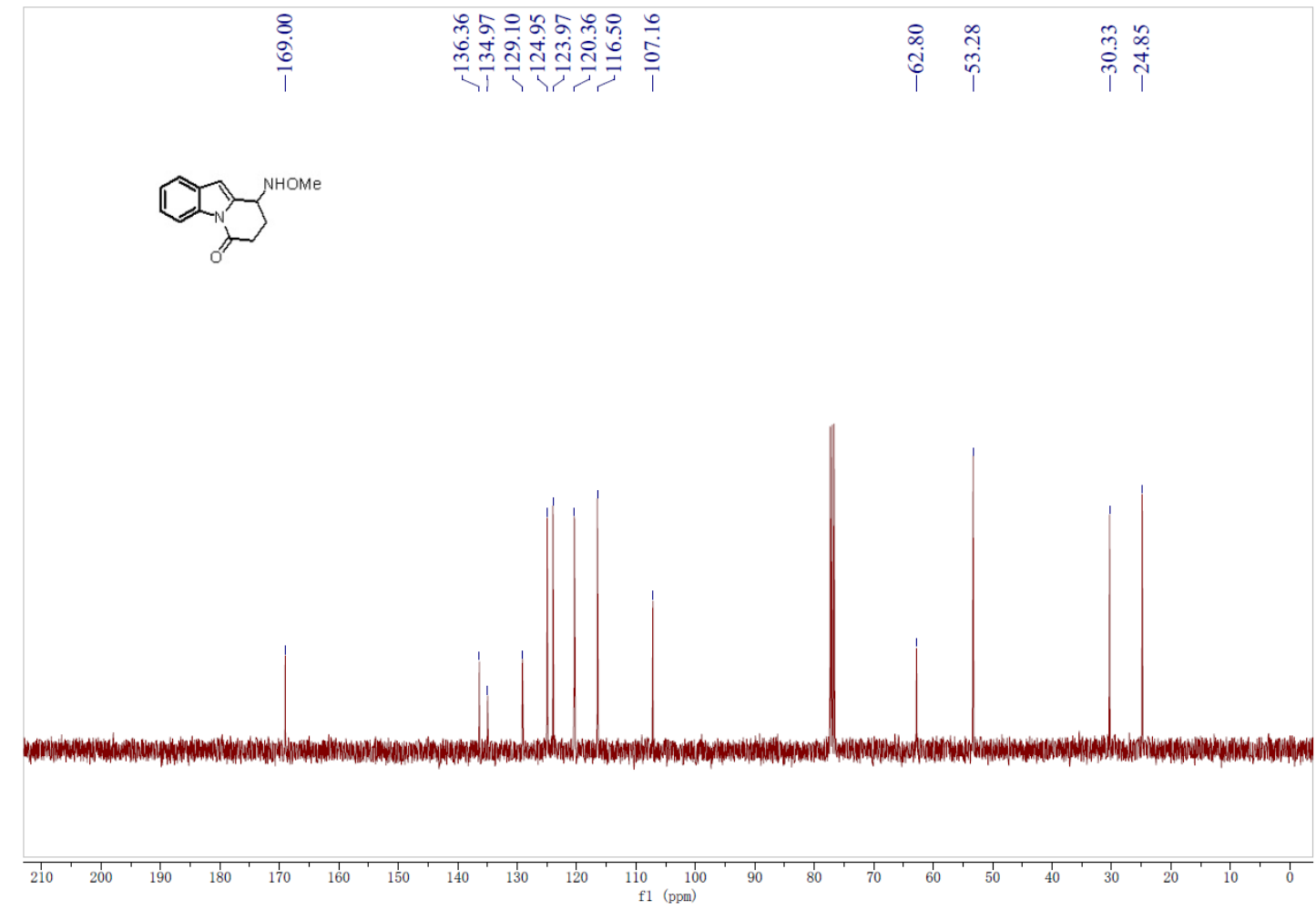


${ }^{1} \mathrm{H}$ NMR Spectrum of 6

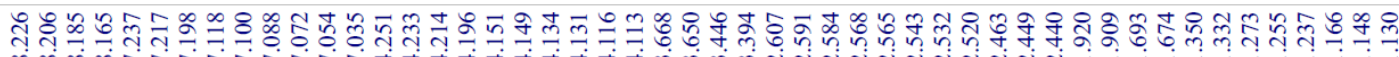

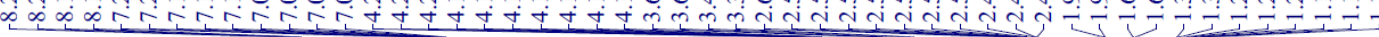
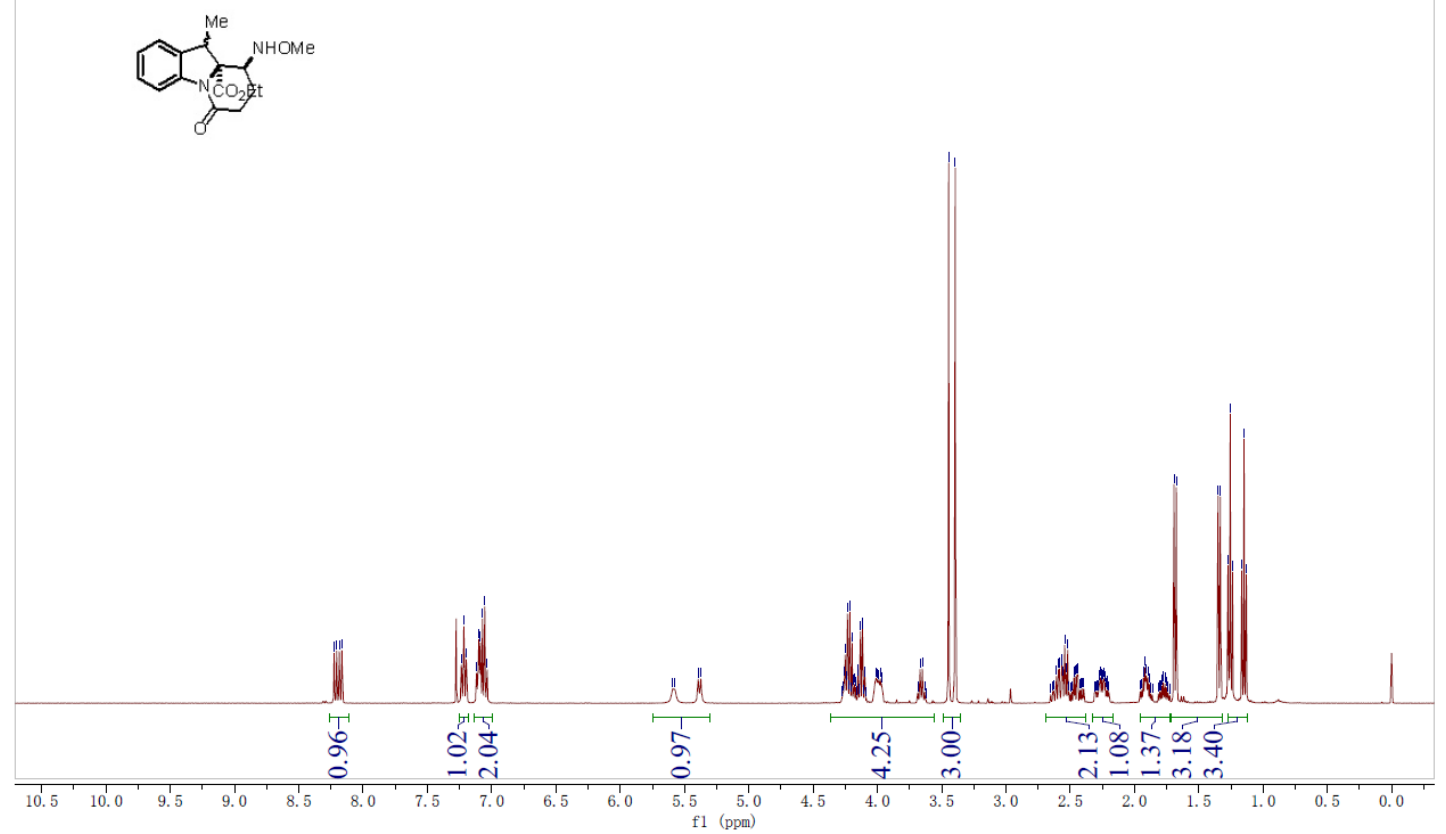

${ }^{13} \mathrm{C}$ NMR Spectrum of 6

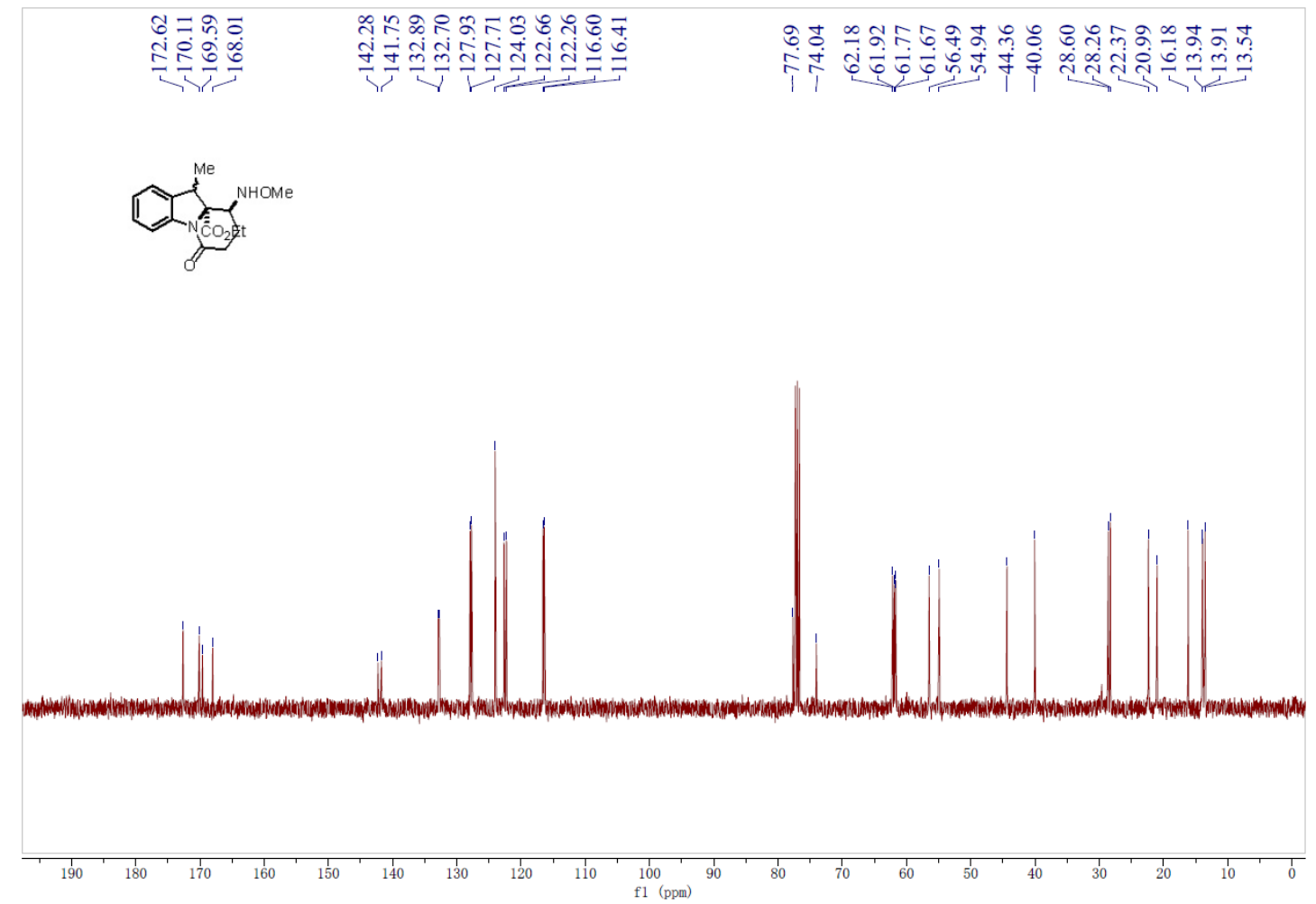


${ }^{1} \mathrm{H}$ NMR Spectrum of 7

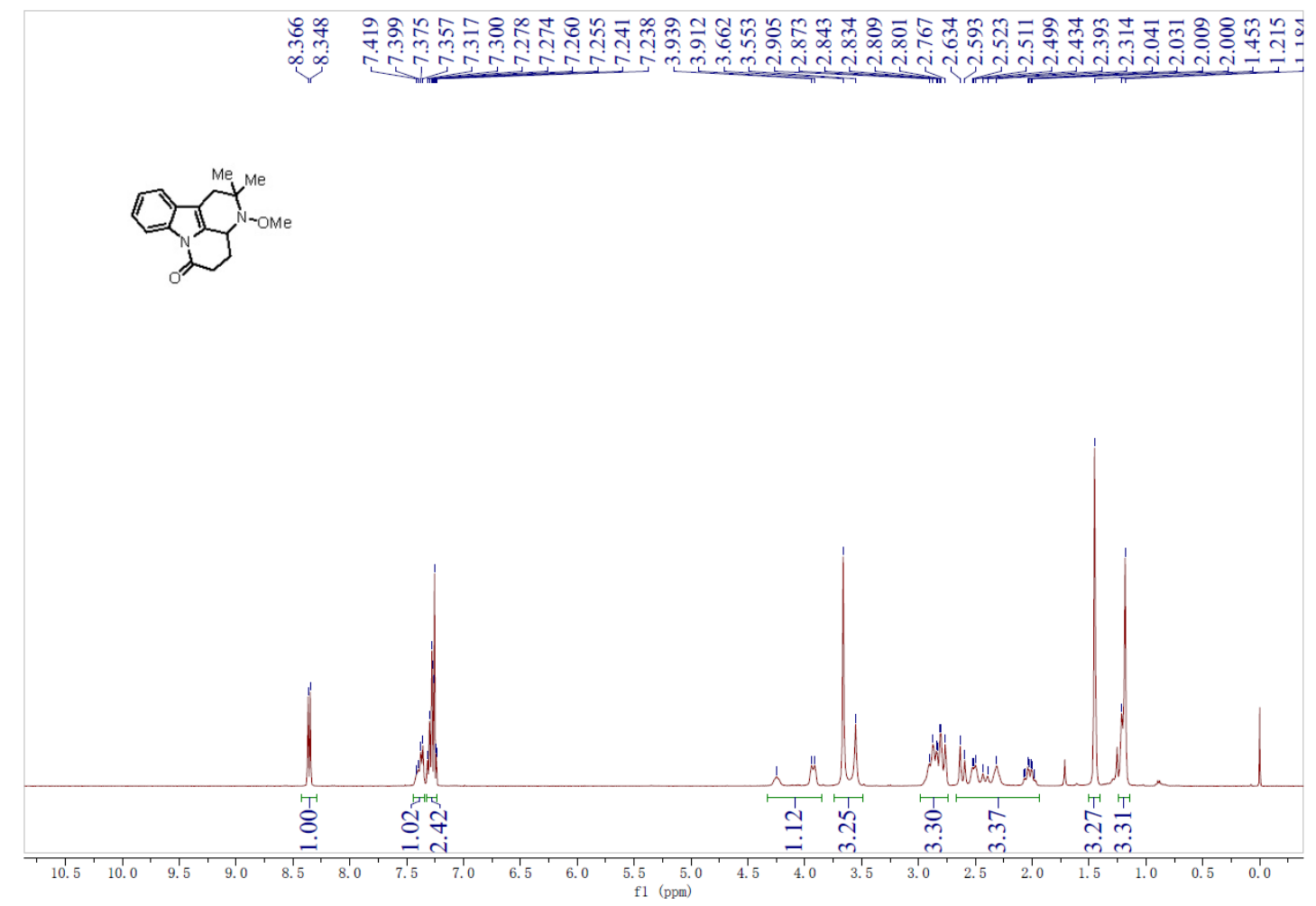

${ }^{13} \mathrm{C}$ NMR Spectrum of 7

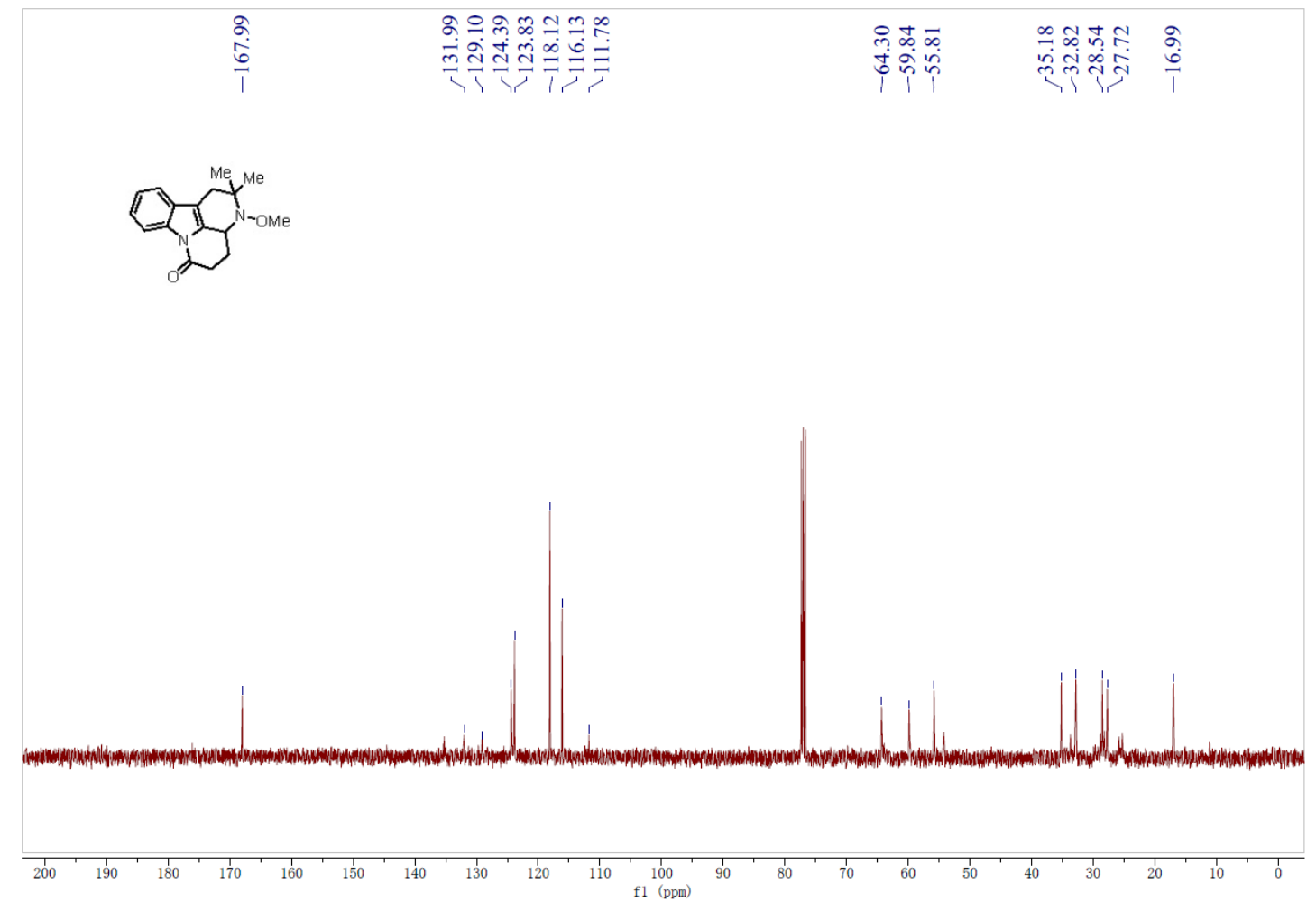

